Fall 1966

\title{
1966 Miracle Yearbook
}

\section{Cedarville College}

Follow this and additional works at: https://digitalcommons.cedarville.edu/yearbooks

Part of the Higher Education Commons, Organizational Communication Commons, and the Public Relations and Advertising Commons

\section{Recommended Citation}

Cedarville College, "1966 Miracle Yearbook" (1966). Yearbooks. 47.

https://digitalcommons.cedarville.edu/yearbooks/47

This Book is brought to you for free and open access by DigitalCommons@Cedarville, a service of the Centennial Library. It has been accepted for inclusion in Yearbooks by an authorized administrator of DigitalCommons@Cedarville. For more information, please contact digitalcommons@cedarville.edu. 

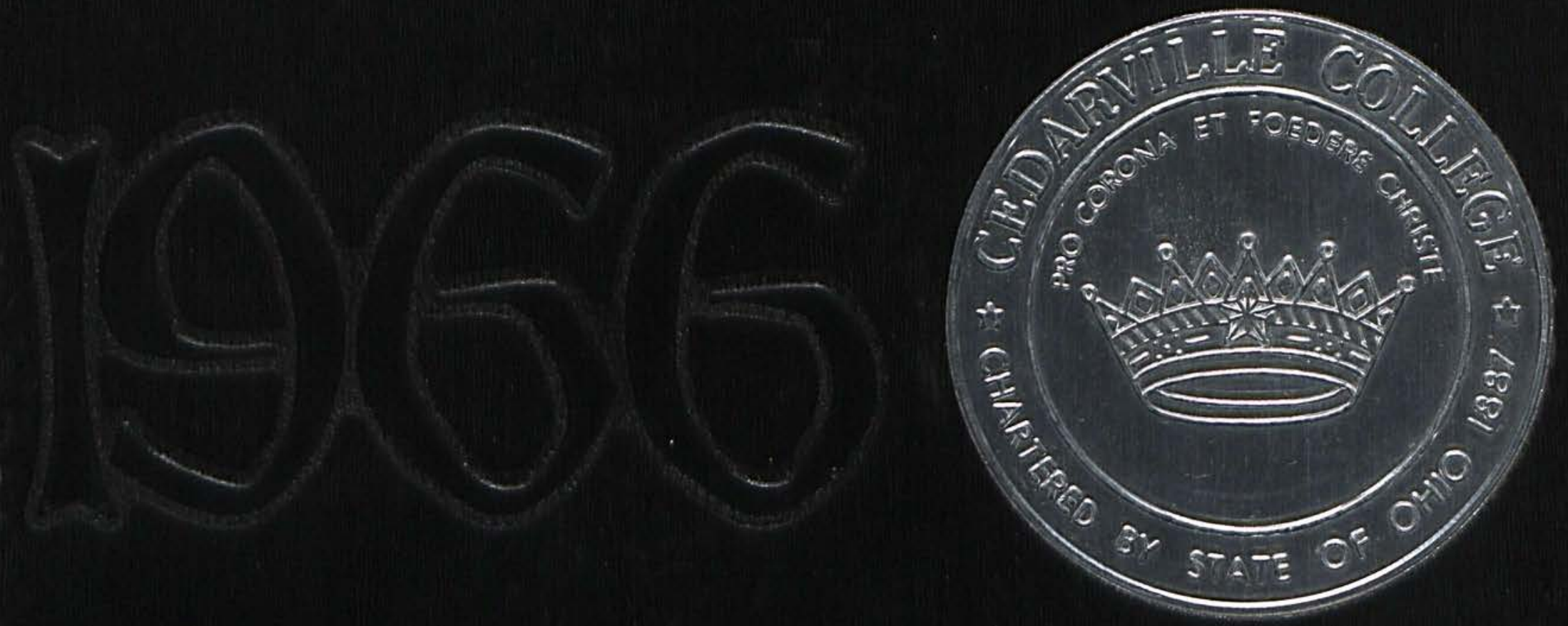
Do Not Remove

Public Relations 

stu'dent (stū'dĕnt; 128; 242), n. [L. studens, -entis, nres. part. of studere to study. See STUDY, $n$.$] 1. A person en-$ gaged in study; one devoted to learning; a learner; a scholar; esp., one who attends a school, or who seeks knowledse from teachers or books; as, the students of an academy or a university; a medical student; a hard student.

2. At Christ Church, Oxford, a member of the foundation. 3. One who studies or examines in any manner; an attentive and systematic observer; as, a student of life. Syn. - See DISCIPLE. 


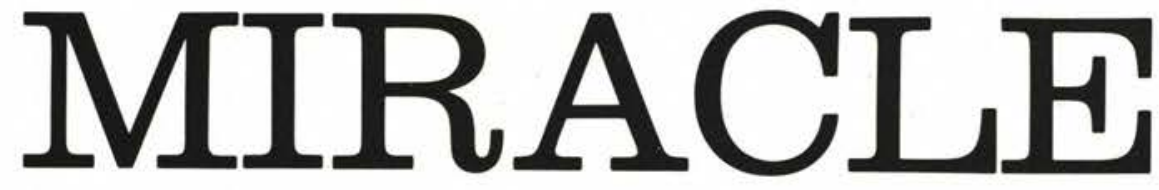

D. Gregor Mehaffey ..... Editor David Trunnell ..... Associate Editor Edward E. Spencer . . . . . . Advisor

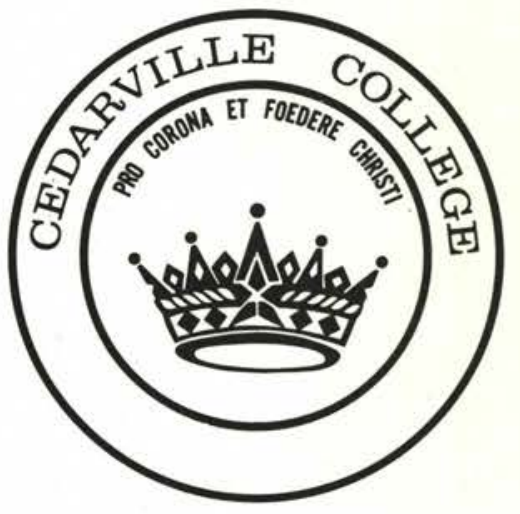




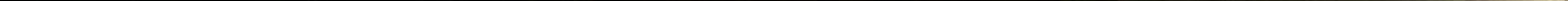




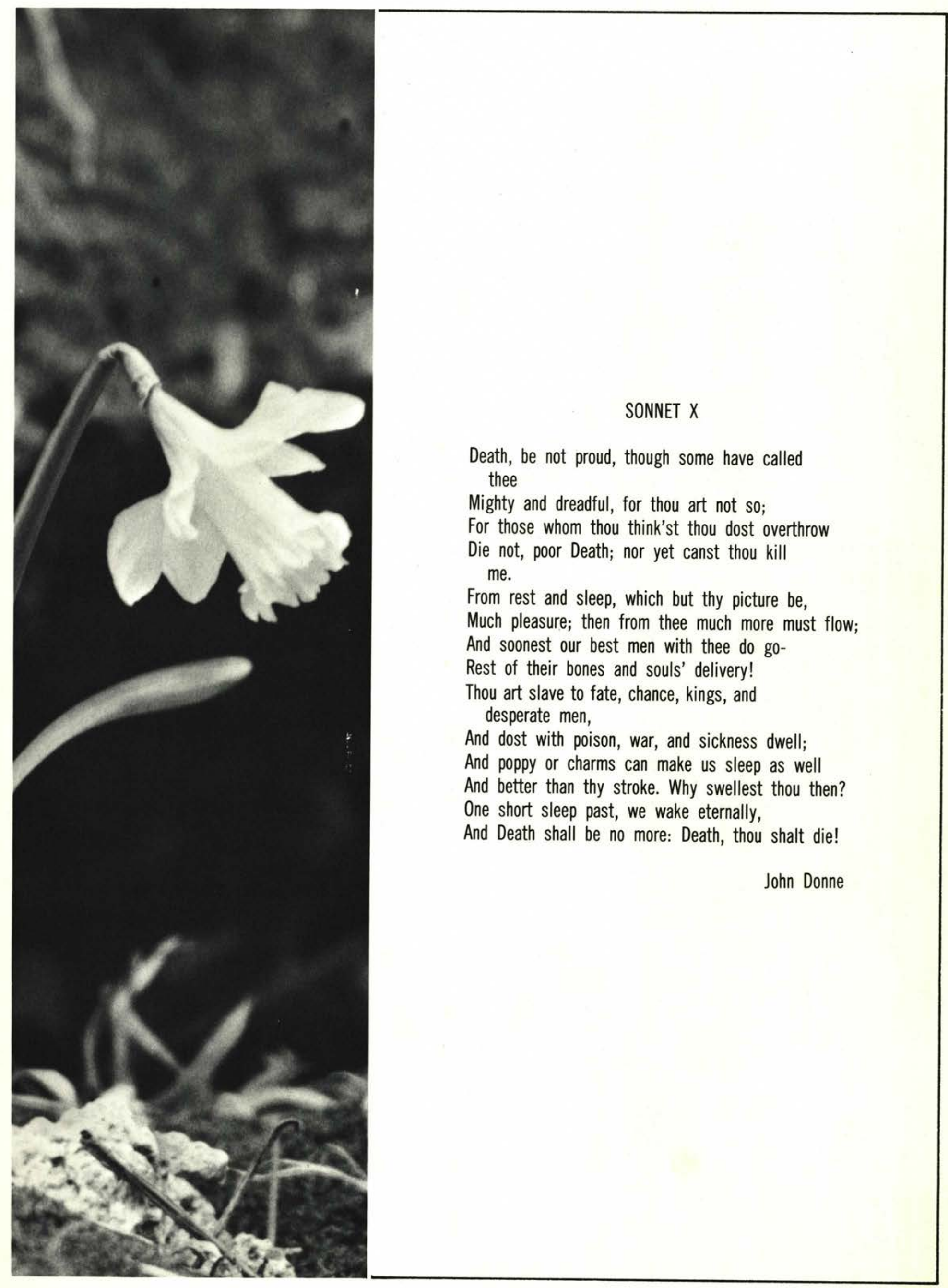





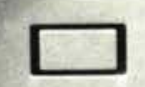

$5=-5$

-

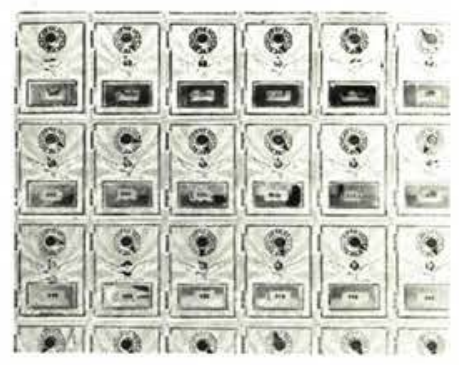

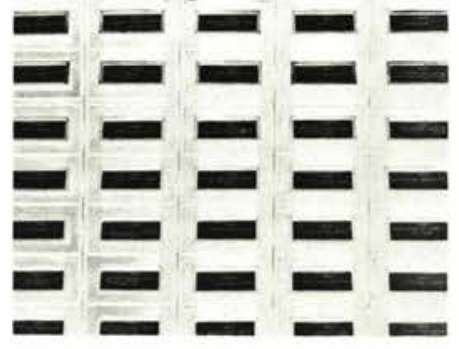
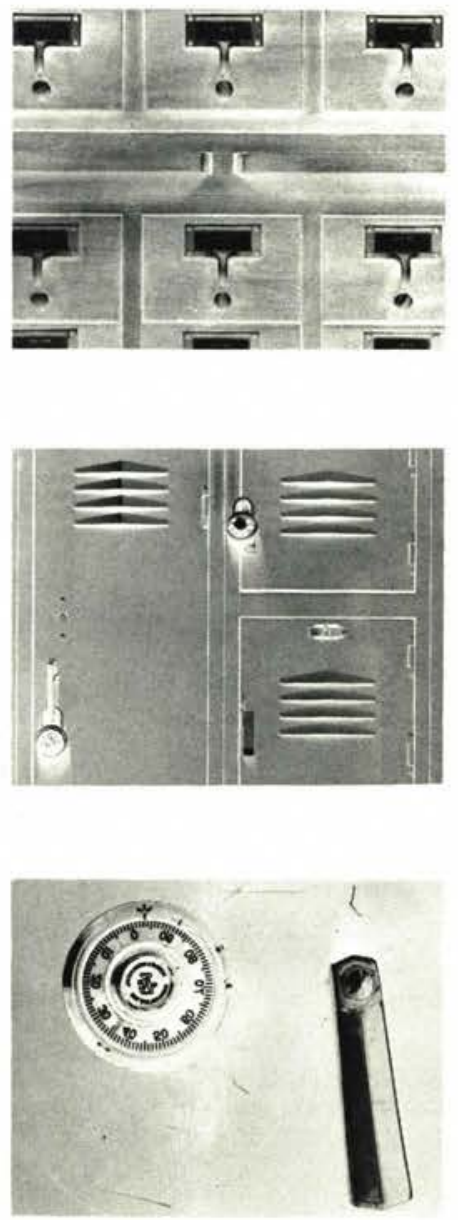

Administration, Faculty and Staff

\section{Campus Candids}

\author{
Students
}

\section{Sports}

\section{Seniors}


Every year thousands of young people stand at the threshold of service upon the completion of the graduation activities. At such a time one is reminded of the words of Christ to the church at Philadelphia, "I have set before thee an open door and no man can shut it" (Revelation 3:8). Phillips translates it, "I have given you a door flung wide open." Every senior leaving Cedarville College on graduation day will face a wide open door for service.

Only four brief years ago this same class entered the doors of Cedarville College. During those years friendships have been formed; knowledge has been obtained; vision has been created; and decisions have been made. Now, these doors close, and new ones open.

Commencement does not imply, however, that all academic pursuits are at an end; but rather it should signify a continuation of study and learning. Every qualified senior will be investigating the possibility of attending graduate school. All Cedarville graduates will continue to make companions of books and a habit of study; otherwise, the full value of a college education will never be fully realized.

Every member of the Cedarville College family prays that the seniors of the class of 1966 will first of all discover the door which the Lord opens for them, and then with all confidence in God's enablement, enter it with genuine sincerity and enthusiasm.

The symbol of the open door suggests opportunity. Paul said, "A door was opened to me of the Lord" (II Corinthians 2:12). He is saying that at Troas he had a God-given opportunity. No two of us ever have exactly the same opportunity, but as we seek the will of God we shall have one which should be the most challenging of anything in our lives. Therefore, it is imperative that we give ourselves to the Lord and accomplish the utmost for Him as we serve.

With every opportunity there comes an inescapable responsibility. Someone has said that "Every right implies a responsibility; every opportunity an obligation; every possession a duty." Simply to face an open door or recognize the challenge before us is not enough. We must walk through it if it is to have its proper use, and we are to receive the benefits of the privileges before us. At the end of life's journey we will give an account of how faithfully we have walked the pathway opened to us by God. Here we must remember that obedience to God is success in His sight even though the world may measure it by some other guideline.

There are also responsibilities of gratitude which every senior must consider. To parents who have sacrificed to make this education possible, and to faculty members who have served because of interest in the student and his future, an expression of deep appreciation is due. It is hoped that through the years every graduate will show a loyal attitude toward the college by giving financially so that others may share the privileges they have enjoyed. A favorable word now and then in behalf of the Alma Mater will be encouraging to all who have labored in love for the education the seniors have completed. These are responsibilities which come as a result of the opportunities found in college days.

Christ may come today; but if He plans it otherwise, may His presence and abundant blessing be with all of you as you serve Him in the years that are ahead. 


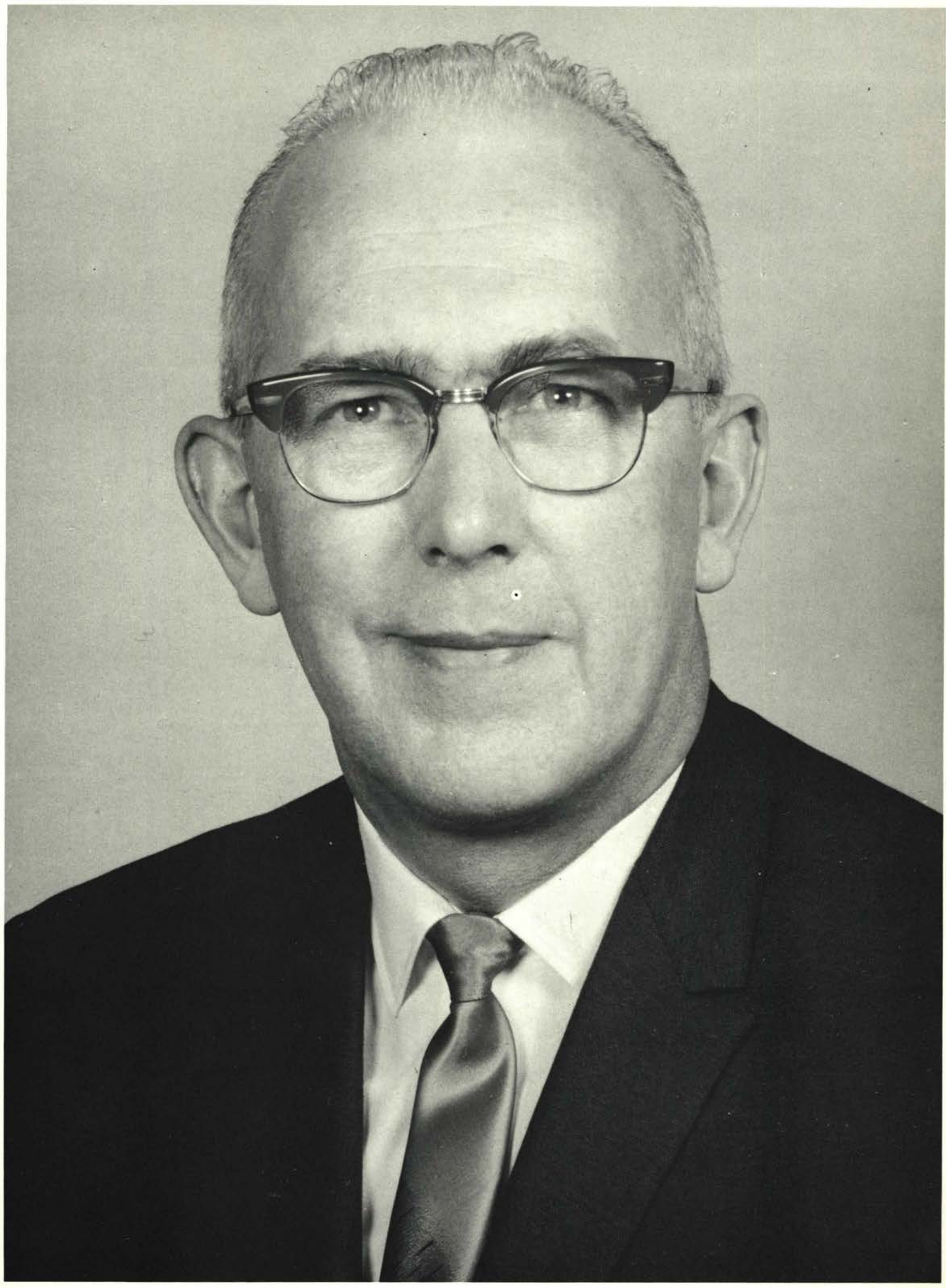




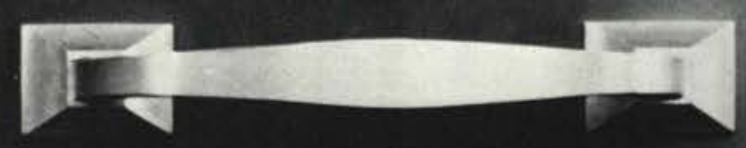

0

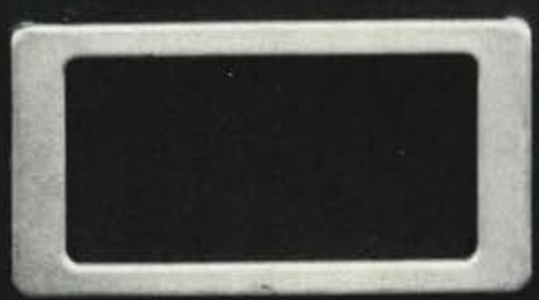




\section{Administration, Faculty, and Staff}


TRUSTEES

Dr. George S. Milner, Chairman; Rev. William Brock, Vice-Chairman; Mr. George L. Boyd, Treasurer; Rev. Thomas Younger, Secretary.

Mr. Charles Barth

Mr. Rudy Bedford

Rev. William Brown

Dr. Jack Cline

Mr. John A. Draxler

Mr. Arthur Dyke

Mr. Vincent Gregg

Mr. Roy Guenin

Mr. Hugh T. Hall

Dr. James T. Jeremiah
Mr. J. Dale Murphy Mr. George O'Bryan Rev. Irwin Olson Mr. William B. Patterson Mr. James Richardson Rev. Gerald V. Smelser Rev. Robert Sumner Rev. Earl Umbaugh Rev. Earl Willets Rev. Donald Woodby

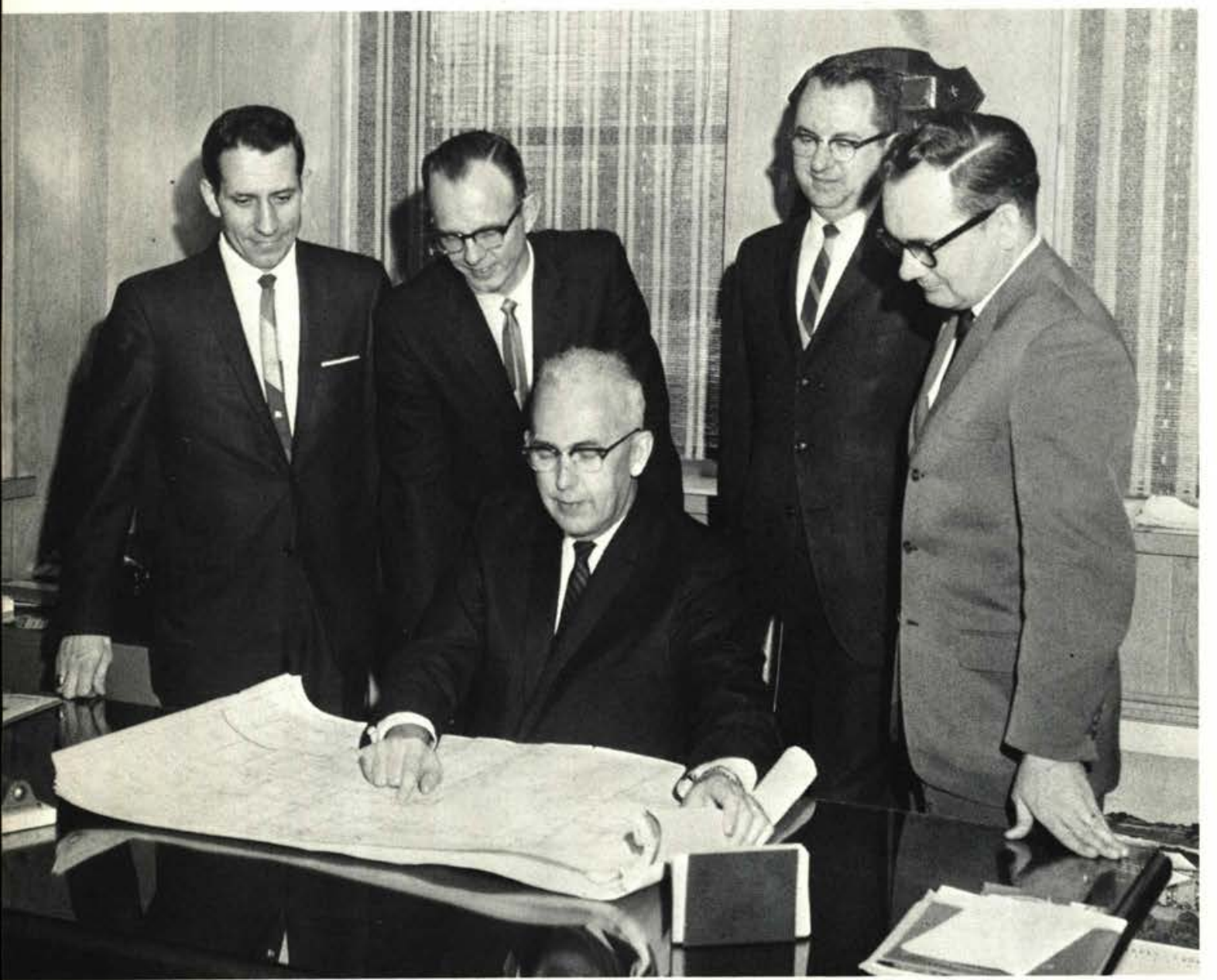

Seated: James T. Jeremiah, President; Standing, Left to Right: Lee Turner, Director of Development; Clifford Johnson, Academic Dean; Richard McIntosh, Dean of Students; Kenneth St. Clair, Business Manager, ADMINISTRATIVE COMMITTEE
George Boyd C.P.A.; Treasurer
Lee Turner Director of Development

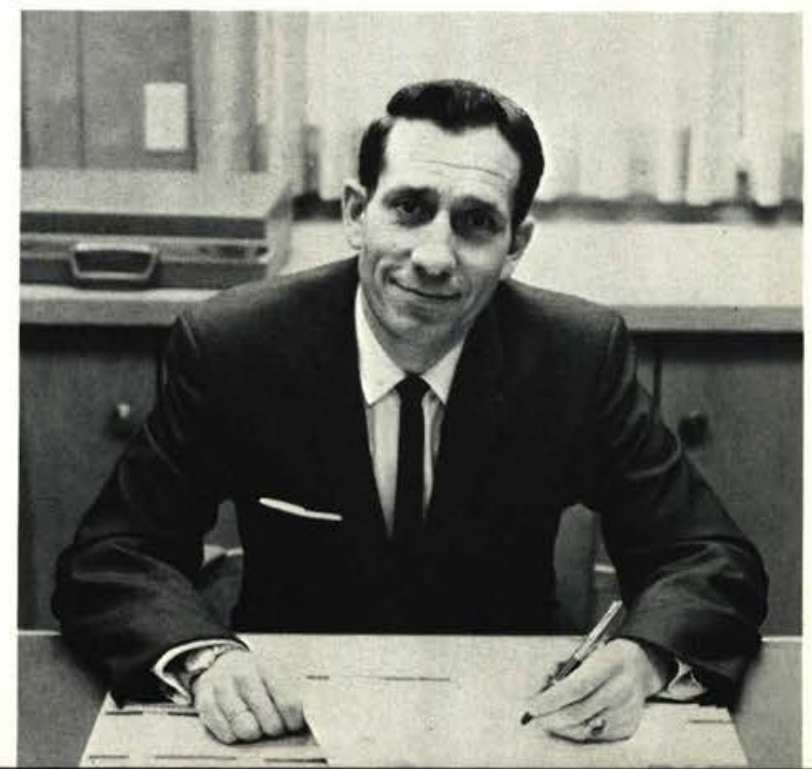



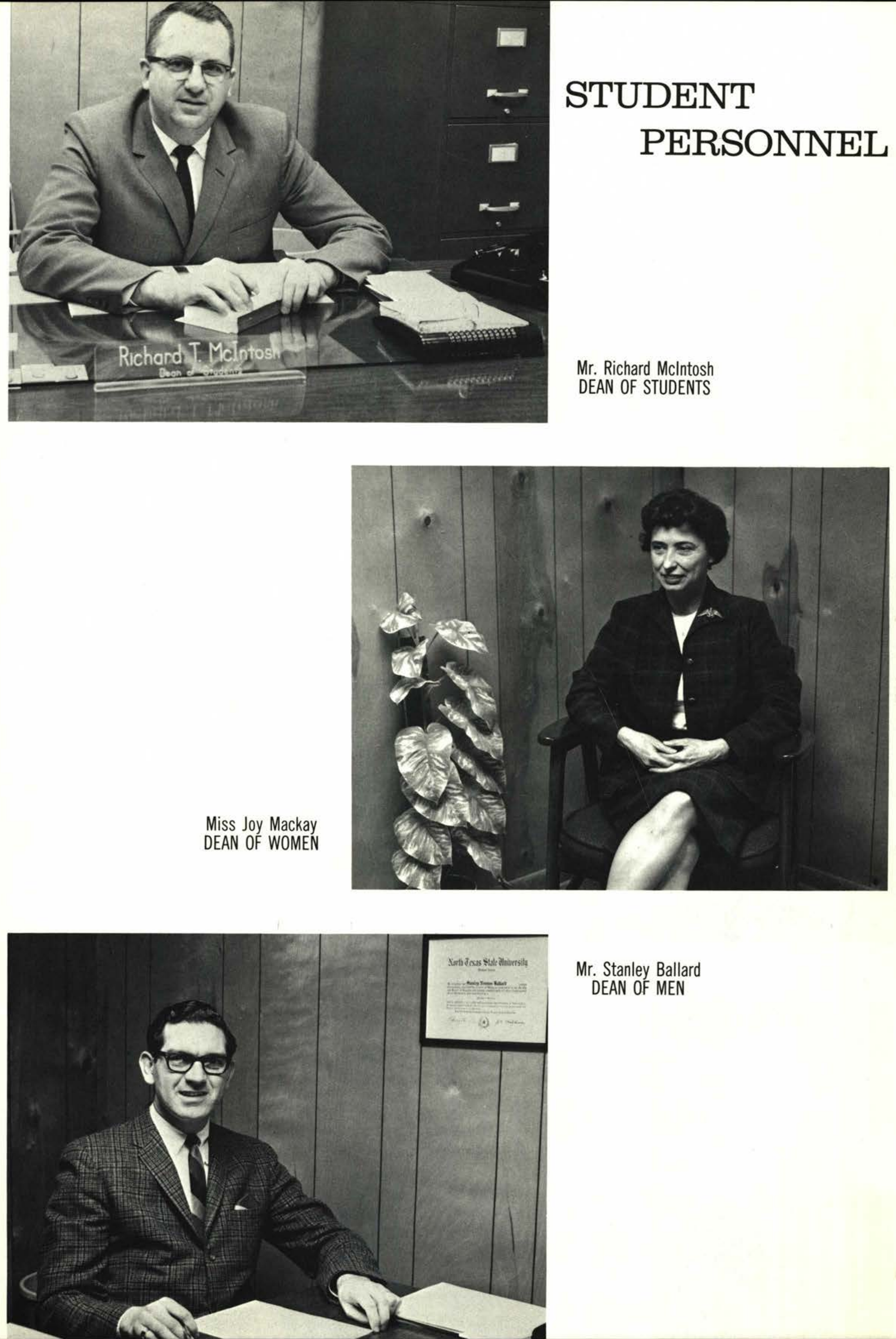

Mr. Stanley Ballard

DEAN OF MEN 


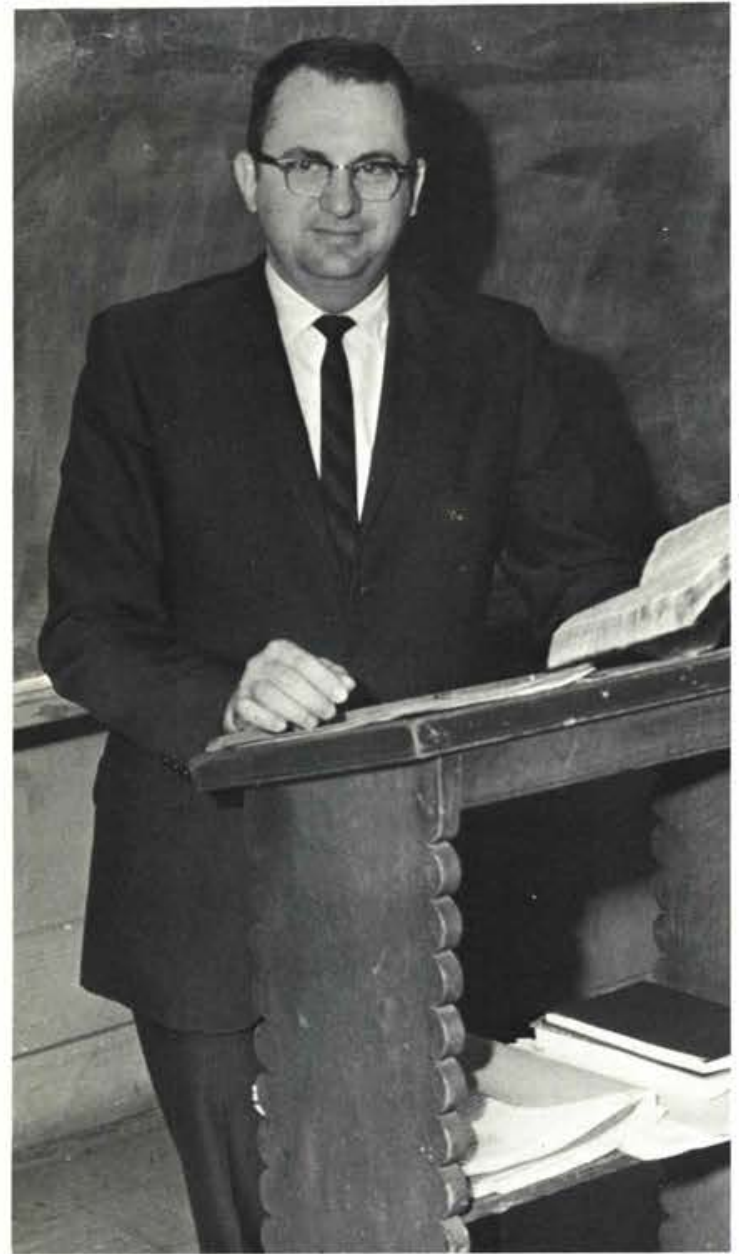

\section{BIBLICAL EDUCATION}

Cedarville College has for its objective the development of the total personality of its students. The Bible Division devotes particular attention to the spiritual development of the individual student. The most effective witness for Christ is one with a well-developed body, an enlightened and disciplined mind, and a spiritual life made vigorous by the operation of the Holy Spirit through the application of God's Word.

To effect this latter objective, the Bible Division provides courses of instruction which are designed to acquaint the student with the facts of Biblical revelation, and to encourage the application of Biblical truth in practical Christian living and effective witness for Christ. Courses are provided which enable the student to study the New Testament in the original language in which it was written to become proficient in its use.

Mr. Richard Mclntosh

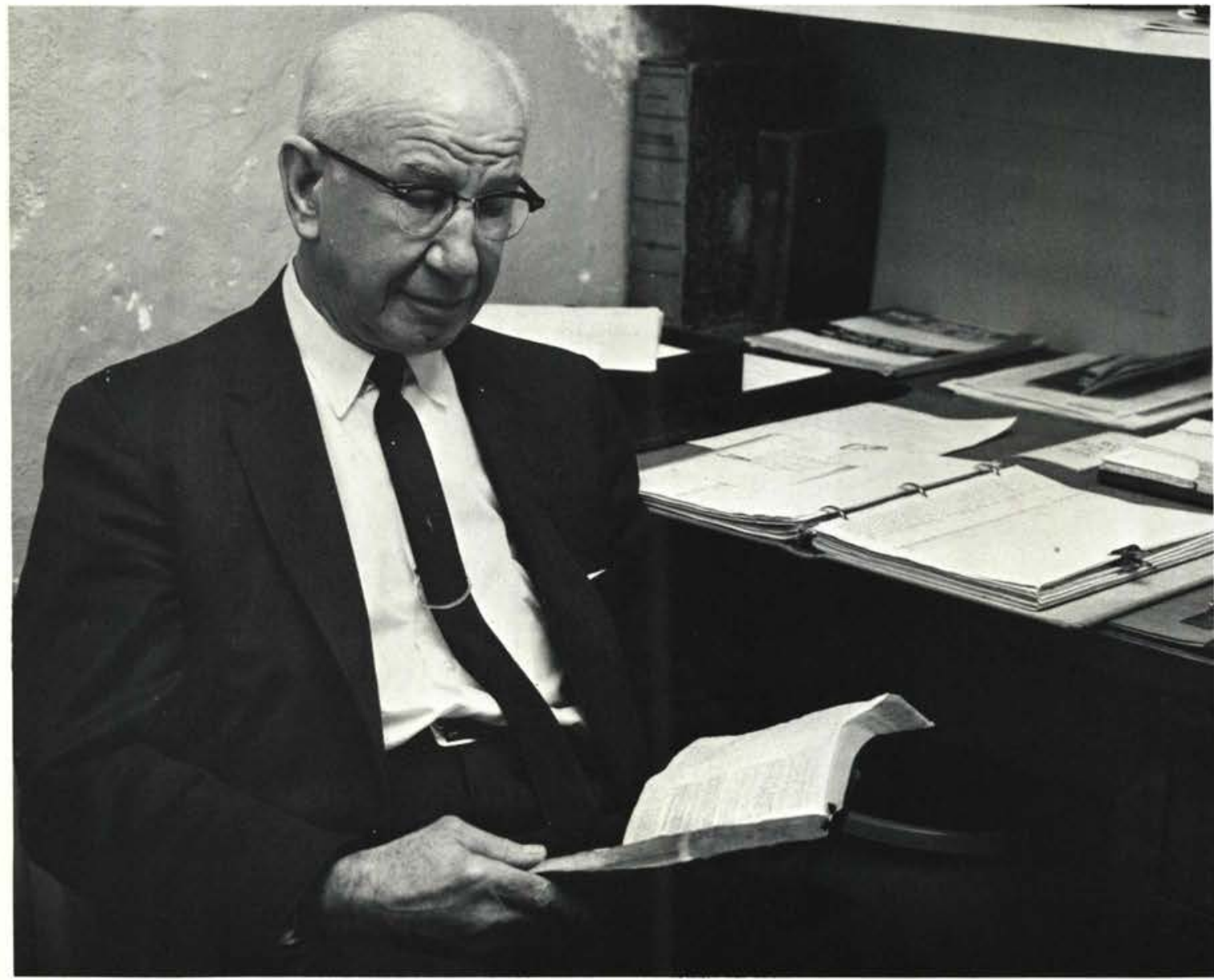

Dr.

Arthur

Williams 


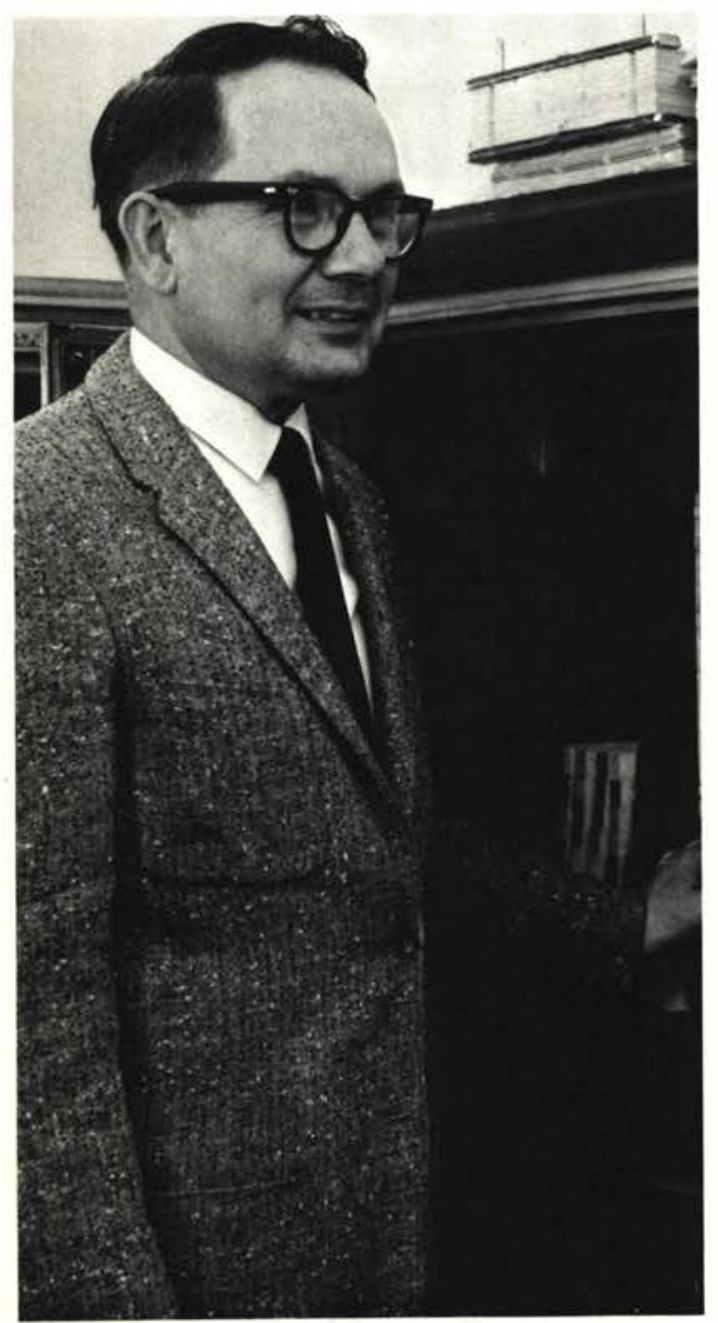

\section{BUSINESS ADMINISTRATION}

The man who serves best is the man who prepares best. These two ideas are literally true in the business world. Realizing this challenge, the Business Department offers excellent preparation to the student desiring a career in business. $\mathrm{He}$ is taught to serve in the business world and in the church as a trustworthy servant of God.

Mr. Kenneth St. Clair

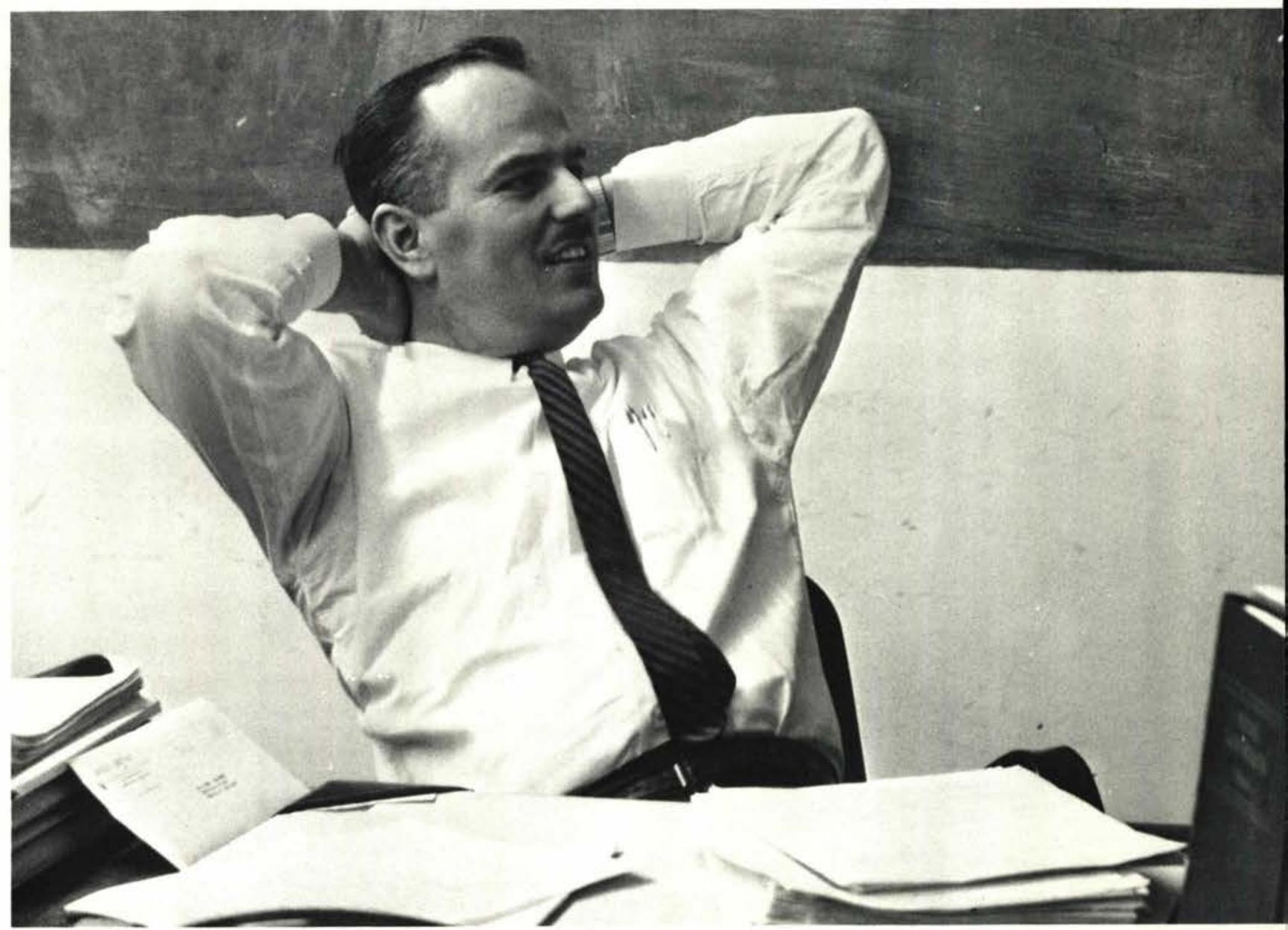




\section{PSYCHOLOGY AND EDUCATION}

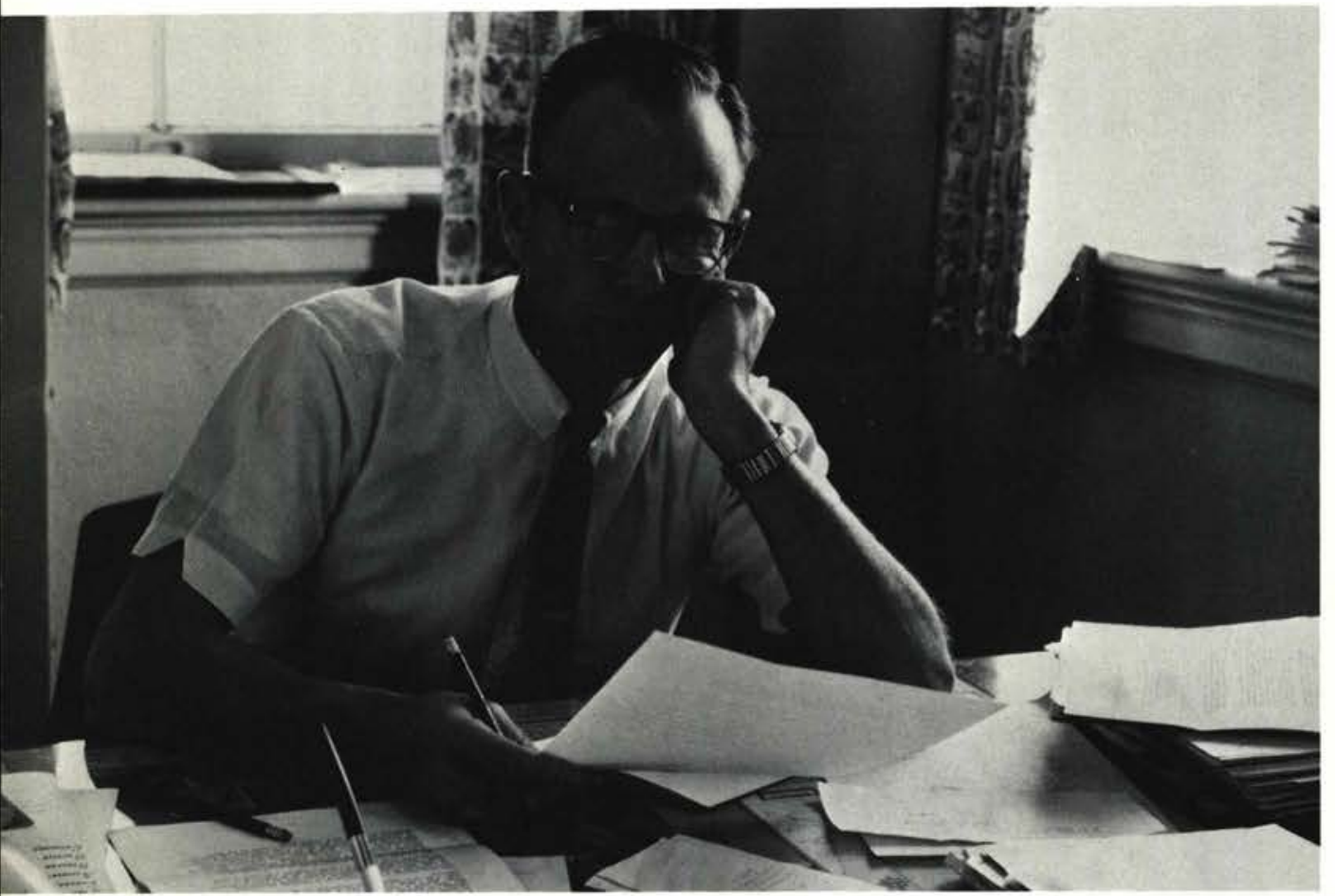

Dr. Clifford Johnson
The wonderful operations of learning, thinking and teaching have long interested man. The Division of Psychology and Education attempts to present instruction in these areas in order to help the student understand principles and concepts which operate in and through him.

Man's behavior has long intrigued scholars and students. Psychology is the discipline which has developed a body of knowledge seeking answers and providing explanations in this area.

Many present day psychologists take philosophical positions which tend to stress the rational acts of human beings as simply a process akin to that used by animals but perhaps a bit more highly developed. The Christian psychologist suggests that factors other than strictly humanistic elements also play an important part in the development and outworking of the human mind.

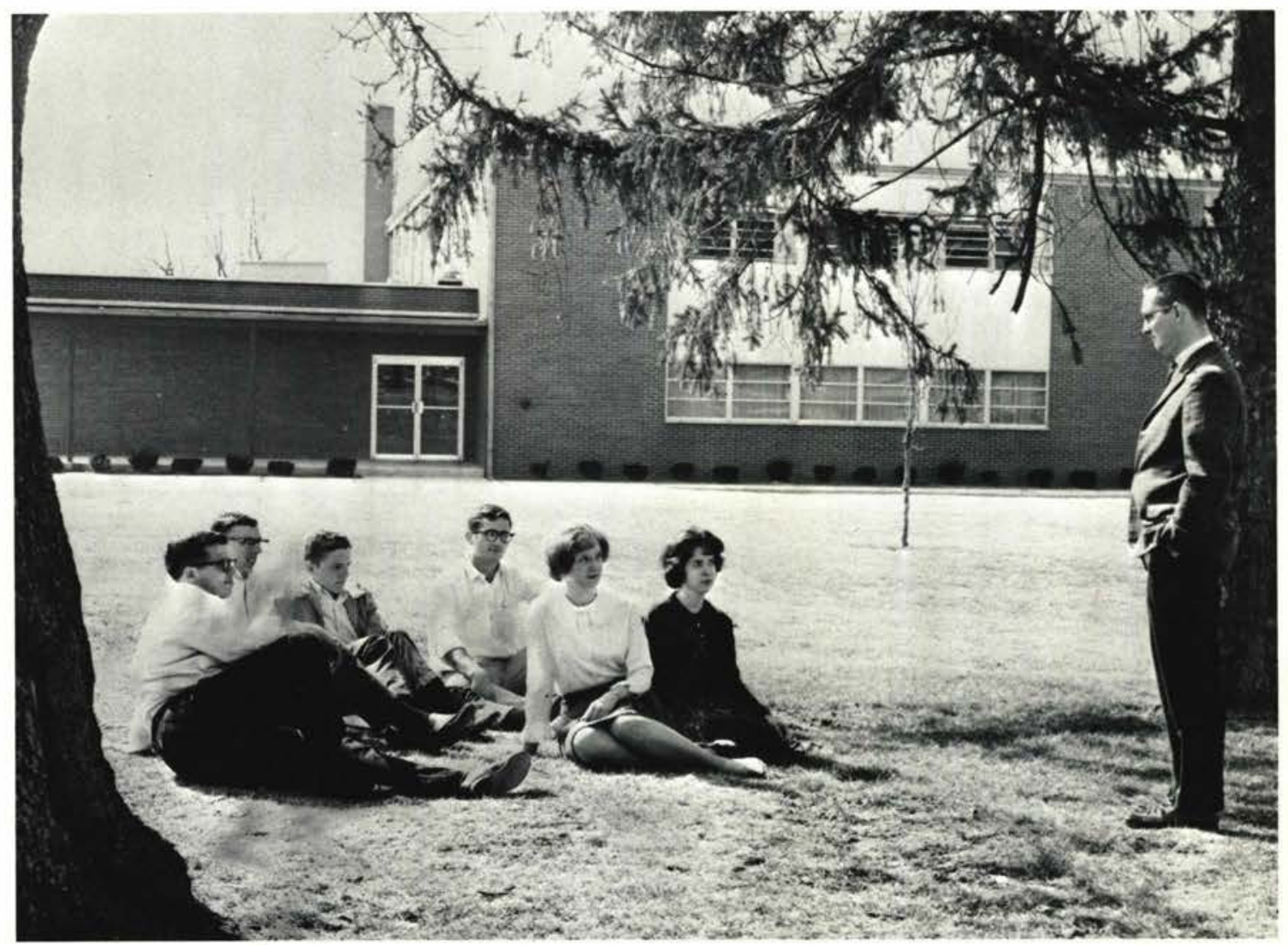




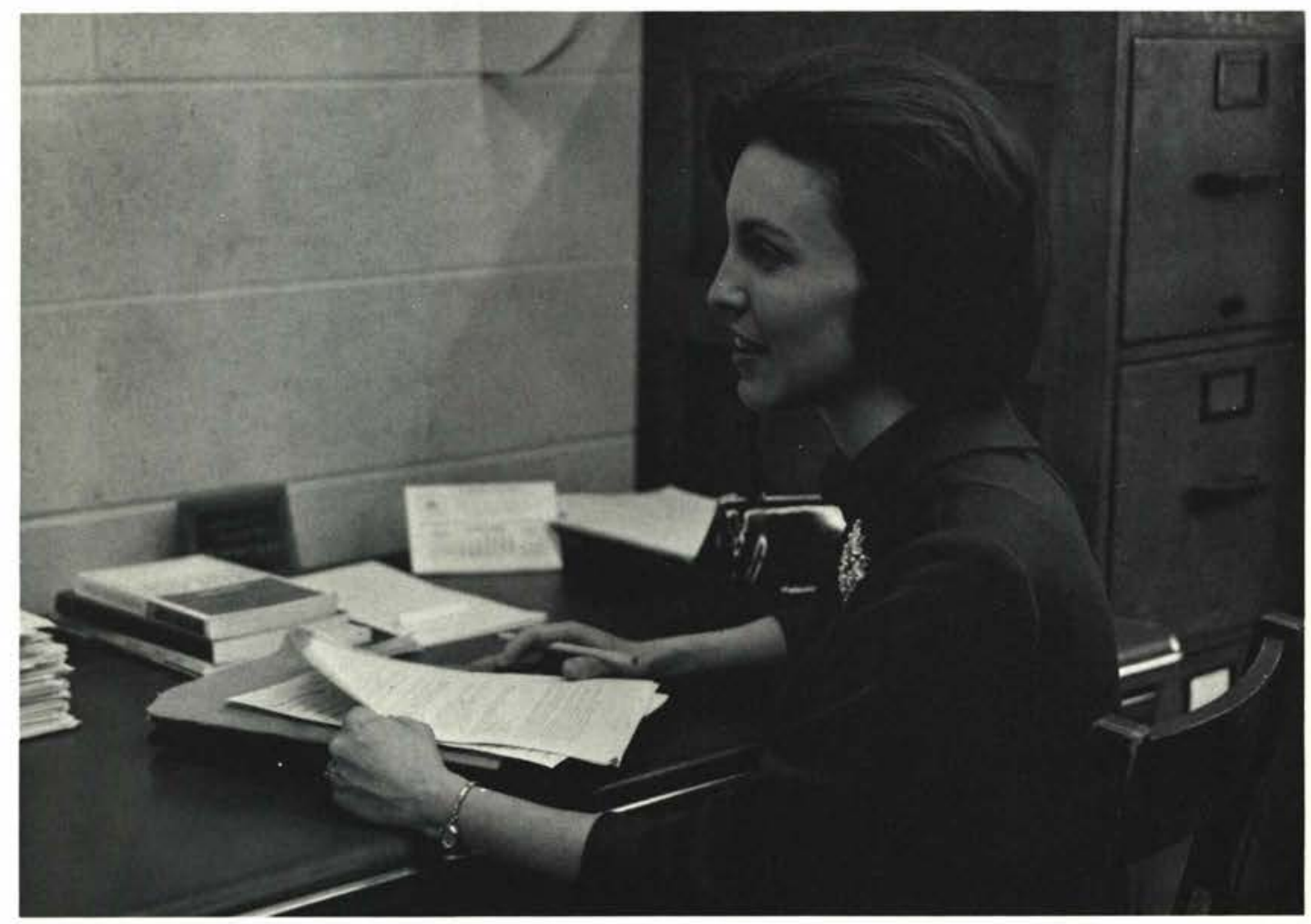

Mrs. Esther Entner

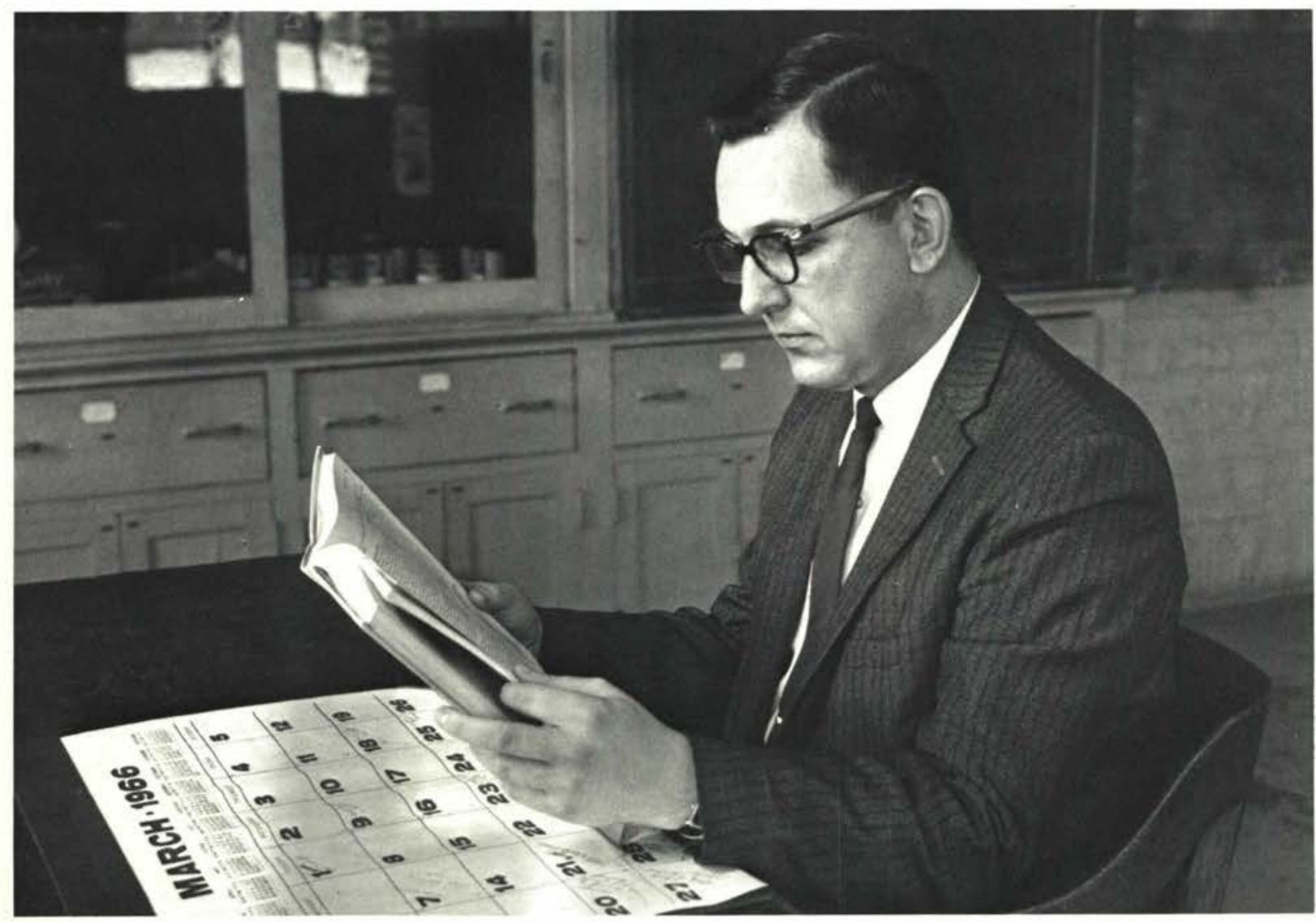

Mr. Max Dowell 


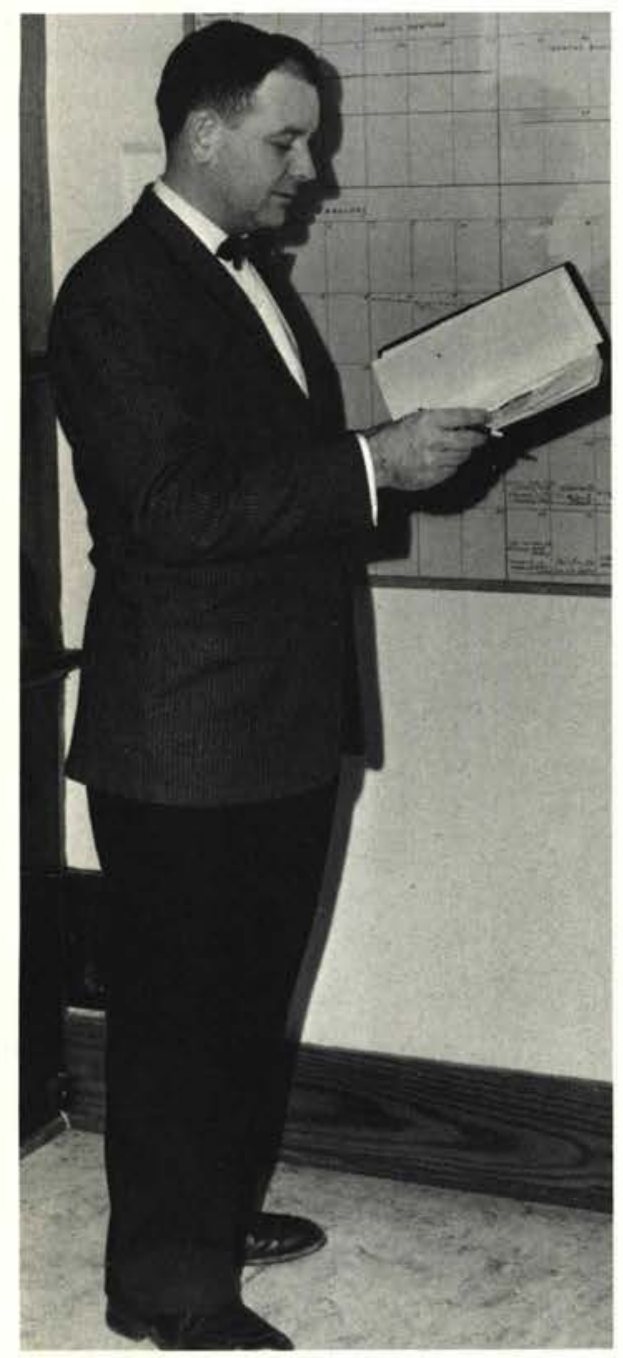

\section{FINE ARTS}

The feelings and emotions of a people are expressed in their art and music. The Division of Fine Arts encourages students to understand people as their values are expressed creatively in art and music.

The Art department, through its various courses of instruction, develops the technical skills of the student with artistic talent. The artistic styles of past eras are studied in art history. Independent study or applied art allows a student to pursue a particular artistic interest.

The music department encourages students to become producers, not just consumers of music.

Music majors learn how to write or arrange music for the church and school. Various styles of music literature are studied in the music history courses.

Mr. Warren Webber

Mr. David Matson

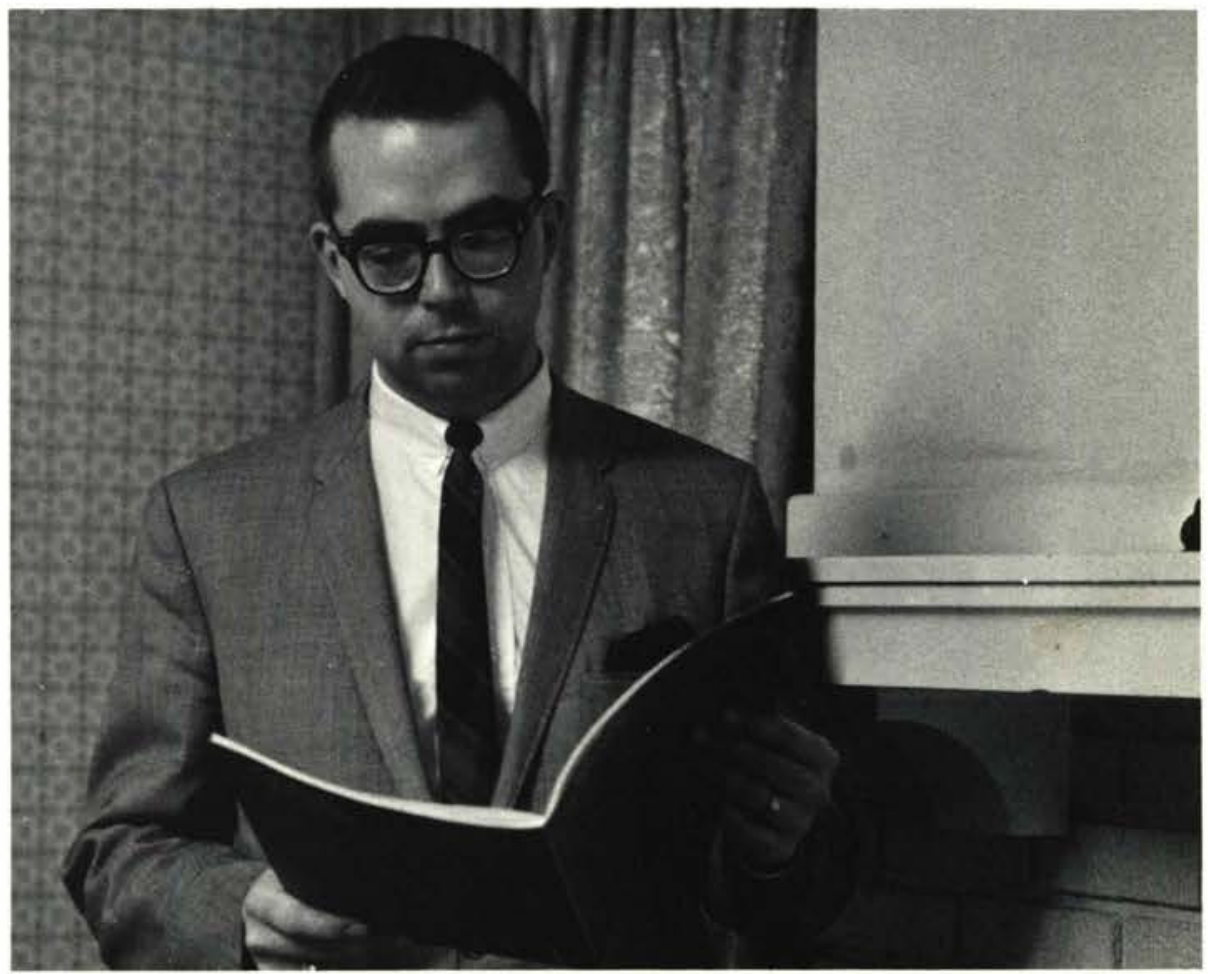




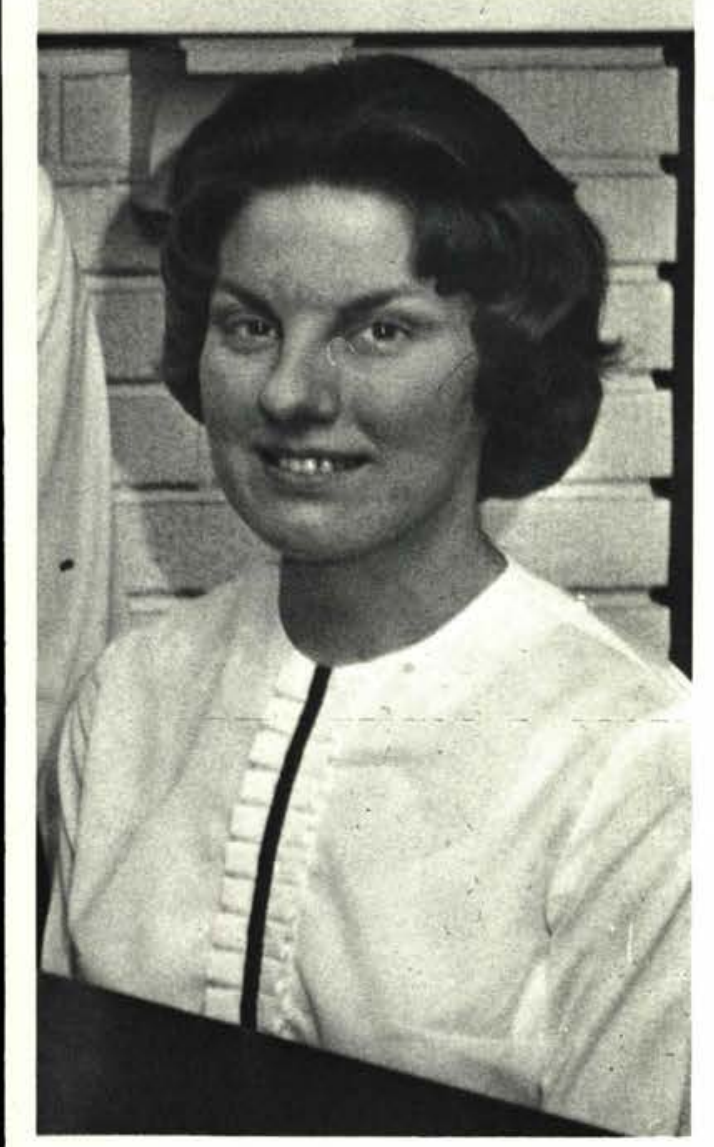

Miss Shirley Byrd

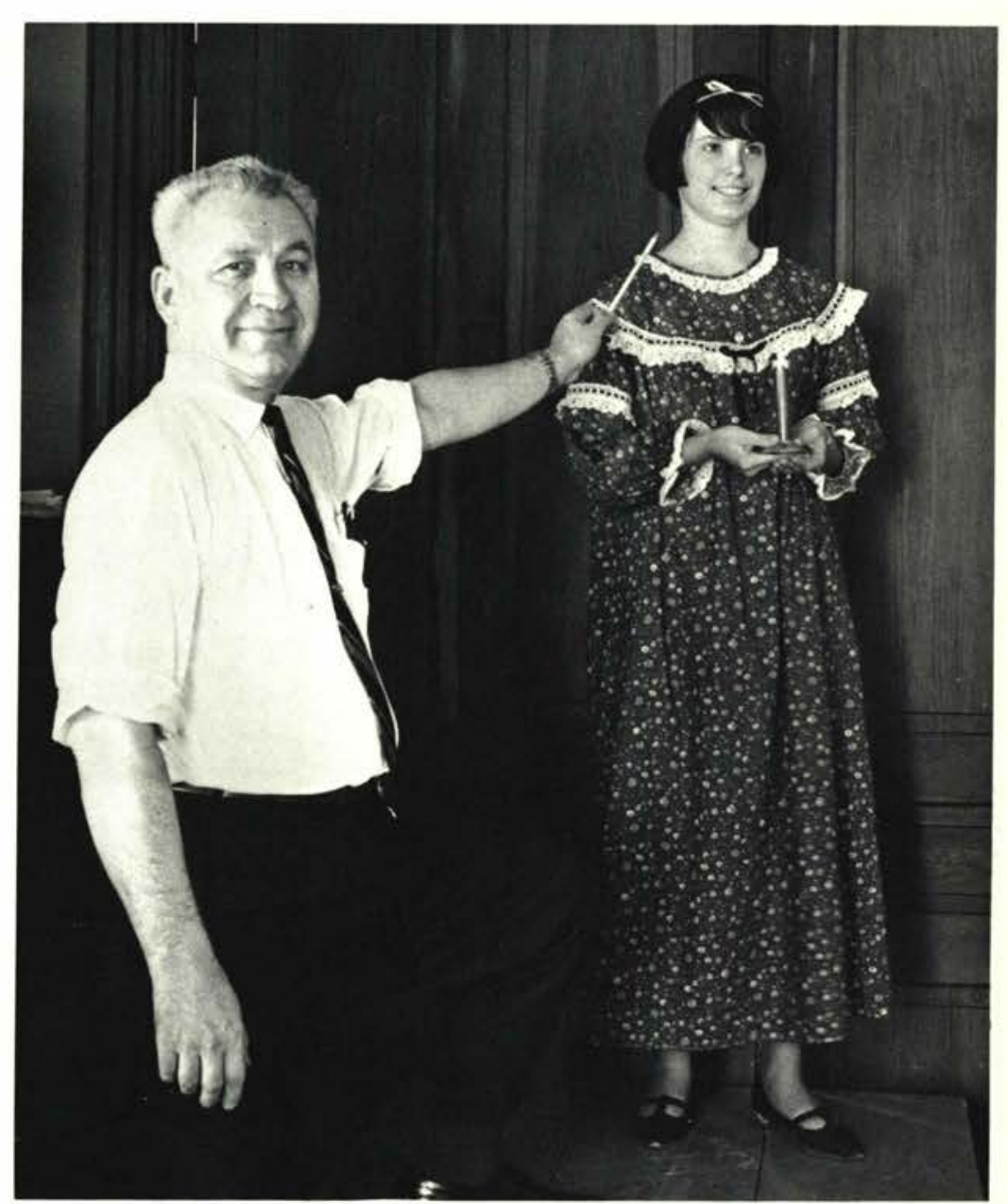

Mr. Emerson Russell

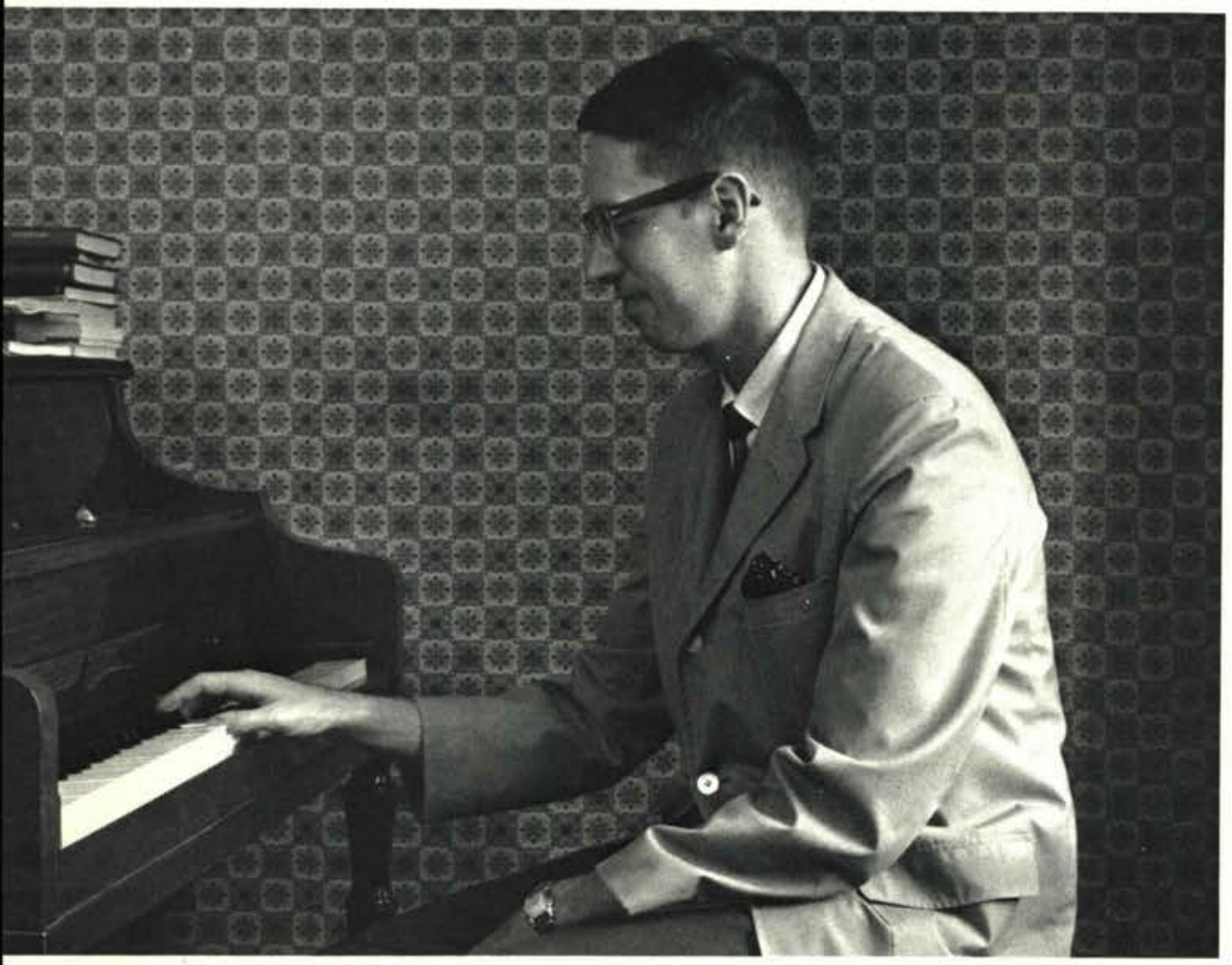

Mr. Paul Vandercoy 


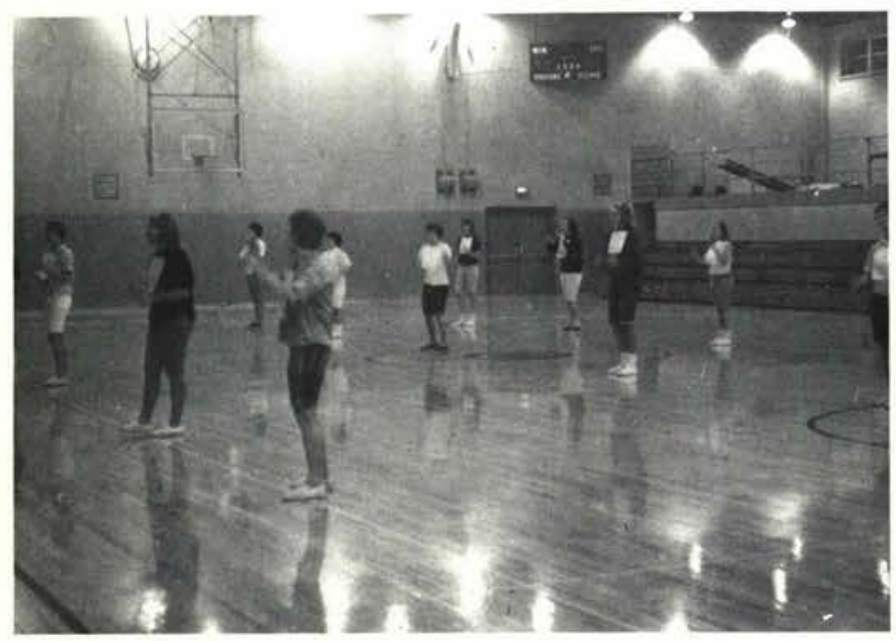

\section{HEALTH AND PHYSICAL EDUCATION}

The Physical Education Department seeks to provide a program of physical development and education in the fundamentals of organized play, a sense of good sportsmanship in the total development of wholesome and effective Christian character; and a course of study designed to aid those looking forward to teaching physical education or coaching athletics.

Mr. Callan's physical education class

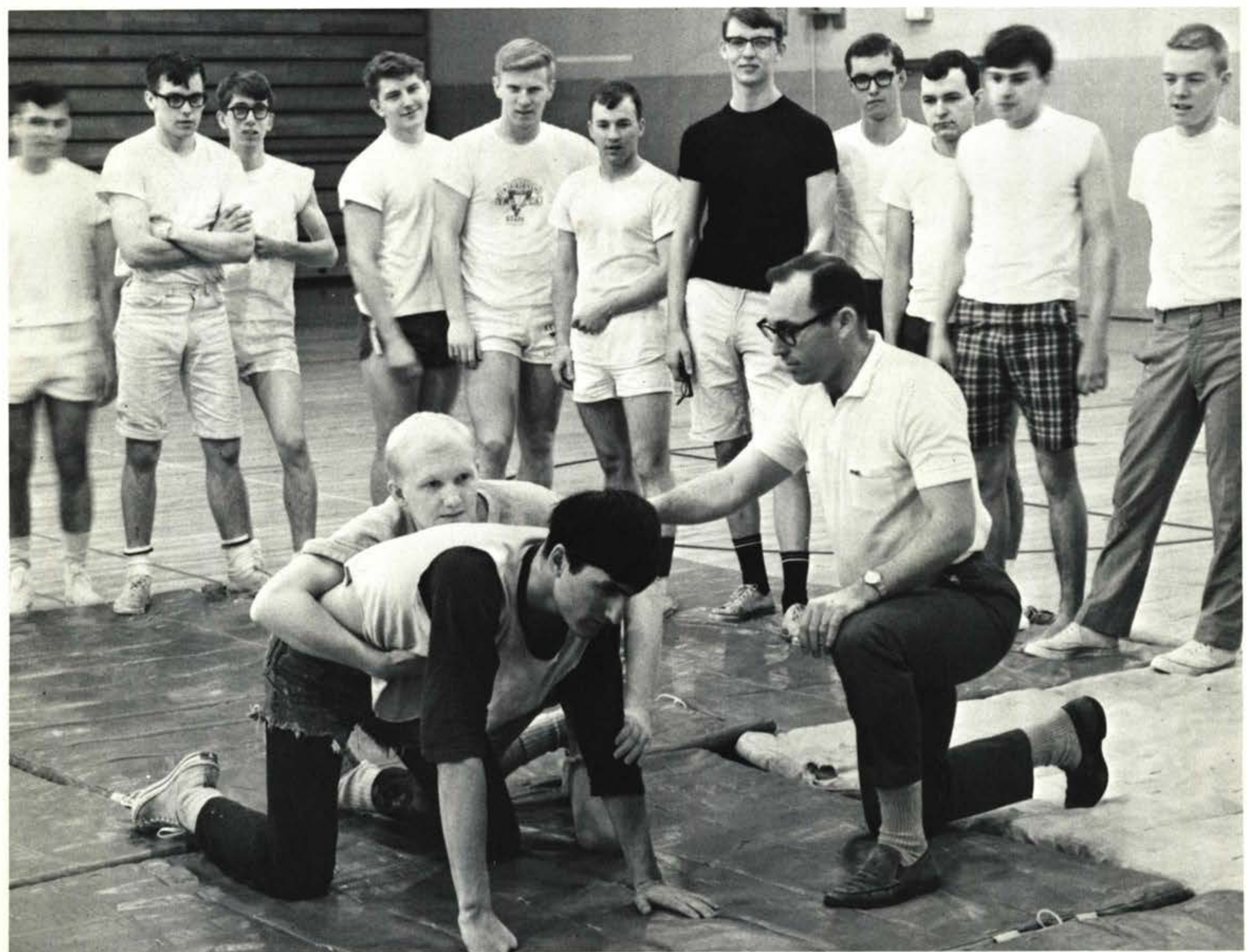




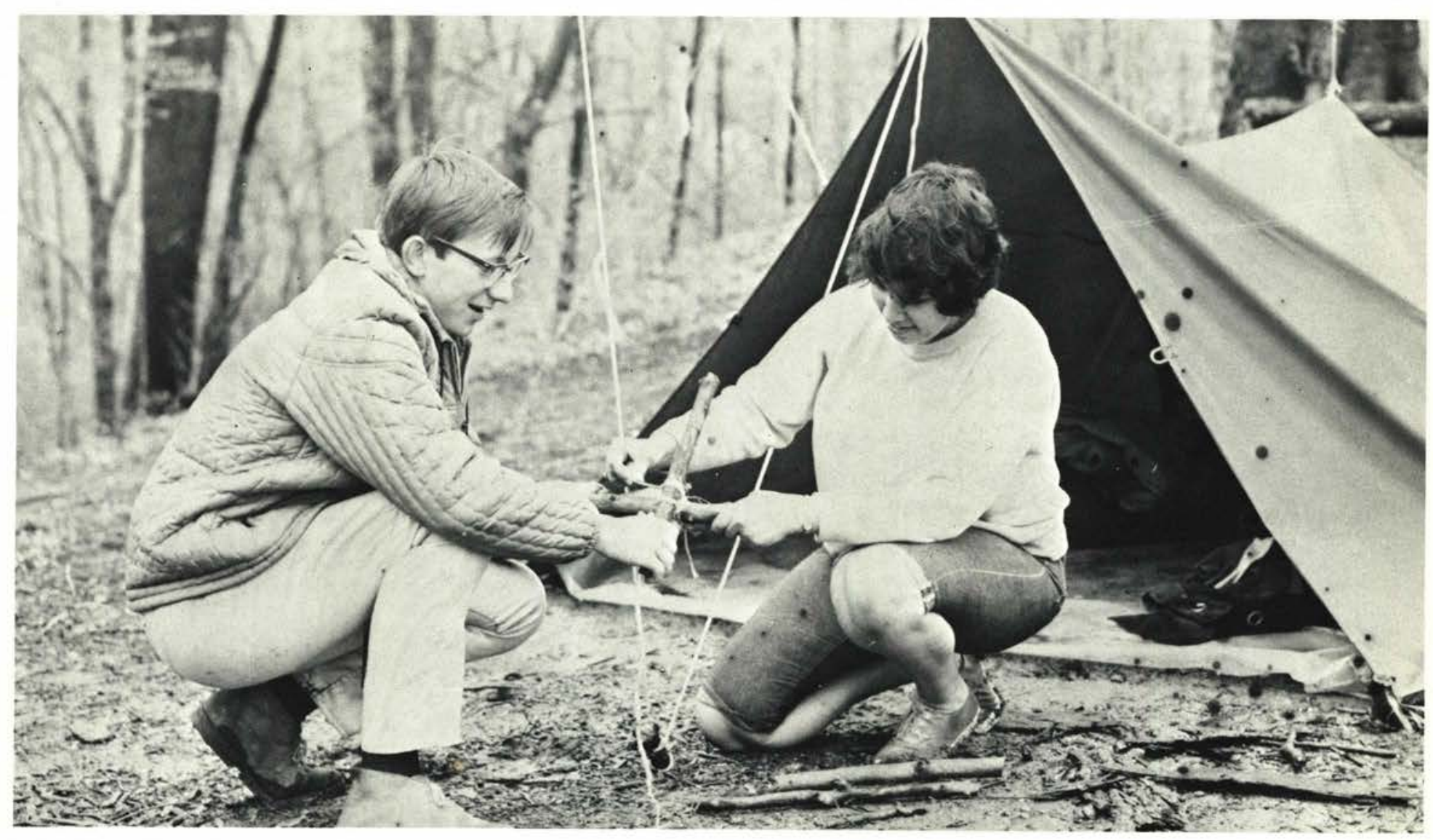

Miss Joy Mackay's camping class.
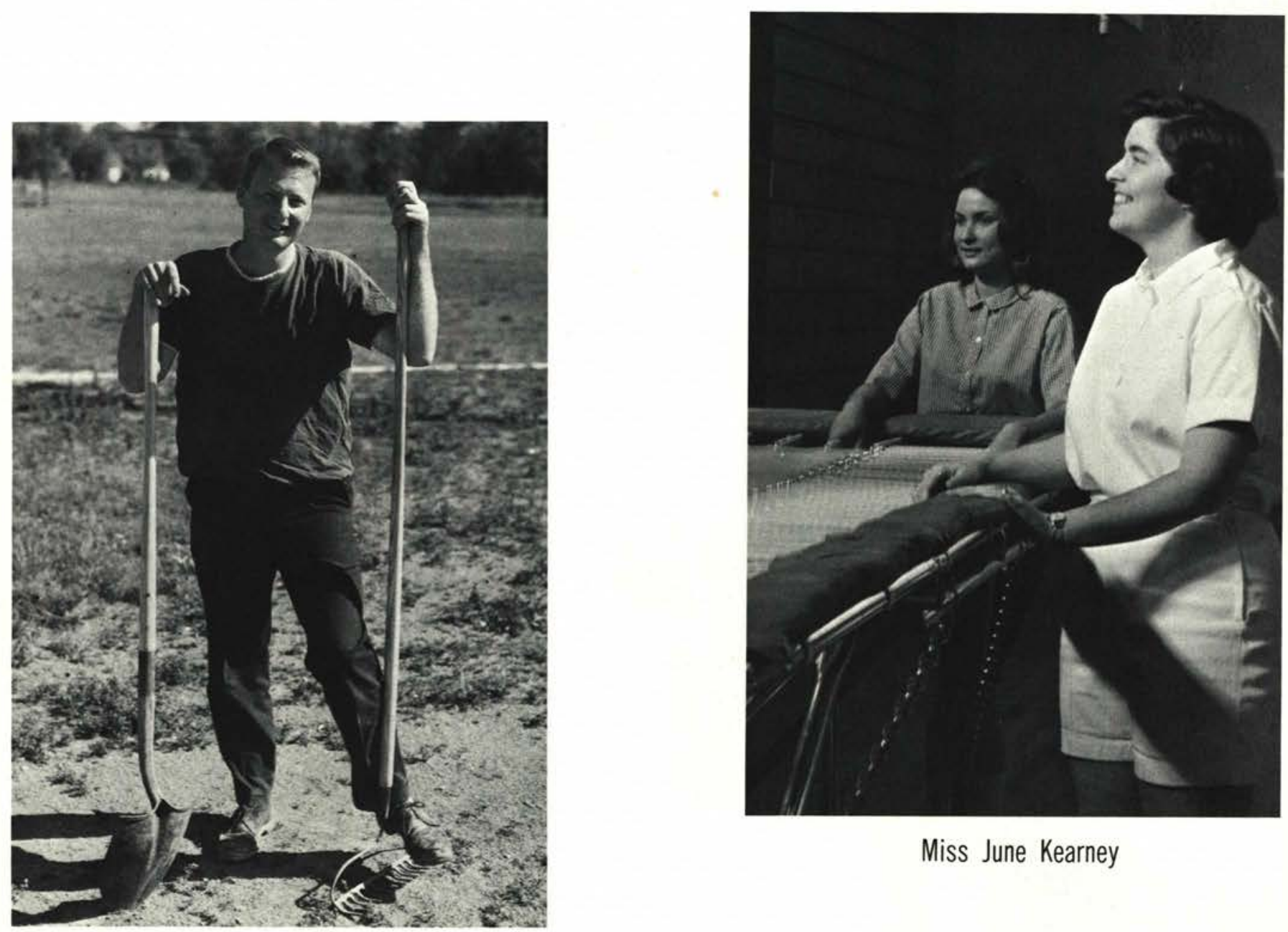

Miss June Kearney

Mr. Dennis Olsen 


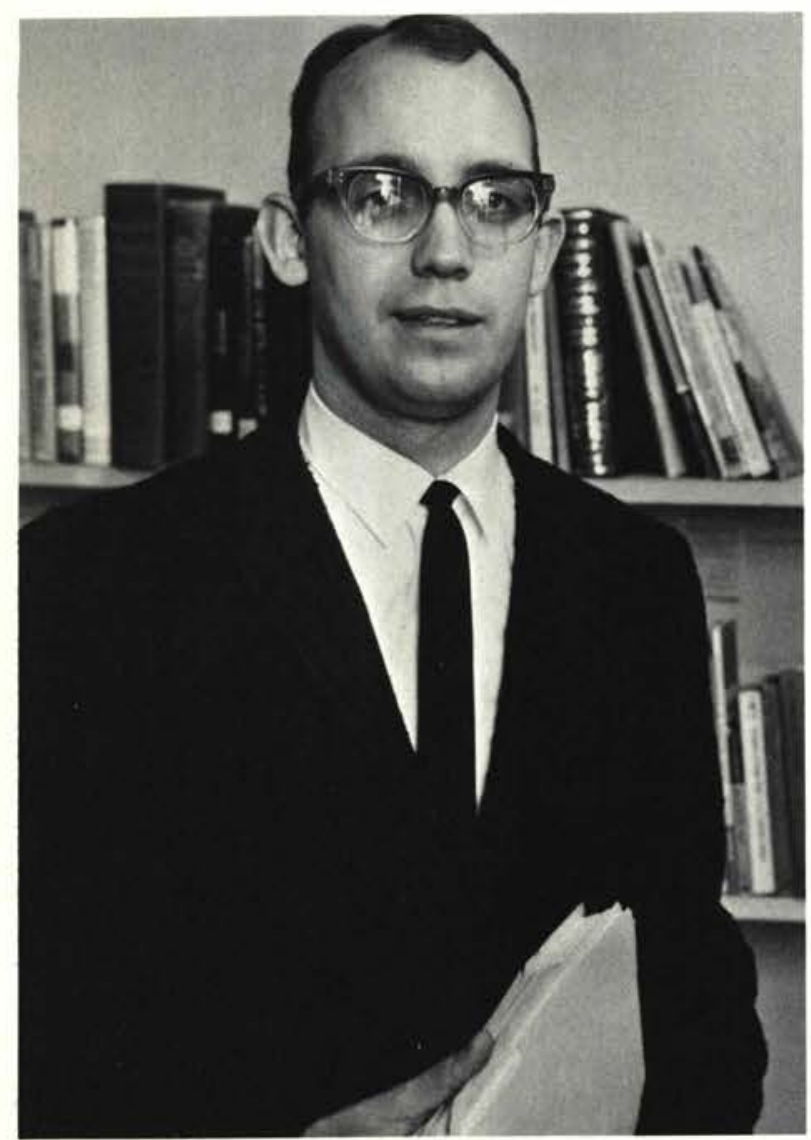

Dr. Raymond Bartholomew

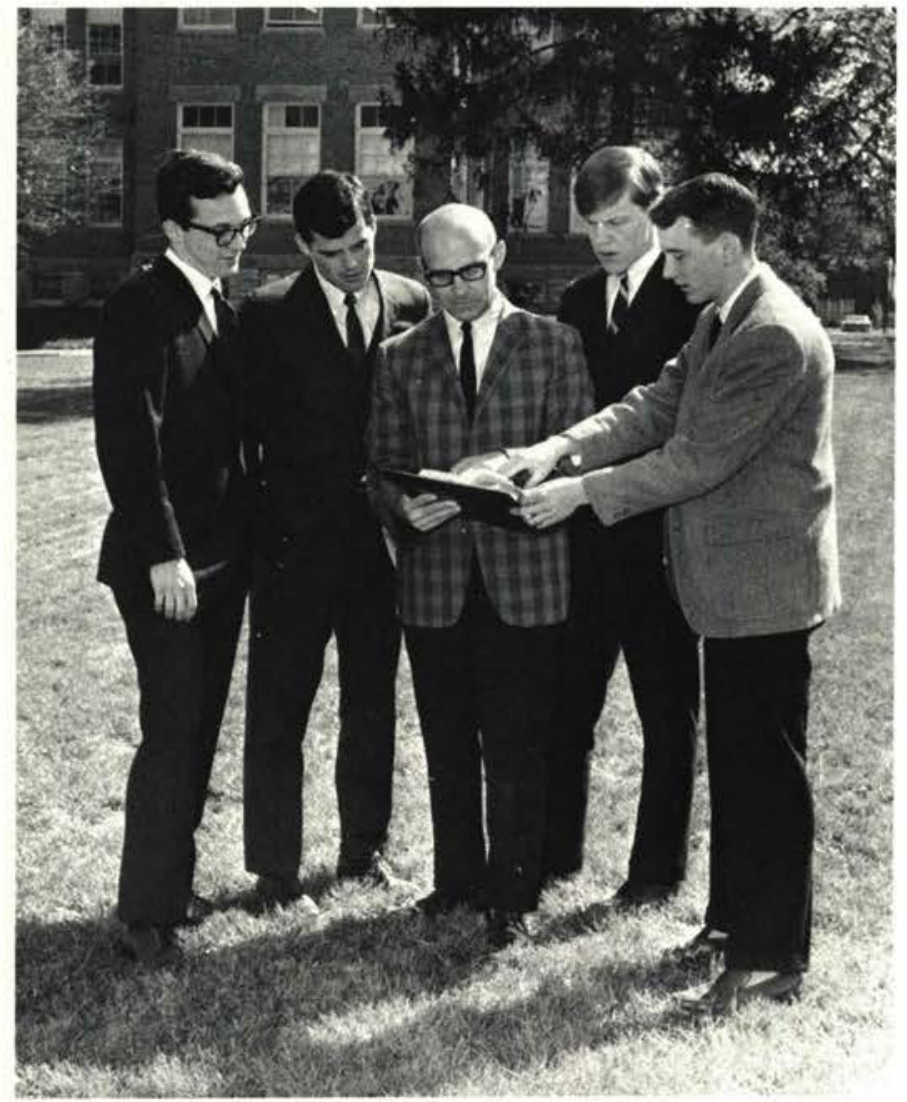

Mr. Edward Spencer

\section{LANGUAGES AND LITERATURE}

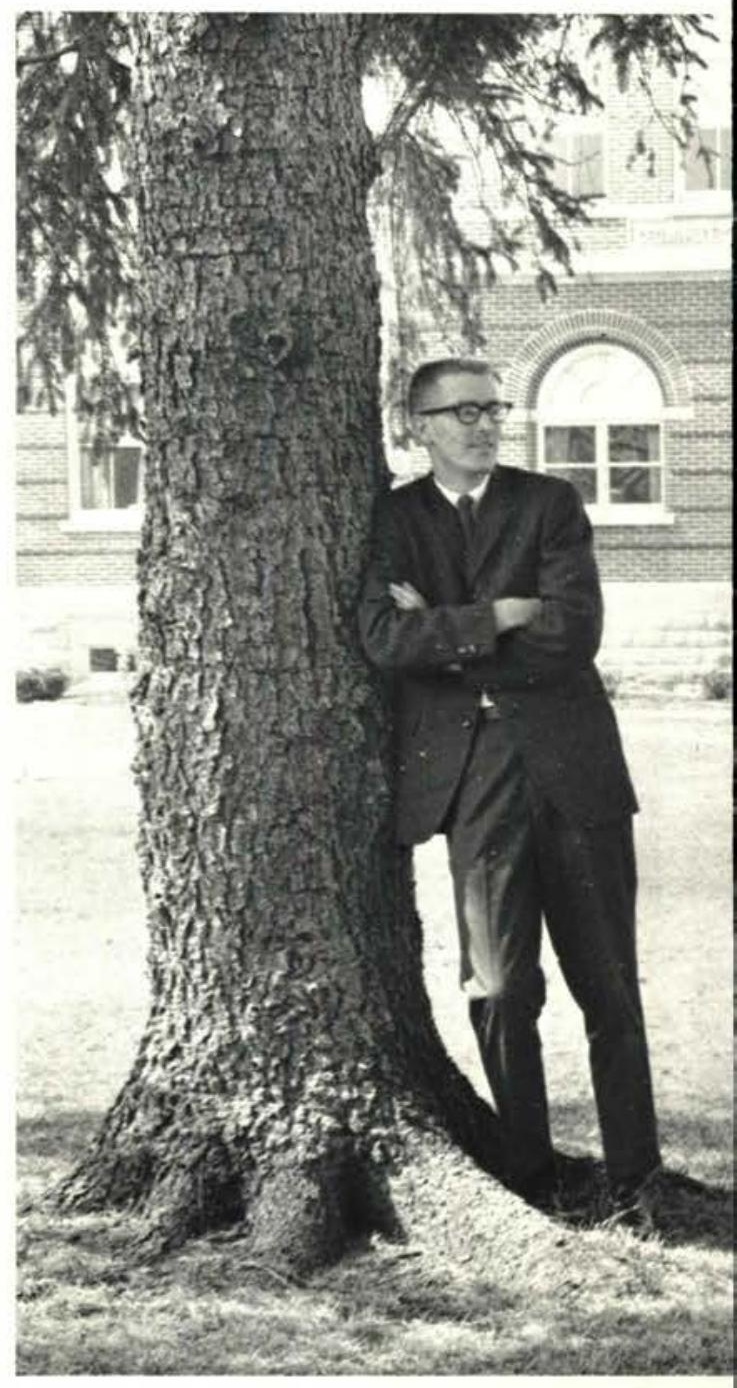

Mr. M. Dean White 
Thoughts into words for communication is the essence of this department. Here the student grows through exposure to the light of great ideas from peoples of all time. These ideas, taken from ancient and modern records, assist him as he grows more adept in communicating with the world around him. His world may someday include those of another language and culture. Therefore, the study of a foreign tongue adds to the growth of the educated individual.

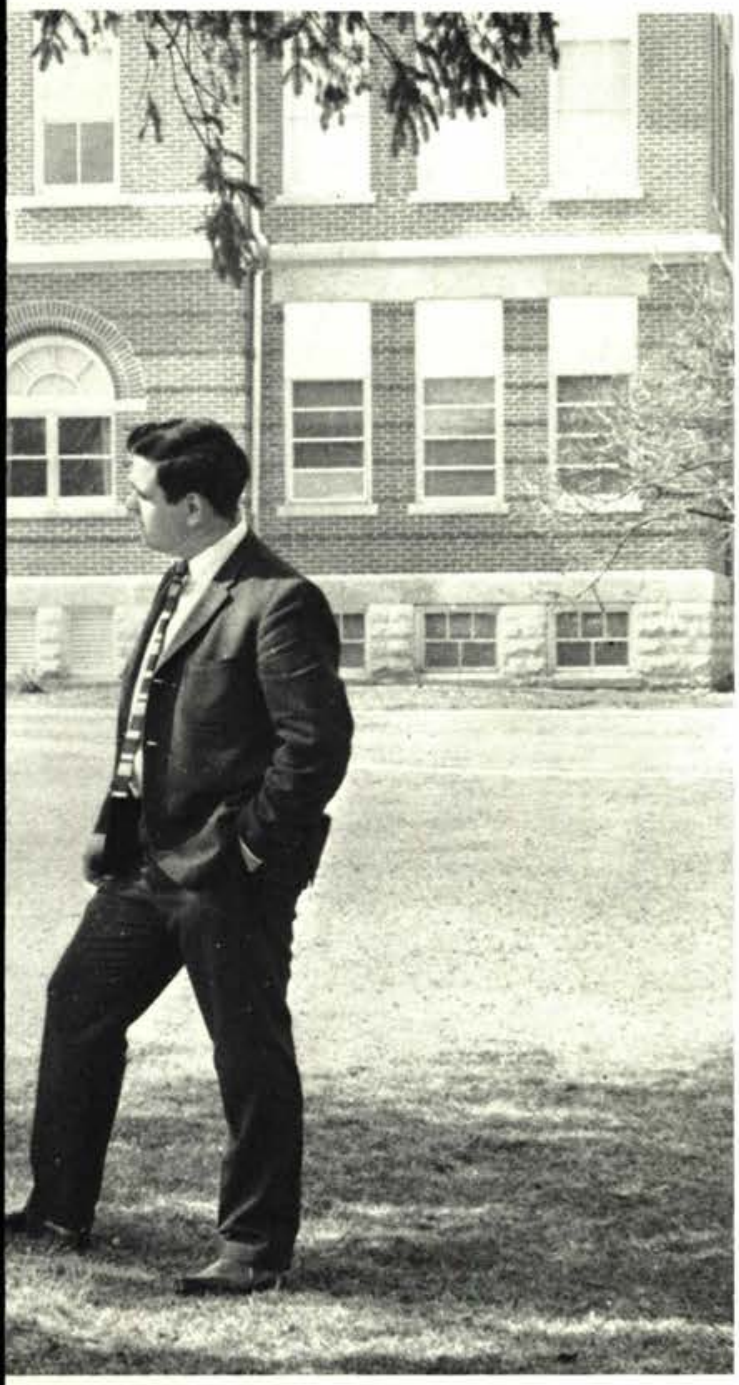

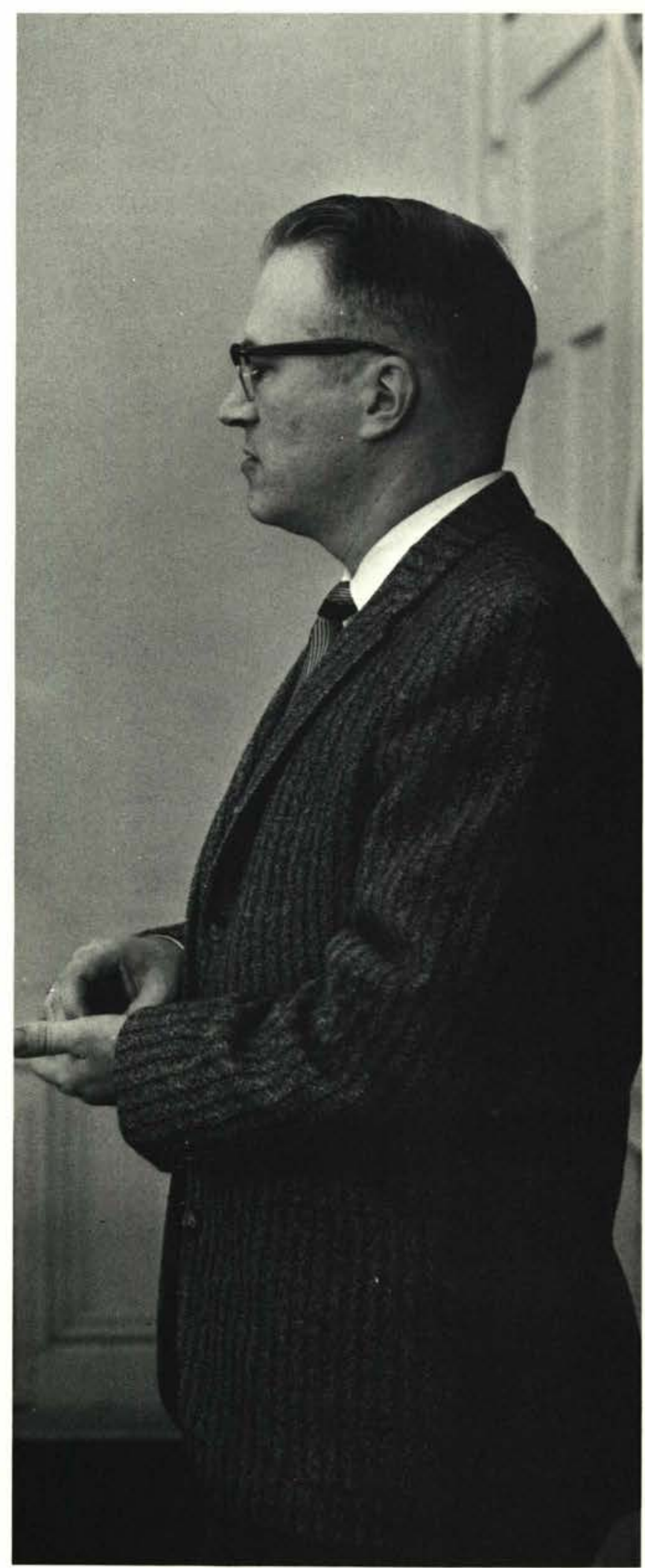

Mr. Edward Greenwood 


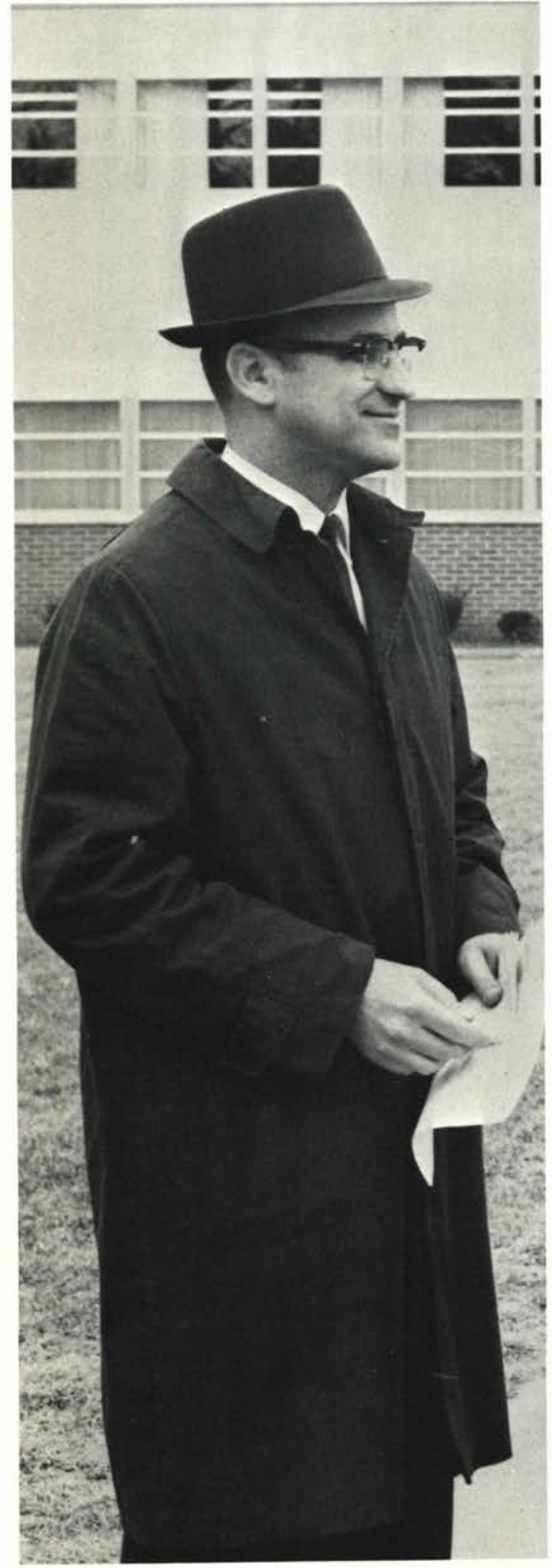

Mr. John Reed

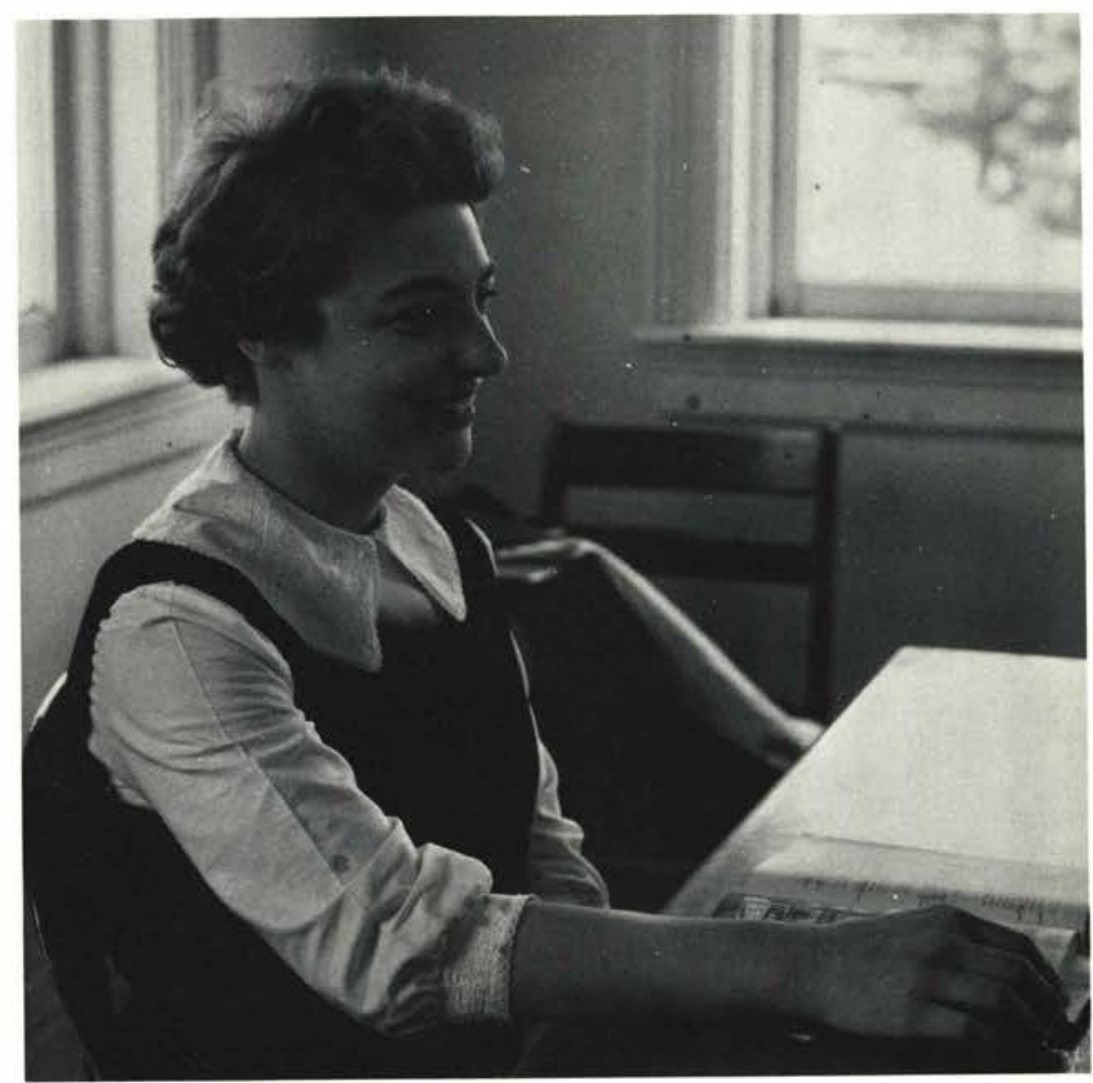

Miss Carla Packard

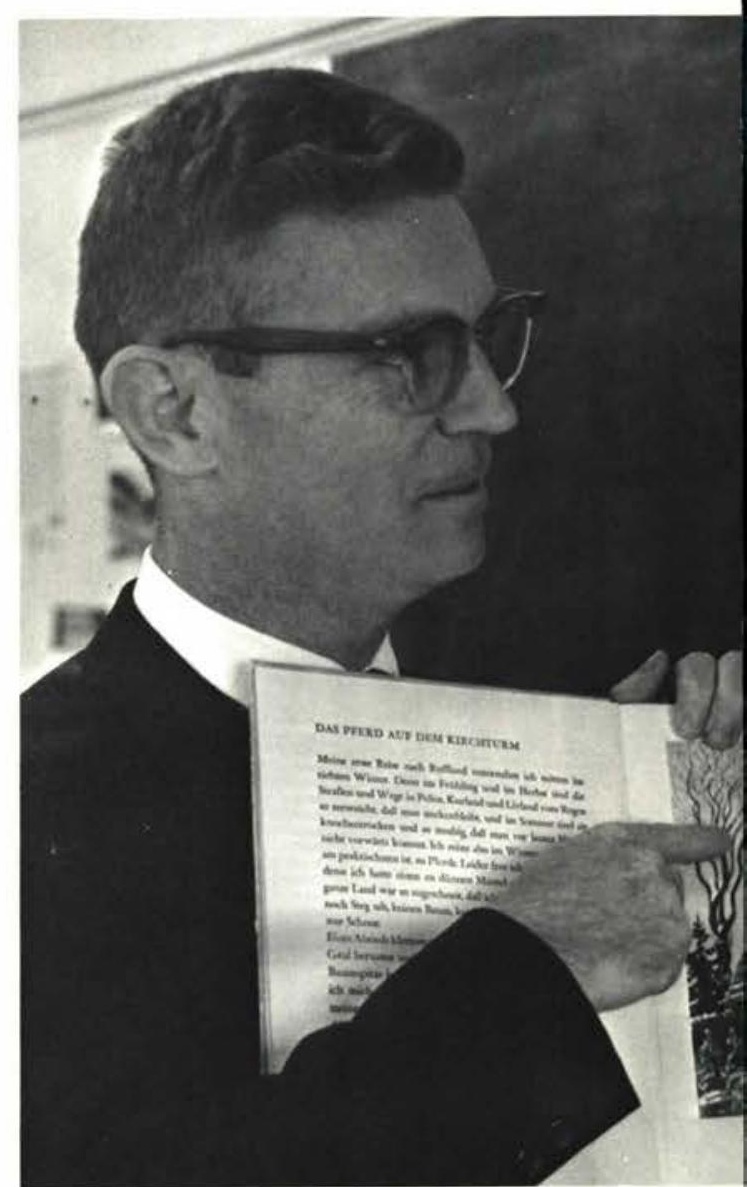

Mr. Harmon Bergen 


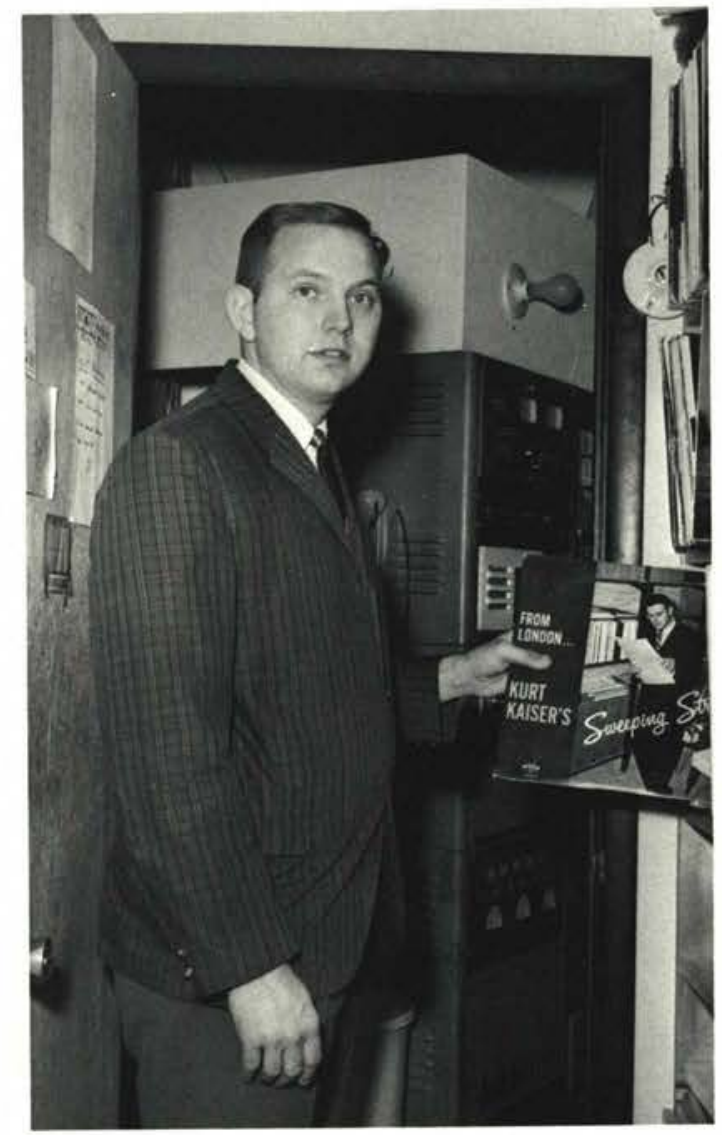

Mr. Paul Gathney

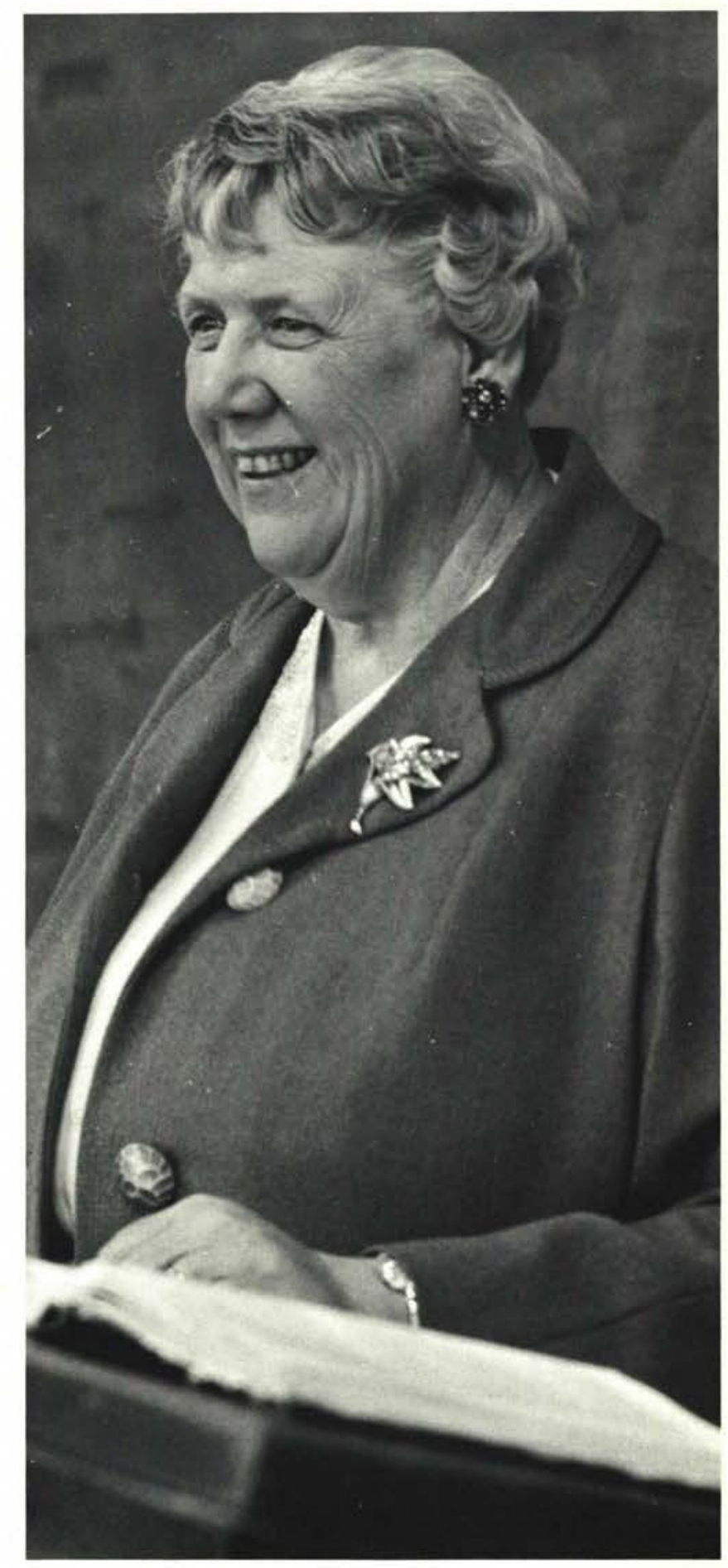

Mrs. Miriam Maddox 


\section{SCIENCE}

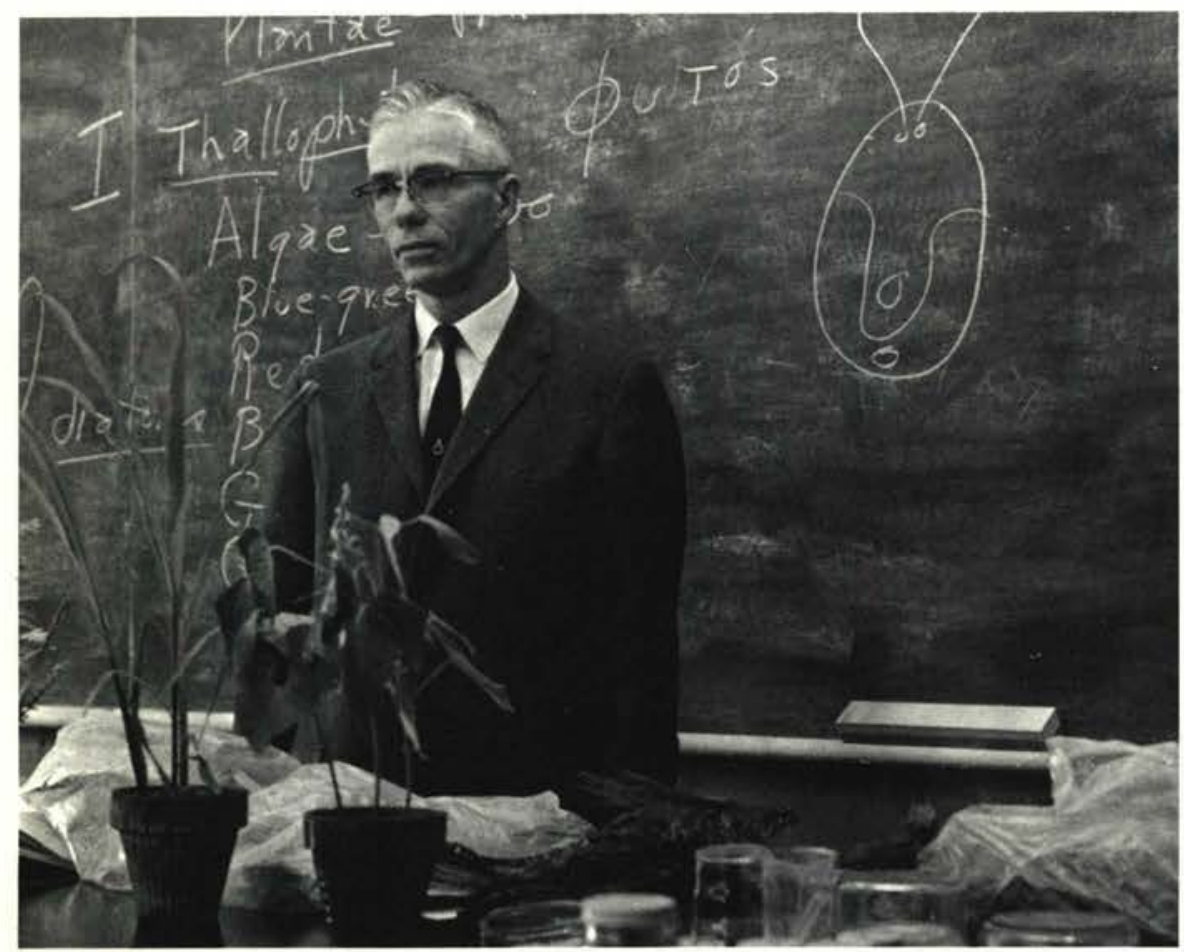

Science is not just a static collection of facts and formulas. It is a dynamic study one whose doors open to those who are willing to explore it. In the Science Department, math and science students are stimulated to delve beyond the formulas and equations to see why things work and behave as they do. This pursuit ultimately leads them to see God working in and through His universe; thus, strengthening their Christian faith.

Mr. Austin D. Elmore

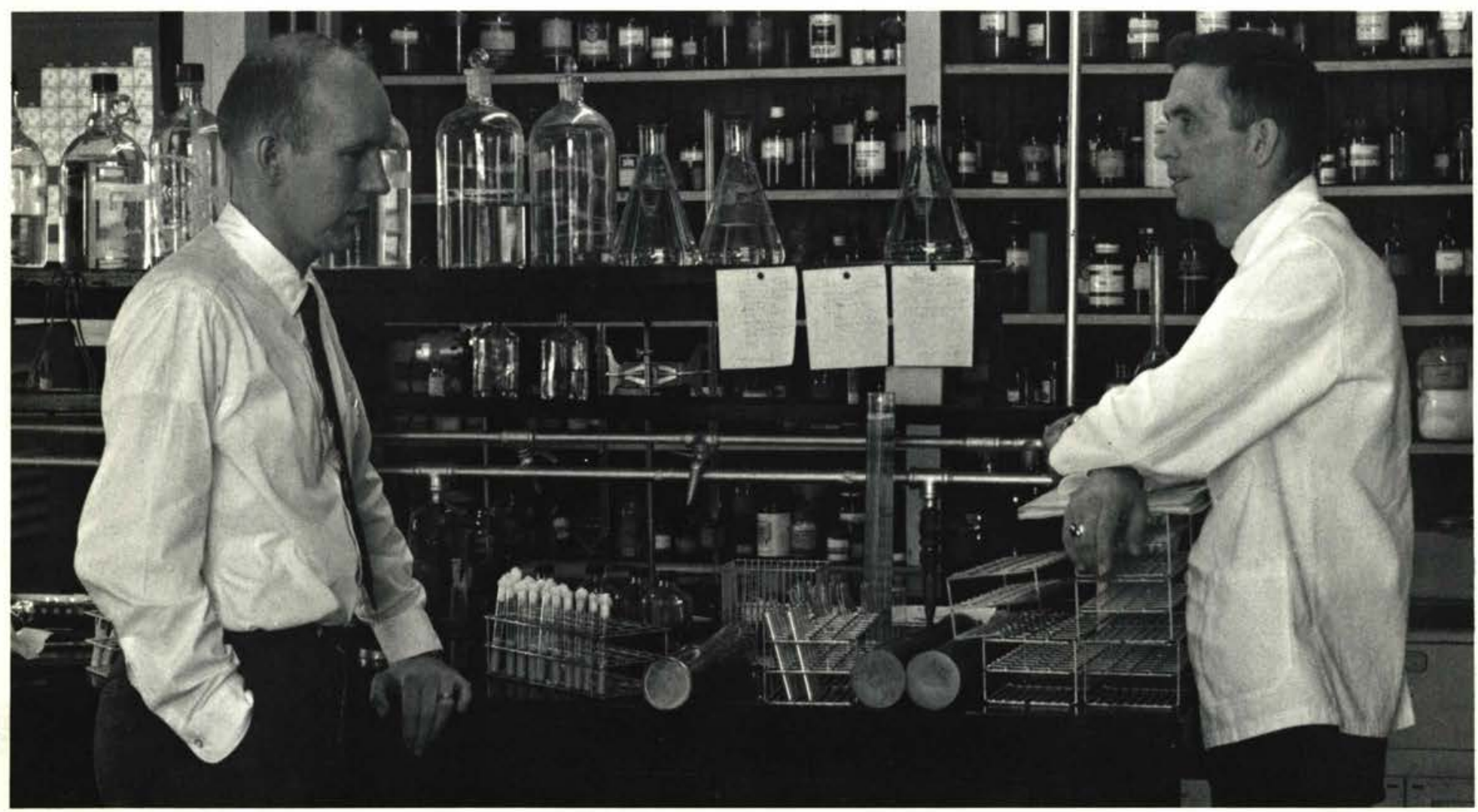

Dr. Donald Baumann and Dr. Dale Thomson 


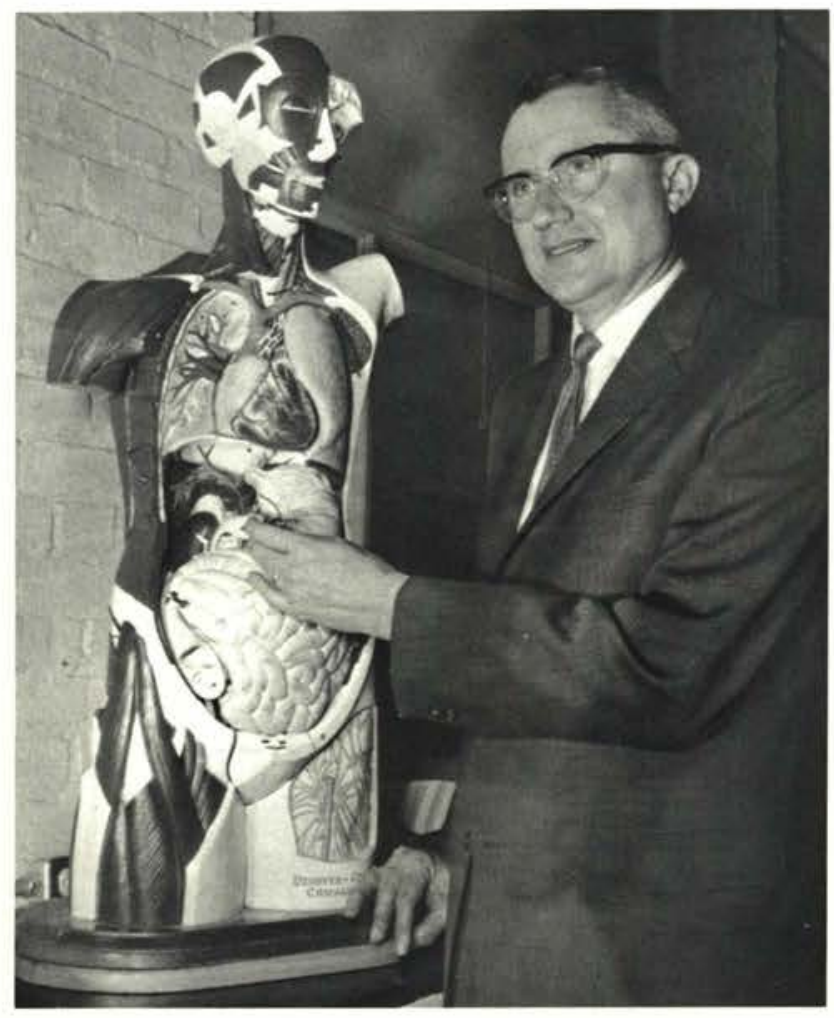

Mr. Howard R. Johnson

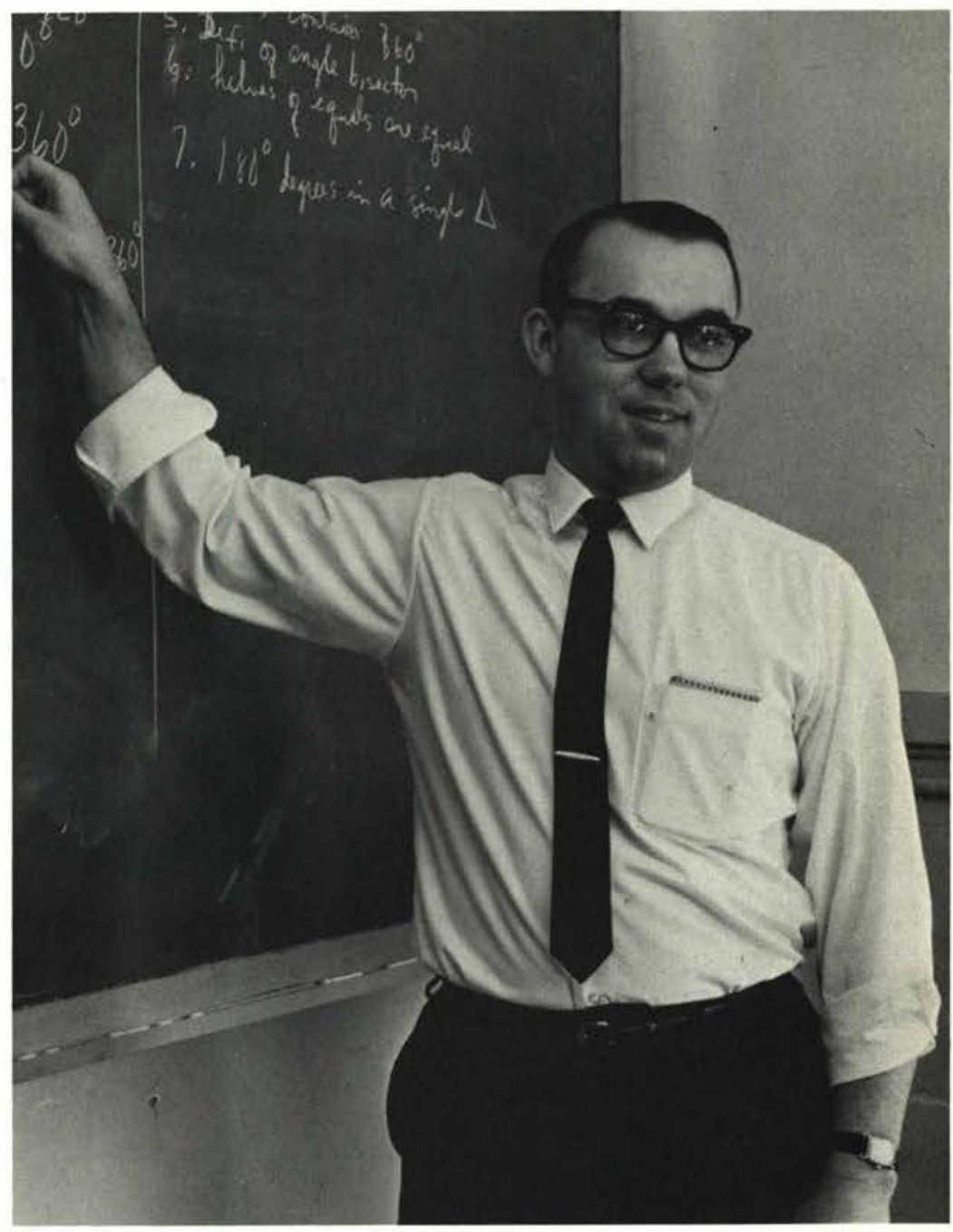

Mr. Daniel E. Wetzel

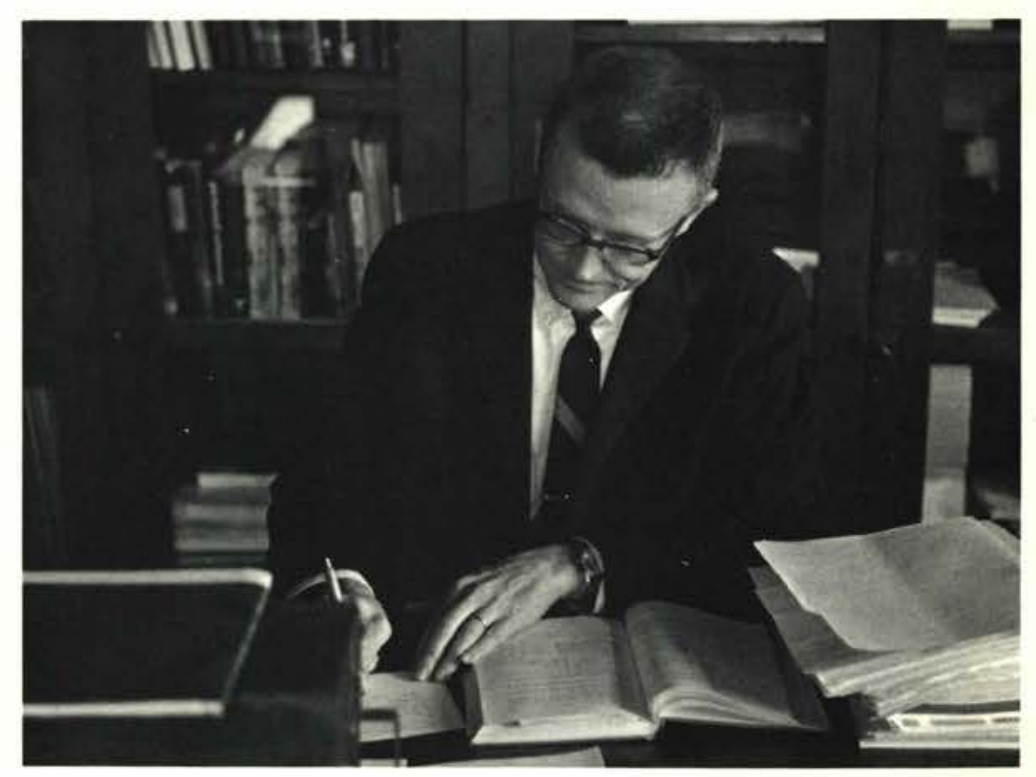

Mr. Bert L. Frye 


\section{SOCIAL SCIENCE}

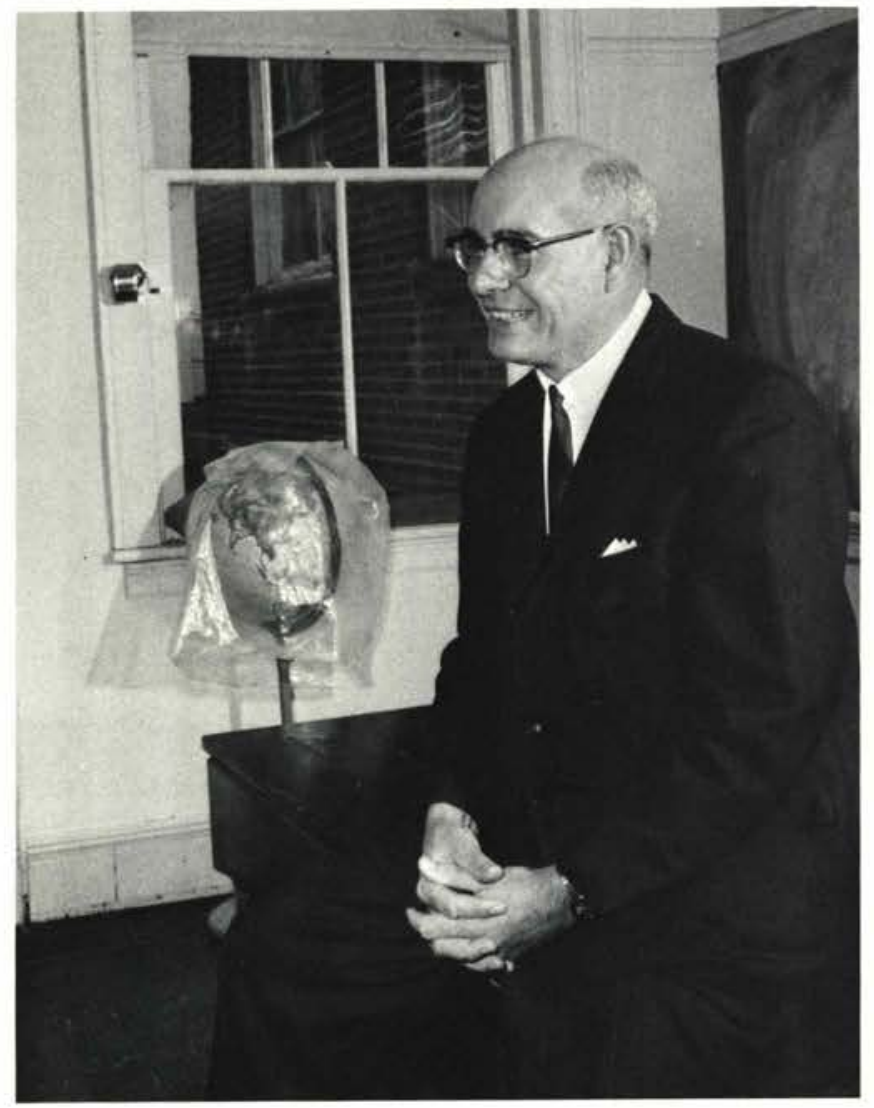

Man through the ages has had social interaction with his environment as well as with other men.

The purpose of the Social Science Department is to show the student the significance of this interaction. Courses are offered in both history and sociology since each area is important in order to understand the total picture of man's reactions and interaction. Once the overall view is achieved, courses of a more specialized nature are studied.

Mr. Cleveland McDonald

Mr. Ralph B. Gale

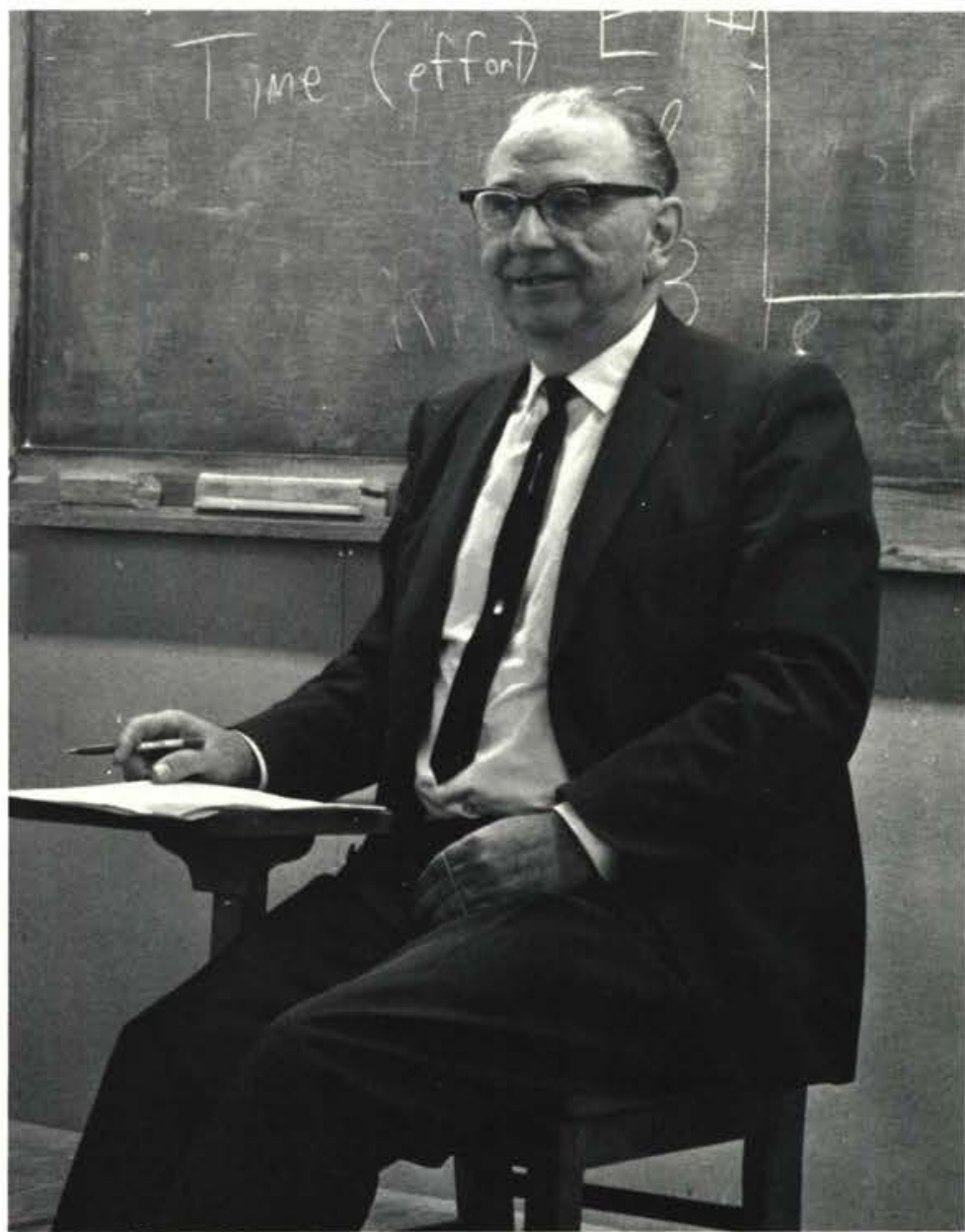




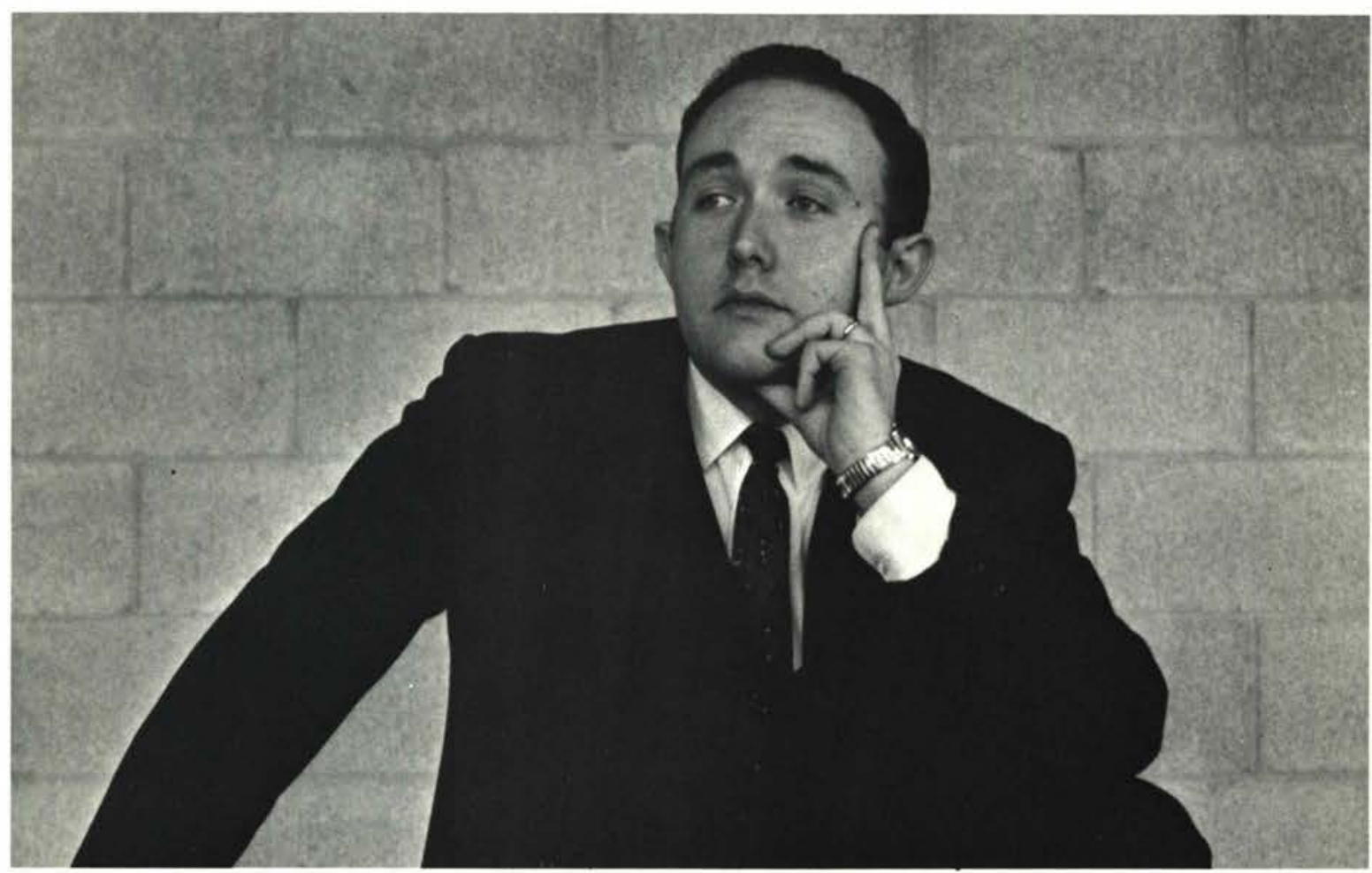

Mr. Murray Murdoch

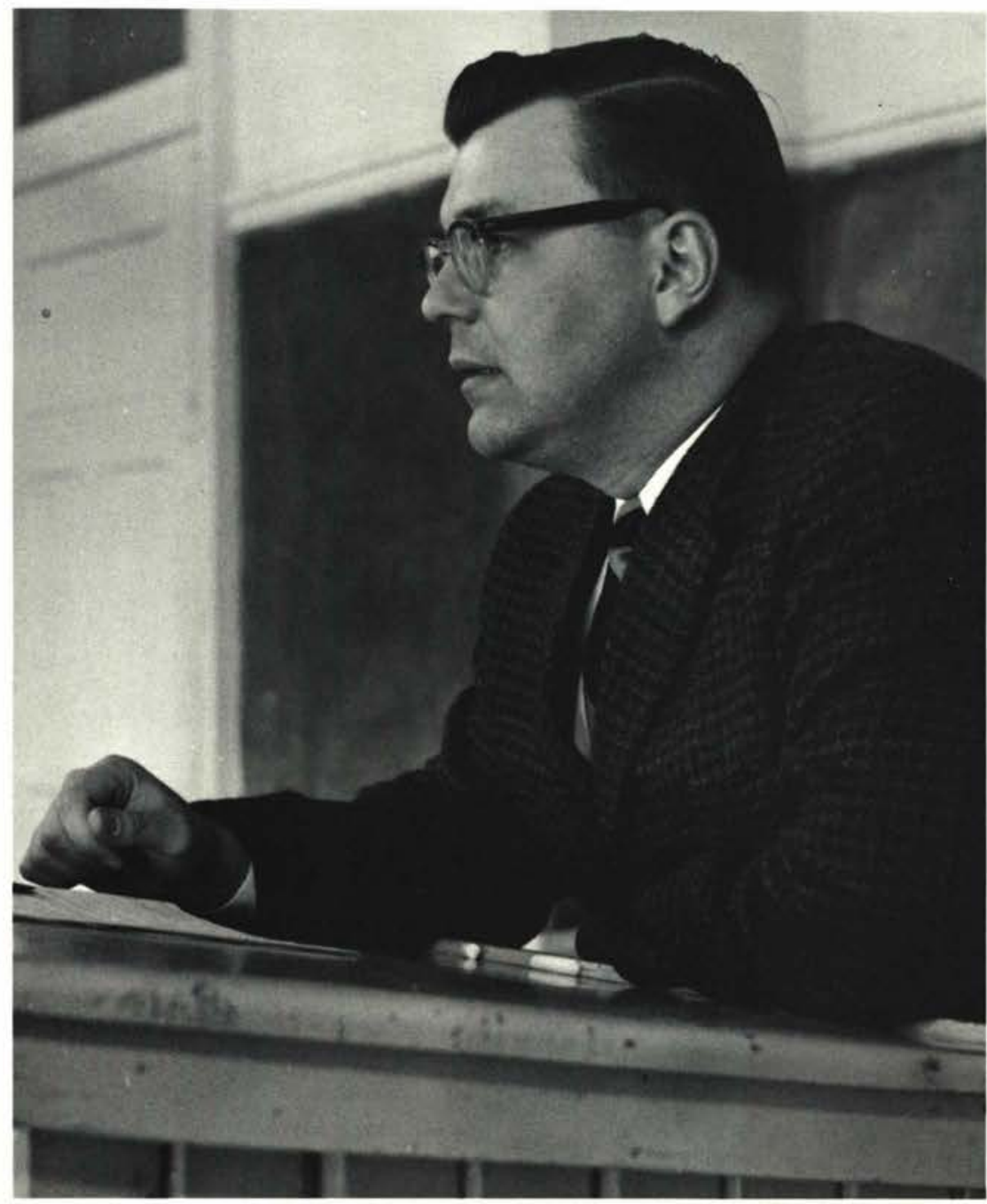

Mr. Al Monroe 


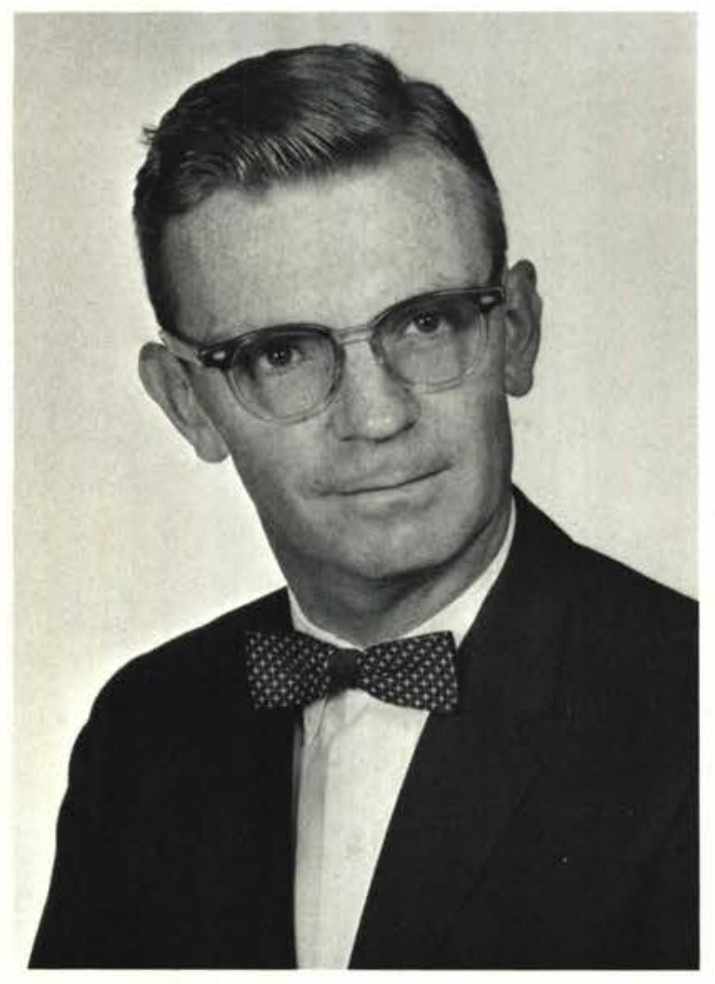

Mr. H. Bergen

REFERENCE LIBRARIAN

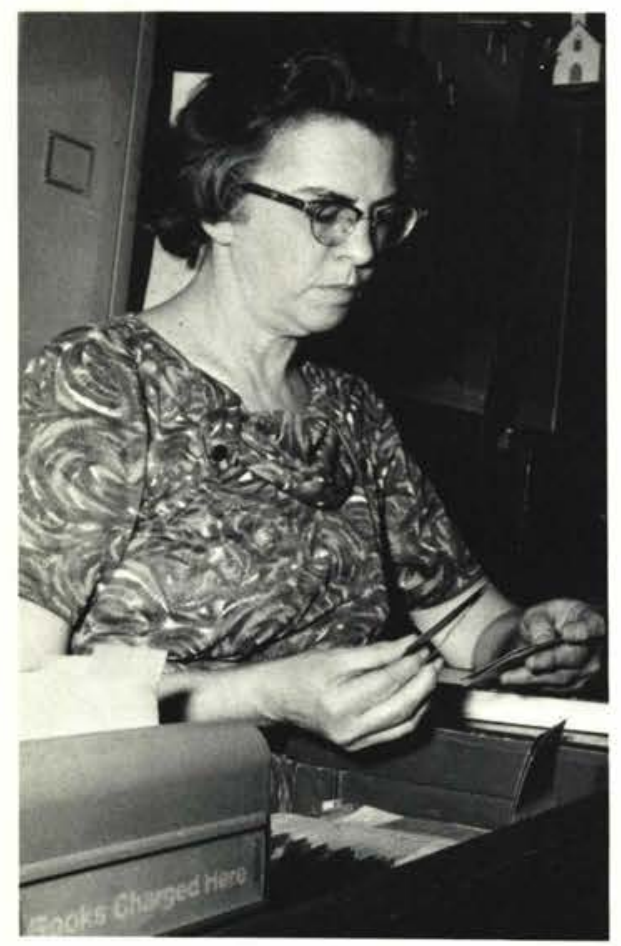

Mrs. Russell

\section{Library Staff}

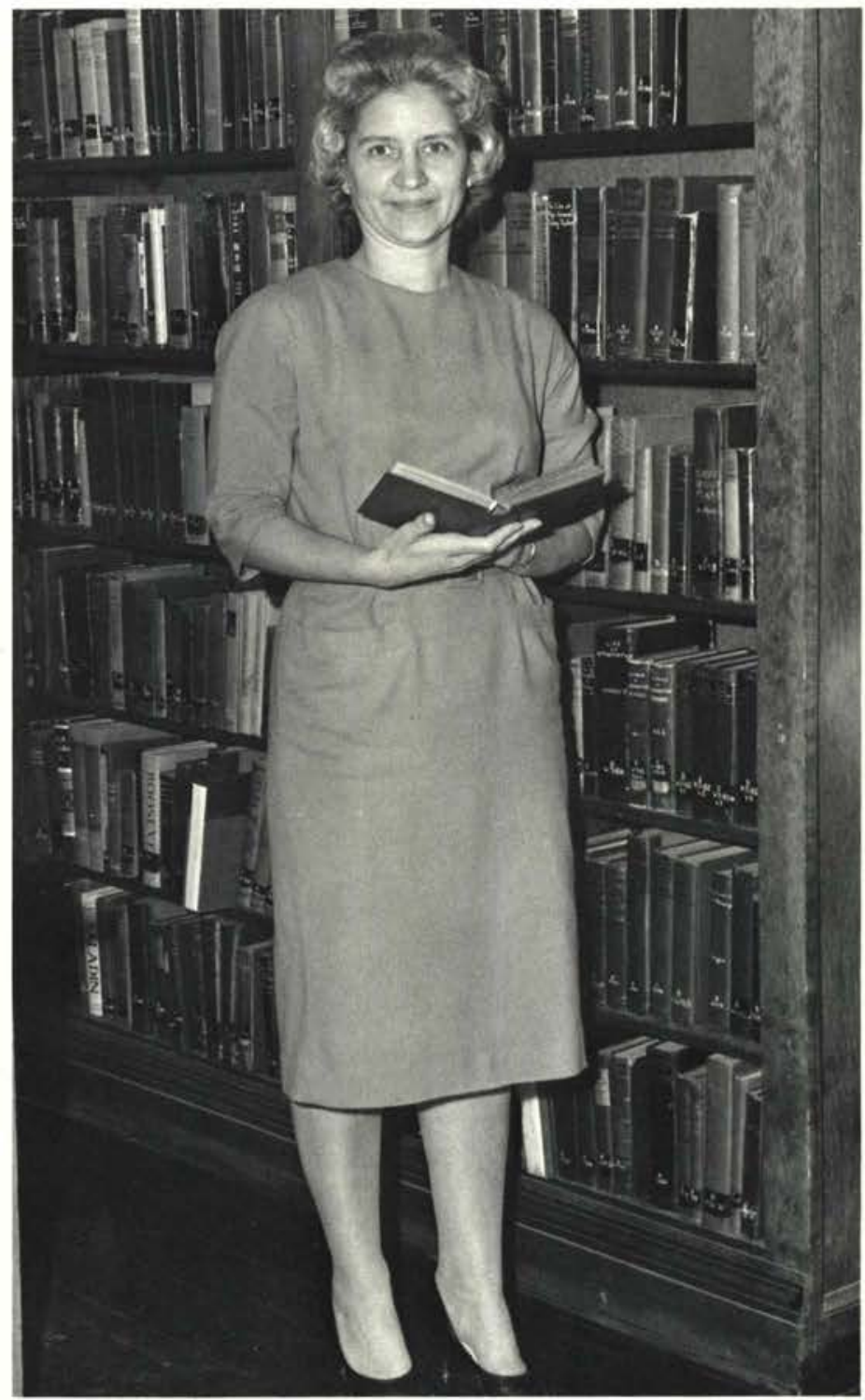

Mrs. A. Chaffee, HEAD LIBRARIAN

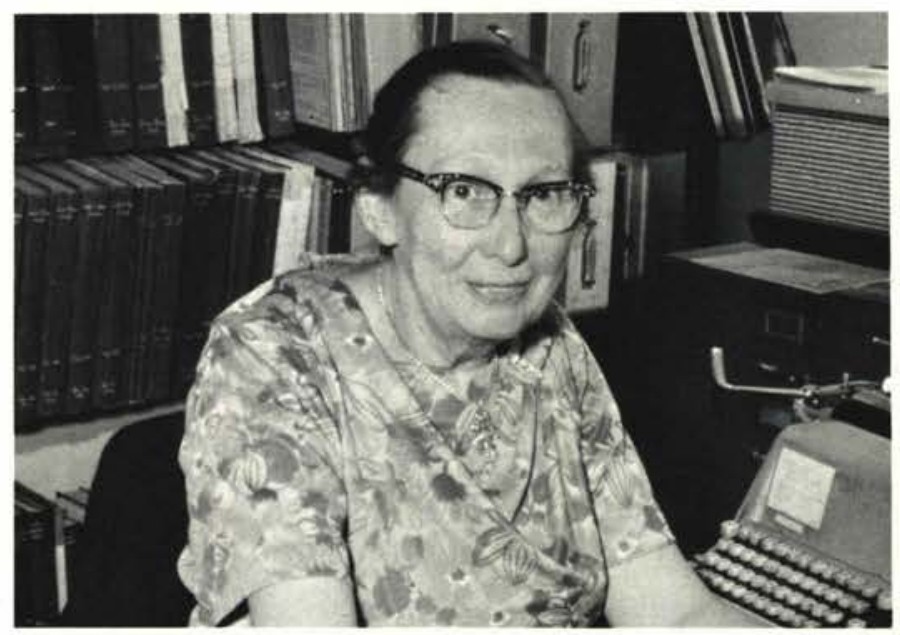

Mrs. E. Ruder 


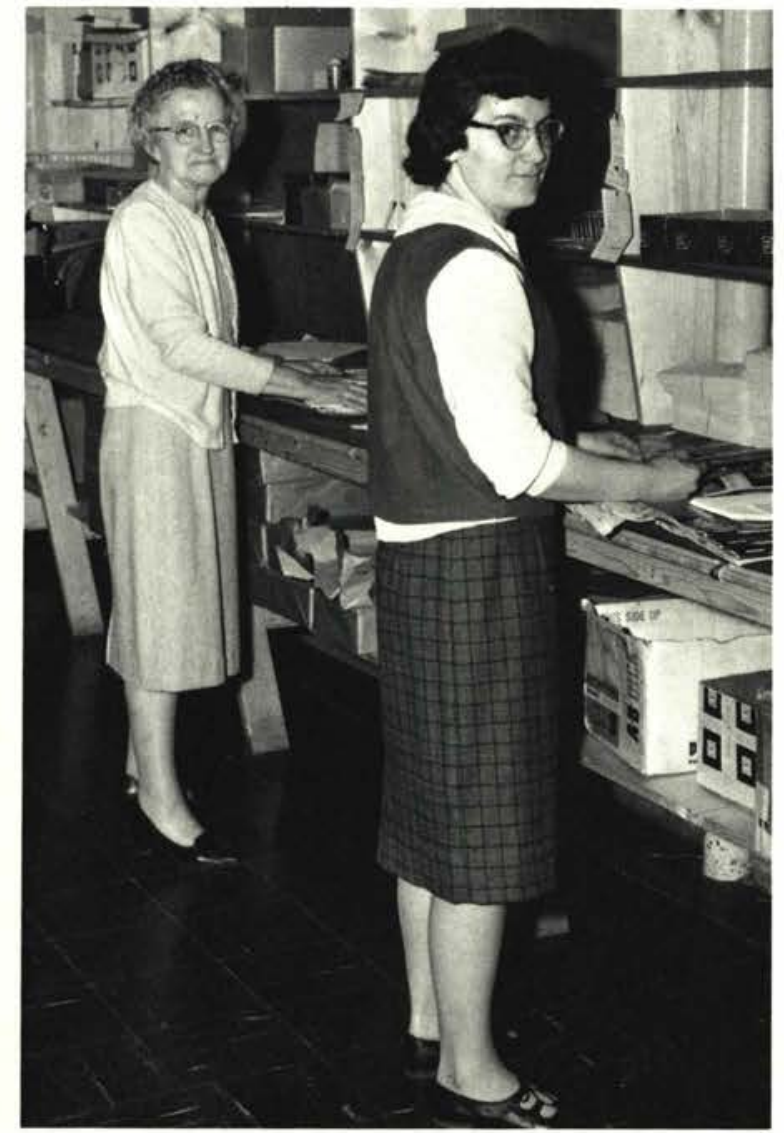

Mrs. Underwood, Mrs. Dillon POSTAL CLERKS

\section{Publications}

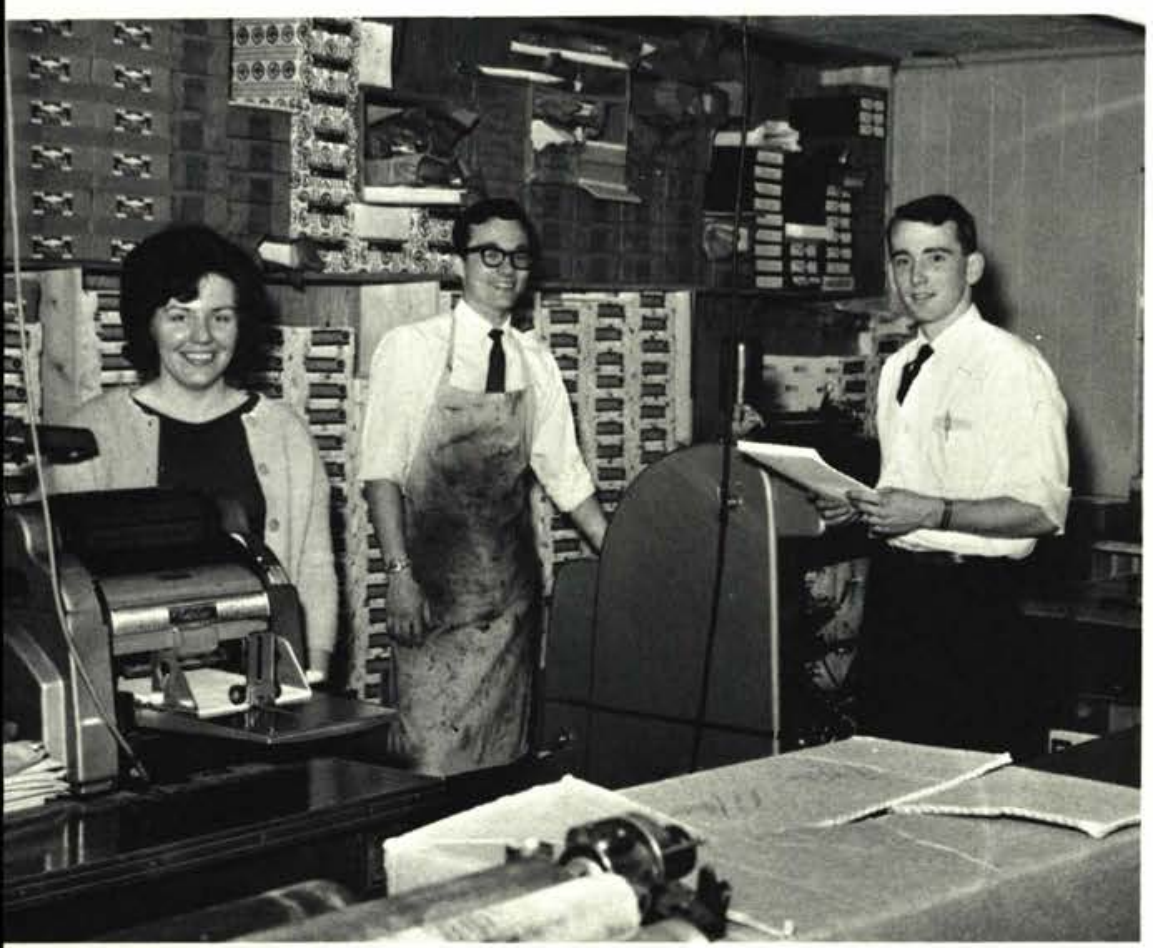

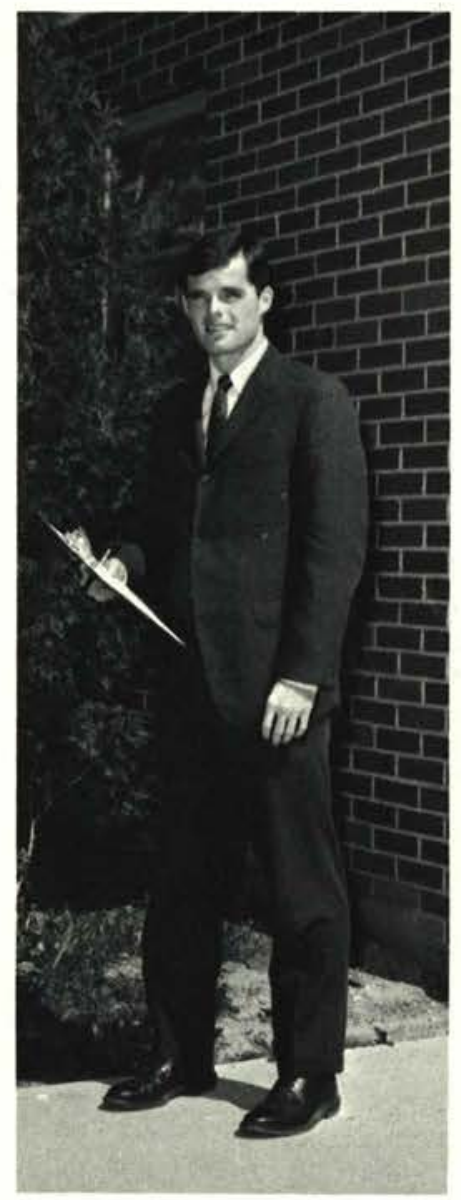

M. Nicholls

DIRECTOR OF ALUMNI RELATIONS 


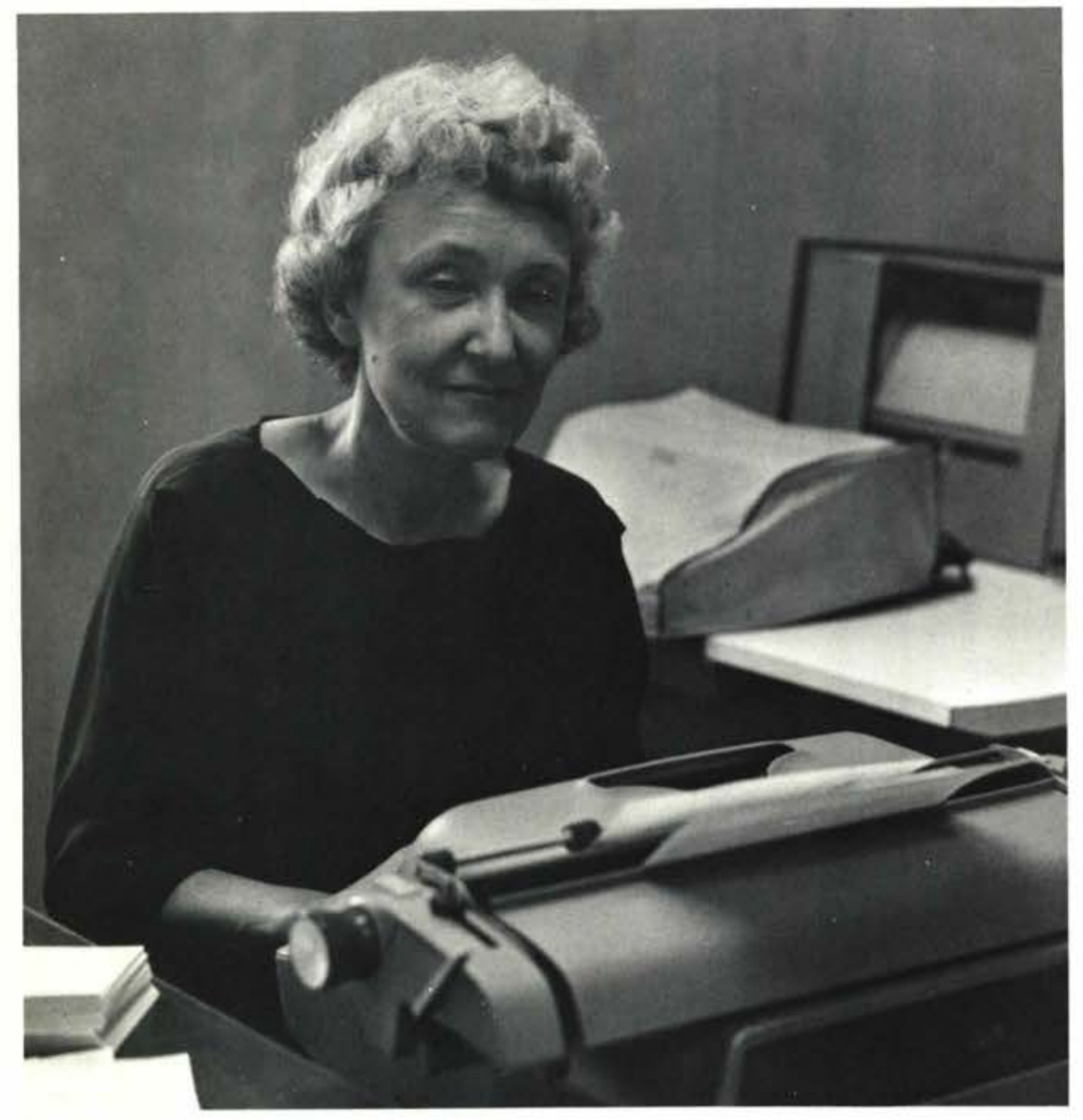

\section{Staff Secretaries and} Business Office

Mrs. Brown, Secretary to President Jeremiah
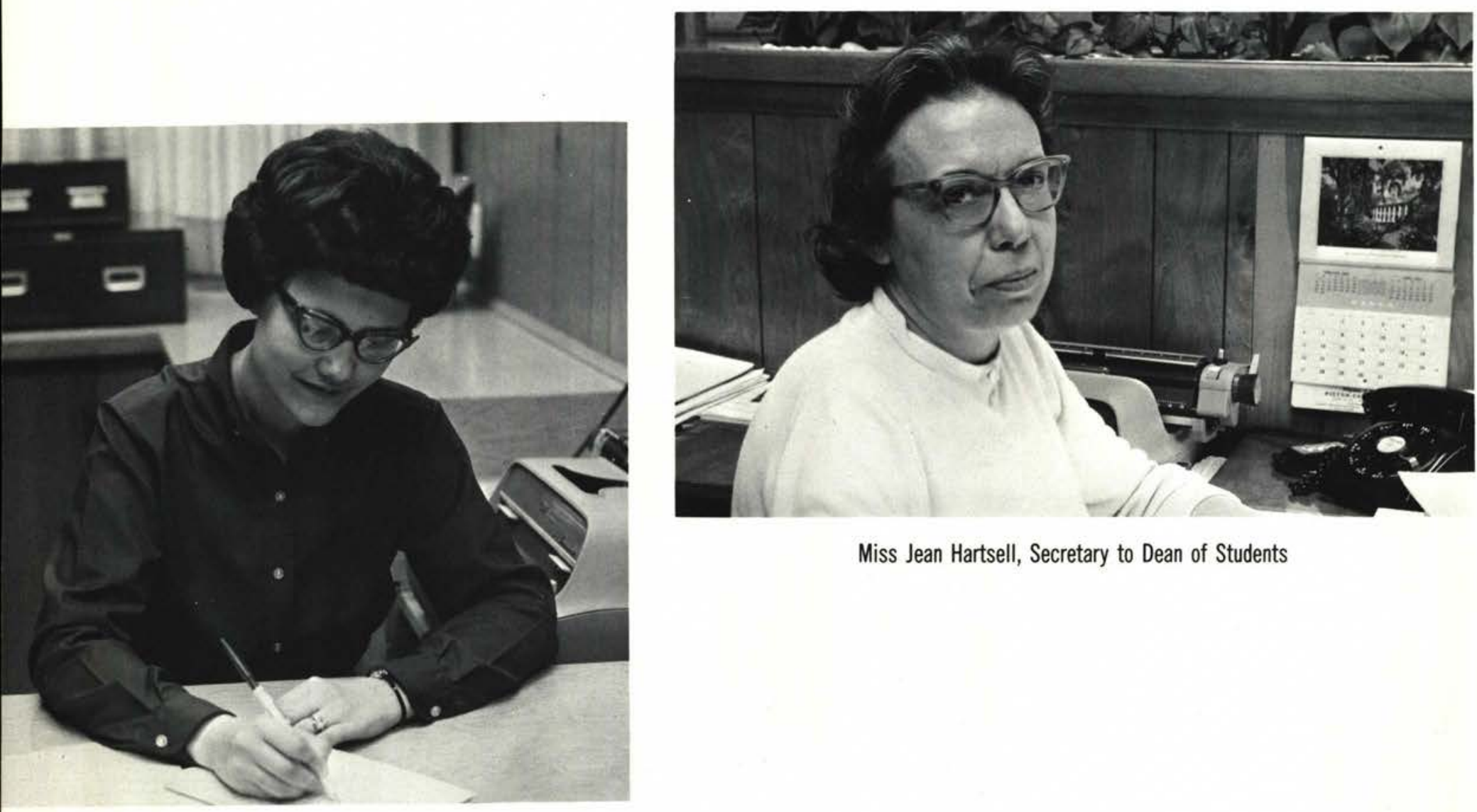

Miss Jean Hartsell, Secretary to Dean of Students

Miss Carolyn Diveley, Secretary to Mr. Turner 


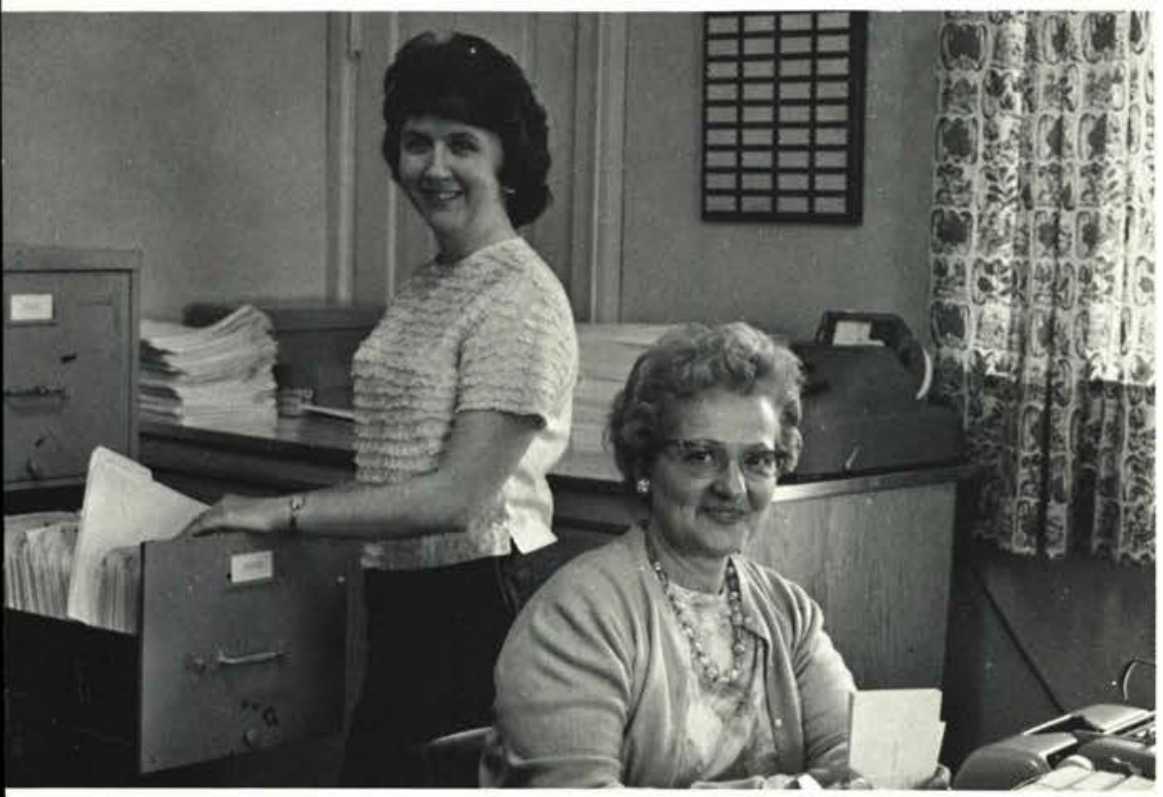

Mrs. Nancy McPheeters

Secretary to Registrar

Mrs. Merle Slabaugh

Secretary to Faculty

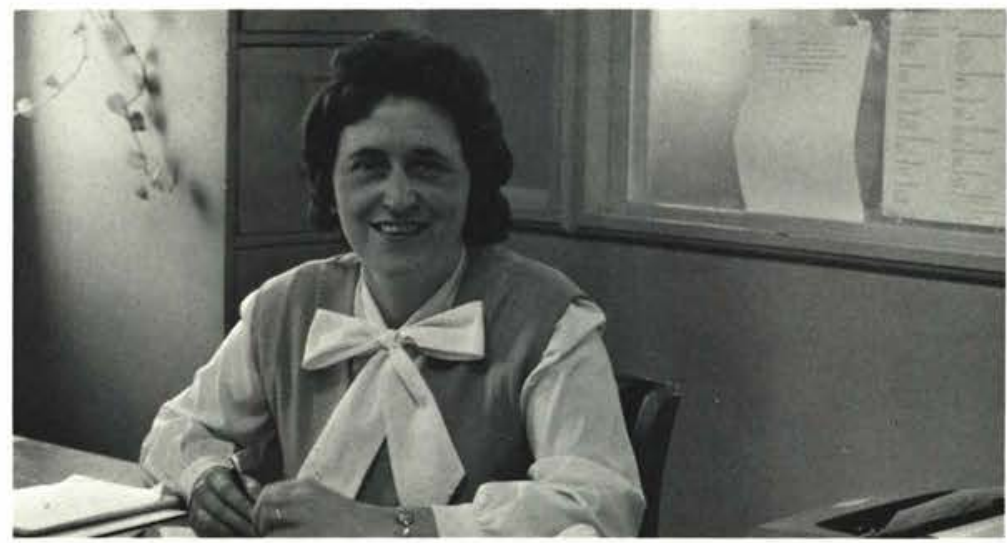

Mrs. May Greenwood

Secretary to Registrar

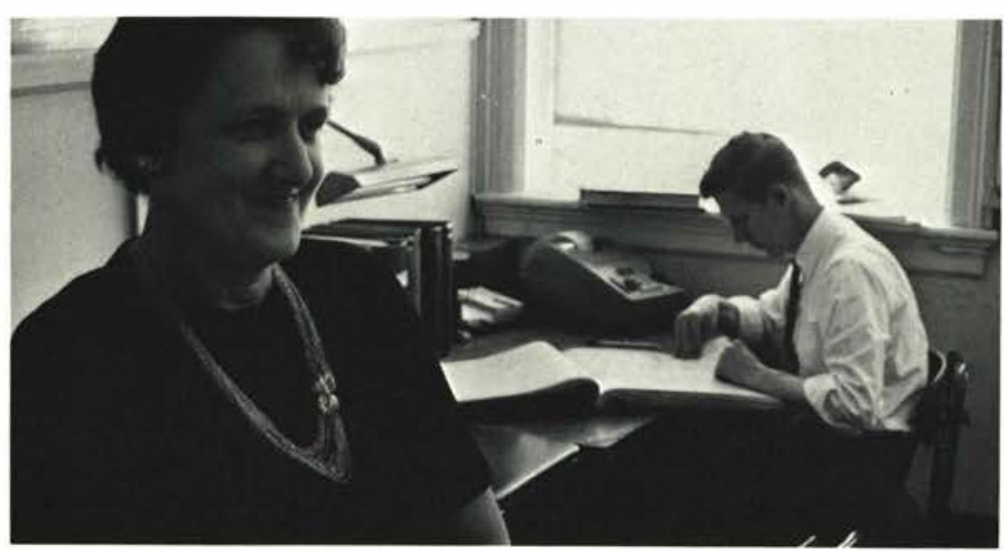

Mrs. Dorothy Spencer, Cashier

Mr. David Gidley, Accountant

Mr. Donald Wilcoxon

Accounts Payable Clerk

Mrs. Marabeth Elmore

Secretary to Business Manager and Receptionist

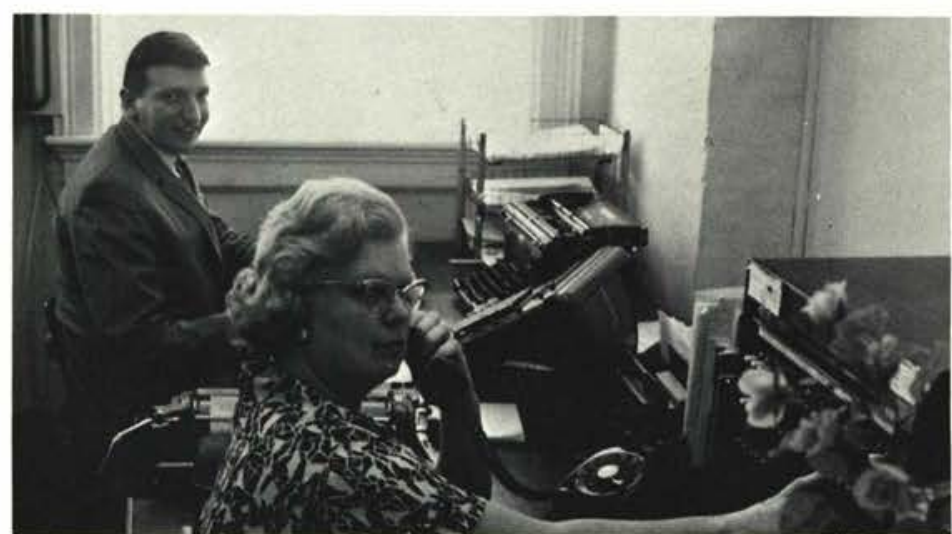




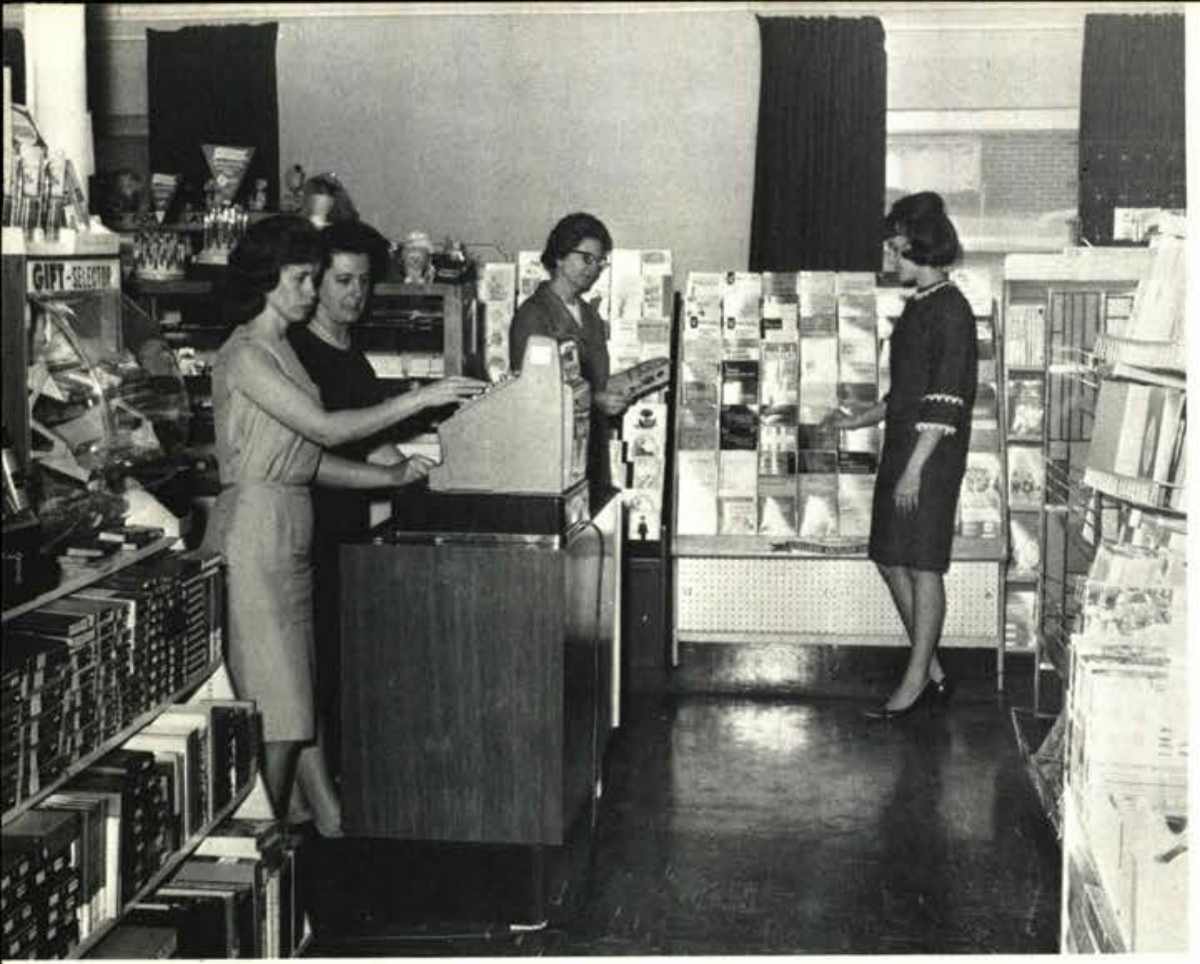

\section{Bookstore Staff}

Mr. D. Gidley, Mrs. B. Bergen, Miss Mick, Bookstore manager, L. Howard

\section{Radio Staff}

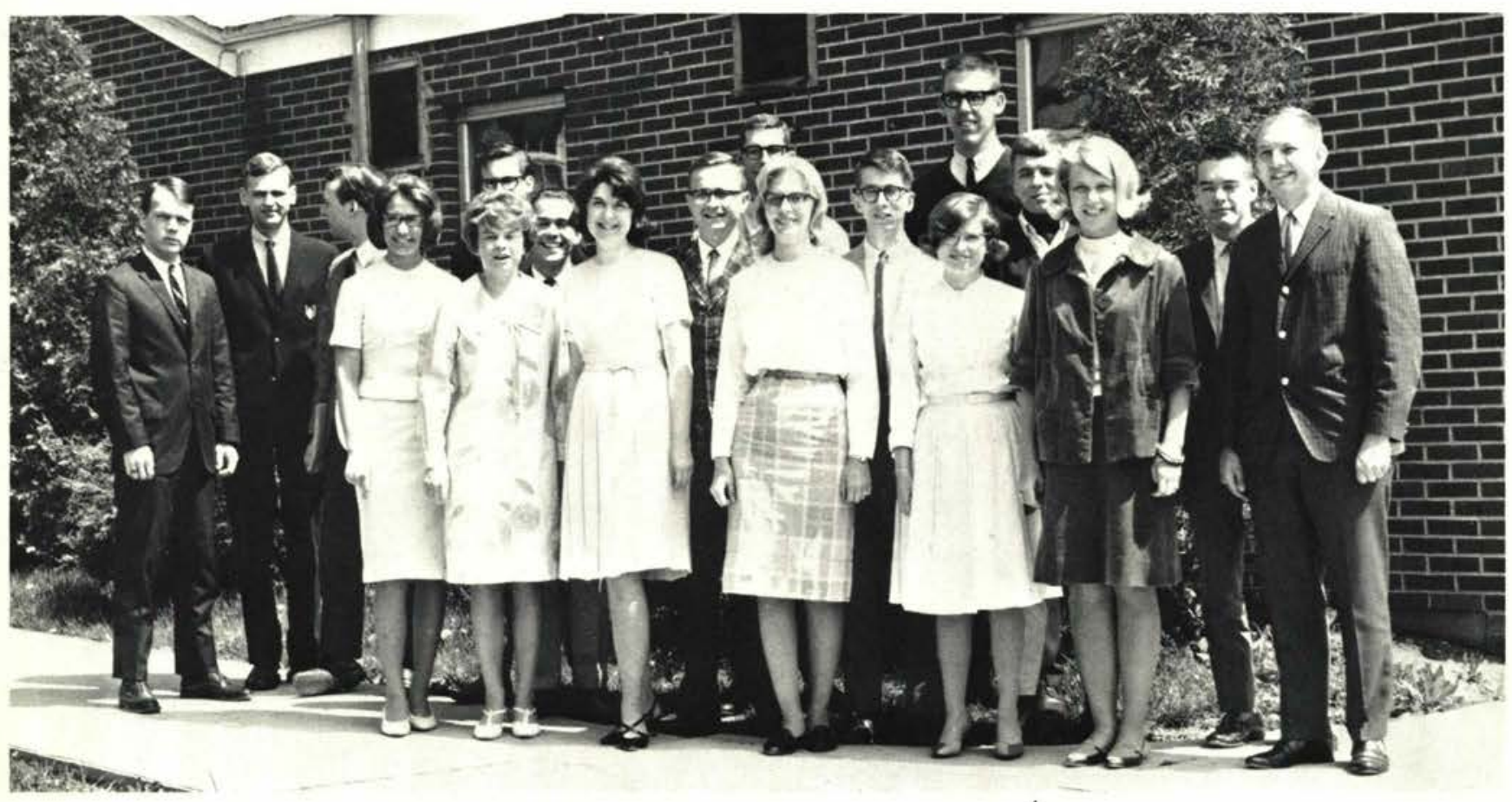

Back row: L. Kytle, A. Whaley, M. Ballard, J. Phillips, S. Griffin, D. Bush. Front row: J. Stockwell, G. Taylor, J. Stockwell, G. Taylor, C. Truxton, W. McVey, L. Waite, C. Hull, G. Goodwin, J. Goodman, J. Blaylock, Mr. Gathney. 


\section{Cafeteria Staff}

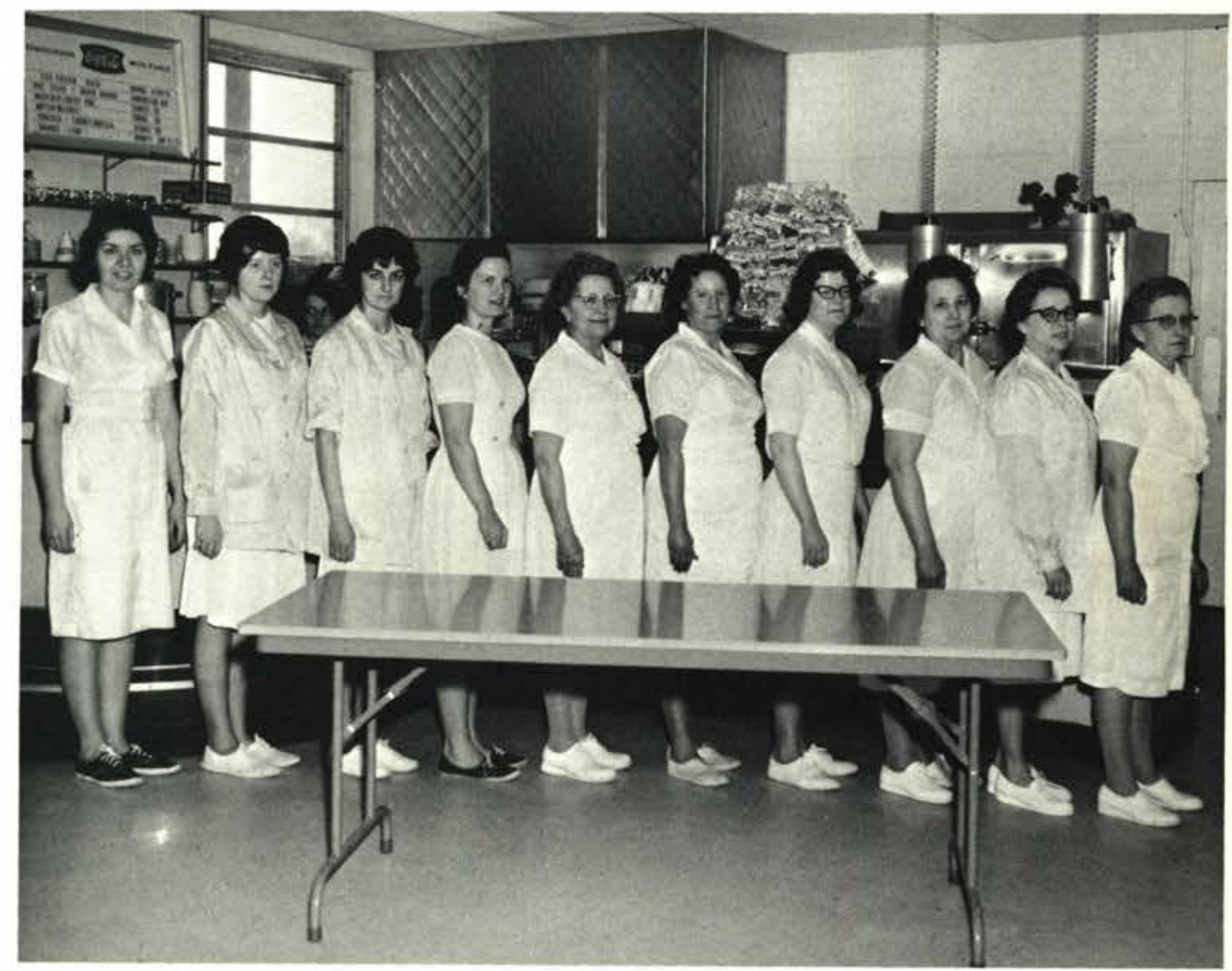

Left to Right: A. Clark, P. Gregg, M. Stuart, C. Baumann, J. Shirley, D. Davis, B. Gentz, P. Taylor, W. Conklin, D. Rasnick.

\section{Maintenance Staff}

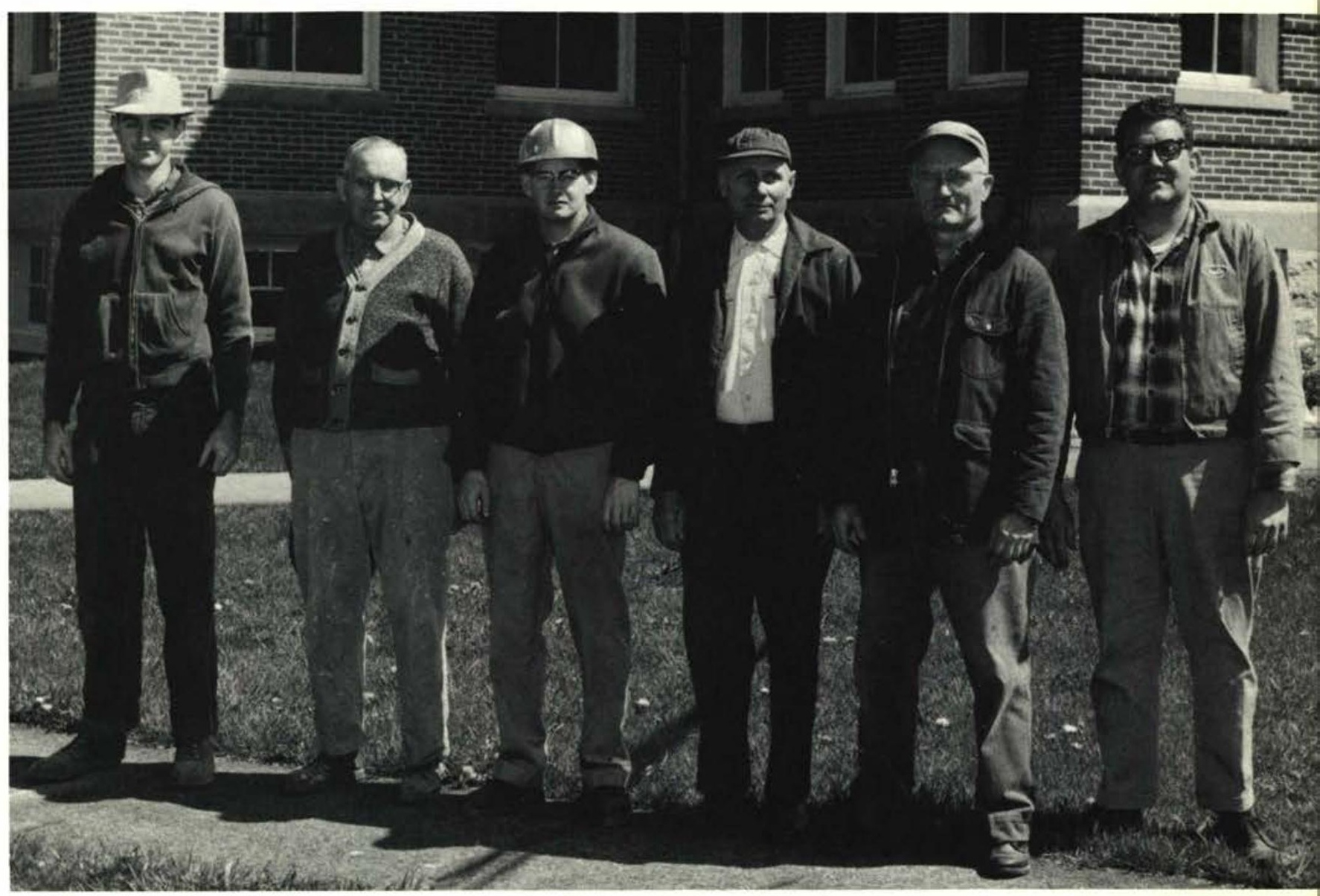




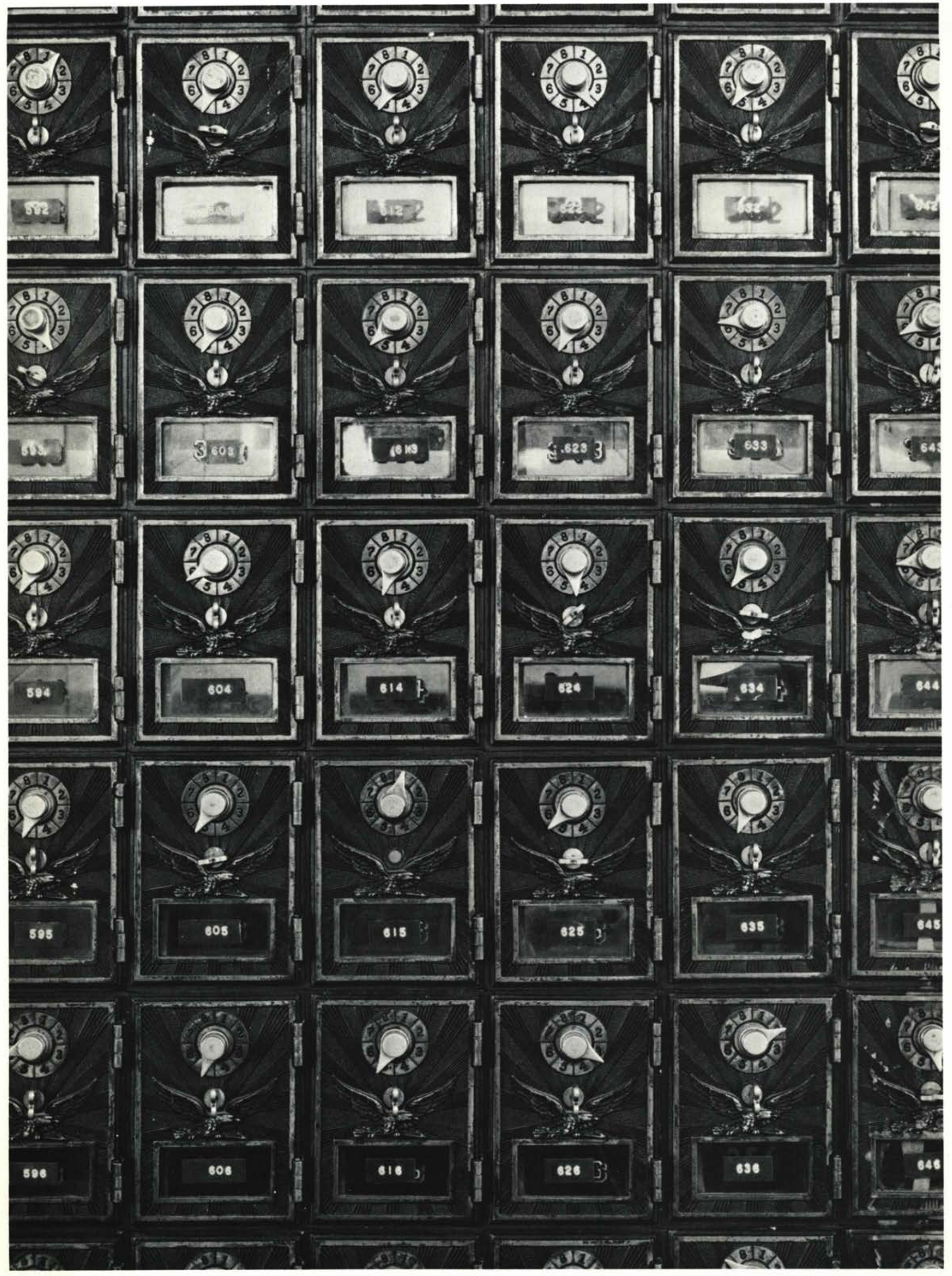




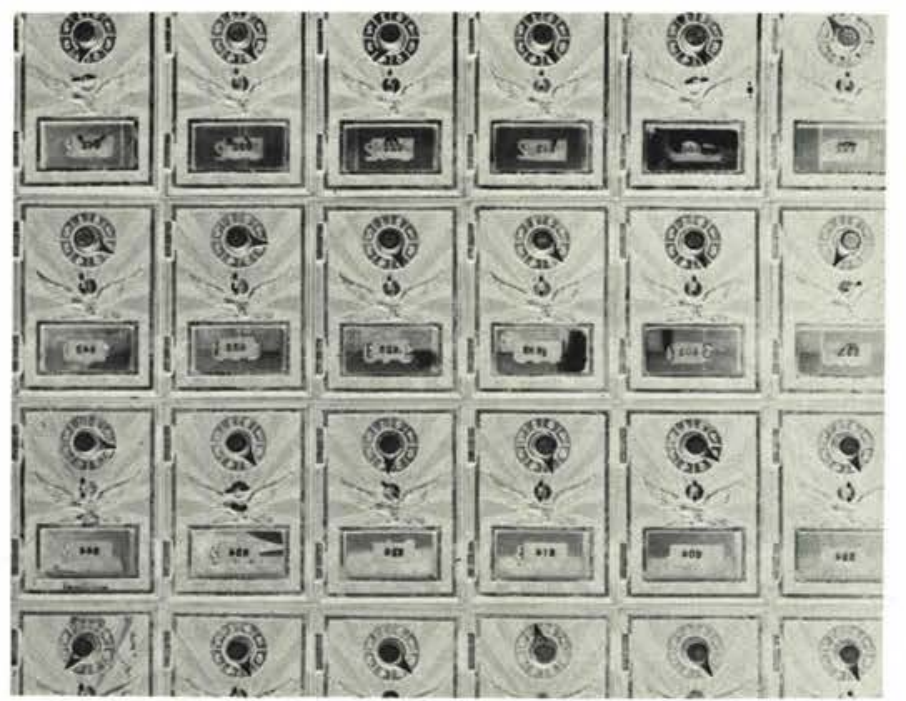

\section{Campus Candids}




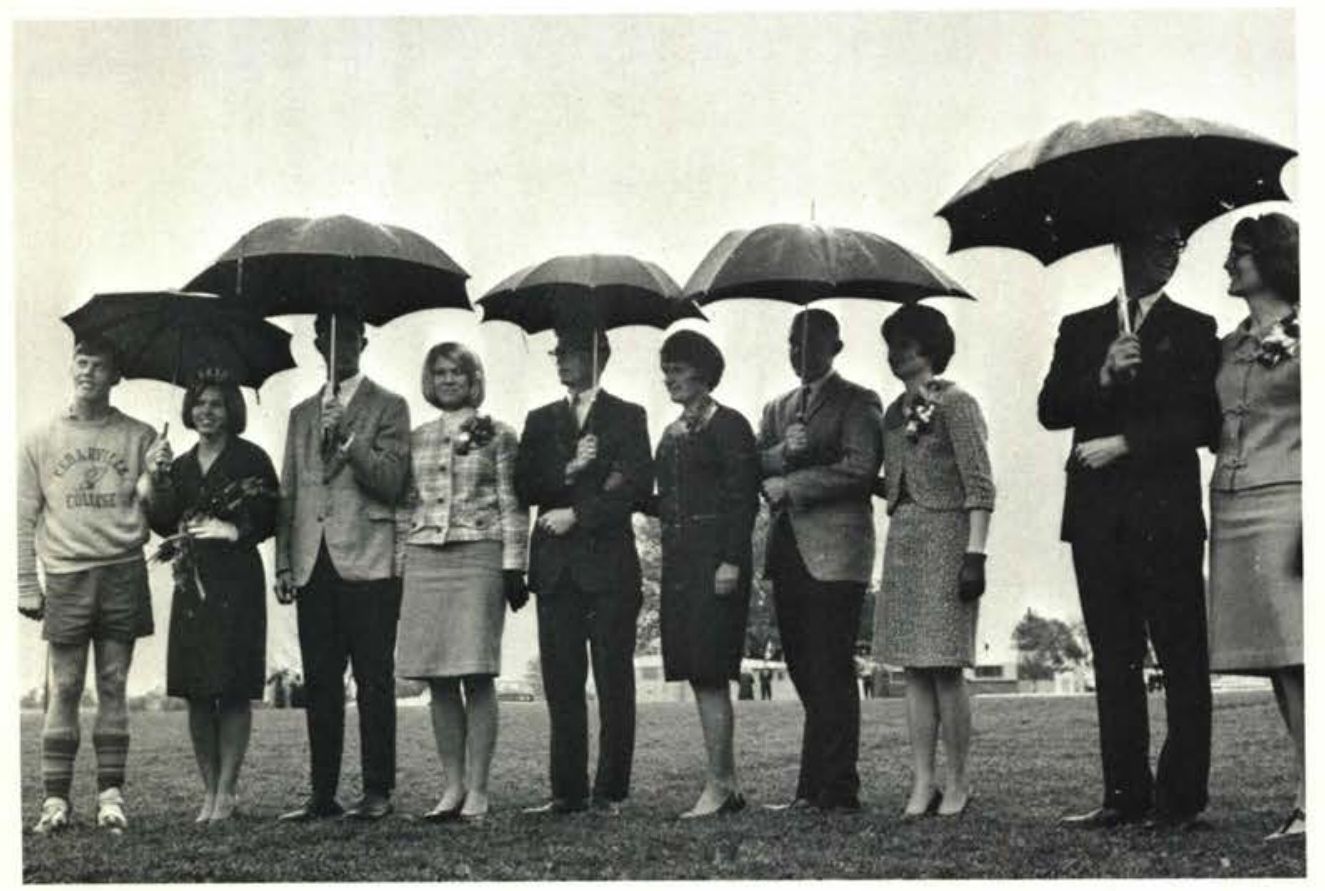

Queen's Court 


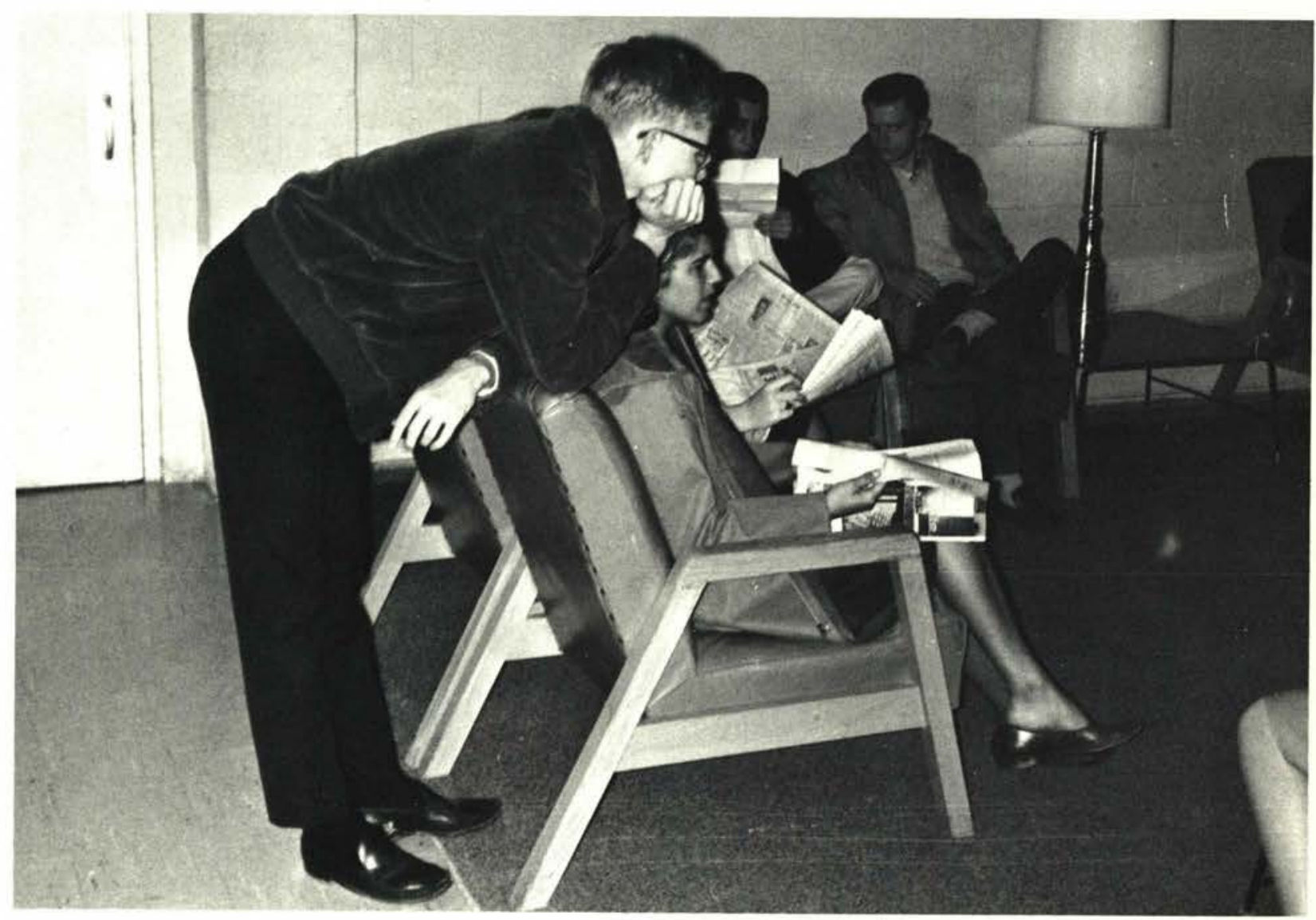

I H

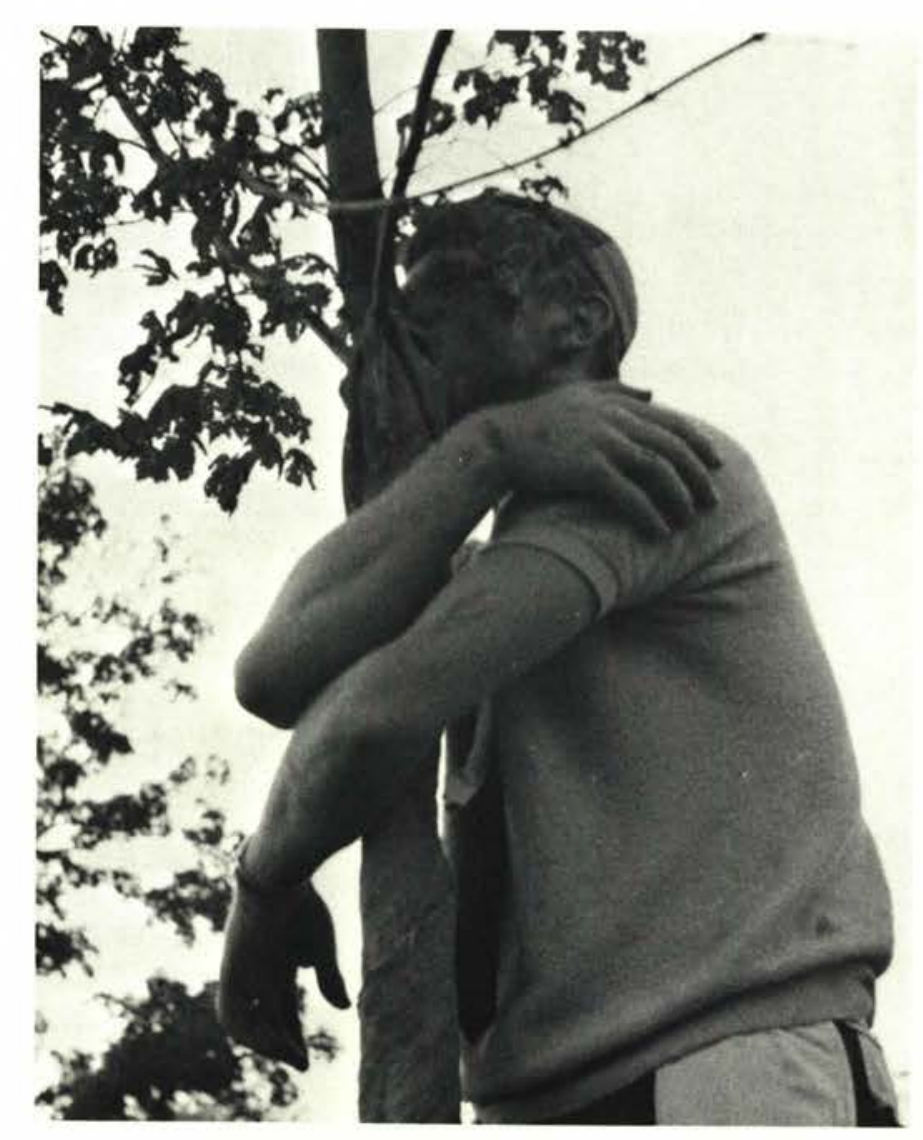



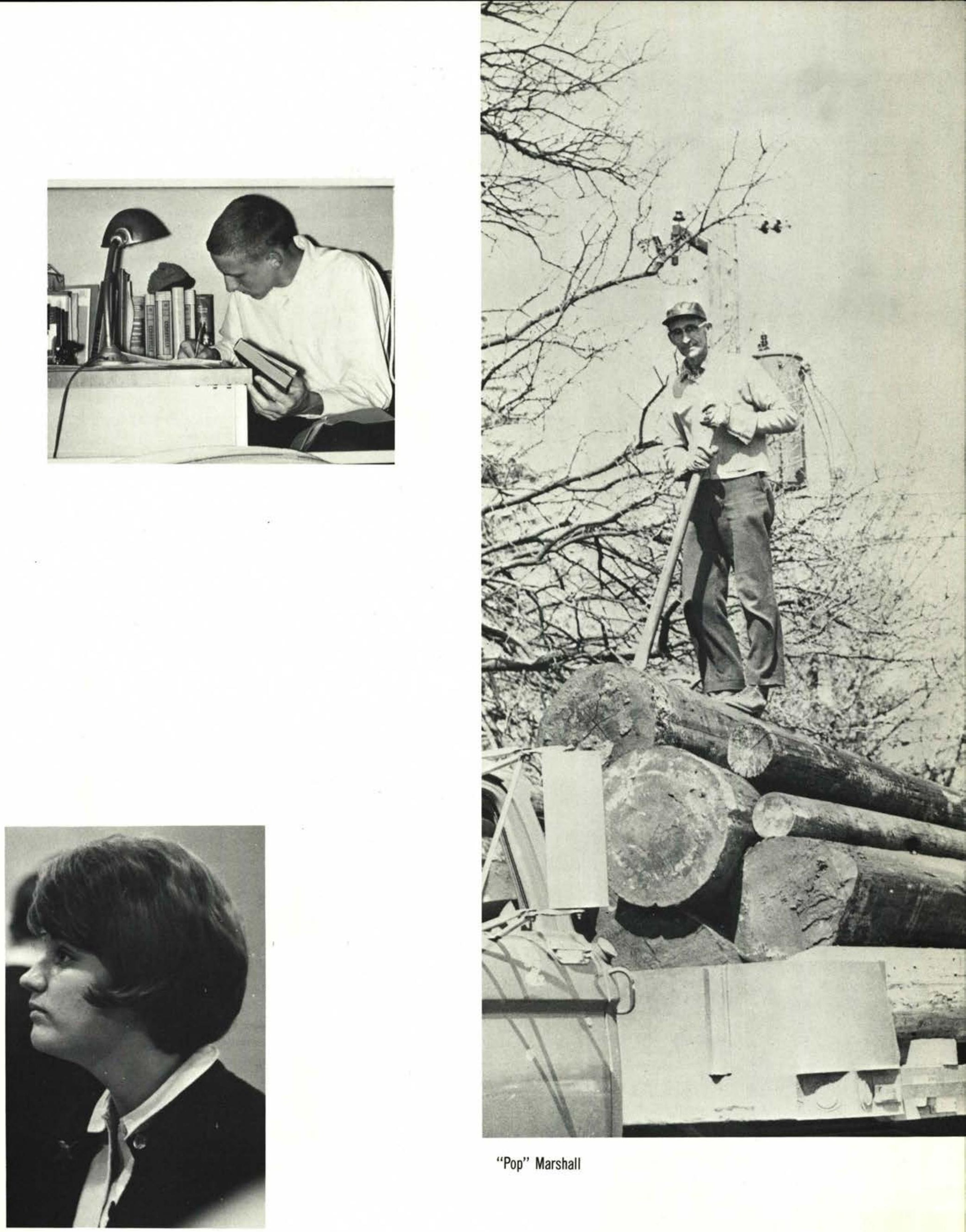

"Pop" Marshall 

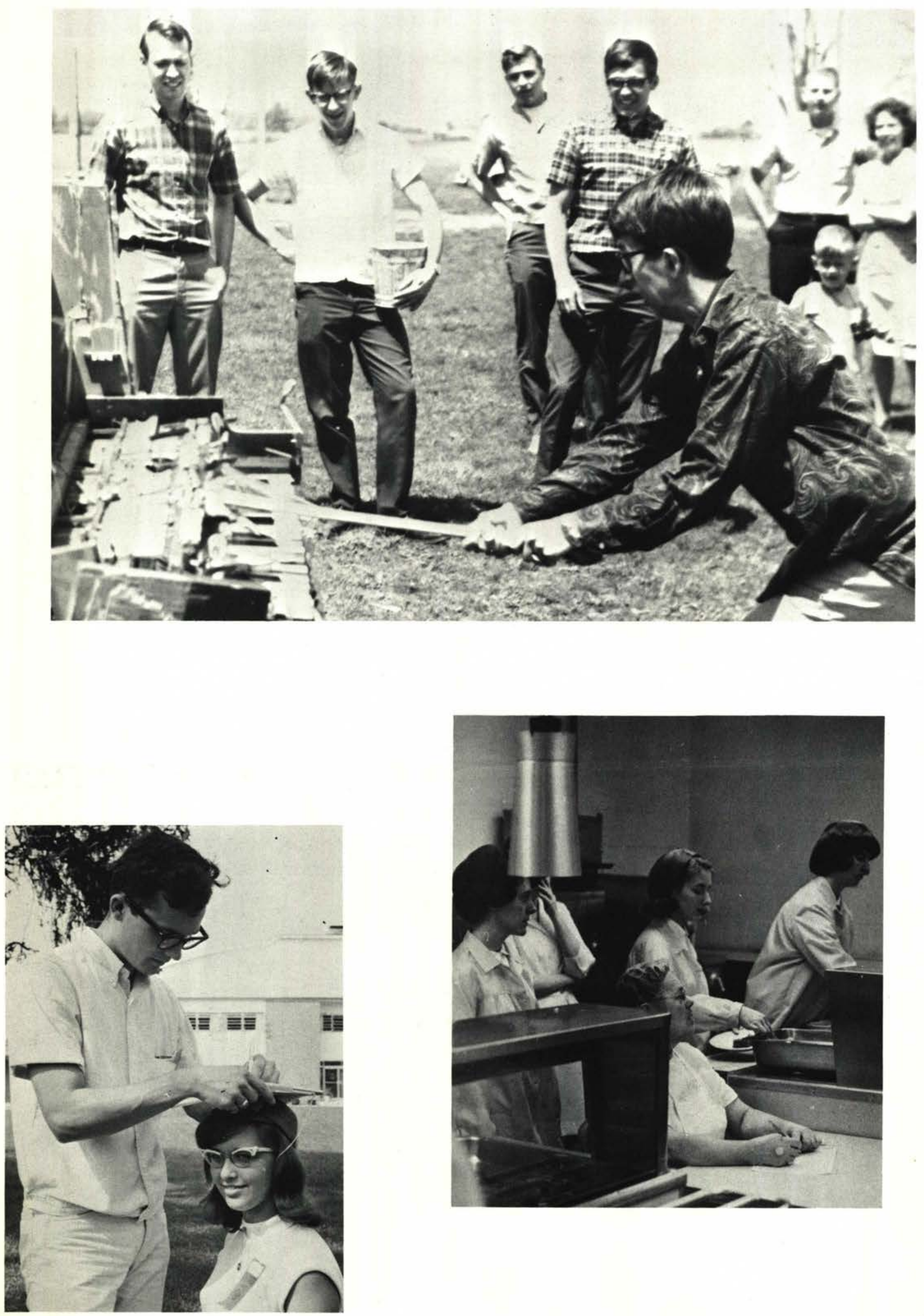

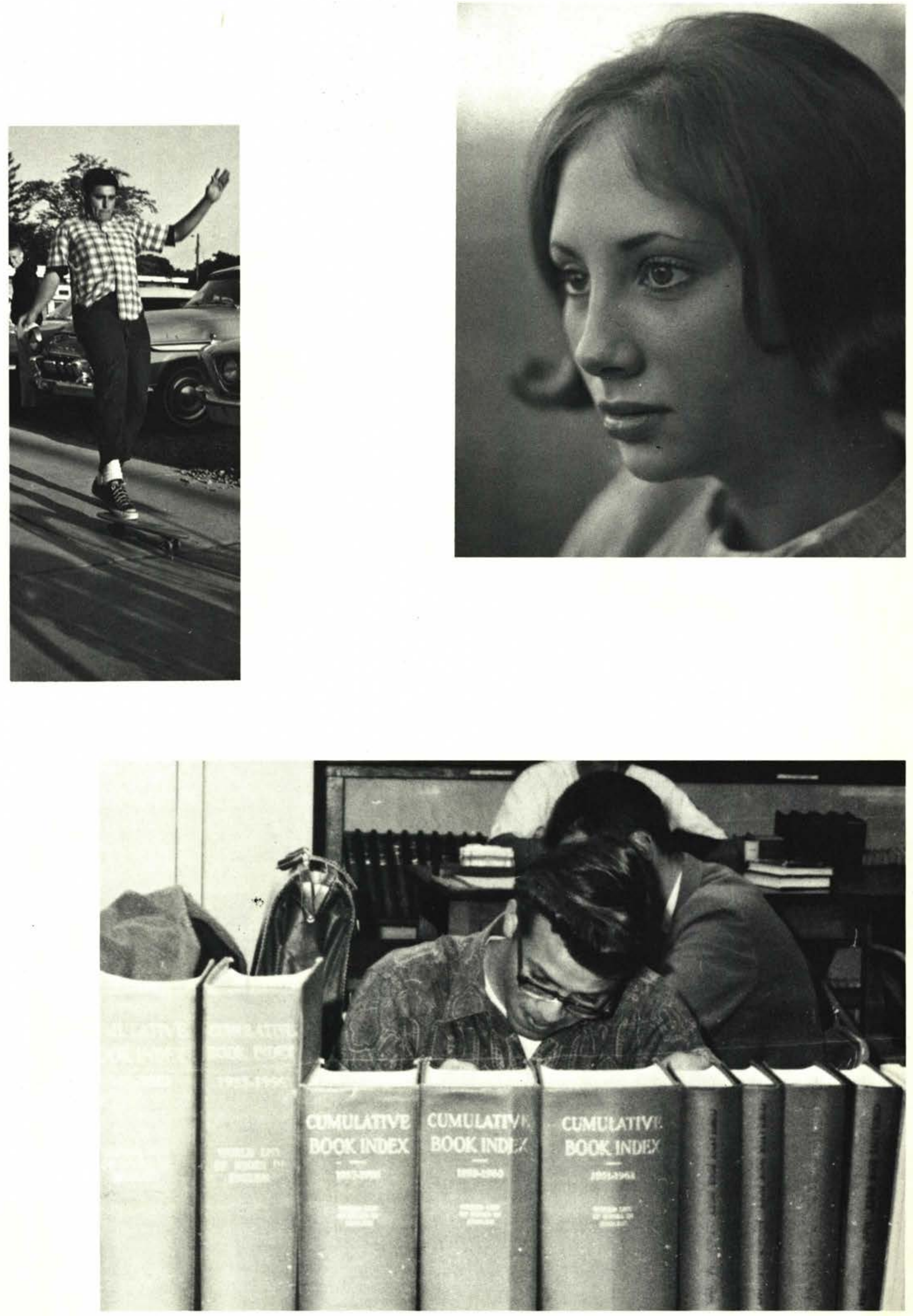

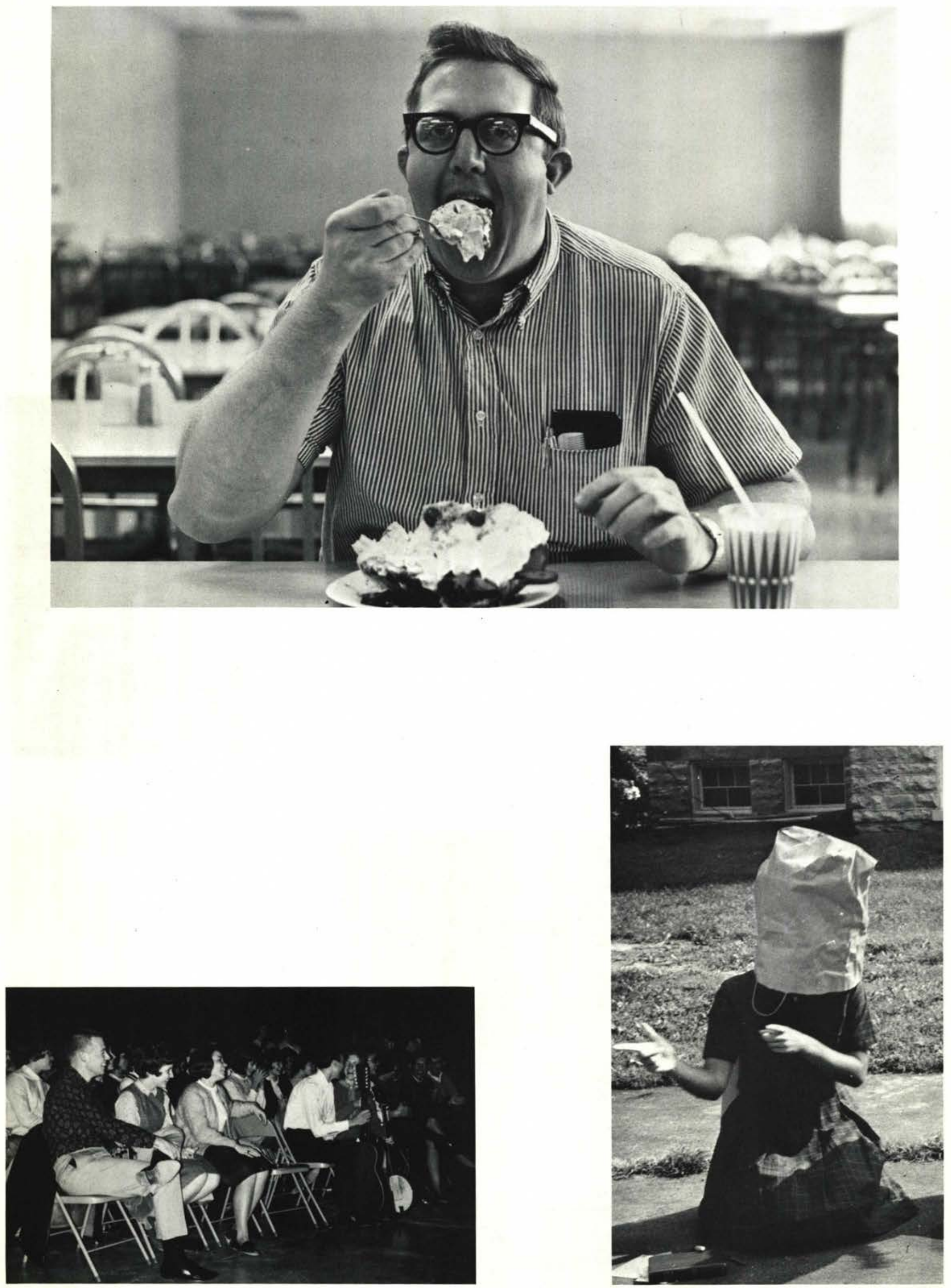

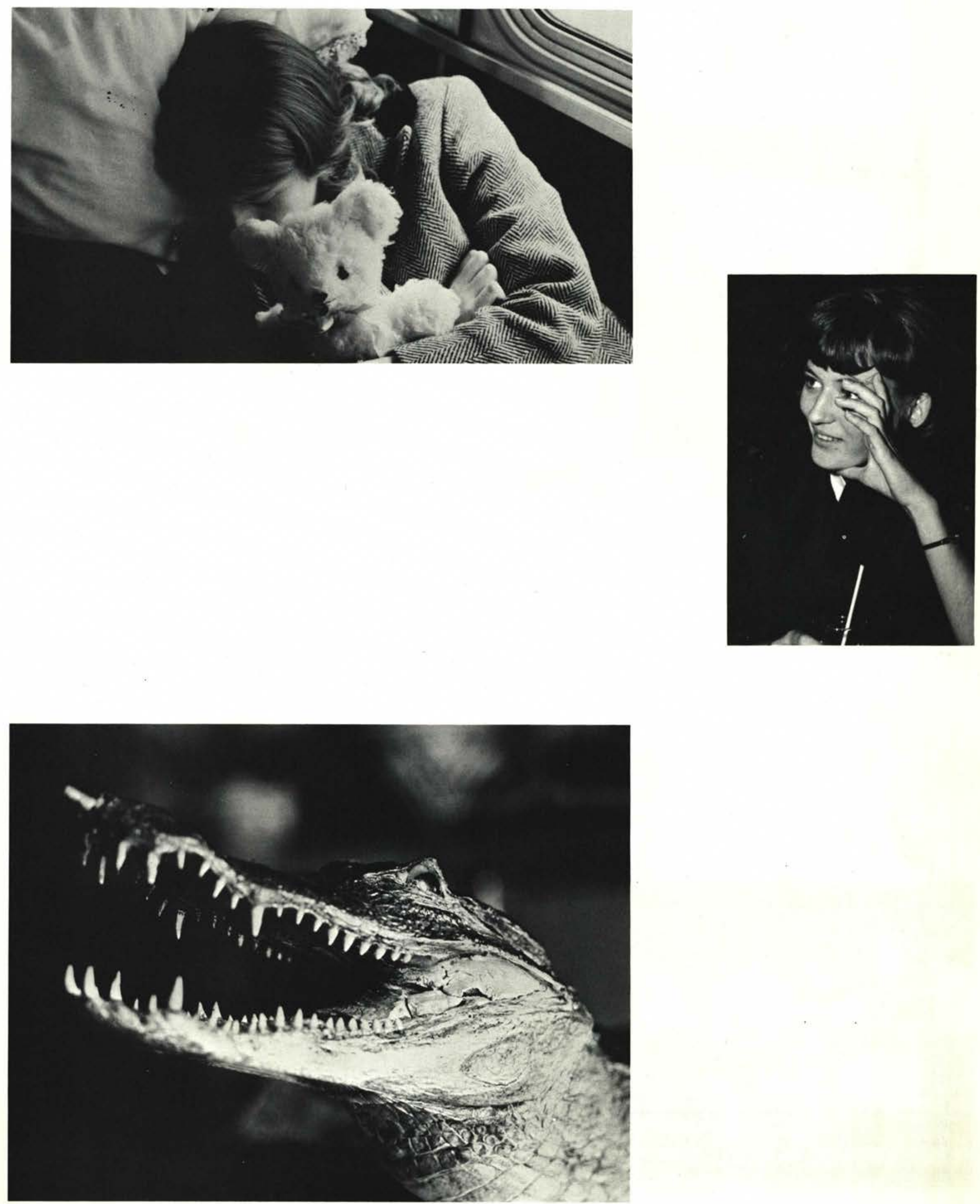

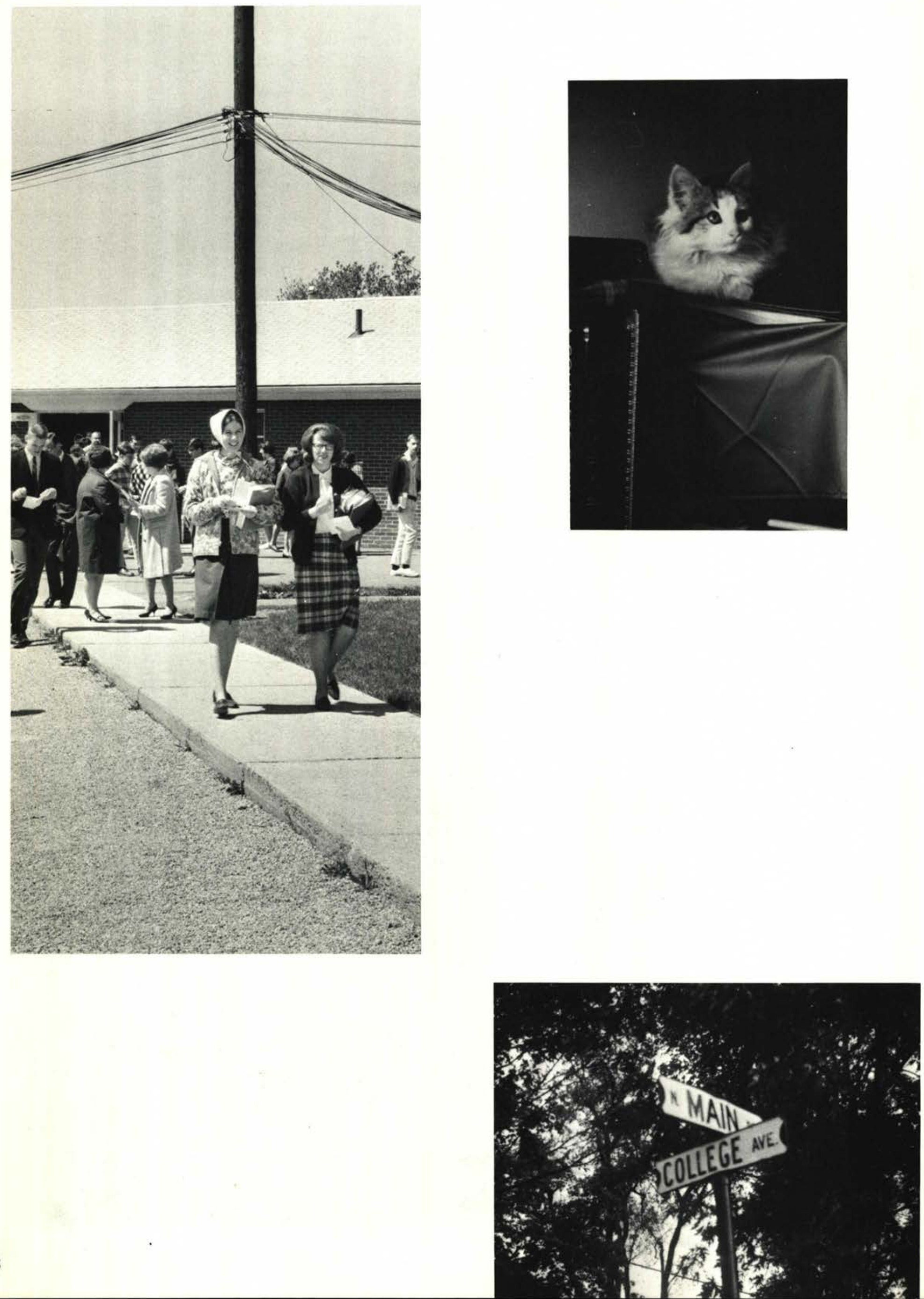


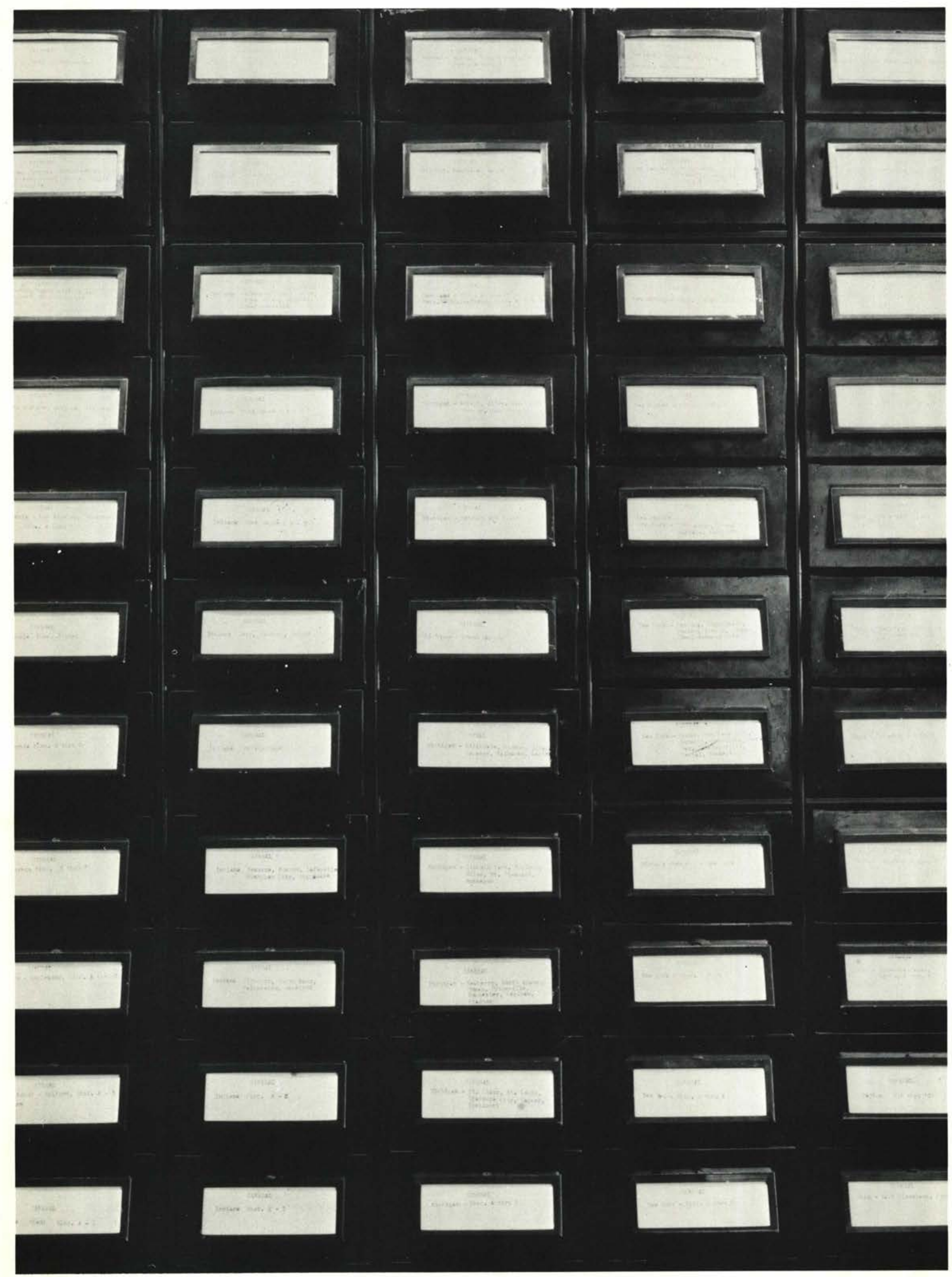




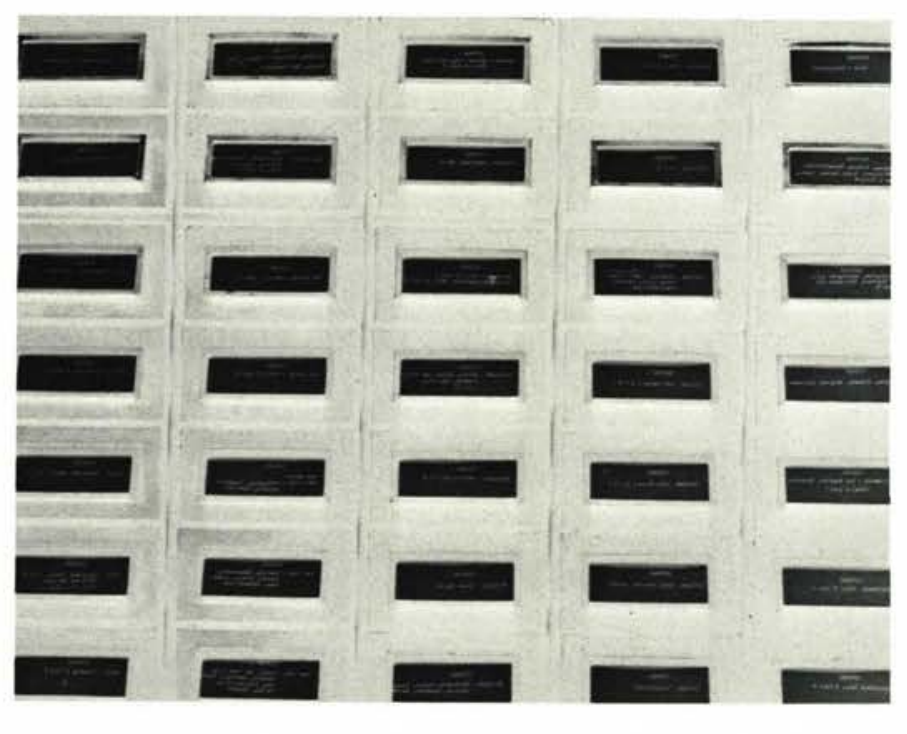

\section{Students}




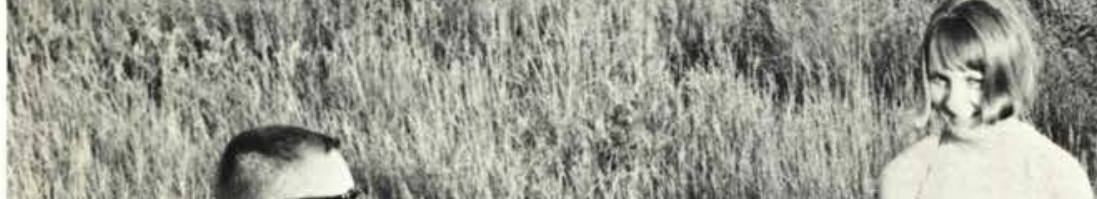

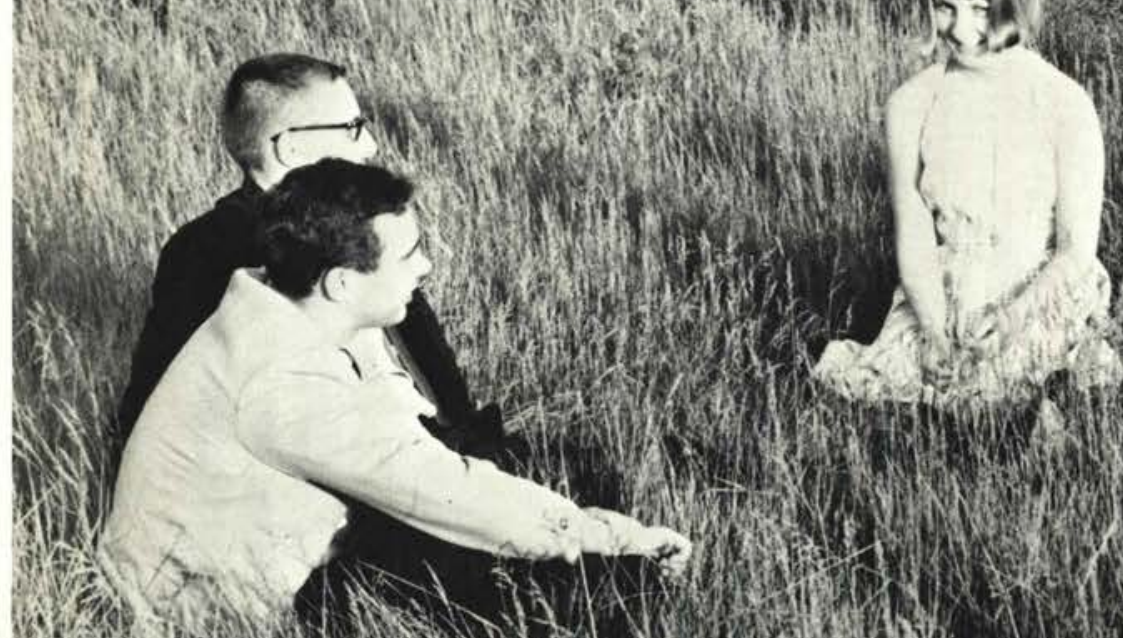

A.t.

Fon. 19 (n) H. $=2$.

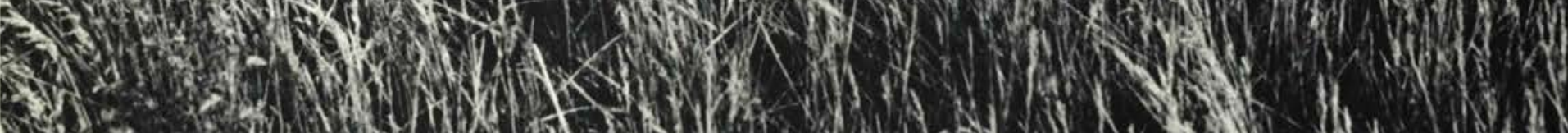

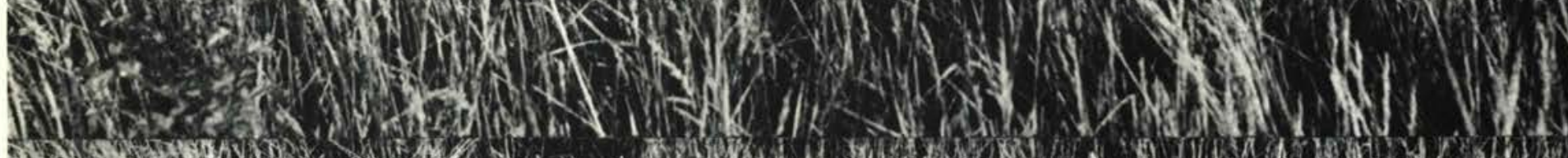

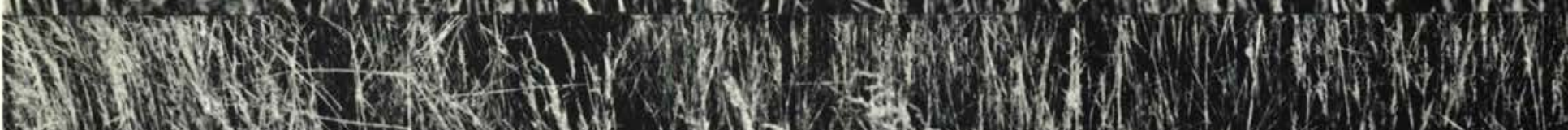
W. 6.5.

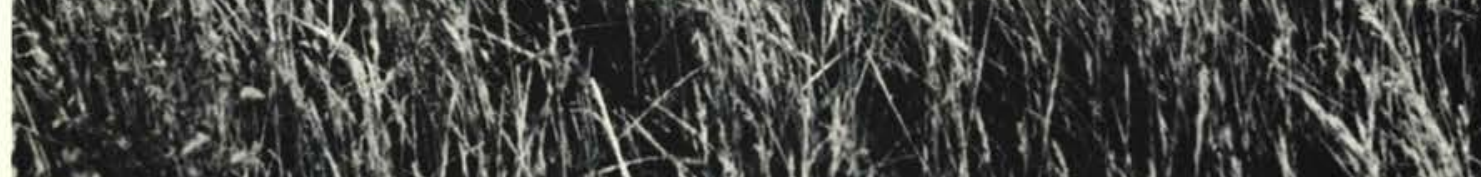

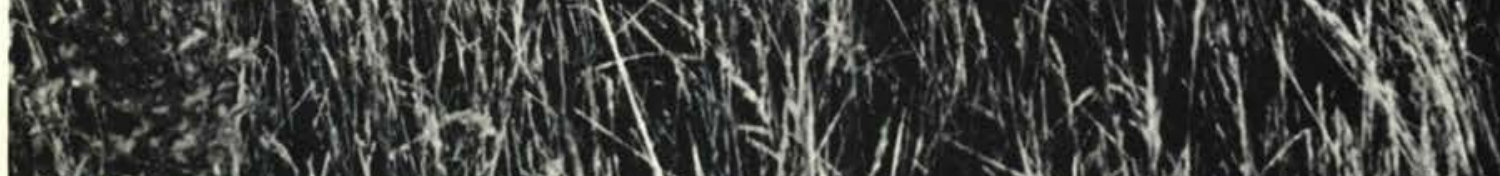

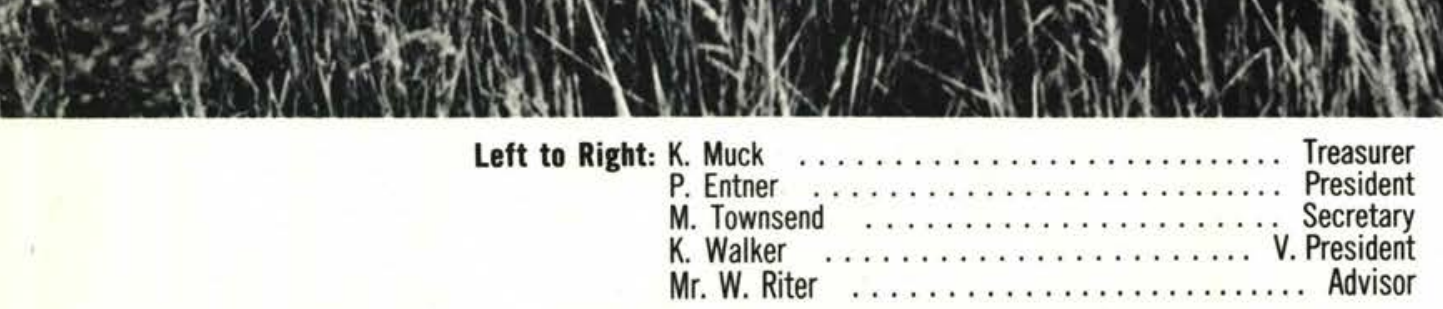


Donald L. Acker

Randolph, New York

Richard R. Adams

New Milford, Connecticut

Charles E. Albright Jr.

Shelby, Ohio

Deane J. Allen

Hurlock, Maryland

Charles B. Anderson

Henry, Illinois

Dave C. Anderson Grand Blanc, Michigan

Margaret S. Anderson

Forsyth, Illinois

Olivia M. Anderson

Berea, Ohio

Gerald P. Auckland Ackport, New York

Lynne C. Babcock Swanton, Ohio

Martha S. Baldock

Rochester, Minnesota

Joanna R. Barcus Valparaiso, Indiana

Roy Barnhart Jr.

Camdan, Ohio

Carol'A. Beerer Elkhart, Indiana

Marilyn A. Beitler

Medina, Ohio

Terry D. Bell

Germantown, Ohio

Loris Bellintani Jr.

Sao Pavlo, Sao Pavlo-Brasil

Cheri L. Benton

East Springfield, Pennsylvania

James L. Berry

Medina, Ohio

Nancy A. Bertram

Boise, Idaho

Janice M. Best

Milwaukee, Wisconsin

Robert H. Bixel

Bellefonte, Pennsylvania

Carol A. Blake

Akron, Ohio
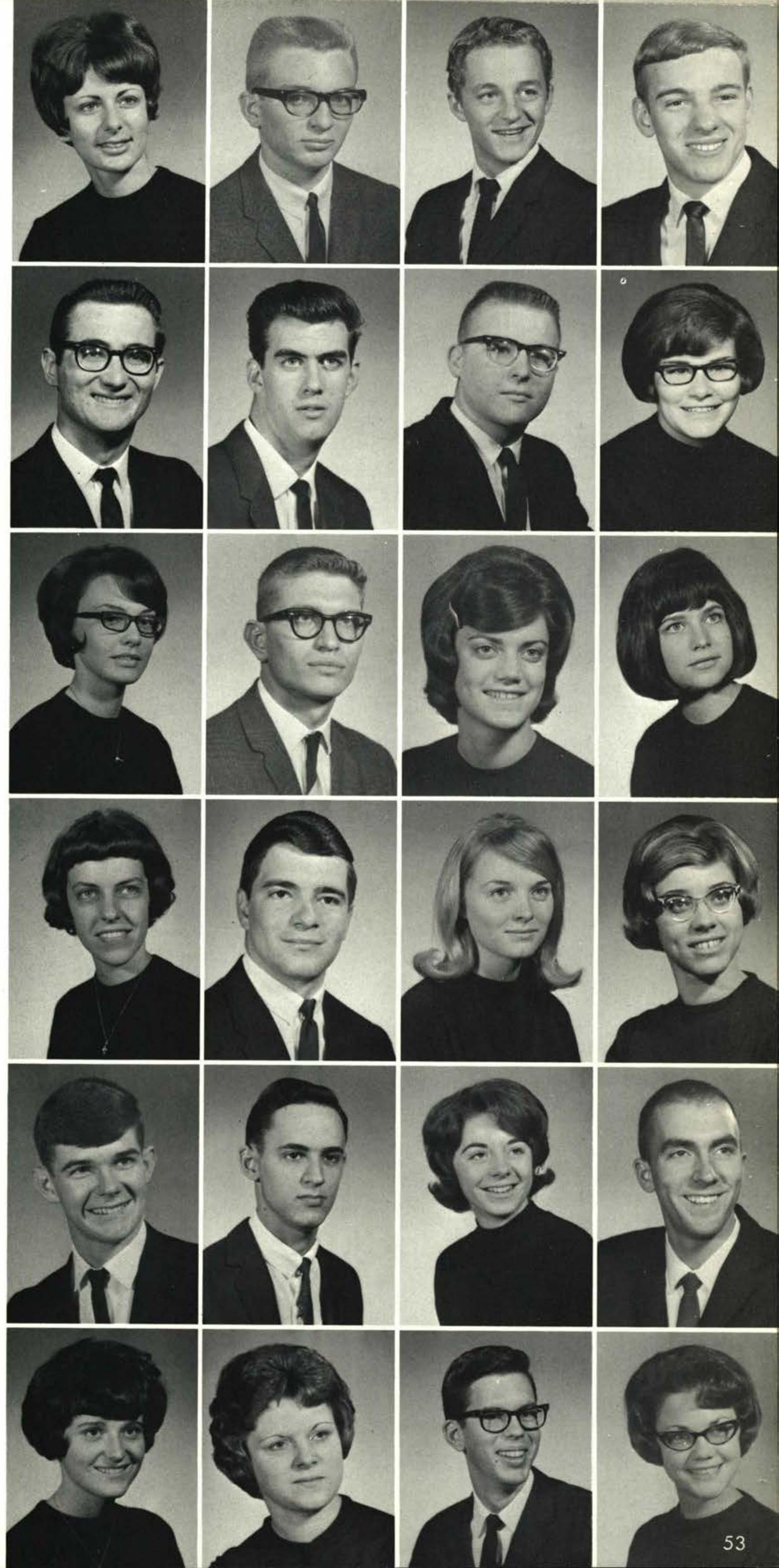
Timothy Carpenter Waterloo, New York

Kathy Carter

Winterset, lowa

M. Kathleen Cartner Markham, Illinois

Diane Chaffe

Cedarville, Ohio

Judith Chapman Gallipolis, Ohio

\section{David Chumbler} Gary, Indiana

\section{Barbara Cleckner} Salem, Ohio

\section{Donley Clithero} Sciotoville, Ohio

Esther Colgan DuBois, Pennsylvania

Cherrill Collins

Sao Paulo, Brazil

James Comden Lakeview, Michigan

Joy Conant

Hasbrook Heights, New Jersey
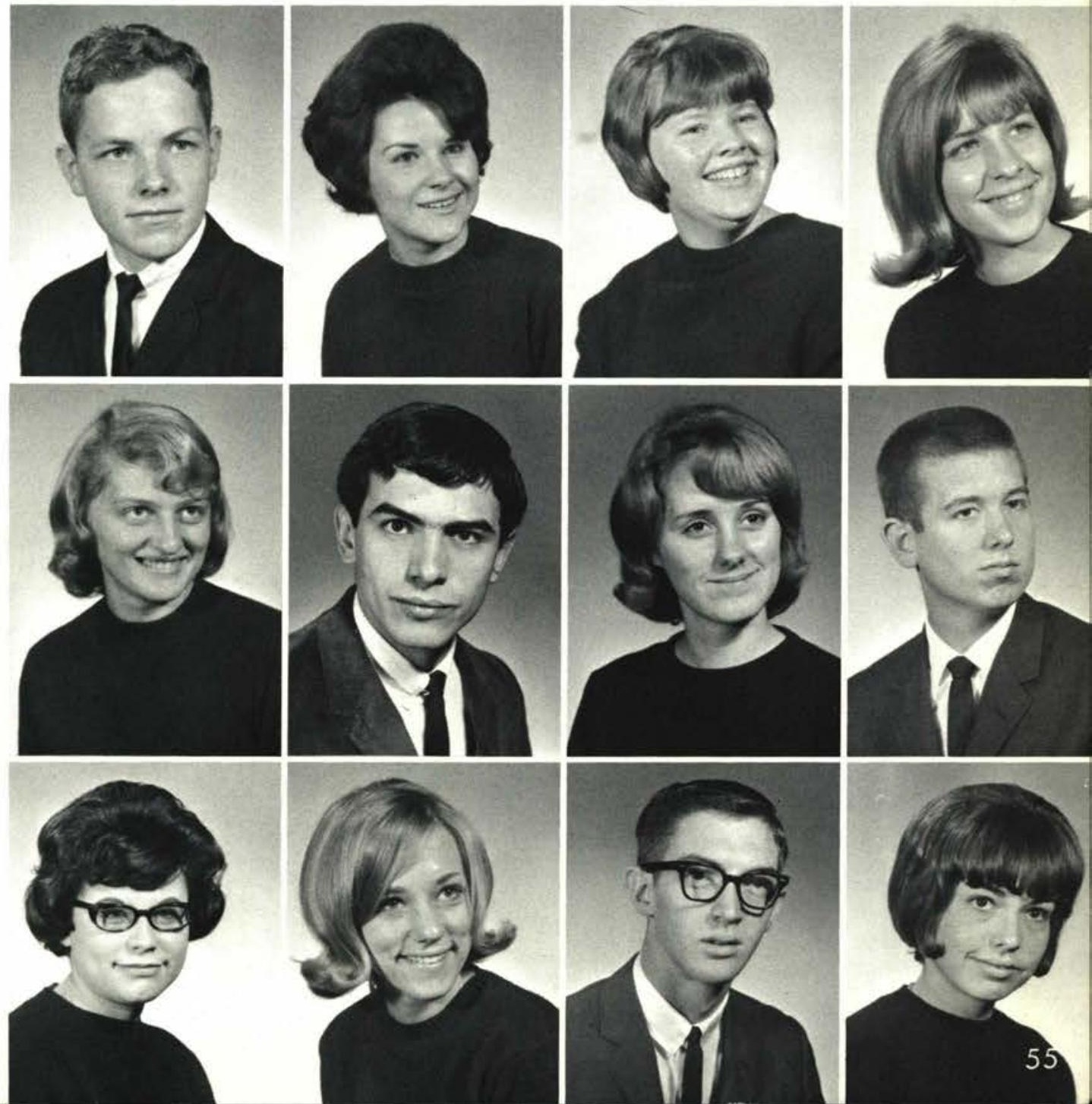

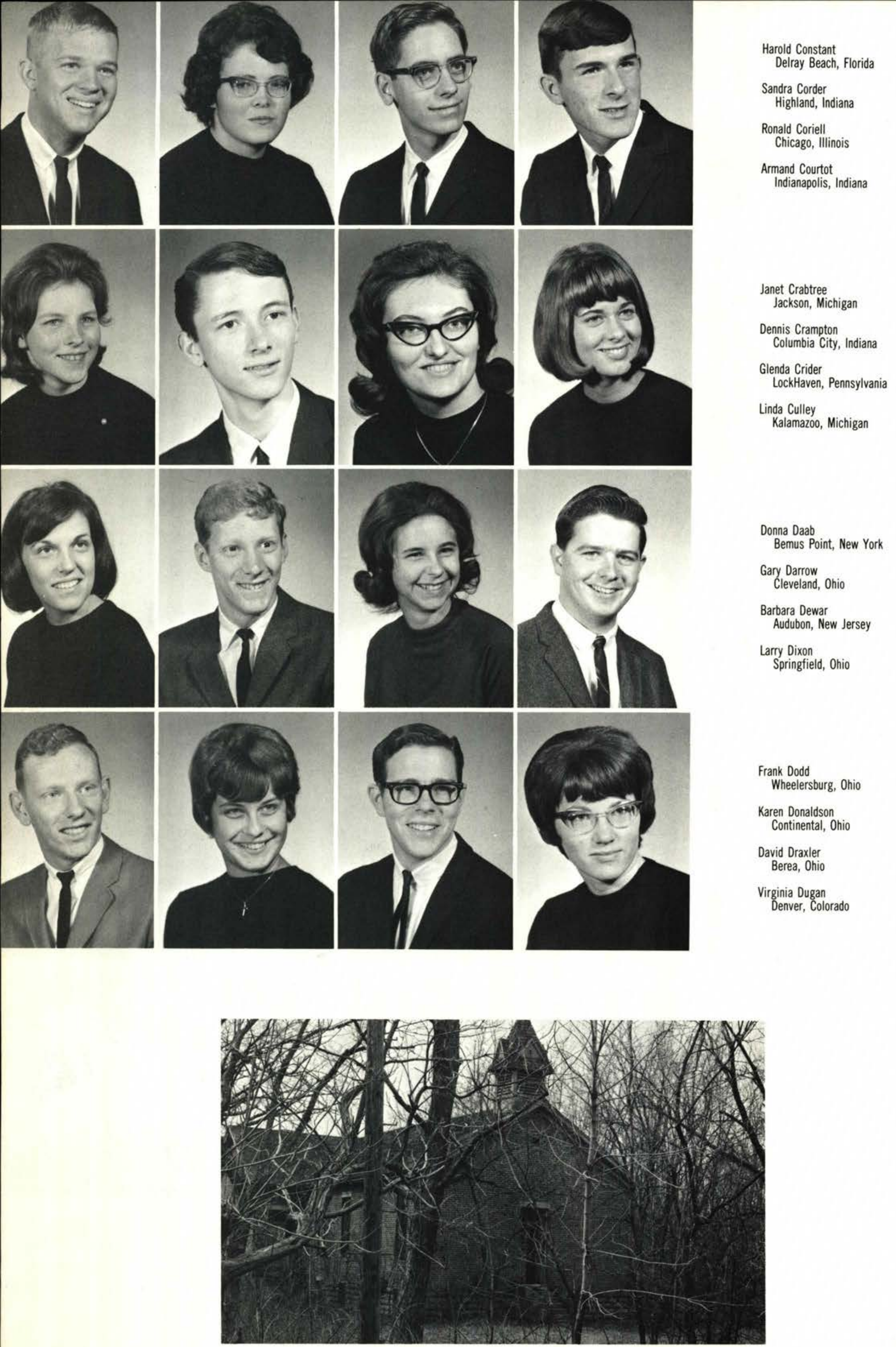


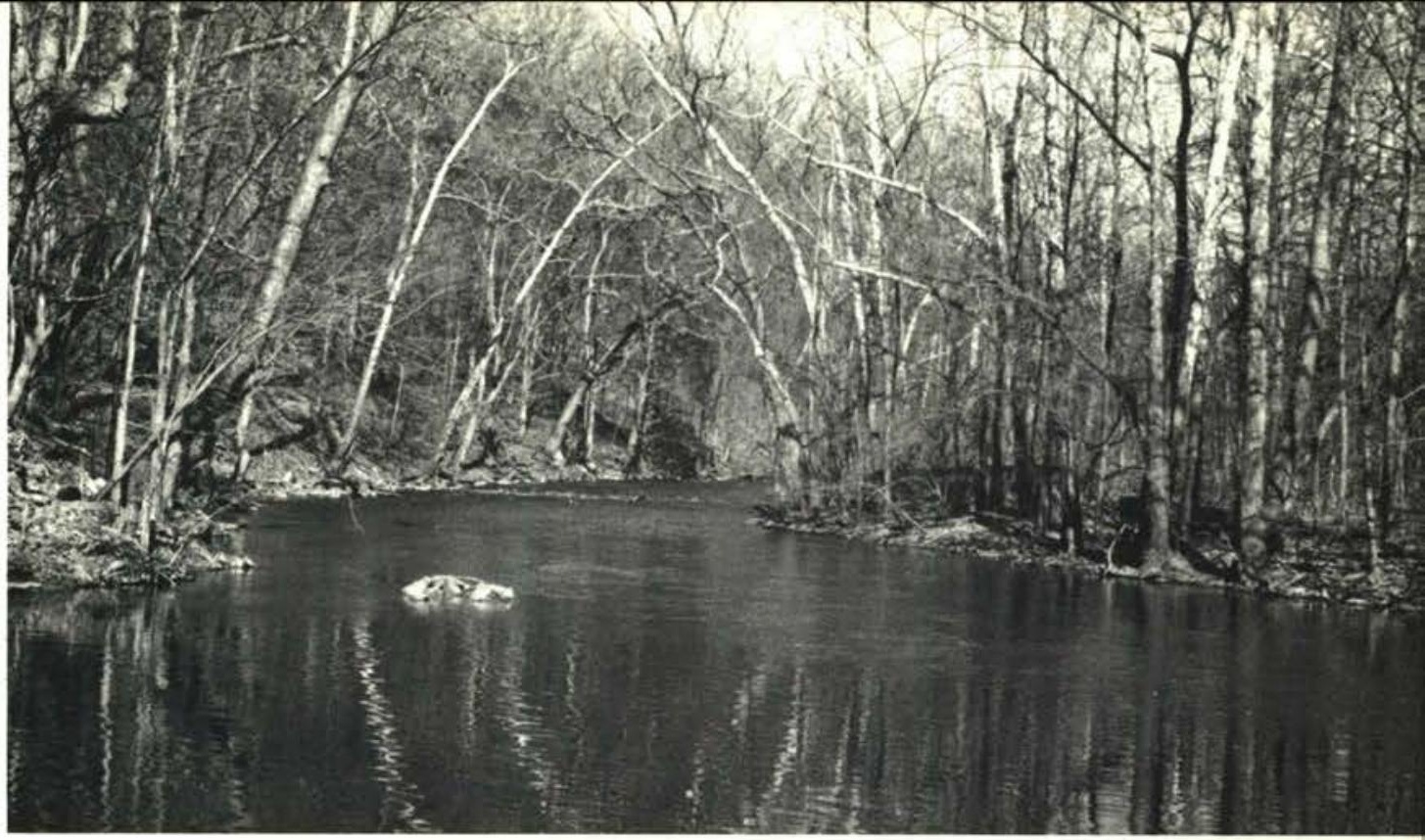

Pamela Dutie

Lima, Ohio

Leslie Edwards Albion, lowa

Carole Emmons Harvey, Illinois

Sarah Empet

Kingsley, Pennsylvania

Emily Entenman

Evansville, Indiana

Paul Entner

Dunkirk, Indiana

Mary Felton

Dayton, Ohio

Steve Fenton

Waterloo, lowa

Joyce Ferris

Sandwich, Illinois

Darlene Fitch

New Castle, Pennsylvania

Barbara Fleming

Lancaster, Ohio

Nancy Freeman

Alberta, Canada
French, Shirley

Kalamazoo, Michigan

Stanley Fries

Maple Shade, New Jersey

Patricia Fulop

Windsor, Ohio

David Gaffner

Williamston, Ohio
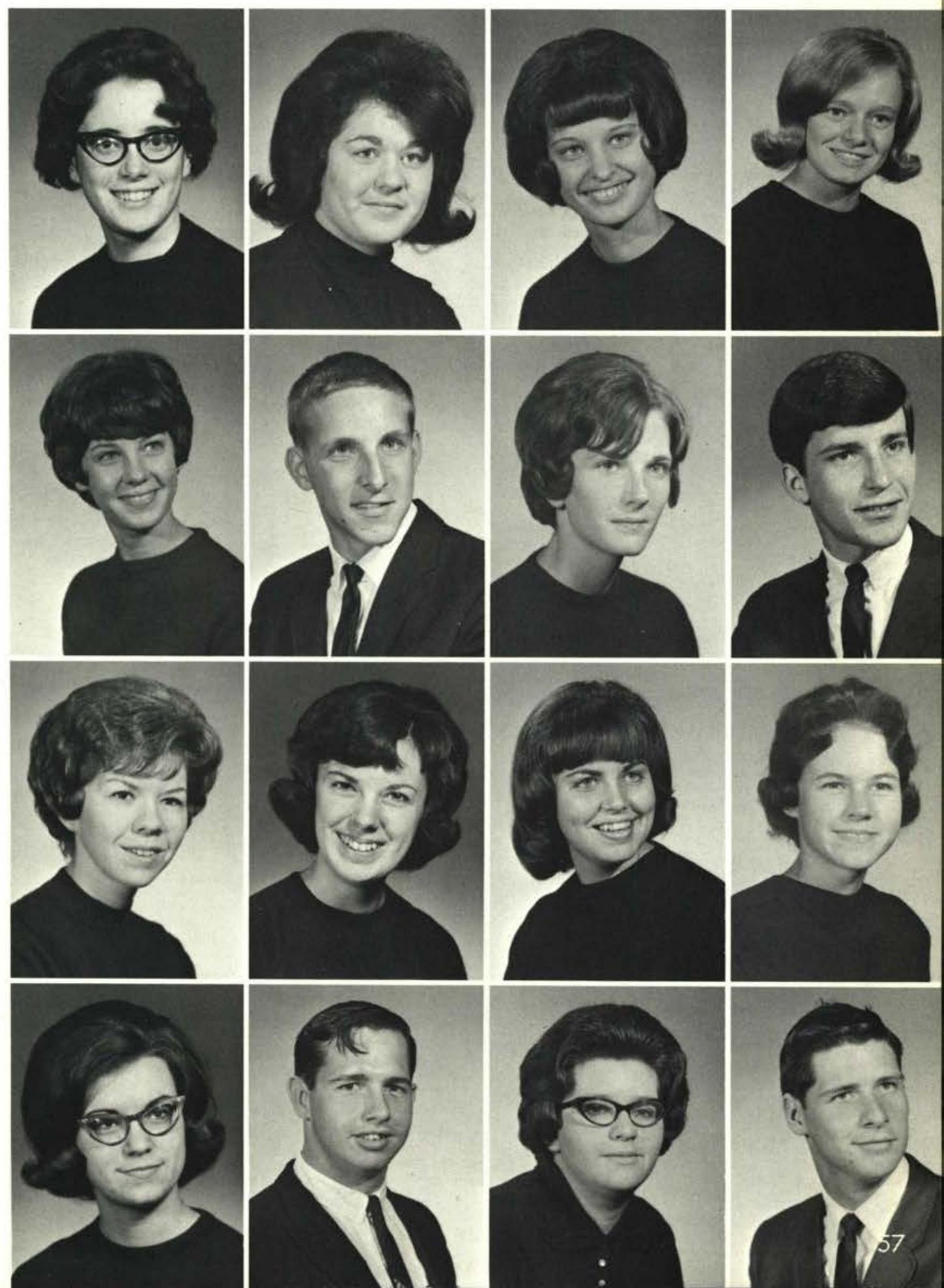


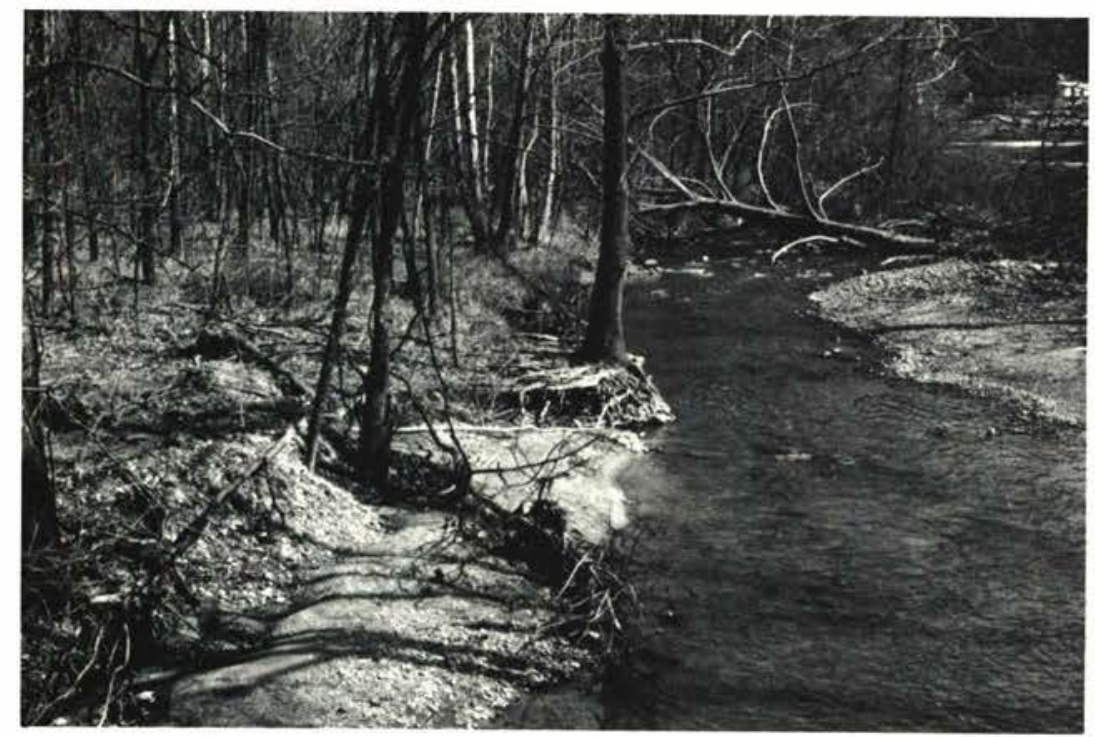

Thomas Greenwood Lorain, Ohio

Julie Gregg

Fulton, Illinois

Eunice Griffes Madison, Ohio

David Haffey

Canal Winchester, Ohio

Terry Hall

Decatur, Illinois

James Hamacher Eau Gallie, Florida

Donna Hamilton Alton, Illinois

David Handyside

Fairport, New York

Cedarville, Ohio

Elaine Hannah

Farrel, Pennsylvania

Ruth Hardy

Mashawaka, Indiana

Carol Hare

Cedarville, Ohio

Nancy Harper

Lorimor, lowa

Gerald Harrison End Well, New York

Ruth Hartikainen Erie, Pennsylvania

Harold Hassenruck Olmsted, Ohio
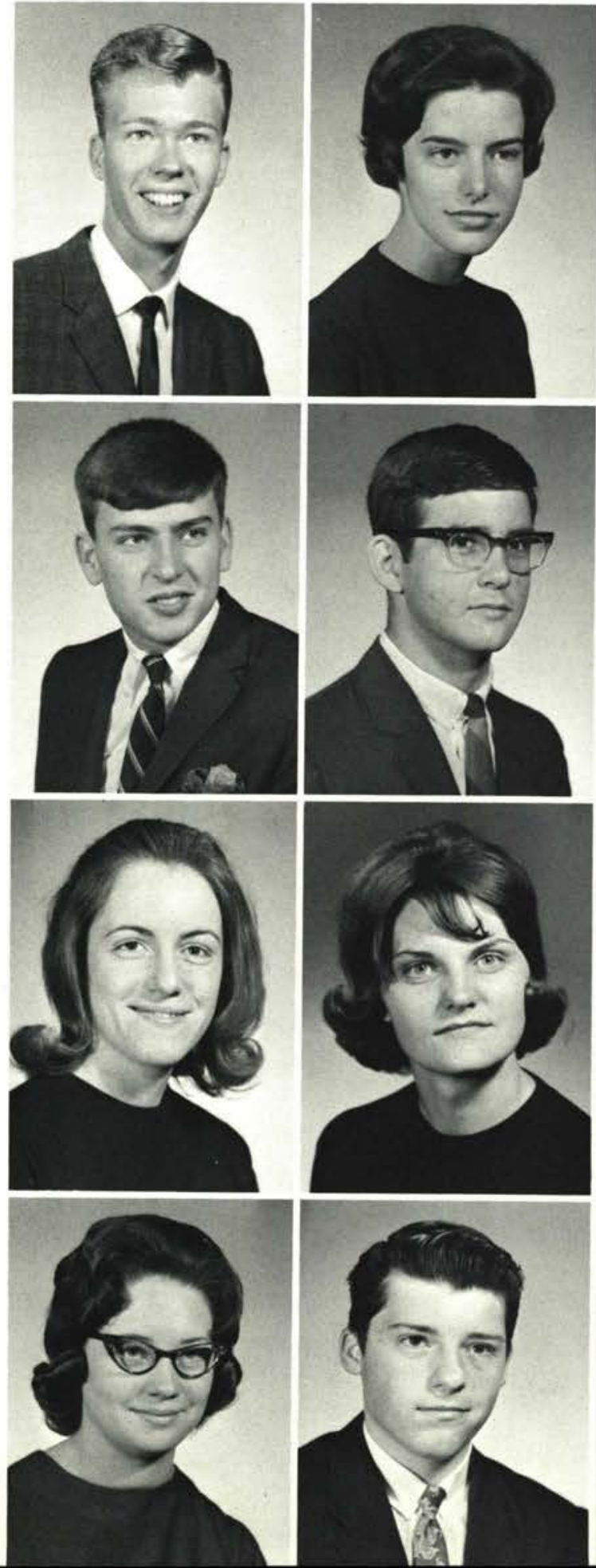
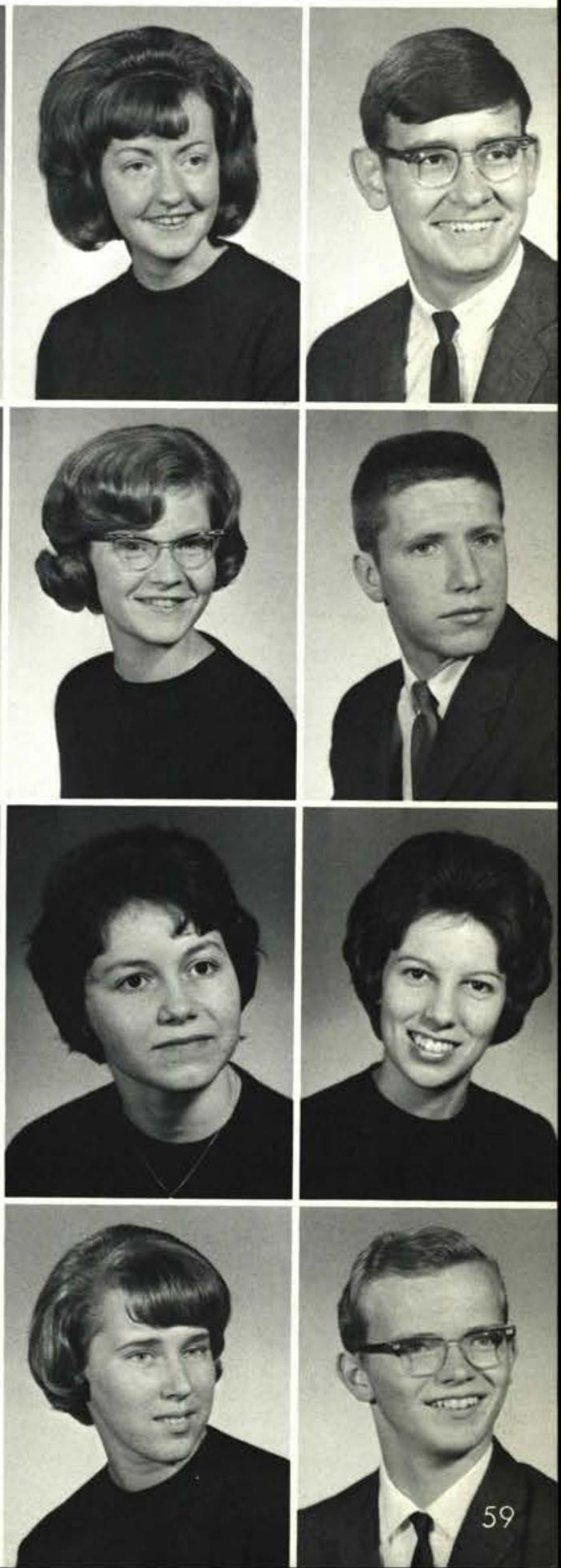

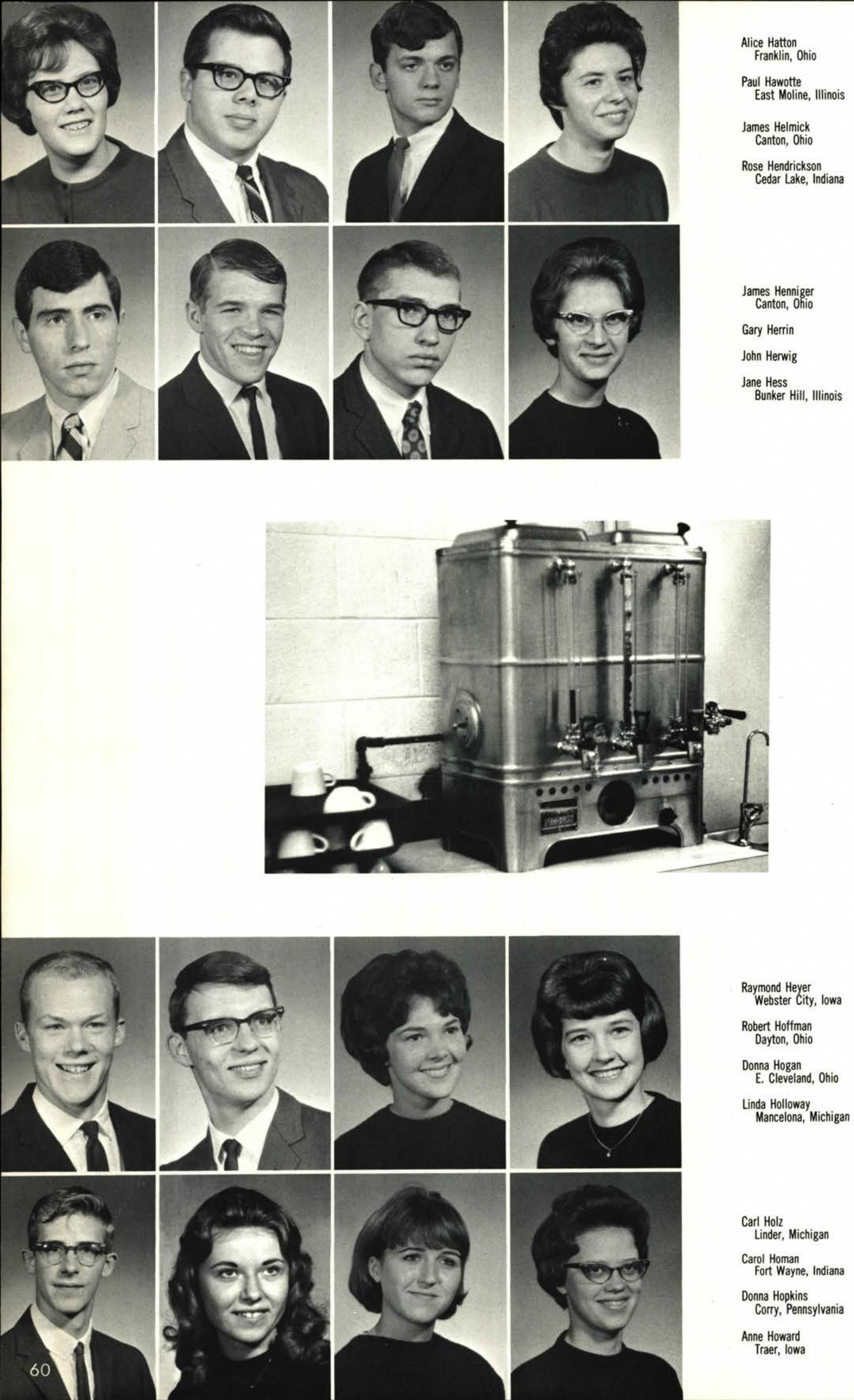

Raymond Heyer
Webster City, lowa

Robert Hoffman Dayton, Ohio

Donna Hogan

E. Cleveland, Ohio

Linda Holloway Mancelona, Michigan

Carl Holz Linder, Michigan

Carol Homan Fort Wayne, Indiana

Donna Hopkins

Corry, Pennsylvania

Anne Howard Traer, lowa 
Bonnie MacPherson

Lynnwood, Washington

Nancy Males

DuBois, Pennsylvania

Charles Marcellus

Detroit, Michigan

Lynnette Marsh

Corry, Pennsylvania

Jayne Masemore

Schererville, Indiana

Judy McClintock

Columbus, Ohio

Bruce McDonald

Lake Orion, Michigan

Kenneth McGhee

Silvis, Illinois

Thomas McGinnis

Springfield, Ohio

Ruth Mcintyre

Audubon, New Jersey

Cecil McMahan

Lincoln Park, Michigan

Donald Mellish

Evanston, Illinois

Ronald Mellish

Ontario, Canada

Barbara Miller

Troy, Ohio

Debra Miller

Xenia, Ohio

Janice Miller

Medina, Ohio
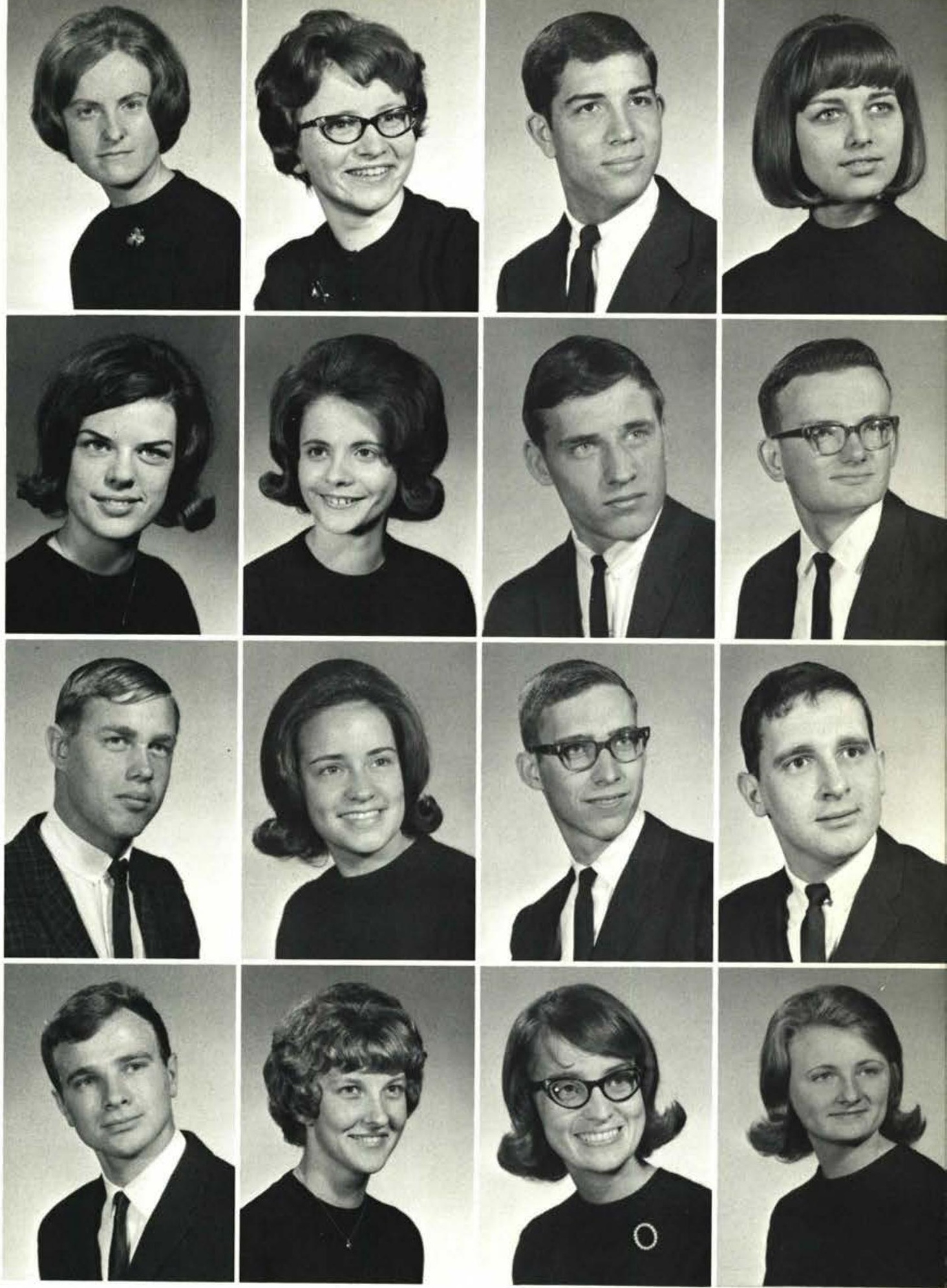

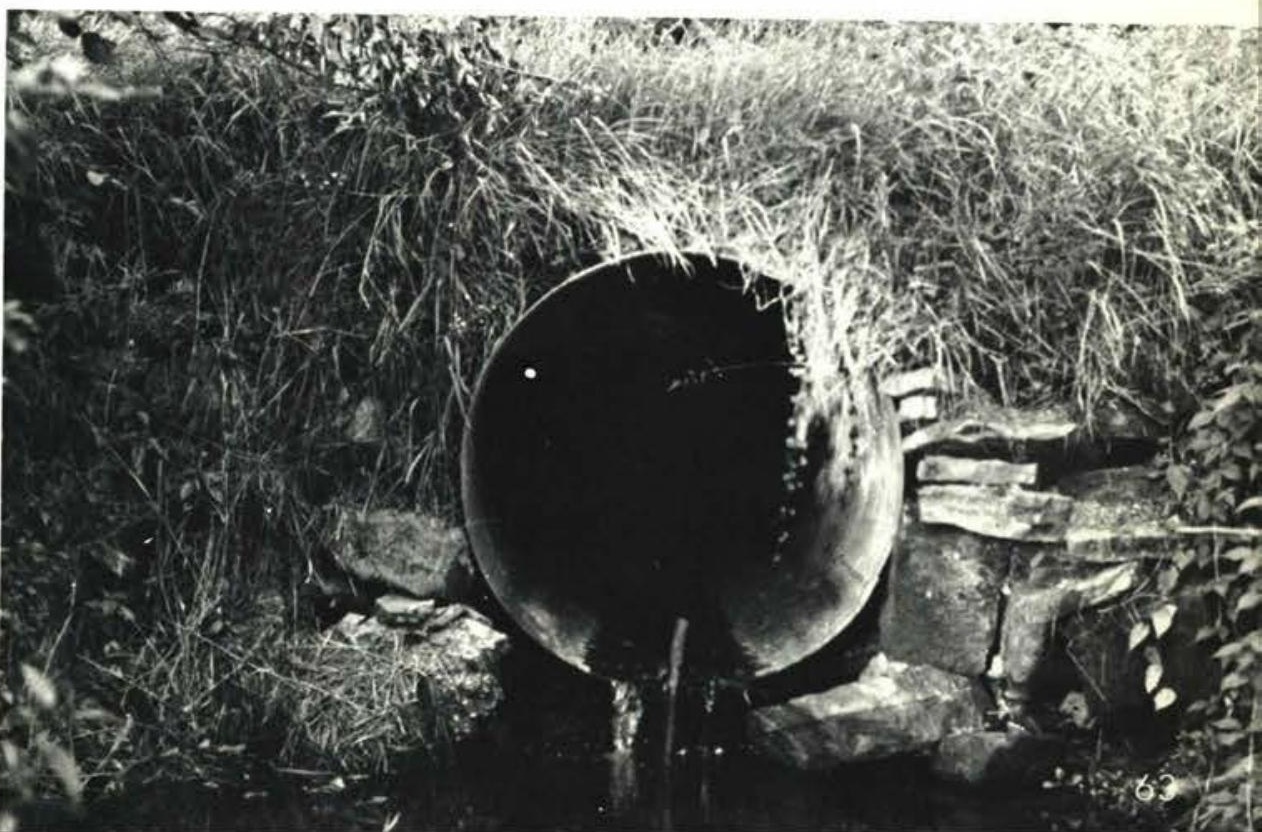



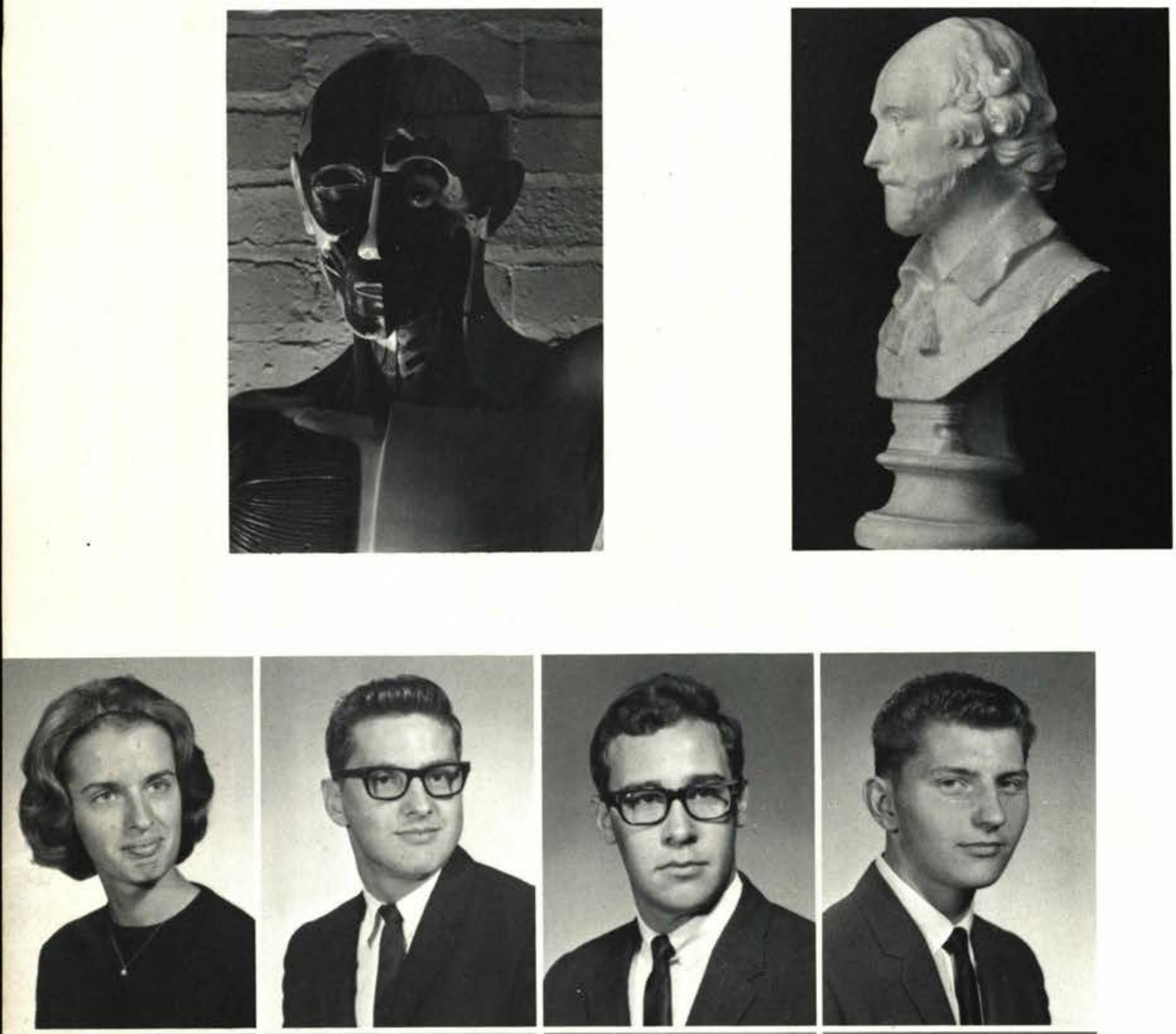

\section{Priscilla Miller \\ Des Moines, lowa \\ Harold Moore \\ Portage, Wisconsin \\ Kenneth Muck \\ Elyria, Ohio \\ Arlan Mulford \\ Troy, Ohio}
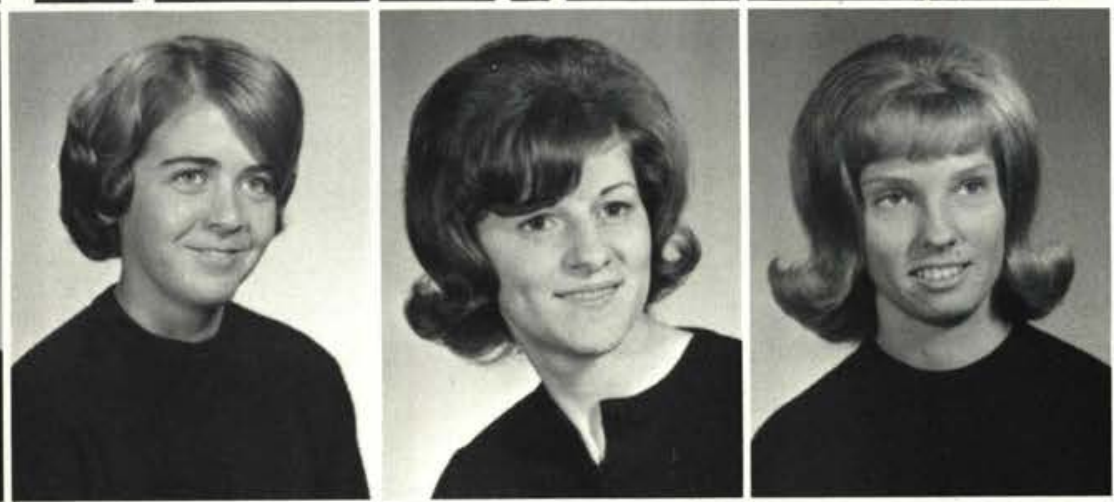

Ernest Music Detroit, Michigan

Susan Myers

Chester, Pennsylvania

Karen Nance

Southgate, Michigan

Charlotte Nash

Hobart, Indiana

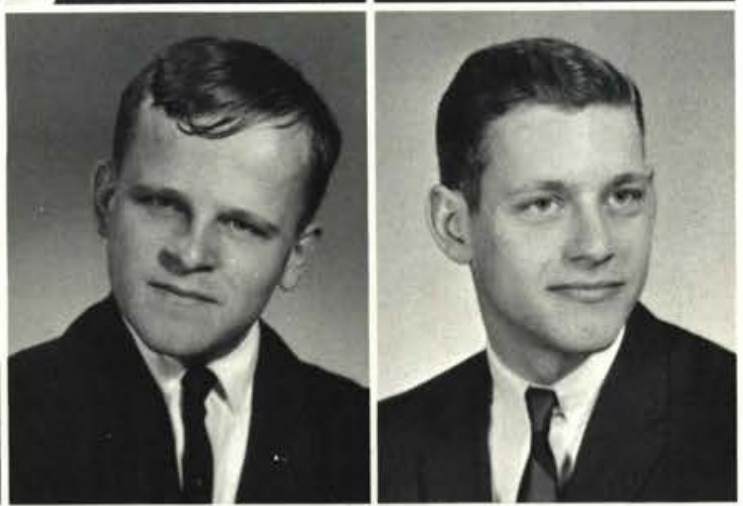

Sydney Nicoson Loveland, Colorado

Nancy Norton Elkins Park, Pennsylvania

Joseph Ogradowski Cedarville, Ohio

Ronald Okresik

Markham, Illinois

Fred Olsen

Lima, Peru

Connie O'Shell

Olmsted Falls, Ohio

Kenneth Overholt

Royal Oak, Michigan

Calvin Packard

Cedarville, Ohio 

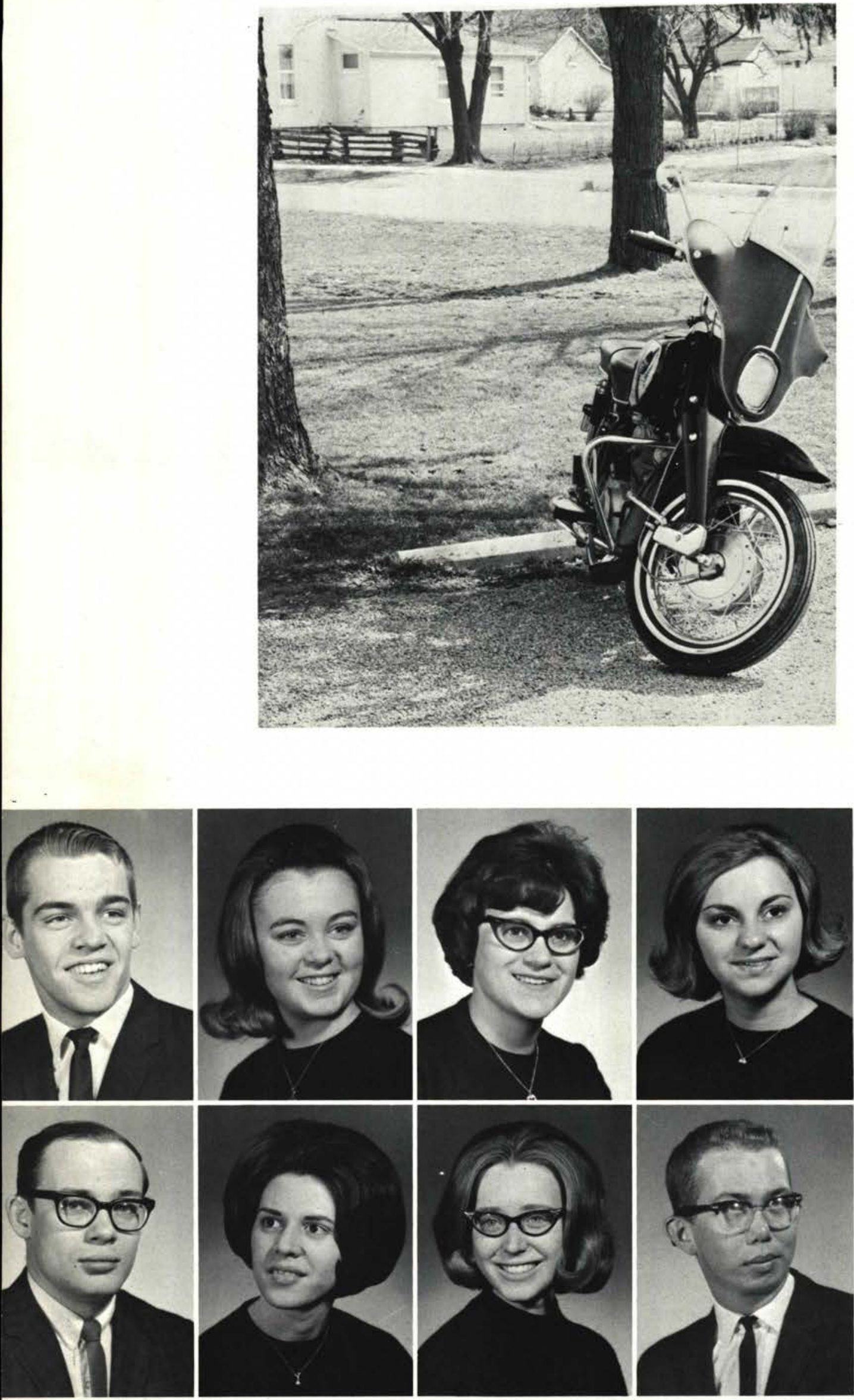

David Rakes Fairborn, Ohio

Joy Ramsey Dayton, Ohio

Karen Rau Palmyra, Michigan

Carol Reed

Niles, Ohio
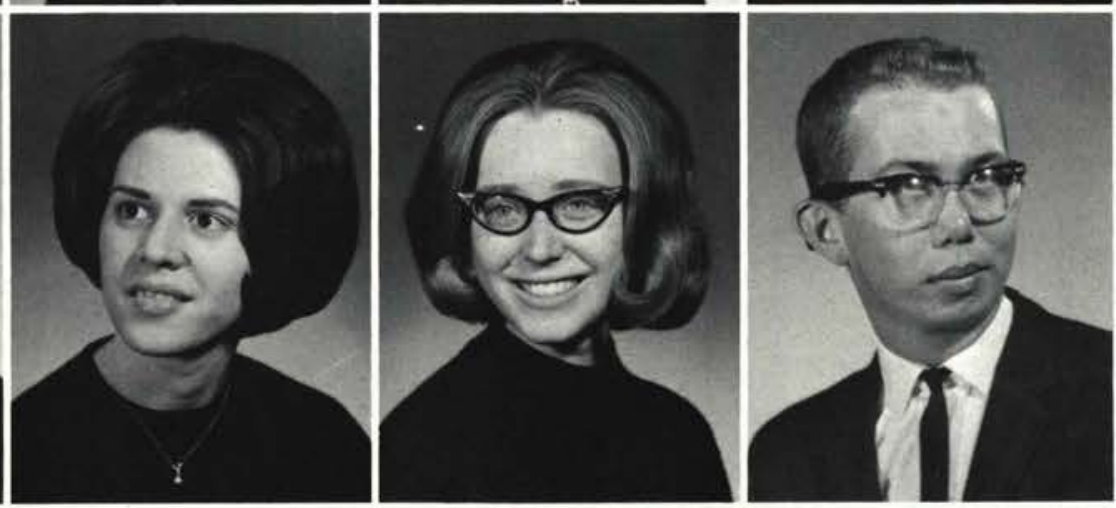

James Rejiester Toledo, Ohio

Jill Renner Kokomo, Indiana

Ruth Ressler Toledo, Ohio

Gerald Richardson Morrow, Ohio

Marlene Rieken Ackley, lowa

Jeanne Risko Kettering, Ohio

Sylvia Rock Roanoke, Virginia

David Rockwell Indianapolis, Indiana 
Stephen Snyder

New Carlisle, Ohio

Sheila Solomon

Argos, Indiana

Kathryn Spencer

Cedarville, Ohio

Marcia Sprunger Berne, Indiana

Maxine Stanberry Peoria, Illinois

Duane Stevens

Inkster, Michigan

Gwendolyn Stewart Indianapolis, Indian

Fred Stickle

Oakland, Minnesota

Craig Stillwell

Oakland, New Jersey

JoAnn Strychalski

Lebanon, Indiana

Lela Tanner

Sherman, New York

Wesley Tansey

Kokomo, Indiana

Delmar Taylor

Cedarville, Ohio

Gary Taylor

Bay Village, Ohio

Laura Taylor

Steamboat Rock, lowa

Linda Taylor

Endwell, New York

Evelyn Terrill

Cedarville, Ohio

JoAnn Tharp

Dayton, Ohio

Susan Thorne

Medford, New Jersey

William Thurlow

Midland, Michigan

Jane Tibbett

Fort Wayne, Indiana

Nancy Towle

Pataskala, Ohio

Marcia Townsend

Greenfield, Massachusetts

Steve Treadway

Xenia, Ohio
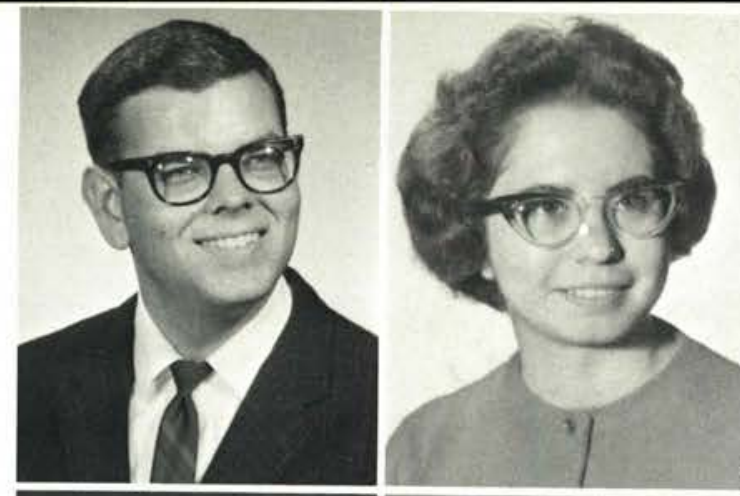

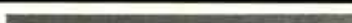
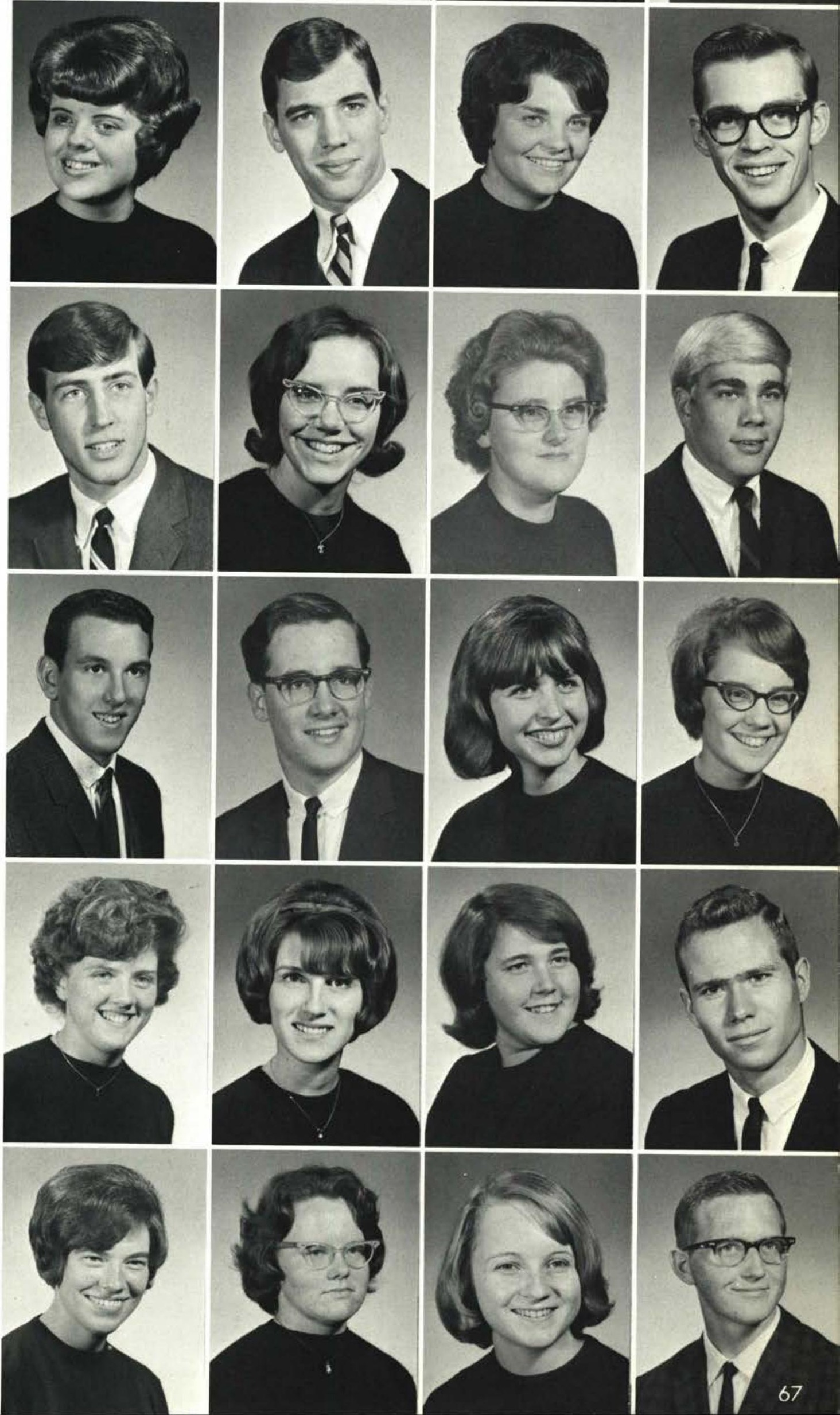

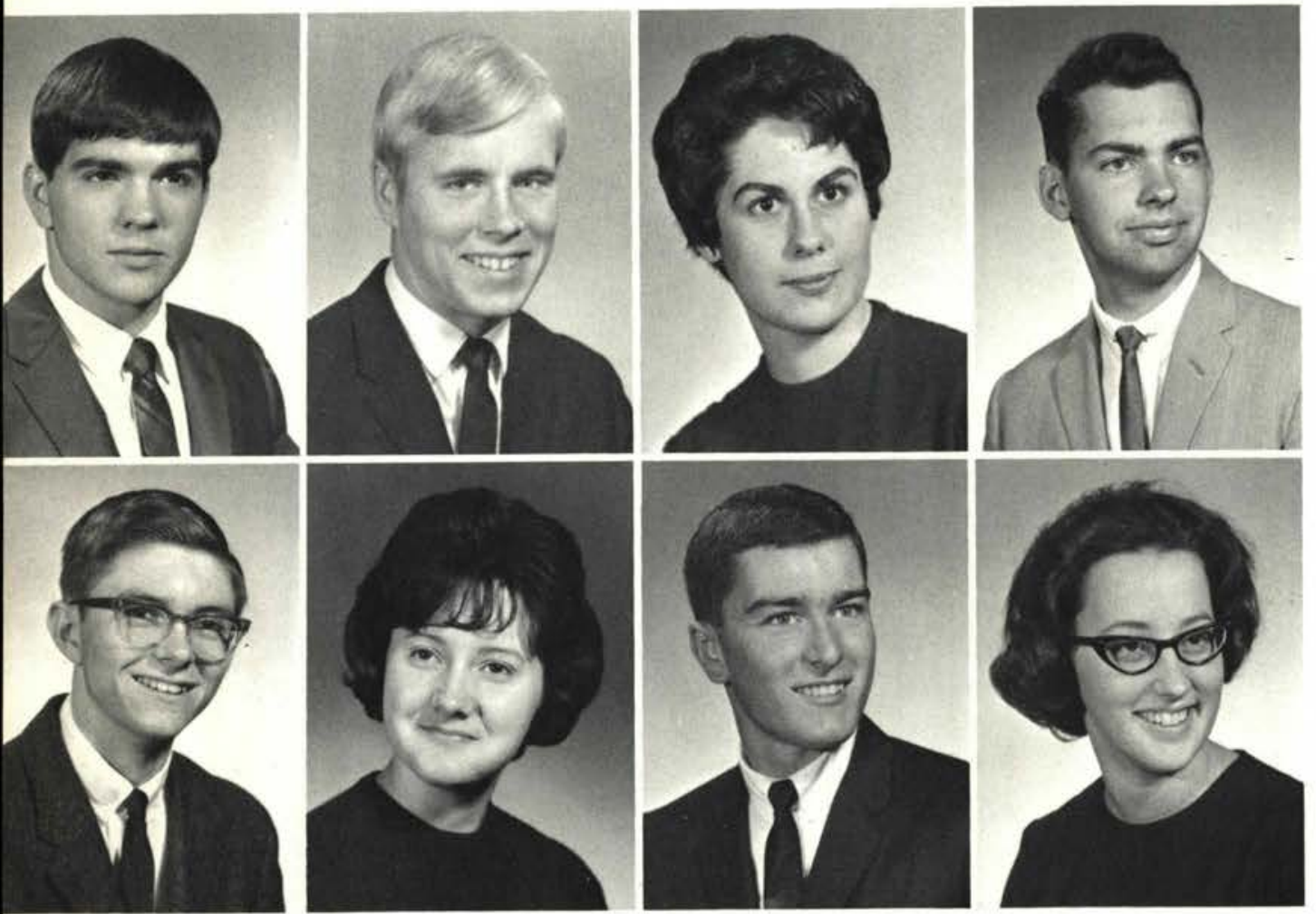

Edwin Tripp

Willowick, Ohio

David Trunnell

Waterloo, lowa

Jeanne Vance

Clayton, Ohio

James Vanderground

Cleveland, Ohio

George Van Voorhis Alton, Illinois

\section{Janice Wakeley} Ripley, New York

Daniel Walborn Wauseon, Ohio

Kathleen Walker Fairbault, Minnesota

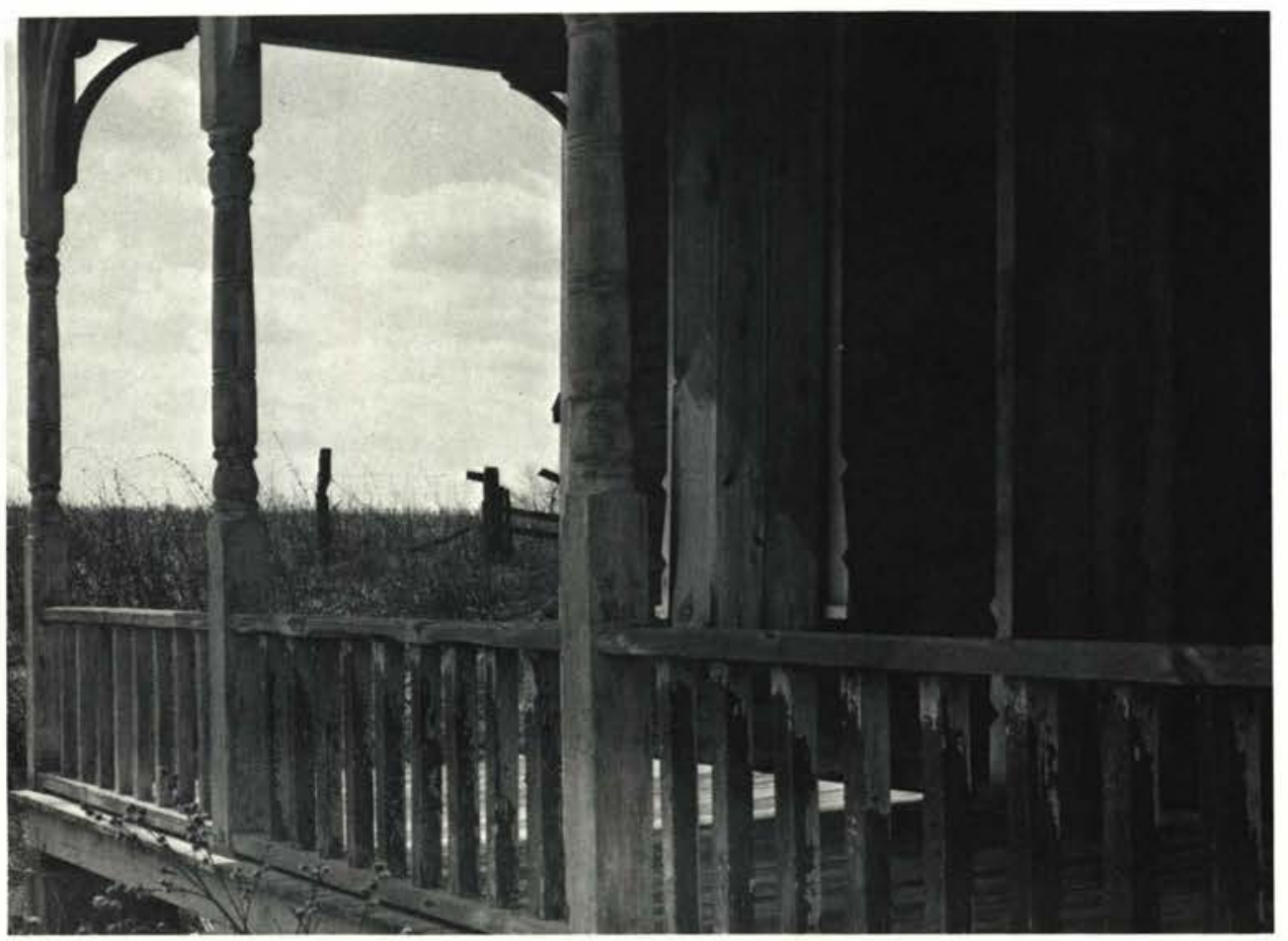


Karen Warden

Painted Post, New York

Timothy Warren Allegan, Michigan

Patricia Weinschenk East Cleveland, Ohio

Susan Weis Rockledge, Florida

\section{Robert Whattoff}

Ames, lowa

Keith White

Cedarville, Ohio

Cheryl Whitney Bernardston, Massachusetts

David Williams

Pontiac, Michigan

Gay Williams

Cuyahoga Falls, Ohio

Patricia Williams

Hammond, Indiana

Ruth Williams

Denver, Colorado

Pamela Willis

East Wenatchee, Washington

Caron Wilson

Fort Wayne, Indiana

Cheryl Wilson

East Cleveland, Ohio

Dale Work

Coudersport, Pennsylvania

Leroy Wright

Saddle Brook, New Jersey

Richard Wright

Pennsboro, West Virginia

Douglas Yoder

West Liberty, Ohio

Jefferson Young

Lorain, Ohio

Michael Zazurskey Lorain, Ohio

George Zellers

South Bend, Indiana

Barry Zimmerman

Silver Lake, Indiana
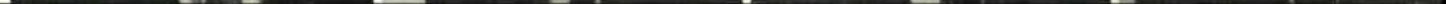

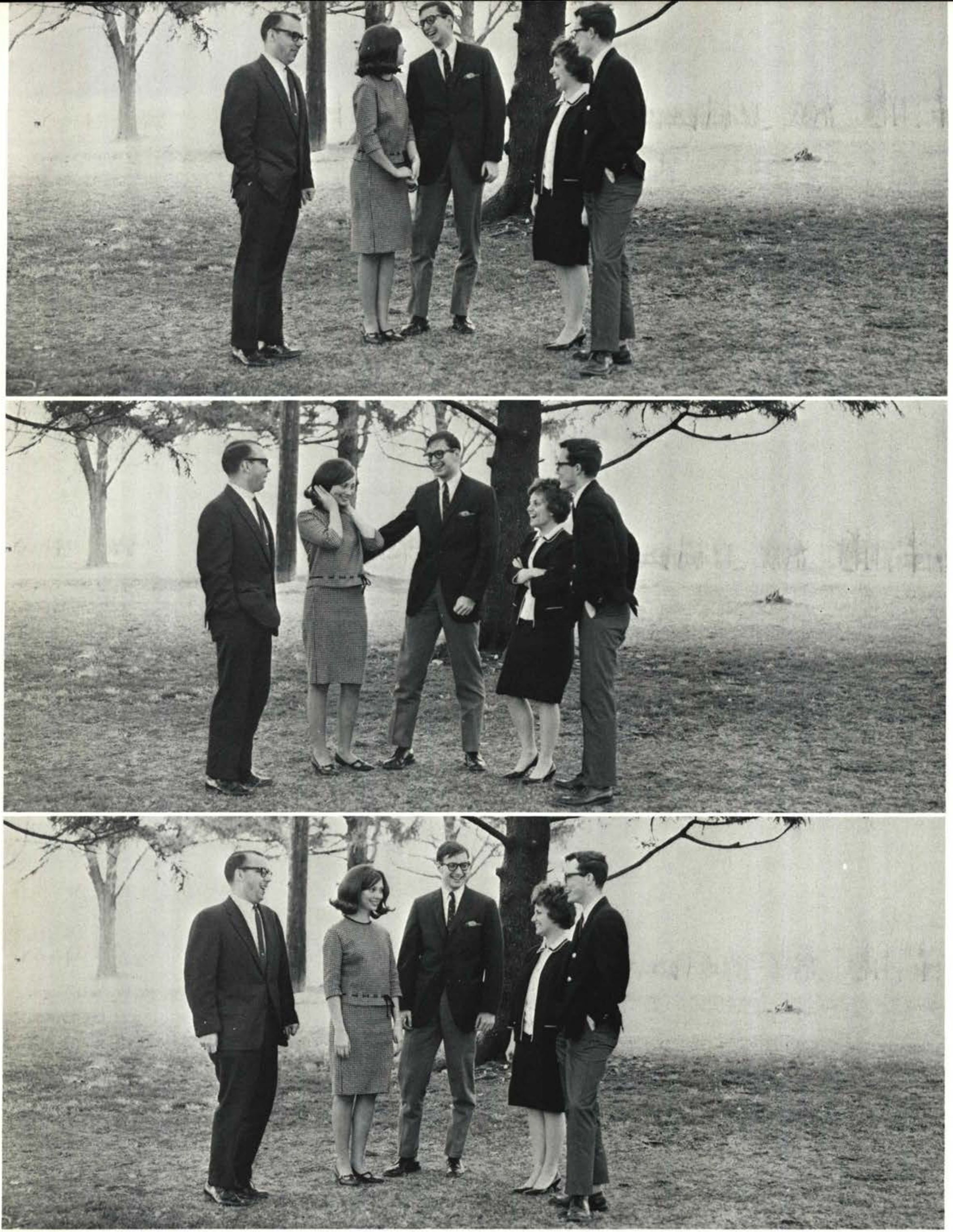

Left to Right: Mr. D. Wetzel . . . . . . . . . . . . . . Advisor

C. Burcham . . . . . . . . . . . . . . . . . . . Secretary

D. Jones ...................... V. President

B. Crosh ........................... Treasurer

M. Hamilton . . . . . . . . . . . . . . . . . President 
Sunday E. Akinyemi Lagos, Nigeria

Ron Allerton

Prospect, Penna.

Lynn Amstutz

Cherry Hill, N.J.

Lois Ancel

Taylors Falls, Minn

Rebecca Baker

Schenectady, N.Y.

Ron Baker

Schenectady, N.Y.

Linda Jane Barkley

Arcanum, Ohio

Donna Barrett

Elkhart, Ind.

Margaret-ann Beiser Jerseyville, III.

Larry Bellew

Cincinnati, Ohio

Carolyn Berg

Stanton, Mich.

Calvin Biddle

Beloit, Ohio

Tom Biller

Gary, Ind.

James Blaylock

Fairborn, Ohio

Valeeta Boelter

Hammond, Ind.

Gregory Boese

Saginaw, Mich.

New Bethlem, Penna.

Dan Boyd

Argenta, III.

Herbert Branch

Cedarville, Ohio

Margaret Brewer

Gallipolis, Ohio

Lynn Brock

Columbus, Ohio

Jon Brown

Cedarville, Ohio

Martha Brown

Holly, Mich.

Carolyn Burcham

Mattoon, III.
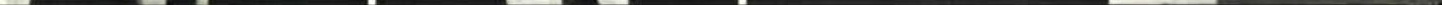

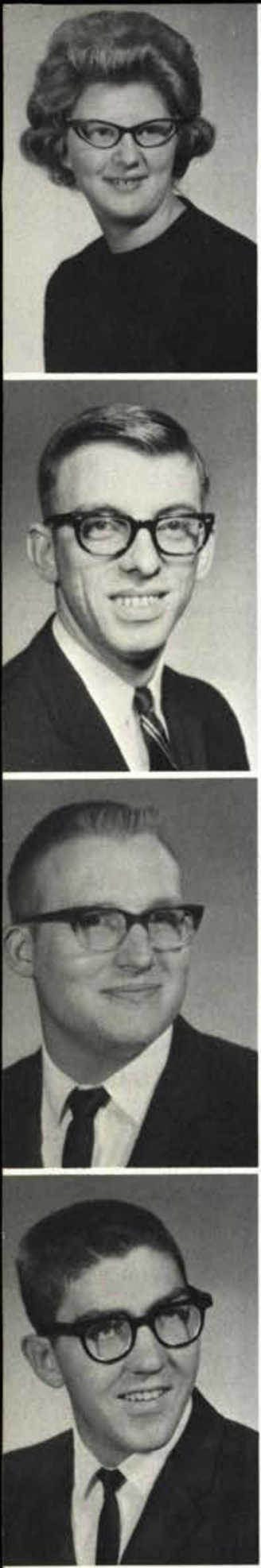
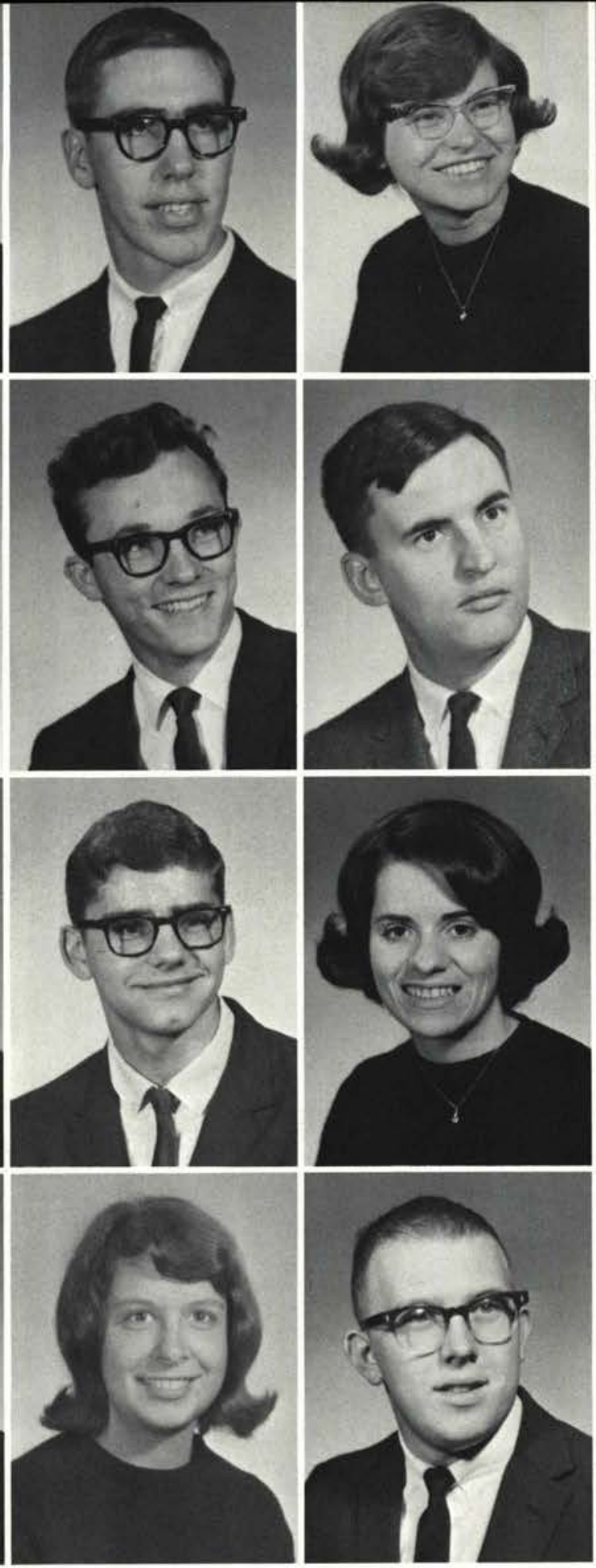
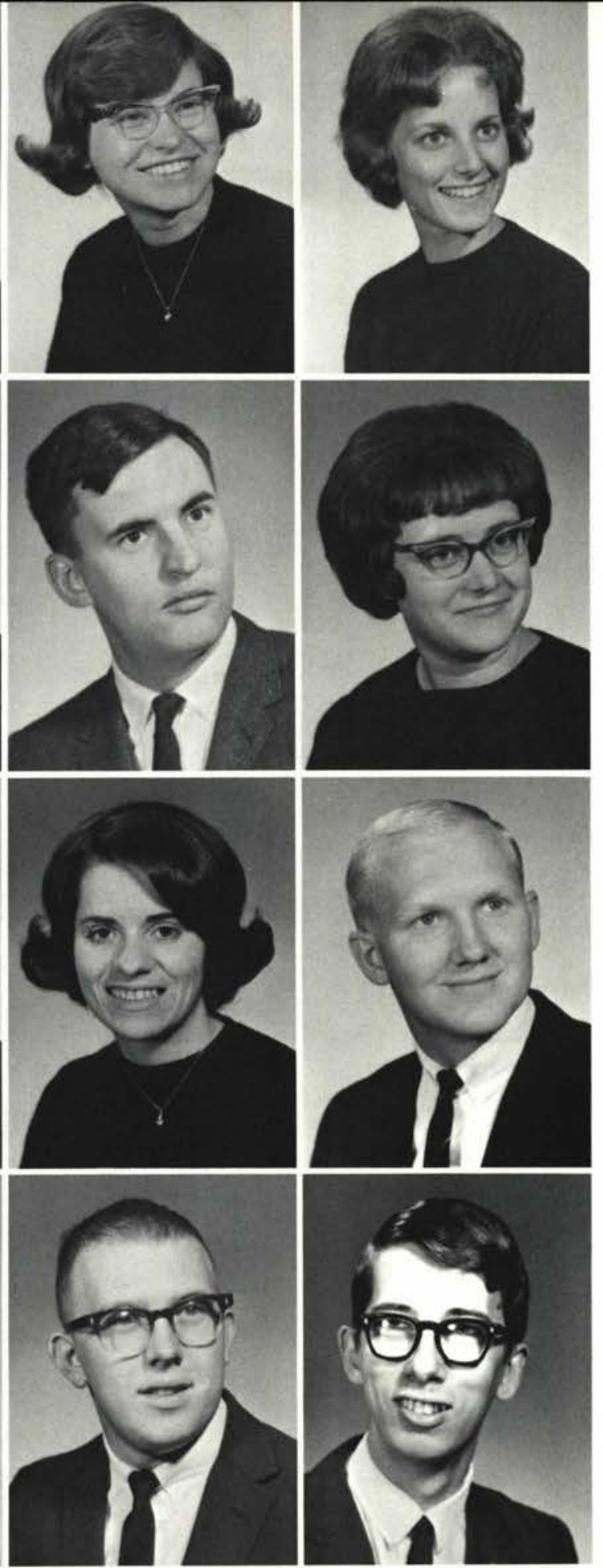

Wynona Geer

Cleveland Heights, Ohio

Jim Goodwin

Oak Forest, III.

Katie Griffin Anderson, Ind.

Barbara Grosh

North Royalton, Ohio

James Gunlock Wheaton, II

Michael Hamilton

Columbus, Ohio

William Hamilton Jefferson, Ohio

Jane Harris

Waterlo0, lowa

\section{Bill Heater} Findley, Ohio

Howard Hillman Adrian, Mich.

Linda Hoffman Gallipolis, Ohio

Joseph Holloway Columbus, Ohio

\section{Ken Hopkins} Parma, Idaho

Cathy Hubbard Glastonbury, Conn.

Glen Huebner

Milwaukee, Wisc.

Charles Hull Cortland, Ohio

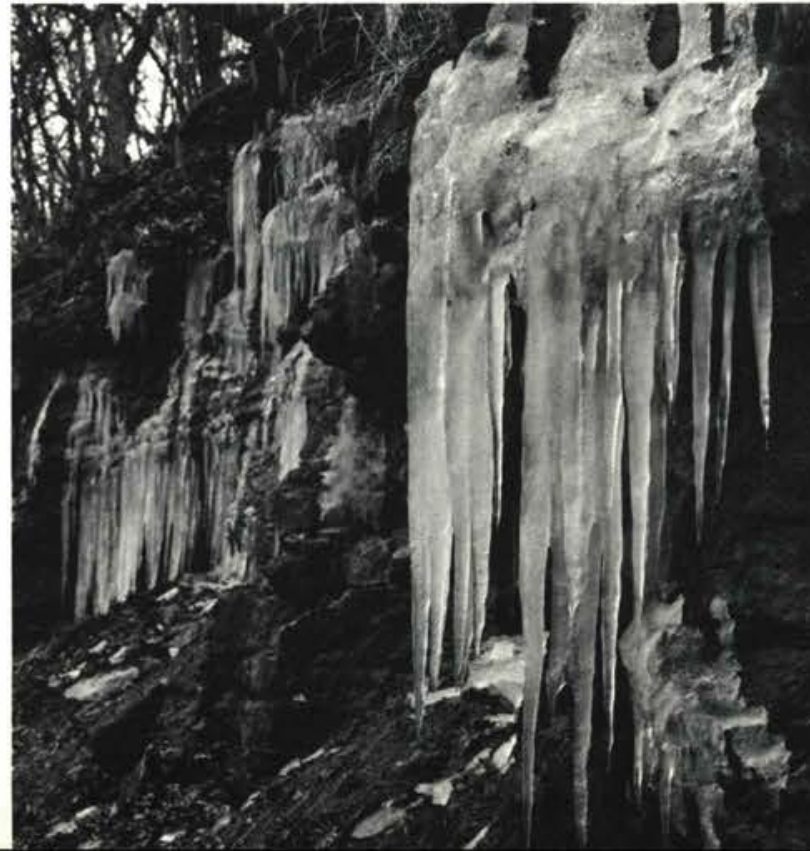



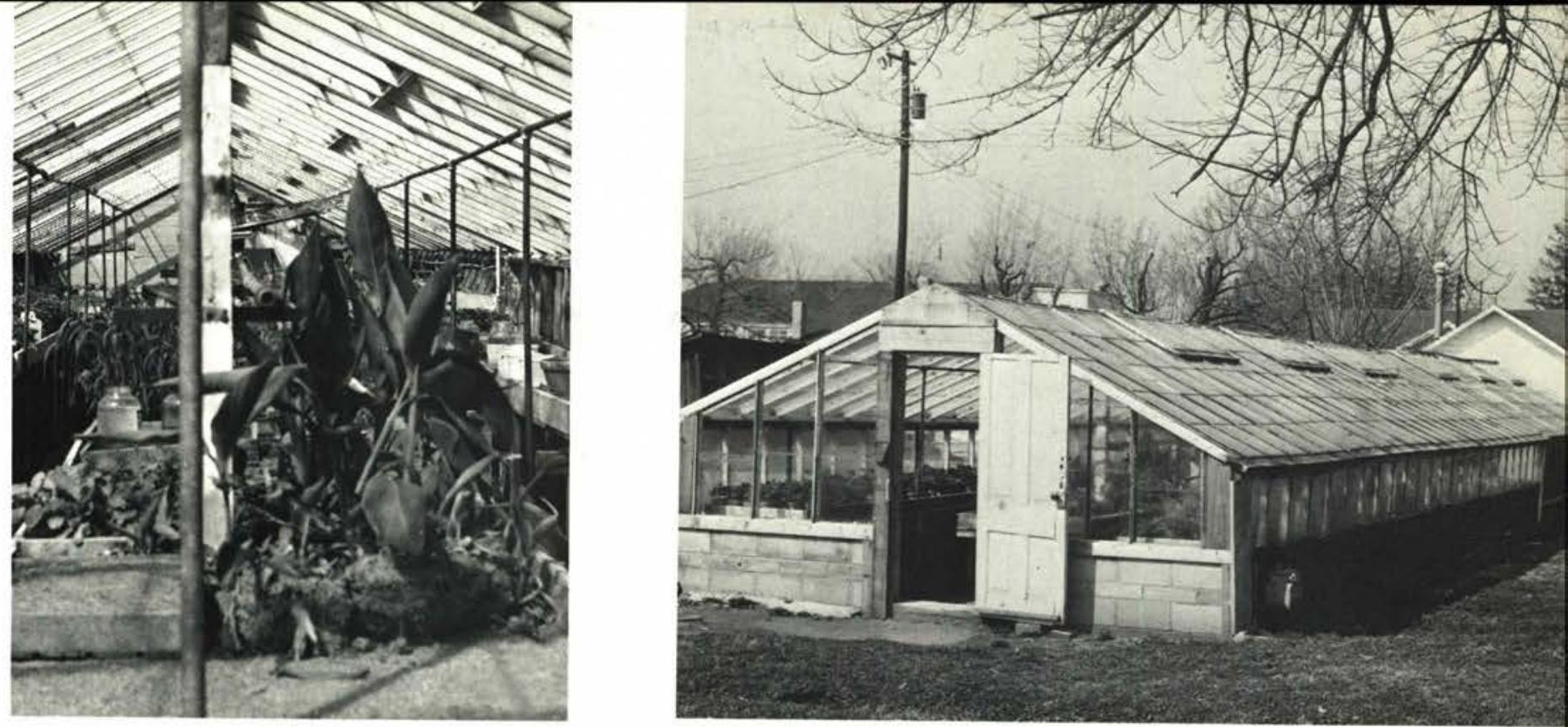

Jim Hume

Dayton, Ohio

Charles Hunter

Jesup, lowa

Sharon Hunter

Decatur, III.

Sharon Jamison Flint, Mich.
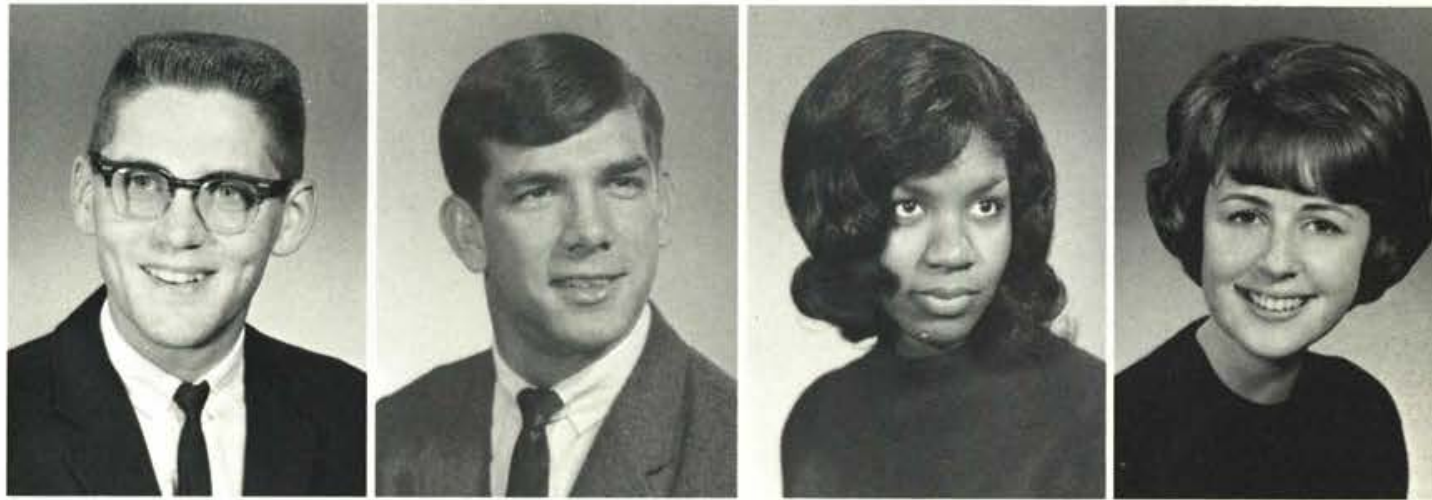

Frank Jenista

Bloomfield, N.

Elaine Johnson

West Palm Beach, Fla.

Joyce Johnson

East Cleveland, Ohio

Kathy Johnson

Seattle, Wash.
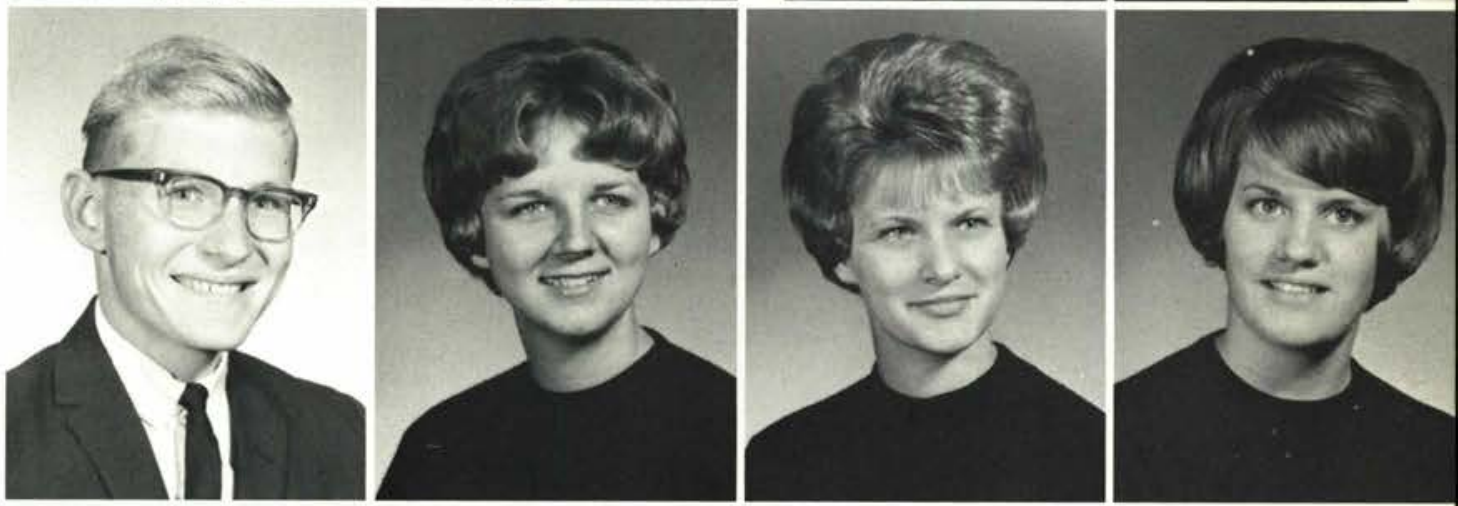

David Jones Arlington, Va.

Sheryl Kaiser

Plymouth, Ind.

Marion Kapp Erie, Pa.

Ruth Kauffold Pana, III.

Janice Keating

Wausaukee, Wisc.

Donna Keib

Shelby, Ohio

Al Keim

Holsobble, Pa.

Barbara Kensil

Decatur, III.
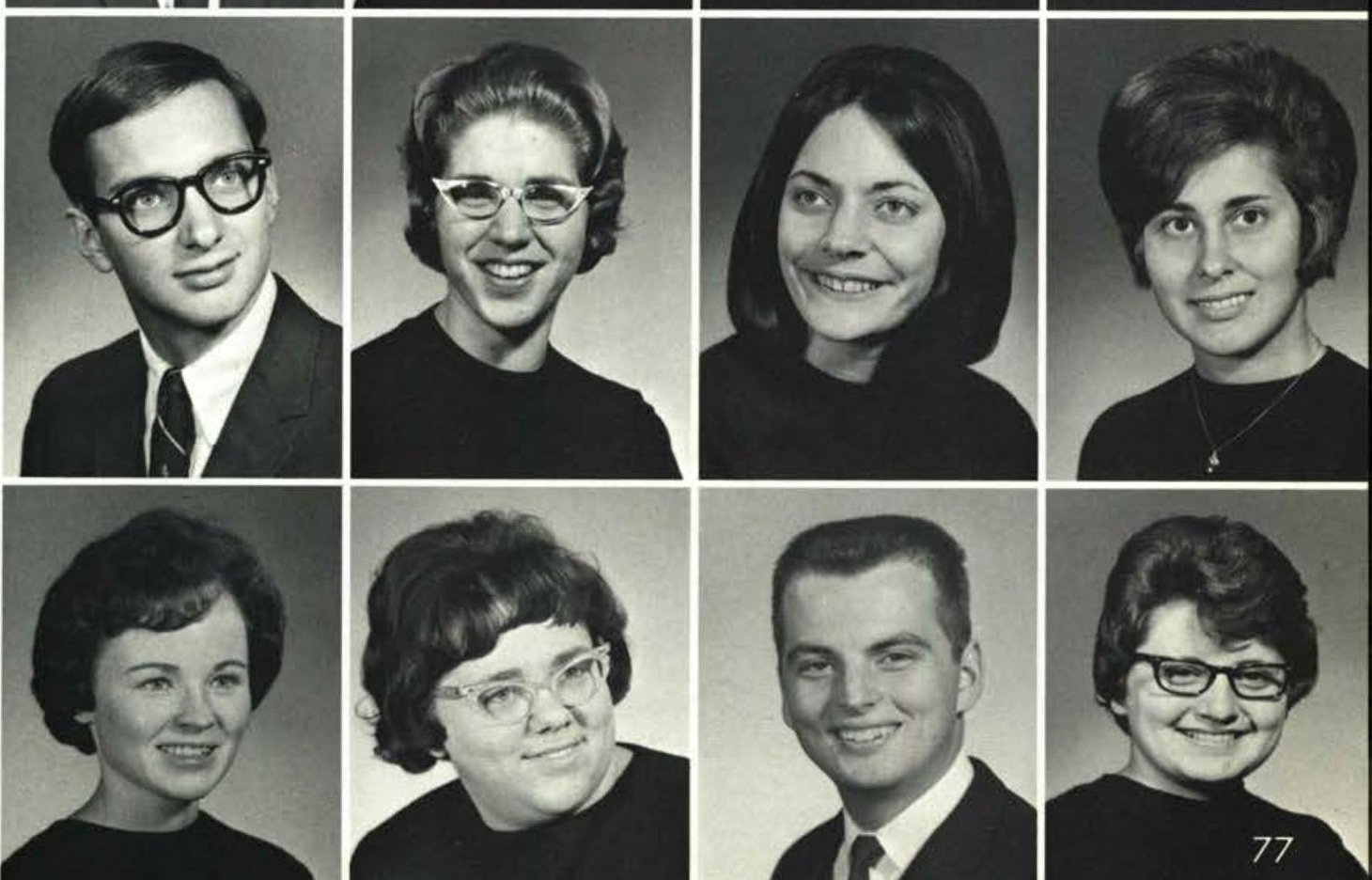

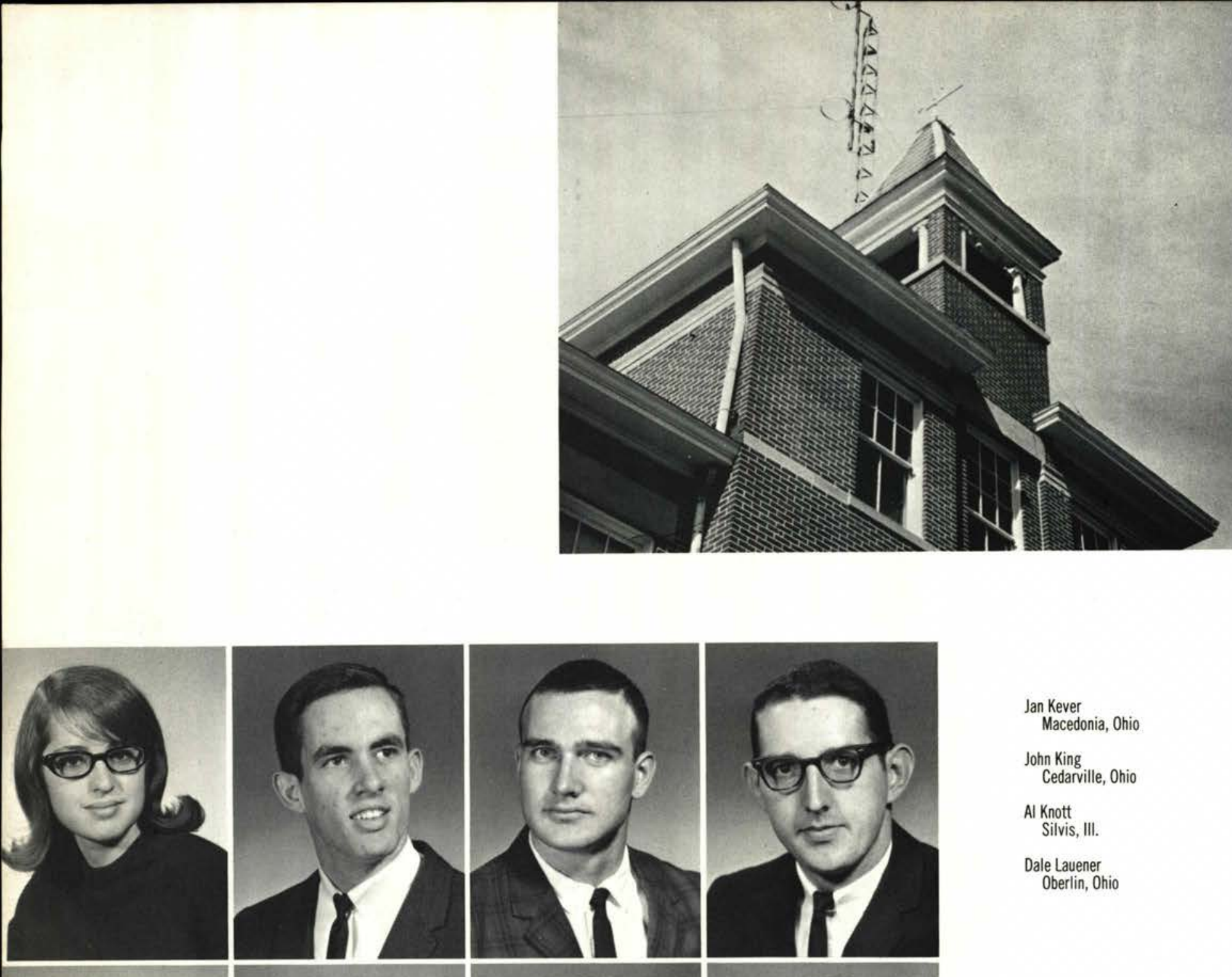

Jan Kever

Macedonia, Ohio

John King

Cedarville, Ohio

Al Knott

Silvis, III.

Dale Lauener

Oberlin, Ohio
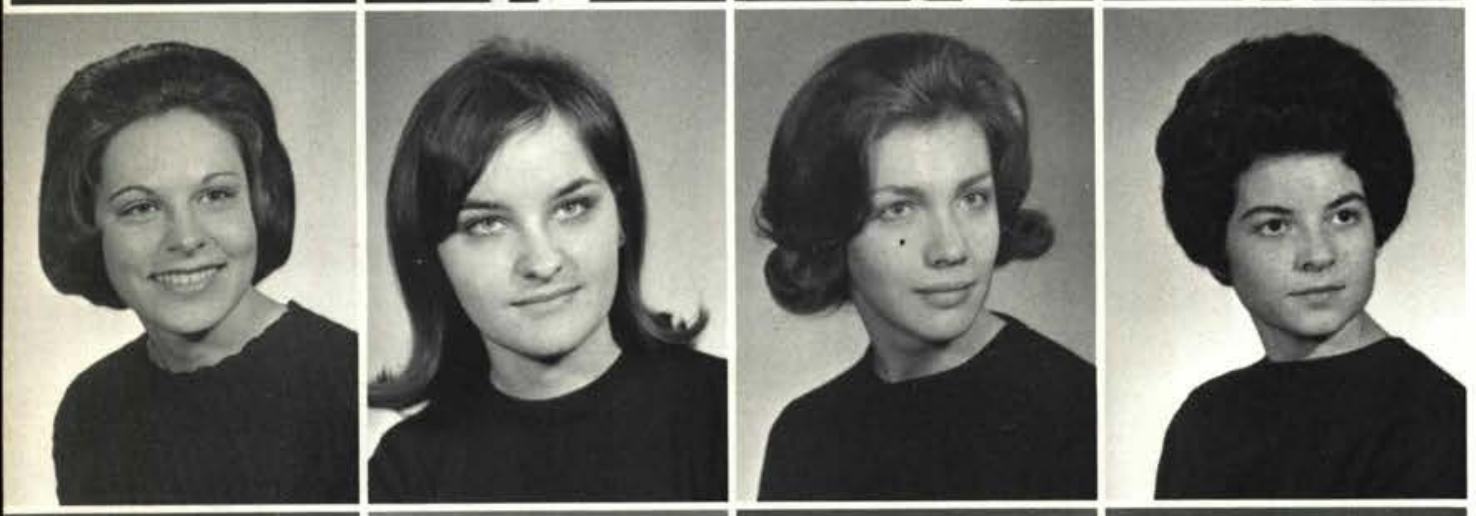

Daphne Lautenschlager

Highland, Ind.

Cheryl Lawhead
Minford, Ohio

Jessie M. Lennox

Cleveland, Ohio

Nancy Lewis

Kokomo, Ind.
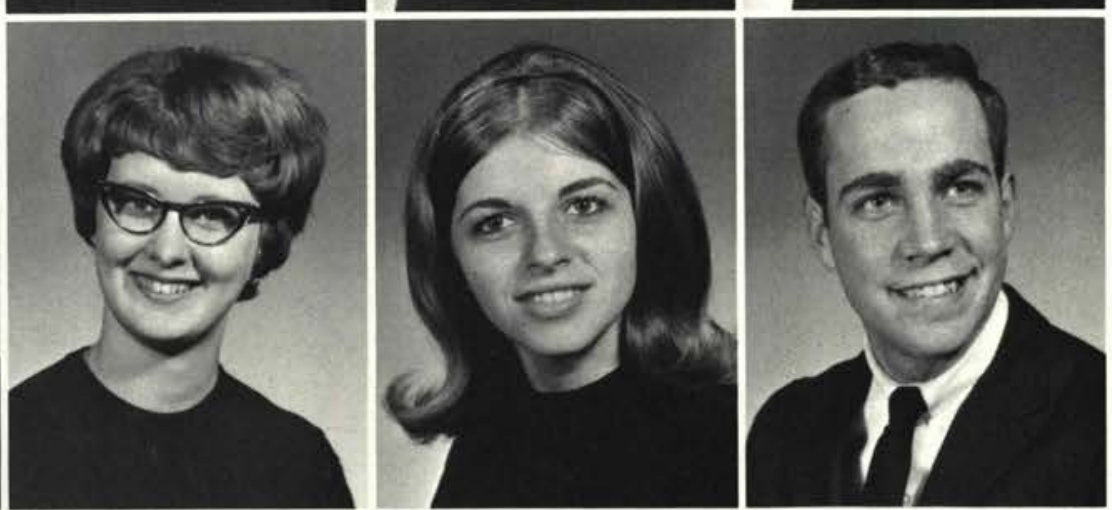

Mary Lockhart

Sciotoville, Ohio

Marsha Louwerse

Decatur, III.

Candice Marsh

Corry, Pa.

\section{Ted Martens}

Haledon, New Jersey

John May

Stroudsburg, Pa.

Cathy McGee Richmond, Va.

Roberta Mclnnes

Columbia City, Mich.

Roger McKenney East Detroit, Mich. 
D. Gregor Mehaffey

Teaneck, N. J.

Betty Meyers

East Moline, III.

Jim Miller

Pontiac, Mich.

Lola Mitchell

Boise, Idaho

Marilyn Mitchell Cedarville, Ohio

Joyce Moore

Mattituck, N.J.

Donna Morgan Charles City, lowa

Beverly Mosely Melvindale, Mich.

James Meyers Van Wert, Ohio

\section{Becky Navorska}

Olmstead Falls, Ohio

Dan Nevitt

Henry, III.

Ken Nichols

Byerville, Ohio

Maynard Norris

Rochester, Ind.

Suzie 0'Shell

Olmstead Falls, Ohio
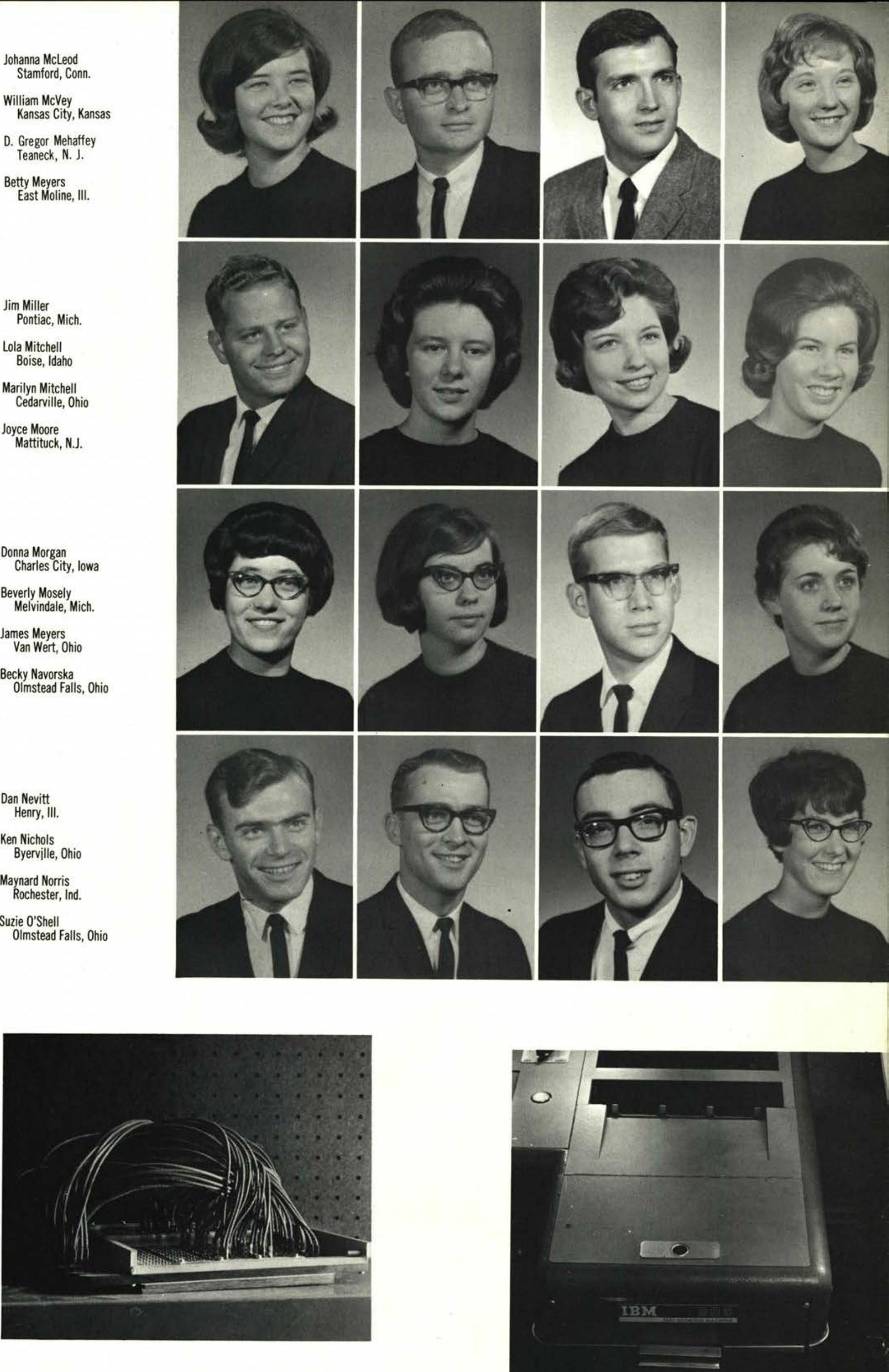
Sheryl Satterblom Highland, Ind.

Marlene Schonscheck Allen Park, Mich.

Mary Ellen Schut Hudsonville, Mich.

Carl Schwanbeck

Monroeville, Ohio

Carol Scott Columbus, Ohio

Marshall Searles

Wellington, Ohio

Byron Shearer Pittsburgh, Pa.

Allen Shriver Ursa, III.
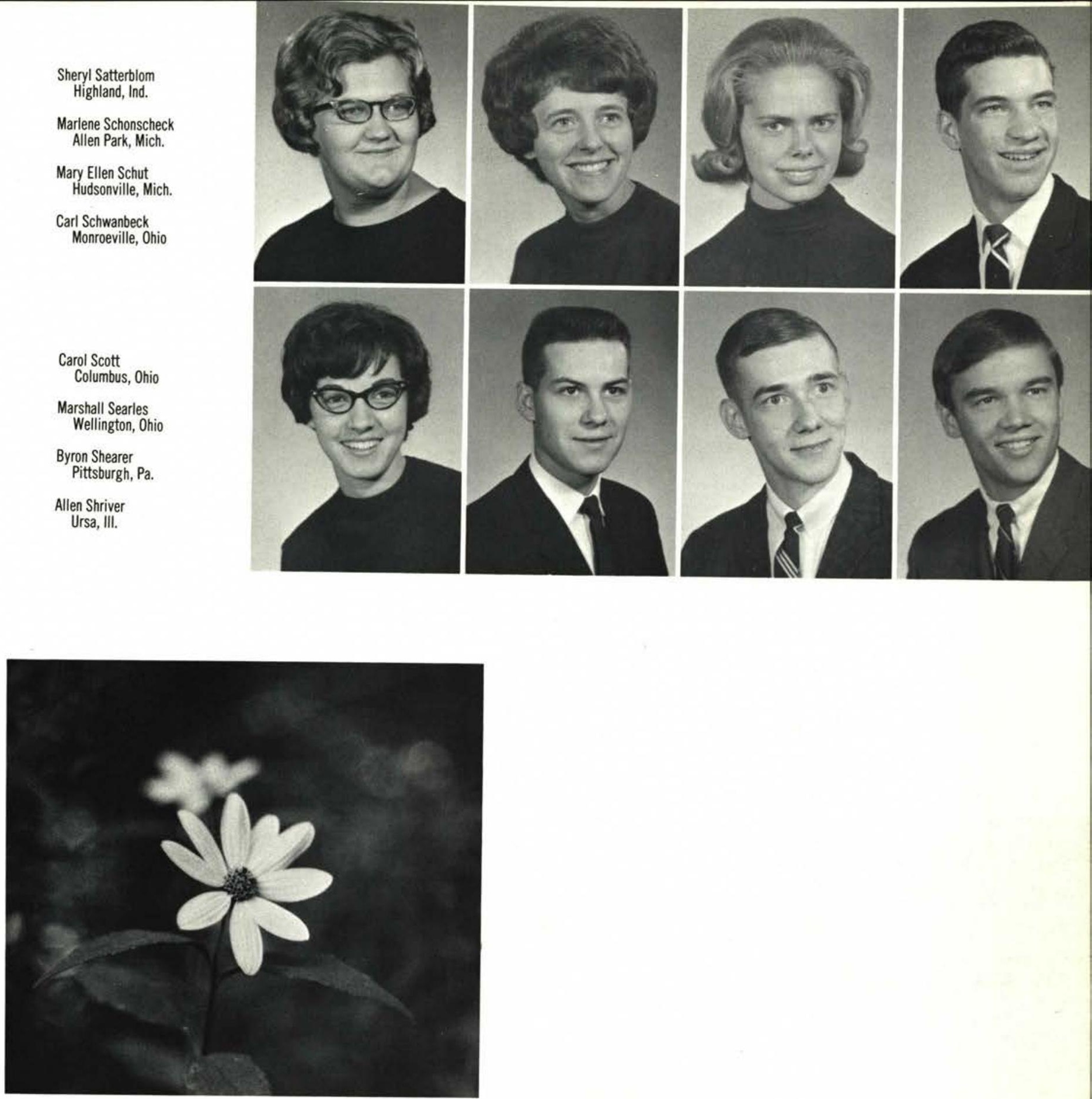

Elma Shugg Pinckney, Mich.

Carol Simonetti Erie, Pa.

Donna Simpson Indianapolis, Ind.

Connie Smith Adrian, Mich.

Linda Spinks

Sherman, N.Y.

Connie Stevenson Baker, Mont.

Roger Stout

Springfield, Ohio

Ronald Stutzman Berlin, Ohio
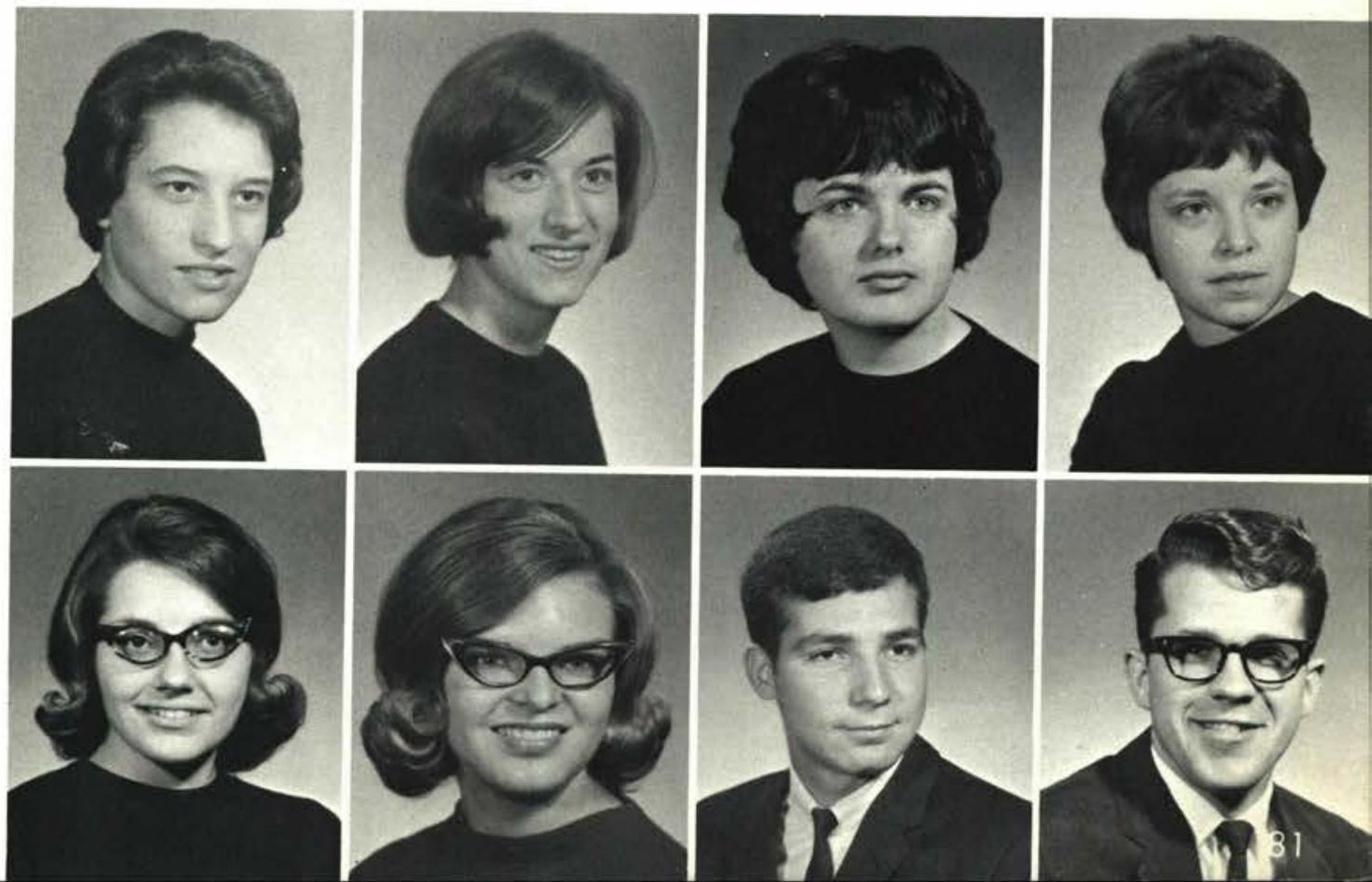

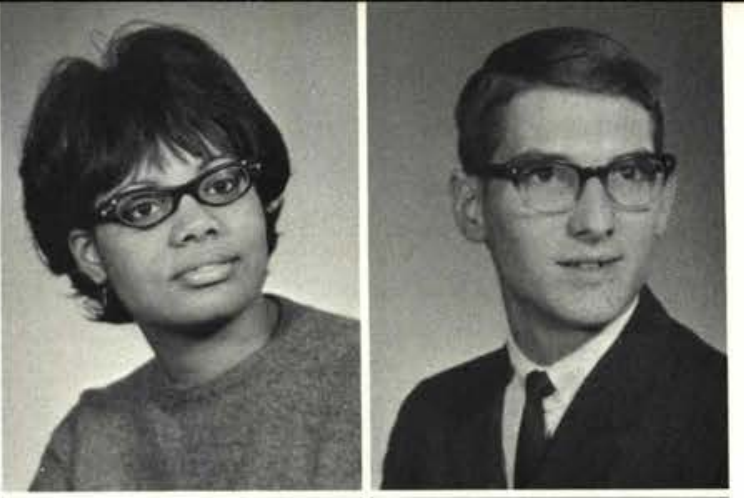

Adrian Sweeney Cherry Hill, N.J.

Sharon Tallman

Hallandale, Fla.

Karen Taylor

Dayton, Ohio

Dan Tillotson

Gary, Ind.

Brenda Thompson Columbus, Ohio

Pat Thompson Pontiac, III.

Gail Tompkins

Sherman, N.Y.

Beverly Vincent Lancaster, N.Y.

Terry Vlug Wyoming, Mich.

Charles Von Hagel Chester, $\mathrm{Pa}$.

Donna Wahl Warwick, R.I.

Larry Waite Hudsonville, Mich.

Leland Walker

Carlisle, lowa

David Wall Dacatur, III.

Diana Ward

East Cleveland, Ohio

John Watson Mishawaka, Ind.

Marlene Werner Columbia Falls, Mont.

Sarah Williams

Cleveland, Ohio

Michael Wilson Rochester, Mich.

Twila Wing

West Winfield, N.Y

Linda Wintrow

Tipp City, Ohio

Joyce Wood

Clarkston, Wash.

Michael Woodend Alexandria, Va.

John Wyngate Corning, N.Y. 


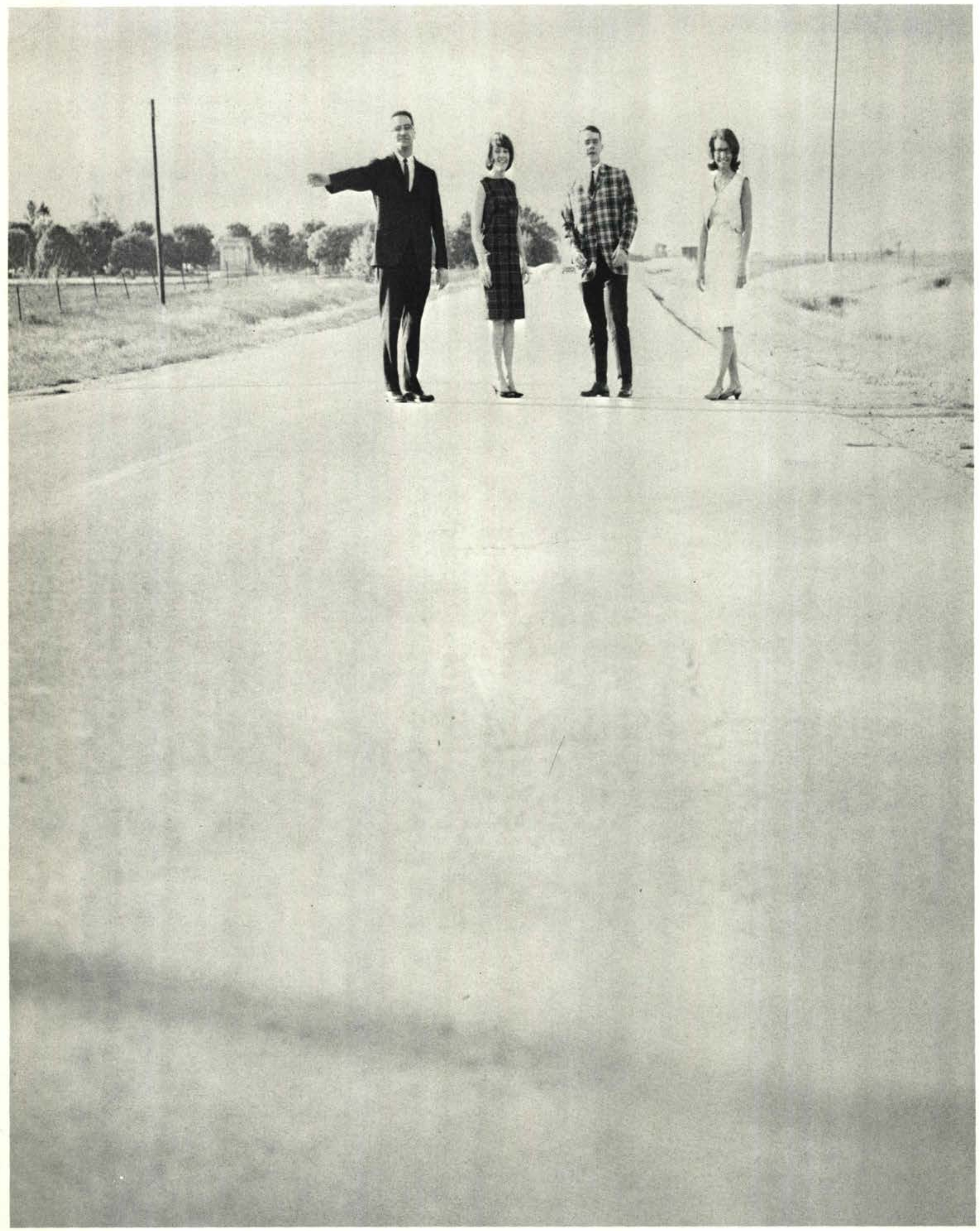

Left to Right: Mr. John Reed $\ldots \ldots \ldots \ldots \ldots \ldots \ldots$ Advisor

B. Smith . . . . . . . . . . . . . . . . . Secretary

T. Timmons $\ldots \ldots \ldots \ldots \ldots \ldots \ldots$ President

L. Kytle $\ldots \ldots \ldots \ldots \ldots \ldots \ldots \ldots$ Treasurer

M. Trimble, Not pictured ........... V. President 
Keith Abshear

Springfield, Ohio

Priscilla Alexander

Fairborn, Ohio

Eunice Anderson

Cheboygan, Mich.

Janie Ashby

Chariton, lowa

Walter Ashley

Avon Lake, Ohio

Mark Averitt

Cedarville, Ohio

Phyllis Bancroft

Adrian, Mich.

Norman Barnard

Kingman, Ind.

Mary Ruth Beerer

Elkhart, Ind.

Russel Bennett

Anchor Point, Alaska

Betty Bertschinger Martins Ferry, Ohio

Judy Bigelow

Cedarville, Ohio

Frieda Bonnema

Perry, Ohio

James Brinckerhoff Cheektowaga, N.Y.

Steve Brown

Kasson, Minn.

Warren Burns

Ft. Wayne, Ind.

Carol Carr

Gerry, N.Y.

Betty Chilton

Des Moines, lowa

Delores Cooper Kettering, Ohio

James Cornett

Arlington, $\mathrm{Va}$.

Al Crabb

Kewanna, Ind.

Ann Damon

Bemus Point, N.Y.

Andy Davis

Charleston, W. Va.

Dennis Dawes

Greenville, $\mathrm{Pa}$.
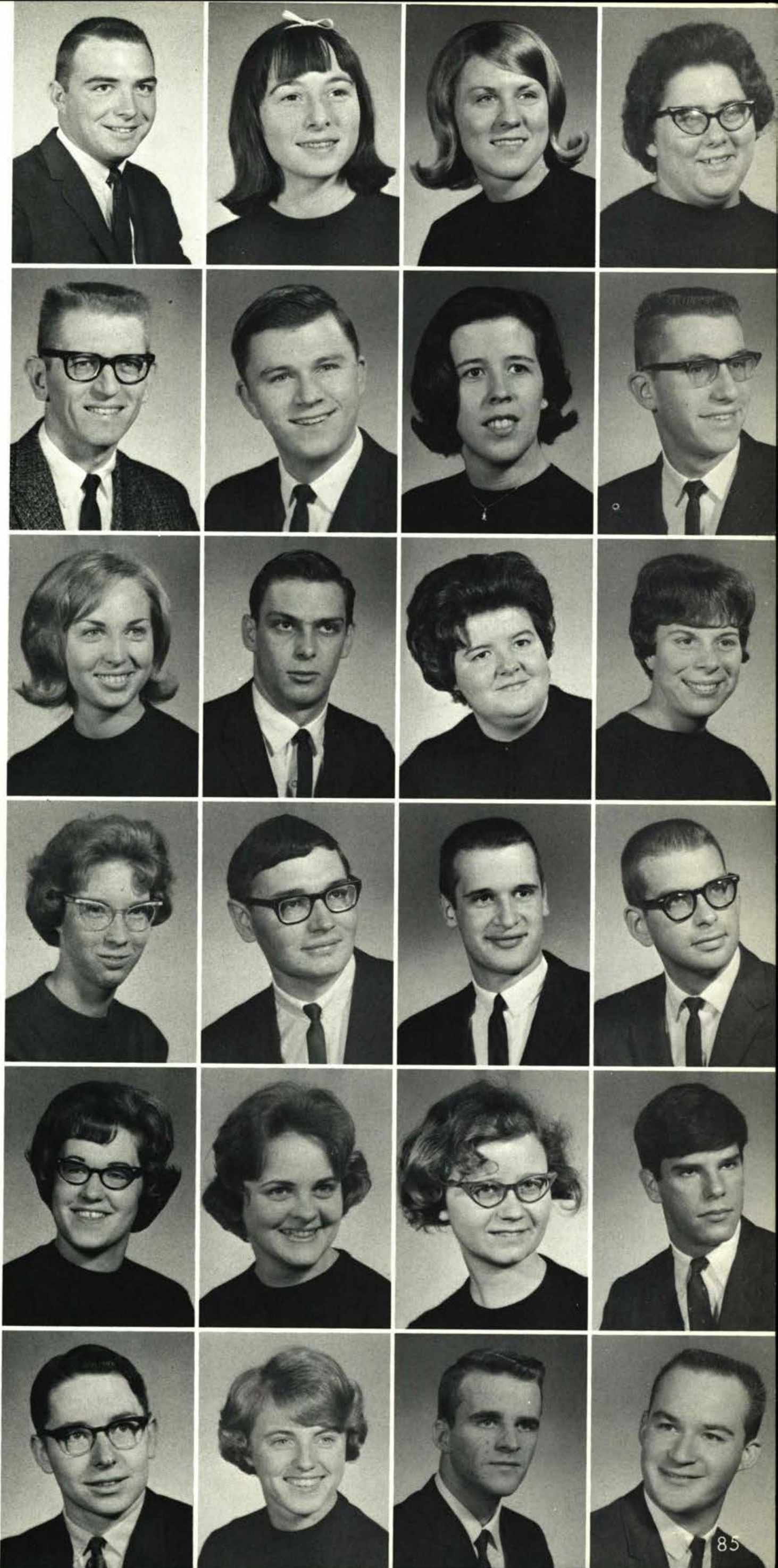

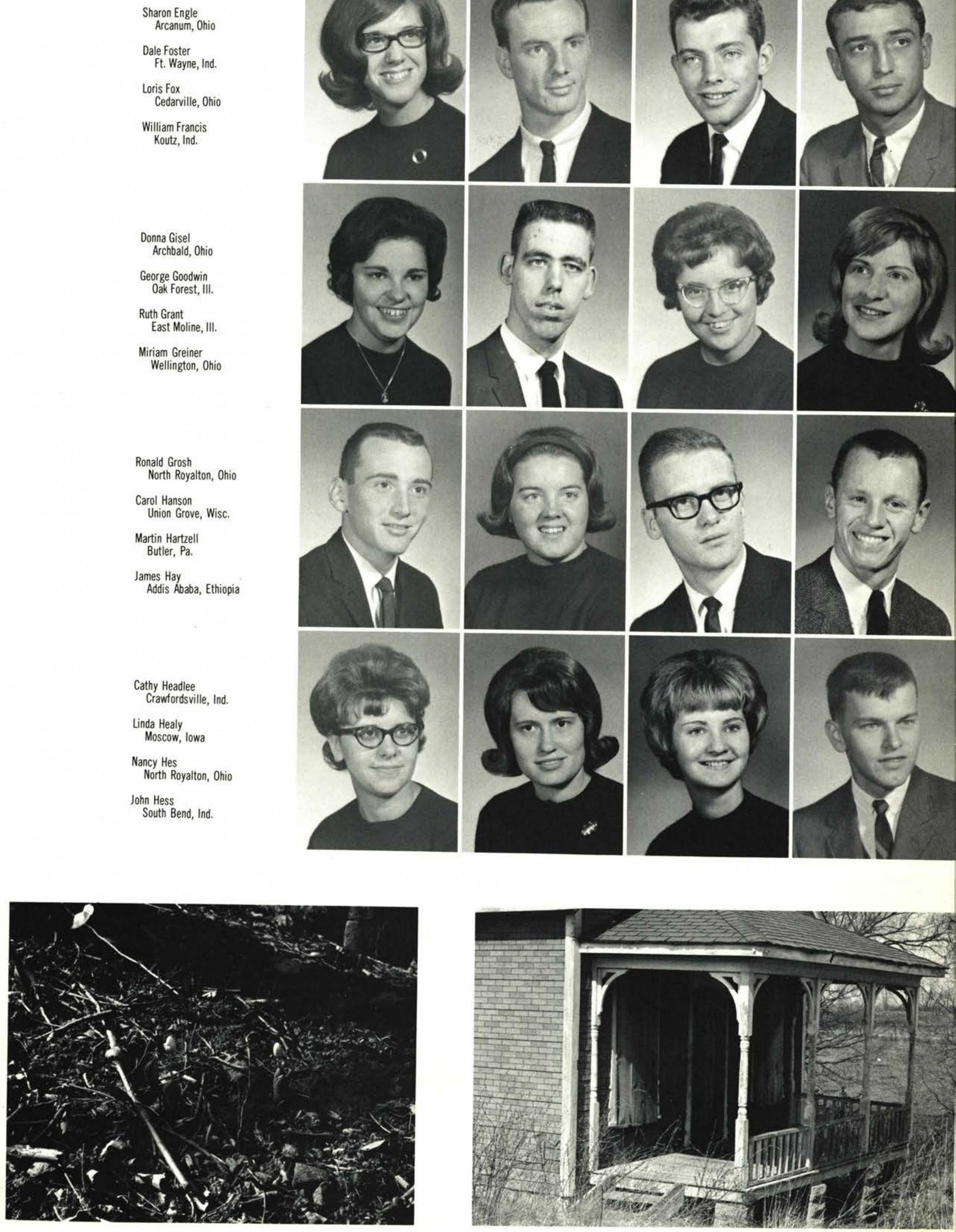

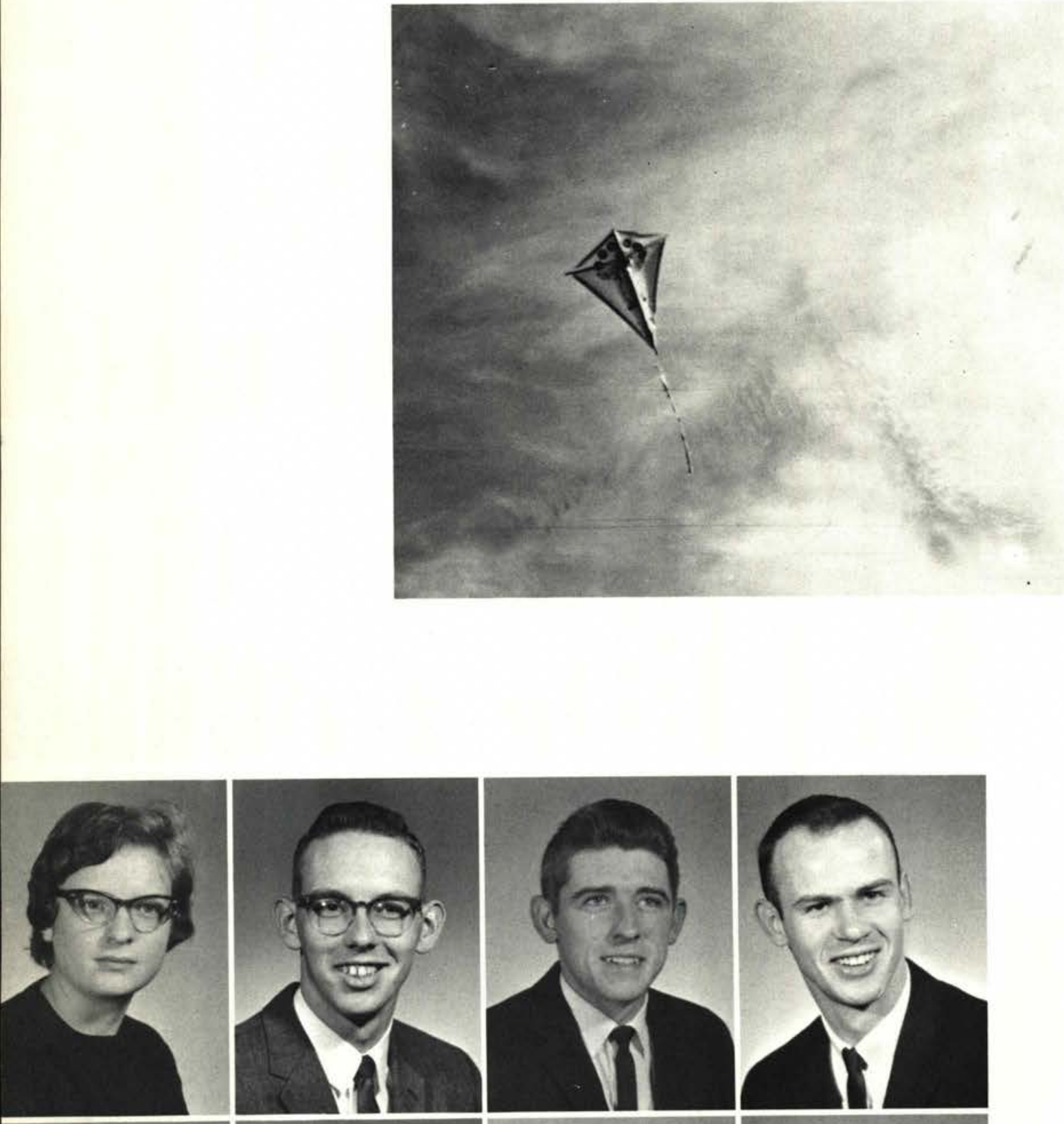

Doris Hiller
Cleveland, Ohio

Dales Holmes

Litchfield, Ohio

Gary Holtz

Miscatine, lowa

\section{Ernie Hopper \\ Xenia, Ohio}
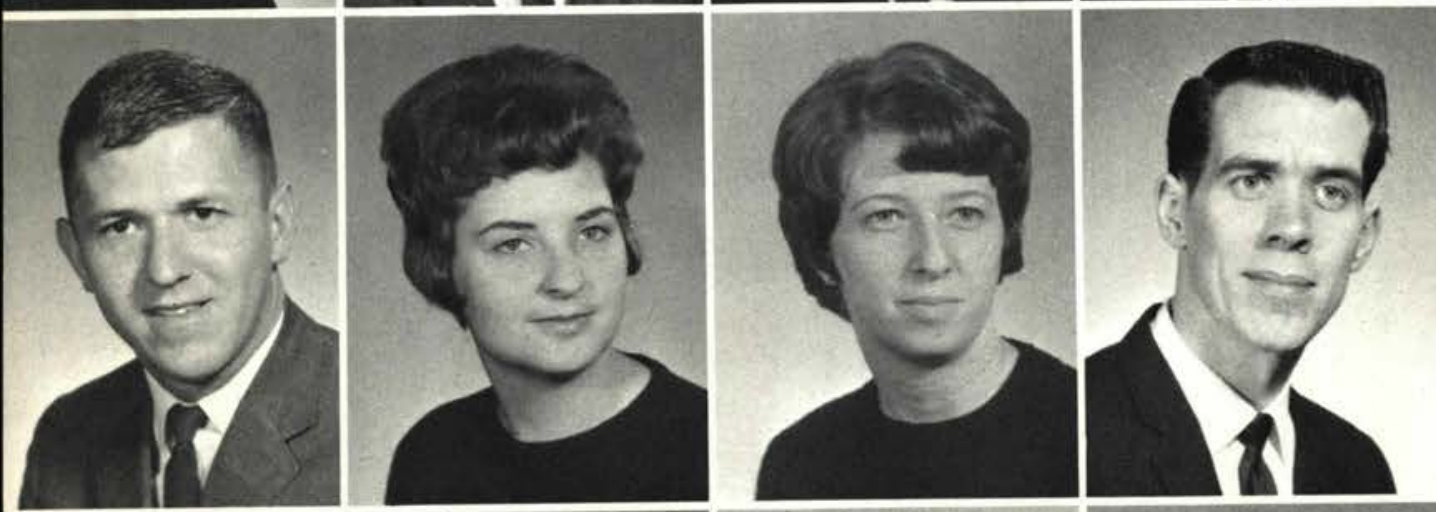

Ted Jager

Wyoming, Mich.

Mary Jones

Richmond, Va.

Sandy Jones Concord, Calif.

\section{Ken Kunkle}

Silvis, III.

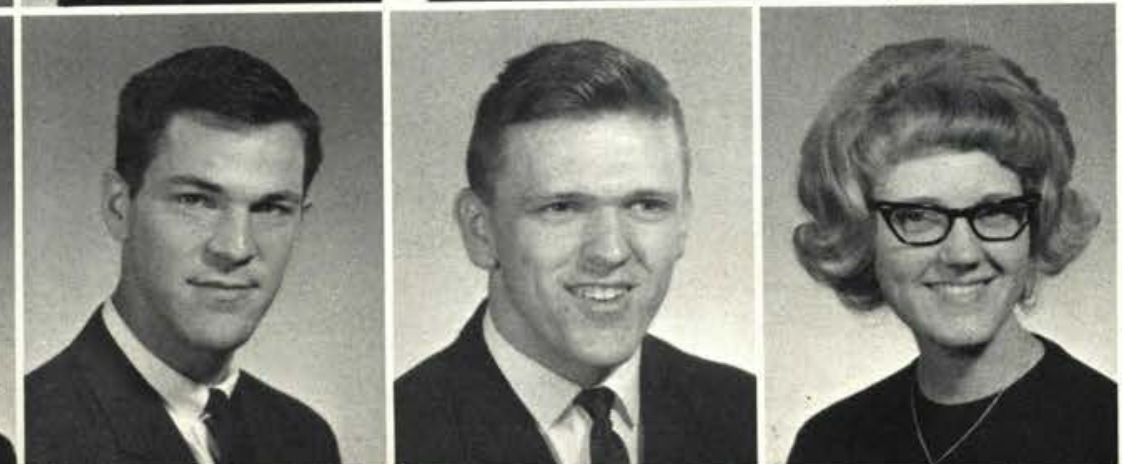

Laurene Kytle Euclid, Ohio

Don Leach East Northport, N.Y.

Doug Lightly

Austin, Minn.

Kathy Mansfield Lorain, Ohio 
David McDonald

Lake Orion, Mich.

Earl McGuffey

Mason, Ohio

James Miller

Valparaiso, Ind.

Linda Miller

Dayton, Ohio

Roberta Miller

Lowellville, Ohio

Bonnie Millikan

Bothell, Wash.

Irene Millikan

Bothell, Wash.

Richard Minier

Cedarville, Ohio

Lawrence Montgomery

Columbus, Ohio

David Newton

Towanda, Pa.

Ted Oakle

Midland, Mich.

James Oswald

Millerburg, Ohio
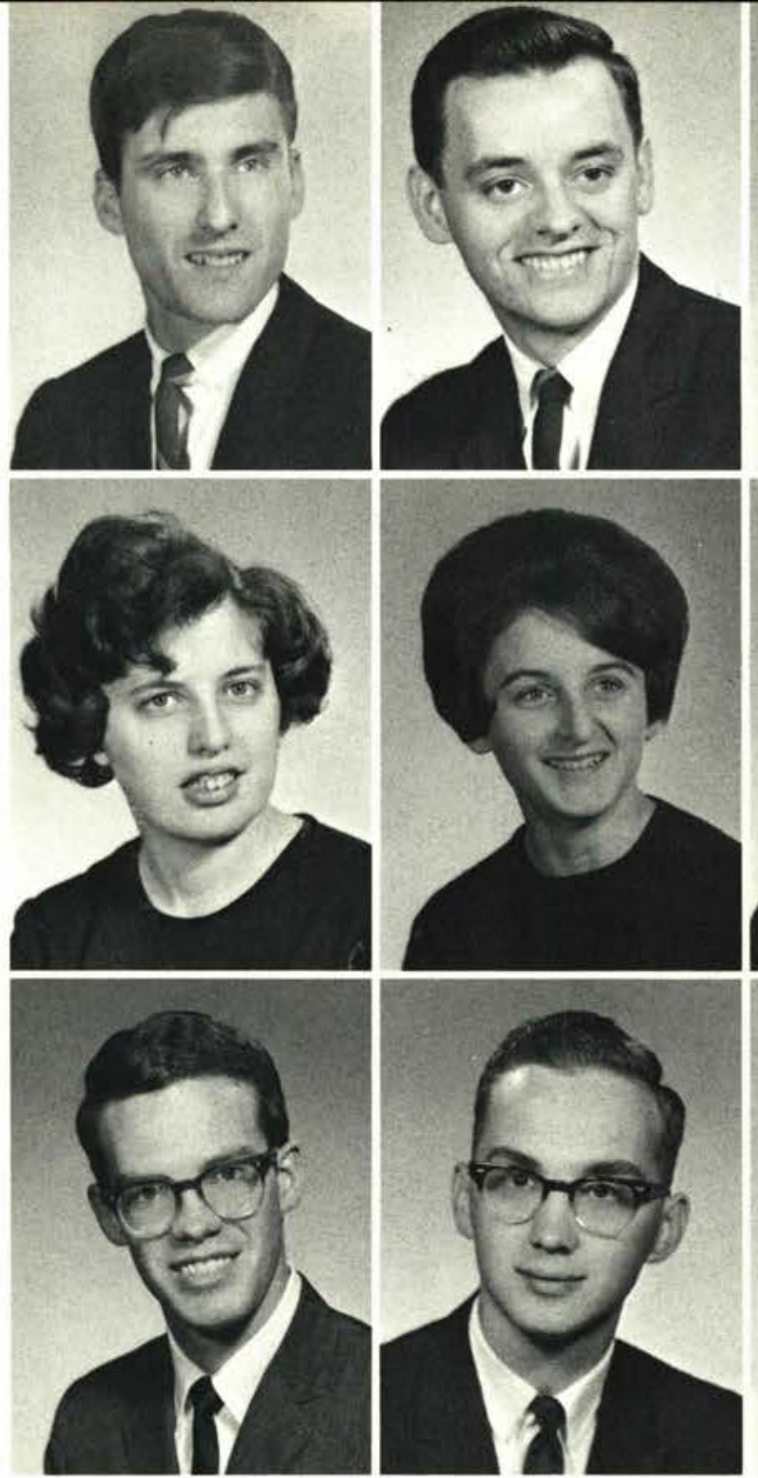
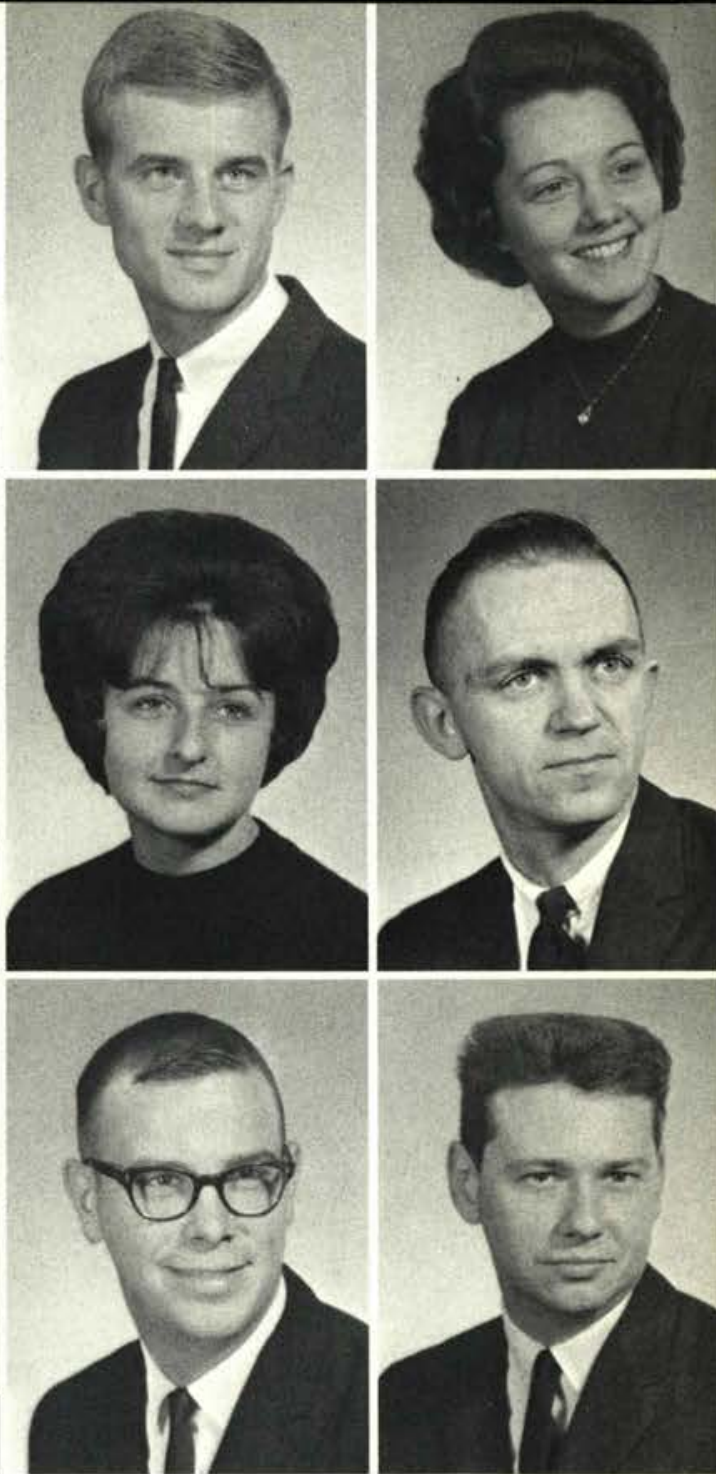

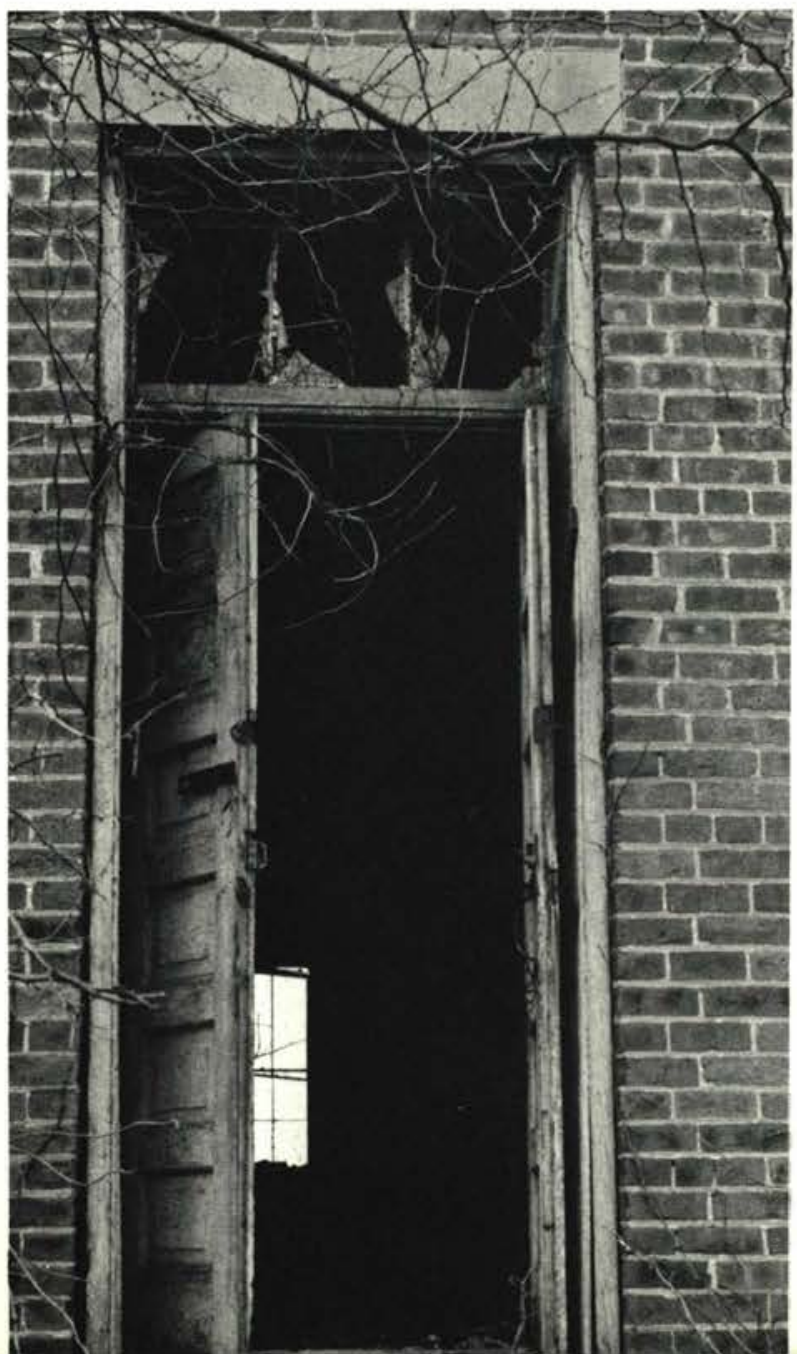




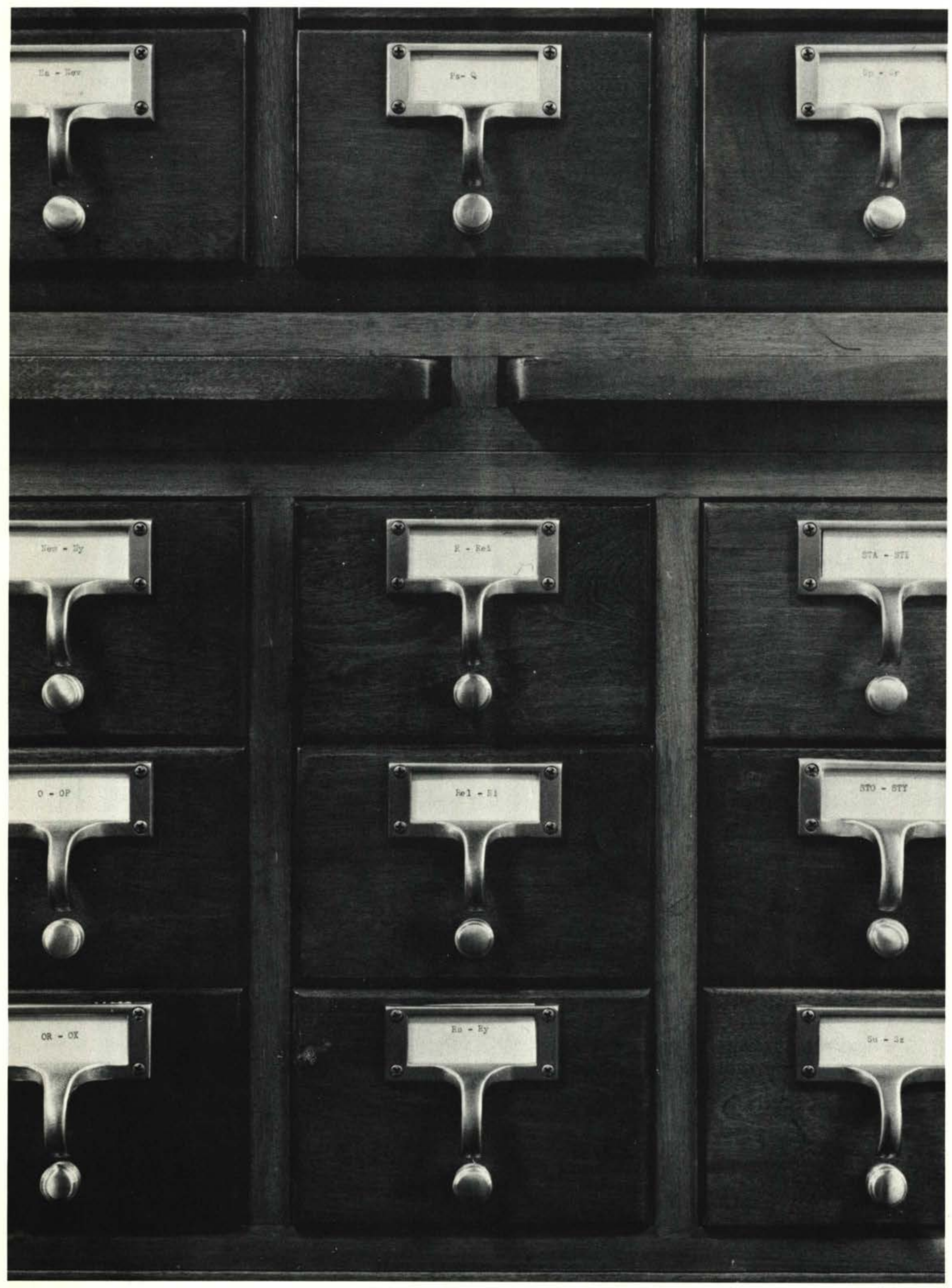




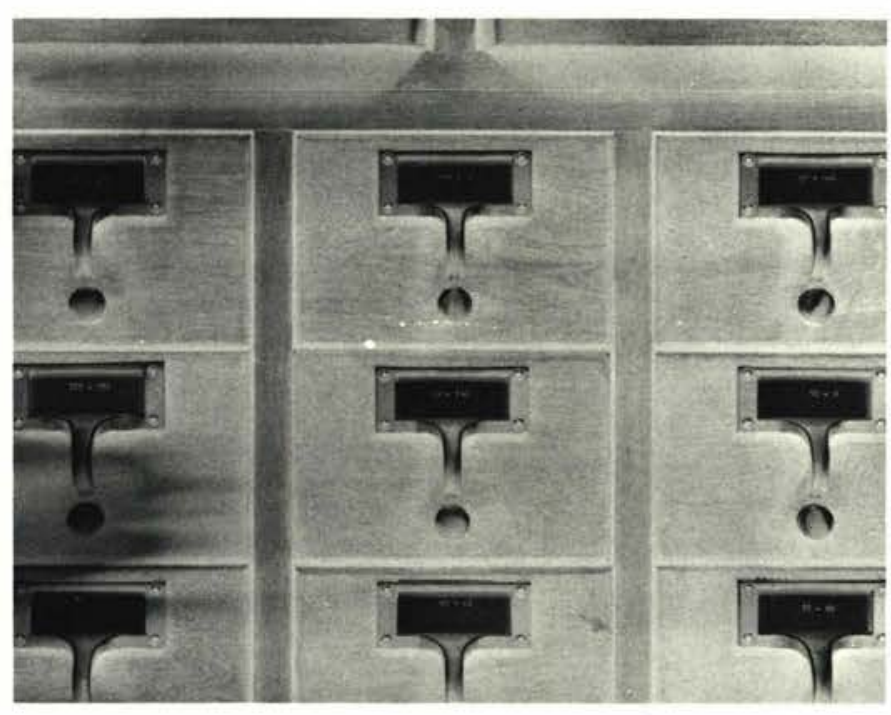

\section{Activities and Organizations}




\section{FALL and SPRING BIBLE LECTURES}

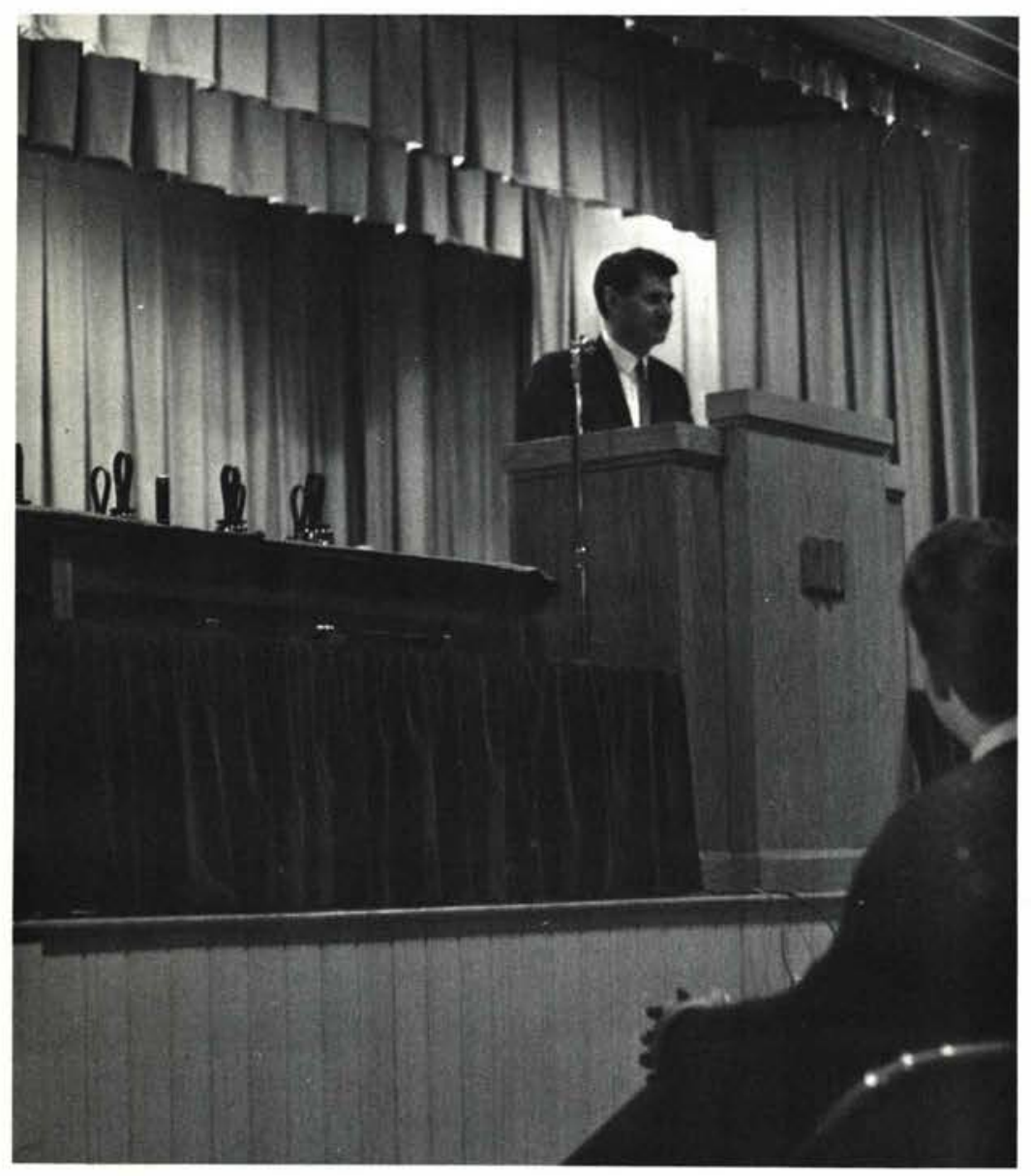

\section{Bible Conferences}

Dr. William E. Kuhnle, pastor of the Garfield Baptist Church in Milwaukee, Wisconsin, opened the fall semester with inspiring messages from the book of Ephesians. Both the Fall and Spring Bible Conferences open with the most important day, the Day of Prayer. Classes are dismissed and faculty and student body together seek the blessing of God from His Word and in group prayer.

Dr. Hugh Horner, pastor of the Calvary Baptist Church, Sandusky, Ohio, challenged each heart as he spoke from the book of Ruth.

\section{Dr. Hugh Horner}

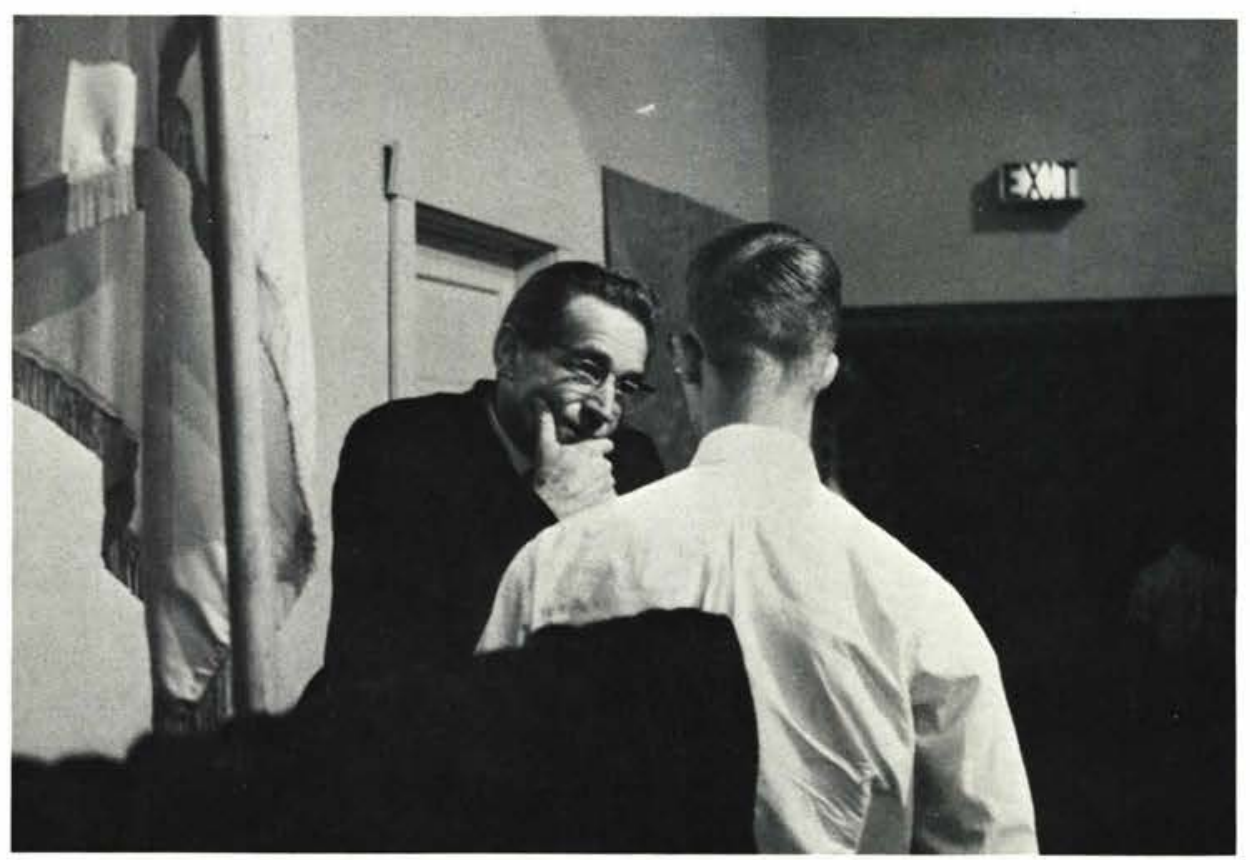

Dr. William E. Kuhnle 


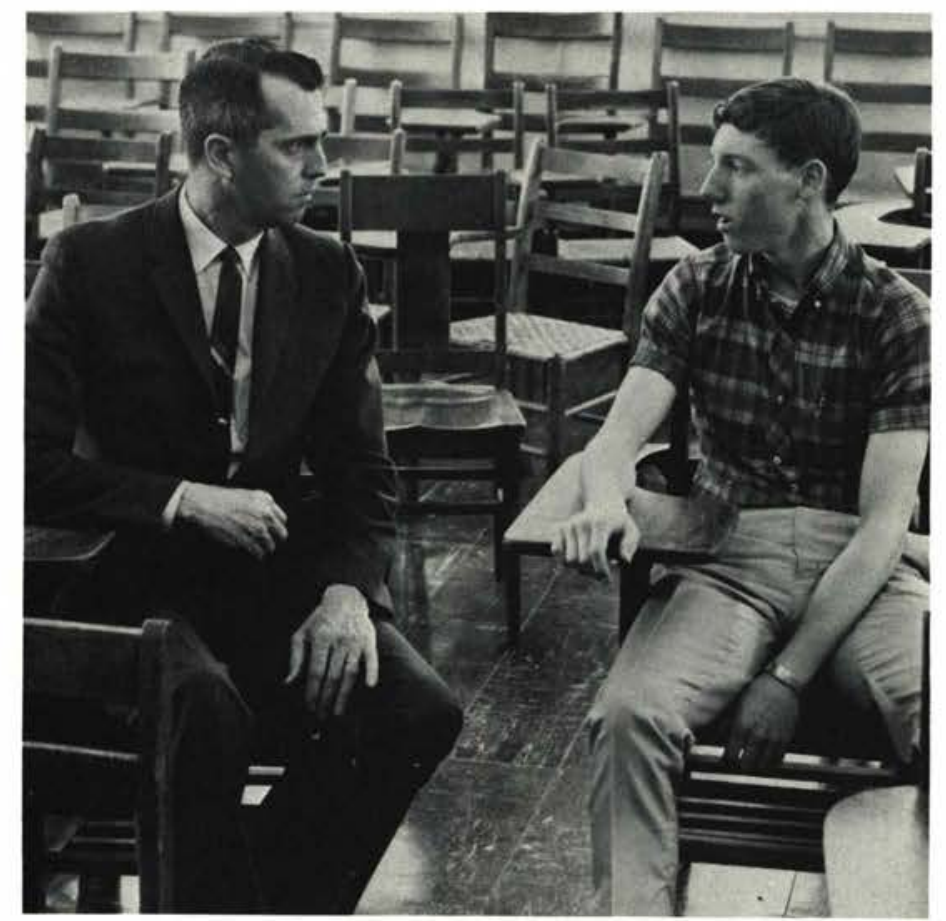

Rev. Richard Durham

\section{Chapel}

"Sweet Hour of Prayer" sets the mood for daily chapel services. The hour set aside for worship usually includes special music and a message from the Word of God. Speakers include pastors of local churches, representatives from Christian organizations, missionaries, laymen from the various professions, faculty and administration from the college, and sometimes students representing various campus organizations.

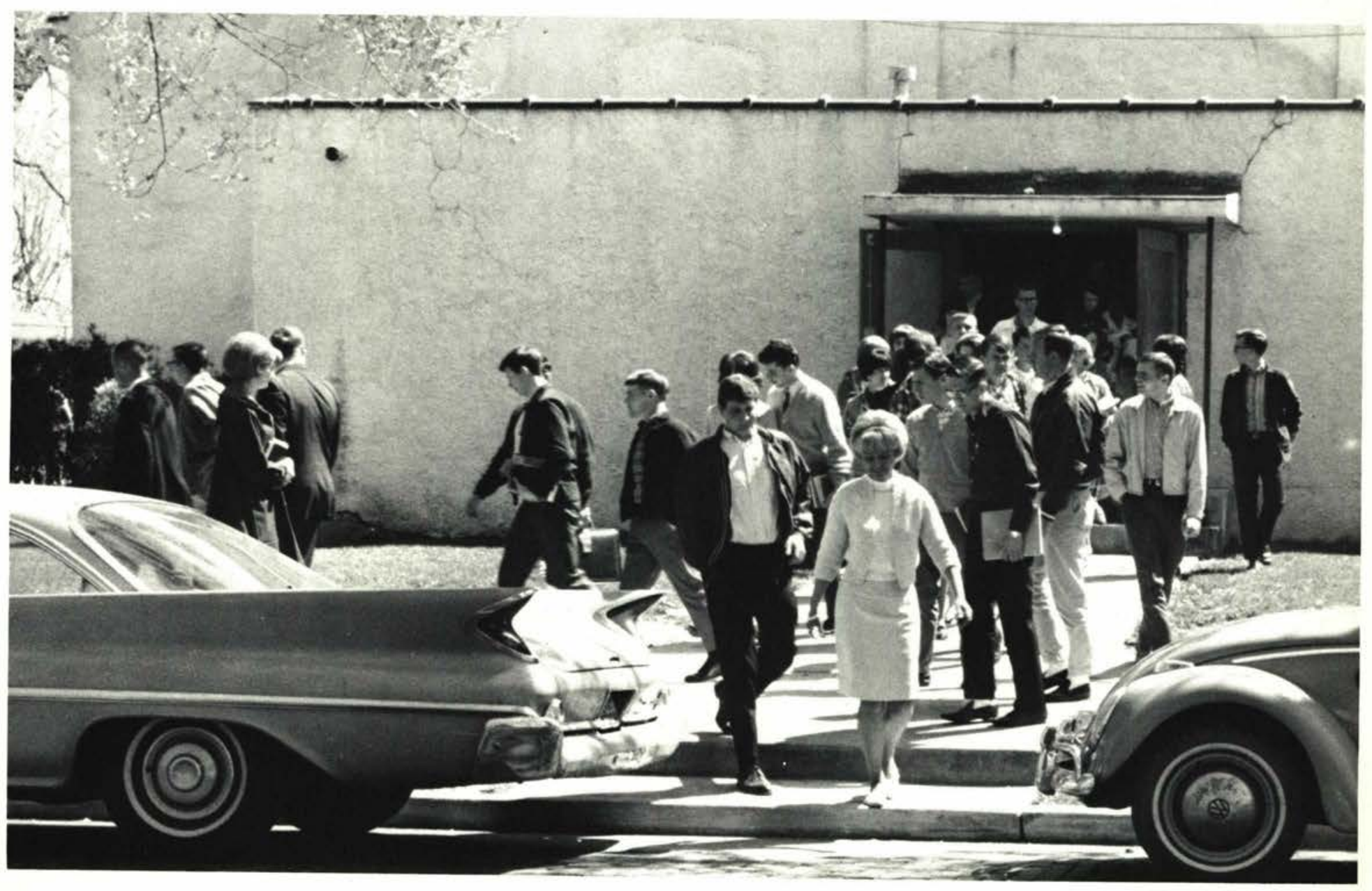



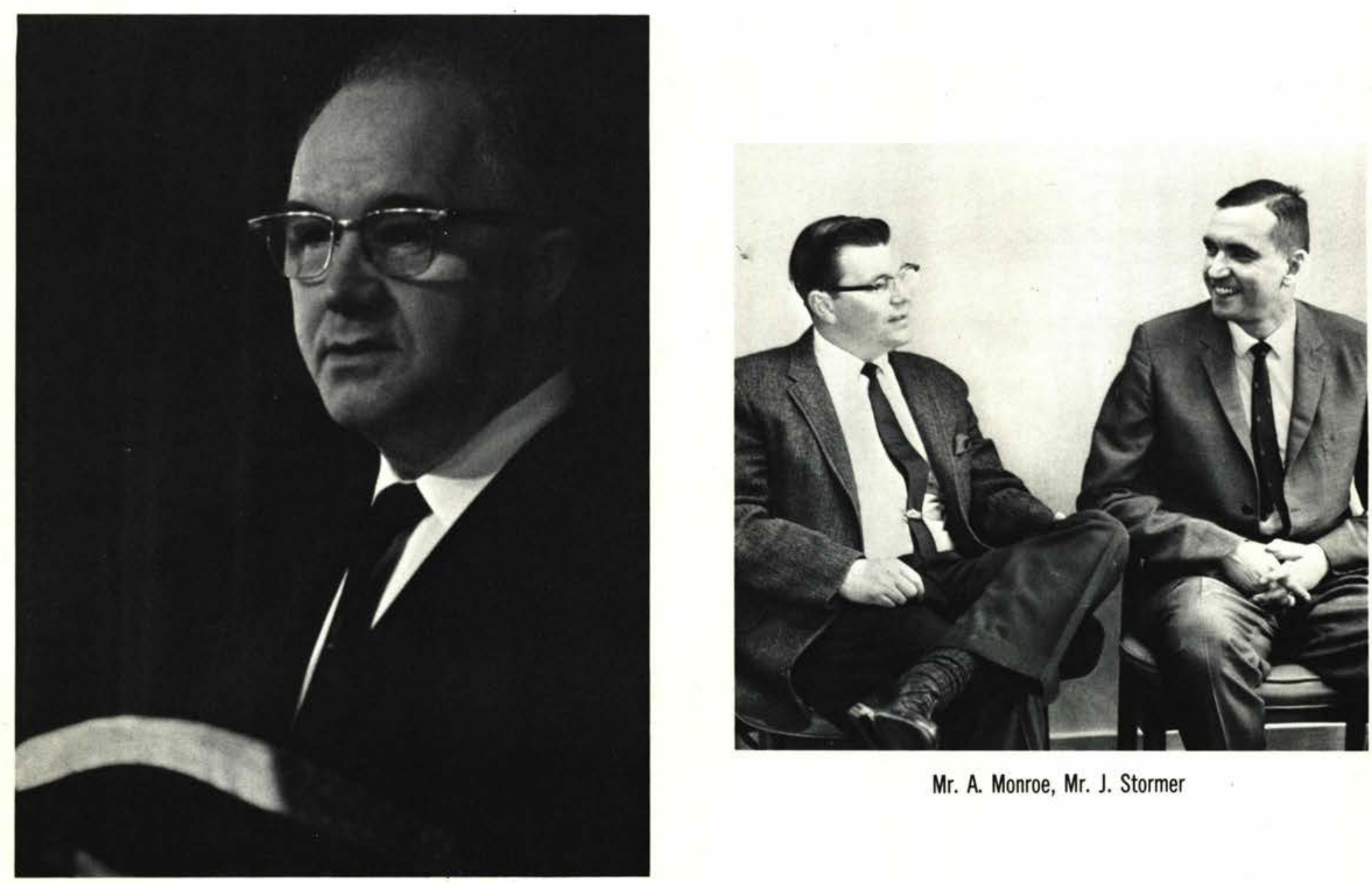

Mr. A. Monroe, Mr. J. Stormer

Rev. Henry Murdock
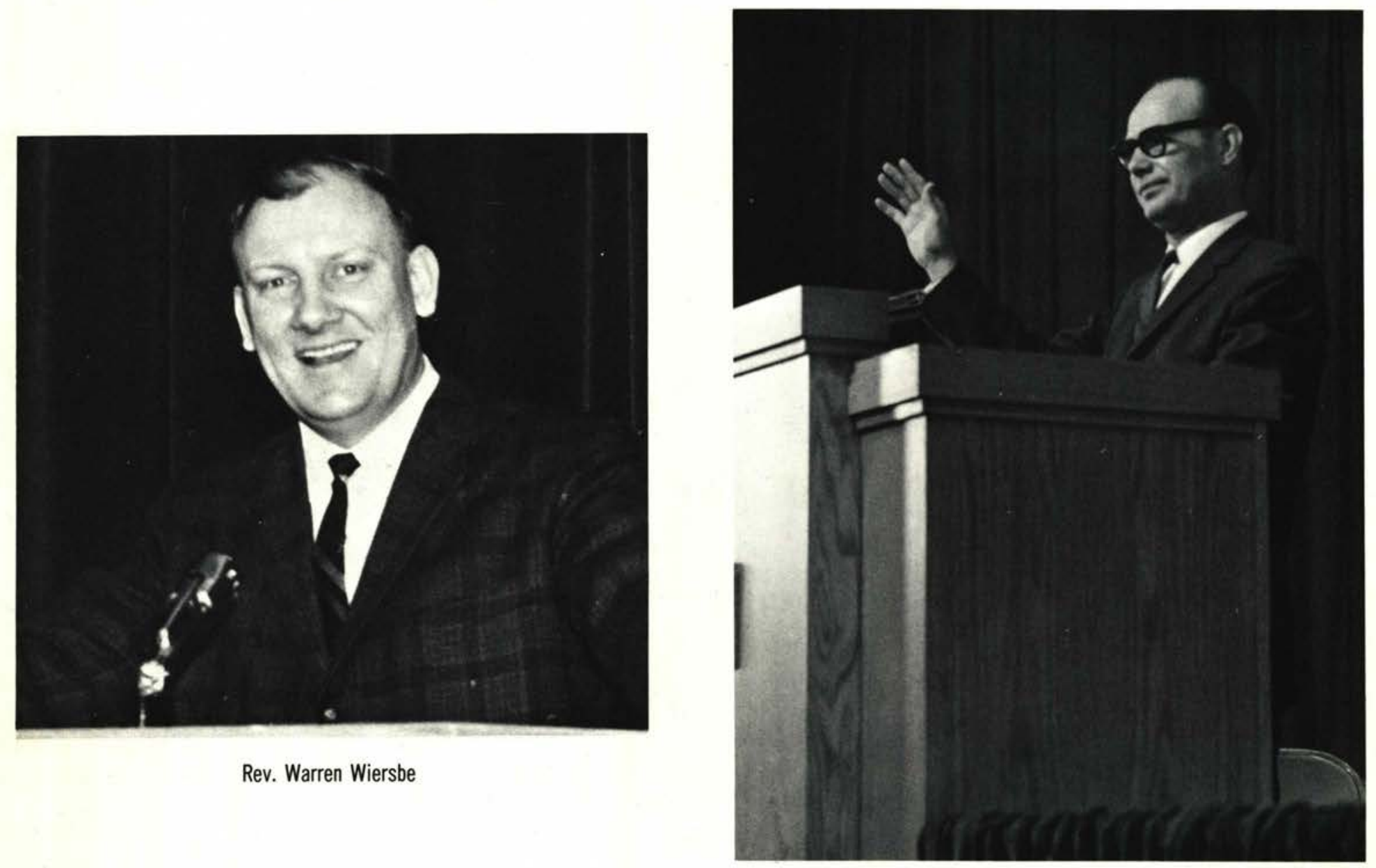

Rev. L. Rowland 


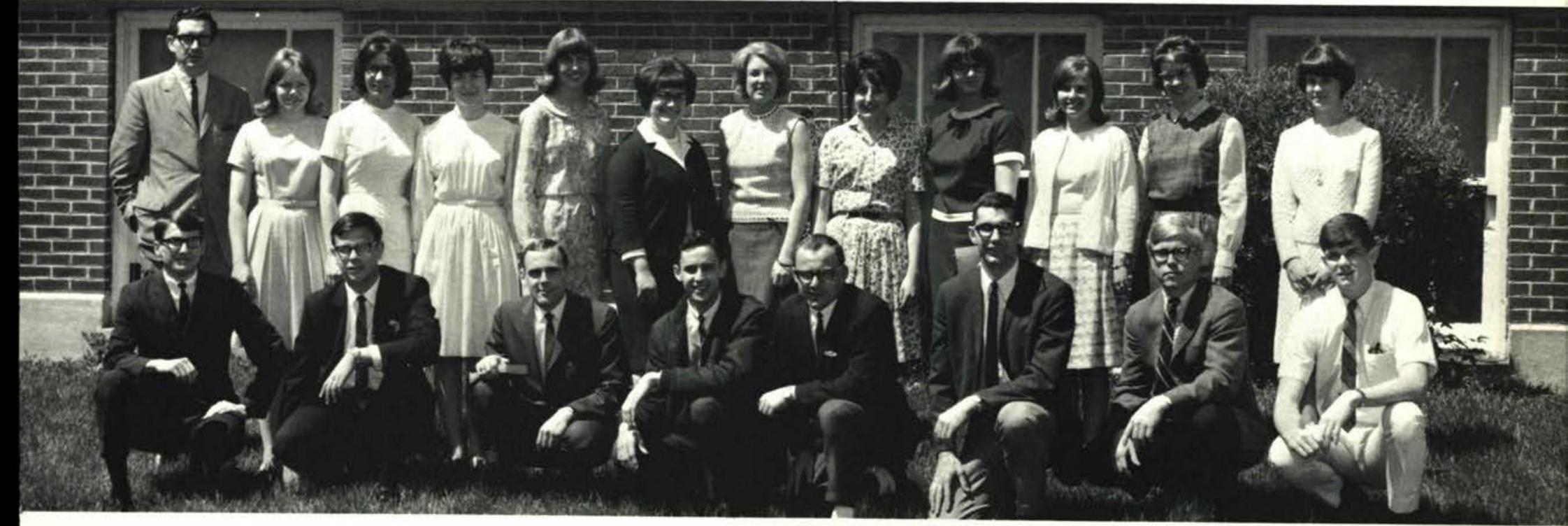

CAMPUS LIFE-Standing: Mr. S. Ballard, S. Empet, L. Kytle, M. Grable, L. Taylor, J. Harris, P. Cannady, I. Millikan, K. Farrell, C. Payne, L. Rulison, E. Entenman. Front: D. Tillotson,

T. Oakley, D. Gordon, L. Bellintani, J. Rueck, L. Waite, D. Trunnell, T. Bell.

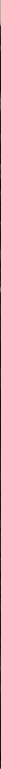

OSSO HOME - Standing: J. Miller, N. Earles, M. Greiner, S. Wells, V. Merryman, A. Keim, N. Freeman, J. Emerson, J. Harris, M. Schulte, D. Dawes. Front: D. Rockwell, L. Waite, M. Hartzell, D. Shue, J. Rueck, T. Clater.
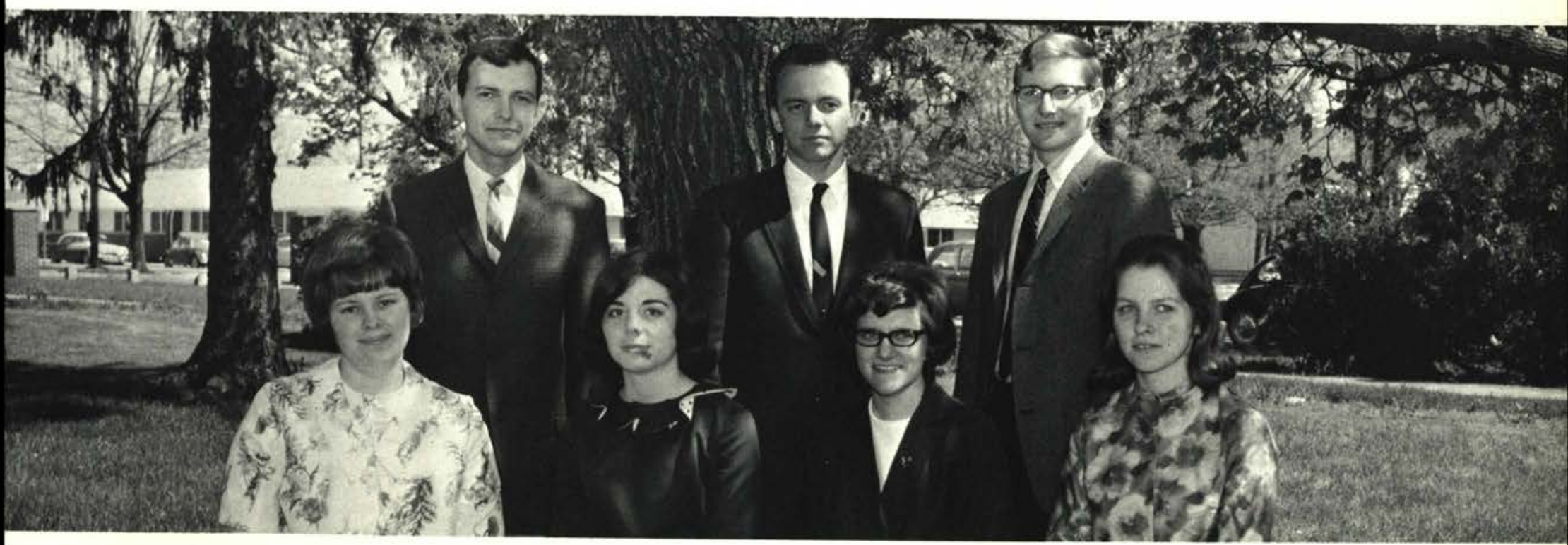

YOUTH GROUPS: D. Wall, R. Allerton, F. Jenista. Seated: K. Carter, S. Benton, D. Durkee, J. Crabtree. 


\section{Artist Series}

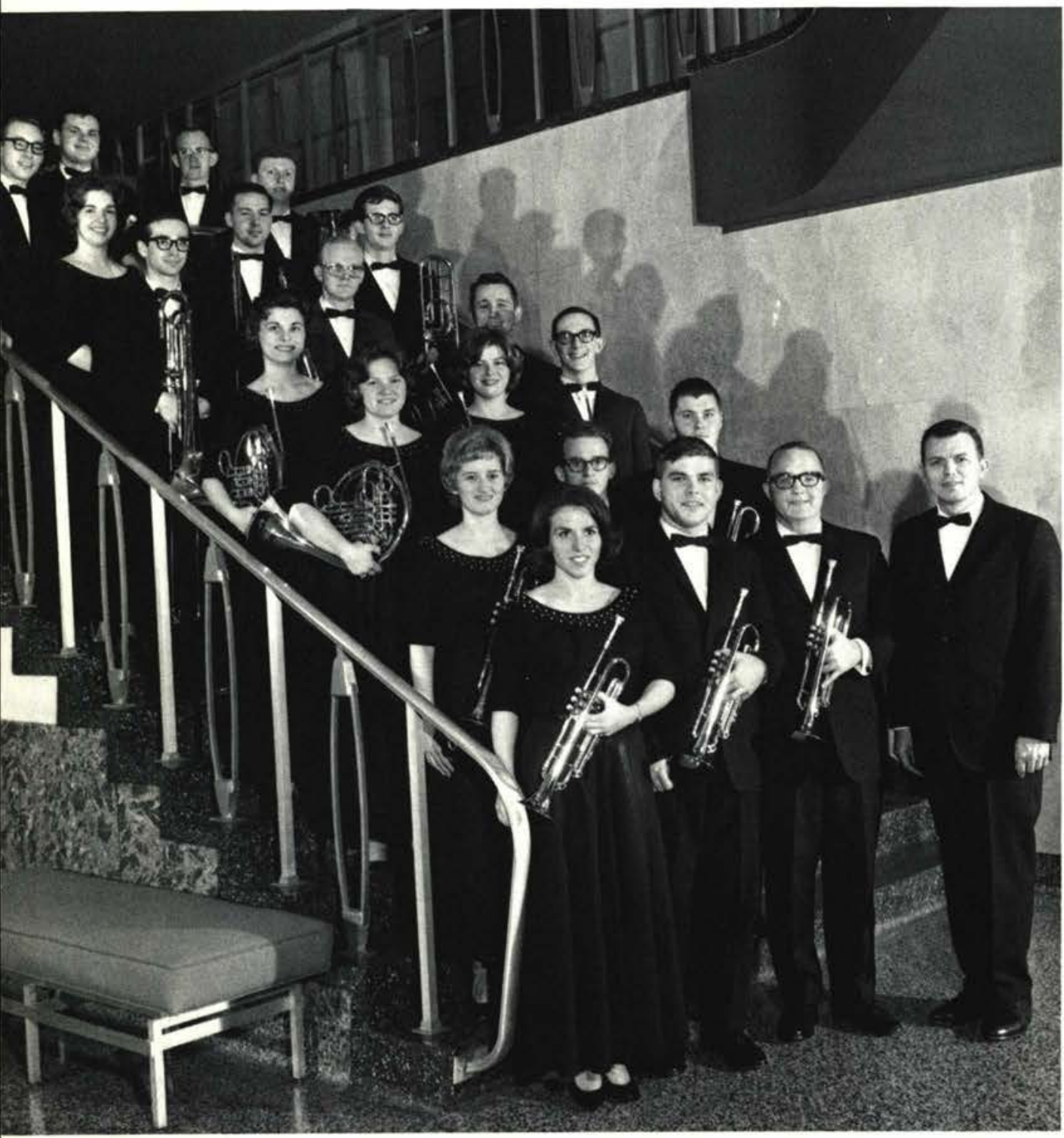

Ohio State Brass Choir

Dept. of Photography, Ohio State University.

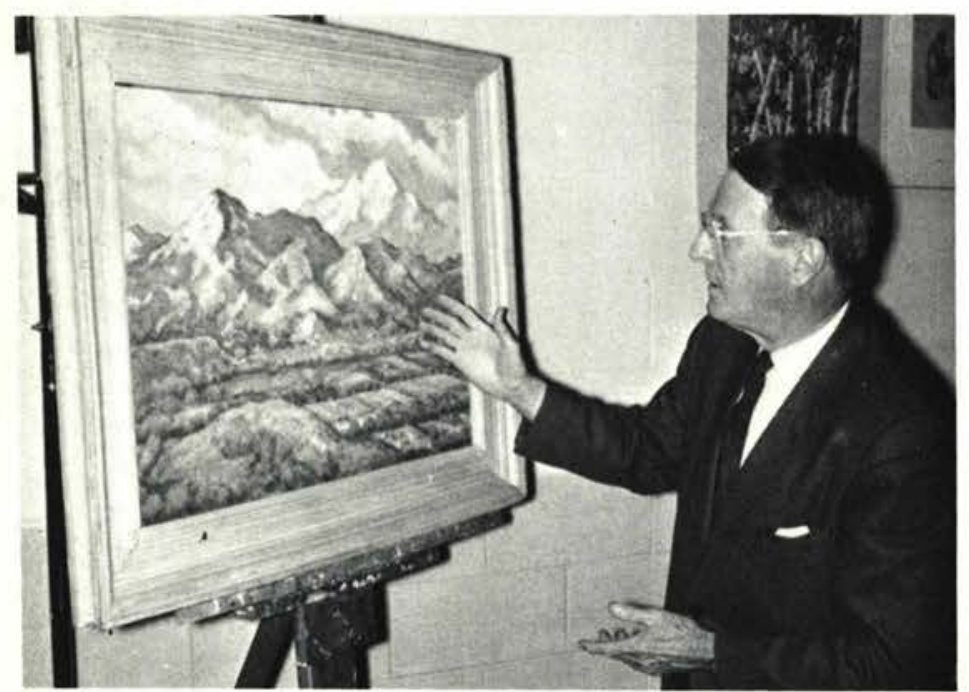

Grant Reynard
Tran Van Choung

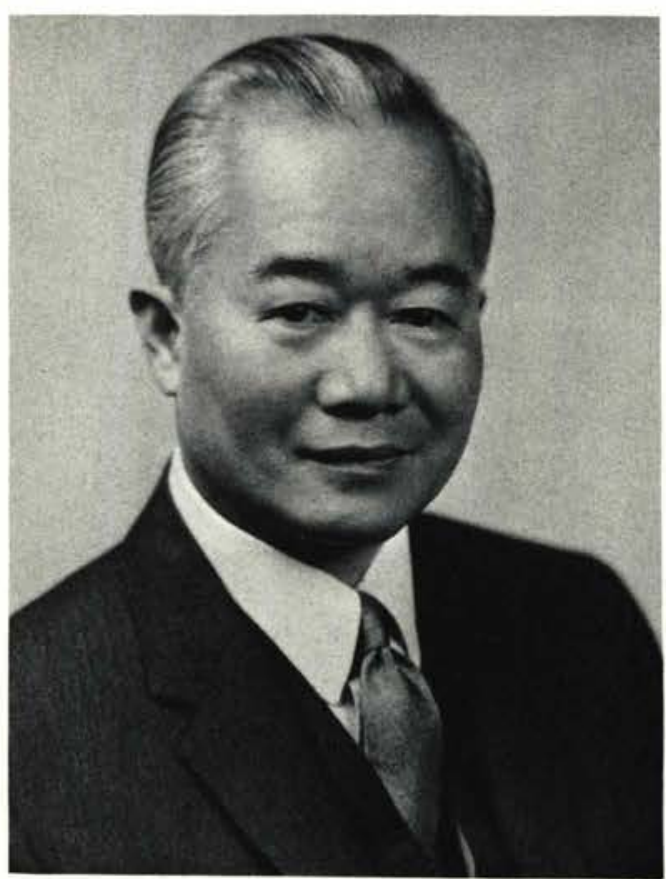




\section{Spring Concert . . . by . . . College Choir}

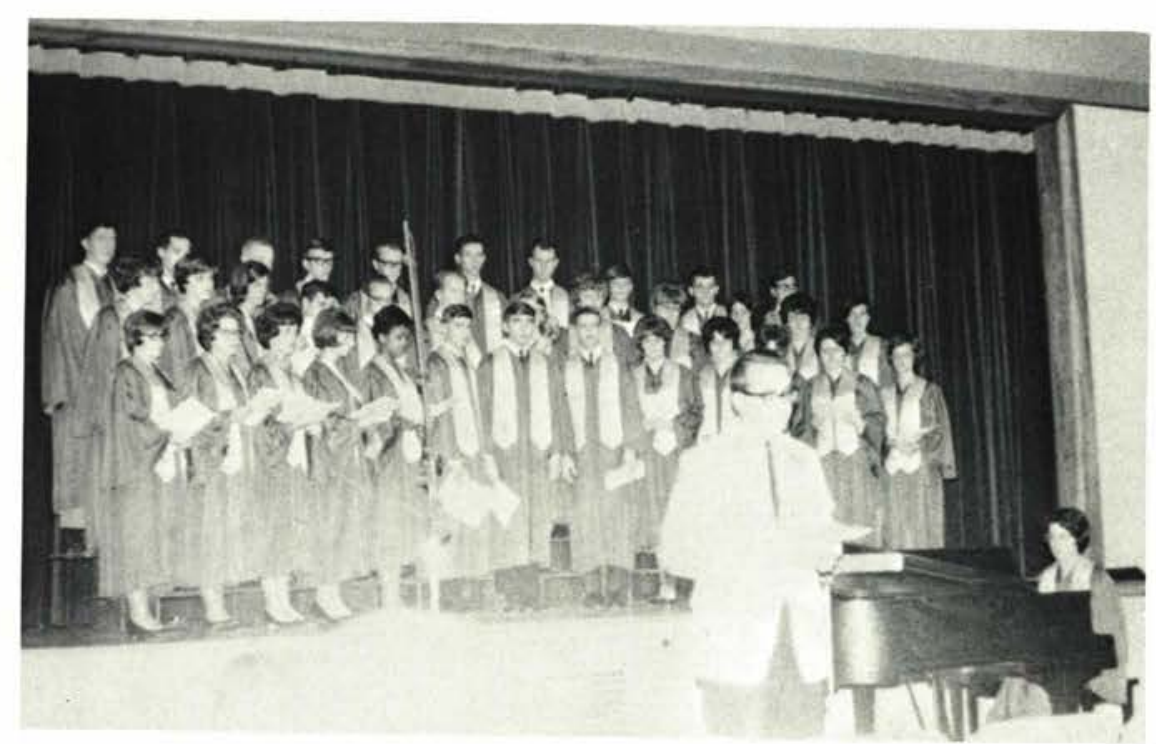

\section{and ... College Band}

and ... Choralaires
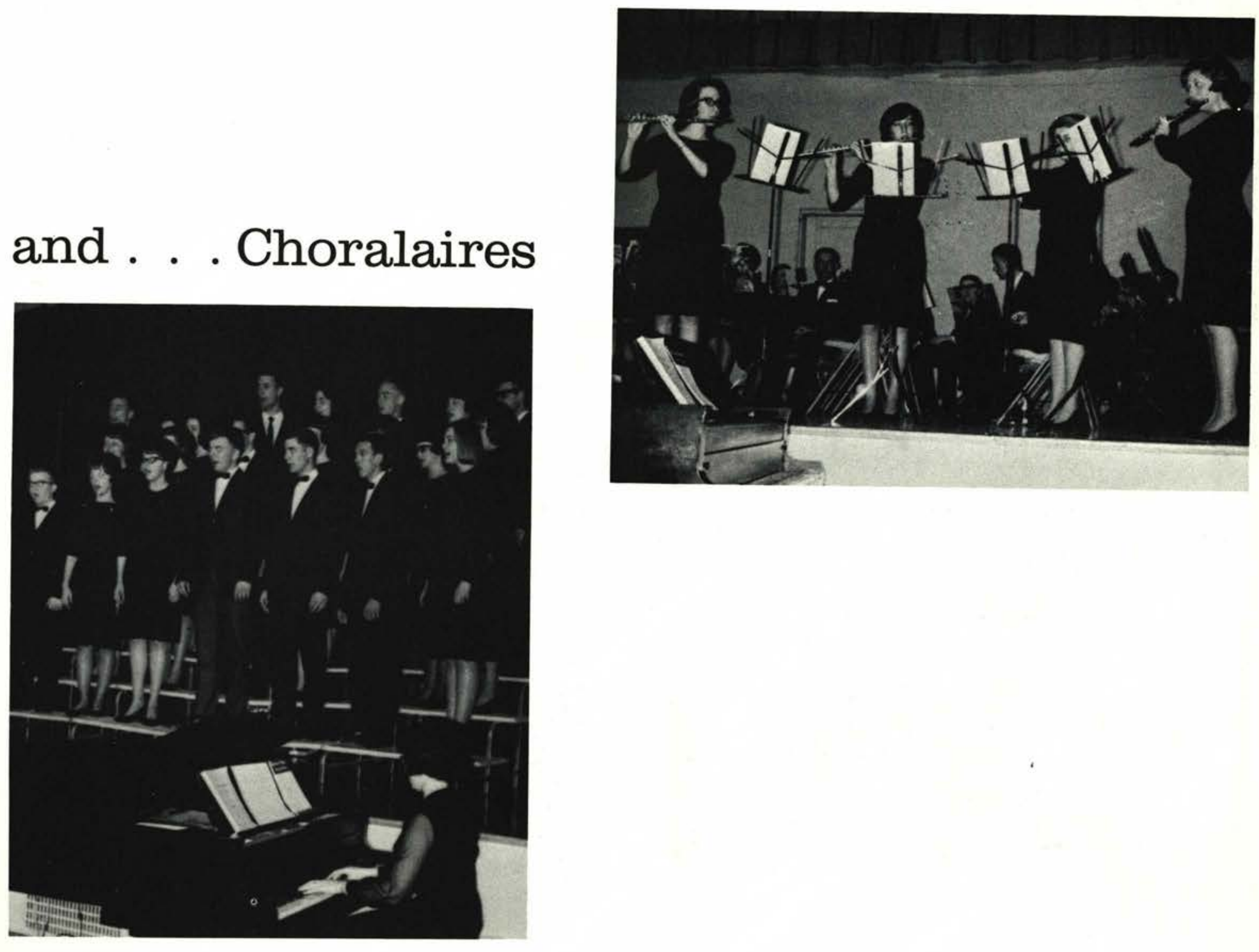

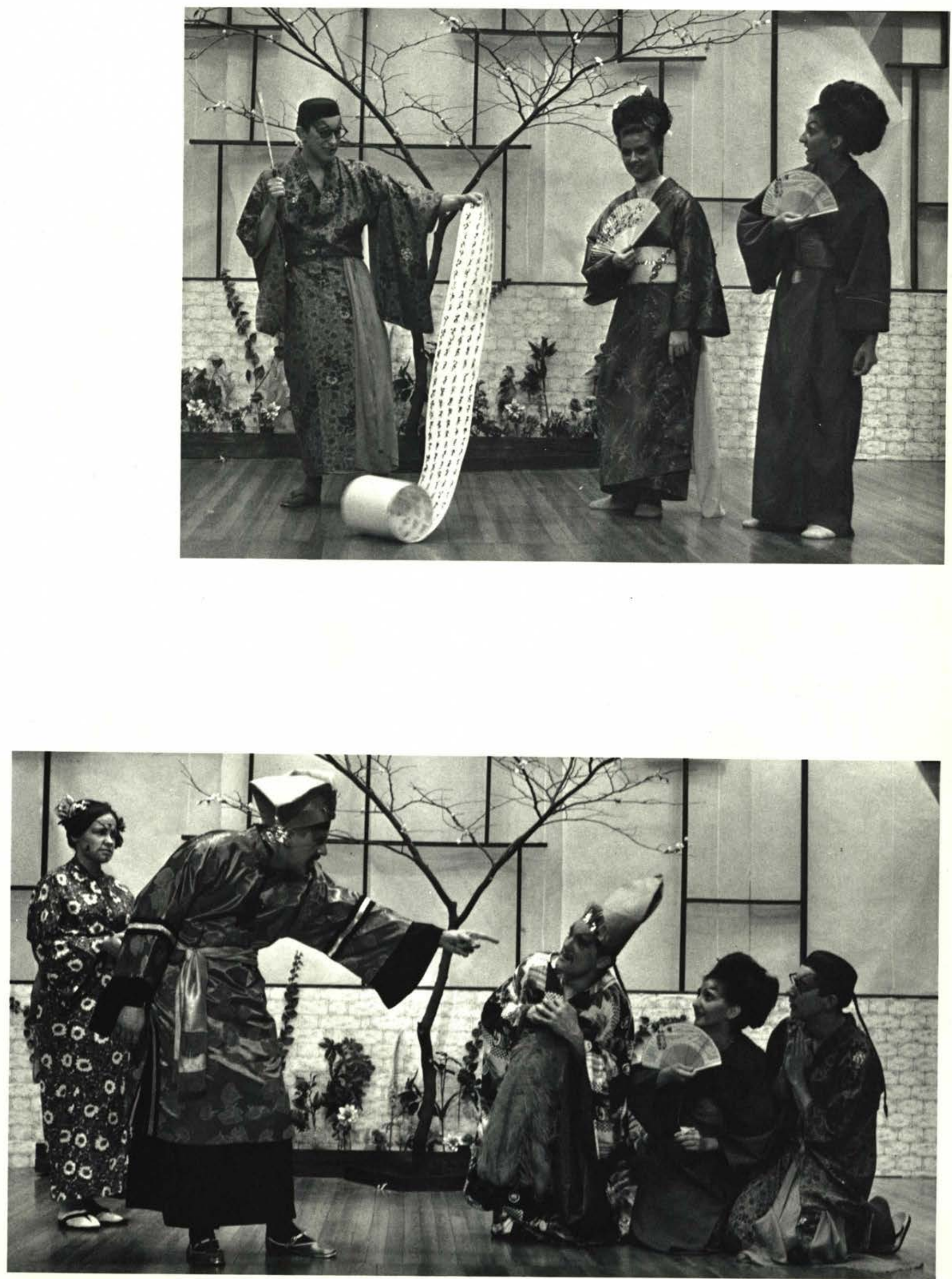


\section{Christmas Party}
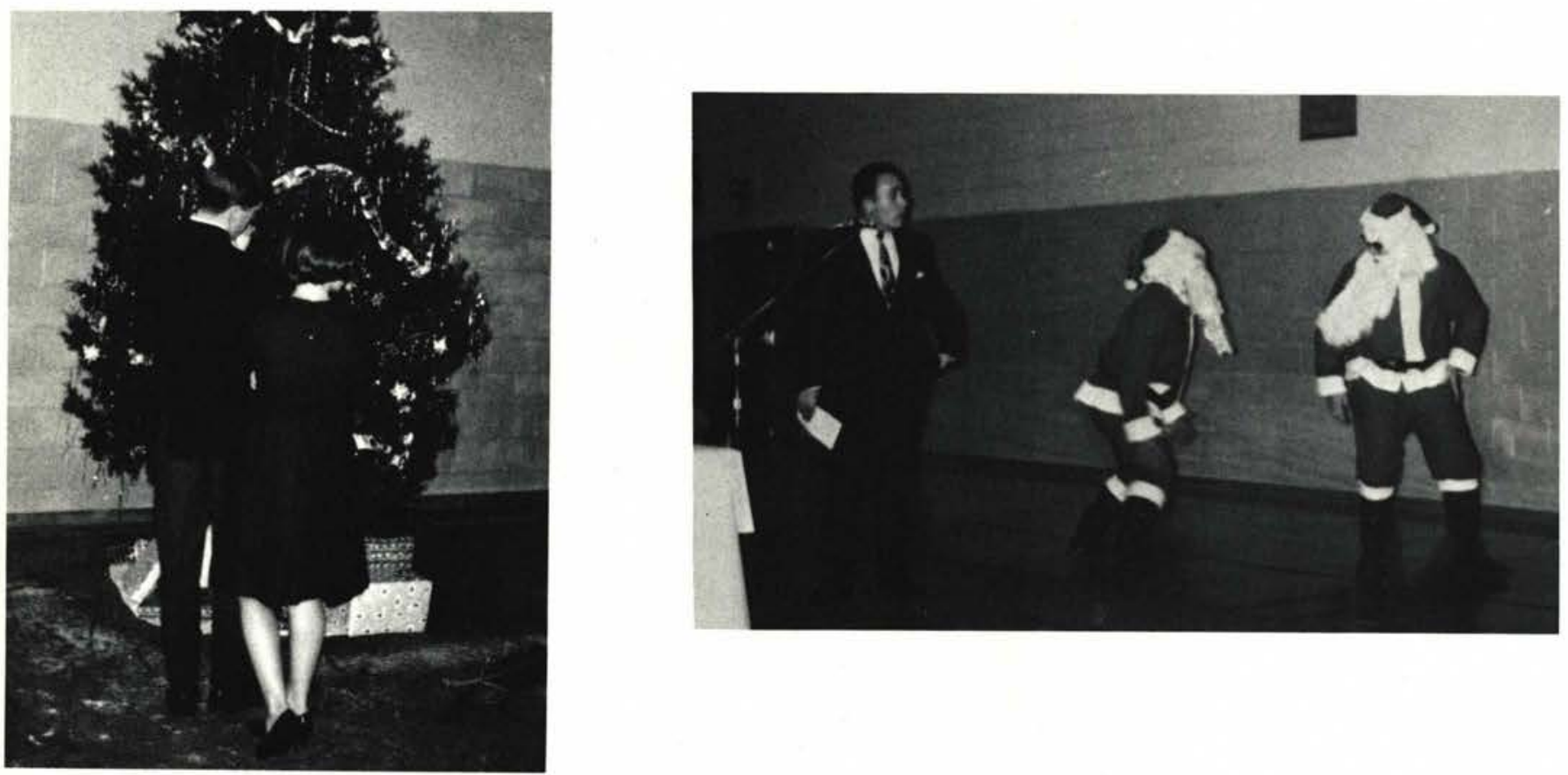

\section{All-School Socials}

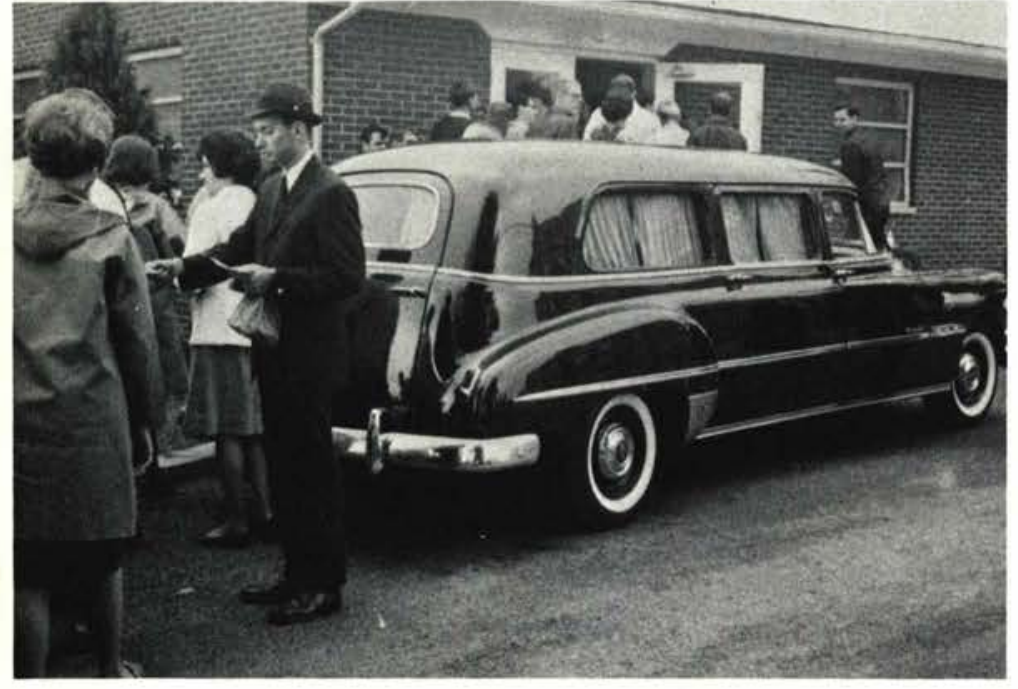

Operation Nightmare

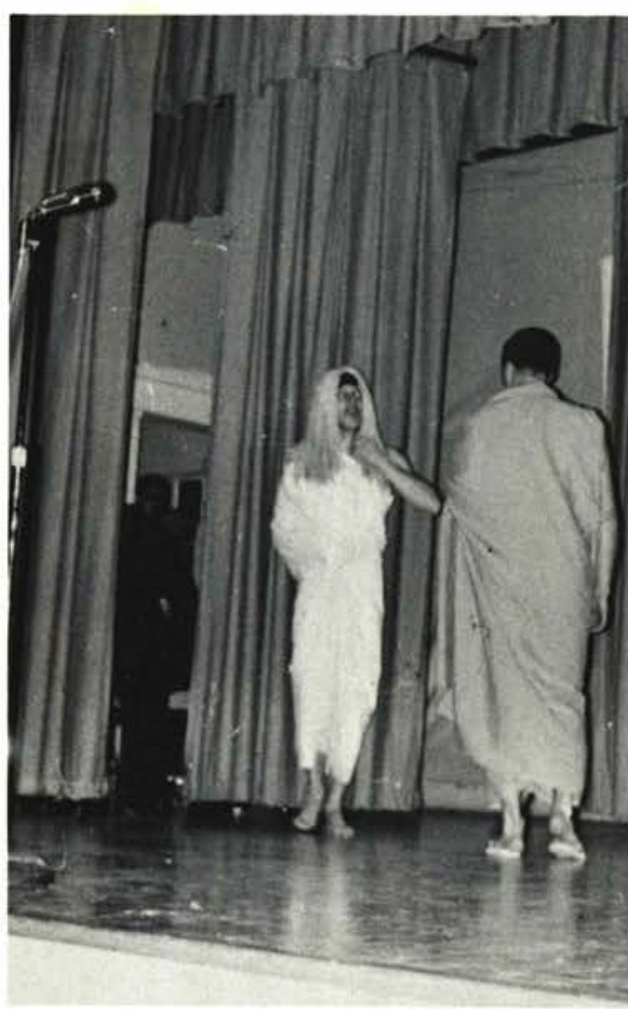

SBP Project Night 

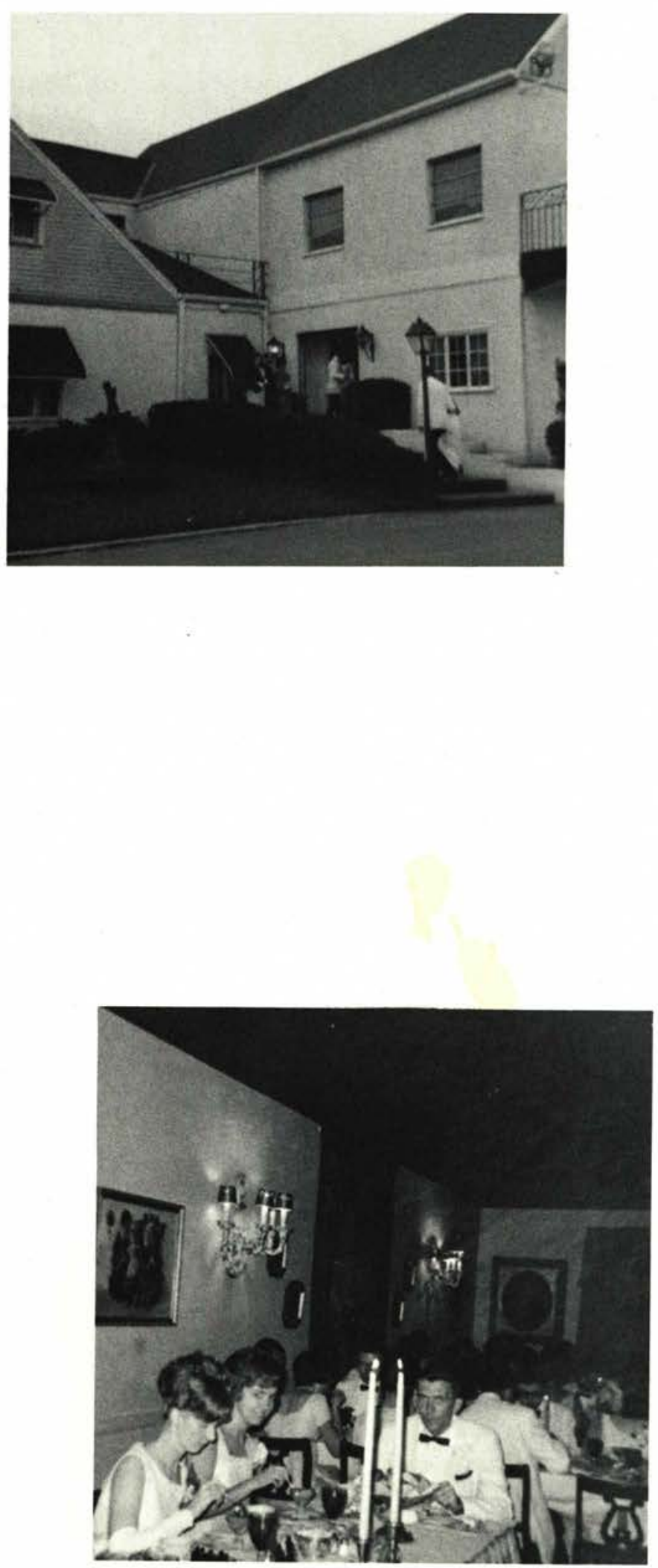

\section{Junior-Senior Banquet}
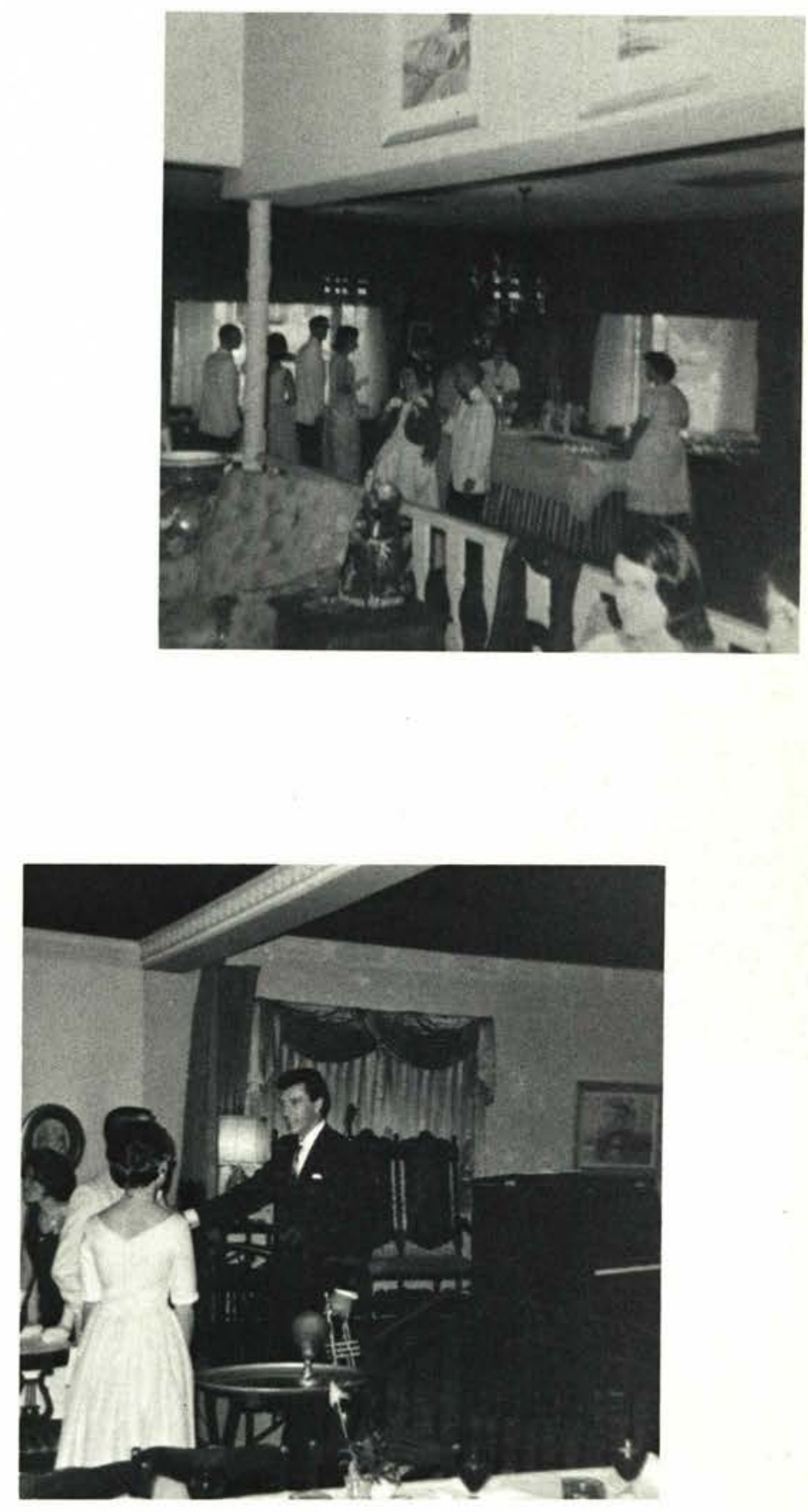


\section{HOMECOMING}
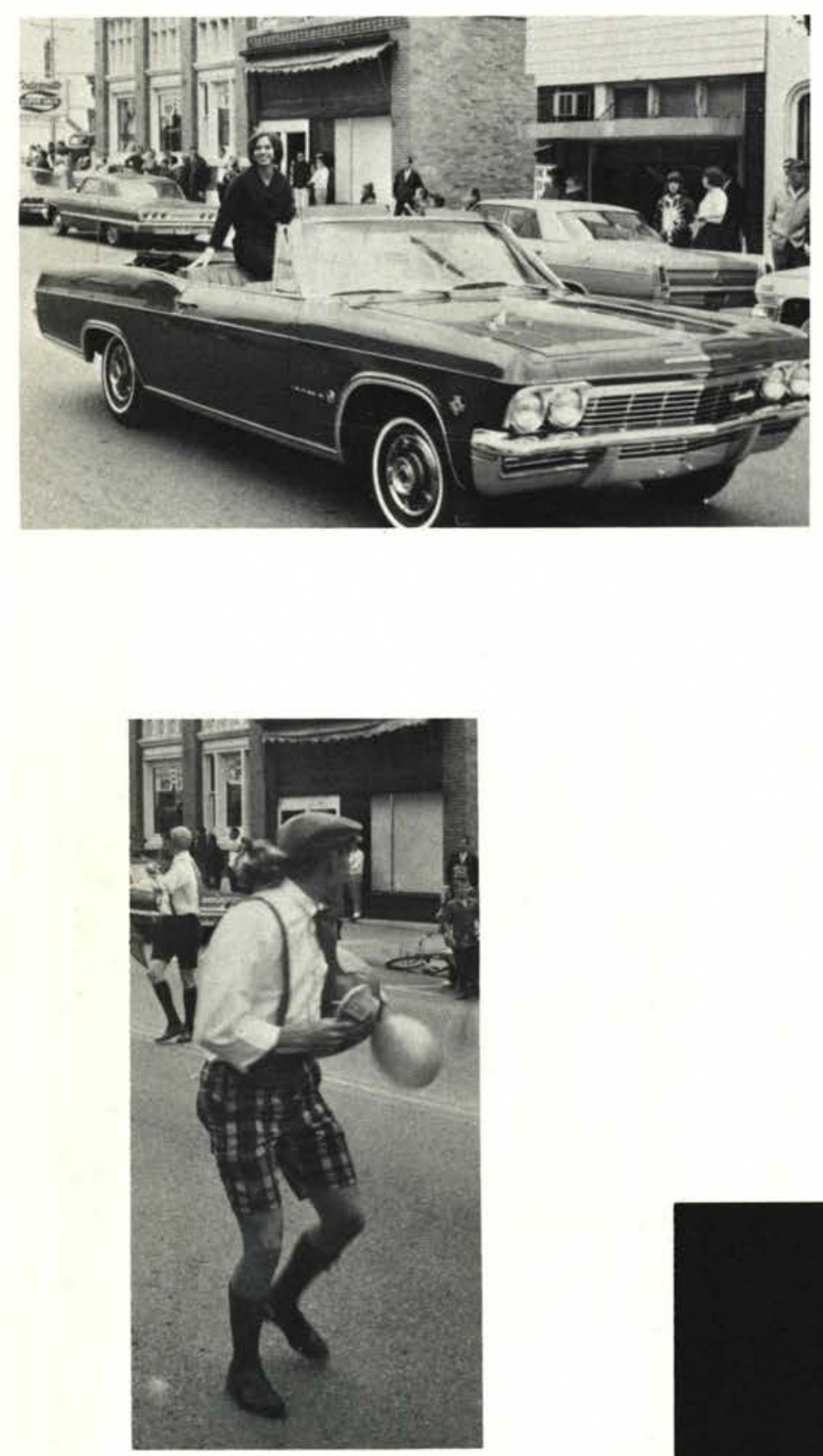
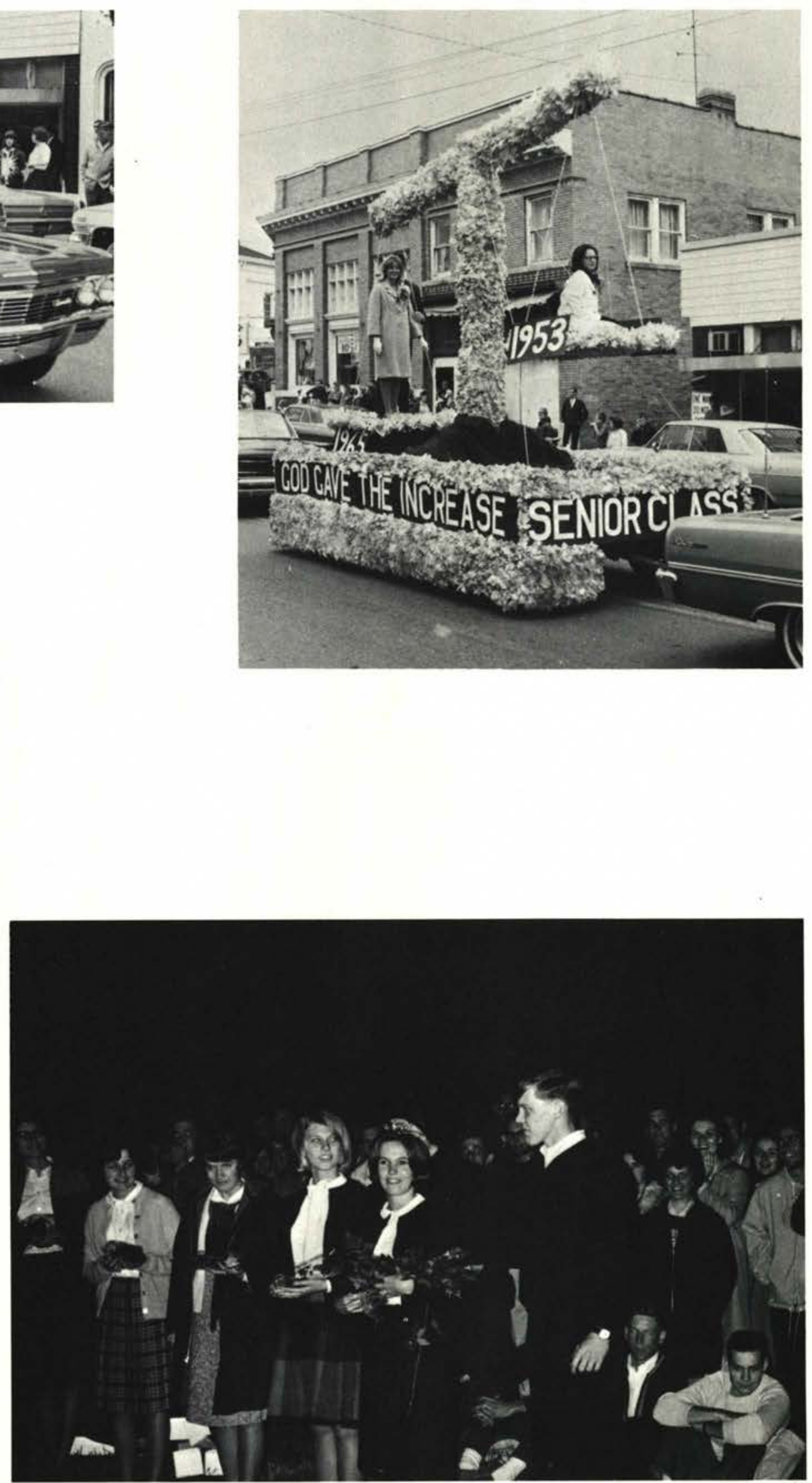

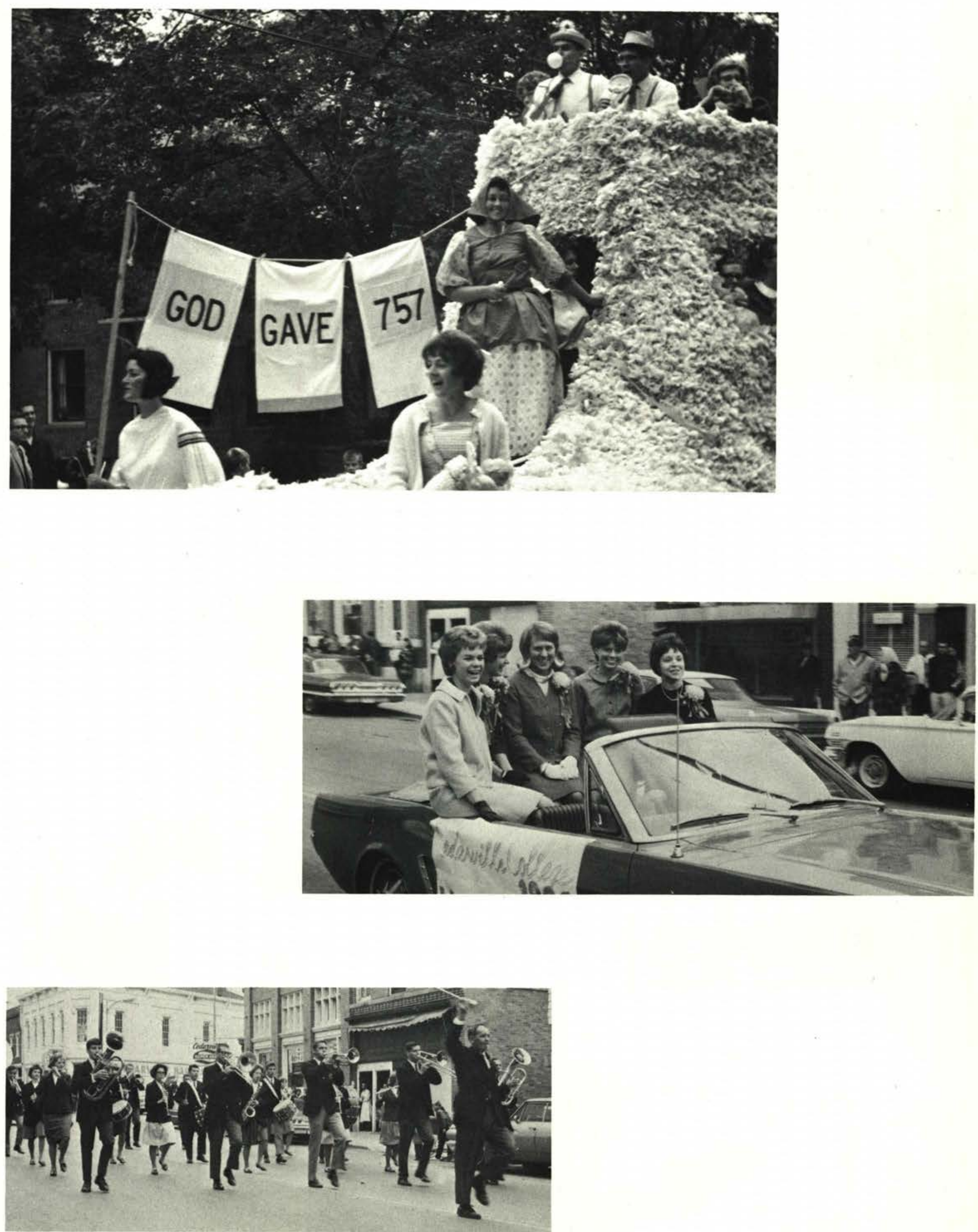

And the band played on.... 

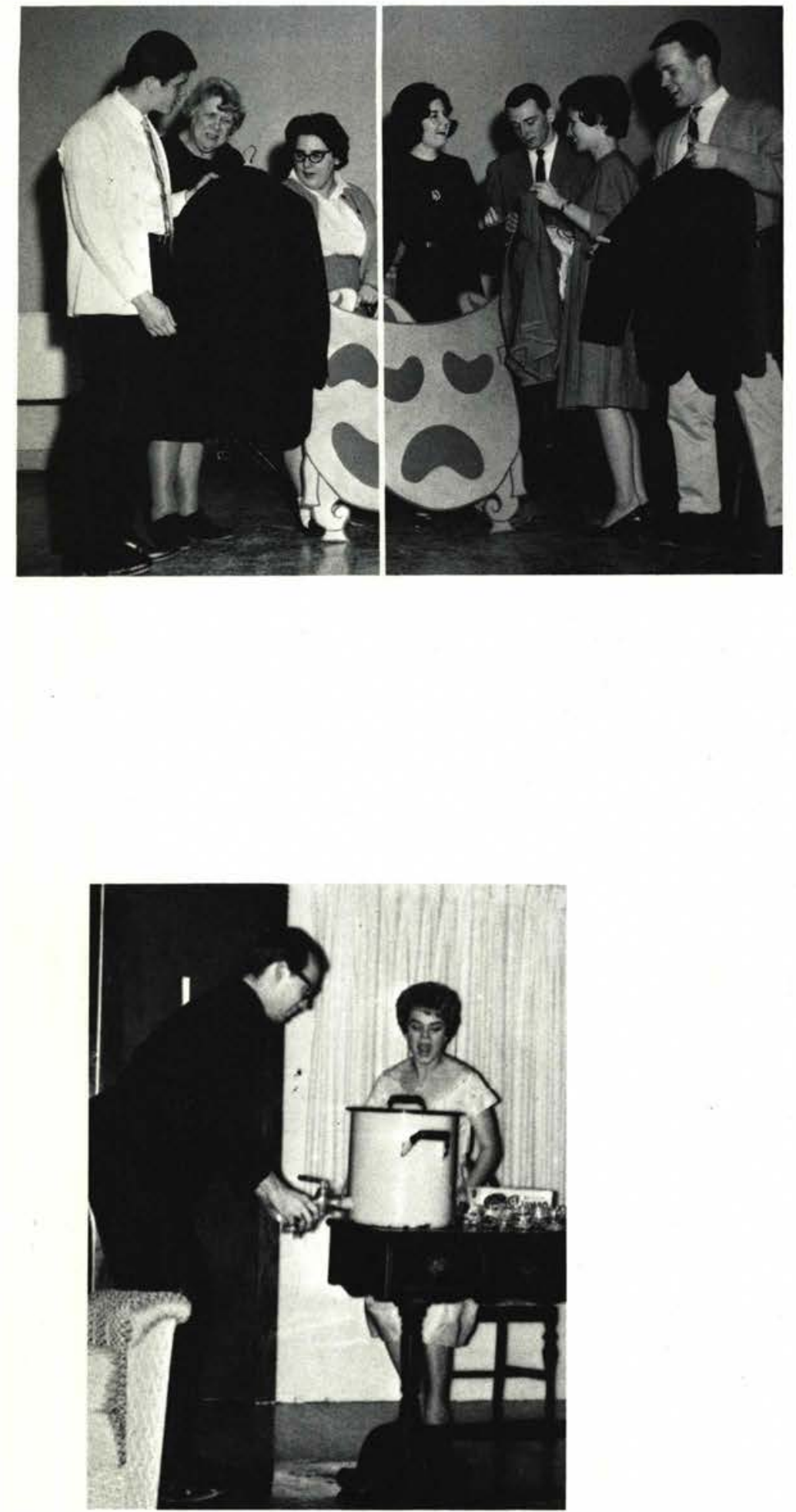

\section{Sock ' $\mathrm{n}$ ' Buskin}

\section{Presents . . .}

\section{Mr. Peepers}

Using the traditional dramatic symbols for their club title, Sock ' $n$ ' Buskin is made up of those students who have participated in campus dramatic productions. Members of this organization do most of the behind-the-scenes work on the annual plays. The Sock ' $n$ ' Buskin also sponsored the one act plays produced by the Introduction to Dramatics class.

This year's officers are John Stockwell, President; John Skillman, Vice-president; Janie Ashley, Treasurer; Carol Mikels, Secretary.

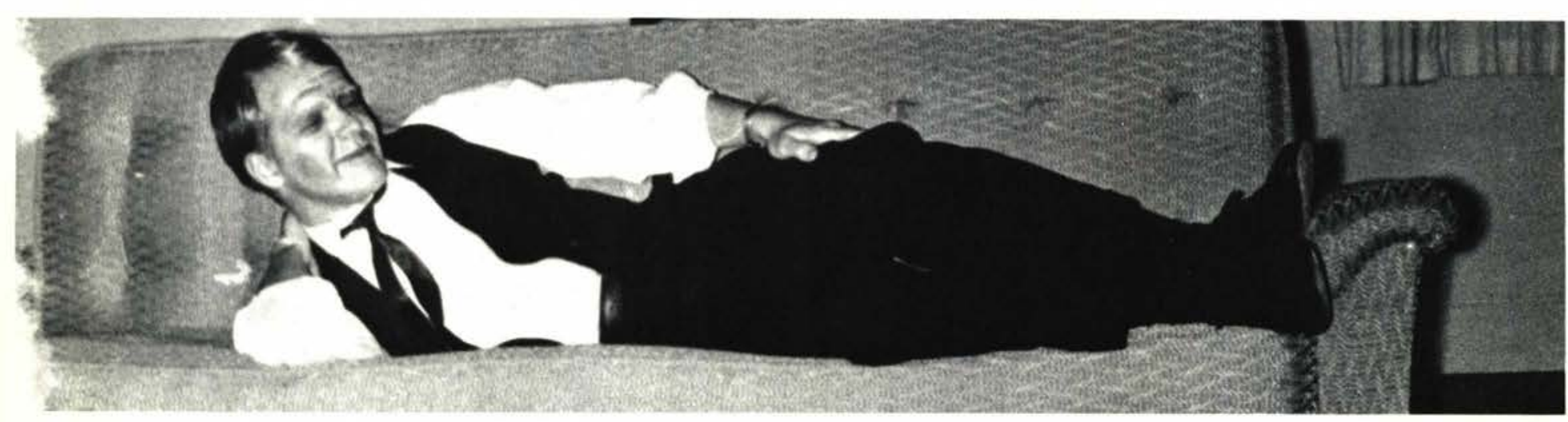




\section{and Thunder on Sycamore Street}
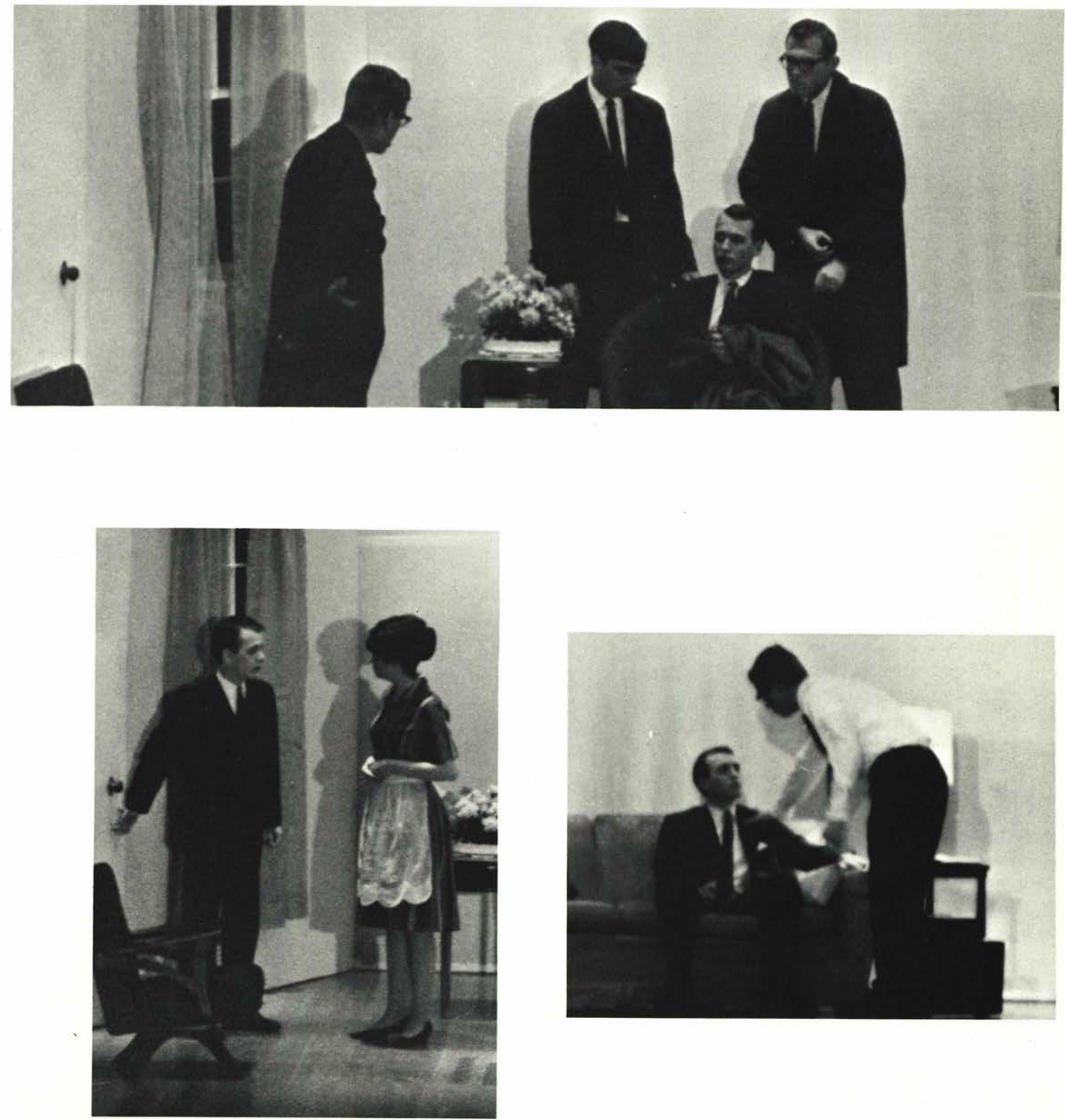


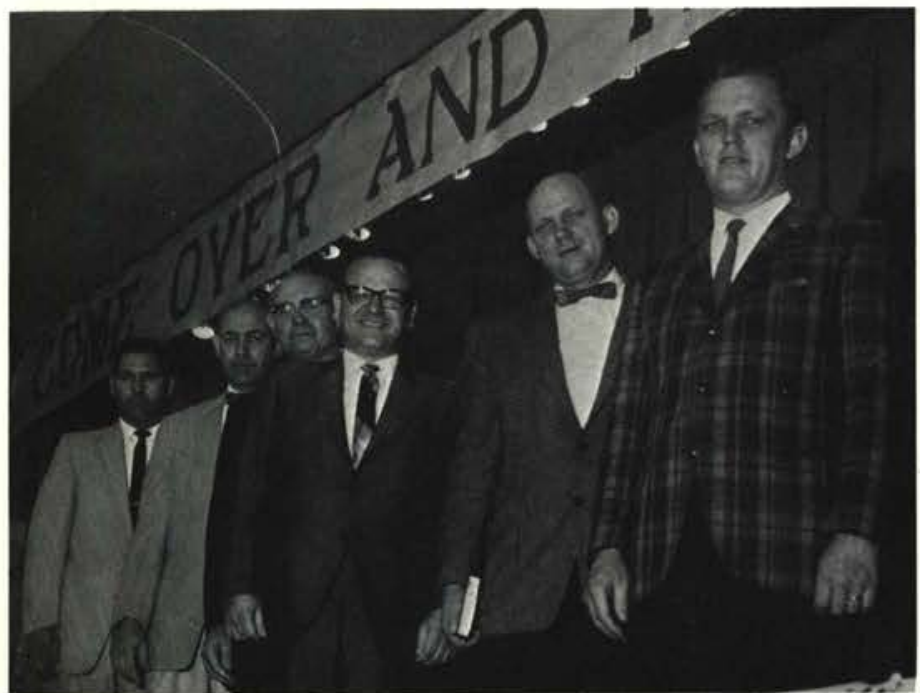

\section{FWM}

What can you do for missions? Fellowship for World Missions has the answer. Each weekday morning the members of this unique organization gather together to pray especially for the needs of our missionaries. The praying is specific and is guided by letters received from the missionaries. Direct aid is given to some missionaries in need by FWM-supported service projects. Their chief contribution to the spiritual growth on our campus each year is the sponsoring of the Missionary Conference.

Left to Right: Mel Pittman, Edward Henry, C. McDonald, William Fusco, William Large, Bud DeVries.

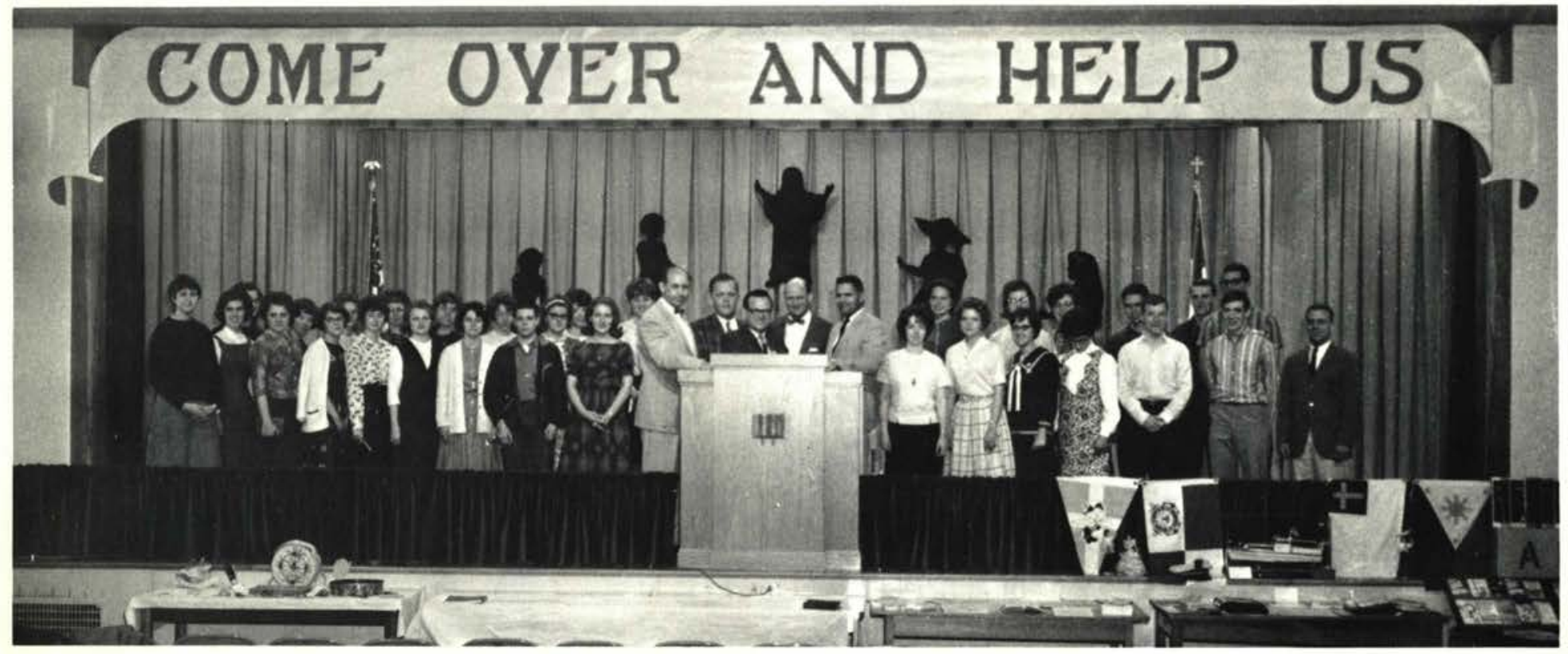

Left to Right: D. Hopkins, J. Van Horn, K. Farell, J. McLeod, L. Smith, J. Hess, B. Bodenmiller, L. Rulison, M. Butler, R. Hardy, F. Bonnema, G. Fisher, L. Tanner, M. Grable, S. Tallman, L. Walker, Rev. Henry, Rev. DeVries, Rev. Fusco, Rev. Large, Rev. Pittman, D. Patton, M. Schut, S. Dufford, S. Gordey, B. Mclnnes, S. Corder, K. Walker, R. Yeater, D. Rockwell, A. Davis, L. Waite, B. Shearer, D. Rockwell. 


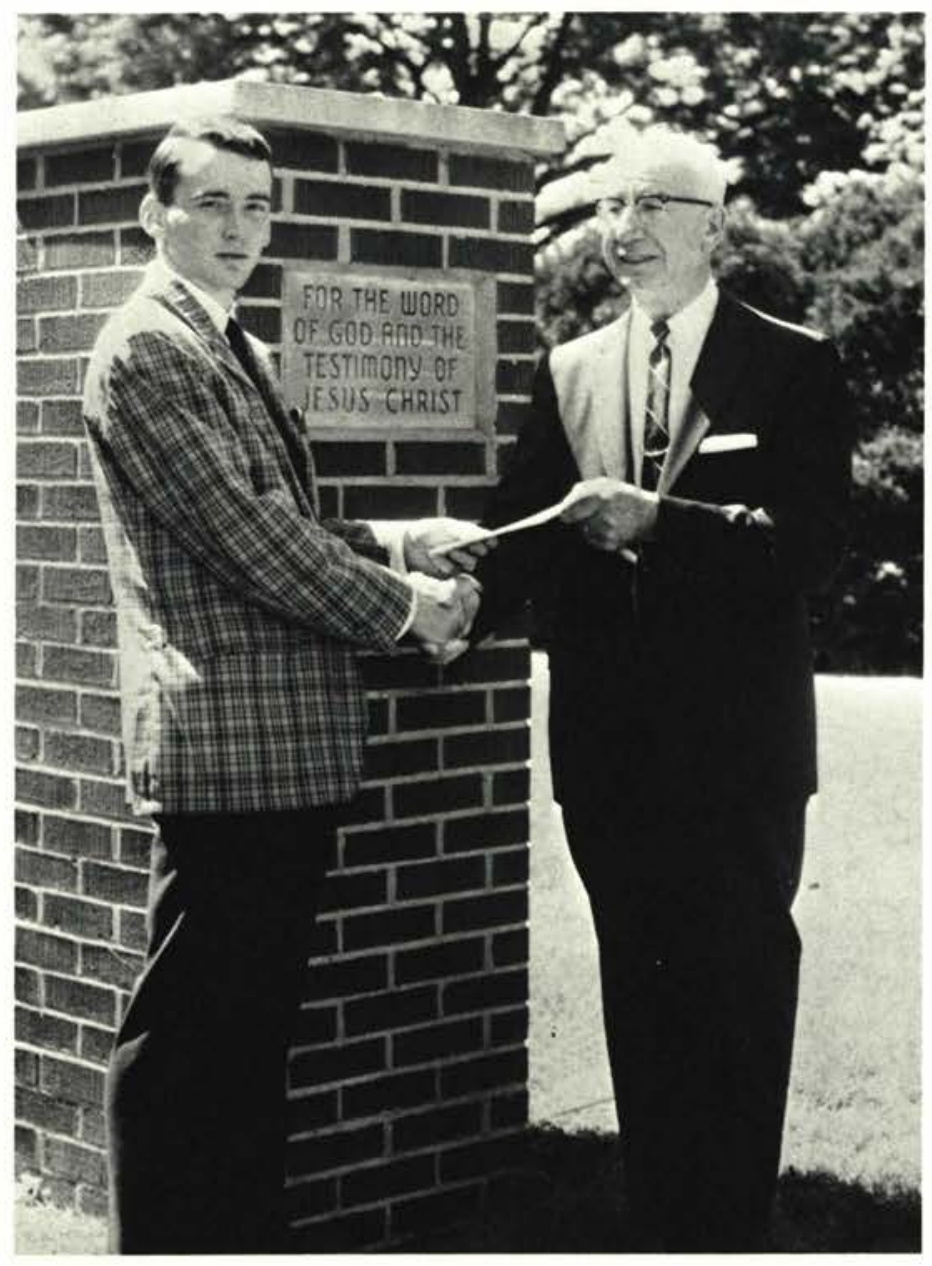

Student Missionary Project Chairman, Ron Grosh, presents check to Dr. Williams.

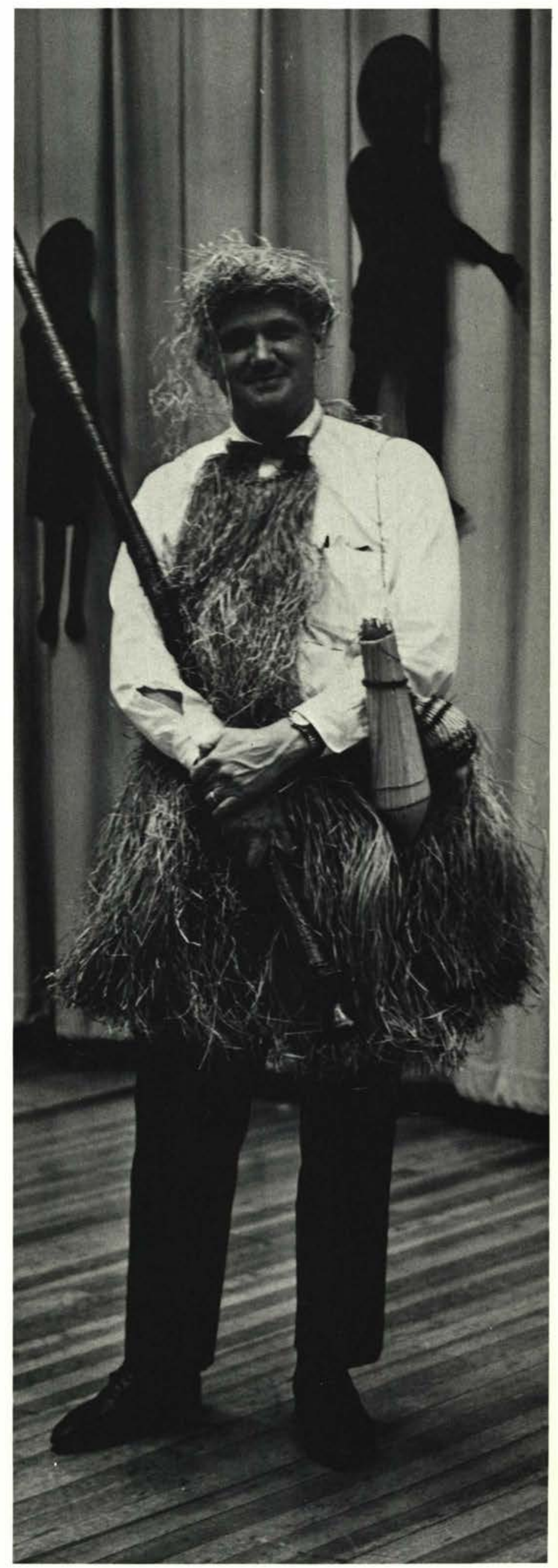

Rev. William Large 


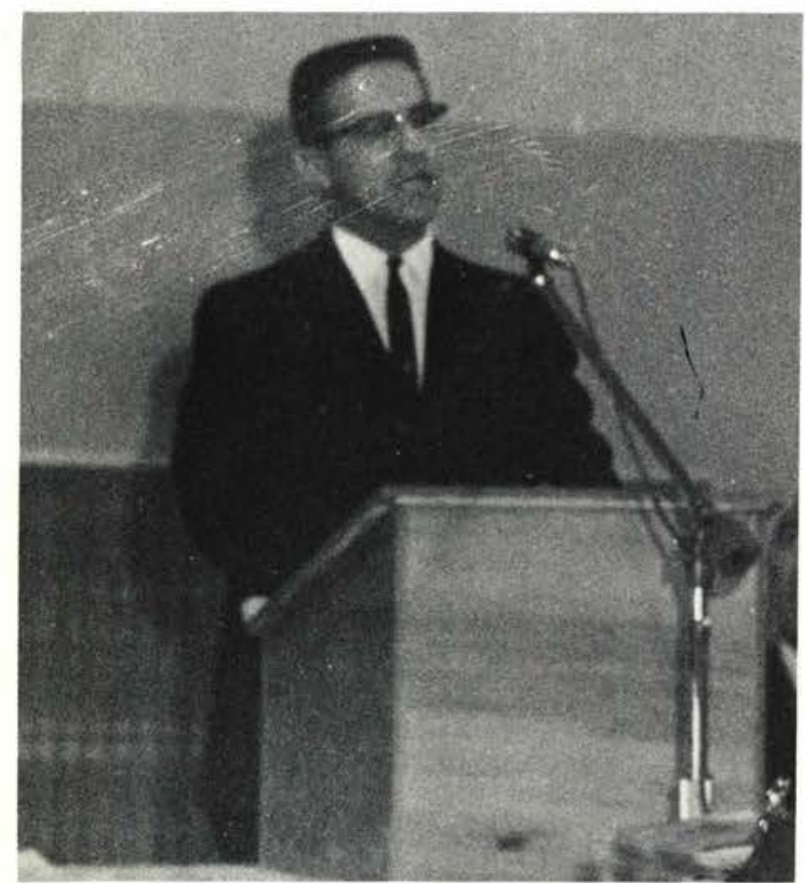

\section{Athletic Banquet}
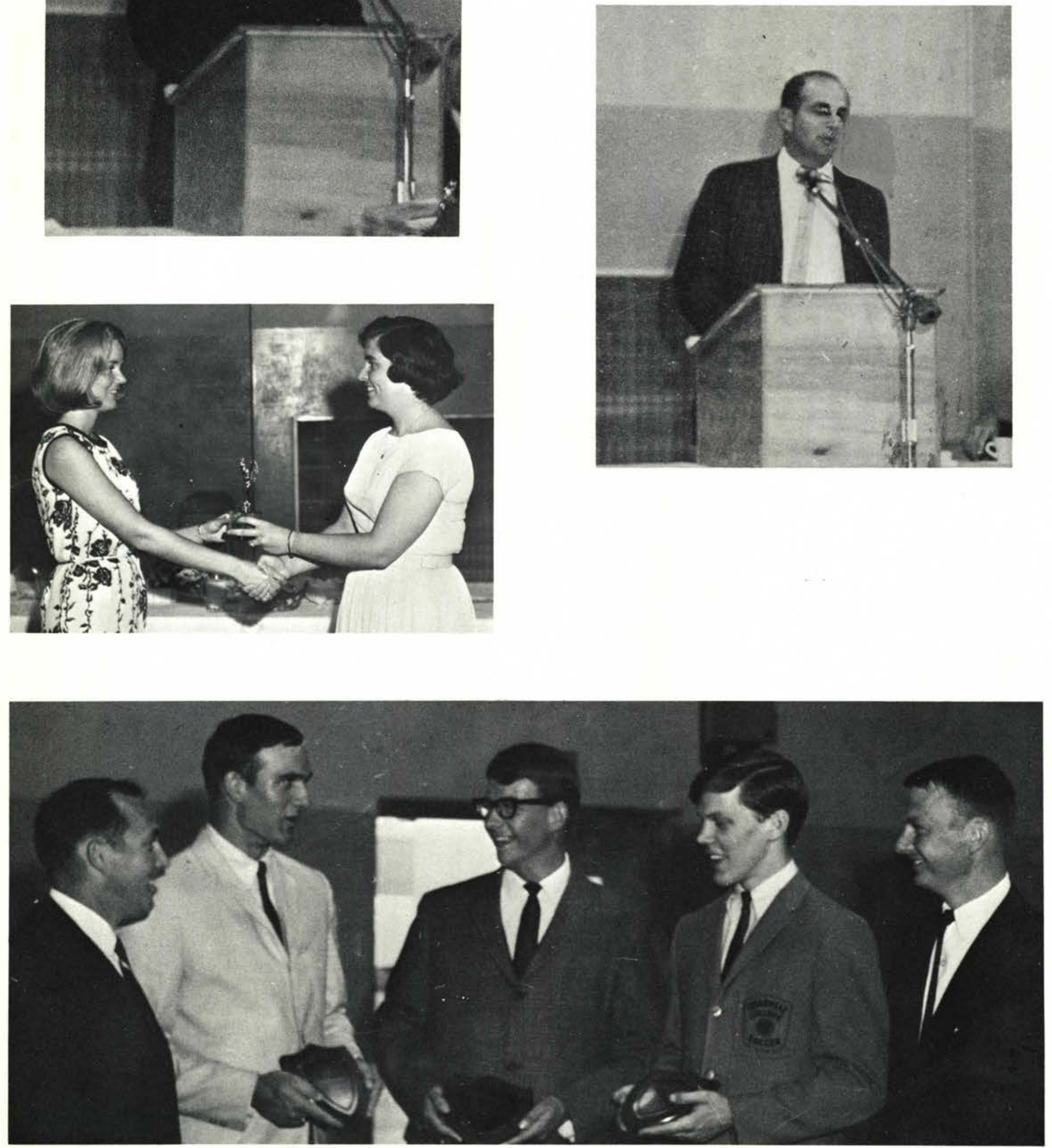


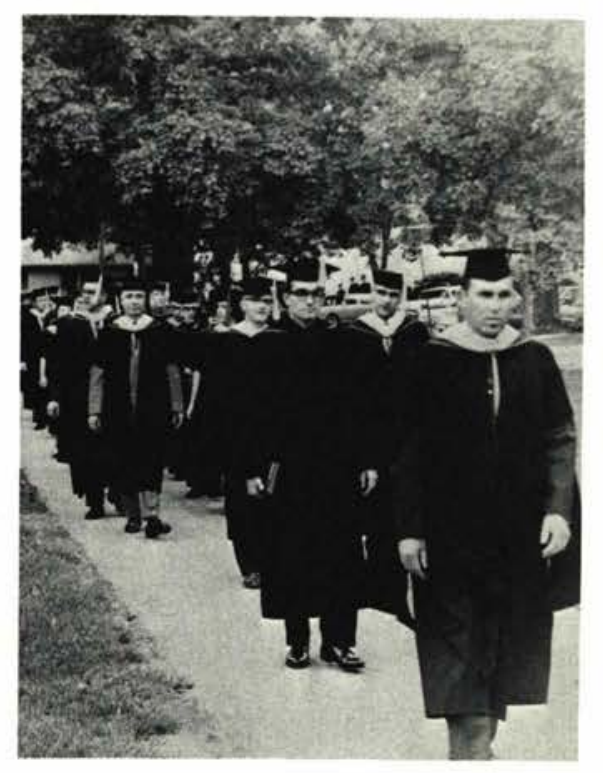

\section{Honors Day}

Honor students were recognized this year at a special chapel service on May 13th. Certificates were awarded to each honored student for academic achievement. A luncheon was also held on Saturday, May 14th, for the honor students and their parents.
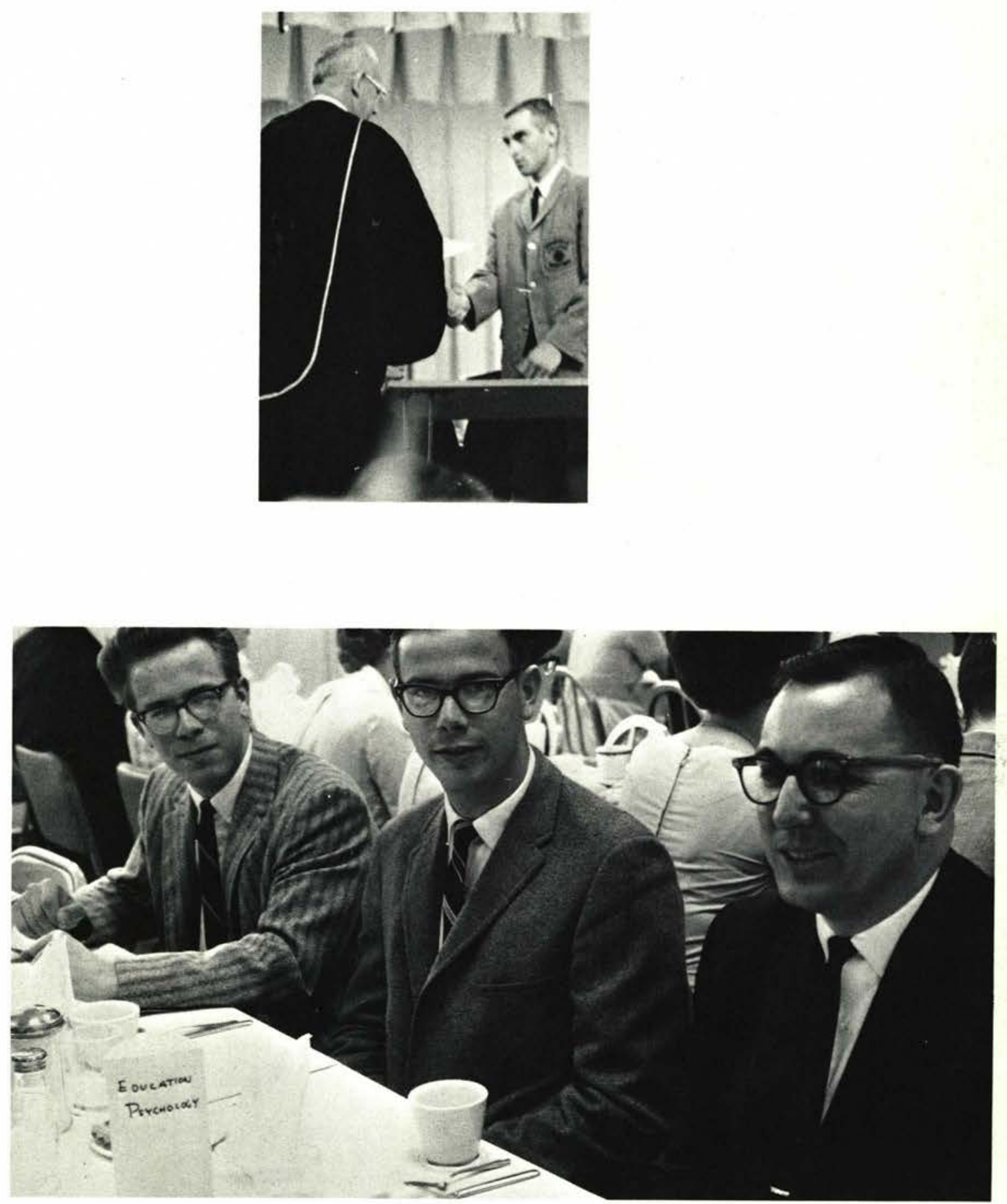


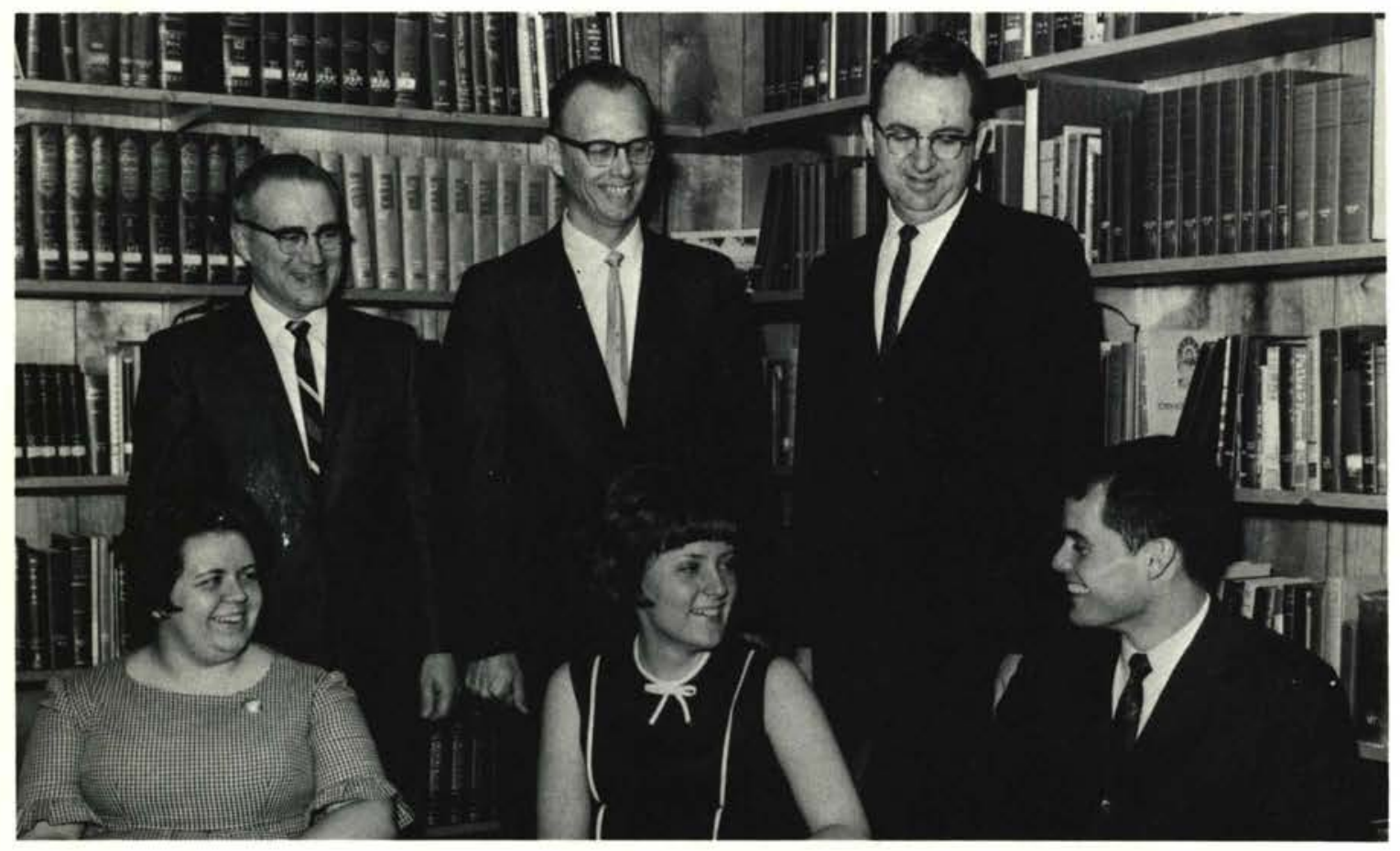

\section{Publications}

\section{Committee}

Standing: Mr. E. Greenwood, Dr. C. Johnson, Mr. R. McIntosh. Seated: J.

Rudduck, S. Lepine, M. Nicholls.

\section{Library}

Committee

Left to Right: Mr. H. Bergen, P. Jackson, Mrs. A. Chaffee, Mr. E. Greenwood, Mr. R. Gale.
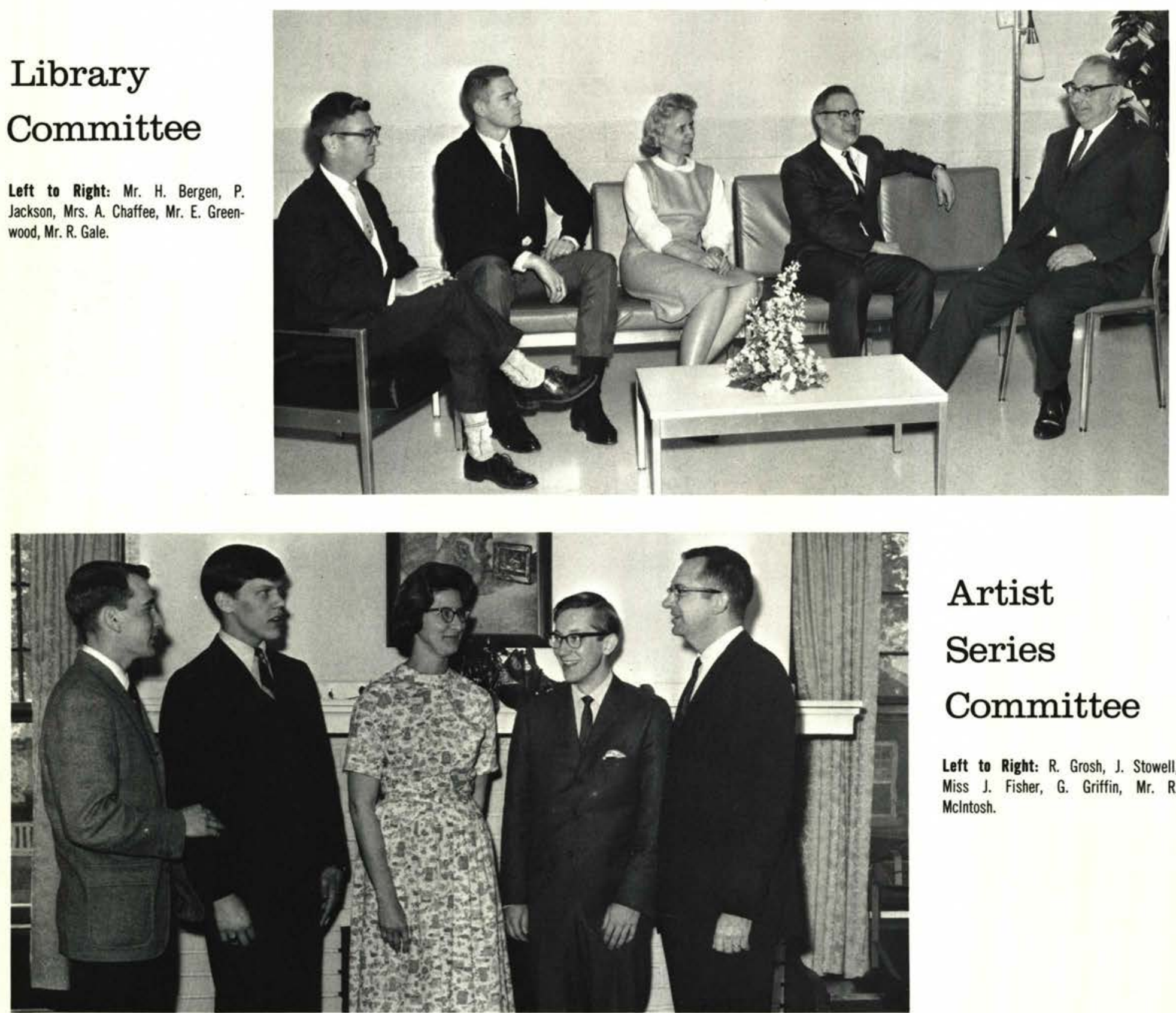


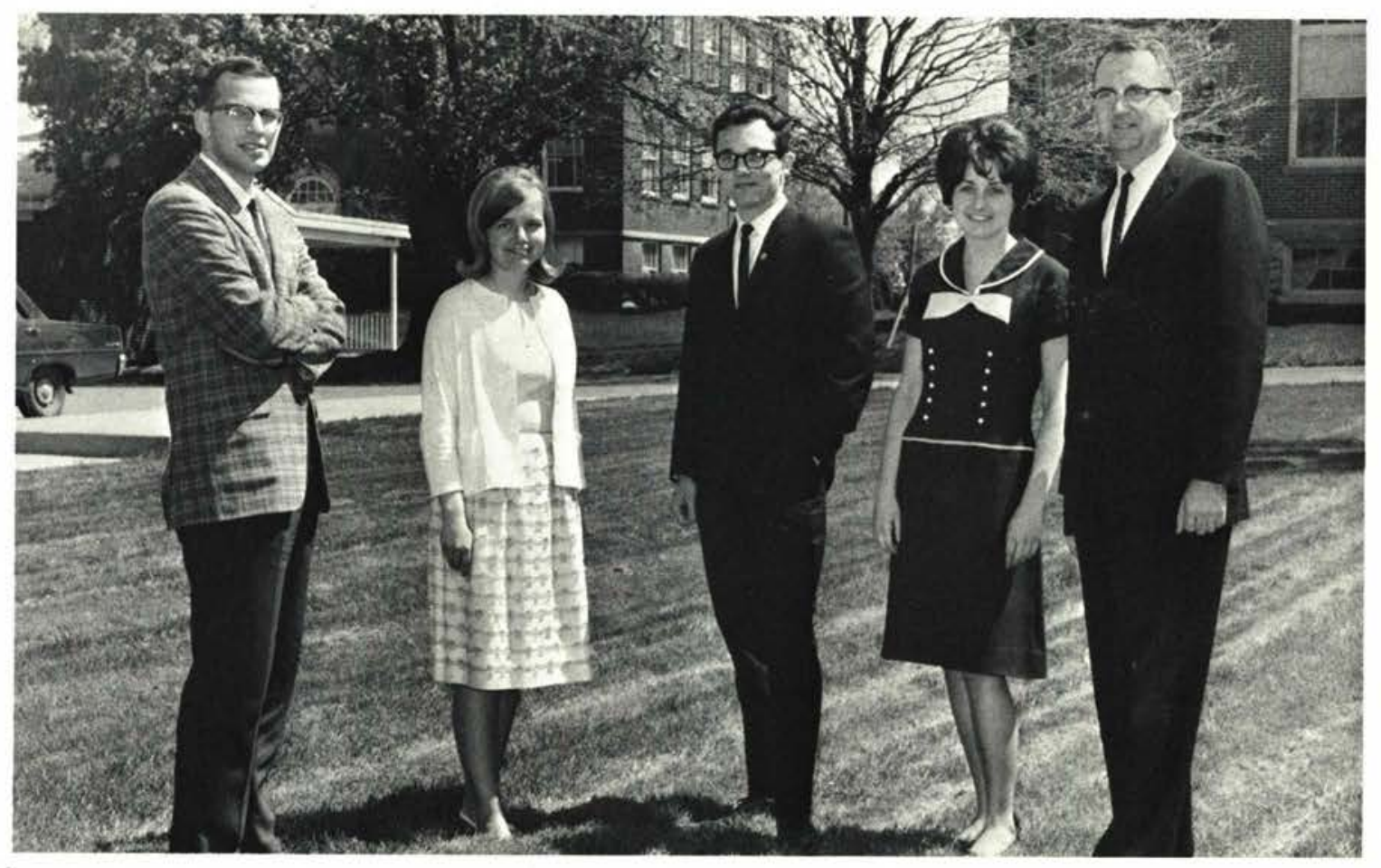

Student

\section{Affairs}

\section{Committee}

Mr. W. Riter, C. Payne, M. Hamilton, I Millikan, Mr. Mclntosh.

\section{Discipline}

\section{Study}

\section{Committee}

Left to Right: Mr. R. Mclntosh, D. Gordon, Mr. S. Ballard, Miss J. Fisher, G. Harris.

\section{Dorm}

\section{Counselors}
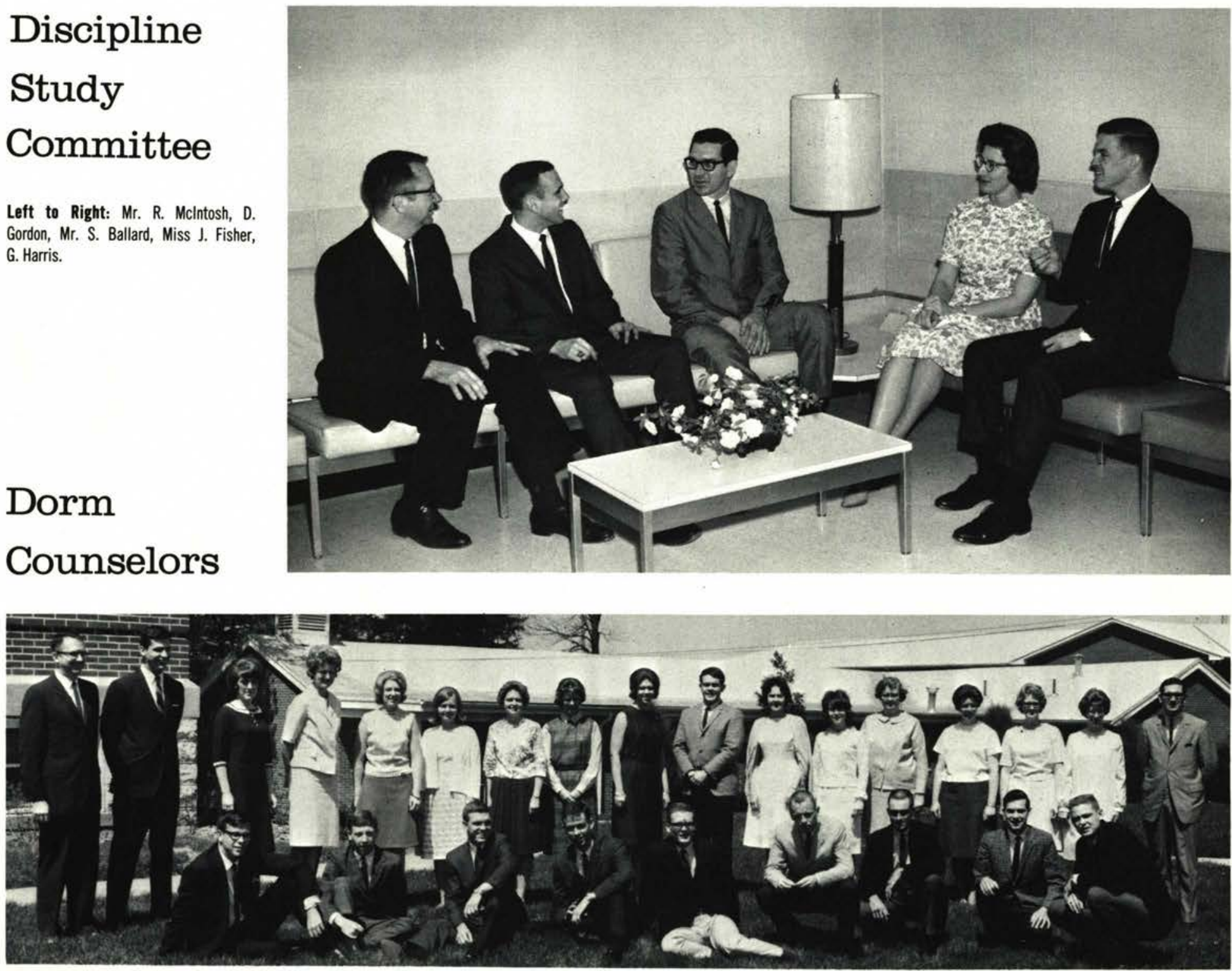

Standing, Left to Right: Mr. R. Mclntosh, M. Jones, B. Smith, C. Michels, P. Cannady, C. Payne, S. Dufford, L. Rulison, M. Schulte, T. Timmons, N. Freeman, J. Fields, L. Ancel, L. Mitchell, K. Mansfield, F. Bonnema, Mr. Ballard. Sitting: T. Oakley, D. Dunstand, G. Harris, M. Crampton, M. Hartzell, J. Hay, J. Brannon, M. Searles, P. Jackson. 


\section{Athletic Committee}

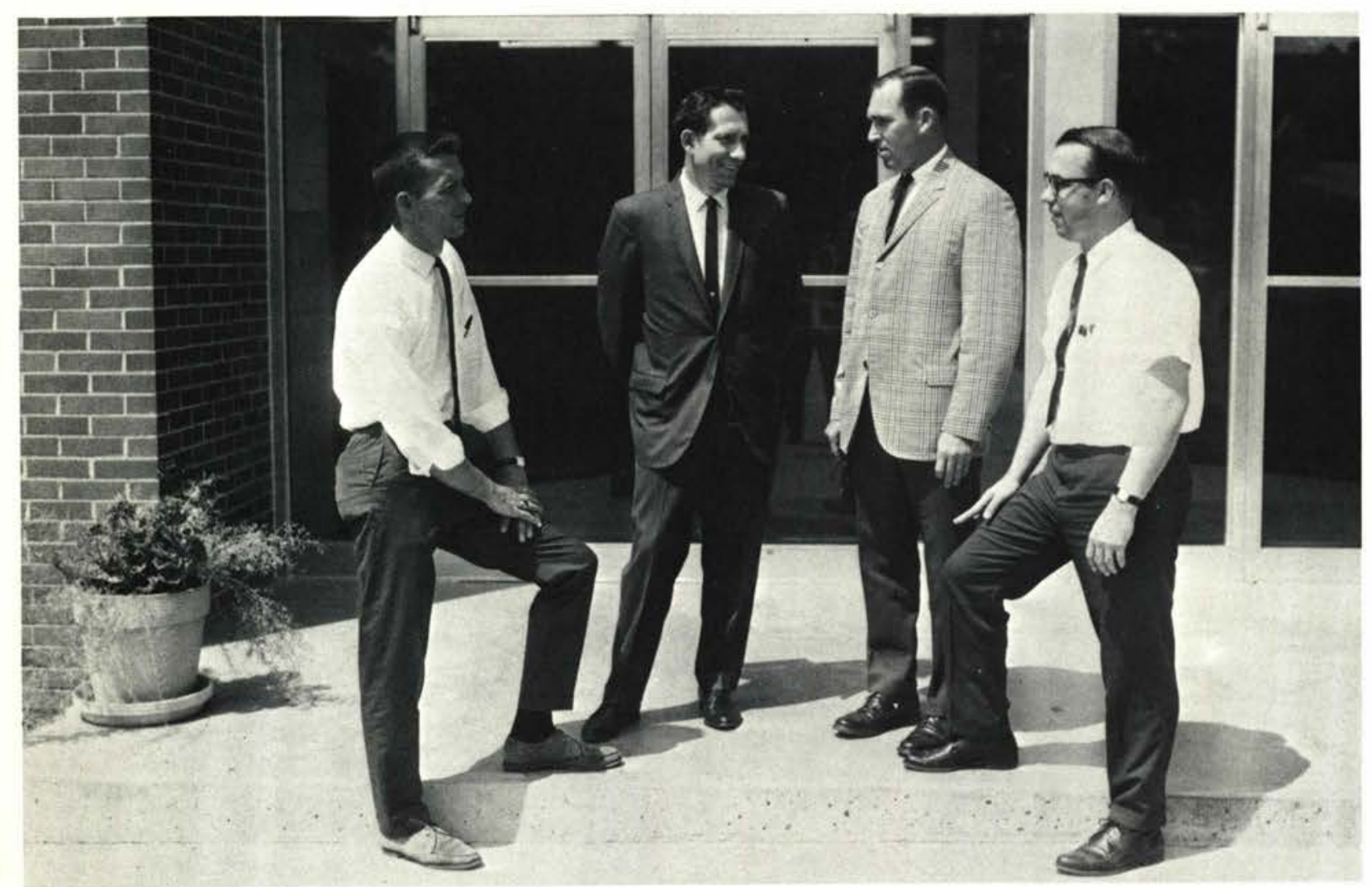

Dr. D. Thomson, Mr. L. Turner, Mr. D. Callan, Mr. D. Wetzel.

\section{Photo Club}

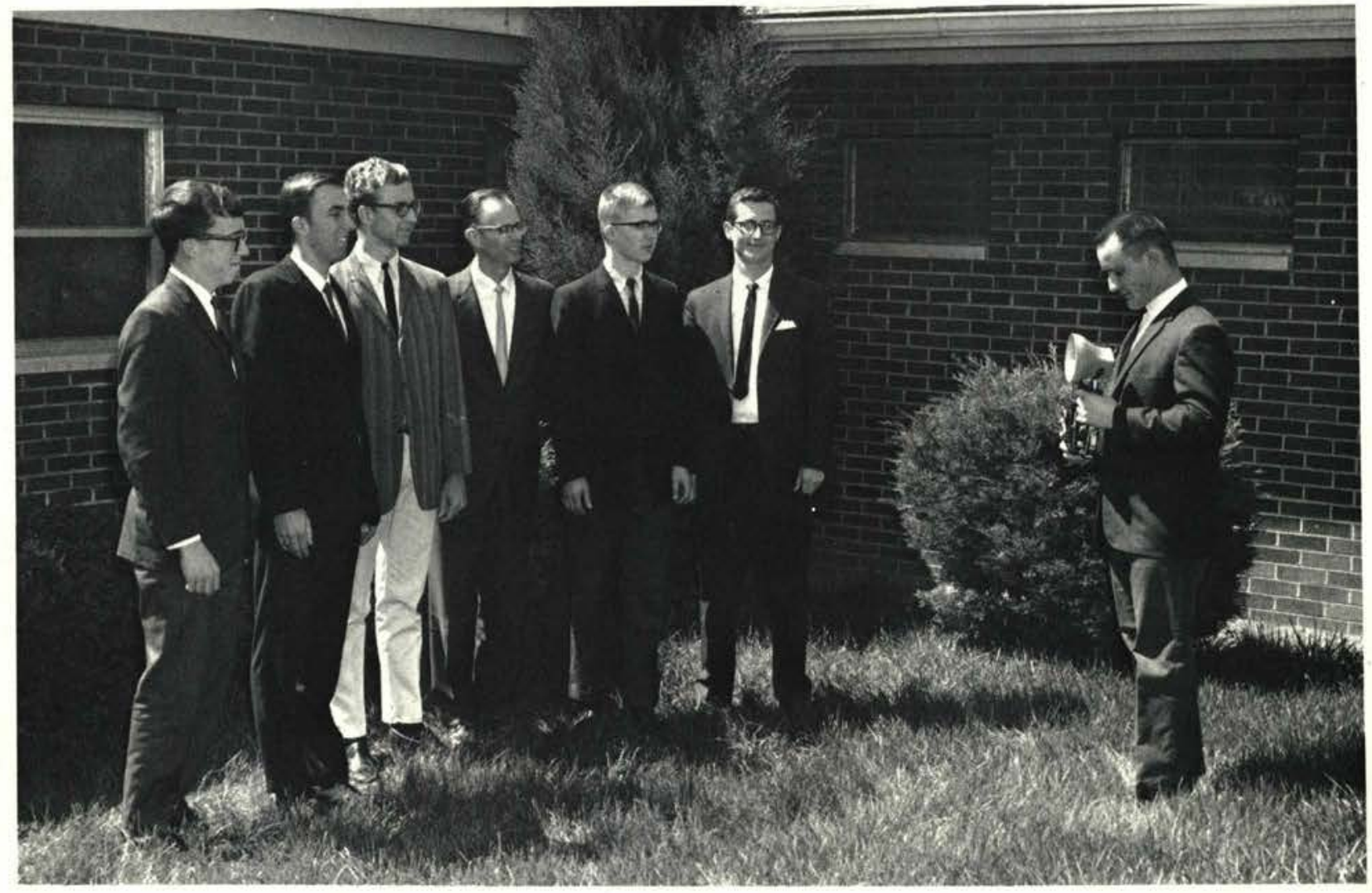

Left to Right: J. Brown, J. Berry, E. Smelser, Dr. C. Johnson, Advisor; F. Olsen, D. Allen, D. Dawes. 


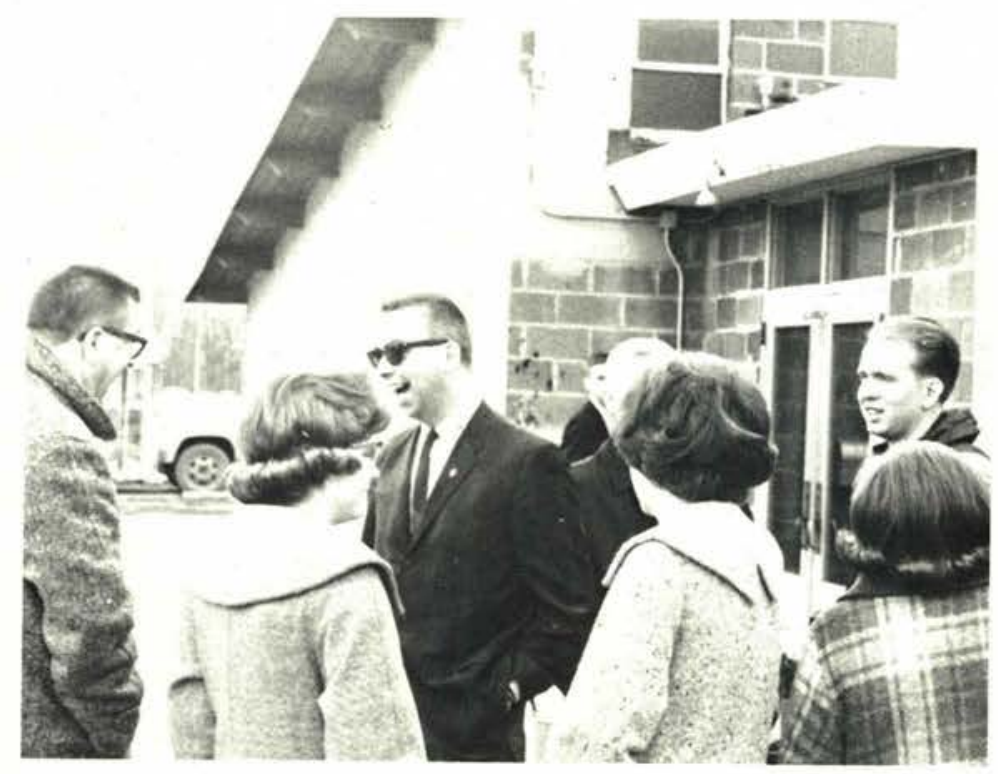

\section{Choir Tour}

The College Choir, under the direction of Mr. David Matson, is an ensemble of approximately forty selected voices that emphasizes the a cappella tradition. The Choir annually makes a Spring tour of many of the supporting churches for the purpose of performing many of the significant sacred choral works of various periods and styles "for the ... testimony of Jesus Christ." In addition, several concerts are given on the campus and in surrounding communities.

Officers of the choir for 1965-66 are: Gary Harris, president; Marshall Searles, vice-president; Irene Millikan, secretary; Ted Jager, student director; Ted Clater, student council representative; Donna Daab and Sarah Williams, librarians; and, Dan Boyd and Dave Draxler, managers.

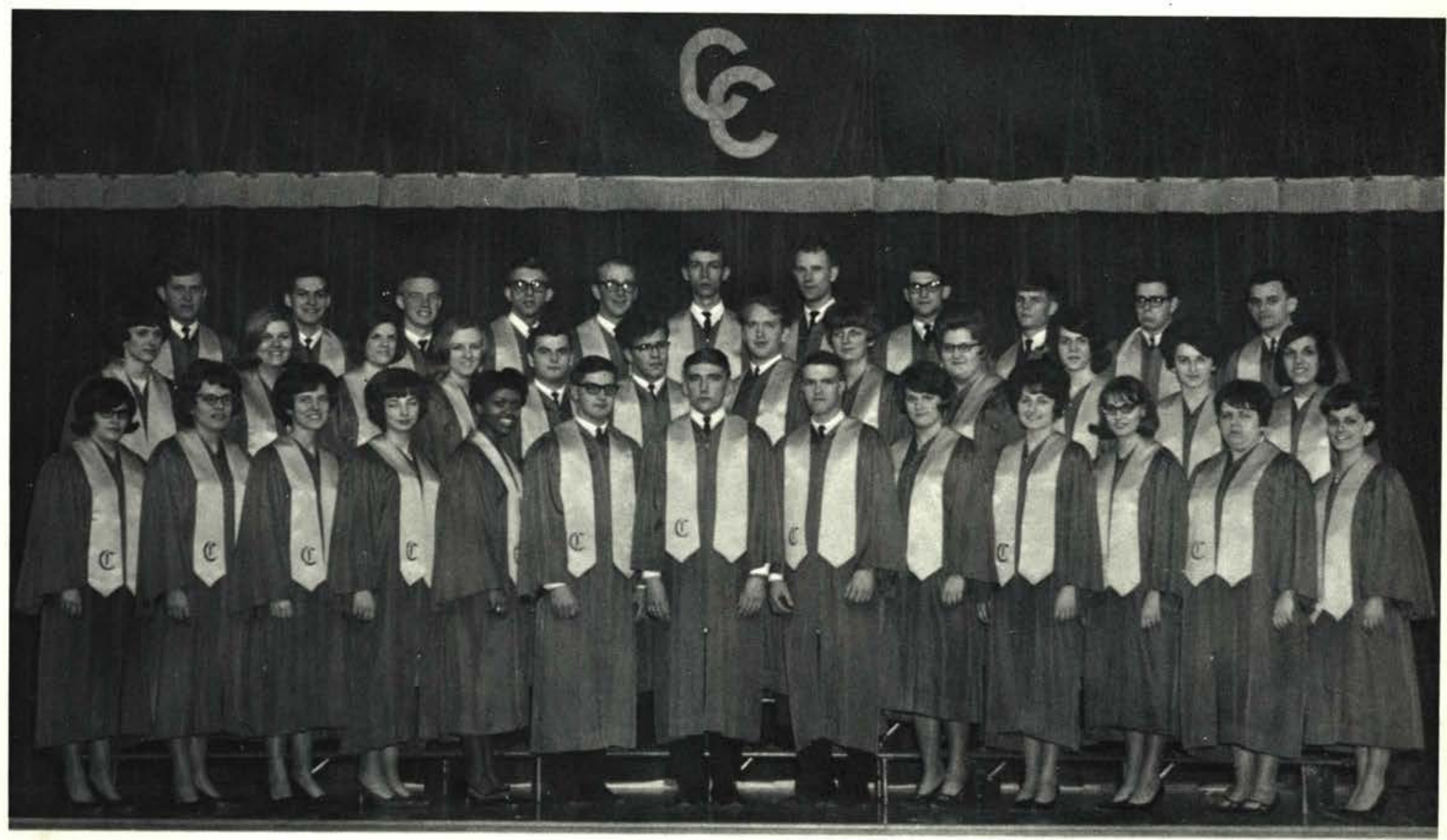

Back row: T. Jager, M. Searles, L. Brock, J. Gunlock, D. Boyd, M. Crampton, D. Nims, D. Tillotson, D. Work, D. Draxler. Second row: M. Cummings, M. Brewer, J. Masemore, L. Riggs, A. Keim, B. Clater, L. Welch, E. Johnson, C. Satterblom, J. Renner, I. Millikan, D. Daab. Front row: M. Anderson, M. Werner, M. Schonschek, K. Salisbury, S. Williams, H. Moore, W. Cushey, G. Harris, K. Johnson, B. Millikan, M. Brown, J. Rudduck, B. Grosh. 


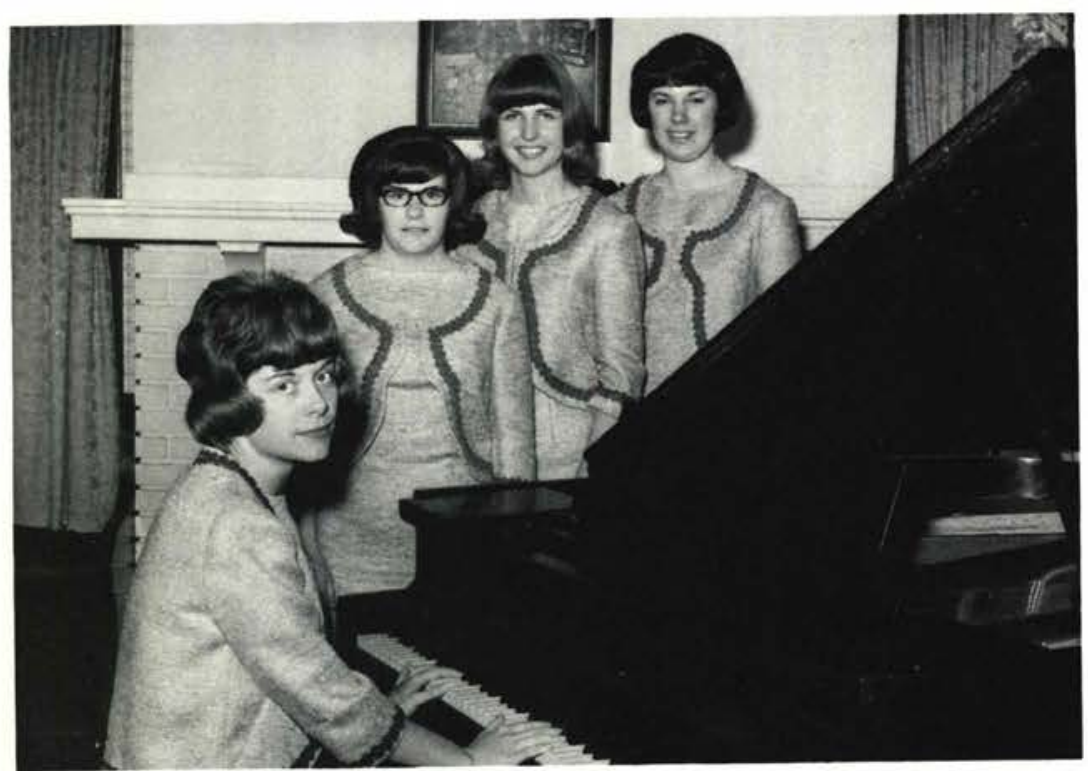

\section{Music Groups}

Representing the Lord Jesus Christ and Cedarville College, music groups left campus nearly every weekend to minister in churches in the surrounding area as well as other states. As the groups presented musical numbers, testimonies and sometimes messages from the Word, many lives were led into a deeper Christian experience.

VICTORS: B. Gothard, pianist, M. Anderson, L. Taylor, D. Fitch.

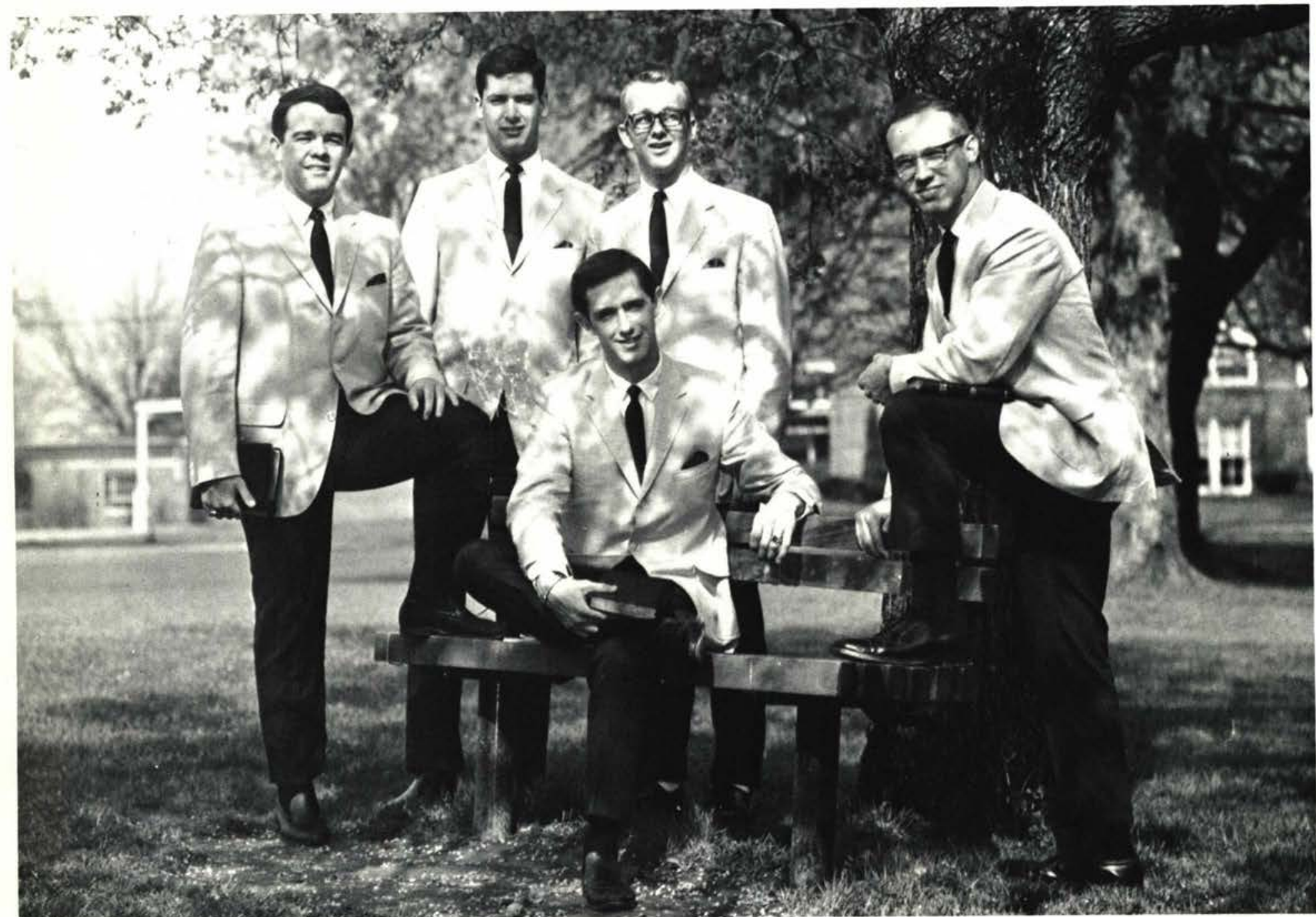

VOLUNTEERS : T. Timmons, M. Jones, D. Boyd, T. Clater, J. Wingate. 


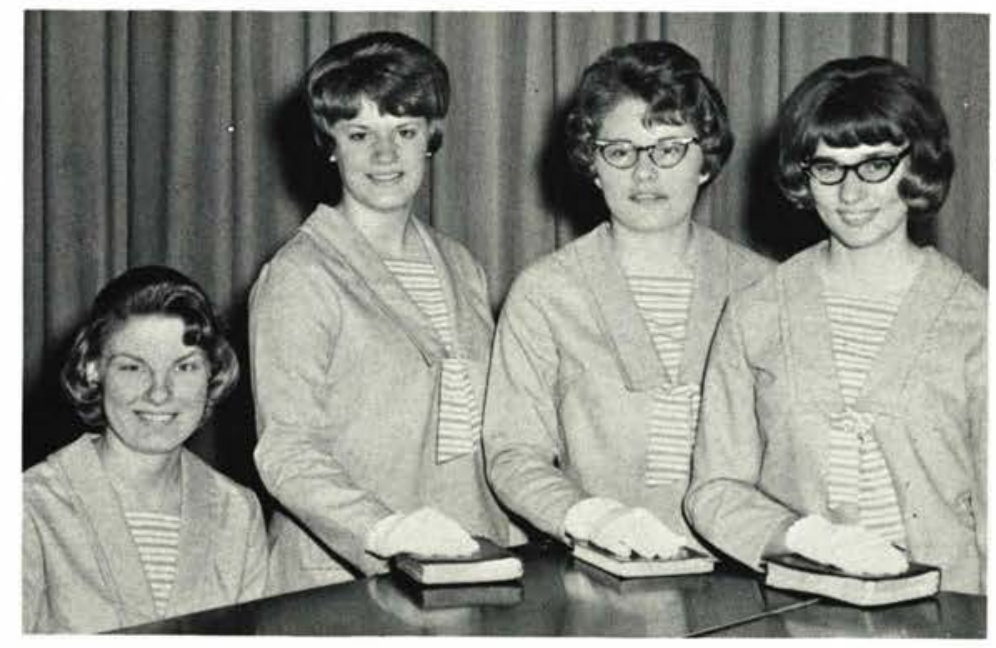

ROYALHEIRS: S. Byrd, K. Johnson, M. Werner, M. Brown.
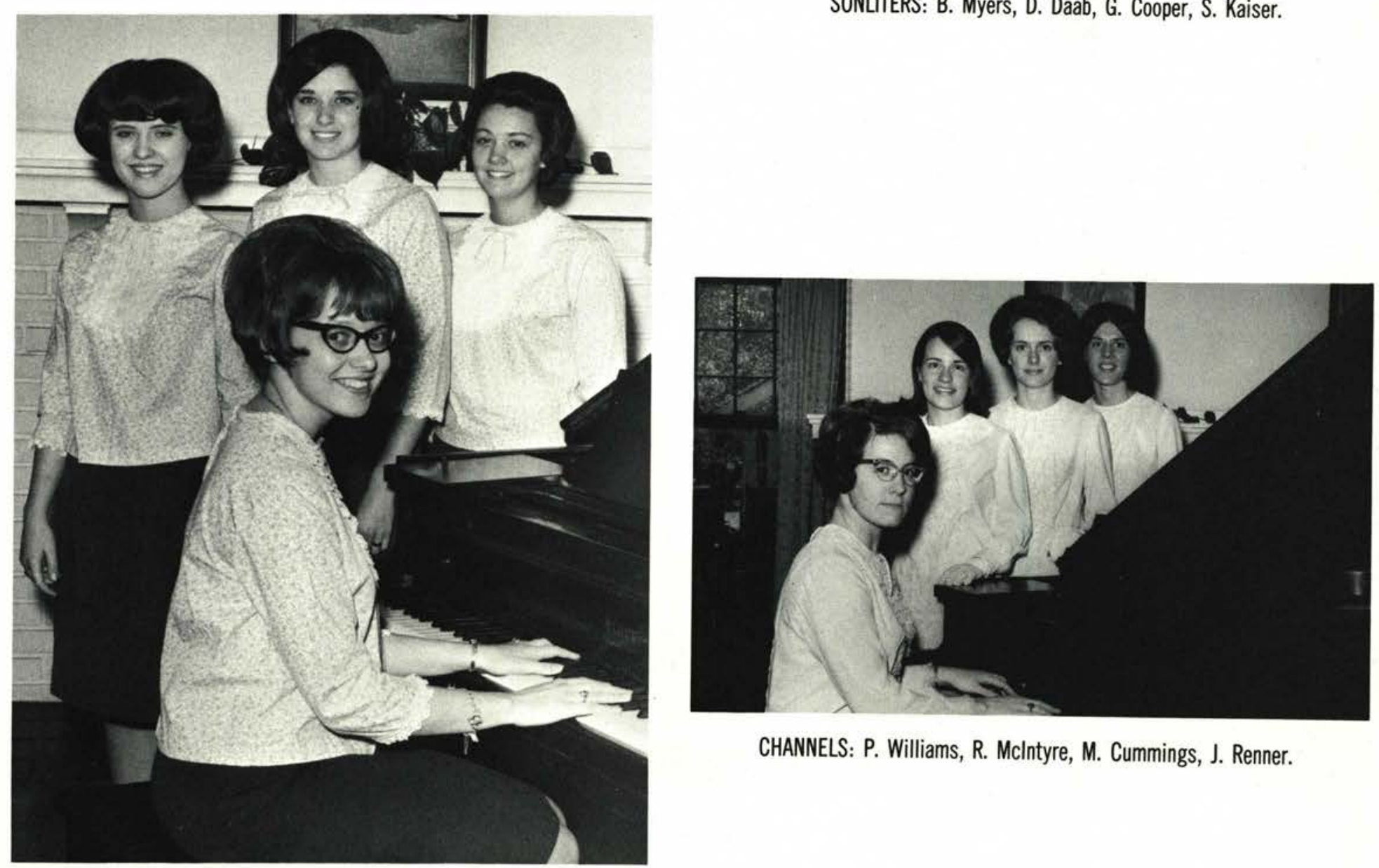

CHANNELS: P. Williams, R. McIntyre, M. Cummings, J. Renner.

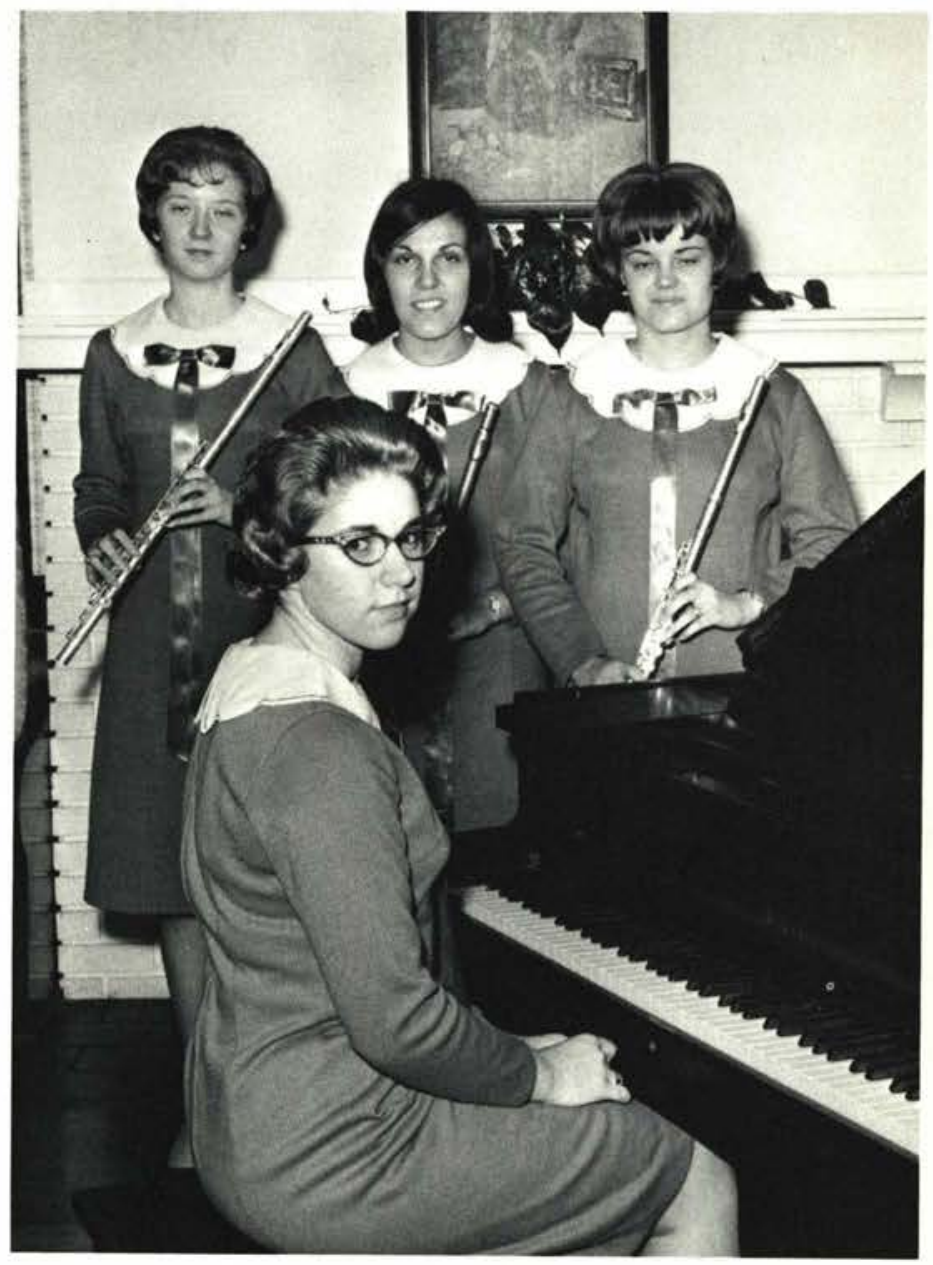

SONLITERS: B. Myers, D. Daab, G. Cooper, S. Kaiser.

MASTERS: P. Perryman, B. Gardiner, L. Miller, C. Jensen. 


\section{College Band}

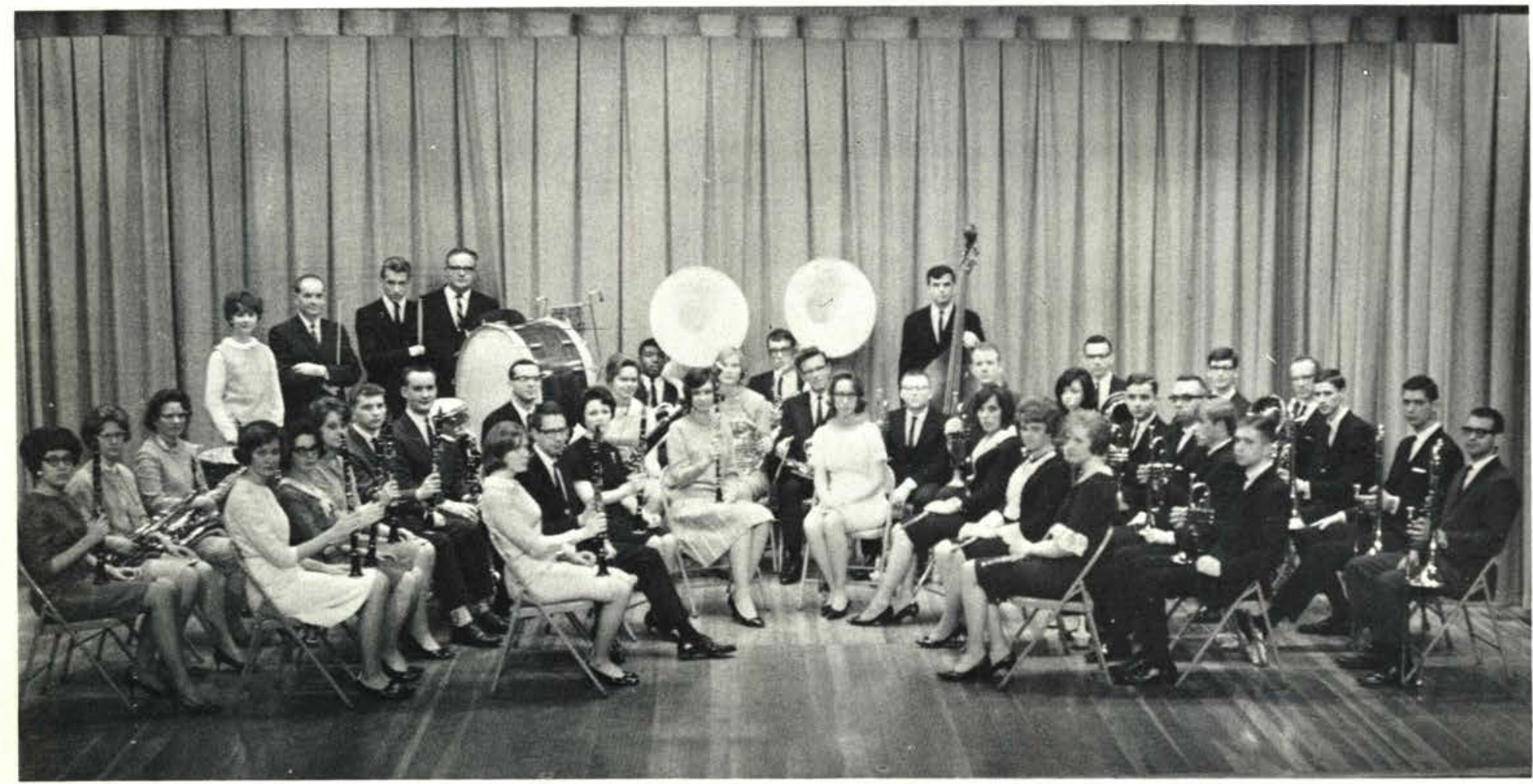

BAND - Front row: V. Merryman, Mr. Vanderkoy, L. Miller, C. Husted, C. Wilson, D. Daab, G. Cooper, B. Meyers. Second row: M. Louwerse, A. Mulford, D. Dawes, Mr. Riter, M. Schut, L. Wessel, B. Clater, D. Anderson, D. Nims, J. Elkins, D. Prizonzano, Mr. Mattson, R. Cannada. Front row: D. Morgan, J. Hess, Mrs. Webber, B. Bodenmiller, Mr. Webber, V. Jensen, B. Griffith, B. Hunter, J. Brown, A. Keim, C. Biddle, S. Fenton, T. Clater, E. Music, M. Jones.

\section{Choralaires}

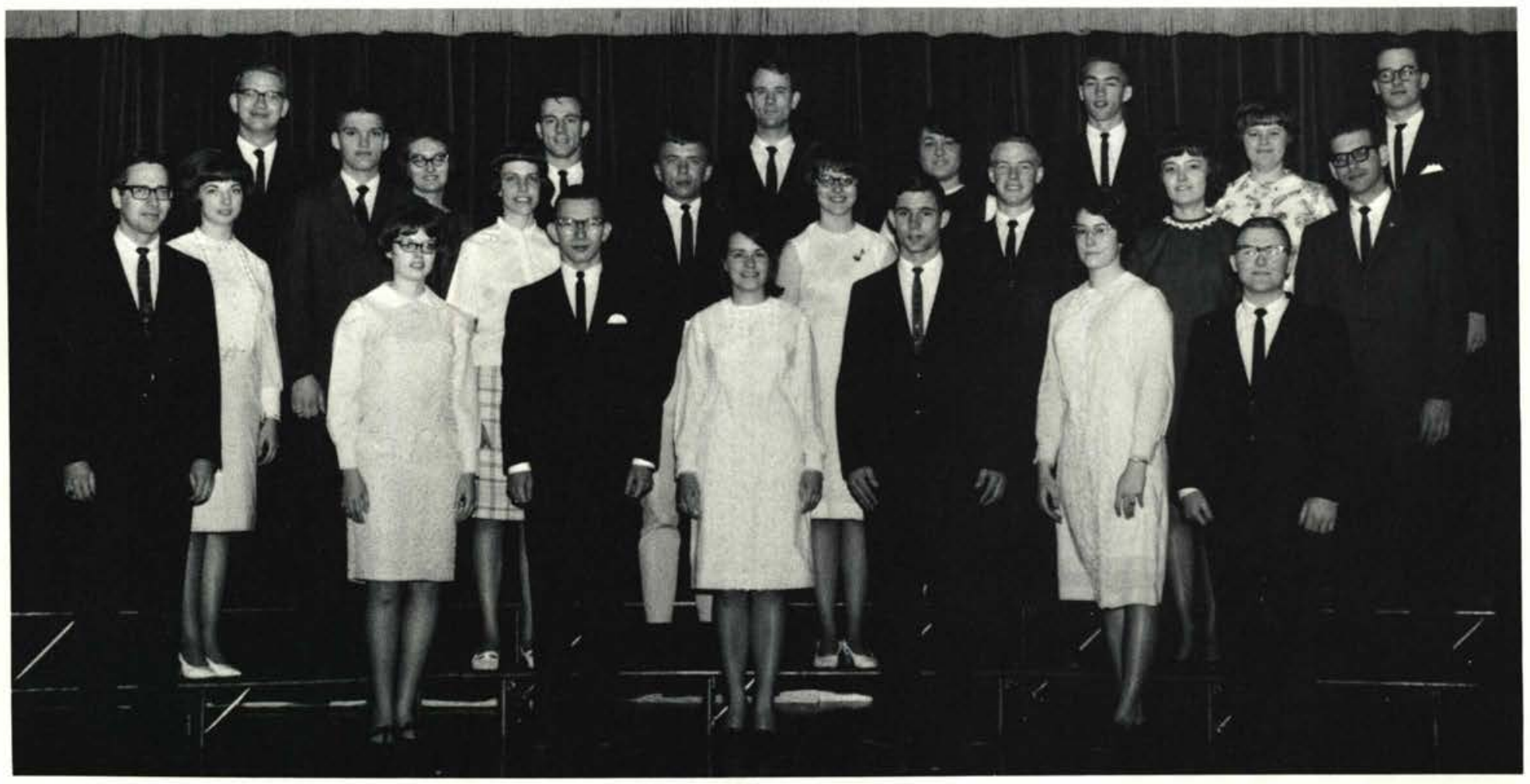

Back, Left to Right: B. King, G. Crider, D. Foster, D. Nims, L. Edwards, D. Yoder, K. Carter, G. Taylor. Second row: K. Salisbury, T. Warren, J. Barcus, J. Goodman, C. Jensen, L. Brock, L. Howard, W. Burns. Front row: Mr. Vanderkoy, C. Biddle, R. Mclntyre, S. Boughier, P. Williams, D. Anderson. 


\section{Debate}

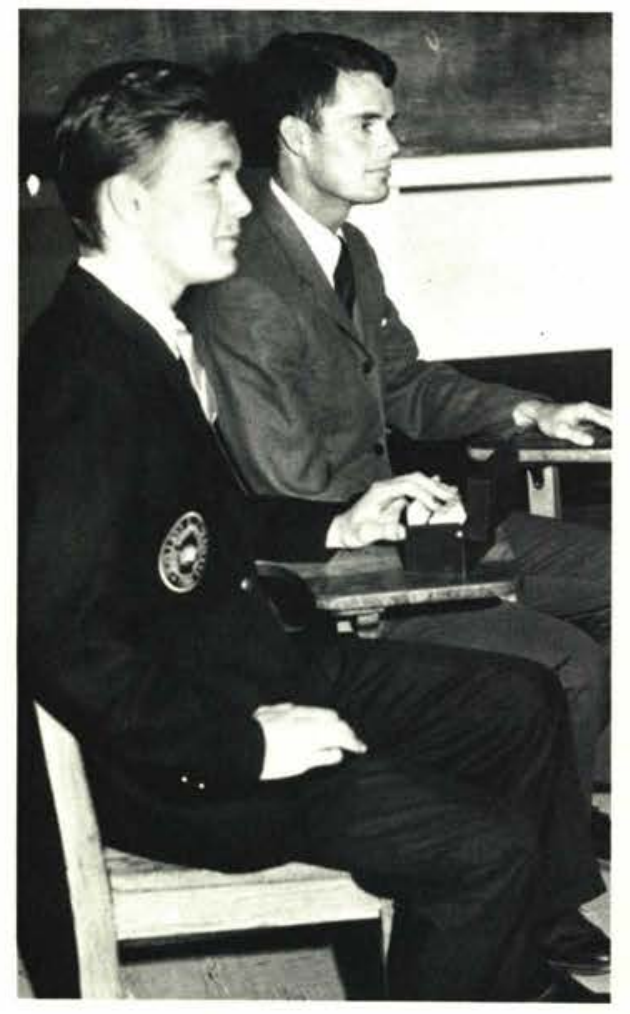

J. Stockwell, M. Nicholls.

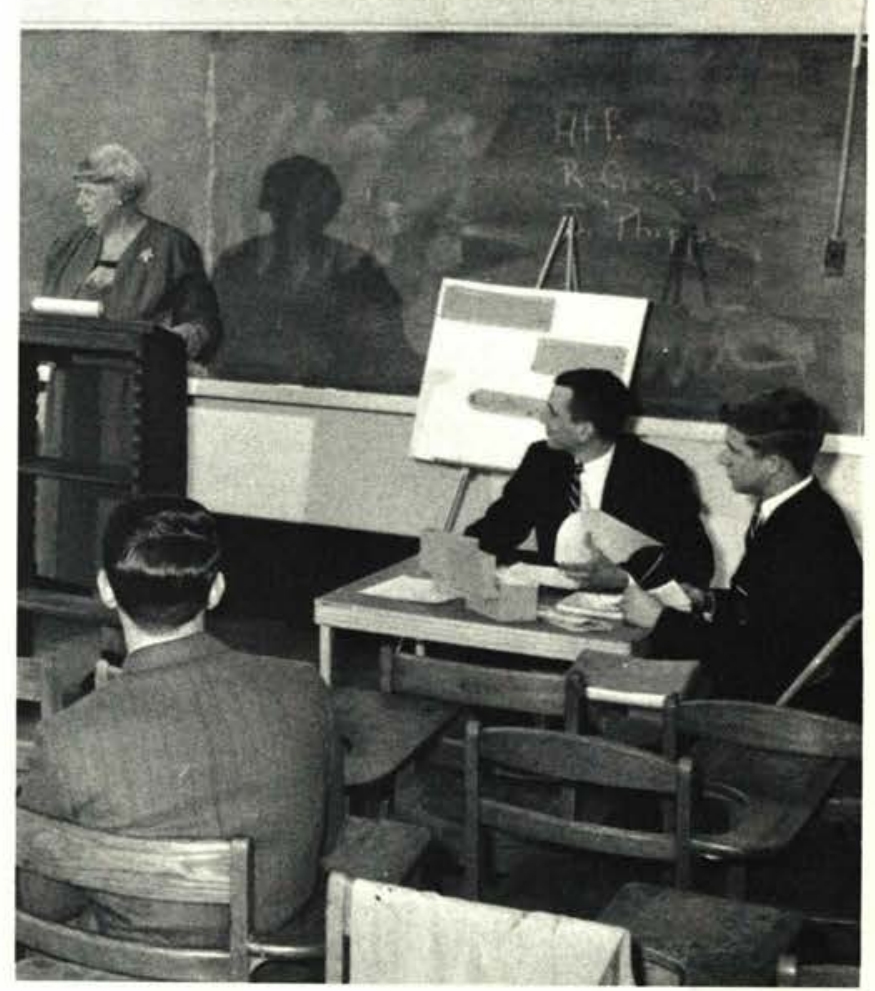

J. Phipps, R. Grosh.

\section{Education Club}

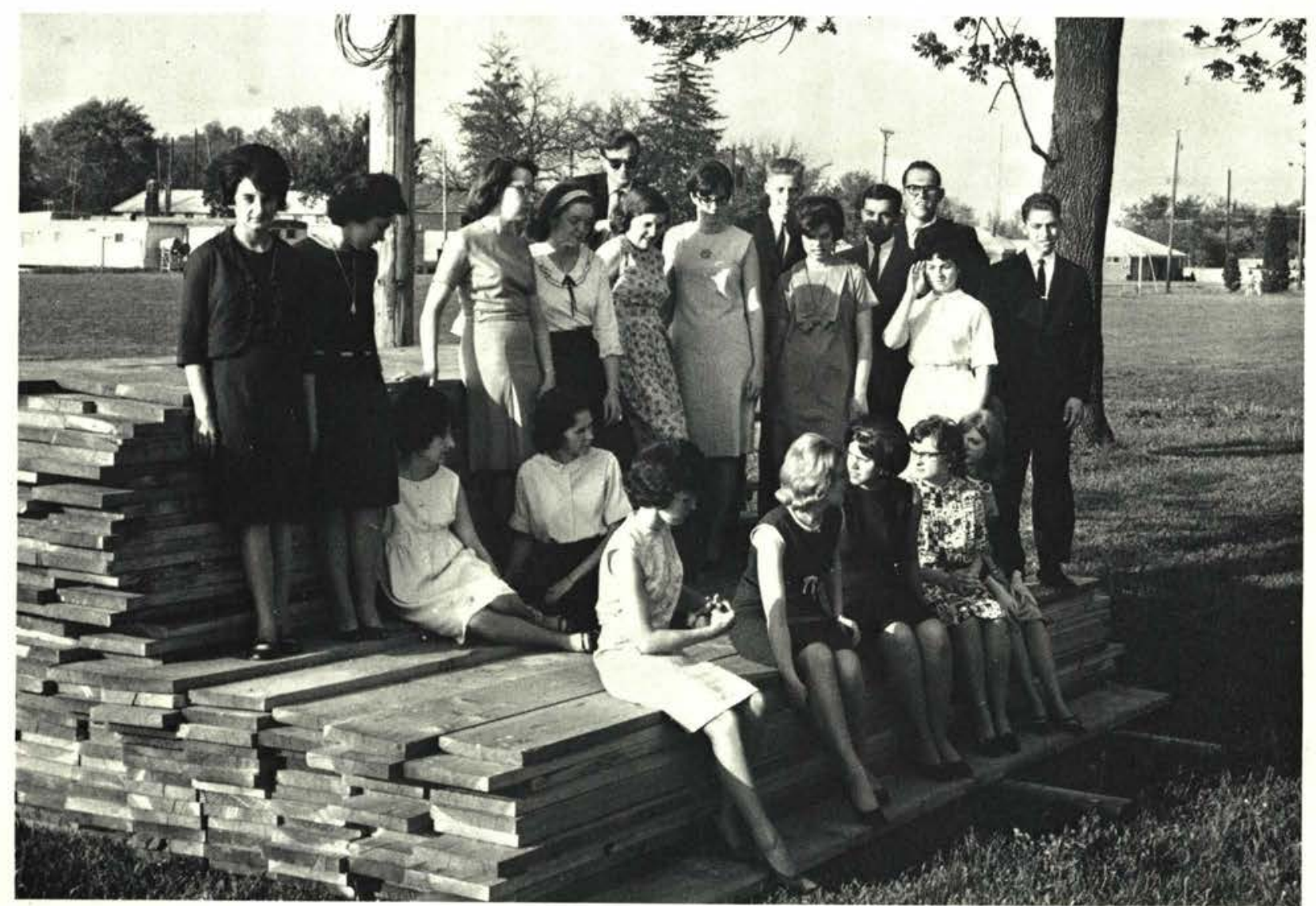

Standing: I. Millikan, L. Miller, S. Gorde, N. Earls, R. Miller, R. Mellish, B. Mosley, S. Marshall, E. Snook, R. Pereira, R. Leach, N. Lewis, R. Yeater. Seated:

B. Millikan, N. Freeman, Mrs. Entner, M. Cannady, M. Schulte, D. Hiller, S. Schwarm. 


\section{Future Business Associates}

Future Business Associates accommodates the students of one of the fastest growing academic departments of the school. The club is organized to promote possible future employment and to provide an opportunity to view the business world in action. In order to do this, speakers from various areas of the business field address the group and tours of plants in the vicinity are taken.

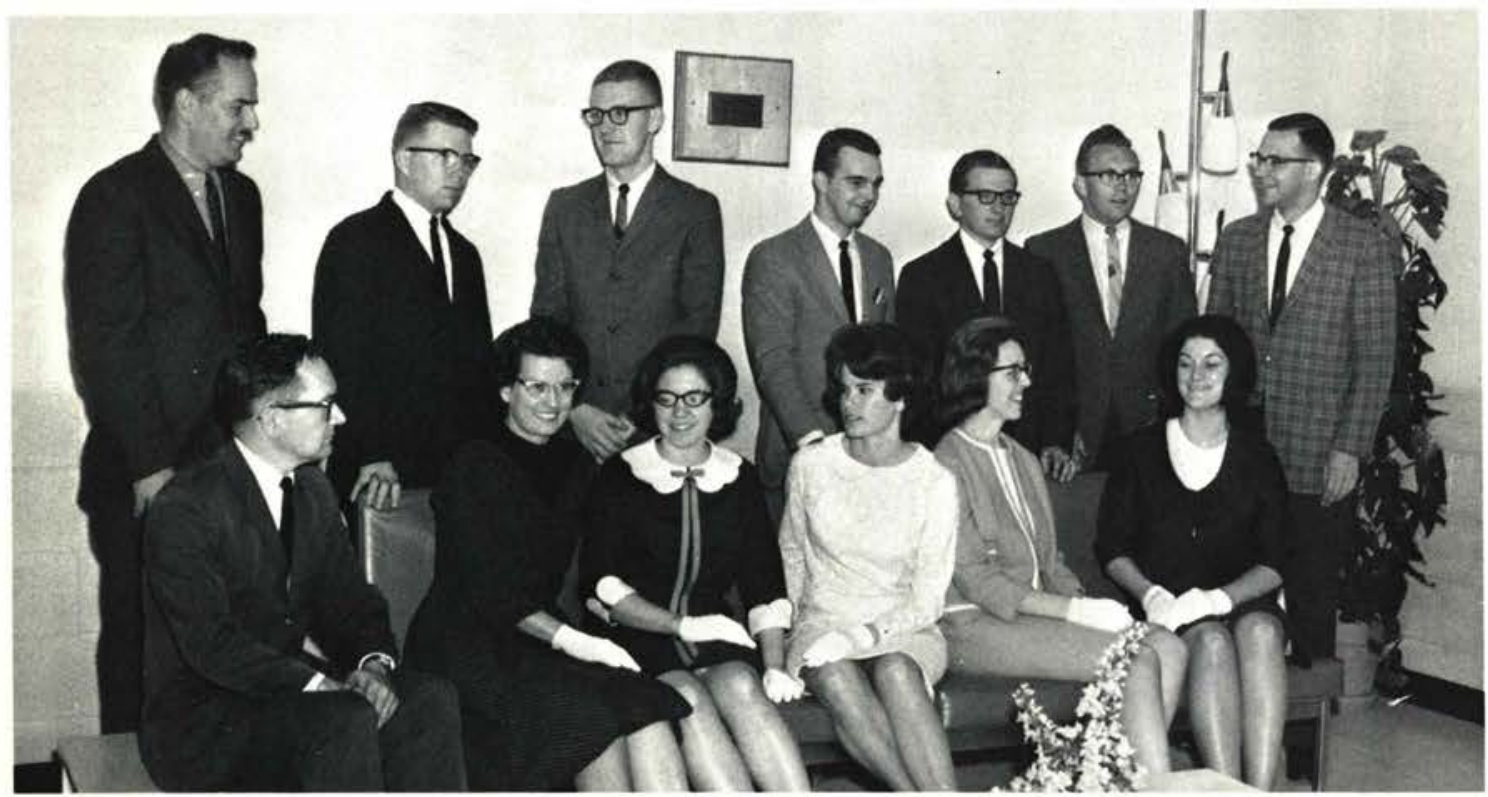

Standing, Left to Right: Mr. Wyse, L. Gavitt, D. Pearce, J. Ziegler, E. Acker, L. Shampoo, Mr. Riter. Sitting: Mr. St. Clair, Mrs. Webber, M. Young, K. McGee, L. Young, M. Goddard.

\section{Conservative Club}

An aired interest in politics is not a prerequisite for membership in the Cedarville College Conservatives; all one needs is an awareness of and an interest in what is occurring around him. The club is basically a discussion group with visiting speakers who examine and perhaps present solutions to many of the current affairs.

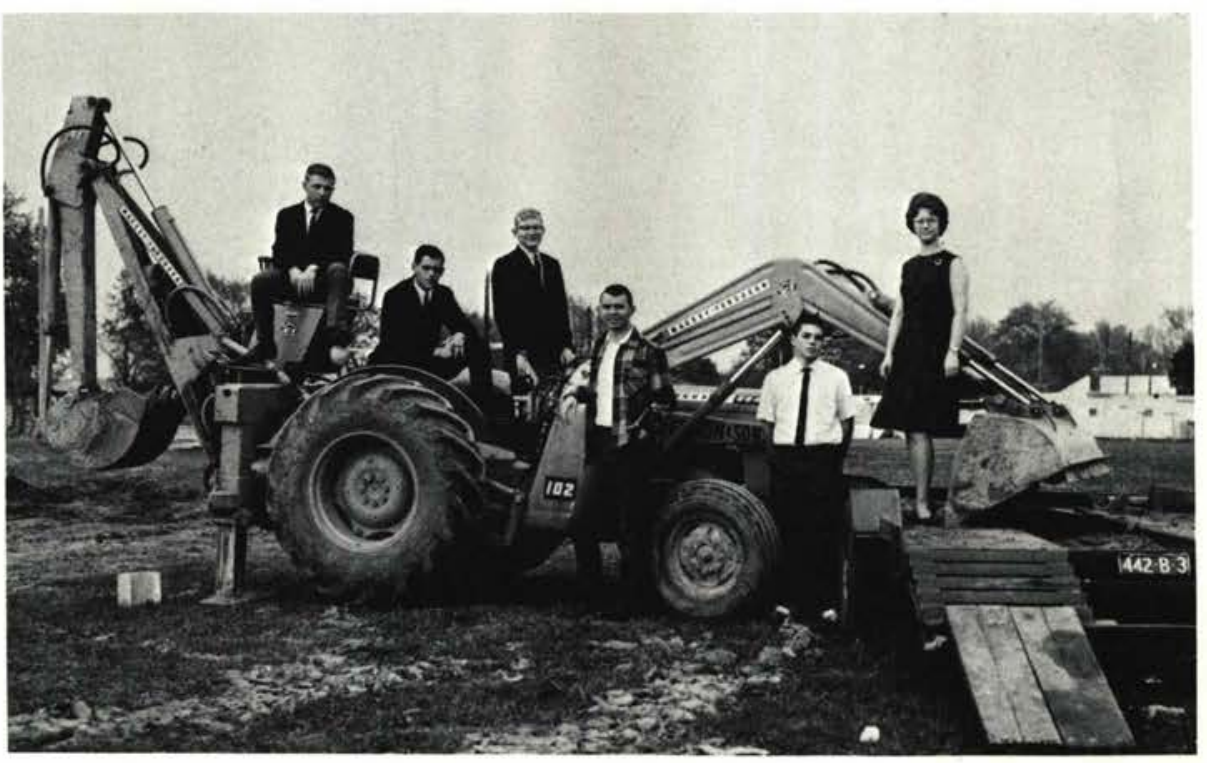

Left to Right: J. Phipps, S. Leiby, F. Jenistra, R. McKnight, G. Fisher, J. Hess. 


\section{Future Pastors' Fellowship}

The Future Pastors' Fellowship is directed toward the understanding of the duties of the modern pastor. Practical experience and sound advice are shared by Dr. Williams as well as other distinguished speakers throughout the year. The organization aims to perpetuate a healthy and sound attitude toward the problems, the

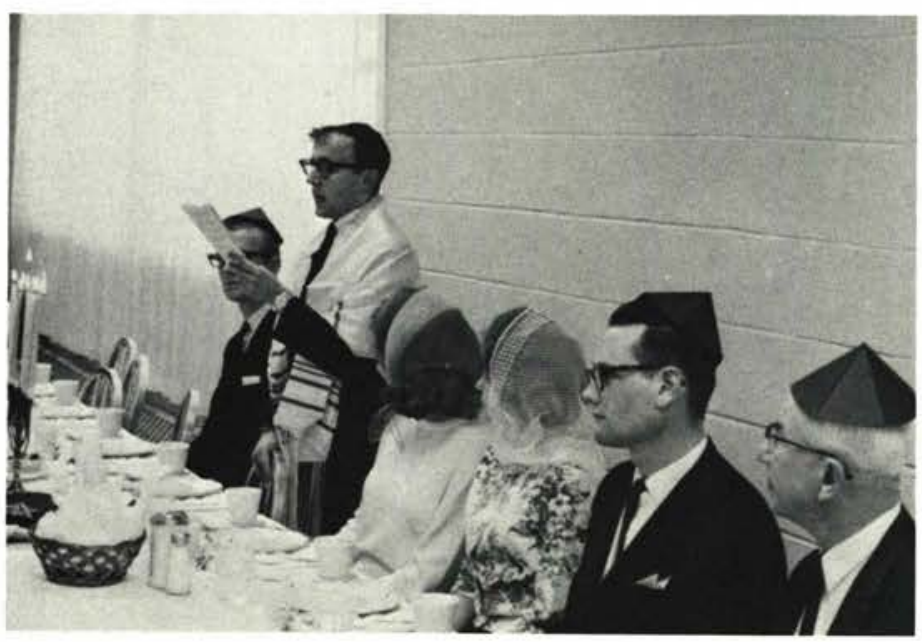
weaknesses, and the responsibilities of the pastor to his people and to the Lord.

Passover Feast

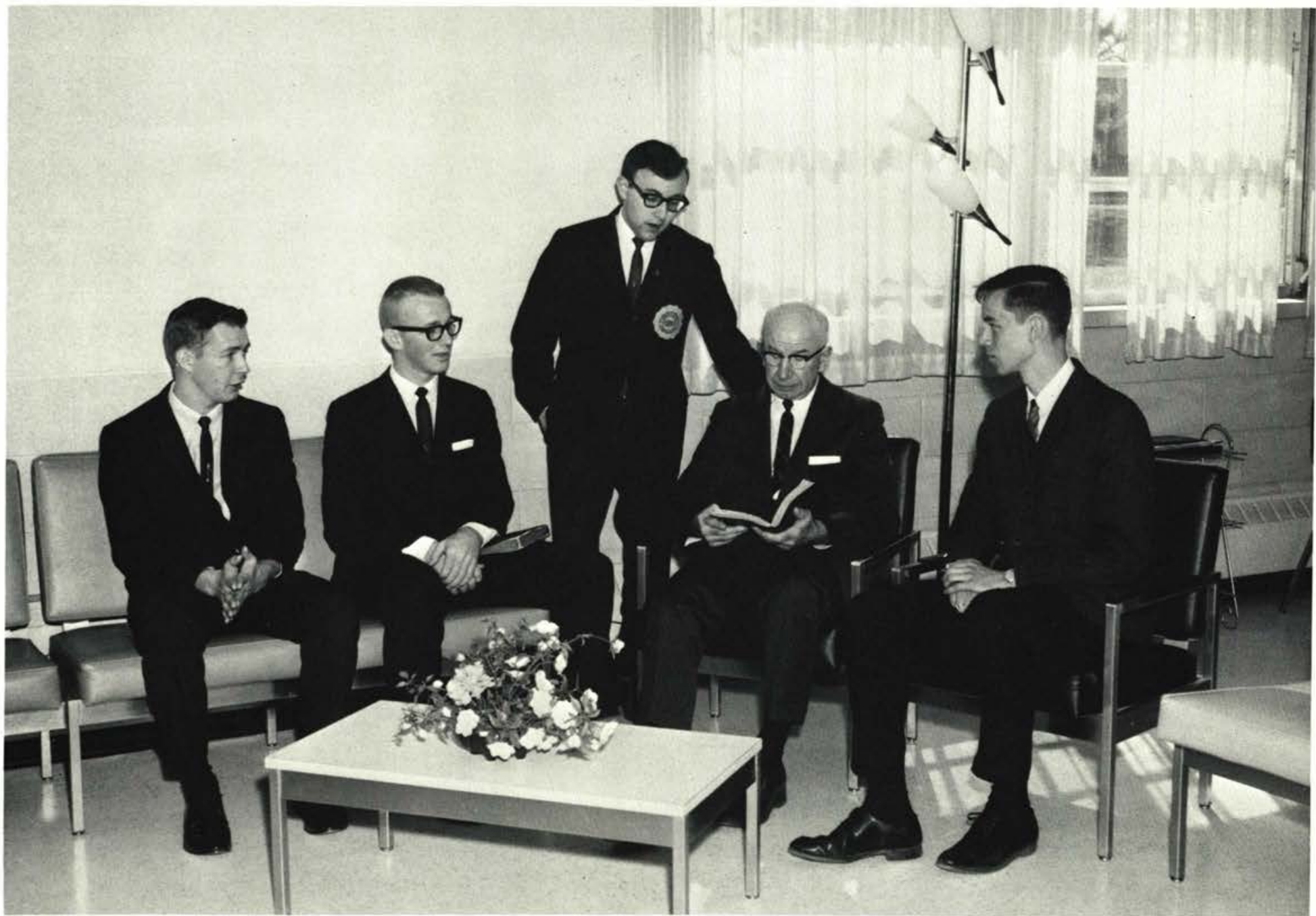

Left to Right: K. Shubert, D. Boyd, A. Fruchtenbaum, Dr. A. Williams, M. Crampton. 


\section{Christian Education in Action}

The main event of this year's club was a weekend trip to Wheaton College, Wheaton, Illinois, to participate in a workshop concerning Christian education.

The Club, which is opened to any member of the student body, was headed by Pat Bird as President, Jan Emerson, Vice-President; Joyce Rudduck, Recording Secretary; Betty Myers, Corresponding Secretary; and Damaris Dunn as Treasurer.

Miss Jean Fisher serves as faculty advisor for the club.

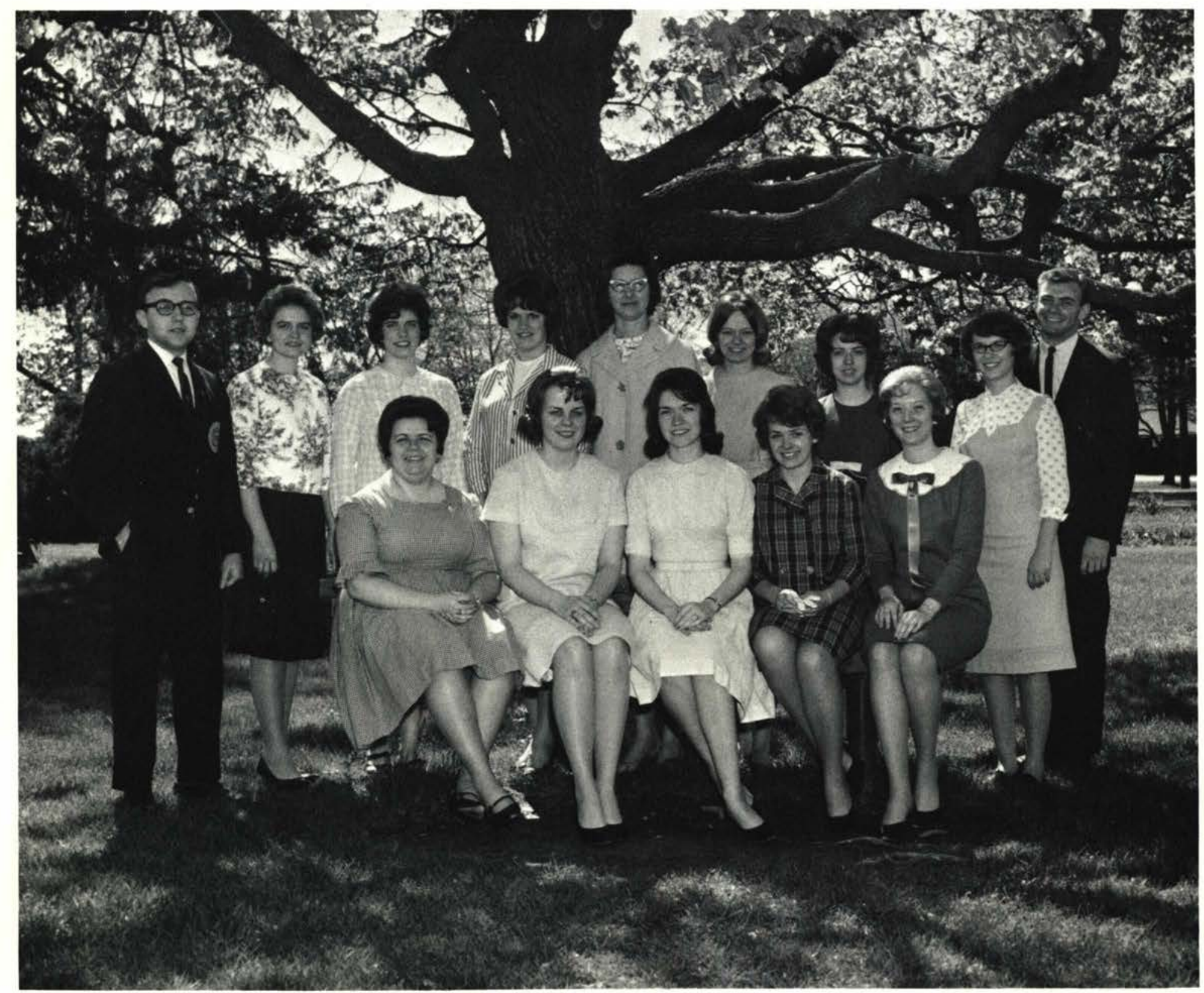

Standing: A. Fruchtenbaum, S. Dufford, B. Perrin, K. Johnson, Miss Fisher, S. Empet, S. Jones, L. Smith, D. Nevitt. Seated: J. Rudduck, D. Dunn, P. Bird, J. Emerson, B. Meyers. 


\section{WRA}

Promoting sportsmanship among the women of the college is one of the major aims of the Women's Recreation Association. The WRA gives interested women an opportunity to enjoy themselves through organized activity. This includes hayrides, overnight parties, etc. WRA acts in the interest of the whole school as well as its members, by using some of its funds to purchase athletic equipment for the college. This growing organization is in its third year of service.

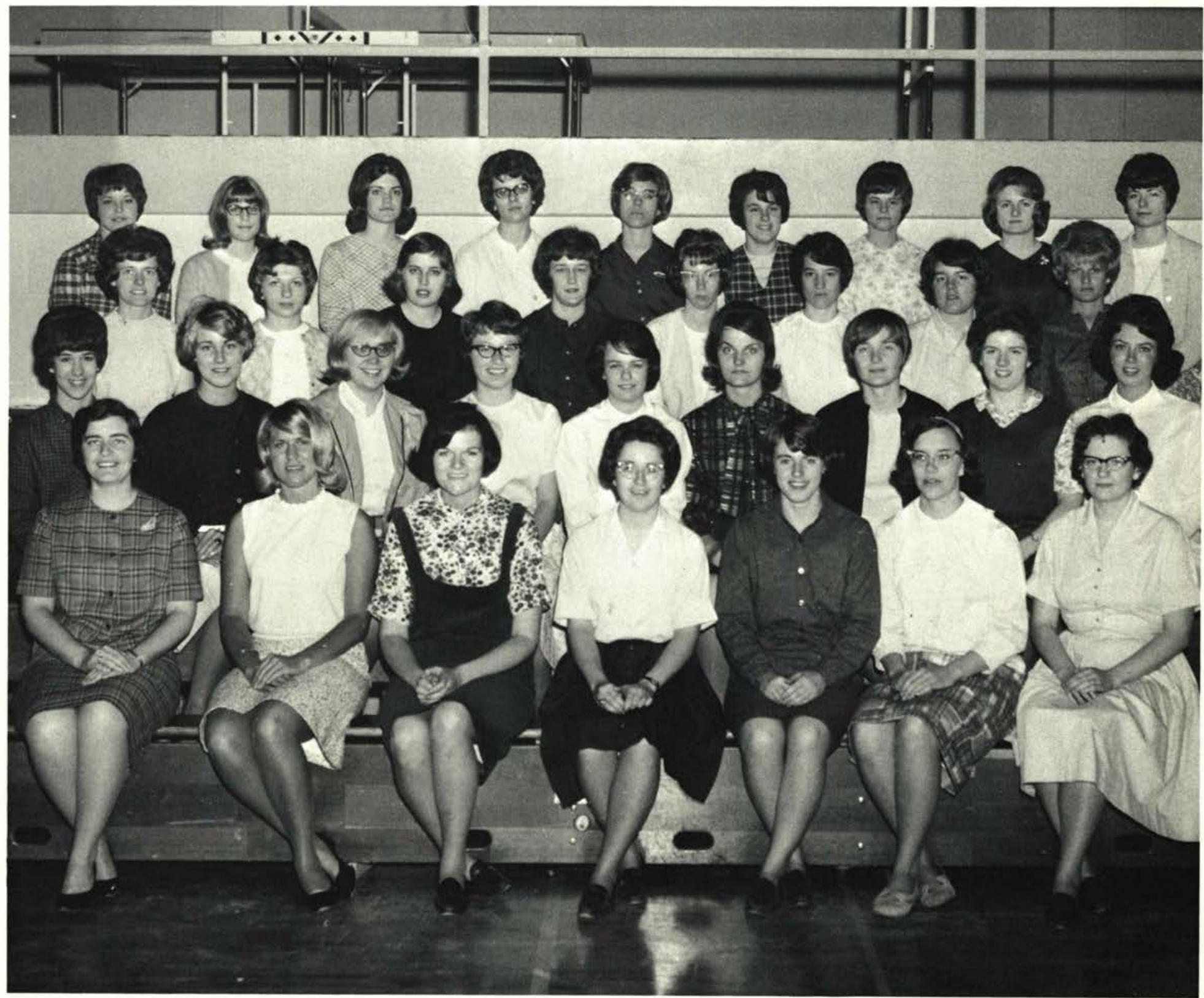

Top row: C. Smith, D. Chaffe, L. Babcock, C. Headlee, M. Beitler, K. Spencer, L. Garlock, M. Grable. Second row: M. Schonscheck, K. Patterson, R. Miller, J. DuBois, F. Bonnema, E. Shugg, C. Hansen, J. Johnson. Third row: C. Wilson, T. Wing, B. Carlson, S. Rader, C. Gail, D. Hannah, L. Wessel, E. Terril, G. Williams. Front row: Miss Kearney, E. Anderson, V Shepherd, R. Grant, A. Damon, J. Strychalski, D. Hiller. 


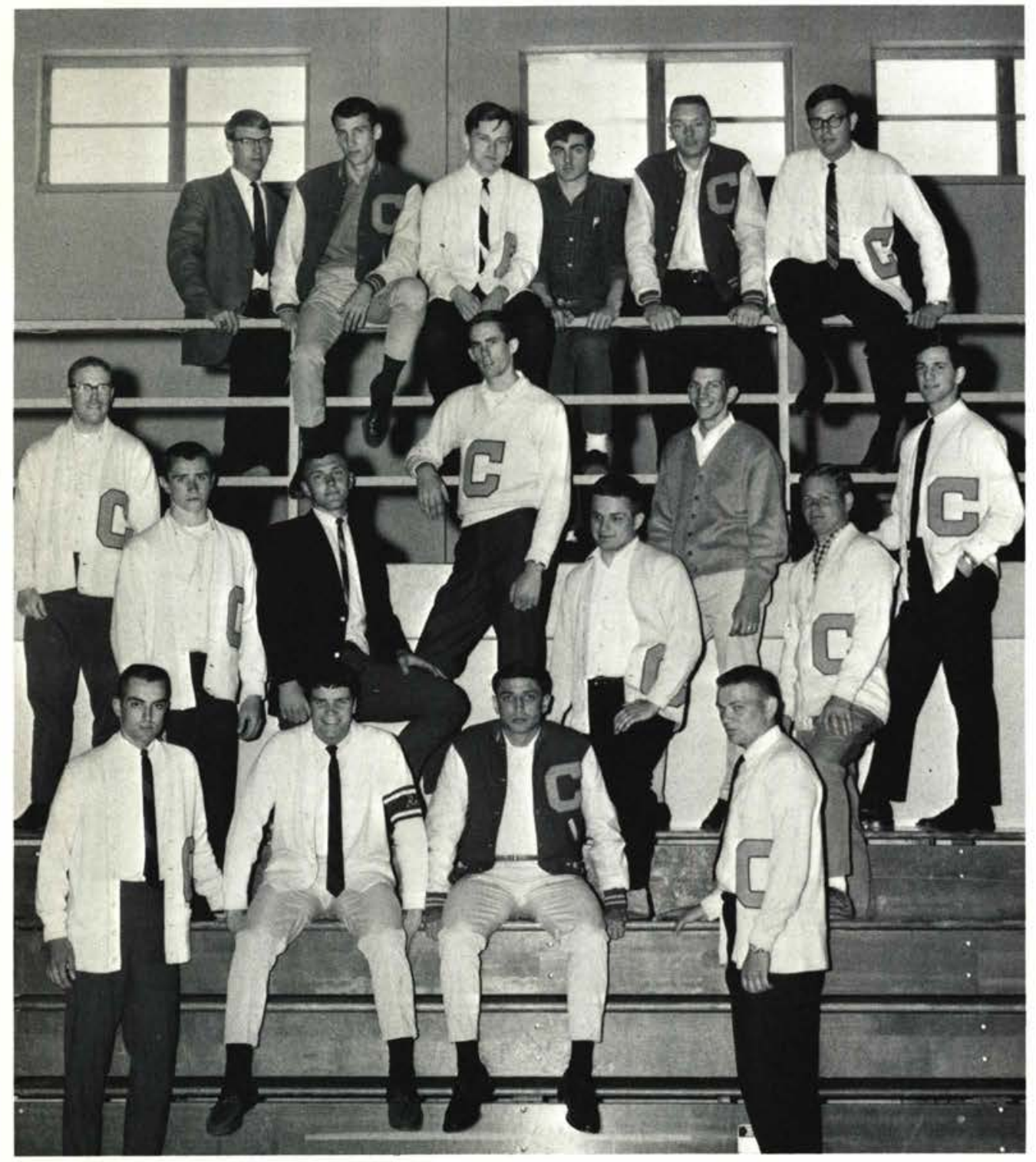

\section{Welcome to Varsity "C"}

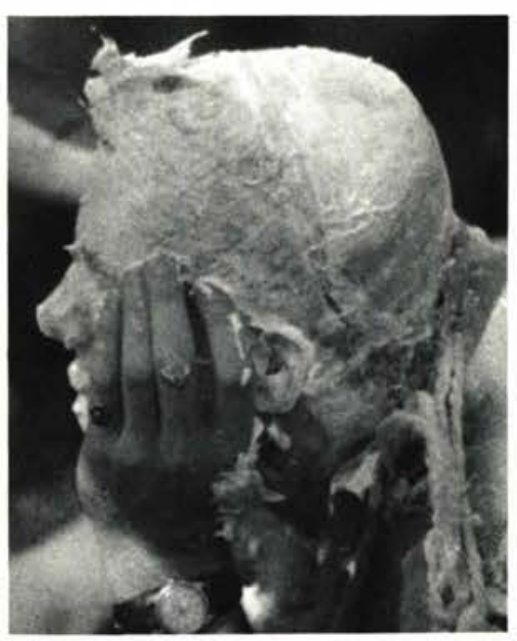

Top row: C. Wilkins, R. Davis, J. Hess, T. Elliott, B. Davis, T. Oakley. Second row: G. Shufelt, D. Cooper, G. Drill, J. King,

T. Duff, J. Englemann, J. Miller, D. Leach. Front row: J. Branon, R. Ross, B. Francis, C. Reed.
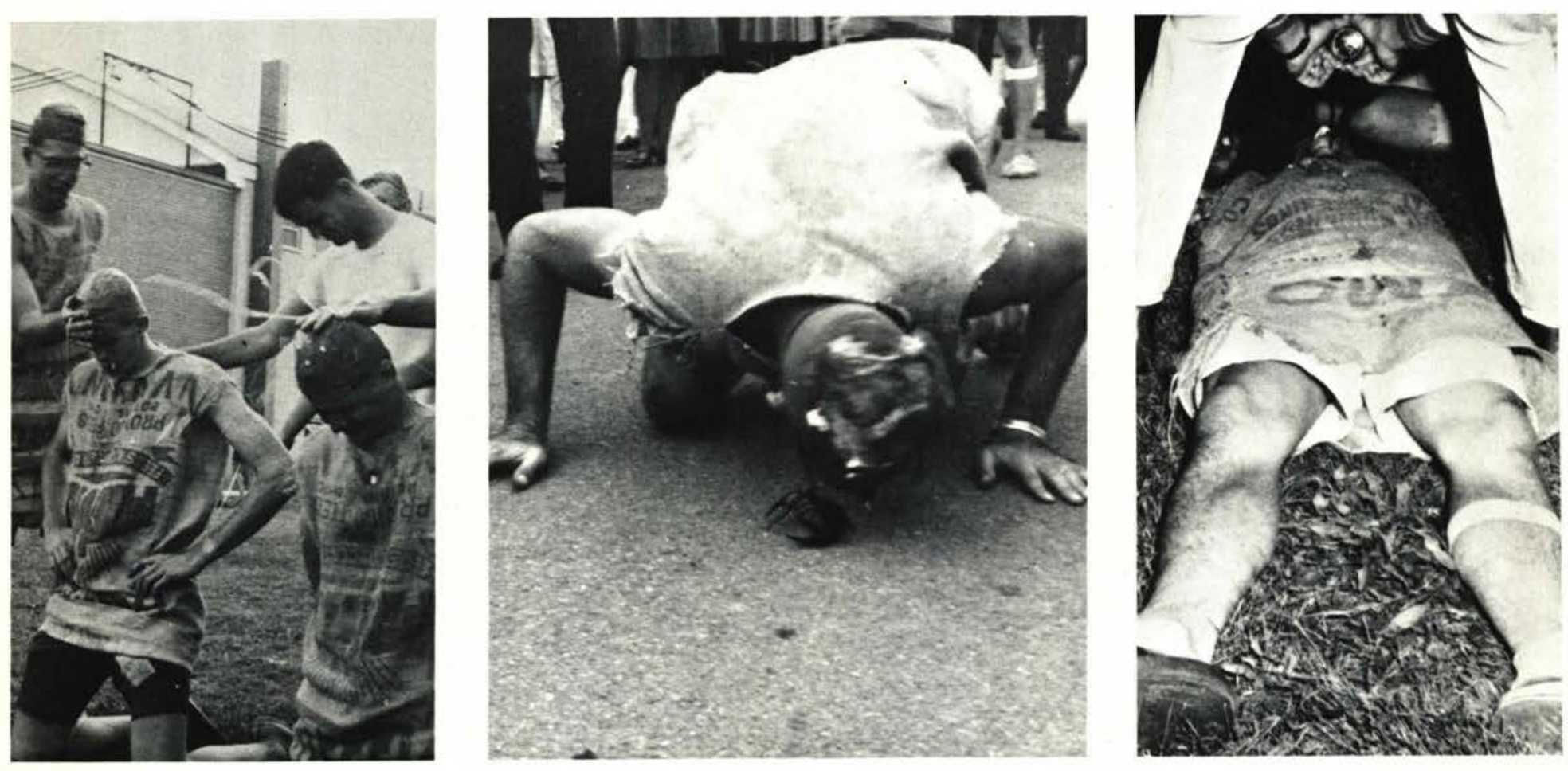
Miracle Staff

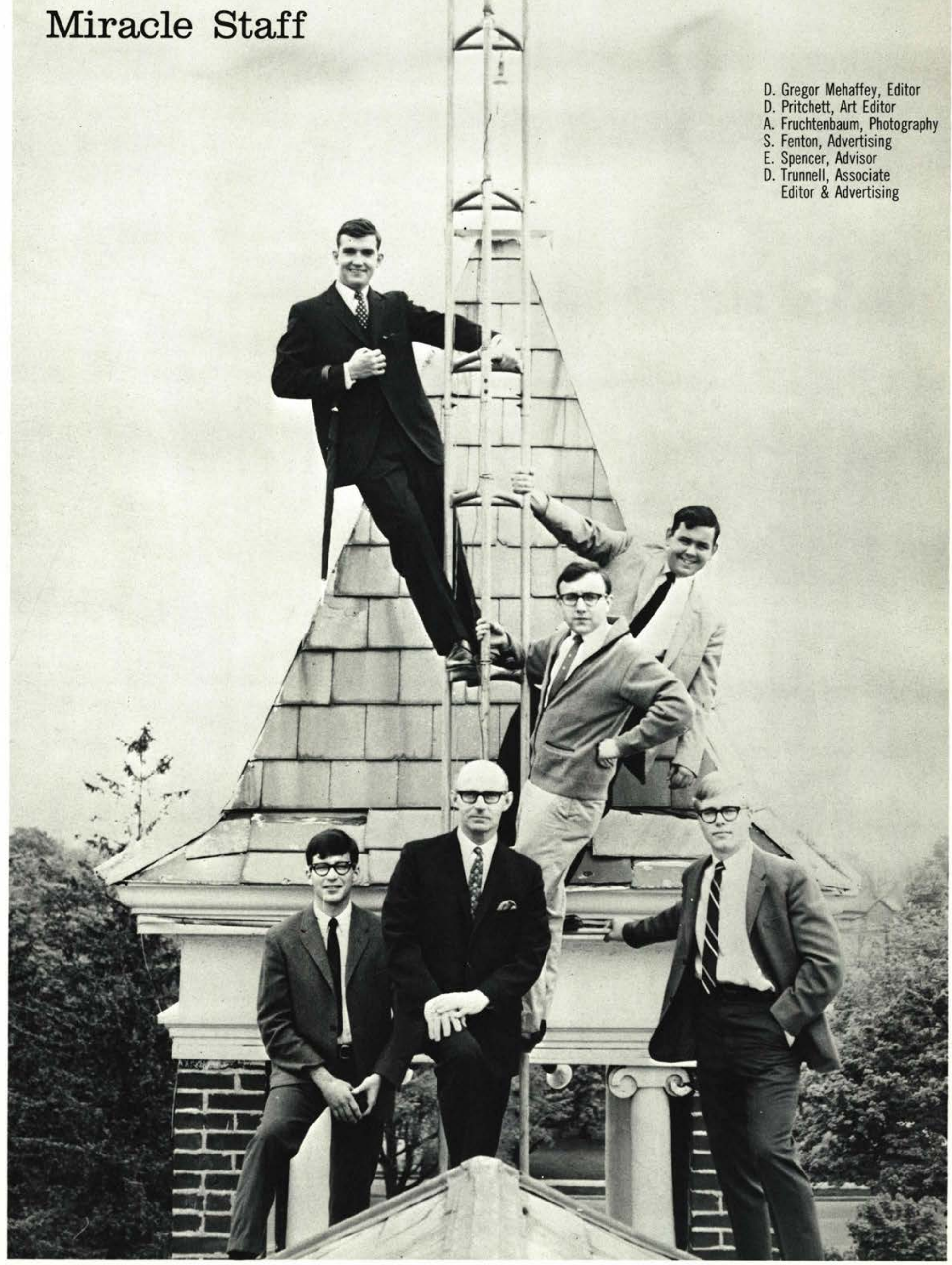




\section{Whispering Cedars}

Under the capable editorship of Michael Nicholls, the Whispering Cedars grew this year to a full-sized college newspaper. Directed by the publications committee, the staff produced twelve issues during the school year. New features in the paper included the 'Faculty Forum,' the 'Question at Issue' column, 'Macs,' and an extensive use of pictures.

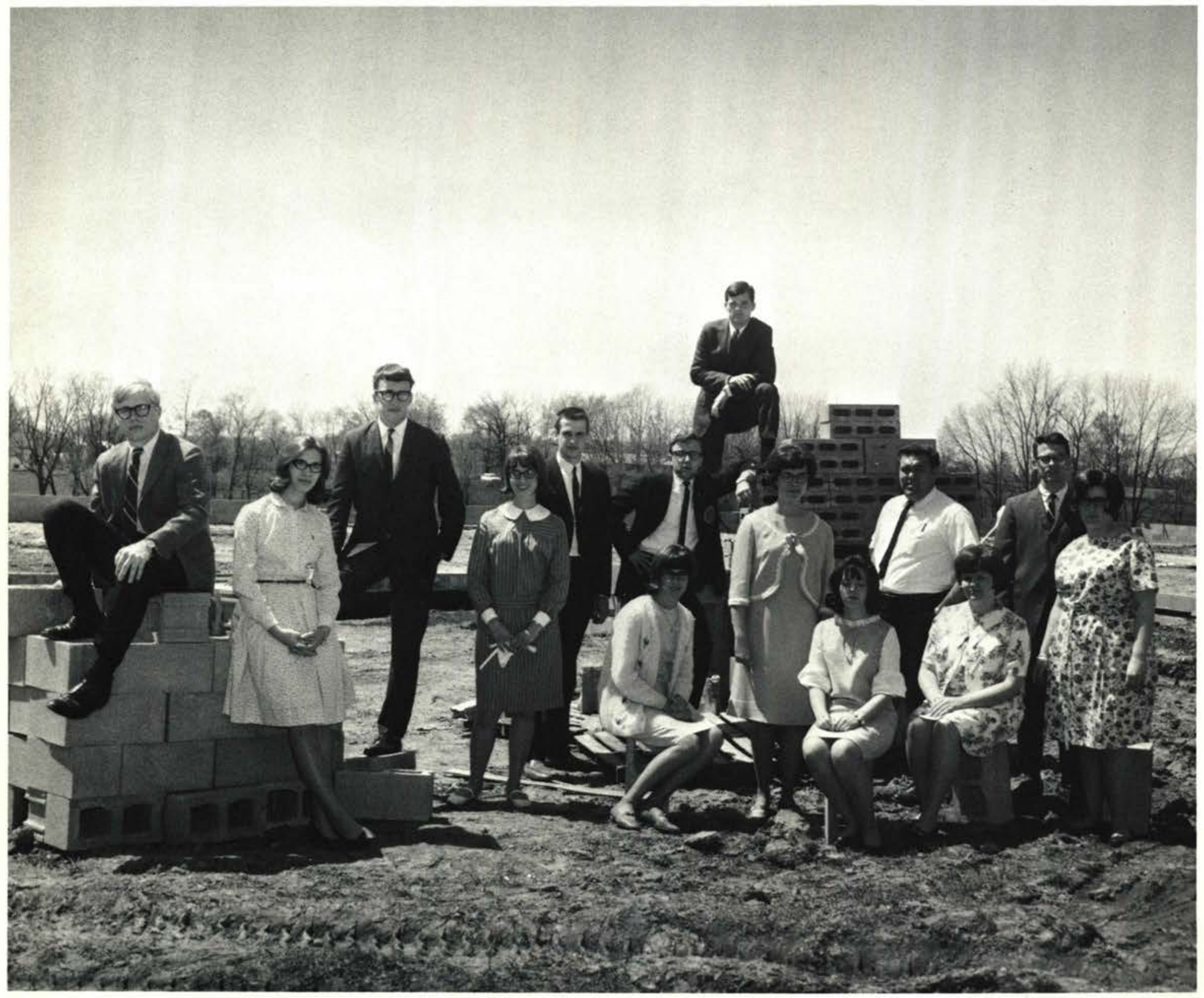

Left to Right: D. Trunnell, J. Lennox, J. Brown, D. Miller, S. Brown, A. Fruchtenbaum, M. Nicholls, S. French, W. Keib, L. Montgomery, P. Fulop. Seated: R. Williams, J. Elkins, K. Cartner. 


\section{Student Council}

Student Council, as a representative body, assists in providing a beneficial extracurricular activities program while still providing the needed voice for the student body in negotiating with the administration. The Student Council plays an important part in inspiring loyalty to the school and its principles and speedily handles actions and problems involved in various school matters. The Student Council sponsors numerous service projects, the more prominent of which are the Bloodmobile visits, the caring for campus vending machines, the Student Body Project, and the Prospective Students' Days.

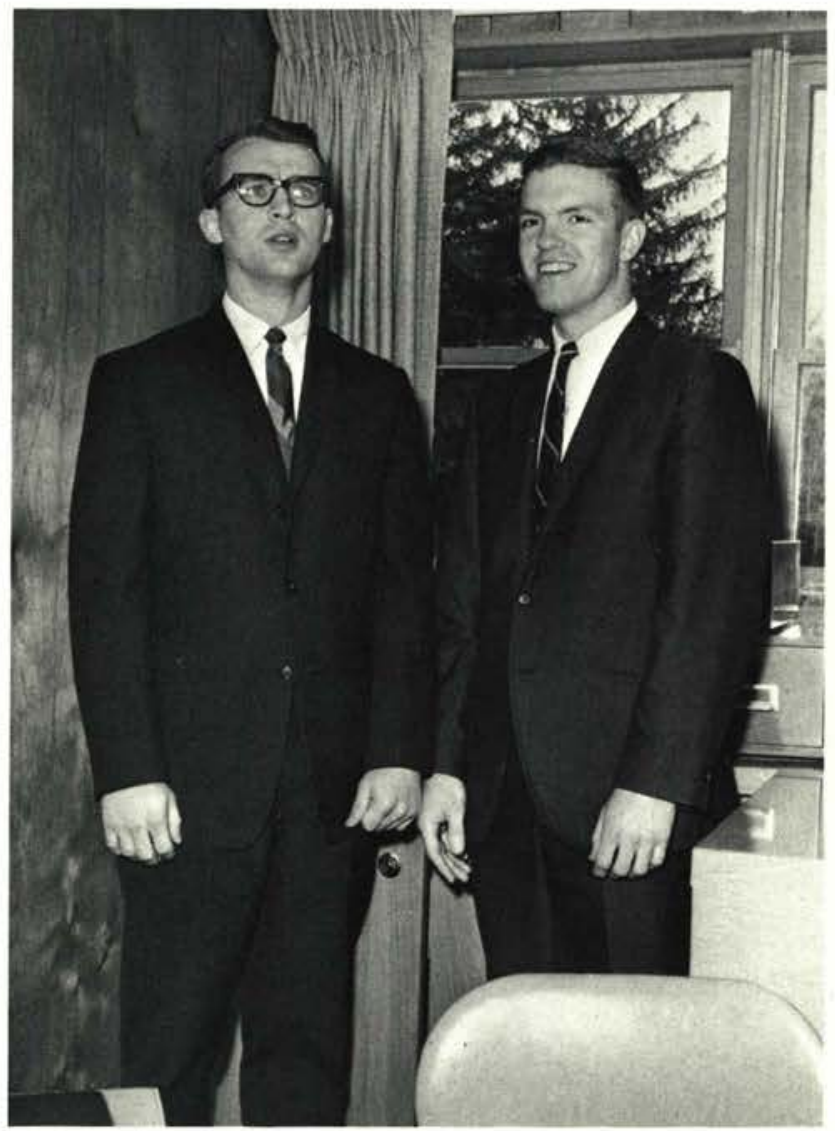

Ken Nichols, Gary Harris.

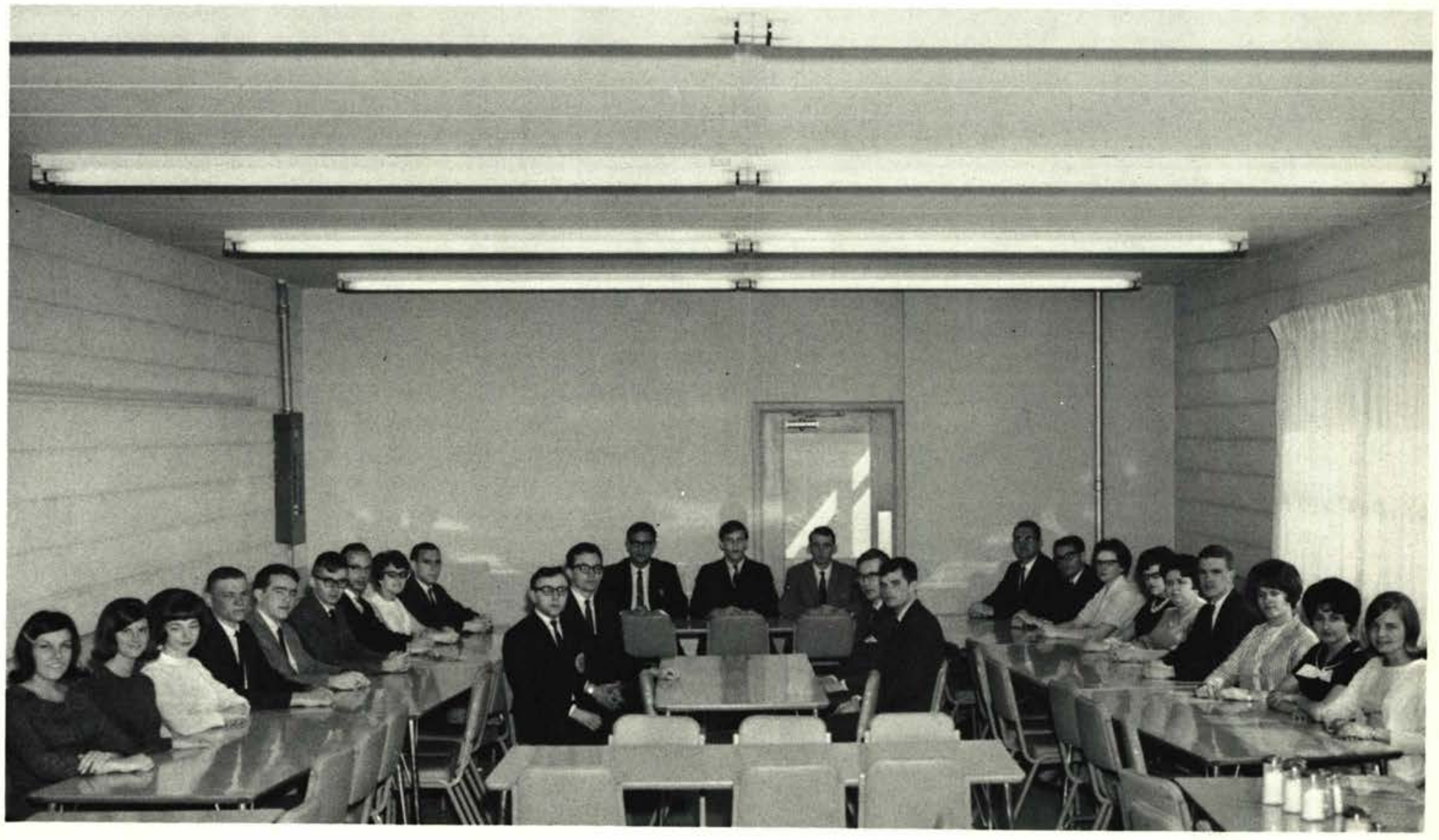

Left to Right: D. Daab, G. Ciehart, K. Salisbury, C. Reed, T. Timmons, J. Skillman, T. Clater, B. Mclnnes, D. Gordon, A. Fruchtenbaum, M. Hamilton, T. Oakely, J. Stowell, R. Grosh, G. Griffin, M. Nicholls, D. McIntosh, J. Roden, D. Hiller, D. Durkee, J. Ruddock, G. Harris, K. Johnson, I. Millikan, C. Payne. 


\section{GAMMA CHI}

The oldest campus literary society for women is Gamma Chi-Women for Christ. Through every activity the organization seeks to stimulate growth and originality in the areas of literature, music, art, and humor. Its aim is to advocate and prepare women of Cedarville College for participation in civic and social affairs.

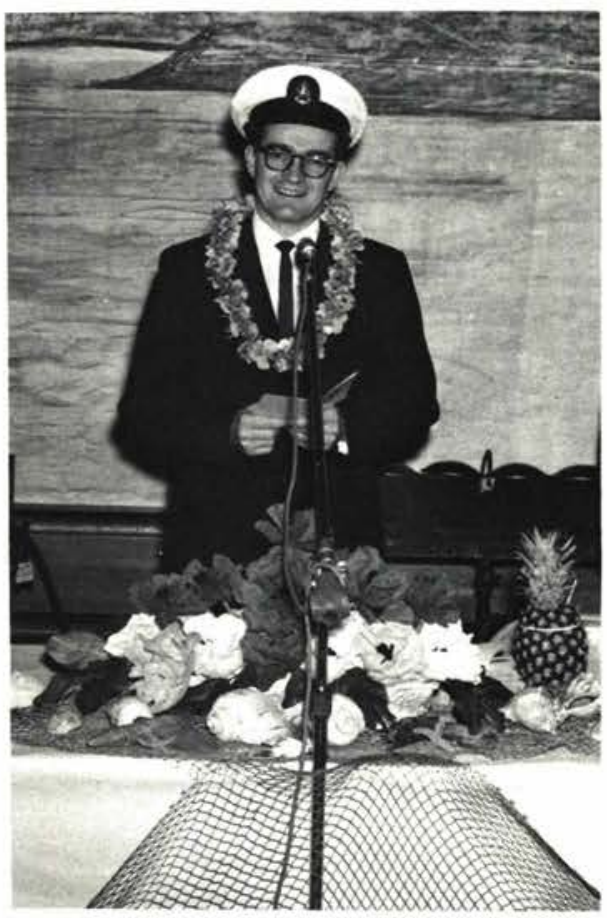

Dr. Robert Gromacki
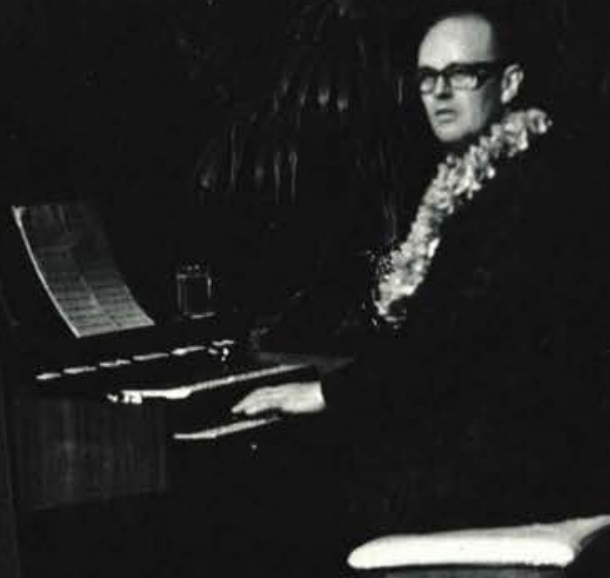

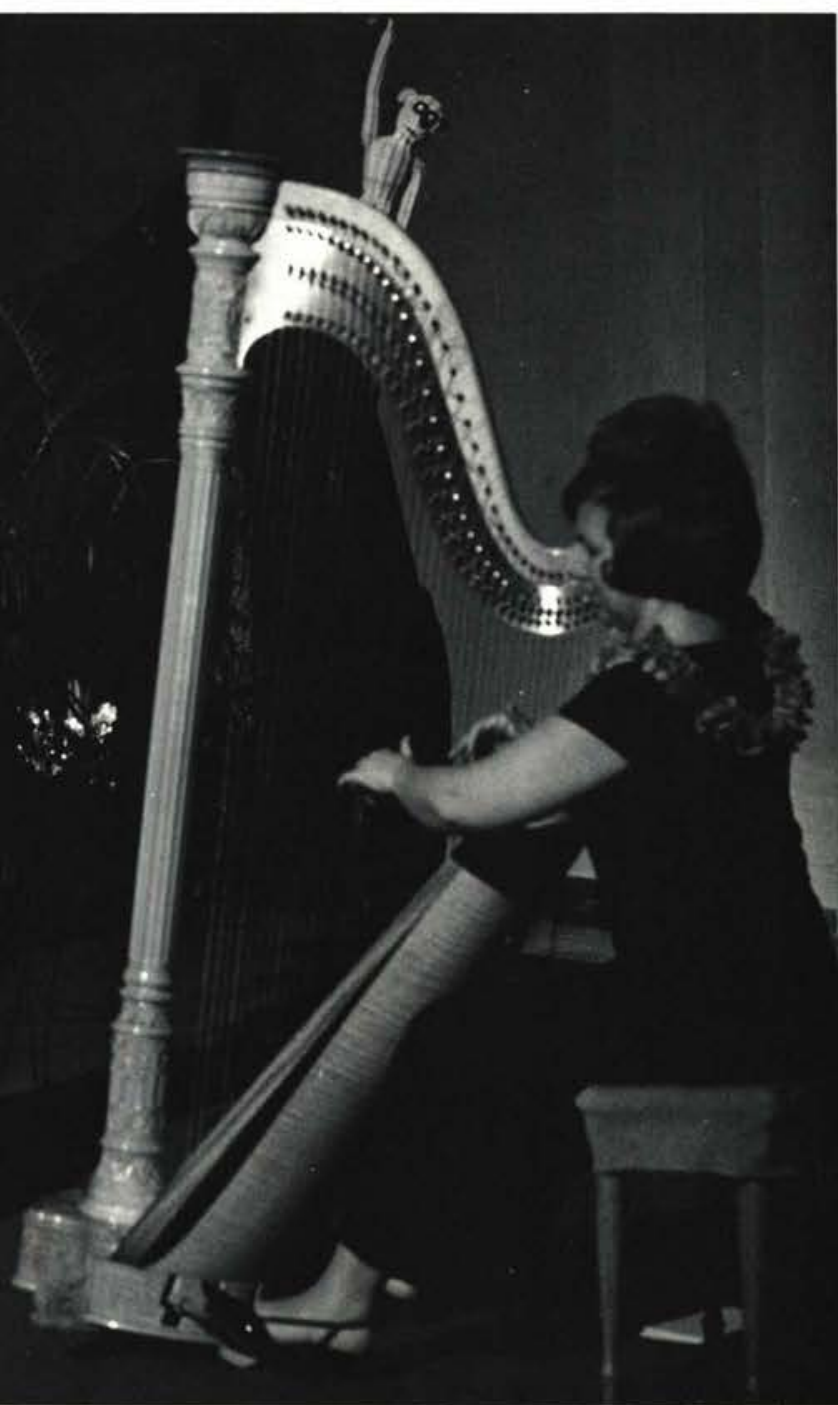




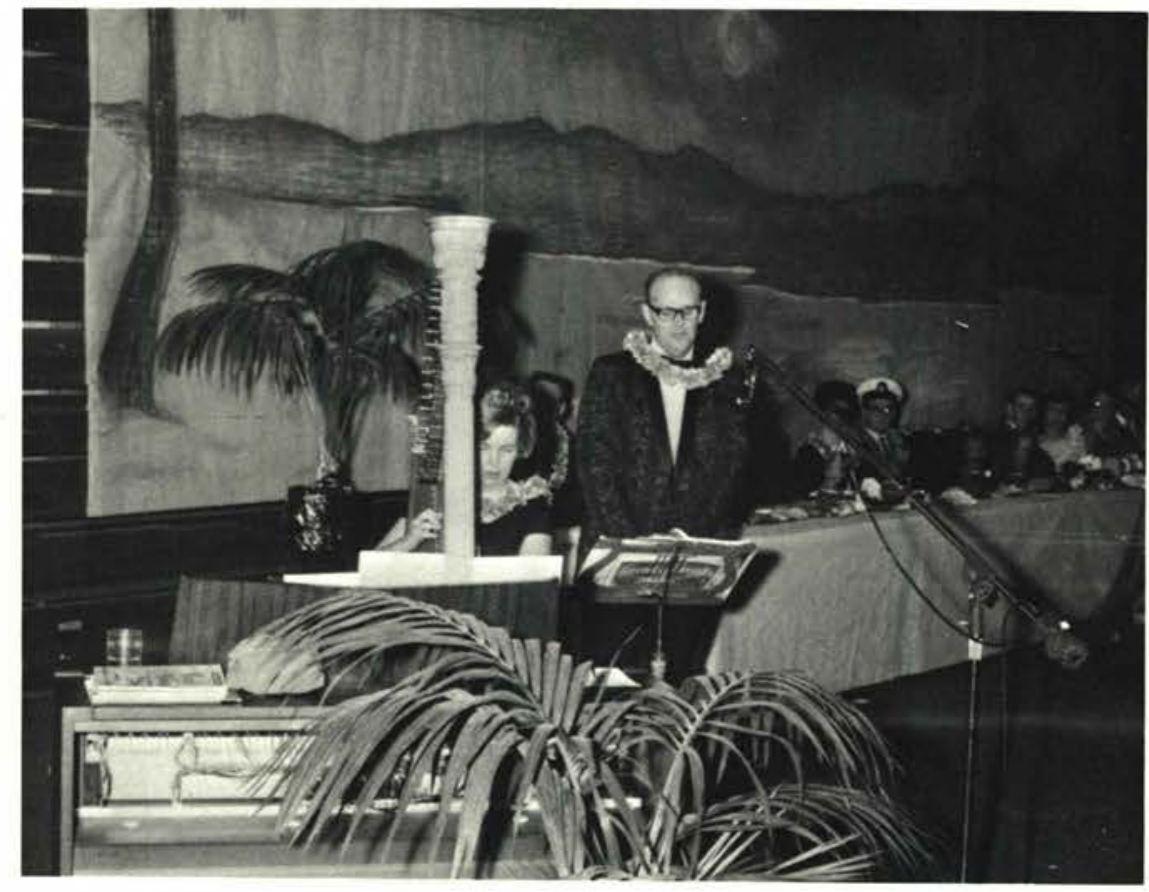

Mr. \& Mrs. John Weyant

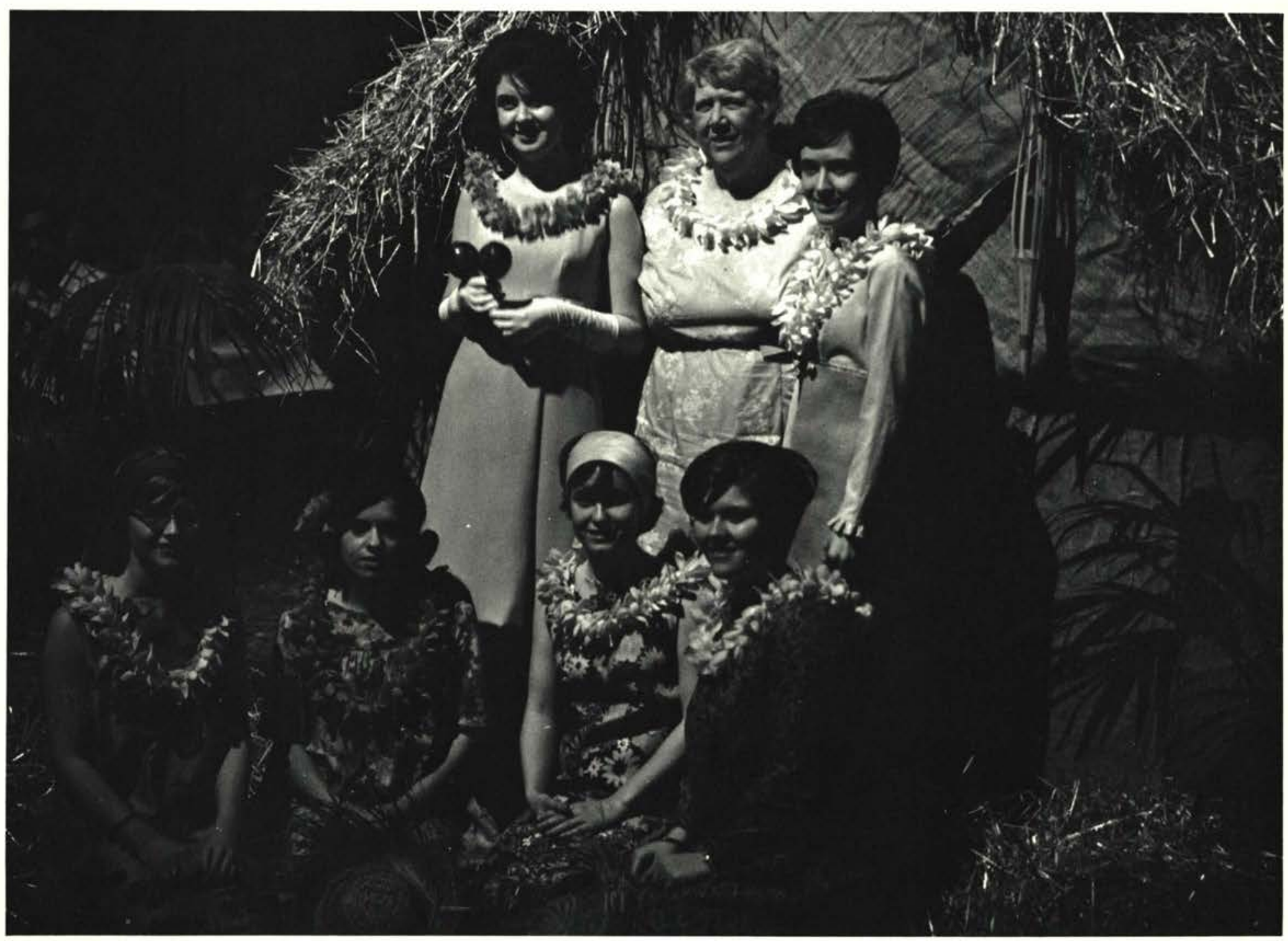

Standing: Mona Ballard, Mrs. Miriam Maddox, Advisor; Jody Ronk. Seated: Donna Hamilton, Linda Hoffman, Jenny Field, Margaret Brewer. 


\section{KAPPA DELTA CHI}

Kappa Delta Chi, a women's literary society, is organized to stimulate an interest in gracious living and to provide an opportunity to practice this type of living in a manner pleasing to God. Members of this organization are exclusively upperclassmen who meet in both formal and informal situations in order to cultivate habits and tastes becoming to Christian young women.
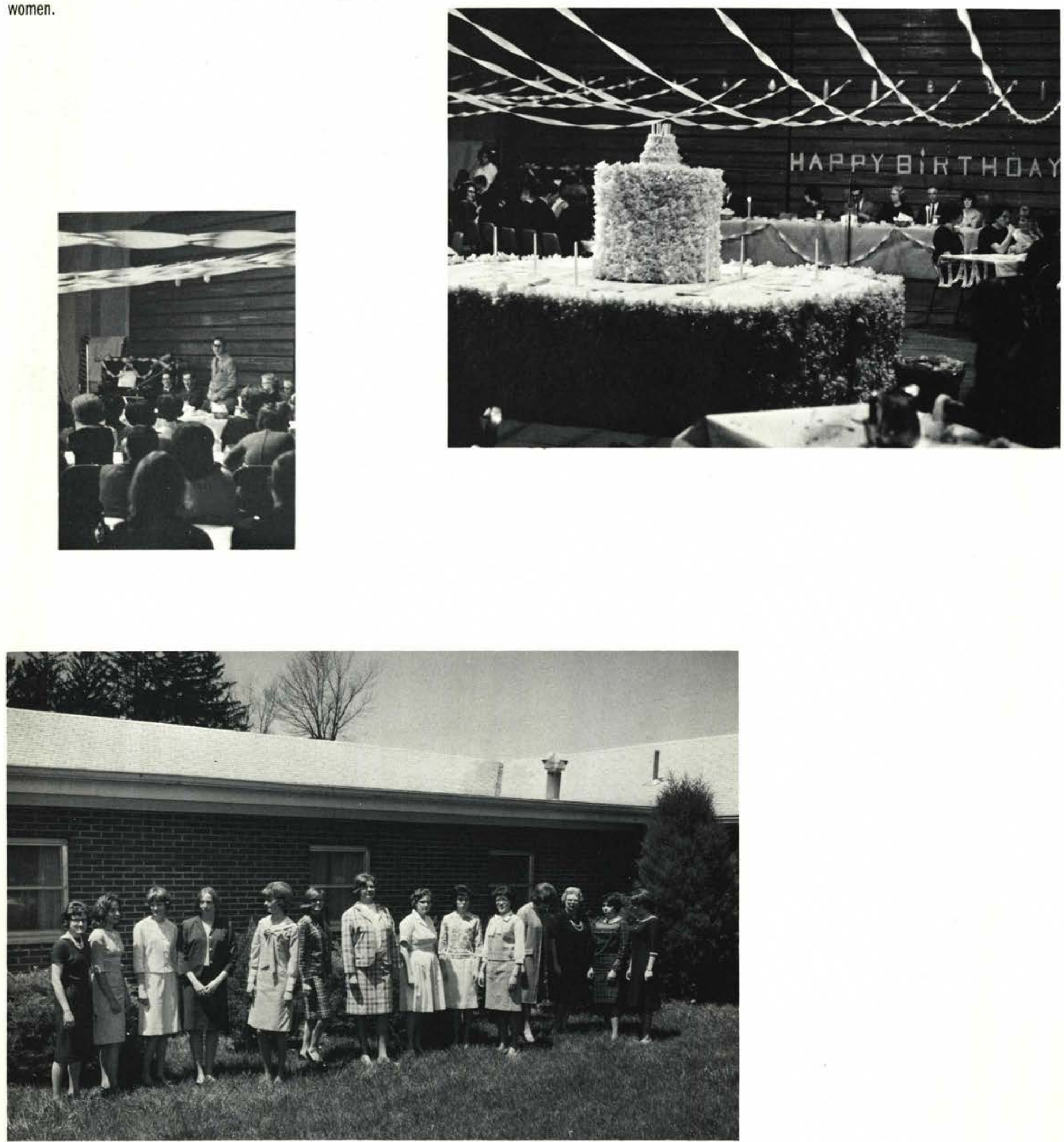

Left to Right: D. Durkee, J. Rogers, C. Barto, M. Beerer, N. Hes, C. Stevenson, M. Rieken, D. Hiller, M. Lockhart, D. Morgan,

M. Jones, Mrs. Elmore, Advisor; G. Liechty, B. Smith. 


\section{ALPHA CHI}

Alpha Chi, the men's literary society on campus, promotes the spirit of refinement and social tastes and also develops poise, personality, and leadership at both formal and informal events. One of the major functions on campus that they sponsor is the annual Thanksgiving banquet. The club also attends other events as a group in order to accomplish the club's purposes.
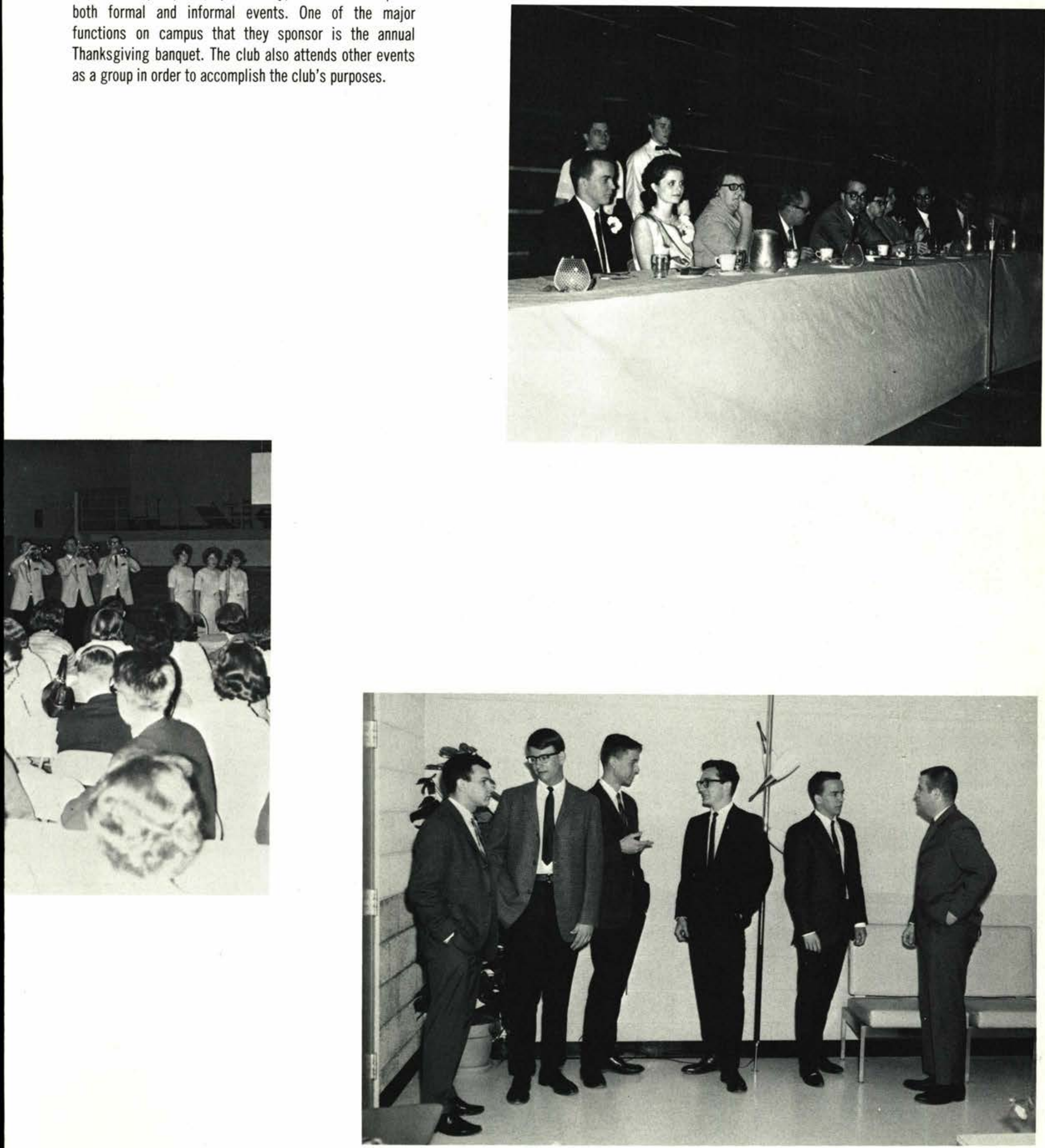

Left to Right: ?. Wall, C. Wilkins, M. Crampton, M. Hamilton, R. Allerton, D. Seigneur. Not pictured: Dr. Raymond Bartholomew, Advisor. 


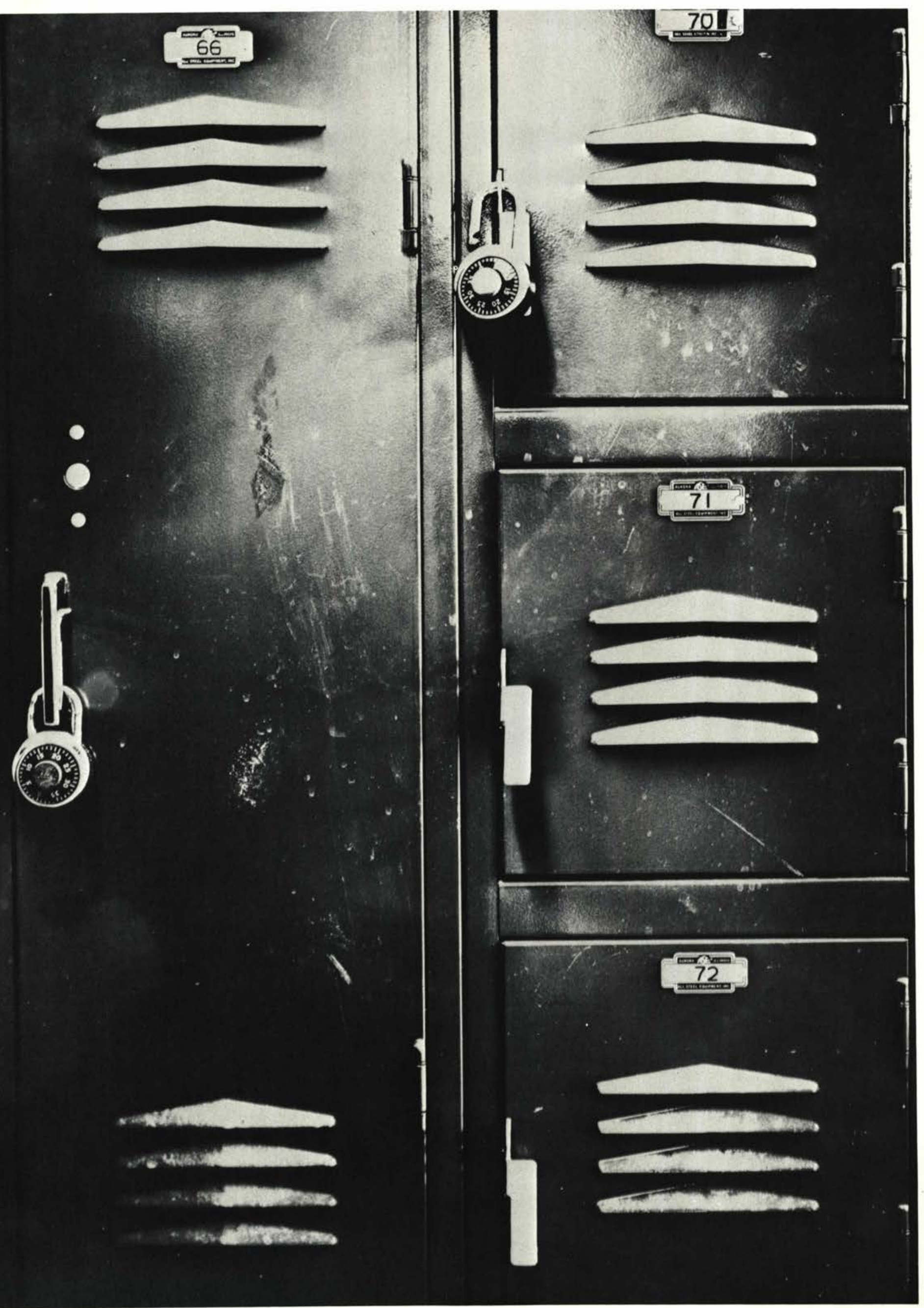




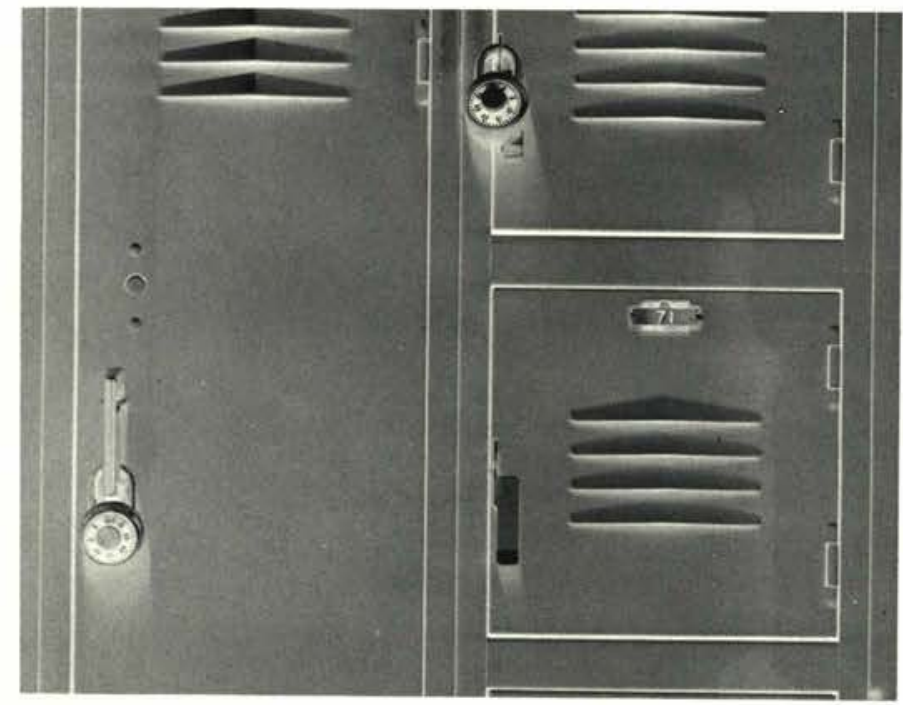

Sports 


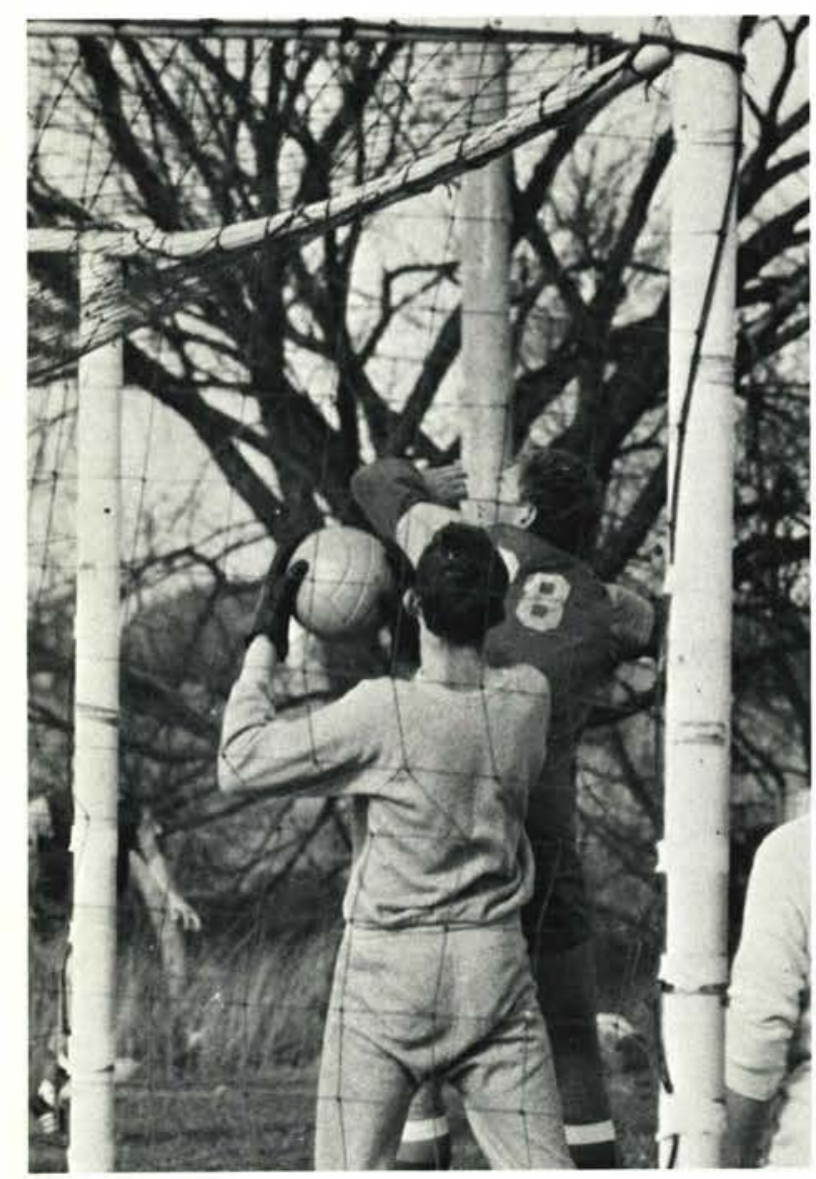

\section{SOCCER}

Greater depth was available and noticed this season as transfer students John Rueck, Mike and Dave Gregory, Bob Worth and Mick Nicholls, all with soccer experience, permitted wholesale substitution without the loss of ability. Led by top scorer Randy Ross and Joe Stowell as captains, the team rolled to an unprecedented 7-2-2 record and a 3-0-2 conference record.

In amassing this record, the Jackets scored 42 goals which led Ohio Conference to their opponents 22 , giving them a 3.8 average against the opponents 2.0. An impressive 307 shots were taken at the opponent's goal while the Cedars defense held the opposition to 150. Both Pereira and Trimble, team goalies, did an excellent job in stopping these.

Special recognition was given to Randy Ross, right wing, when he was announced Ohio's scoring champ with a total of 20 points and was chosen for the AllOhio second team. Joe Stowell, fullback, received distinguished recognition by being named to the AllOhio first team and by being named a National Soccer Coaches Association's All-Midwest Selection.

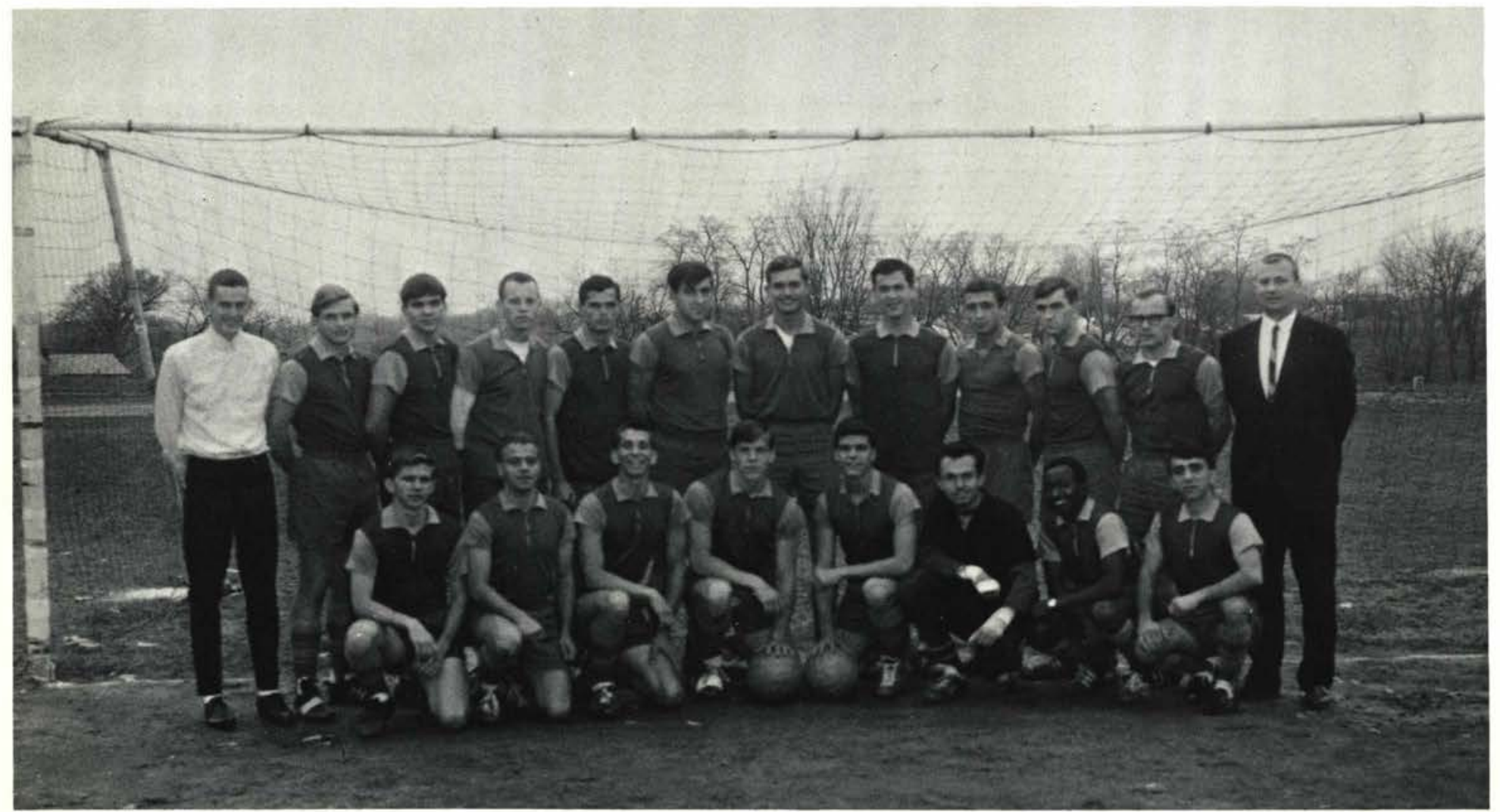

SOCCER-Standing: K. Pearce, K. Daniels, E. Tripp, J. Hay, M. Nicholls, R. Worth, D. Leach, W. Francis, M. Gregory, J. Rueck, Mr. Dennis Olsen, Coach. Kneeling: M. Trimble, D. Rockwell, J. Engelmann, J. Stowell, R. Ross, M. Pereira, S. Akinyemi, J. Falci III. 

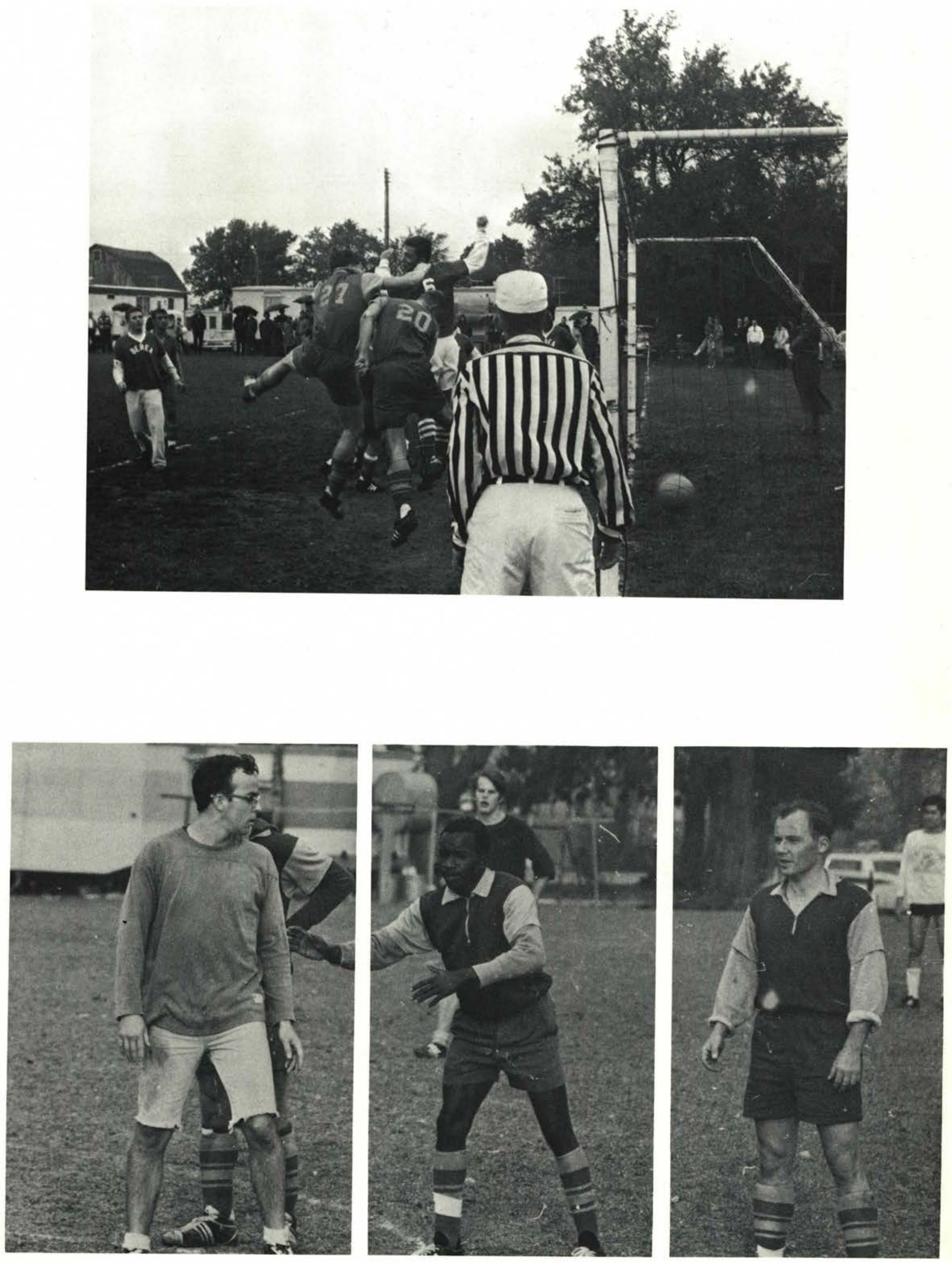

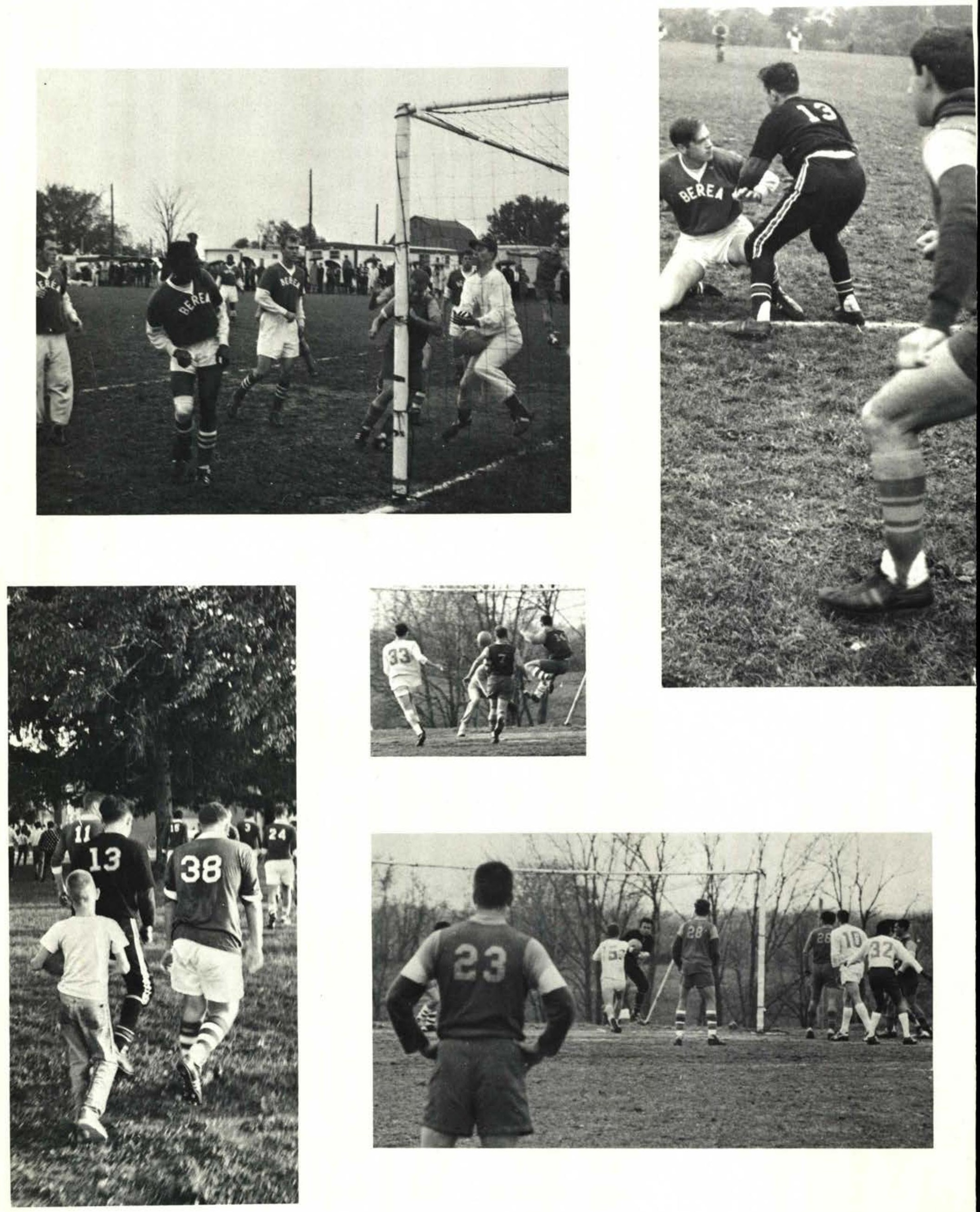

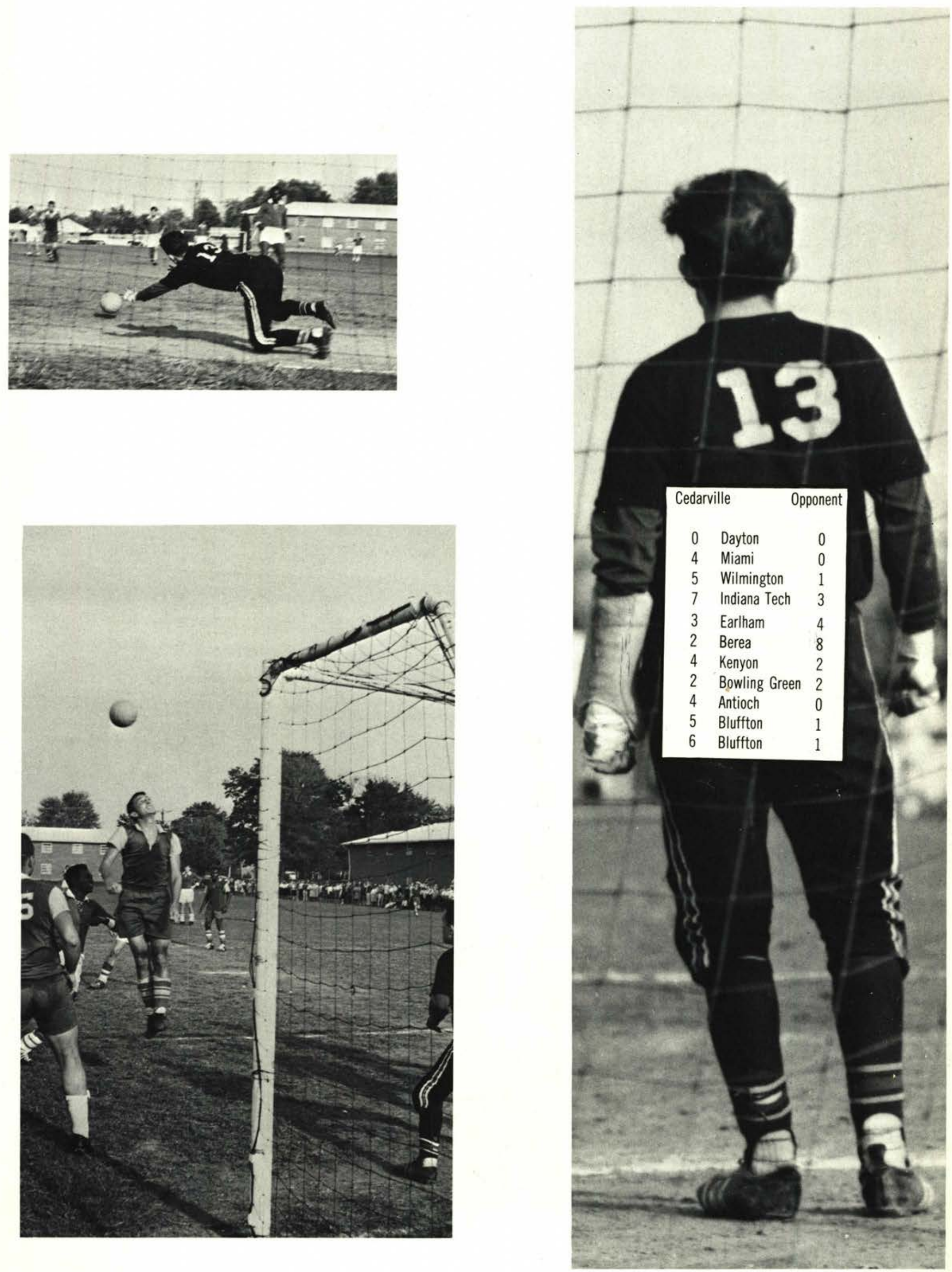


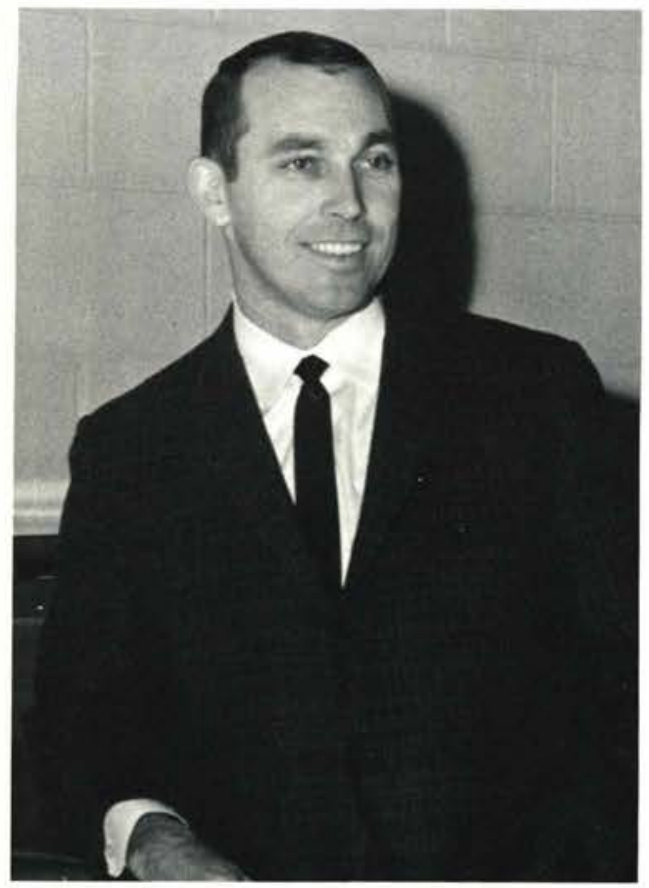

\section{BASKETBALL}

Cedarville's basketball team finished the season this year with 12 wins and 11 losses. Under the direction of Coach Don Callan, the year saw the team building for the future. Prospects are good for next year, for the team members are all underclassmen.

Al Knott, sophomore forward from East Moline, Illinois, was named to First Team berths in both the Mid-Ohio and District Teams. He led both Conference and District in scoring with 521 points and a 22.6 point per game average. This pushed his two-year total to 1035.

Sophomores Gary Drill from Winchester, Indiana and Larry Waite also received Conference honors. Gary won a berth on the Second Team while Larry Waite from Hudsonville, Michigan, was awarded an Honorable Mention.

Coach Callan

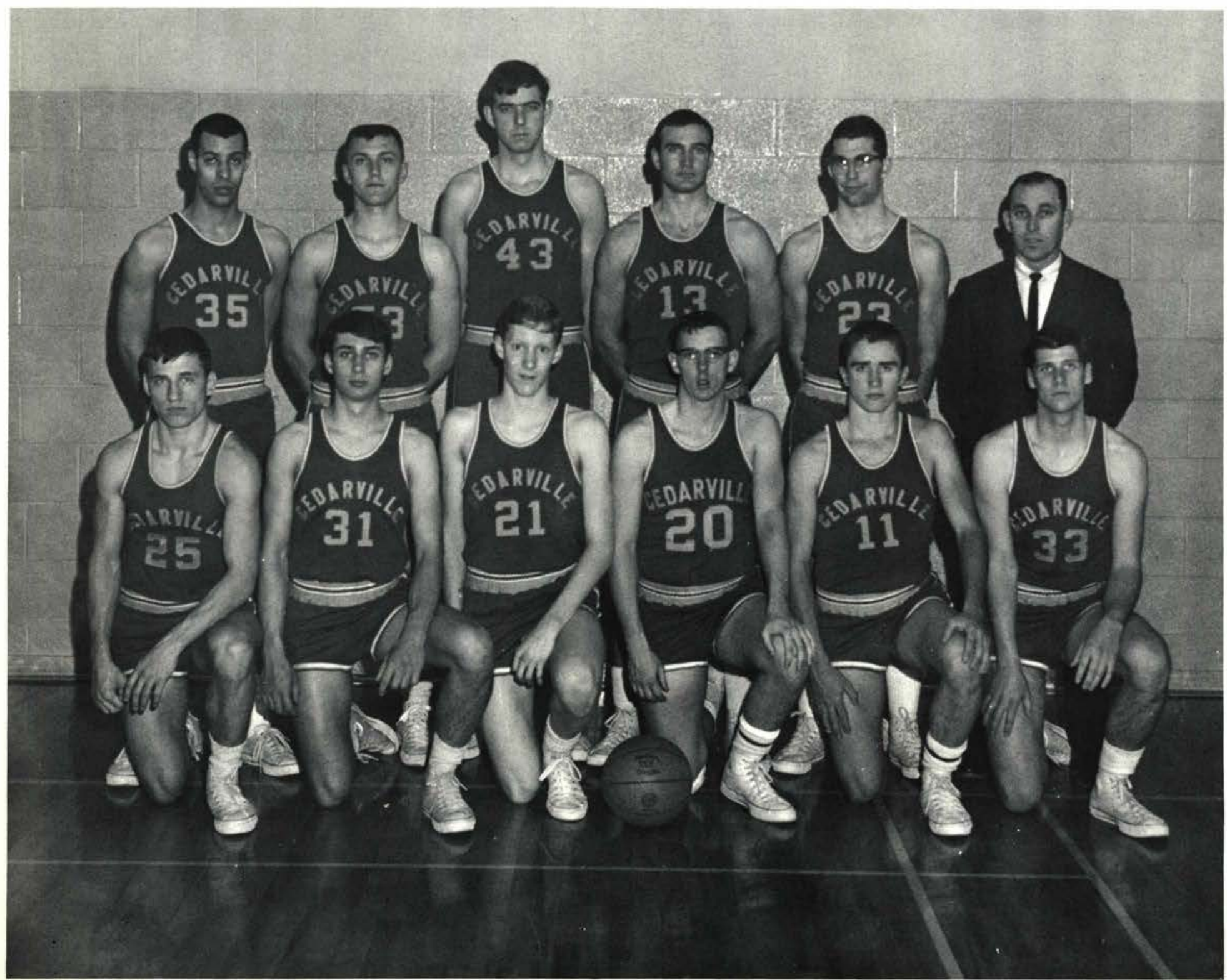



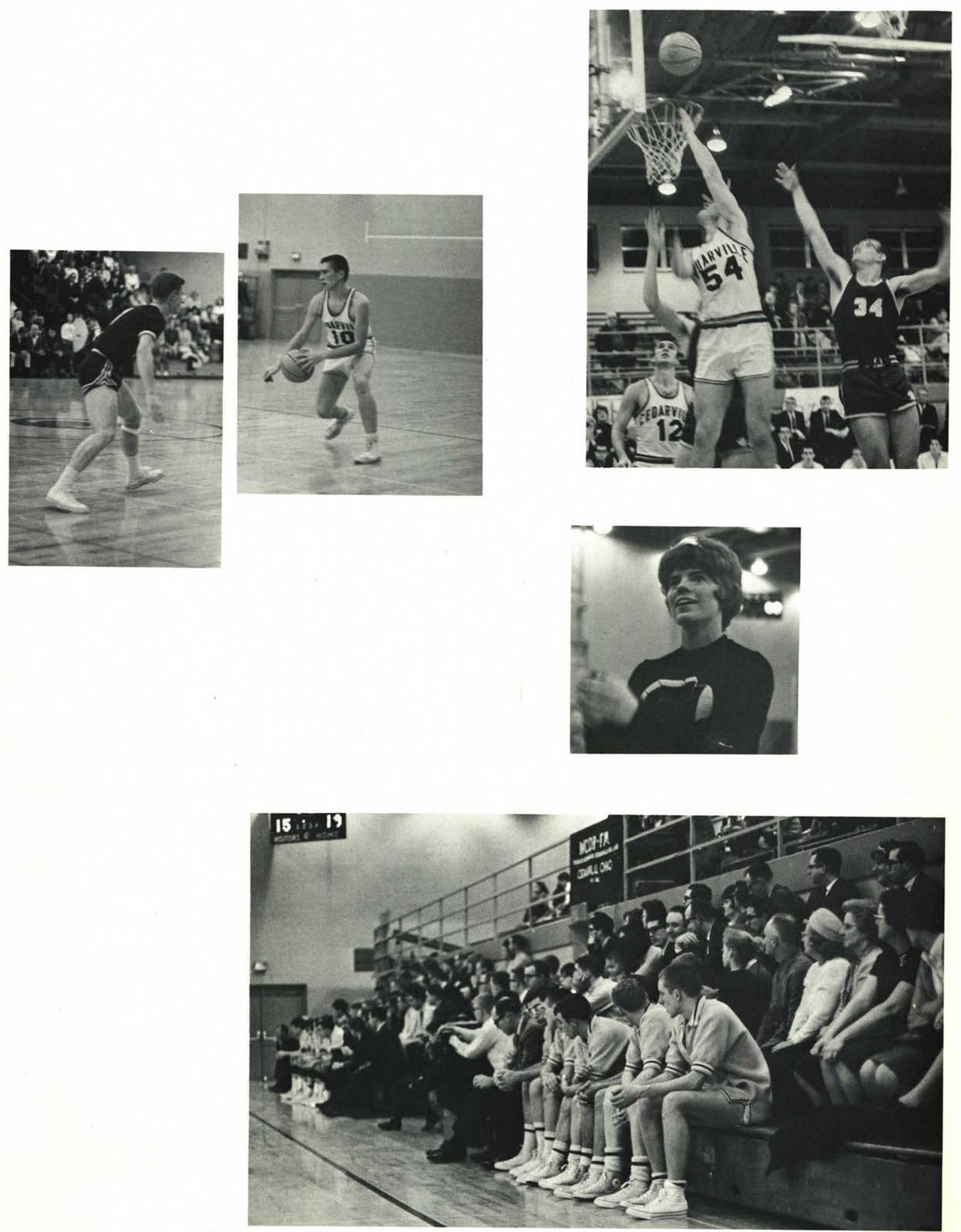

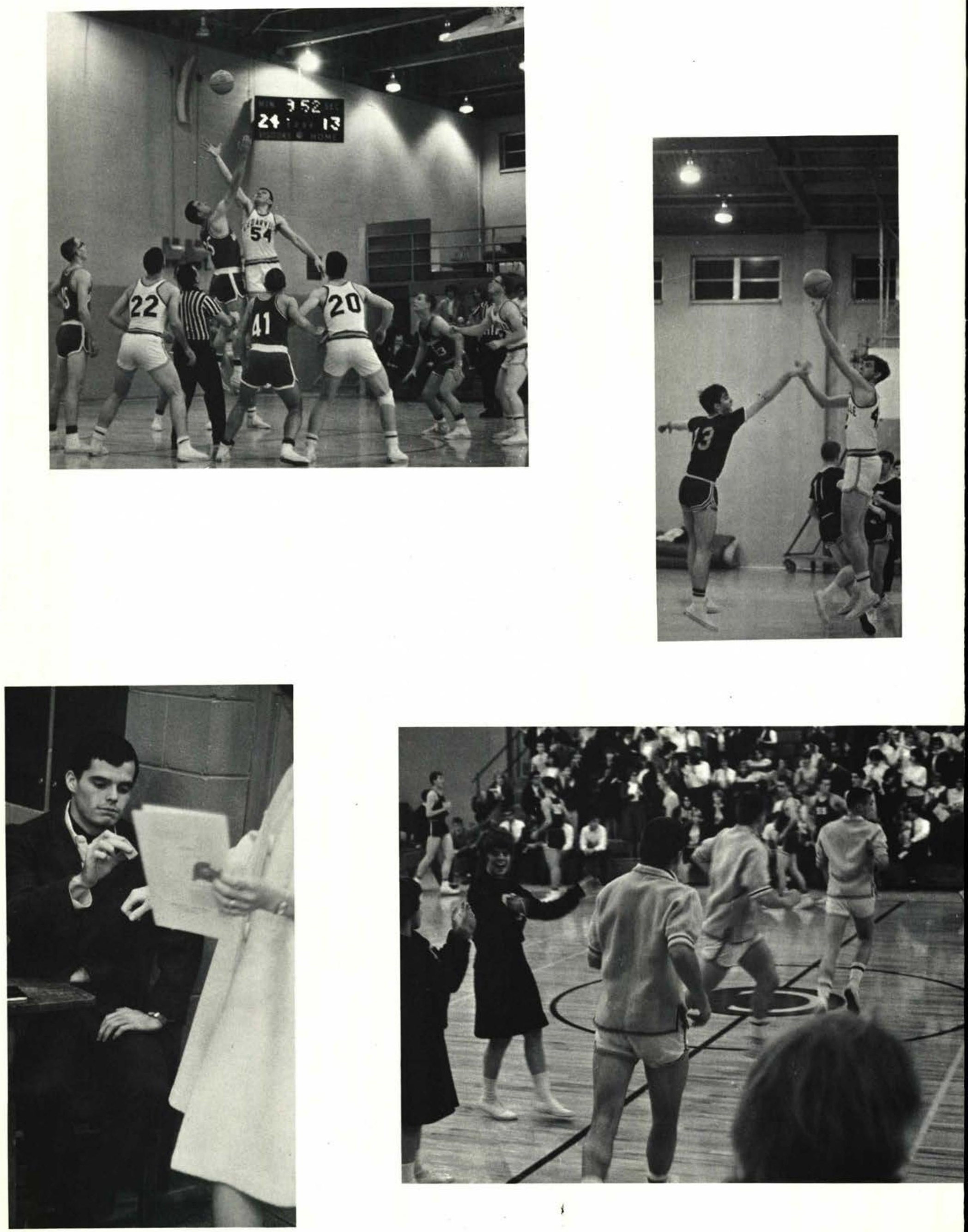

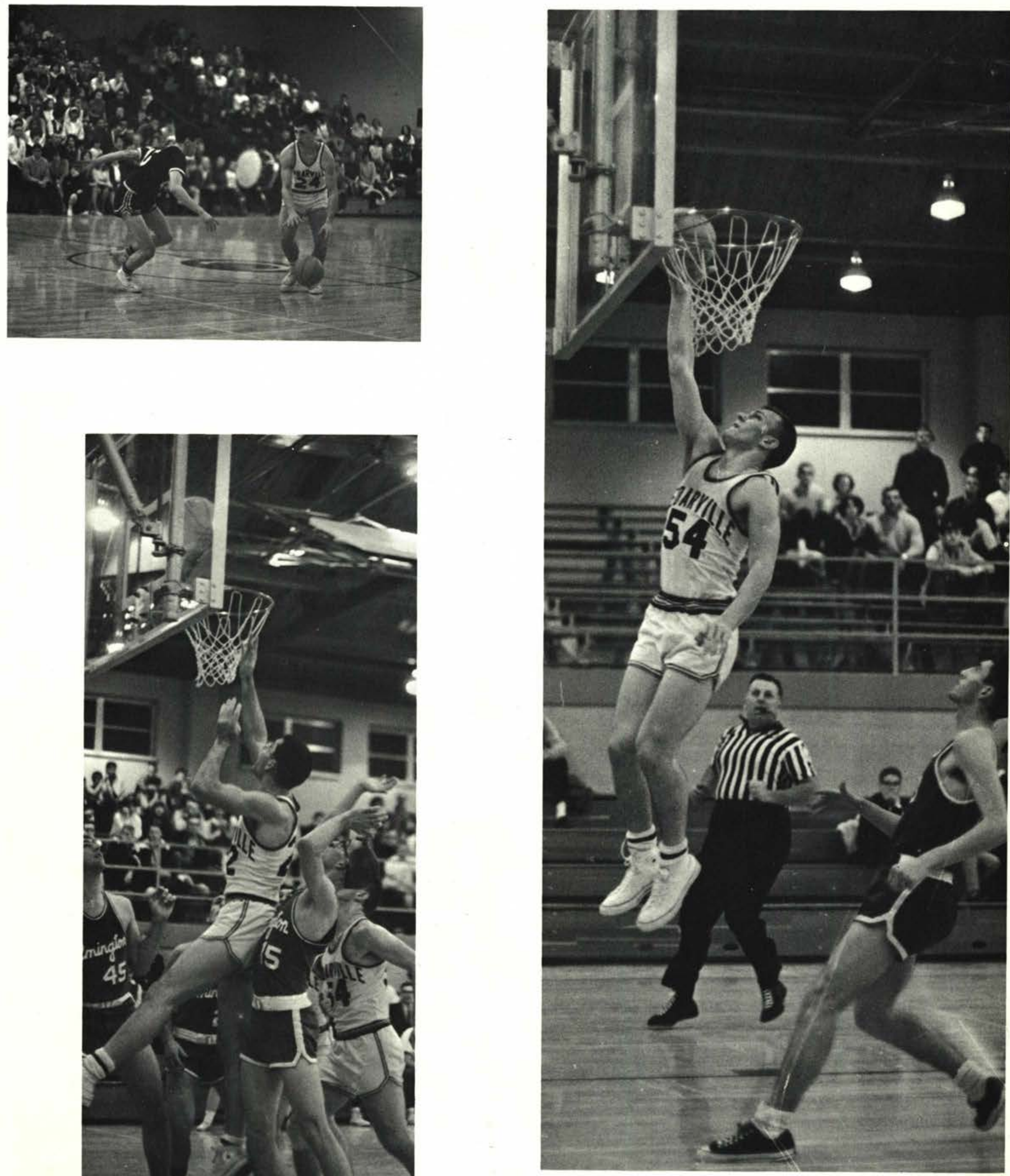

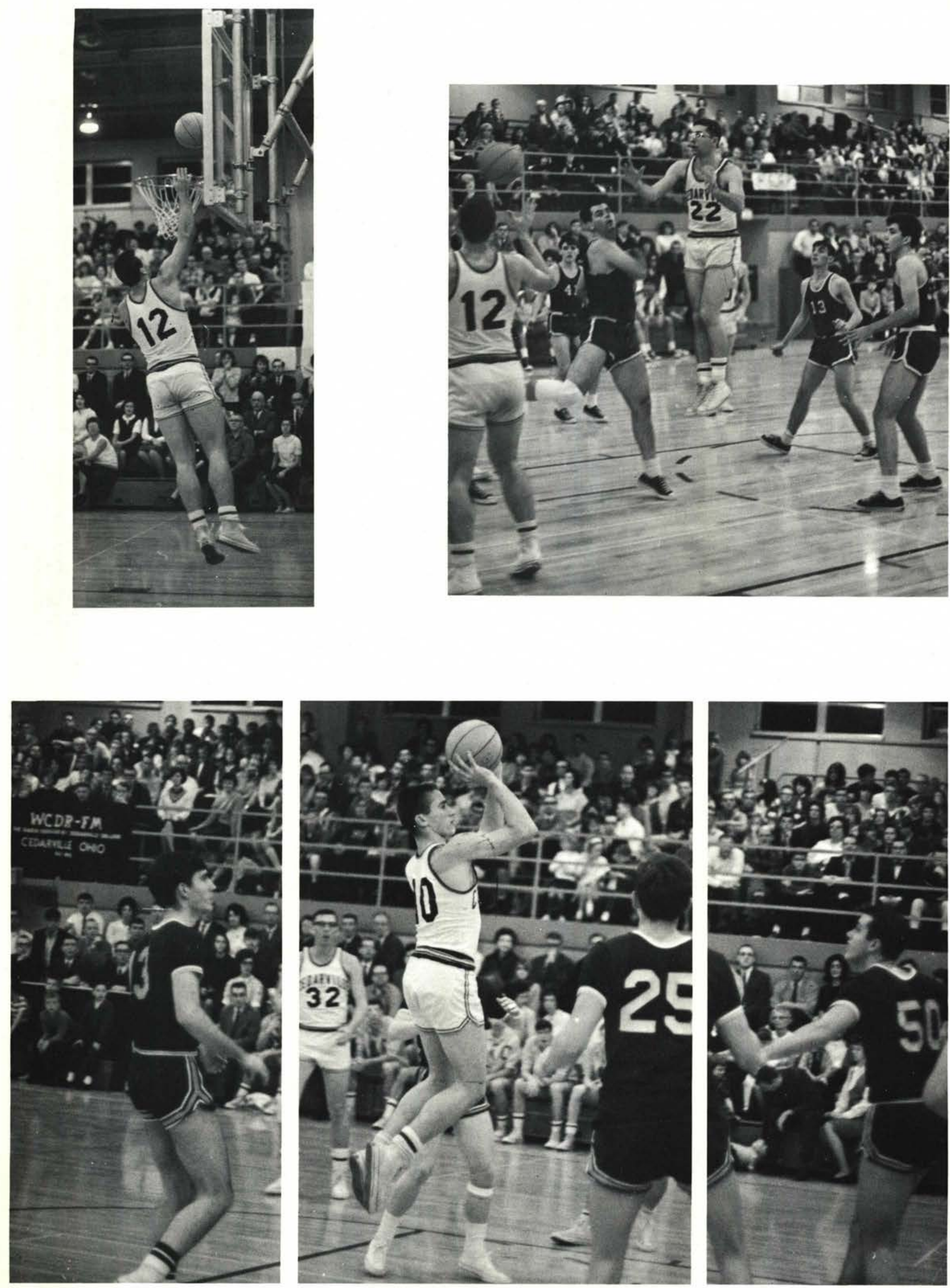

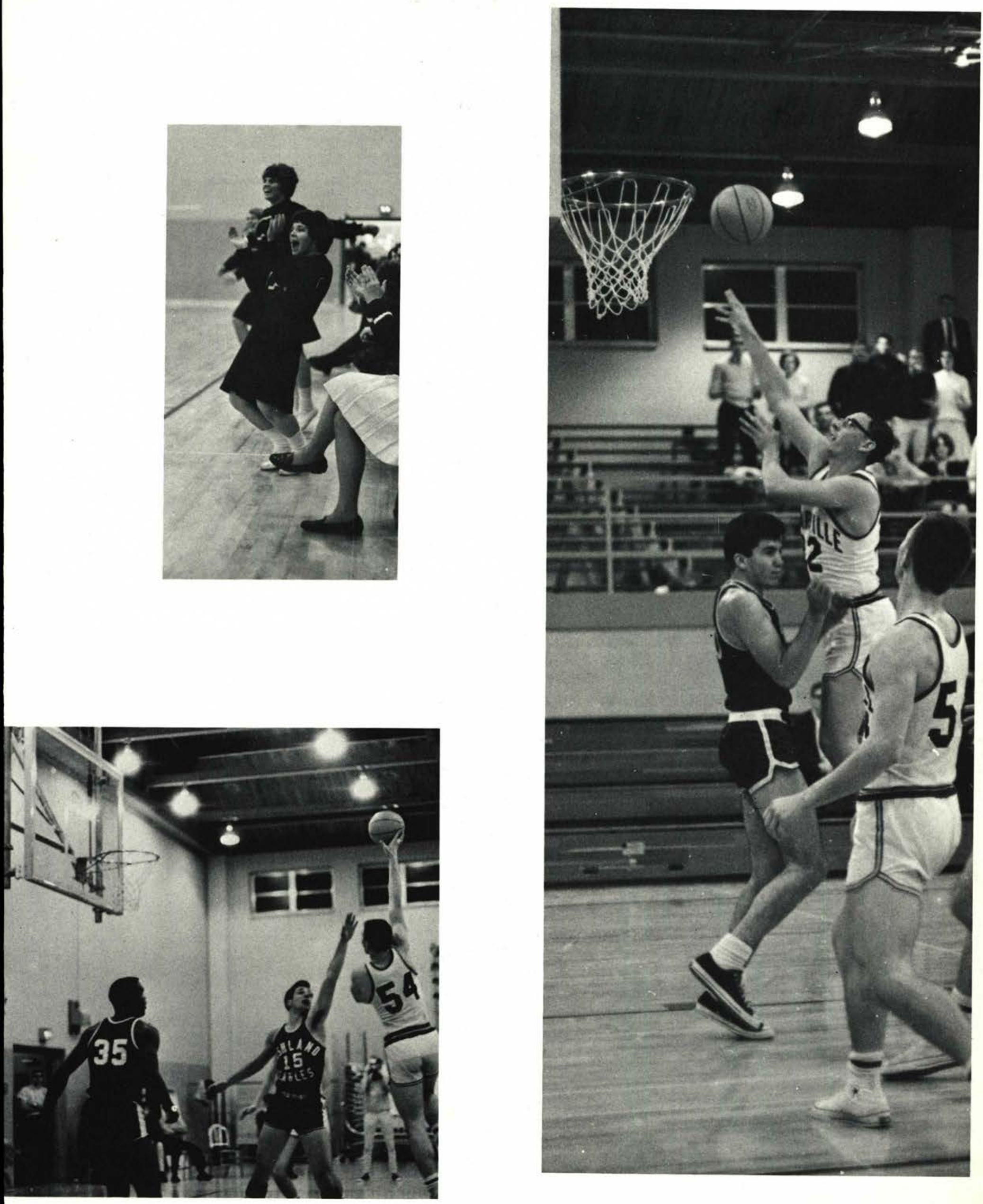


\section{Cheerleaders}

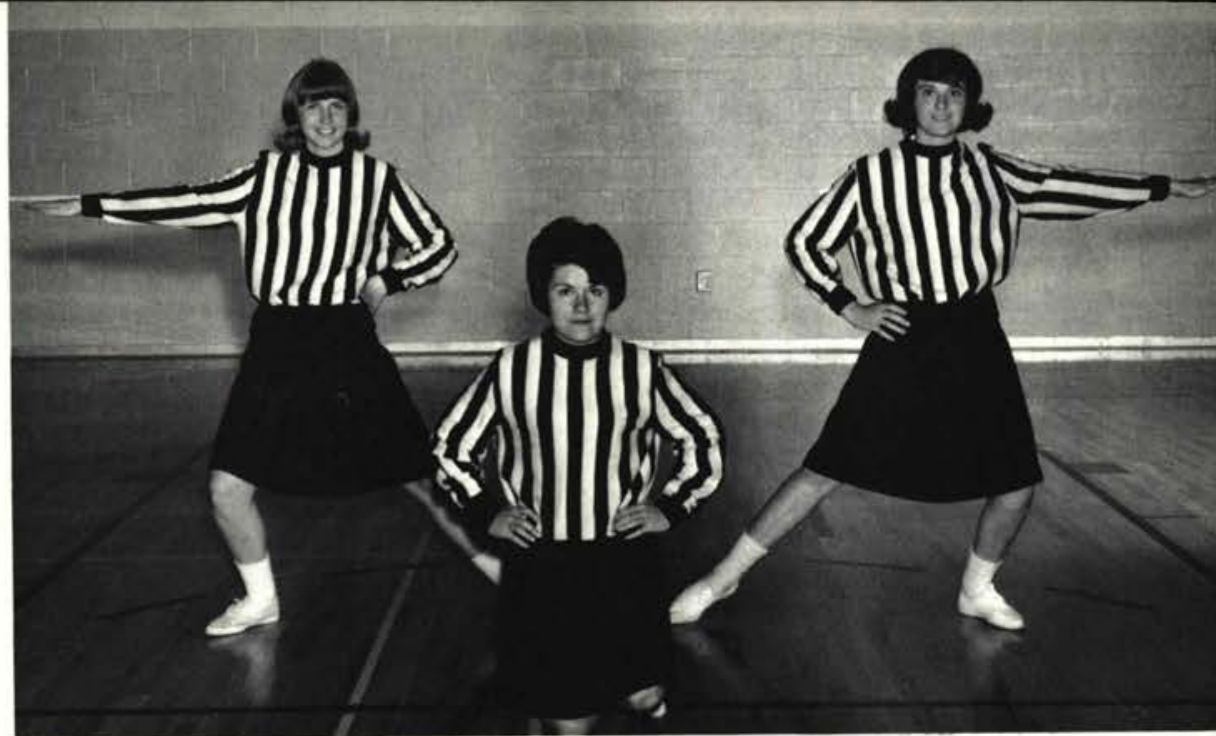

Laura Taylor, Cathy Carter, Nancy Bertram.

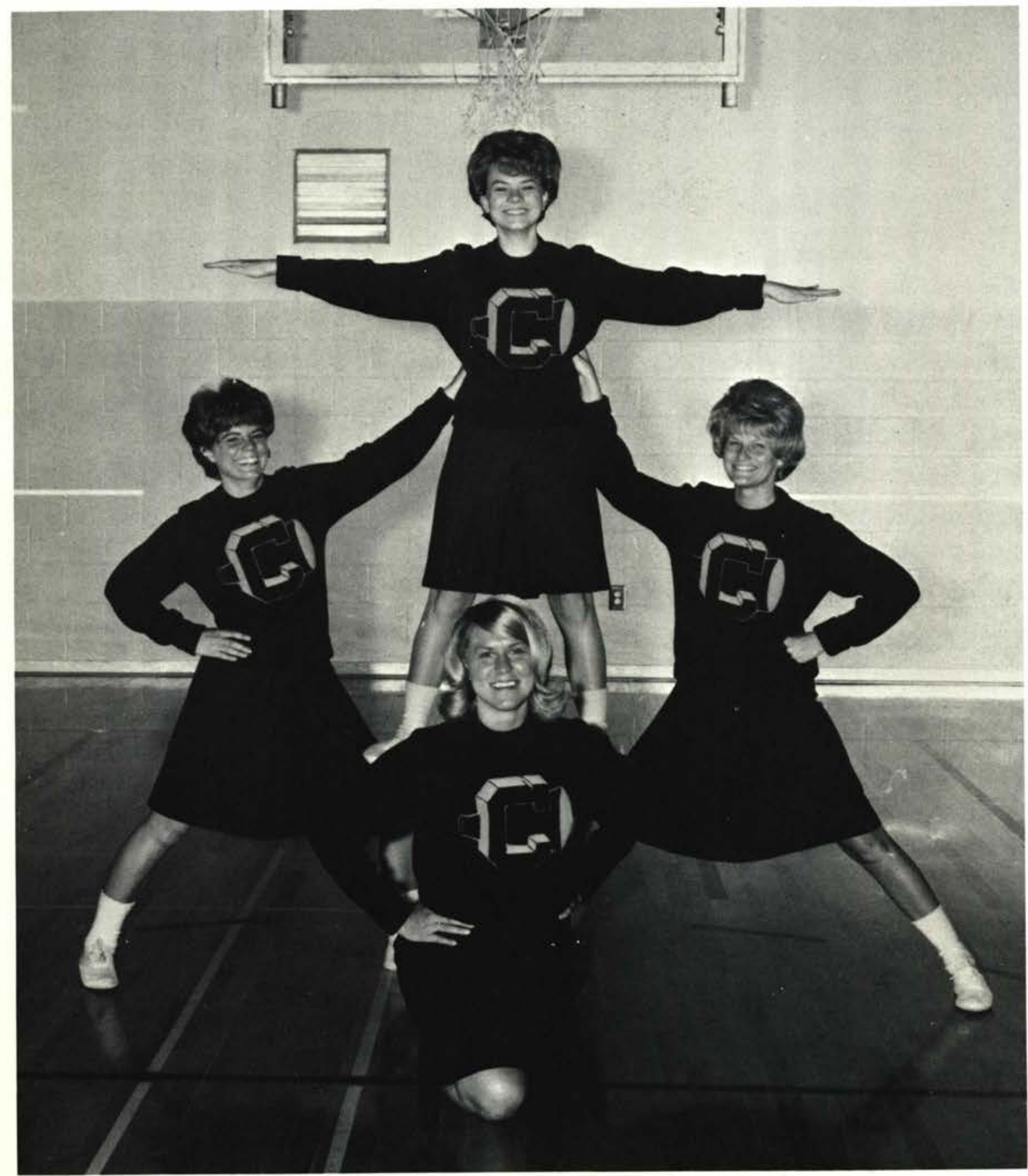

Marianne Schlecter, Annette Whaley, Eunice Johnson, Joyce Johnson, Connie Smith (not pictured). 


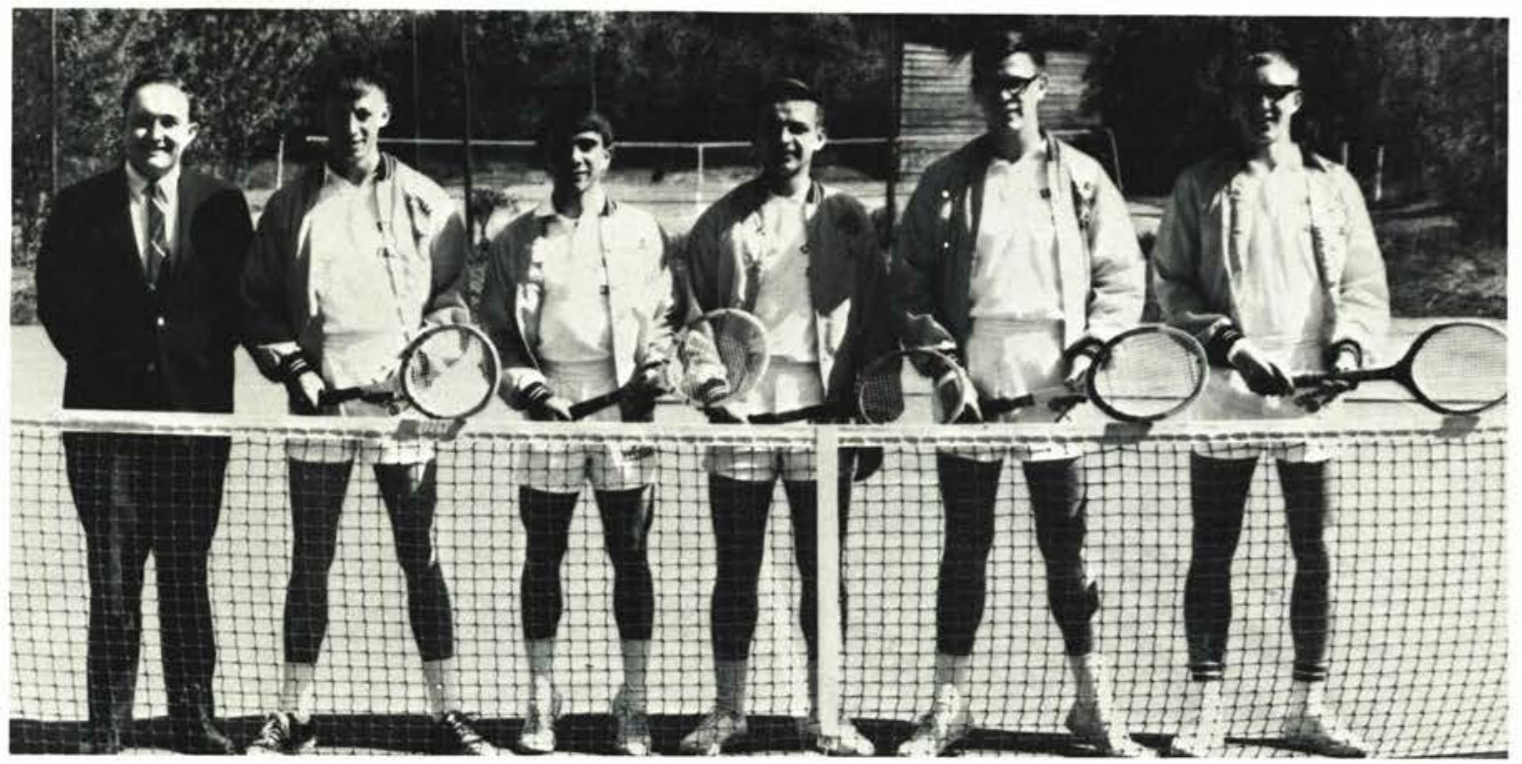

Left to Right: Coach M. Murdoch, Captain D. Petitt, J. Falci, R. Baker, T. Oakley, P. Entner.

\section{Tennis and Golf}

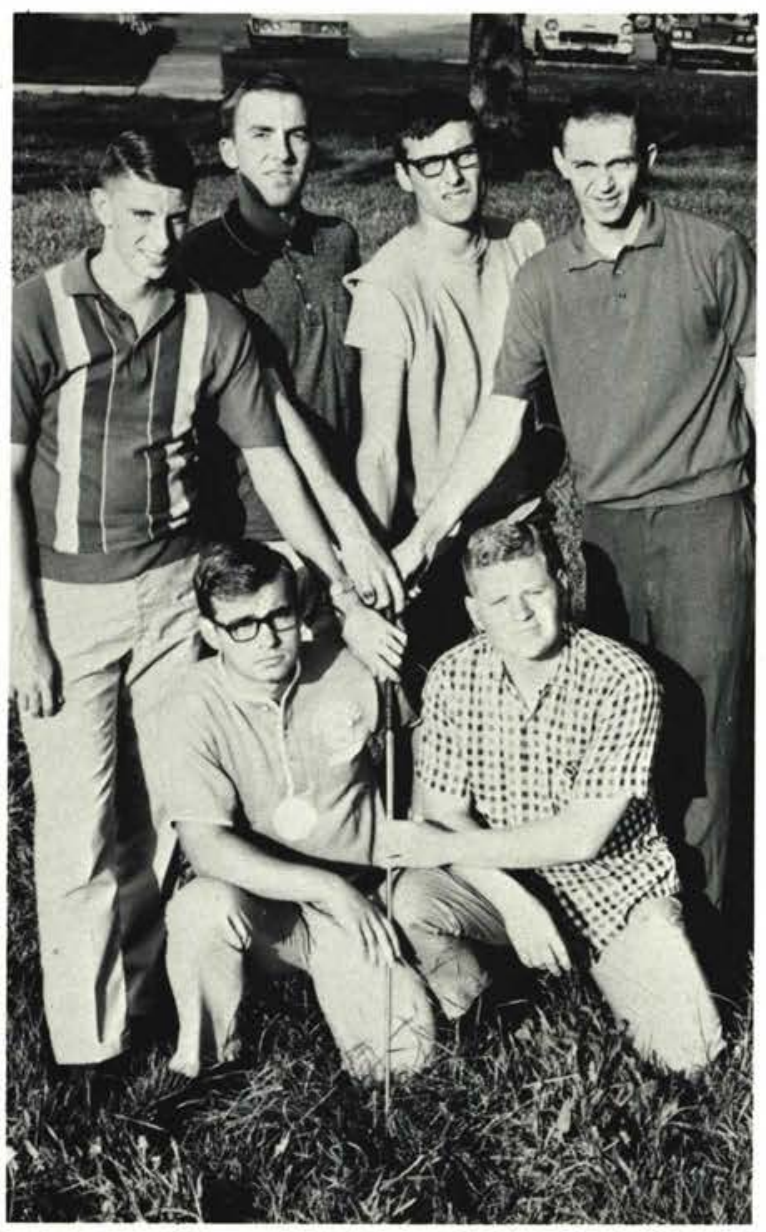

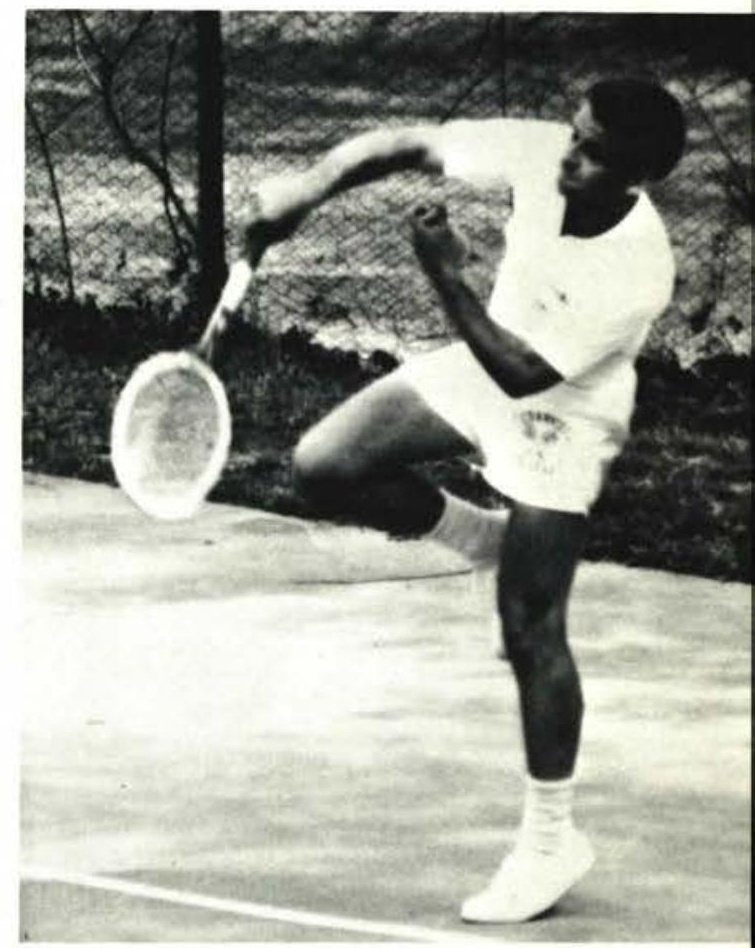

Ron Baker

Left to Right: D. Carroway, J. Berry, D. McDonald, R. Minier, B. Schultz, J. Miller. 


\section{Track}
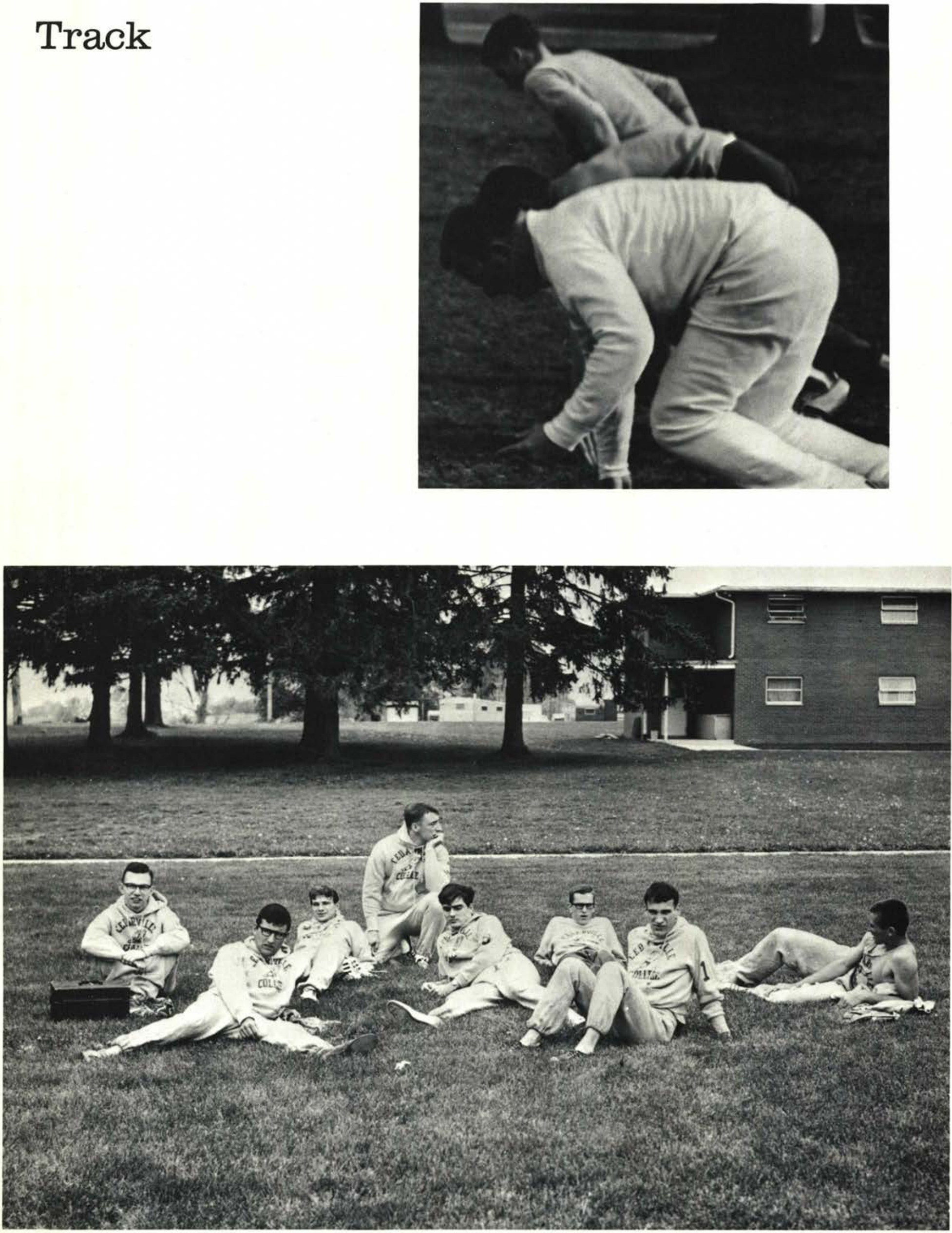

C. Biddle, D. McDonald, R. Mellish, C. Packard, T. Elliott, K. Pearce, R. Davis, D. Cooper. 


\section{Girls' Basketball}
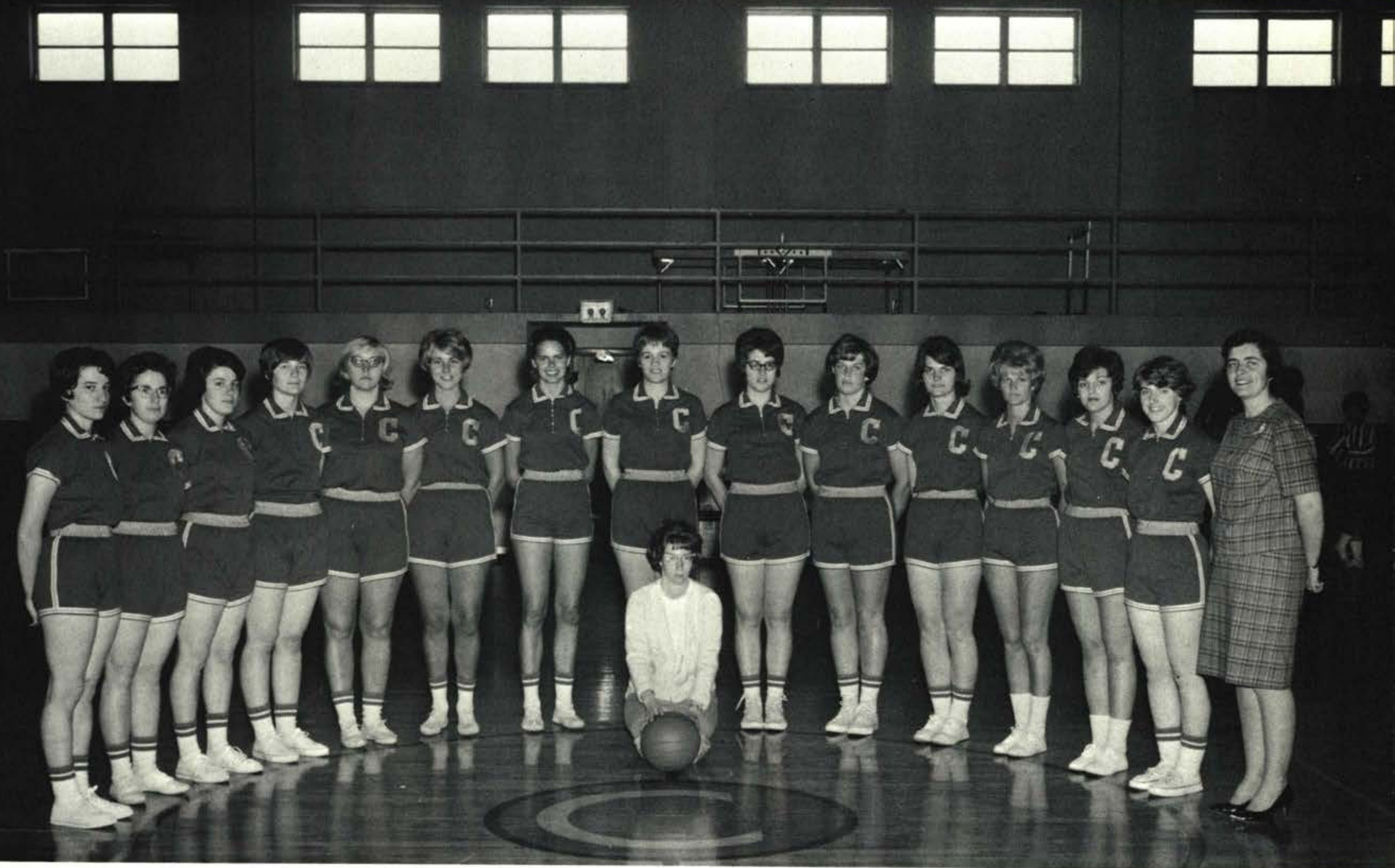

Left to Right: E. Shugg, R. Grant, K. Spencer, L. Wessel, B. Carlson, T. Wing, M. Schut, L. Walker, P. Engles, S. Wescott, D. Hannah, D. Hannah, J. Johnson,

G. Tompkins, A. Damon, Miss Kearney. 


\section{Field Hockey}
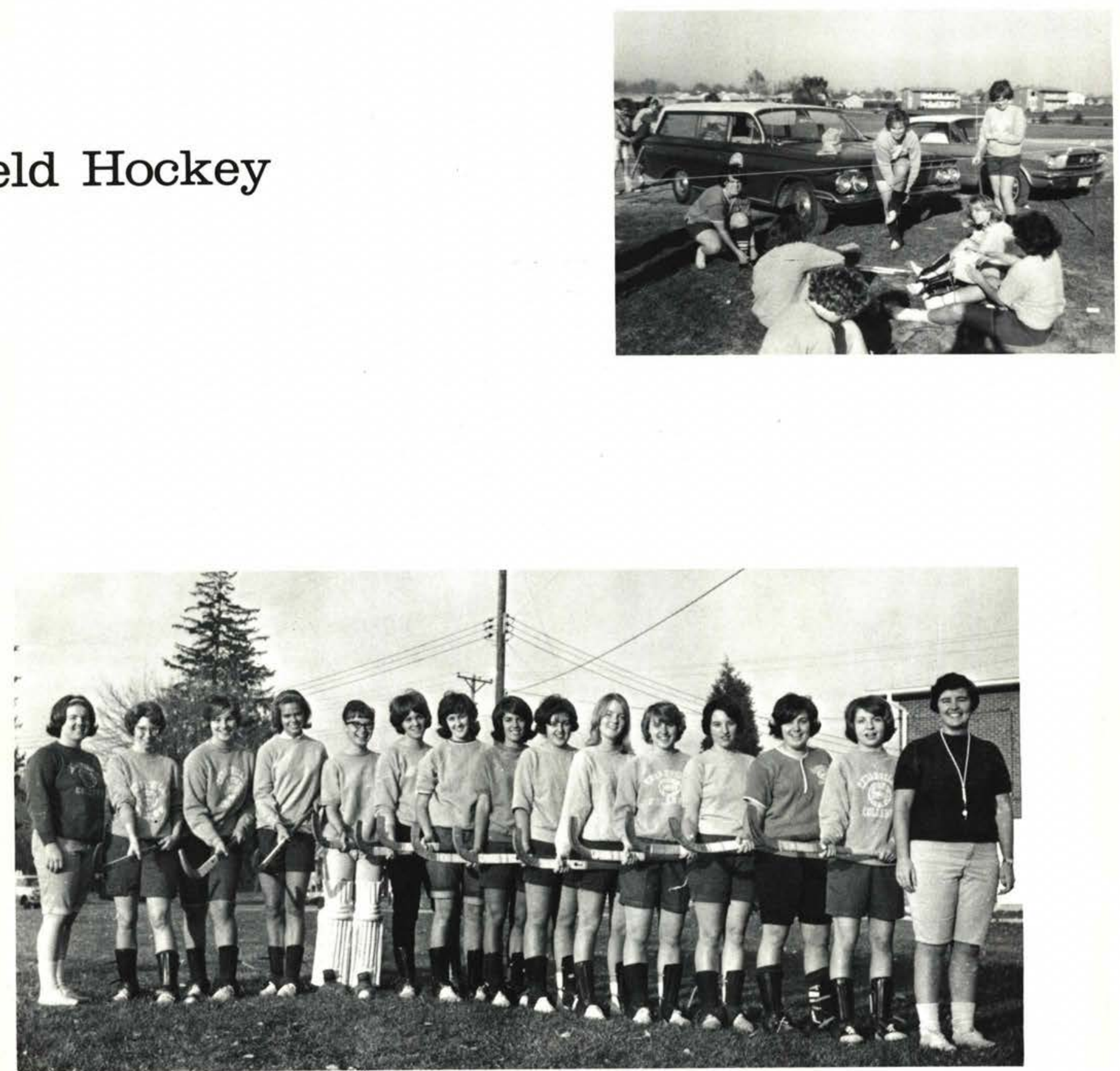

Left to Right: C. Hanson, F. Bonnema, T. Wing, M. Schut, S. Rader, L. Walker, J. DuBois, R. Rogers, G. Tompkins, L. Wilson, A. Damon, E. Shugg, K. Spencer, K. Patterson, Miss Kearney.

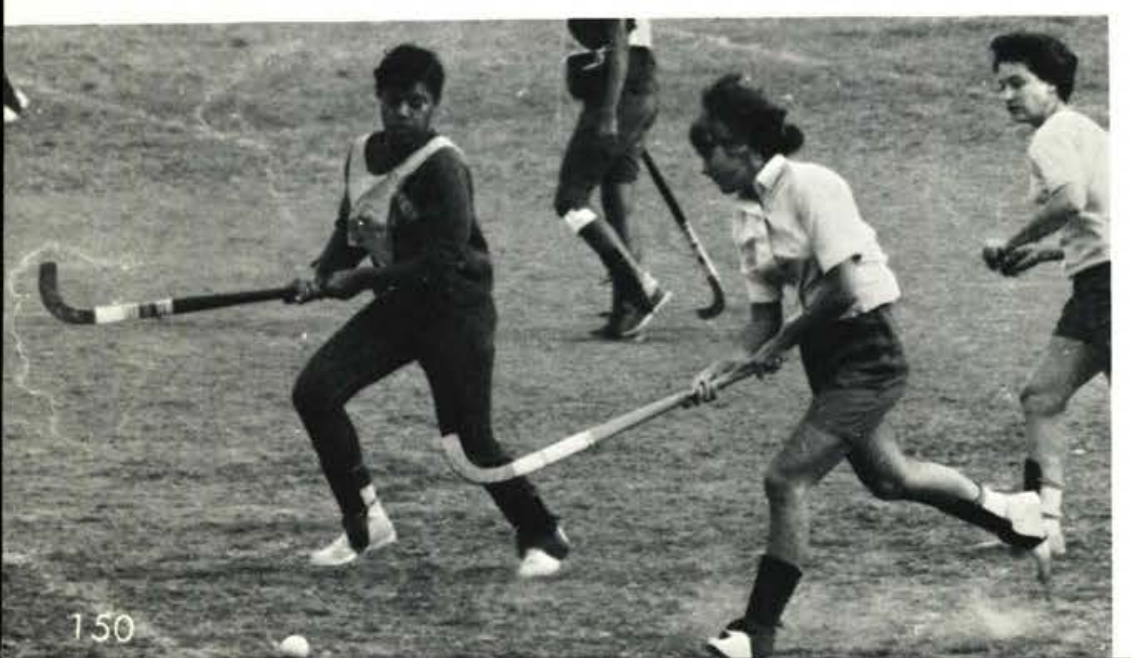




\section{Girls' Volleyball}

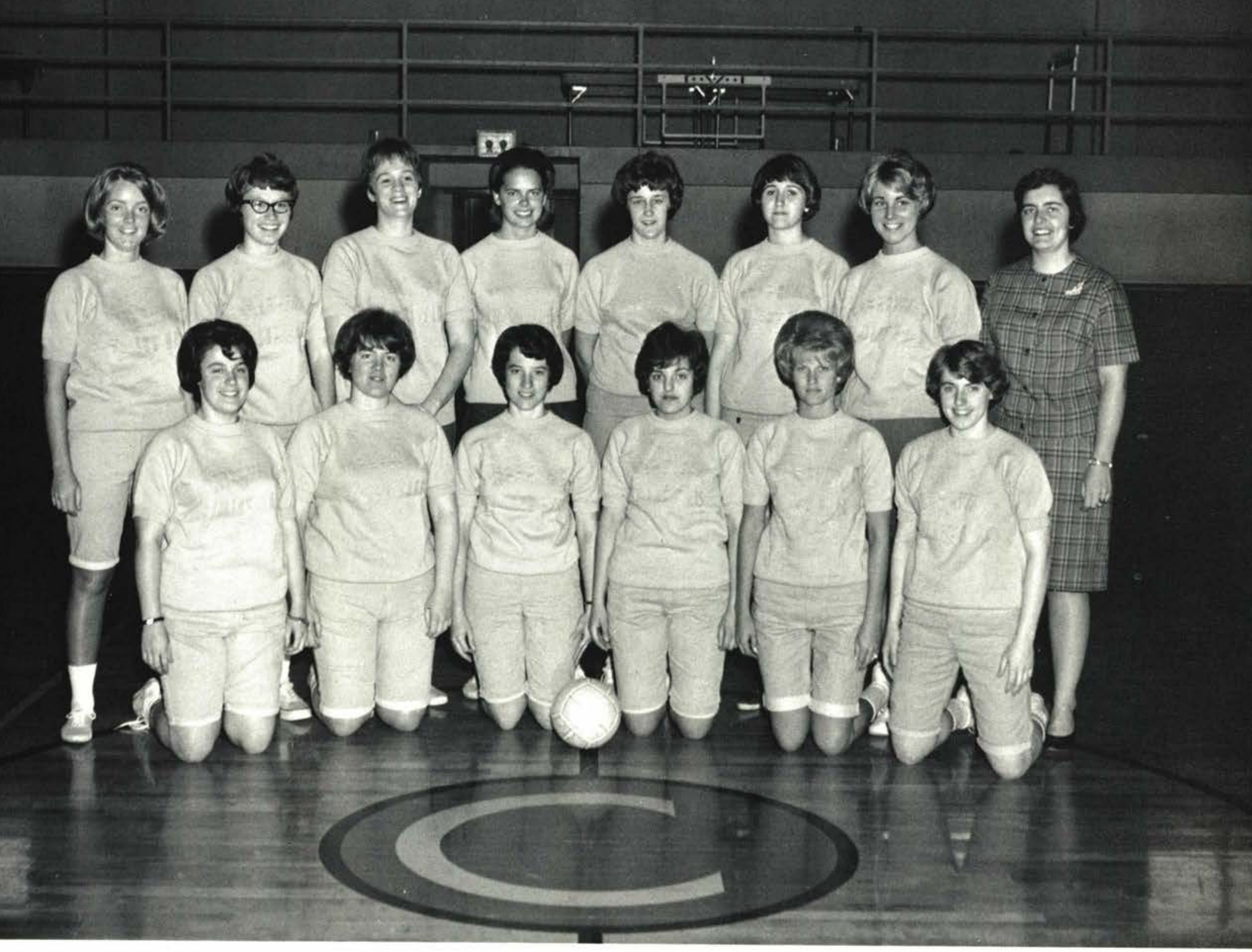

Standing: L. Wilson, S. Rader, L. Walker, M. Schut, J. DuBois, D. Hopkins, T. Wing, Miss Kearney. Kneeling: K. Spencer, C. Hanson, E. Shugg, G. Tompkins, J. Johnson, A. Damon. 


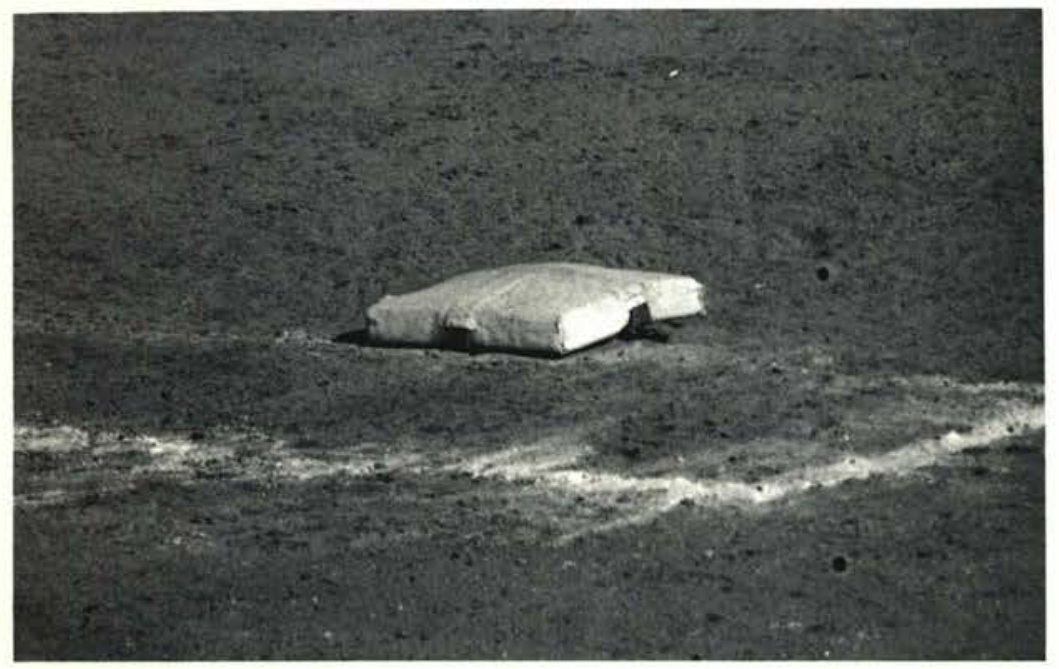

\section{Baseball}

Under the direction of Coach Dennis Olson, baseball led the way with an outstanding season. The team finished the season with a 13-5 record. Eight of the wins went to Charlie Wilkins who was named to the N.A.I.A. All-American N.A.I.A. 22 team. He posted a 8-1 record while leading the nation in strike-outs with a 15.5 average per nine inning game. He was also ranked fifth nationally with a 1.09 earned run average. Charlie also received the Most Valuable Player award.

Other athletes receiving national, district, or conference mention are the following: Roger McKinney, Honorable Mention AllAmerican in baseball; in All-District N.A.I.A., G. Shufelt, J. Brannon, and C. Wilkins; All-Conference N.A.I.A., C. Wilkins, V. Ashley, B. McDonald, G. Shufelt, R. McKinney, and Al Knott.

The team batted an average of 300 .

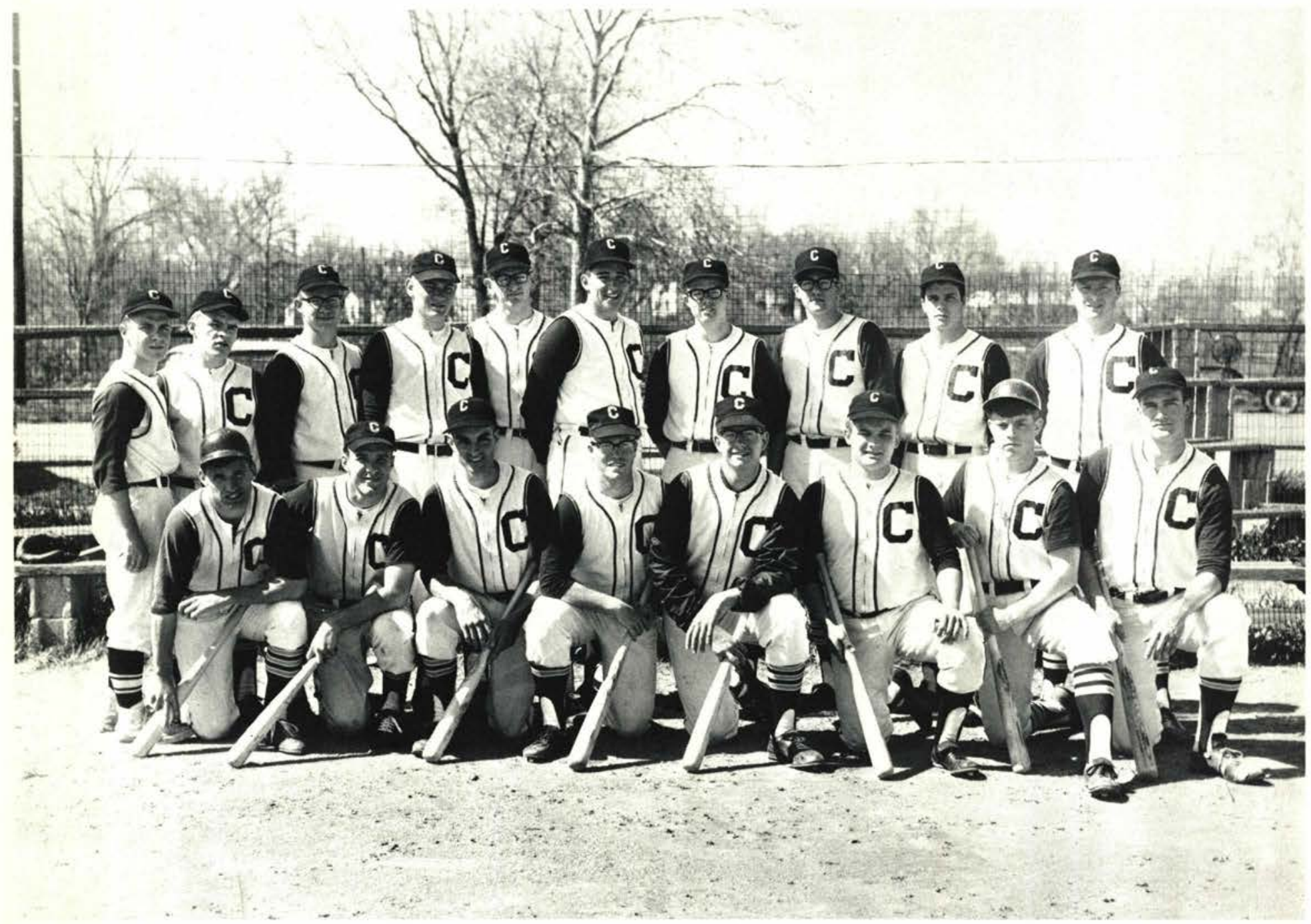

Standing: J. Phipps, G. Herrin, M. Kintz, C. Reed, V. Ashley, M. Woodend, F. Hall, D. Holland, R. Ross, Mr. Olsen. Kneeling: B. McDonald, R. McKinney, J. Brannon, G. Shufelt, C. Wilkins, T. Duff, P. Johnson, A. Knott. 

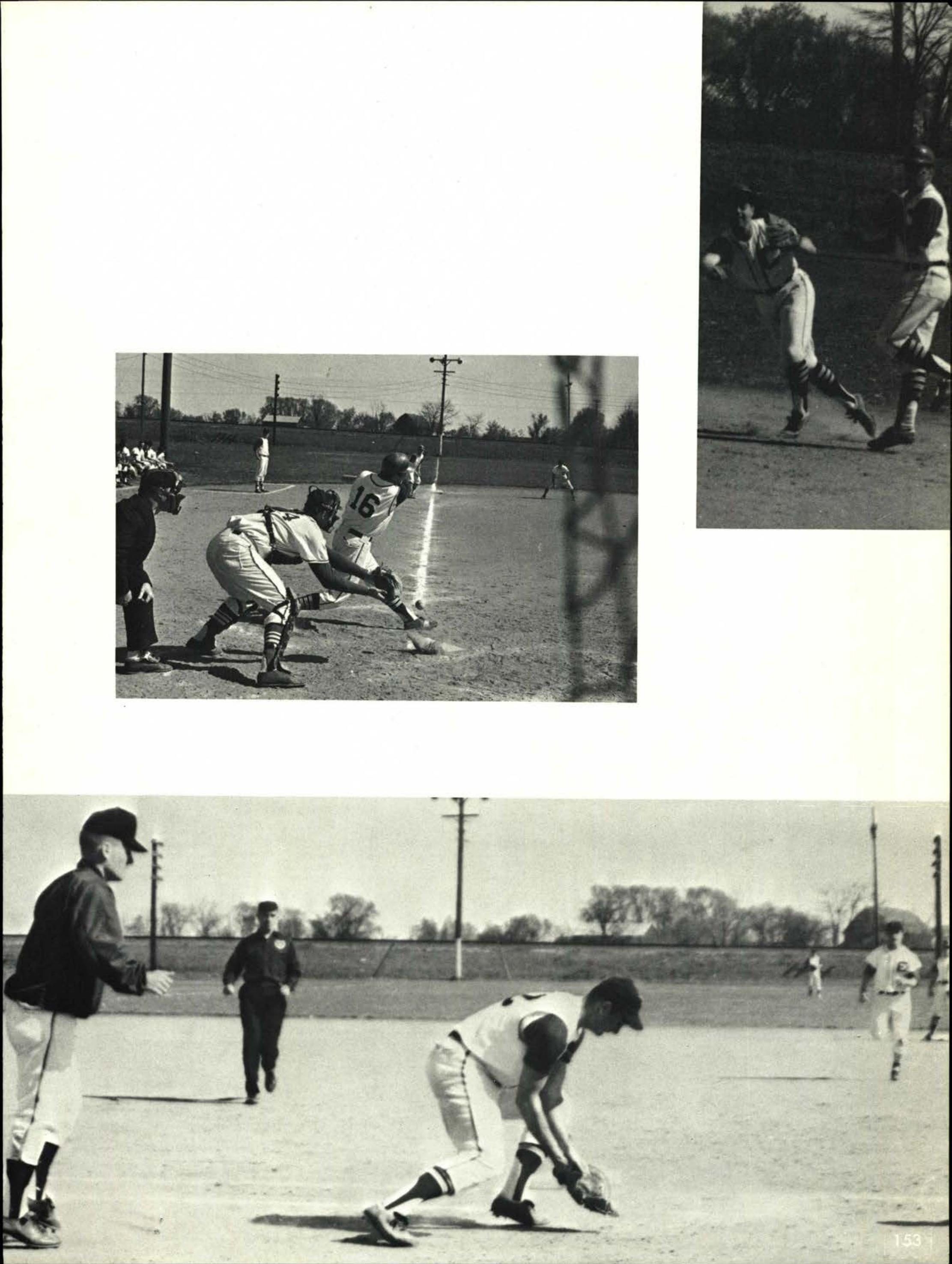

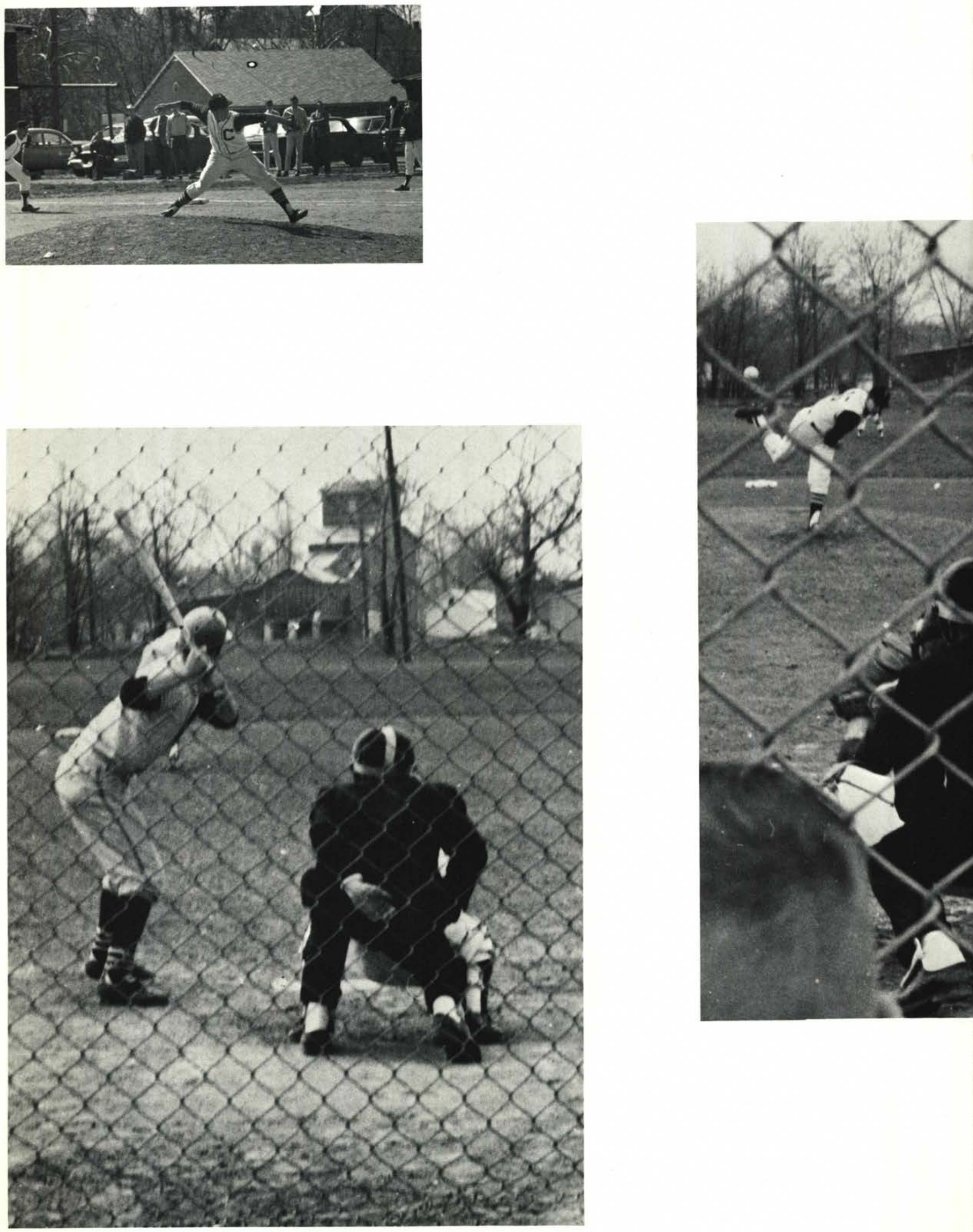

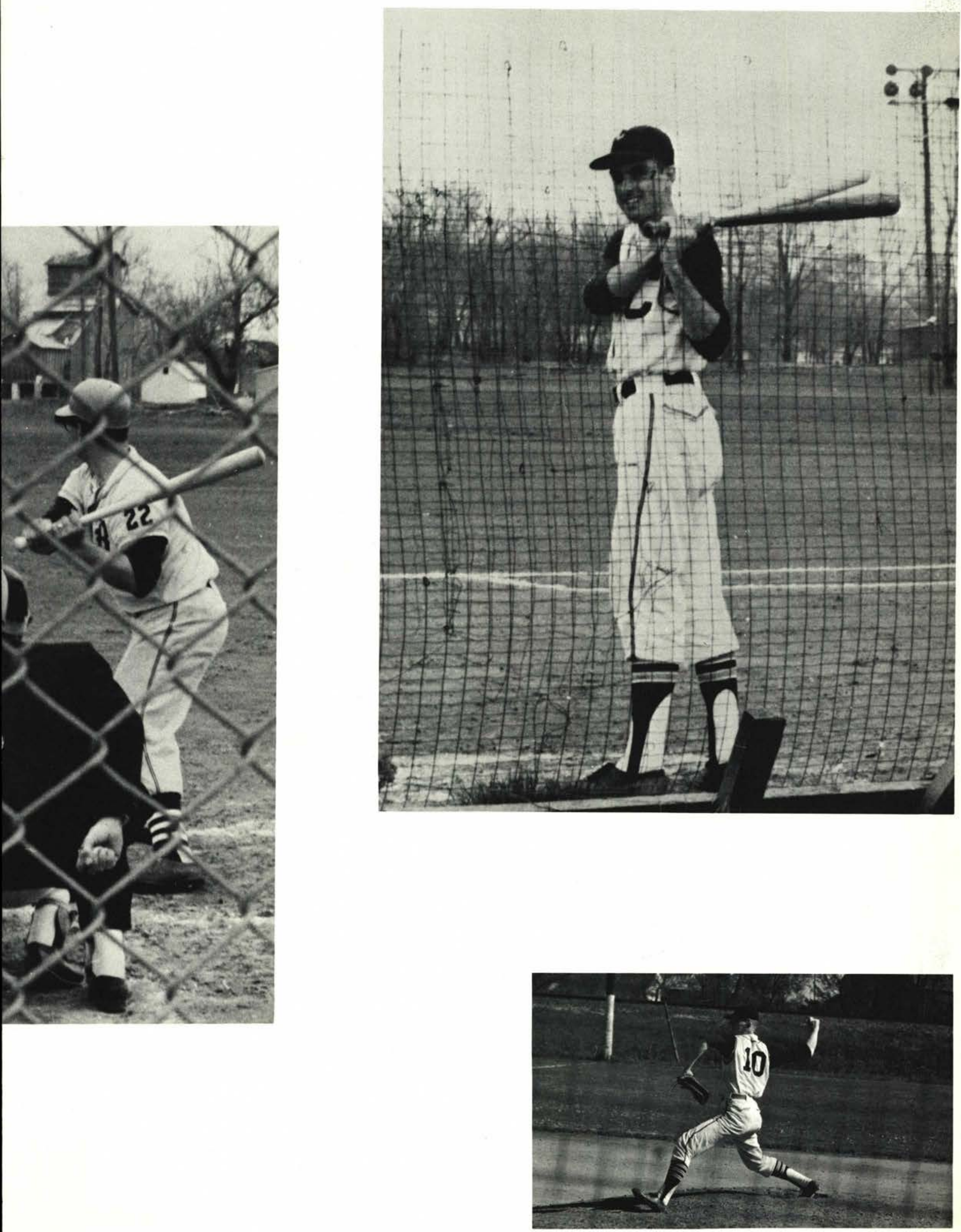


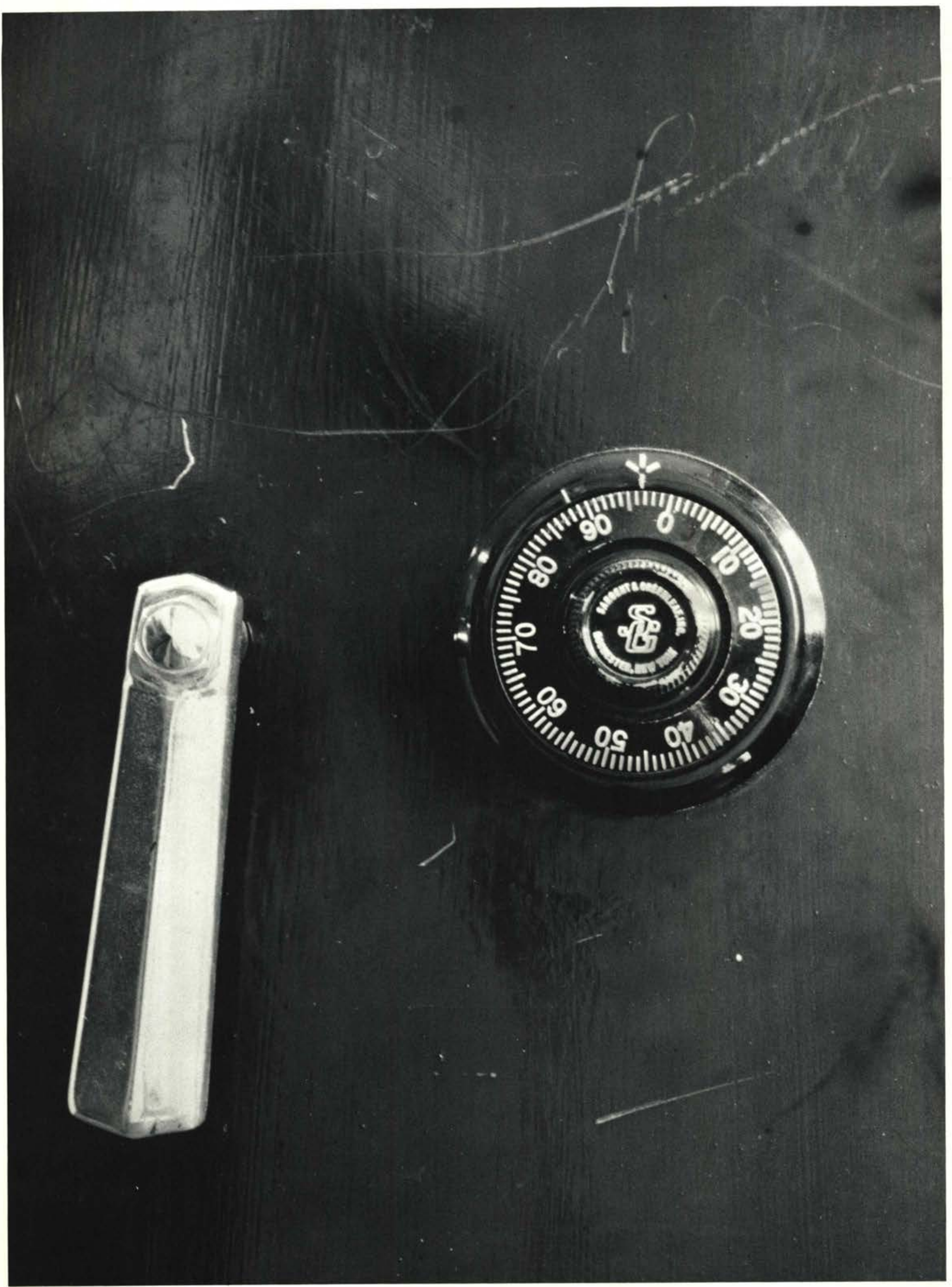




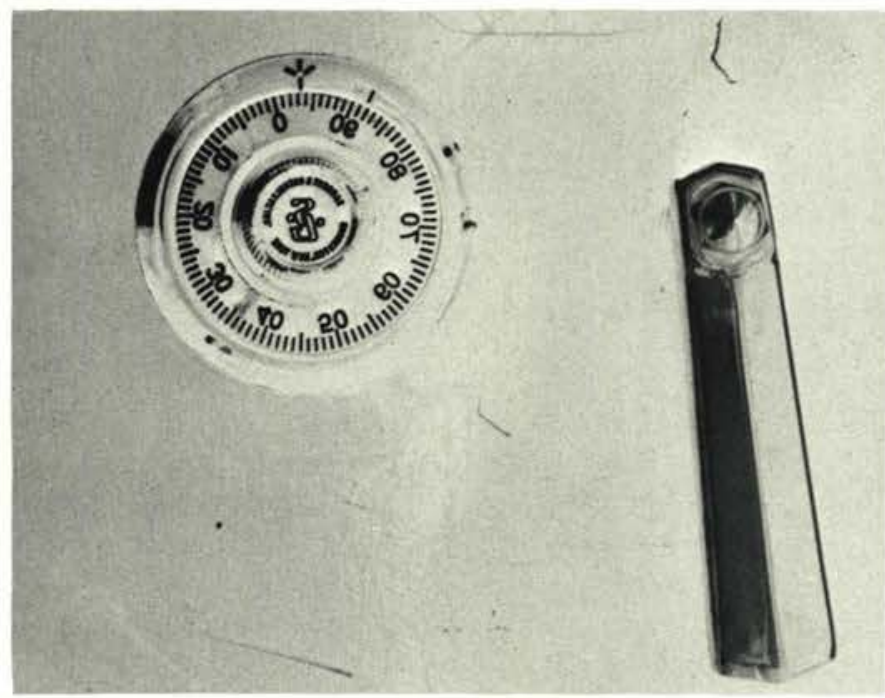

Seniors 

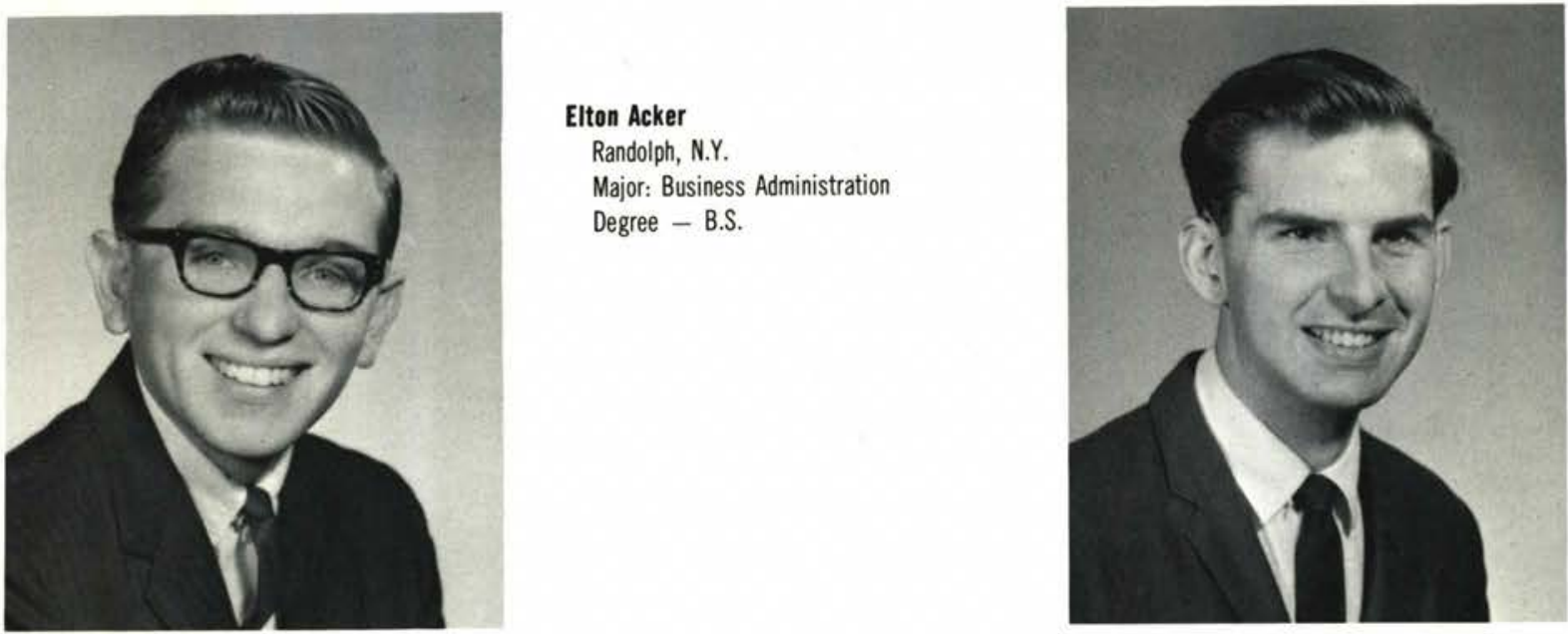

Richard Amos

\section{Elton Acker}

Randolph, N.Y.

Major: Business Administration

Degree - B.S.

Major: Elementary Education Degree - B.S.

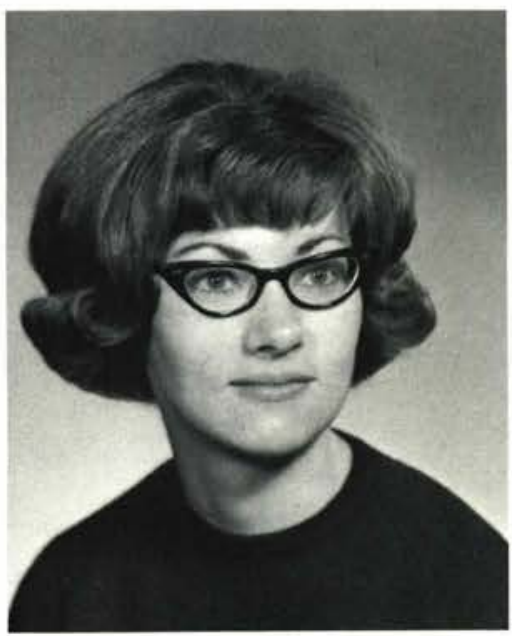

\section{Grace Averitt}

Cedarville, Ohio

Major: Church Music

Degree - B.M.

Mona Ballard

Belleville, Mich.

Major: Speech

Degree - B.A.

Stephen Benedict

Boise, lowa

Major: General Science

Degree - B.S.
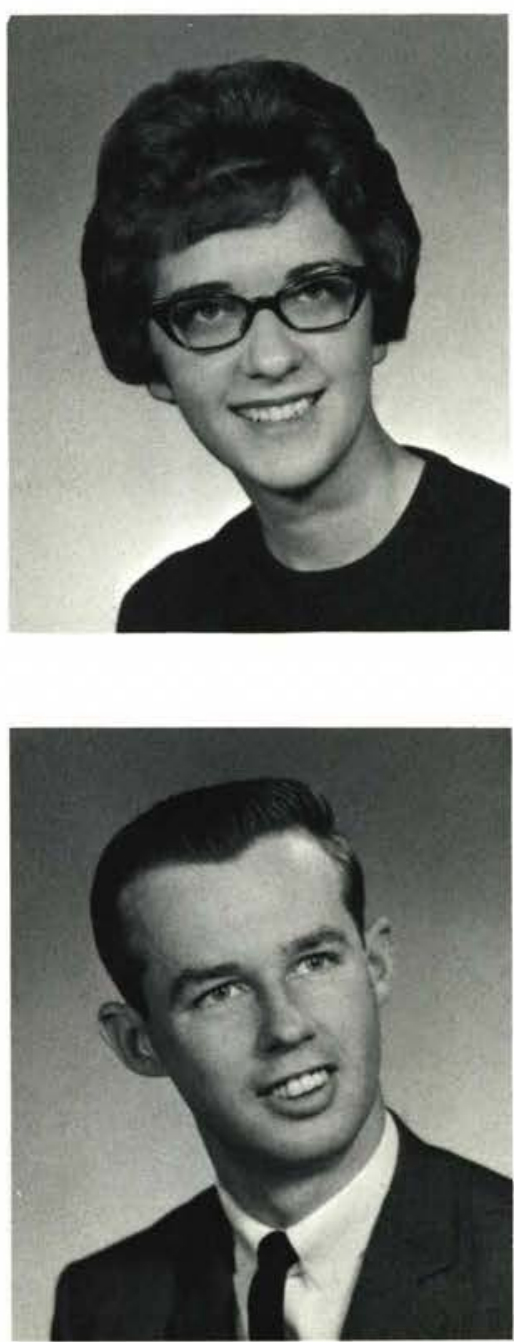

\section{Betty Beikert}

Butler, Penna.

Major: Elementary Education

Degree - B.S.

\section{Marvin Bergsma}

Grand Rapids, Mich.

Major: History \& Government Degree - B.A. 
Patricia Bird

Saline, Mich.

Major: Christian Education

Degree - B.A.
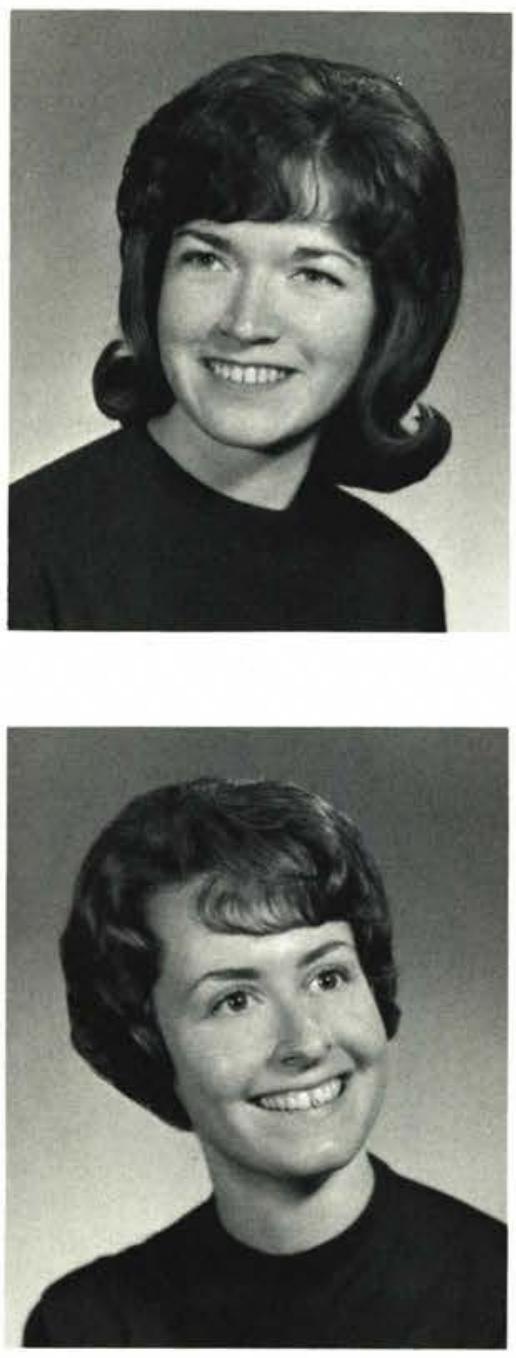

th Ann Boonstr

Garfield, N.J.

Major: Biology Comprehensive

Degree - B.S.

\section{Donald Brake}

Colchester, III.

Major: Pre-Seminary Bible

Degree - B.A.

\section{Dorinda Bush}

Cuyahoga Falls, Ohio

Major: Elementary Education

Degree - B.A.
Martha Bennett

Cleveland, Ohio

Major: English; Degree, B.A.
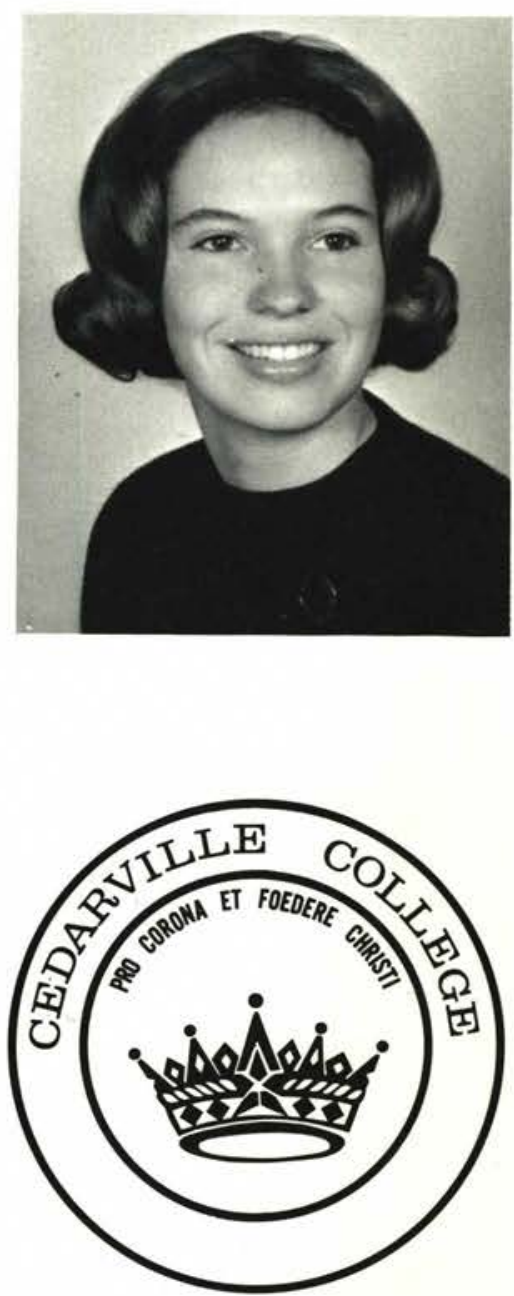

\section{Marian Buckle}

Lockhaven, Penna.

Major: Social Studies

Degree - B.S.
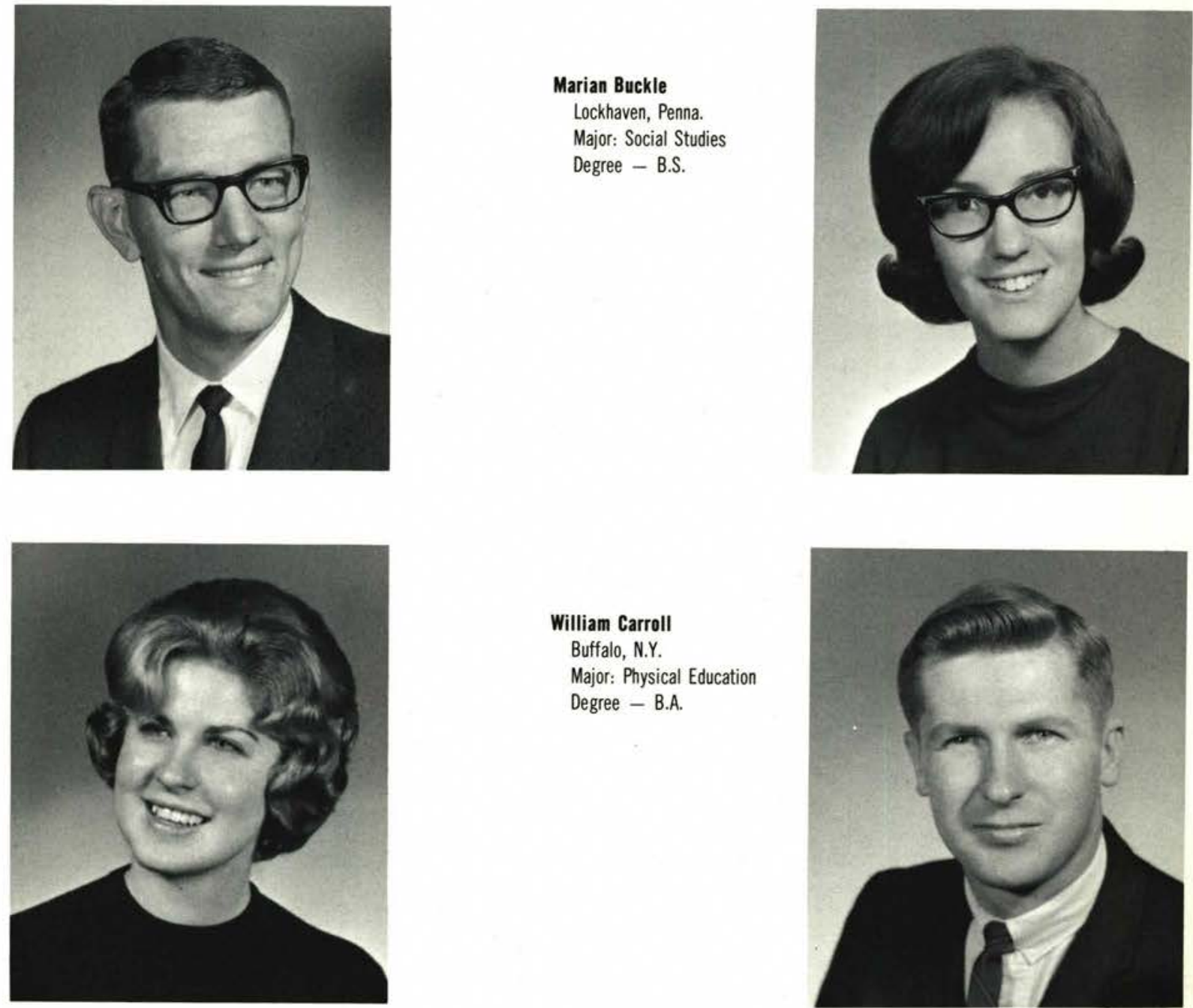

Buffalo, N.Y.

Major: Physical Education

Degree - B.A. 

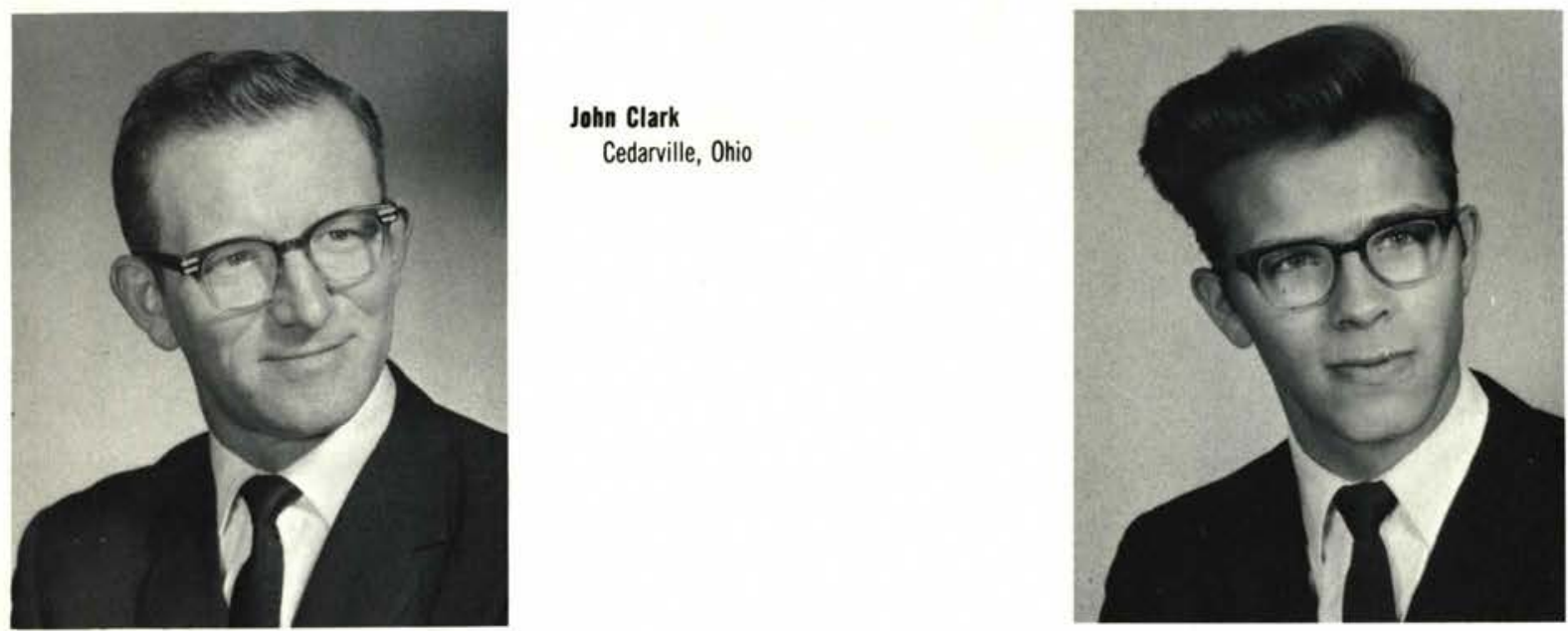

Robert Clater

John Clark

Cedarville, Ohio

Major: Psycholog

Degree - B.A.

Ronald Eugene Culley

Kalamazoo, Mich.

Major: Biology

Degree - B.S

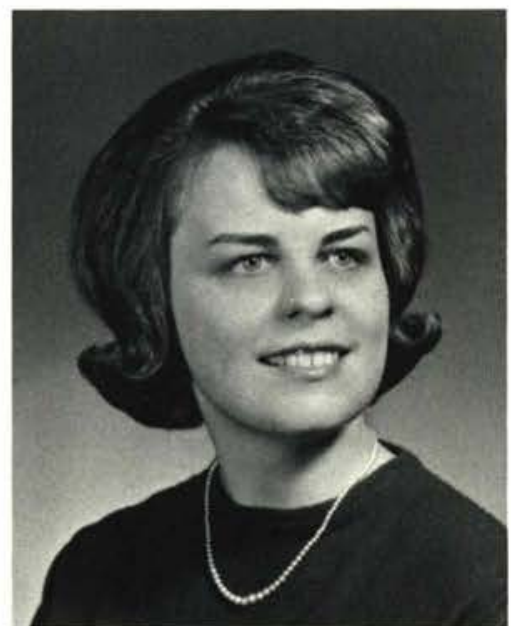

\section{Pamela Earl}

Hasbrouck Heights, N.J.

Major: Elementary Education

Degree - B.S.

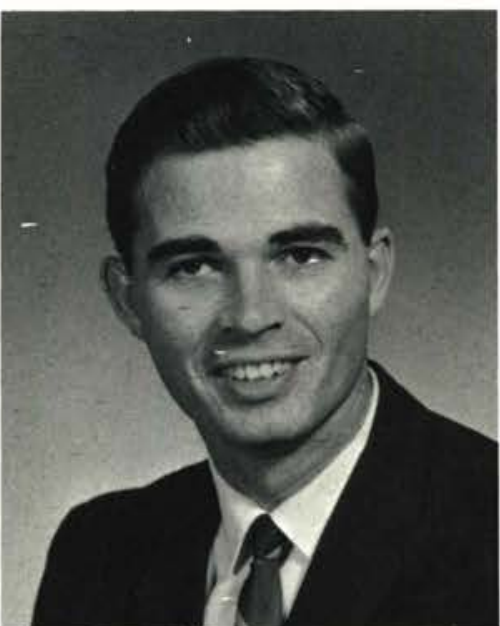

David Elmore

Cedarville, Ohio

Major: Bible Comprehensive Degree - B.A.

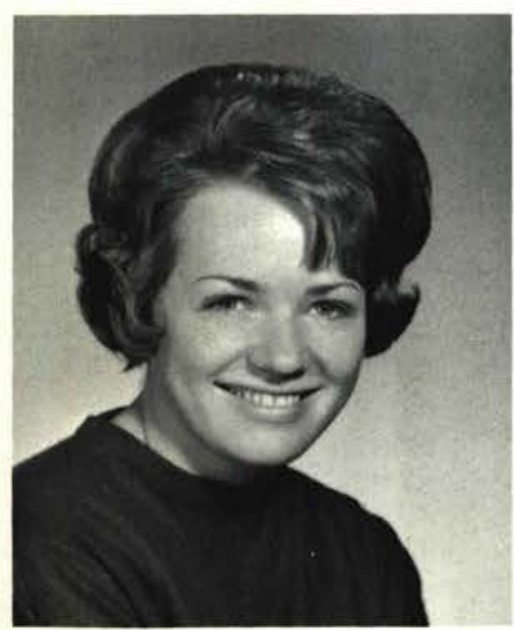

\section{Janice Emerson}

Brooklyn, Mich.

Major: Christian Education

Degree - B.S.

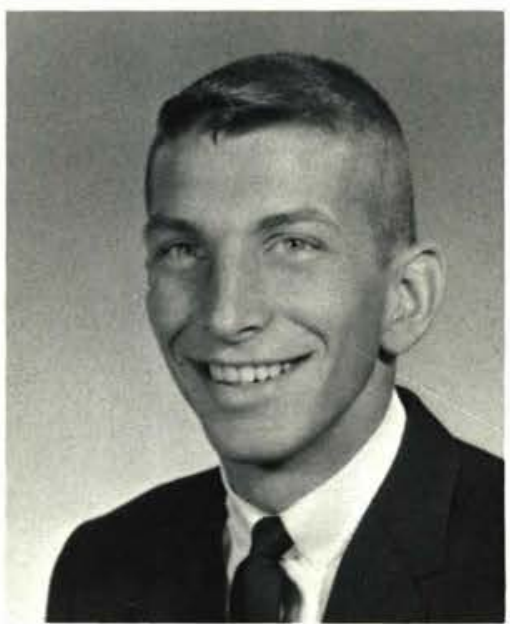

Euclid, Ohio

Major: Christian Education

Degree - B.S.

\section{James Engelmann}

Flint, Michigan

Major: General Science

Degree - B.S. 


\section{Gerald Allen Fisher}

Lebanon, Indiana

Major: Foreign Language - Degree B.A.

\section{Sheryl Joan Fox}

Cedarville, Ohio

Major: Sociology - Degree B.A.

Arnold Genekovich Fruchtenbaum

Levittown, New Jersey

Major: Pre Seminary Bible - Degree B.A.

\section{Verna L. Glander}

Random Lake, Wisconsin

Major: Bible-Degree B.A.
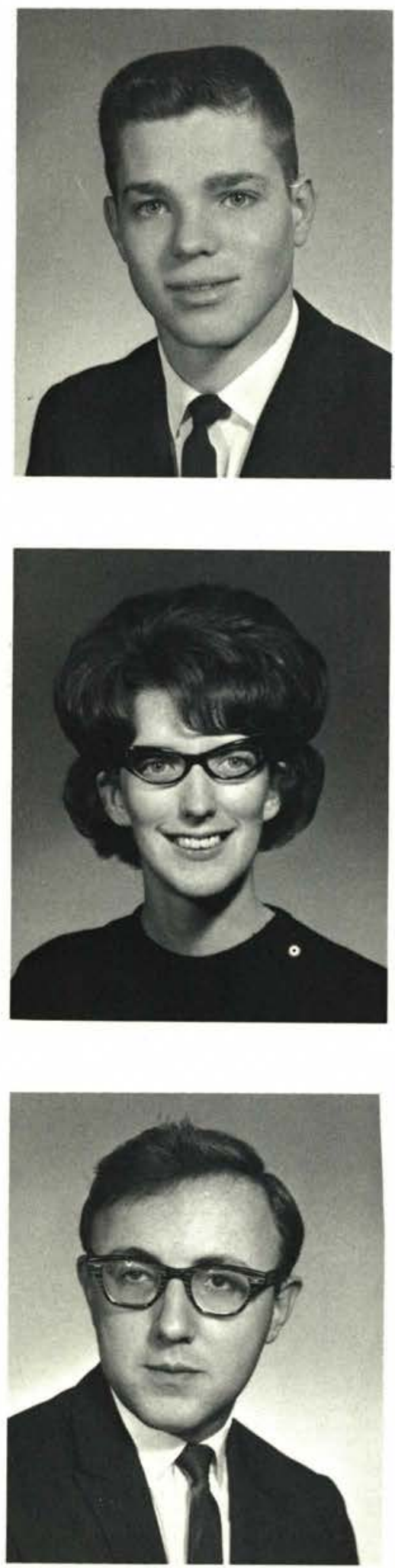

\section{Elizabeth Louse Geritz}

Coudersport, Pennsylvania

Major: Pre Seminary Bible -

Degree B.A.

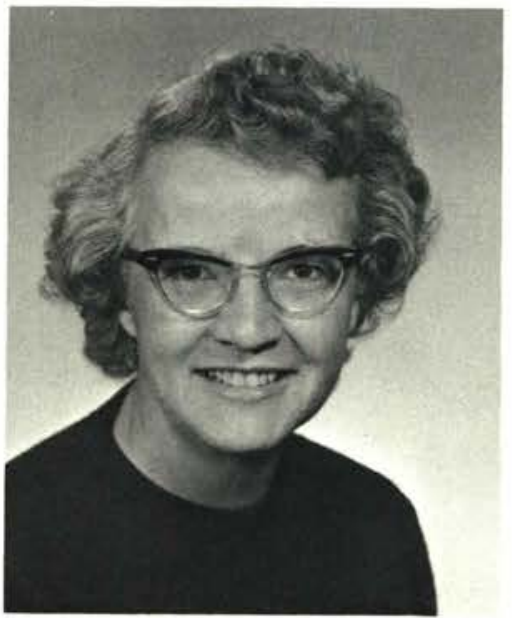

\section{Marilou Goddard}

Portsmouth, Ohio

Secretarial Science - Degree B.S.
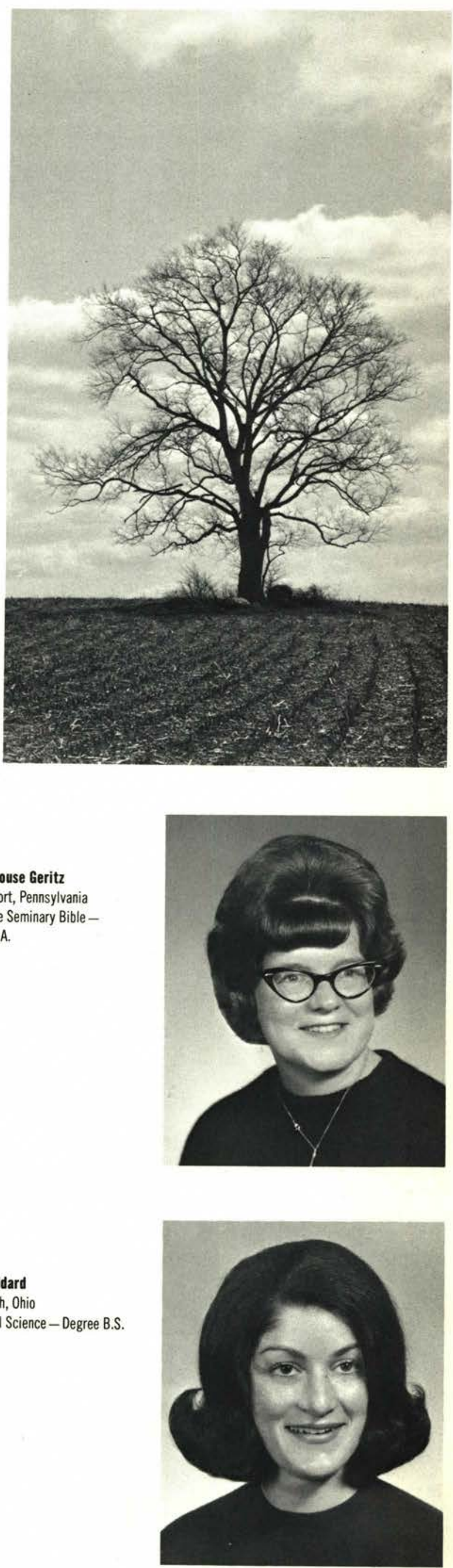


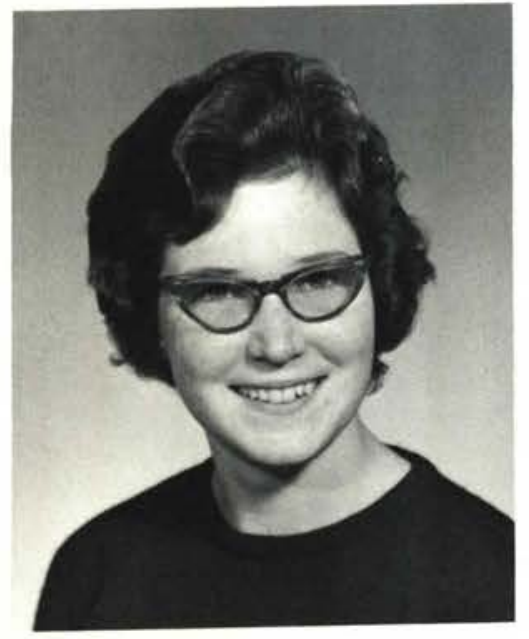

Patricia Carol Good

Eggertsville, N.Y.

Major: Elementary Education

Degree - B.A.
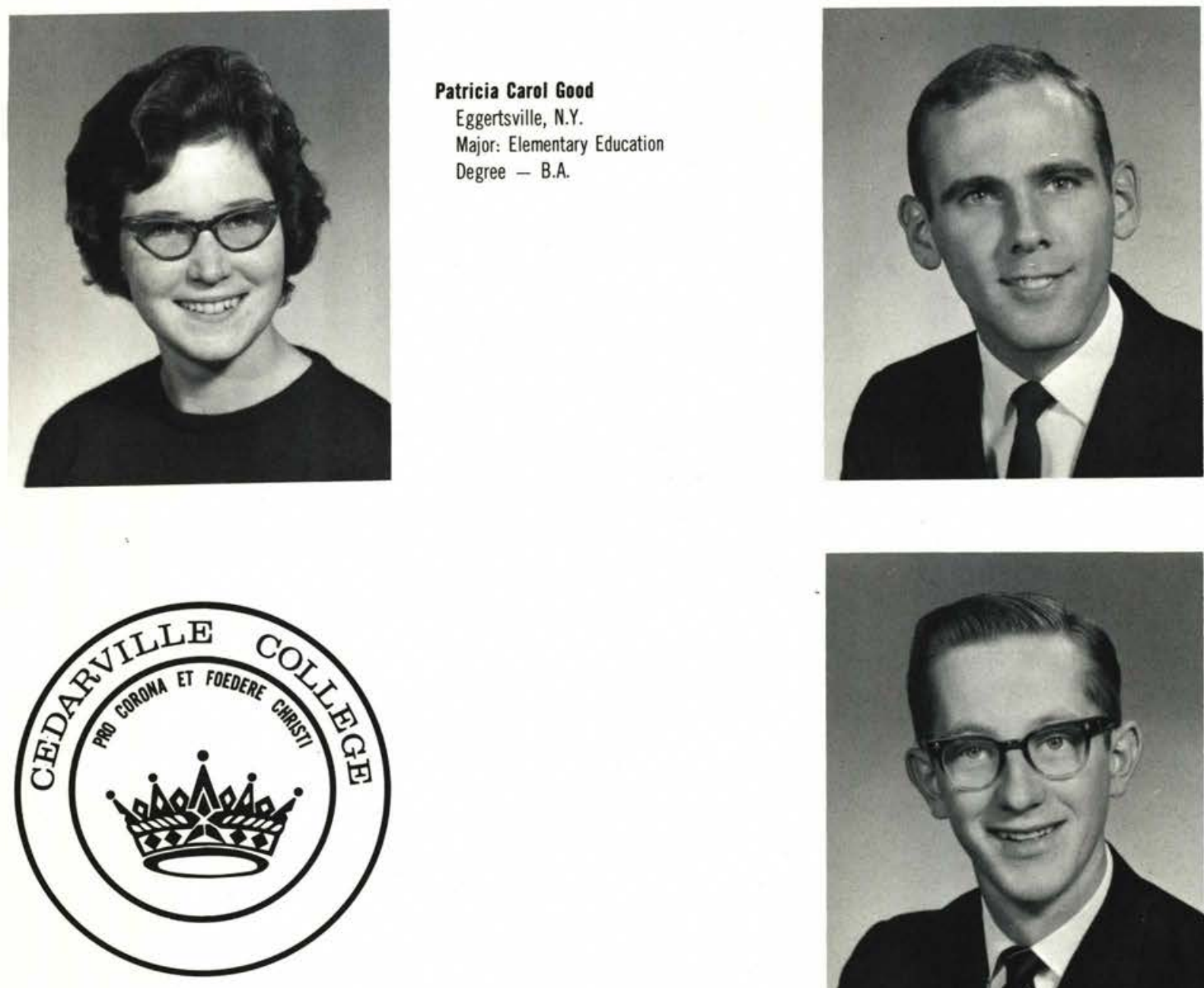

Edward David Gordon, Jr.

Garretsville, Ohio

Major: Pre-Seminary Bible

Degree - B.A.

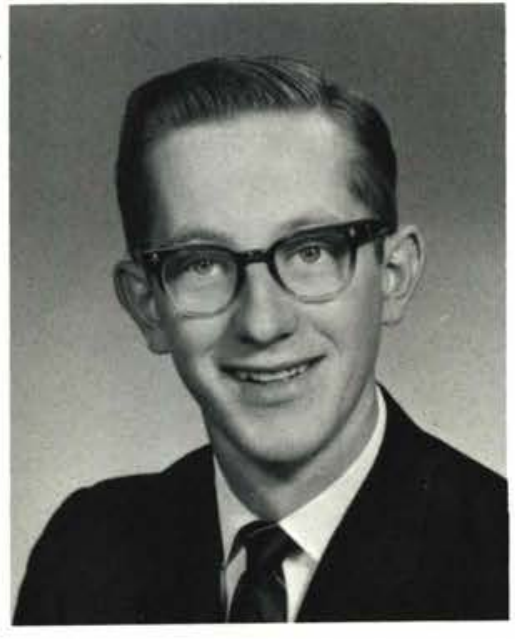

Philip Daniel Grisso Springfield, Ohio

Major: Social Studies Comp.

Degree - B.S.
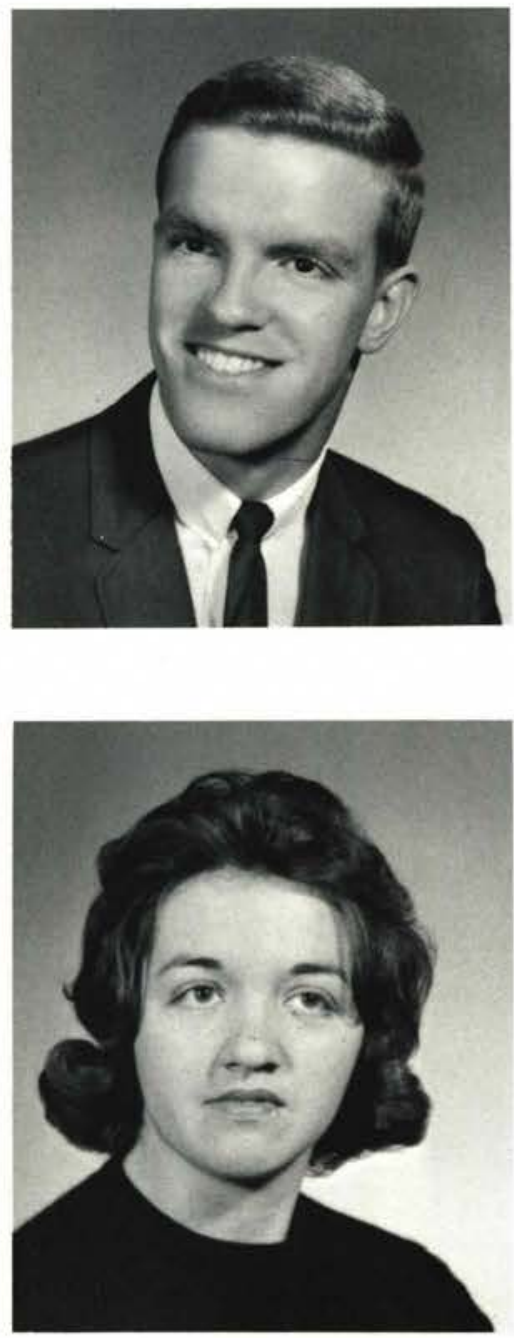

Gordon Donald Griffin

Lincoln, Nebraska

Major: Piano

Degree - B.A.

\section{Gary James Harris}

Beech Grove, Indiana

Major: Pre Seminary Bible

Degree - B.A.

\section{Arlene E. Hoyte}

Whitney Point, N.Y.

Major: Elementary Education

Degree - B.A. 
Lloyd L. Lane

Coldwater, Mich.

Major: General Business

Degree - B.S.

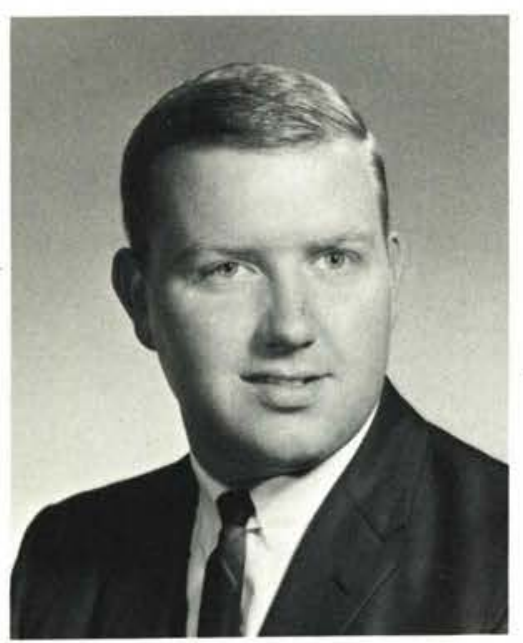

\section{Suzanne Ruth Lepine}

Cheektowaga, N.Y.

Major: Sociology

Degree - B.A.

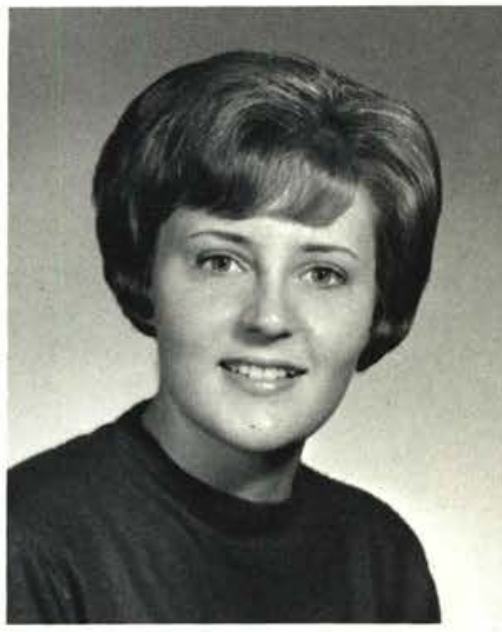

\section{Gretchen R. Liechty}

Hobart, Ind.

Major: Elementary Education Degree - B.A.
Buster Mayhew McPheeters Quincy, III.

Major: Social Studies Comp. Degree - B.S.
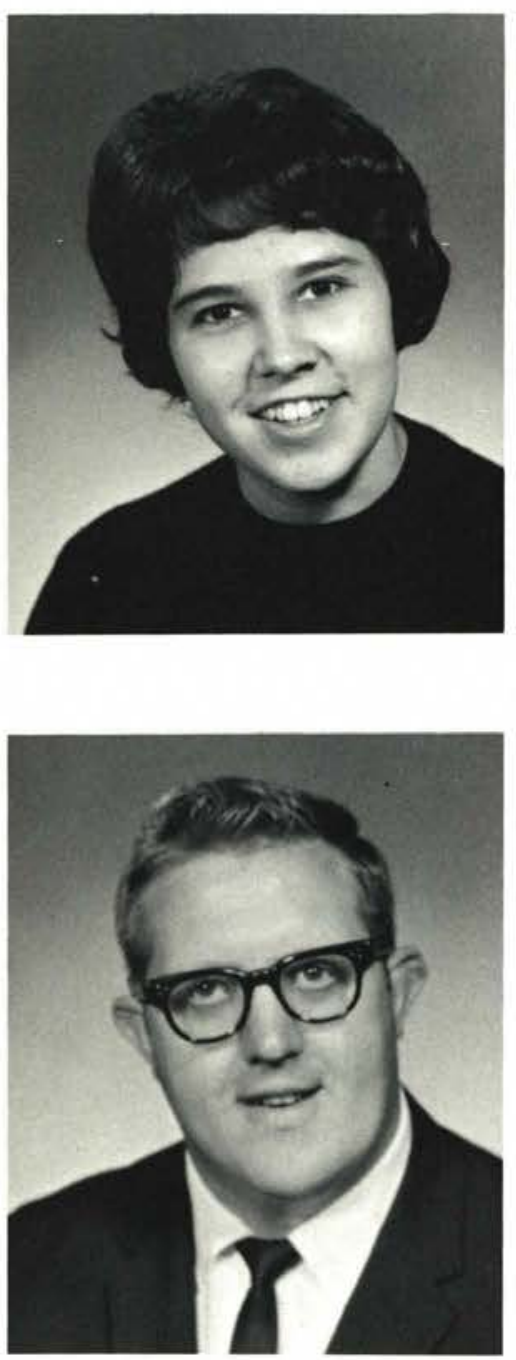

Valerie Lee Merryman Teaneck, N.J.

Major: Biology

Degree - B.S.

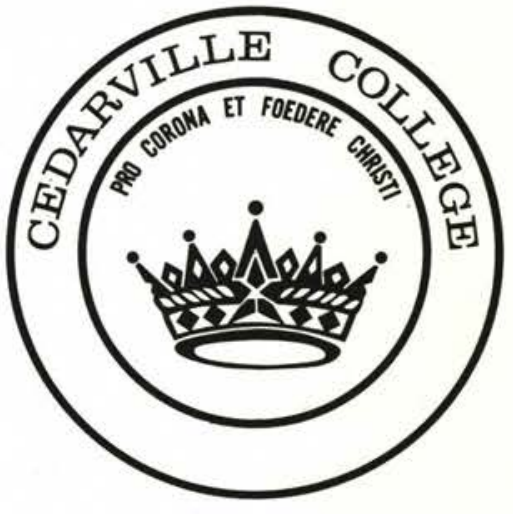

Joyce Elaine Micka Ridgefield Park, N.J.

Major: Elementary Education Degree - B.A.

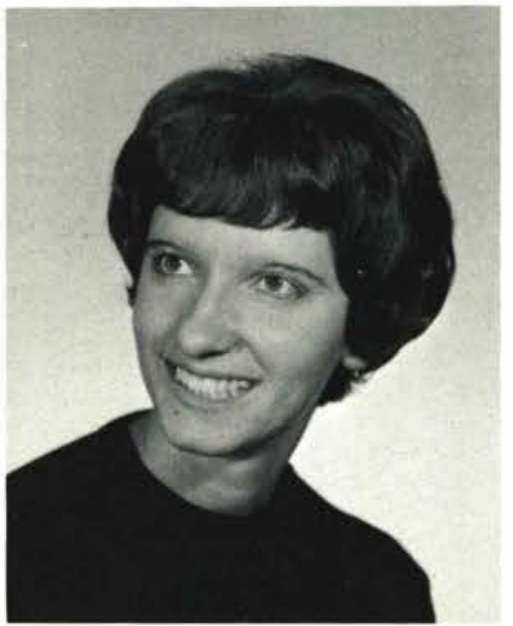

Carol Joyce Mikels Indianapolis, Indiana Major: Business Education Degree - B.S.
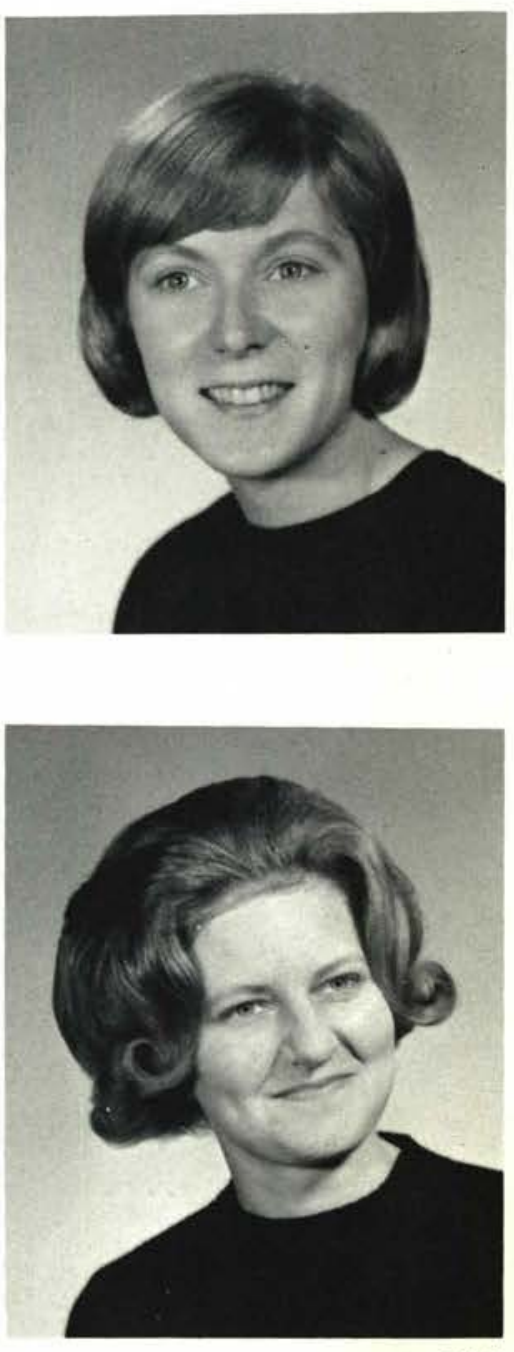


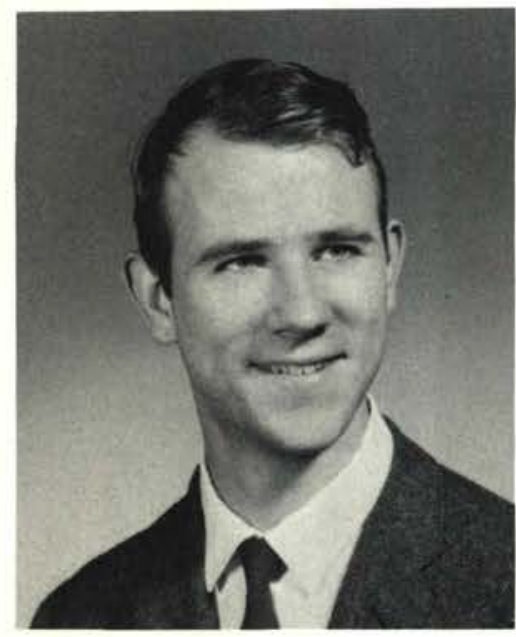

David Nims

Keene, N.H.

Major: Music

Degree - B.S.

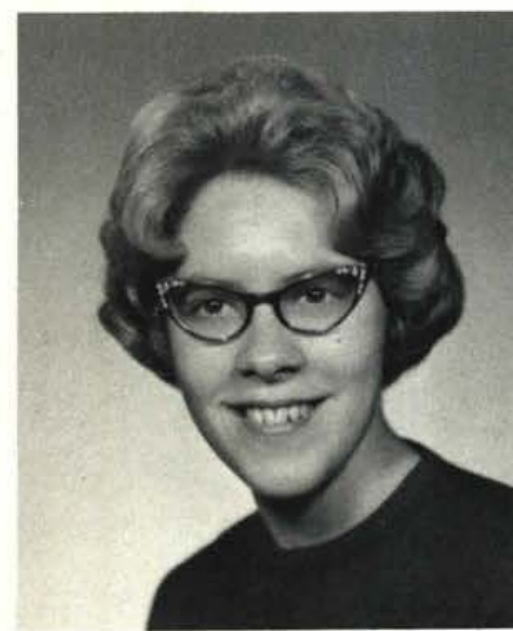

\section{Rebecca S. O'Keefe}

Parma, Ohio

Major: Elementary Education

Degree - B.A.

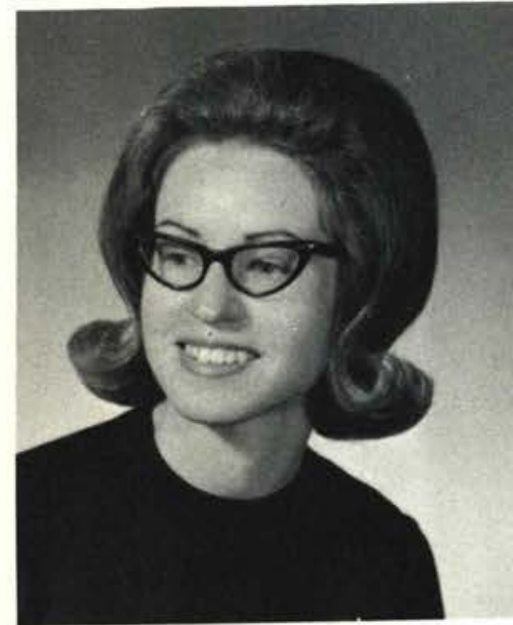

\section{Shirley Otto}

Carroll, lowa

Major: Math., Physical Science Degree - B.S.

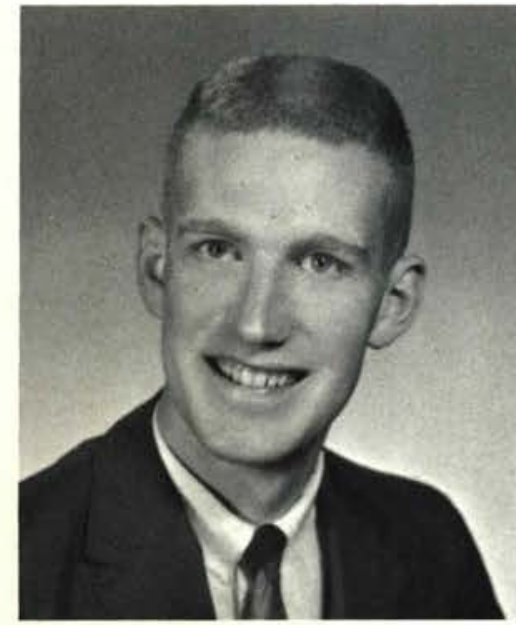

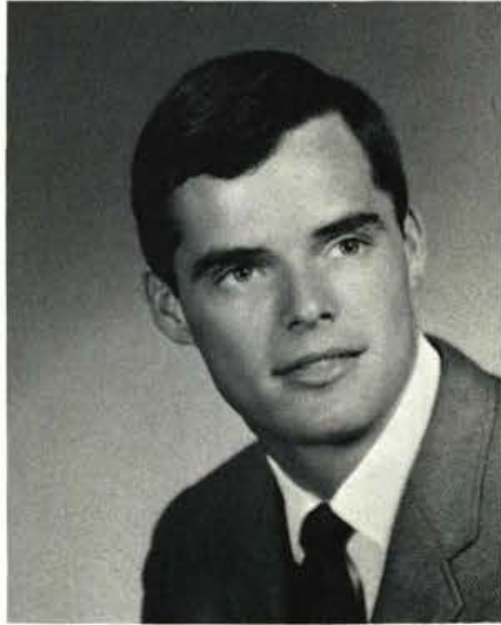

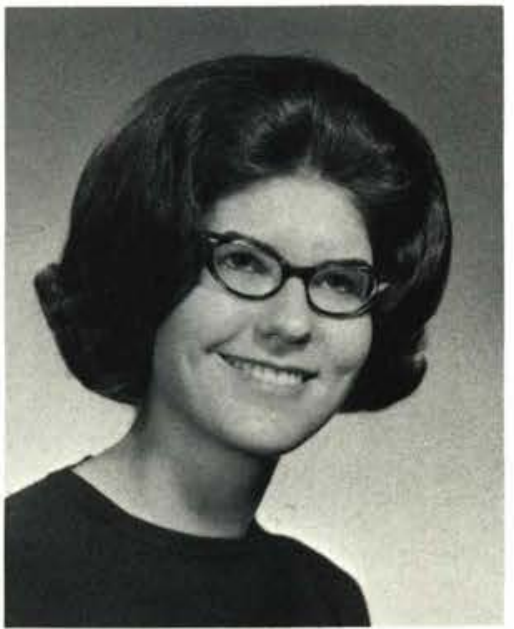

Dorothy Olsen

Berea, Ohio

Major: English

Degree - B.A
. Nicholis

Carlisle, lowa

Major: History

Degree - B.A
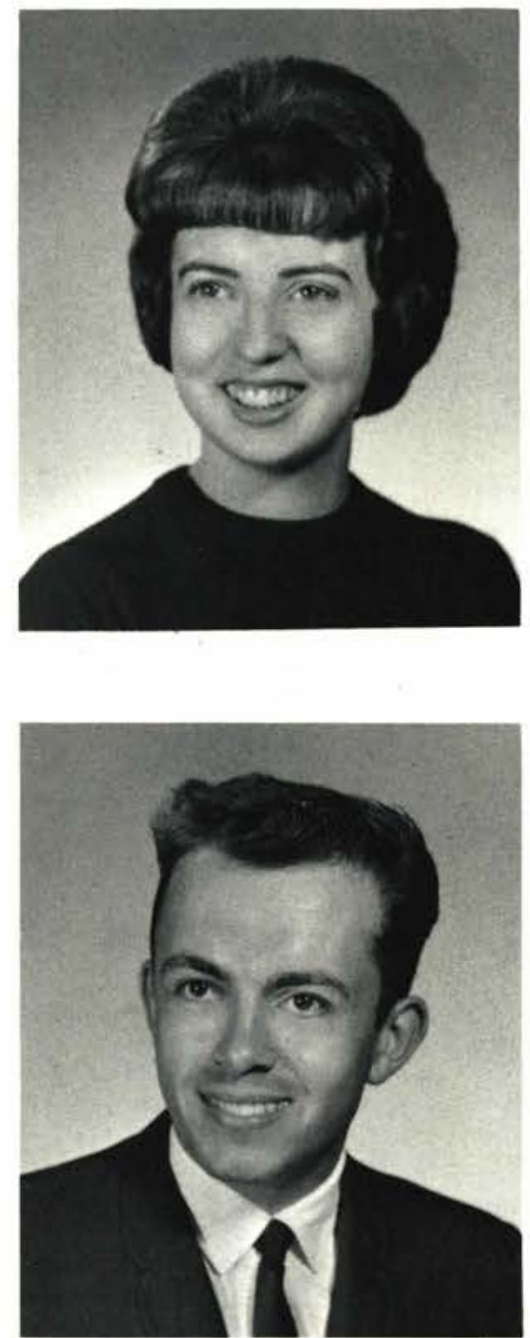

\section{Darlene Patton}

Cleveland, Ohio

Major: Christian Education

Degree - B.A.

\section{Dennis Pearce}

Cedarville, Ohio

Major: Accounting

Degree - B.S. 


\section{David Prosser}

Warren, Ohio

Major: Math

Degree-B.S.

\section{Carolyn Retie}

Major: Elementary Education

Degree-B.S.

\section{Denis Rockwell}

Indianapolis, Indiana

Major: Bible Comprehensive Degree-B.A.

\section{Joyce Rudduck}

Cedar Falls, lowa

Major: Christian Education

Degree-B.A.
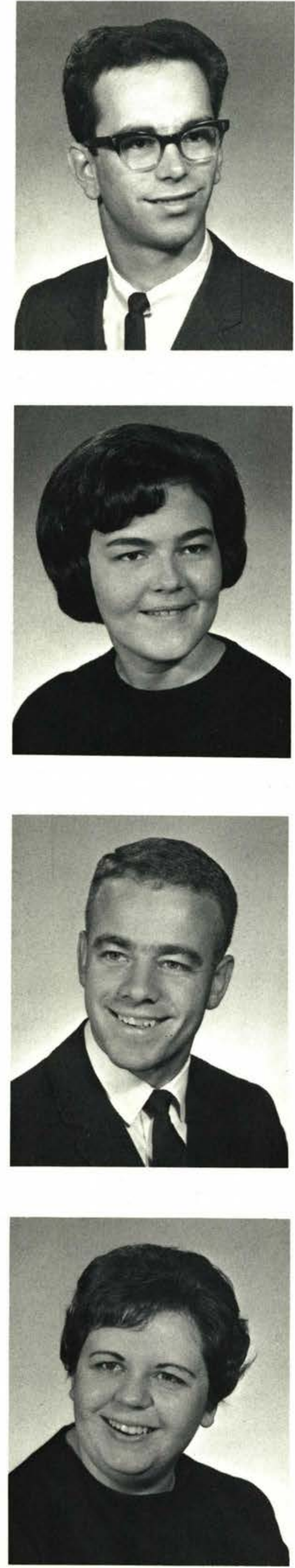

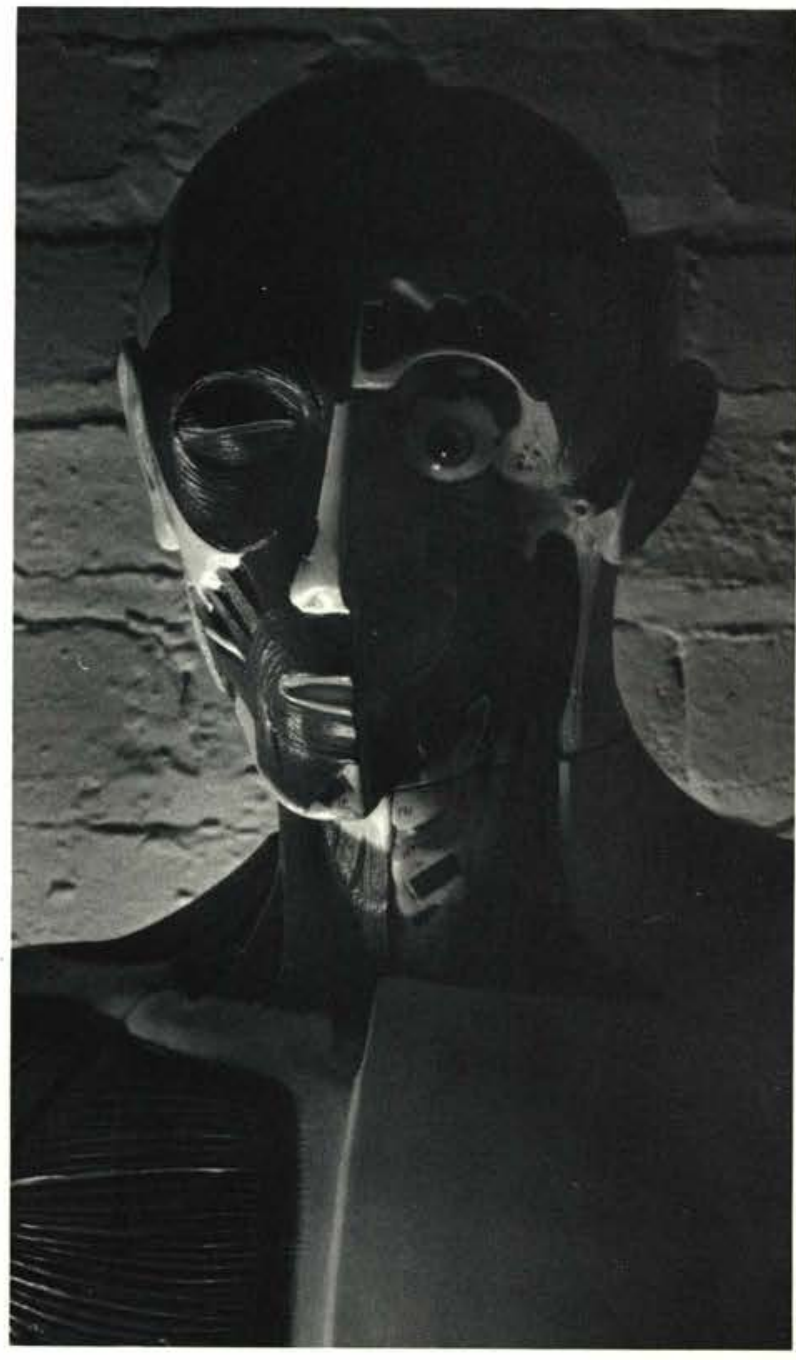

Phyllis J. Ronk

Richmond, Va.

Major: Speech

Degree-B.A.

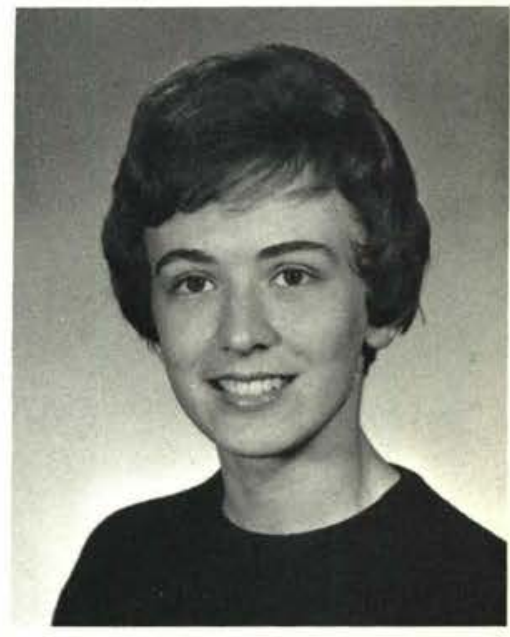

Larry Shampoe

Clymer, N.Y.

Major: Accounting

Degree-B.A.

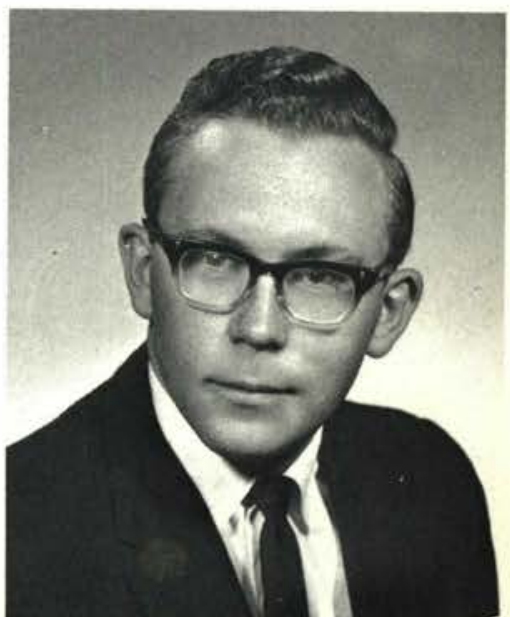




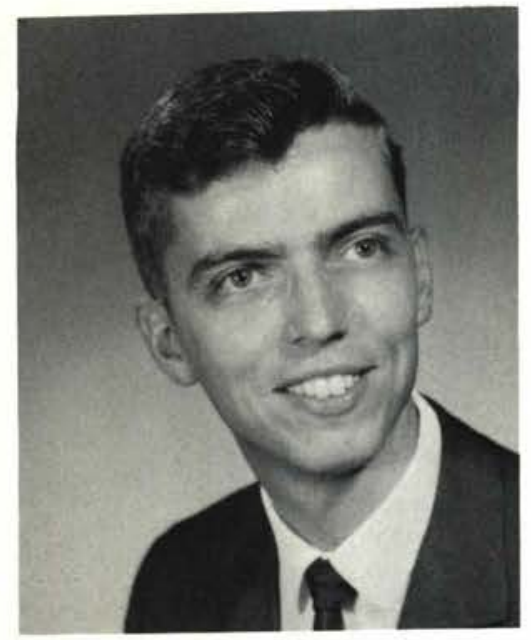

Larry Sharpless

Fostoria, Ohio

Major: Christian Education

Degree - B.A.
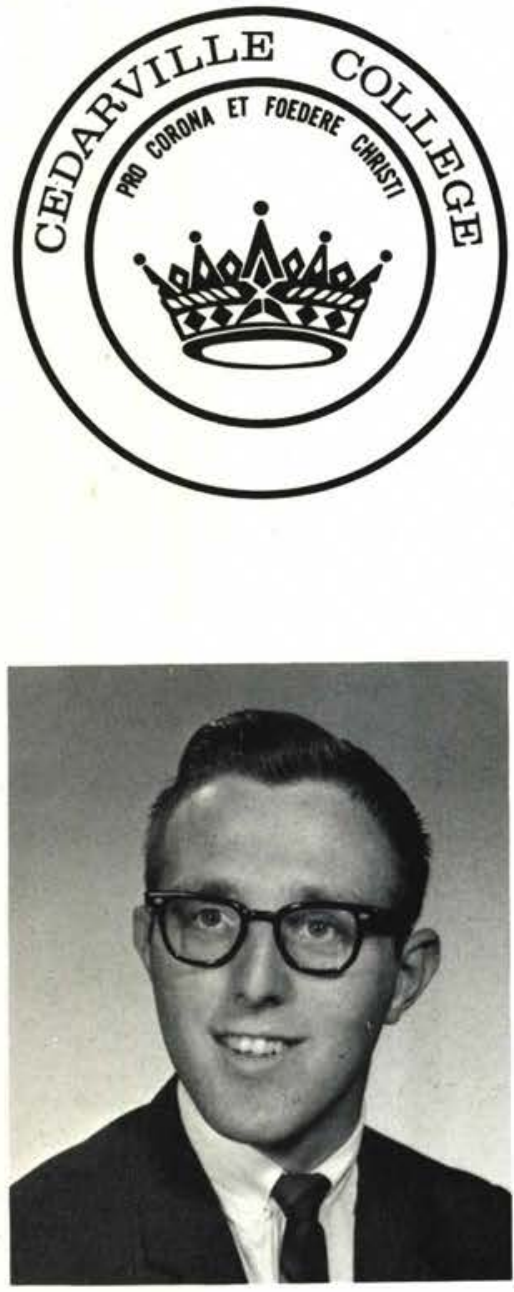

\section{David Staple}

Spring Valley, Minn.

Major: Physical Education

Degree - B.S.

John C. Stockwel

Cedarville, Ohio

Major: Speech

Degree - B.A.
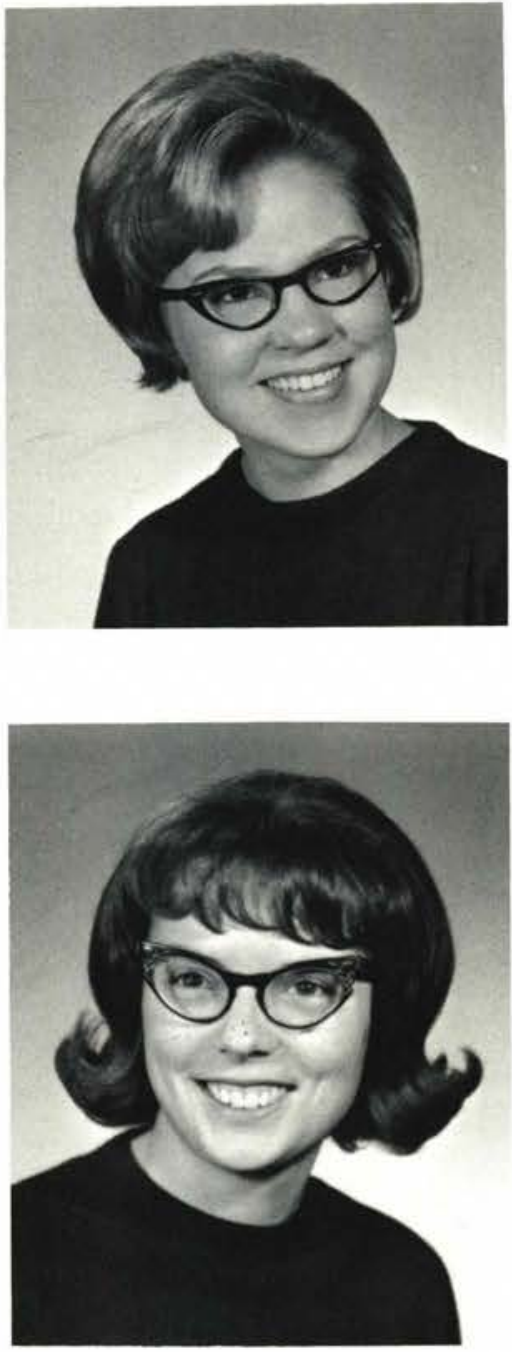

\section{Judy Ann Spear}

Decker, Mich.

Major: Elementary Education Degree - B.S.

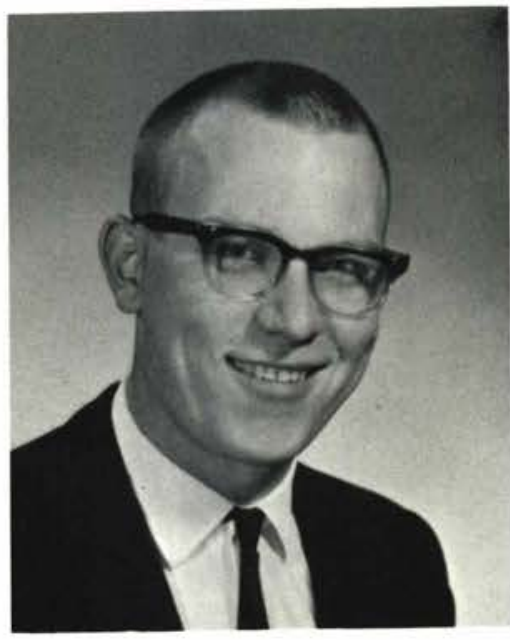

Dan E. Stephens

McMillan, Mich.

Major: Accounting

Degree - B.S.

\section{Ron Stuart}

Horseheads, N.Y.

Major: Bible Comprehensive Degree - B.A. 
Joseph Mishael Stowell, III Hackensack, N.J.

Major: English

Degree - B.A.

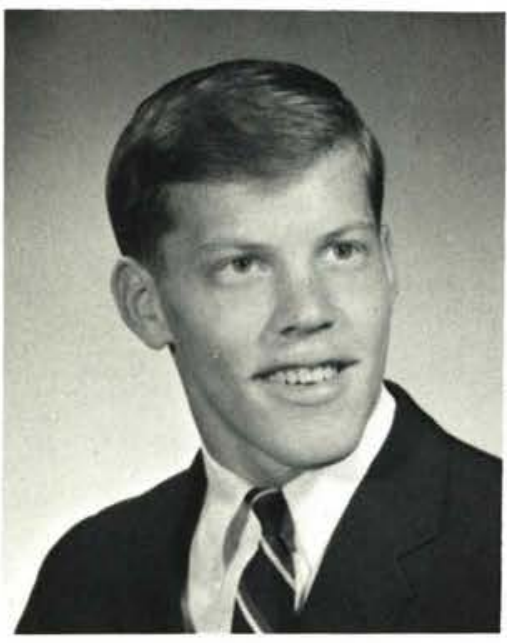

Gordon Allen Taylor

Steamboat Rock, lowa

Major: Speech

Degree - B.A.
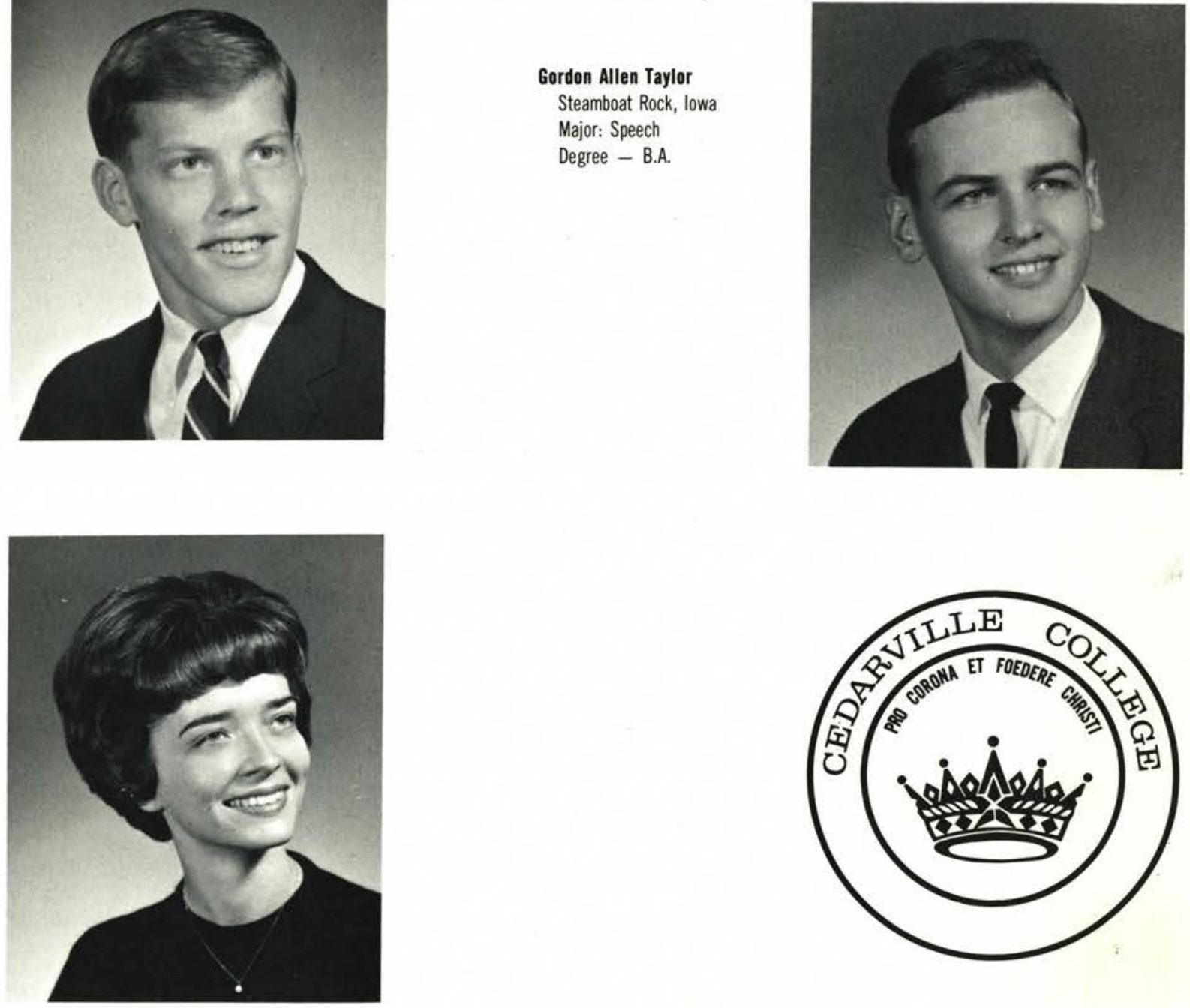

Degree - B.A.

\section{Janice Gale VanHorn}

Plainfield, Illinois

Major: Elementary Education Degree - B.A.
Sharon Elaine Vance

Marion, Ohio

Major: Elementary Education Degree - B.A.
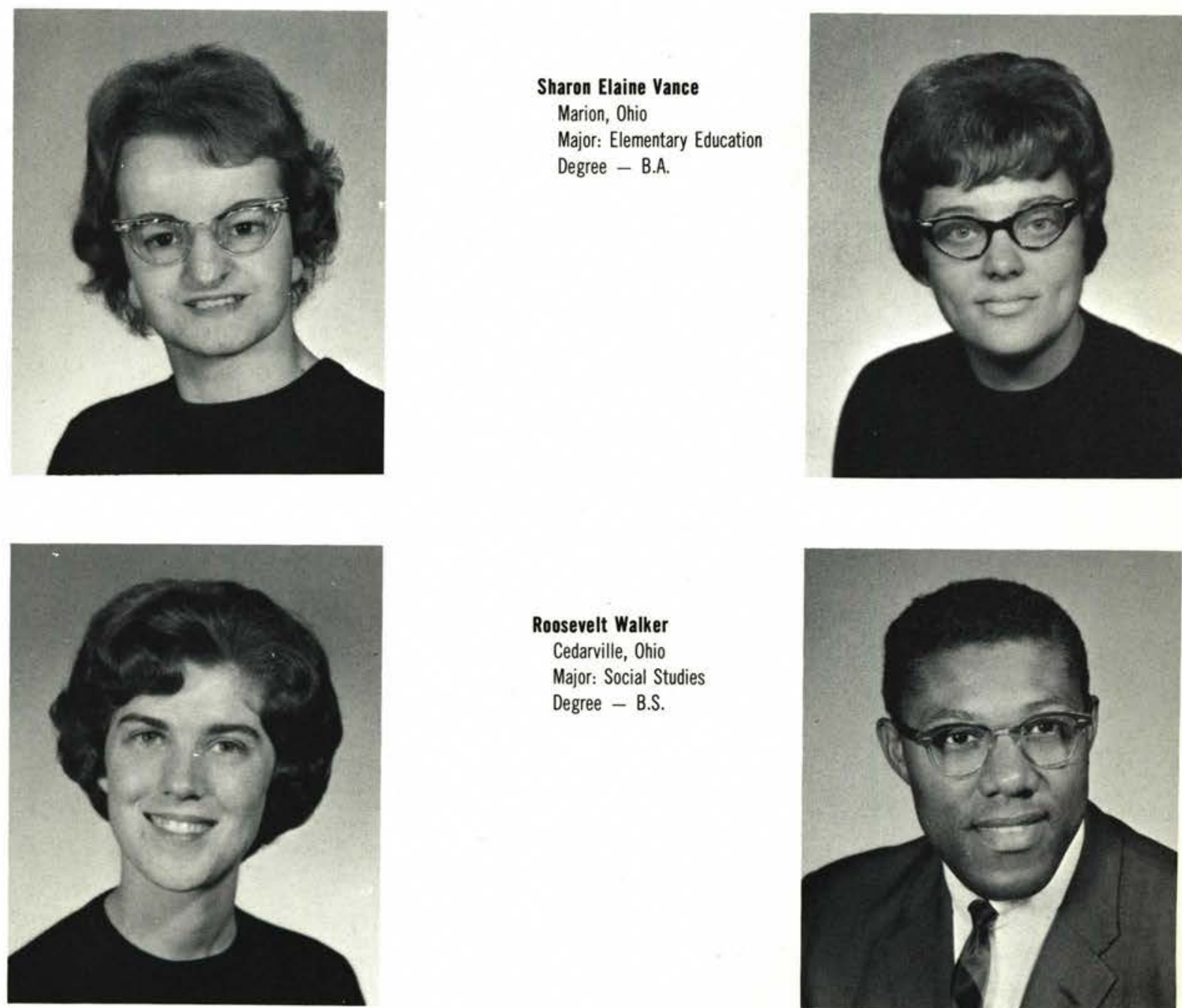

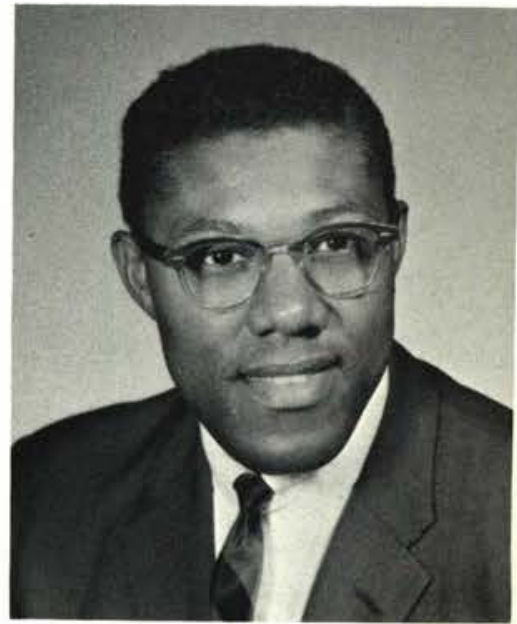

Roosevelt Walker

Cedarville, Ohio

Major: Social Studies

Degree - B.S. 


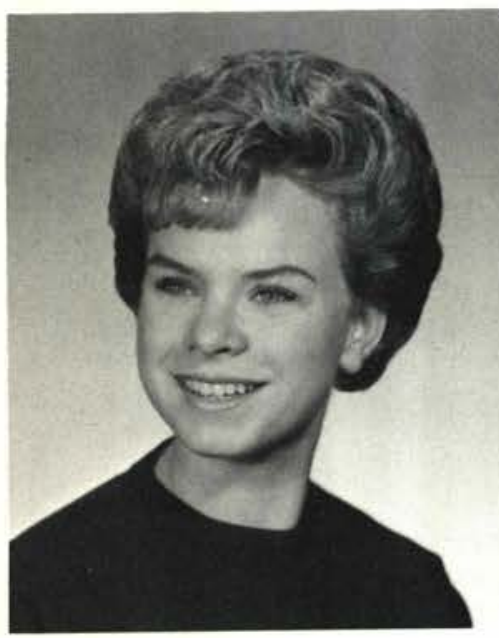

Teresa Annette Whaley

Woodland Hills, Calif.

Major: General Science

Degree - B.S.

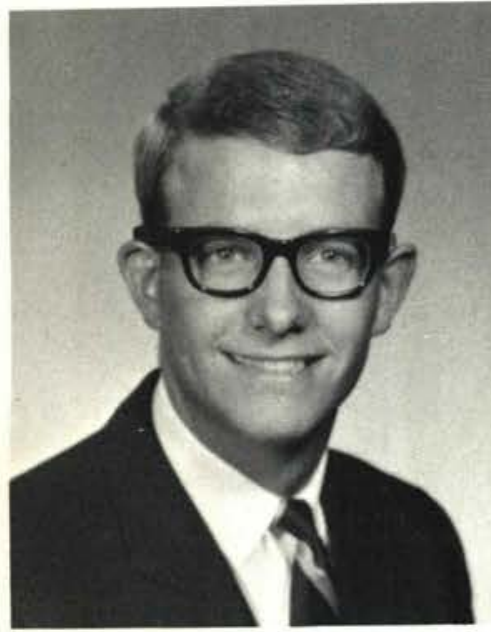

\section{Charles Lloyd Wilkins}

Columbus, Ind.

Major: History, Physical Science

Degree - B.S.

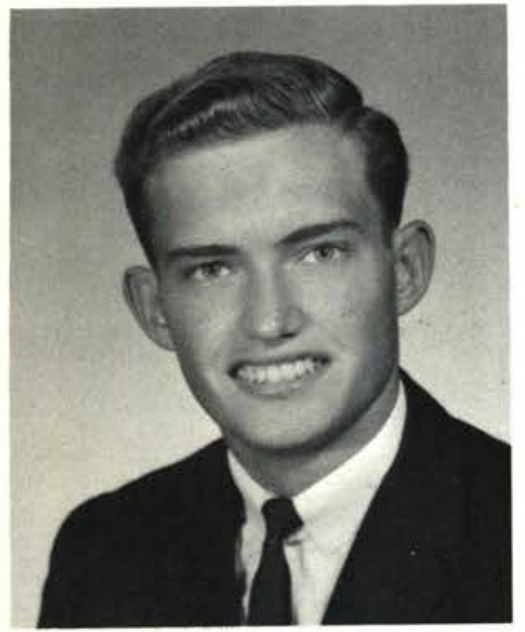

Robert C. Worth

Medford, N.J.

Major: Biology

Degree - B.S.

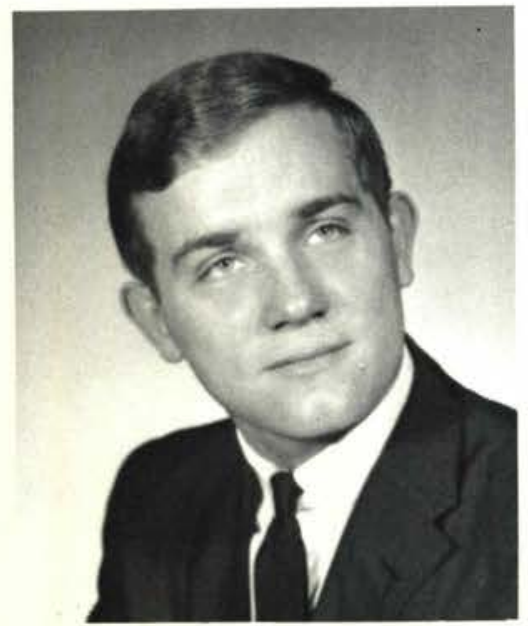

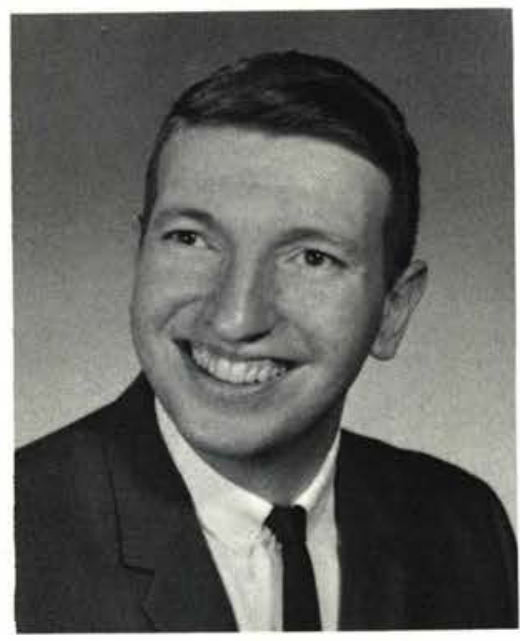

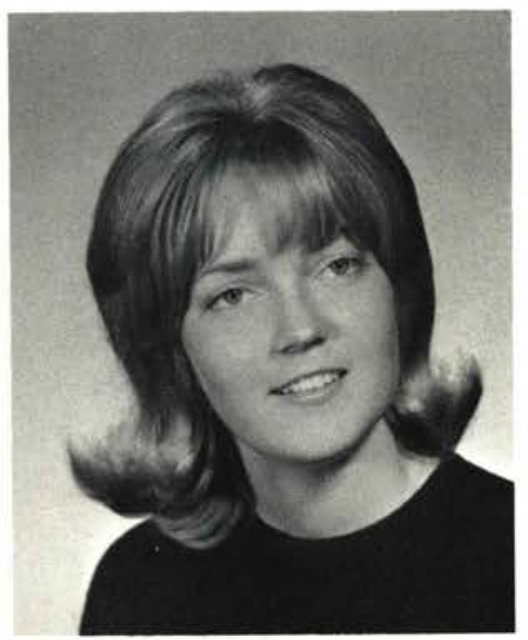

Linda Jean Wilson

Shafter, Calif.

Major: Biology

Degree - B.A.

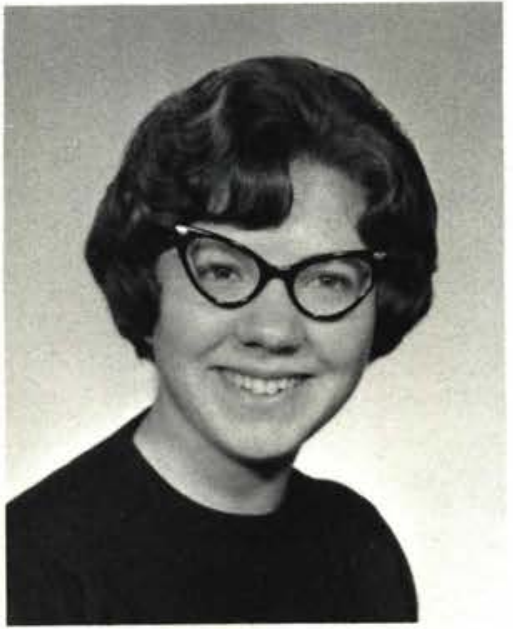

\section{E. Maxine Young}

Mt. Pleasant, Mich.

Major: Secretarial Science

Degree - B.S.
Richard Lynn Zandstra

Grand Rapids, Mich.

Major: Biology

Degree - B.S.

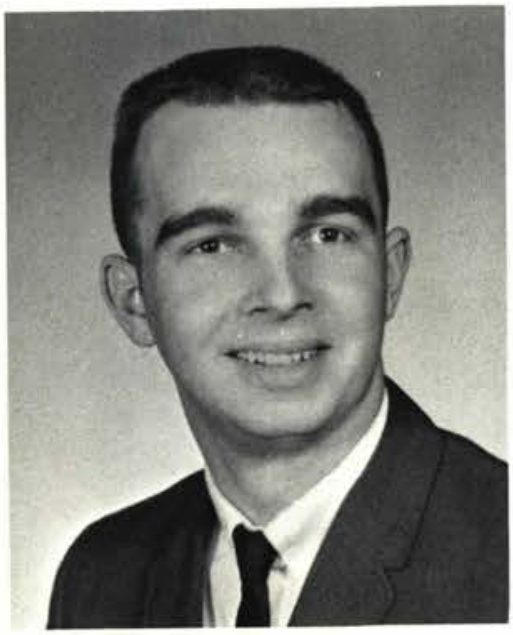

James Doran Zeigler

Turnersville, N.J.

Major: Accounting

Degree - B.S. 


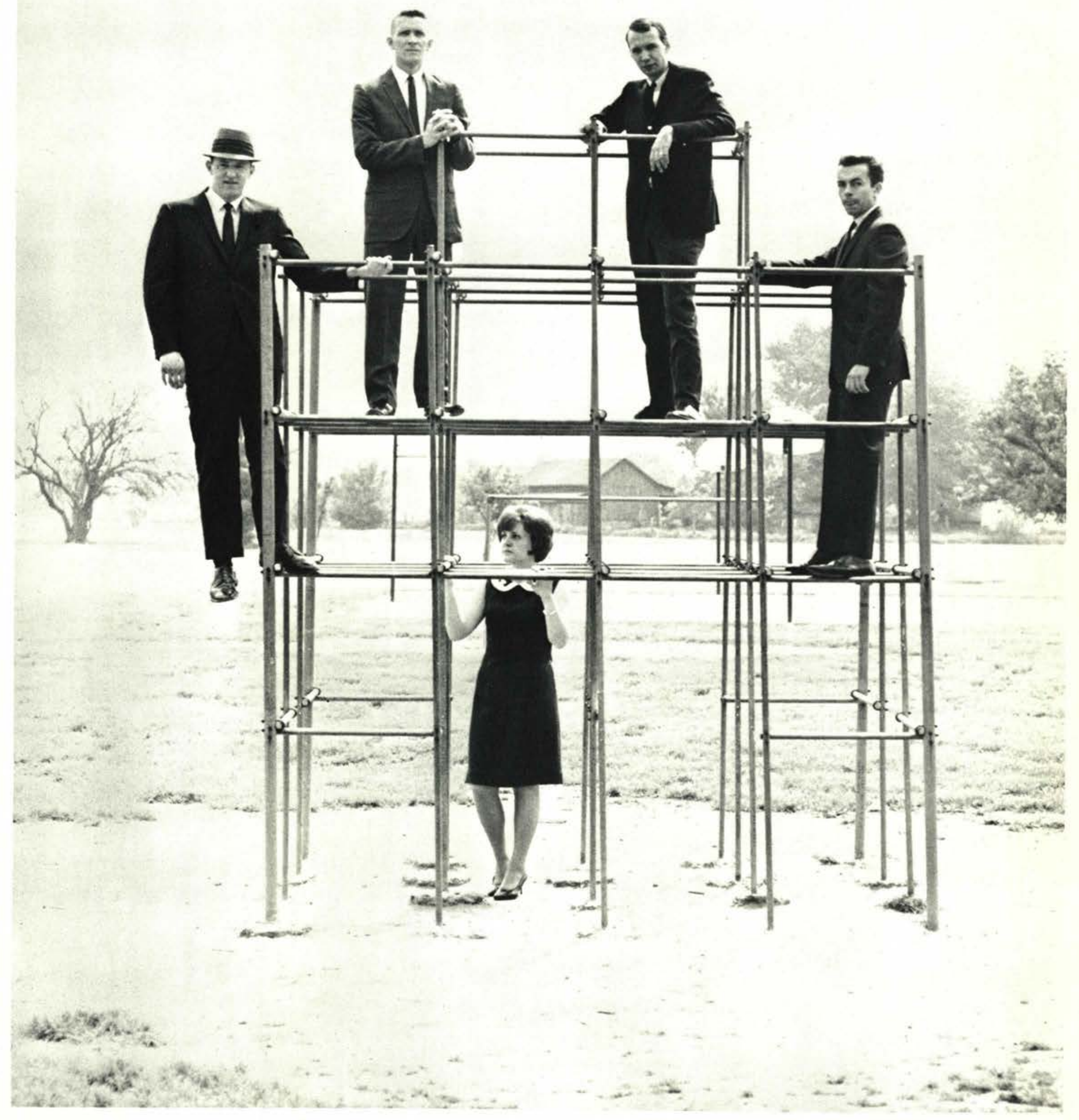

Left to Right: T. Jager ................... V. President

Dr. Dale Thomson ........................ Advisor

J. Stockwell . . . . . . . . . . . . . . . . . . . .

M. Pereira . . . . . . . . . . . . . . . . Treasurer

S. Lepine $\ldots \ldots \ldots \ldots \ldots \ldots \ldots \ldots$. Secretary 


\section{Baccalaureate}

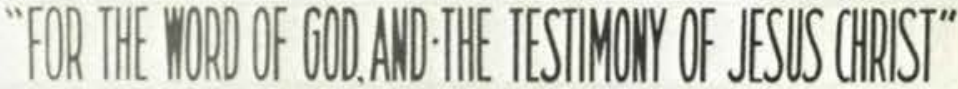

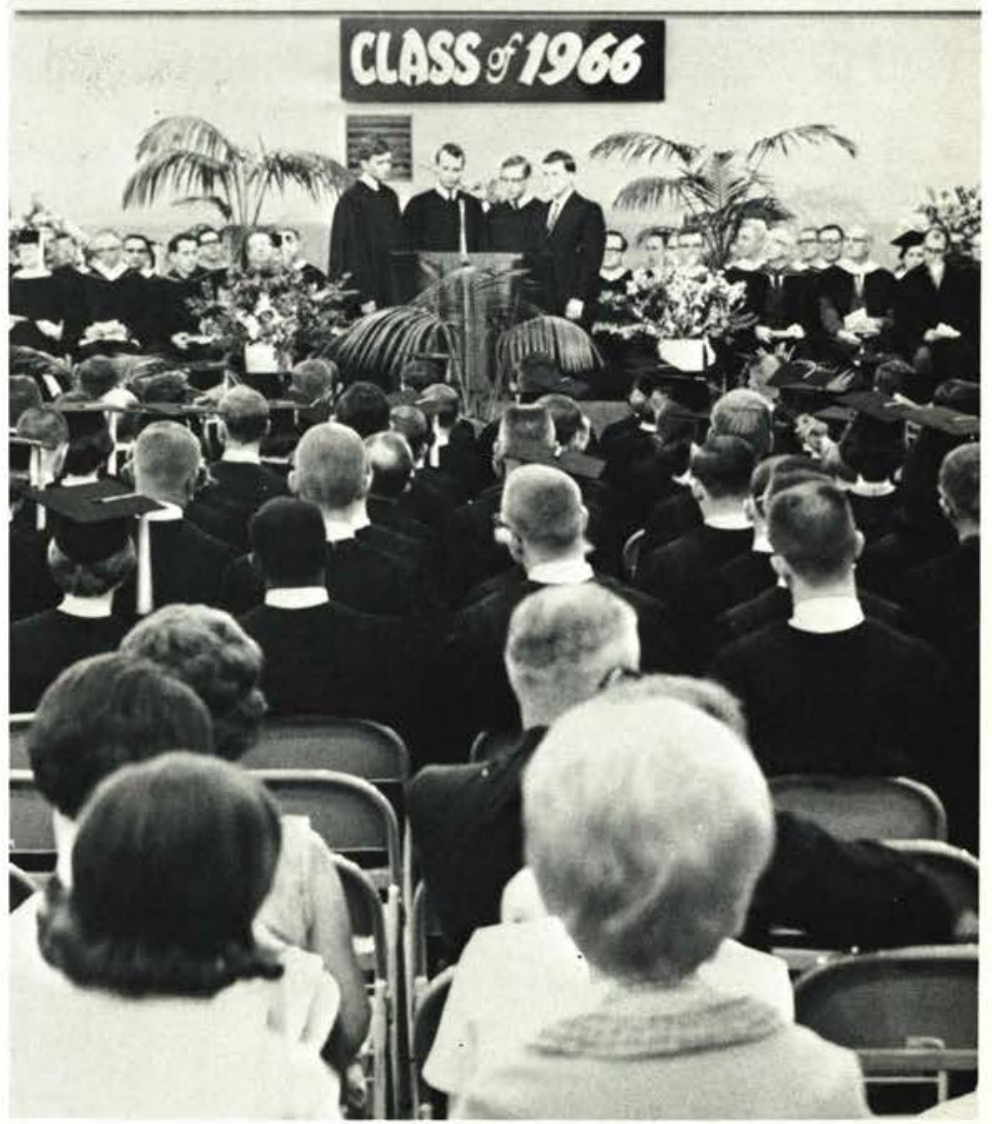

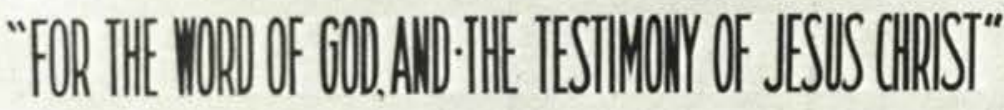

\section{Class of 1966}

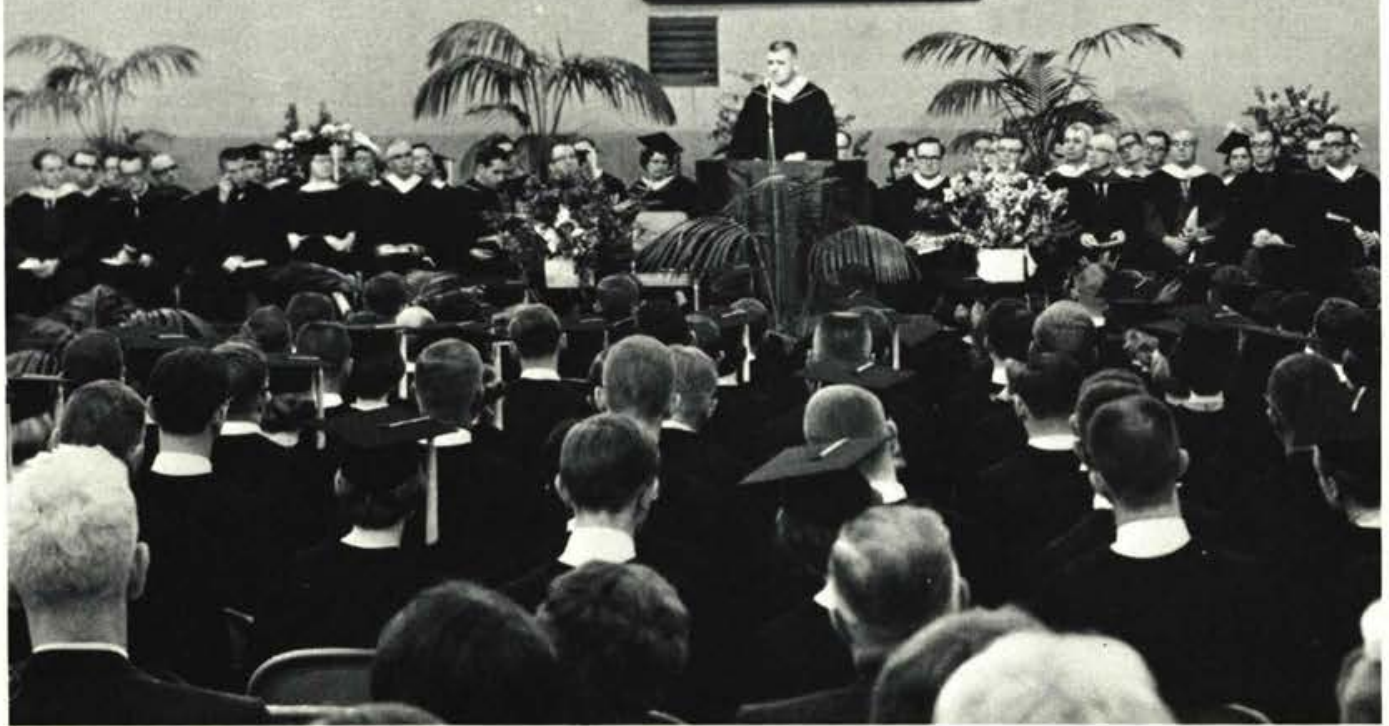




\section{Commencement}

An impressive Commencement service was held on May 28, 1966, in the Student Center. Leading the procession were four Junior girls: Louise Stutesman, Lisa Rulison, Ruth Grant, and Irene Milikan.

After a trio number by Grace Averett, Dorothy Olsen, and Mona Ballard, Dr. Joseph M. Stowell, pastor of First Baptist Church, Hackensack, New Jersey, presented a challenge to the graduates and audience.

The Doctor of Divinity Degree was conferred upon Rev. Roy G. Hamman, Mishawaka, Indiana.

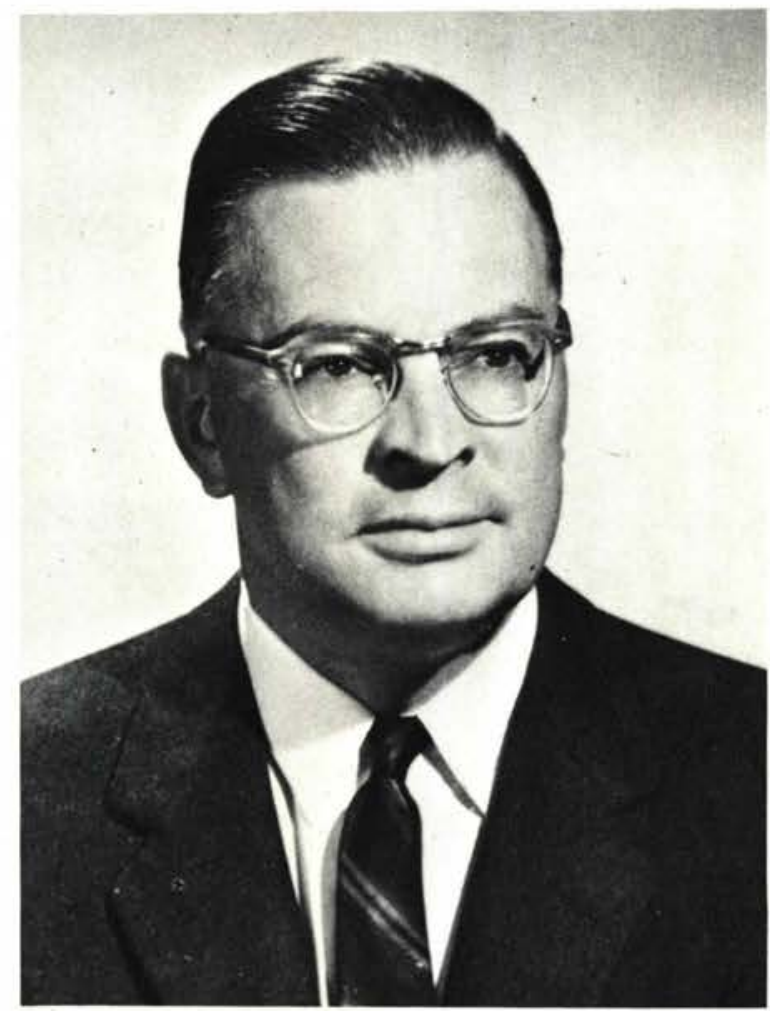

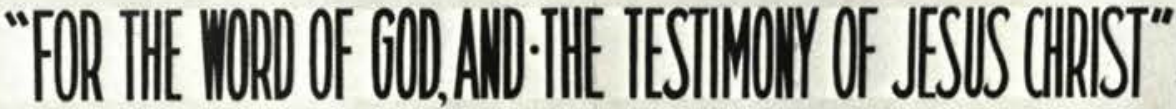

\section{Class of 1966}

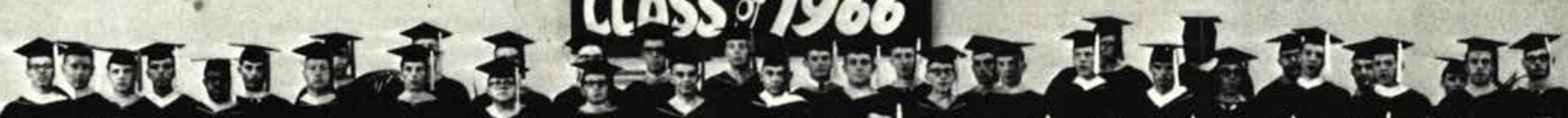

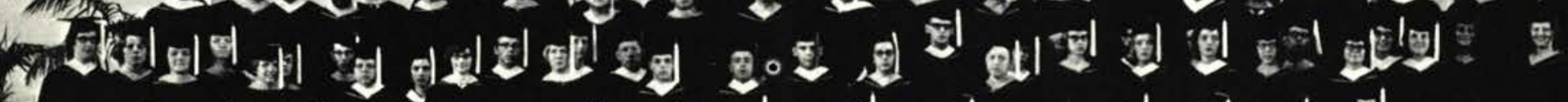

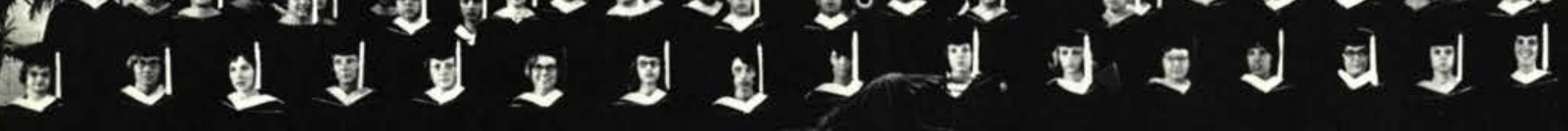

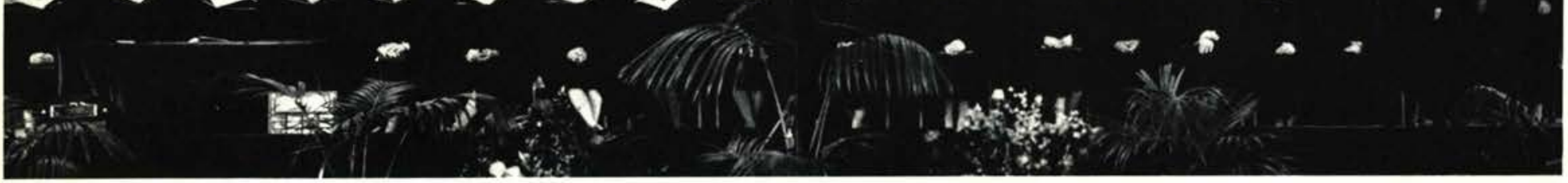



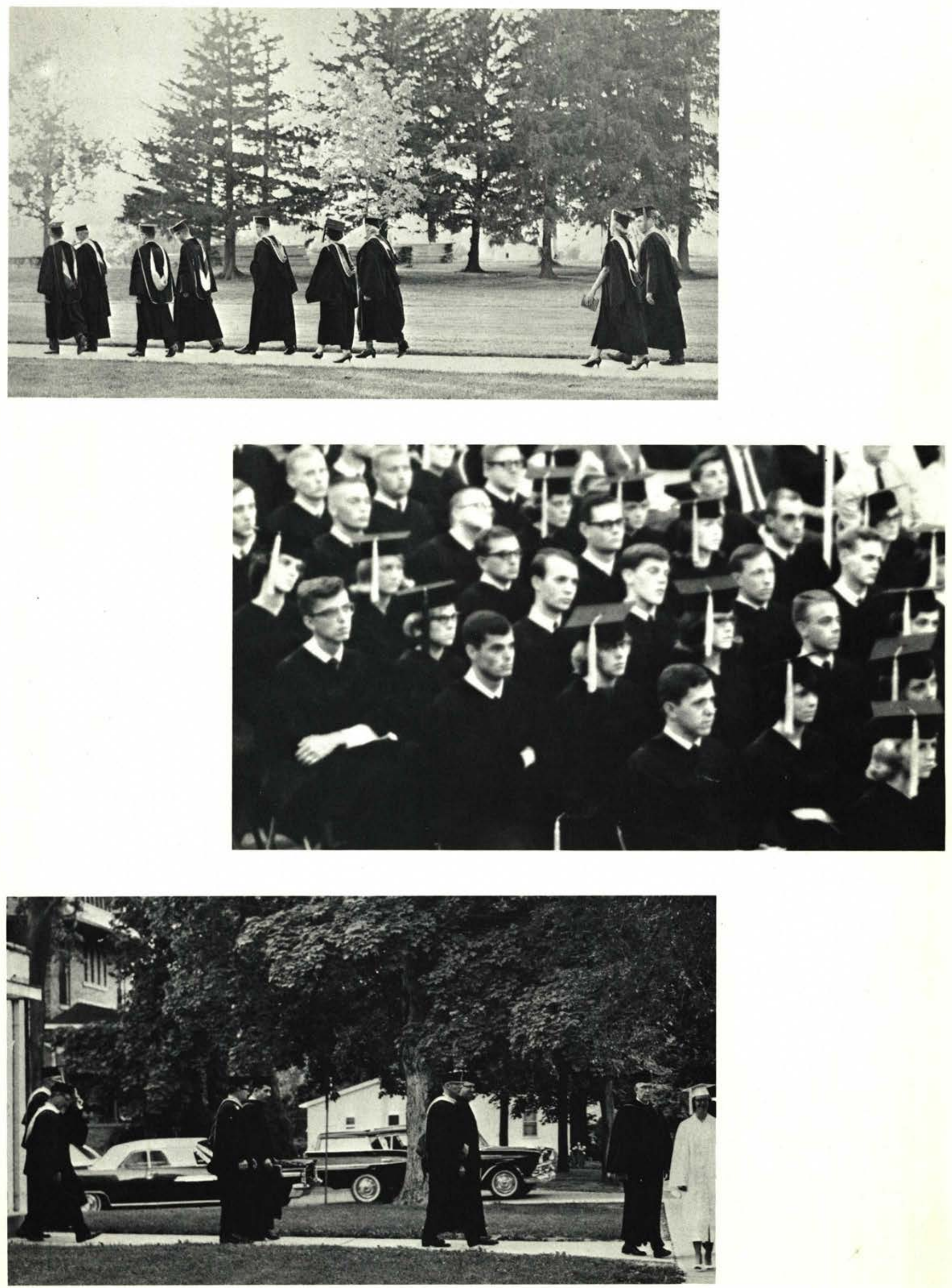


\section{Seniors . . Remember . . .}

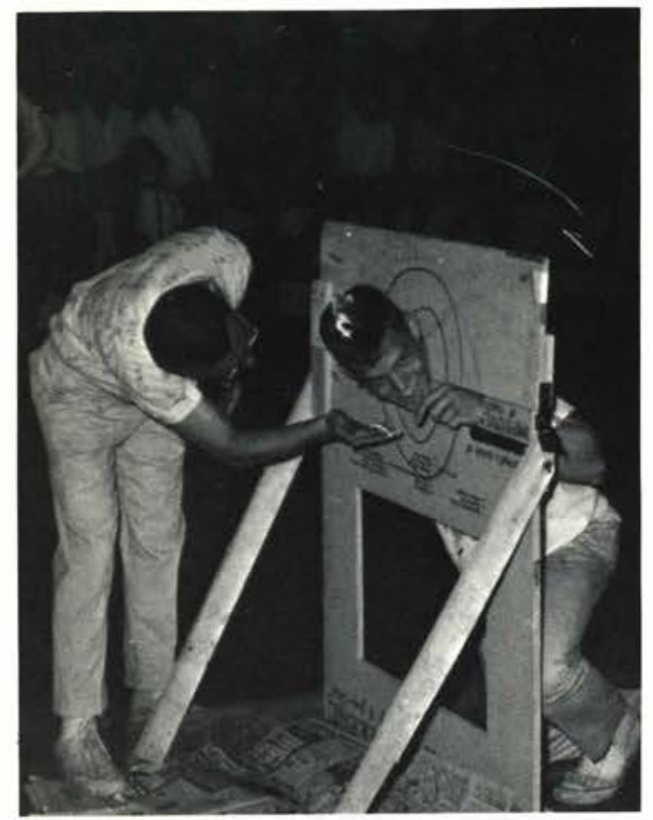

Freshman Initiation

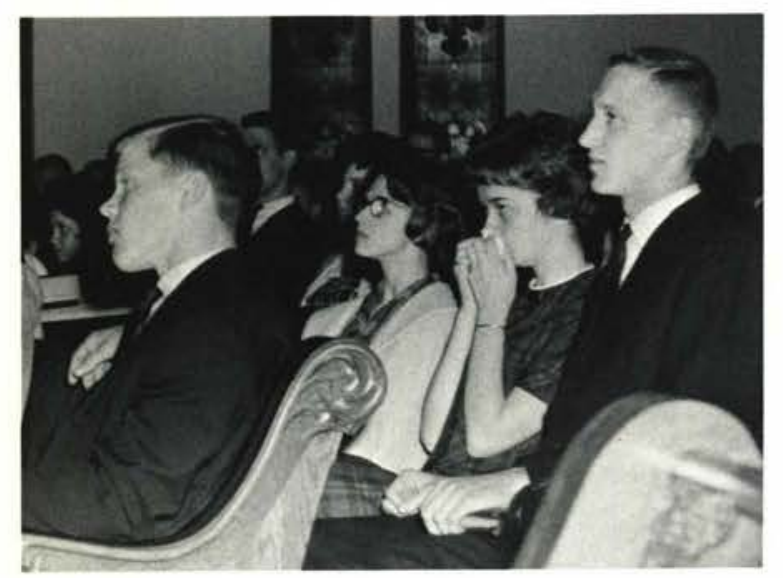

Grace Baptist Church

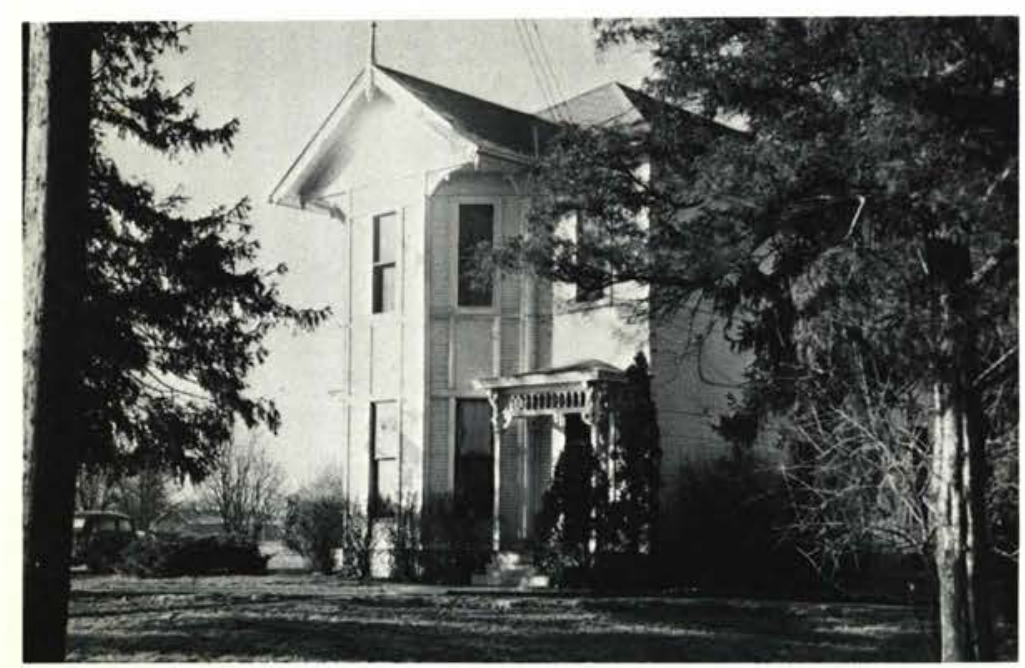

Rife Hall

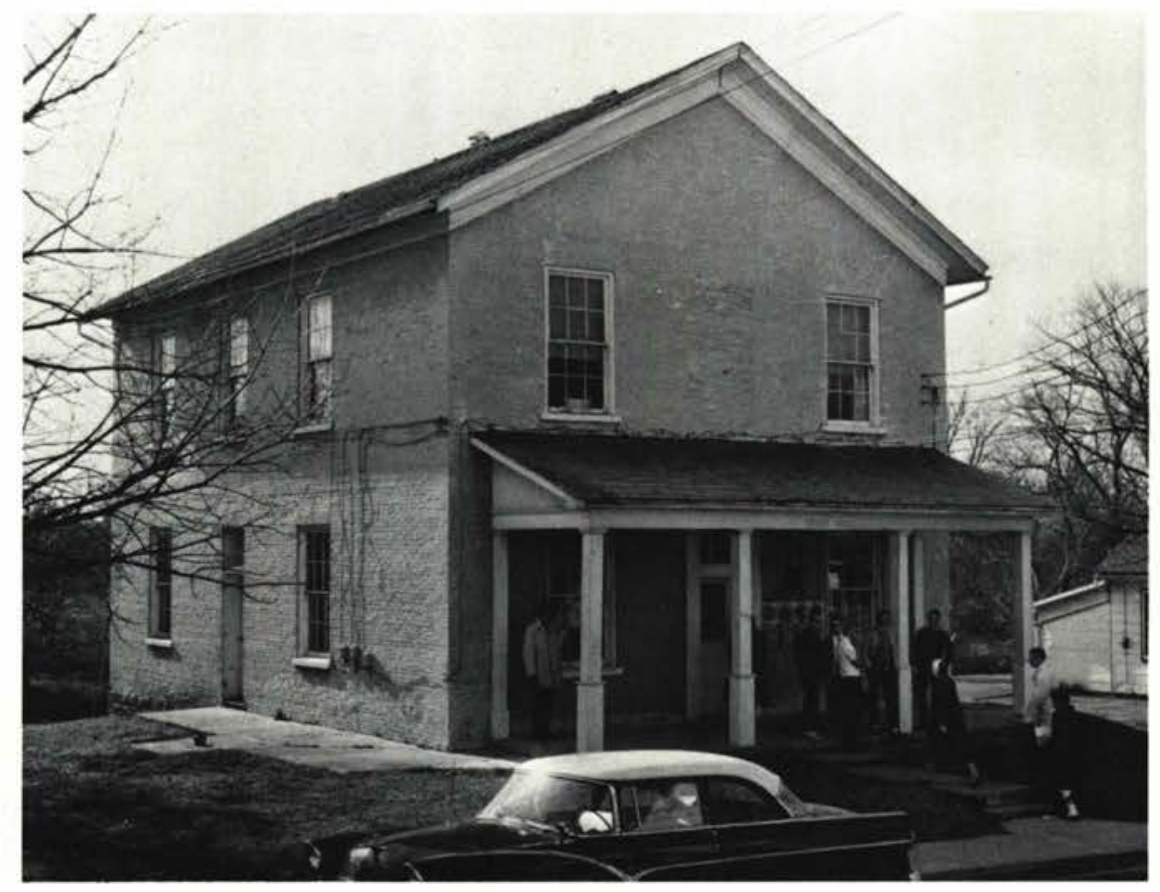

Cedar "Palace"

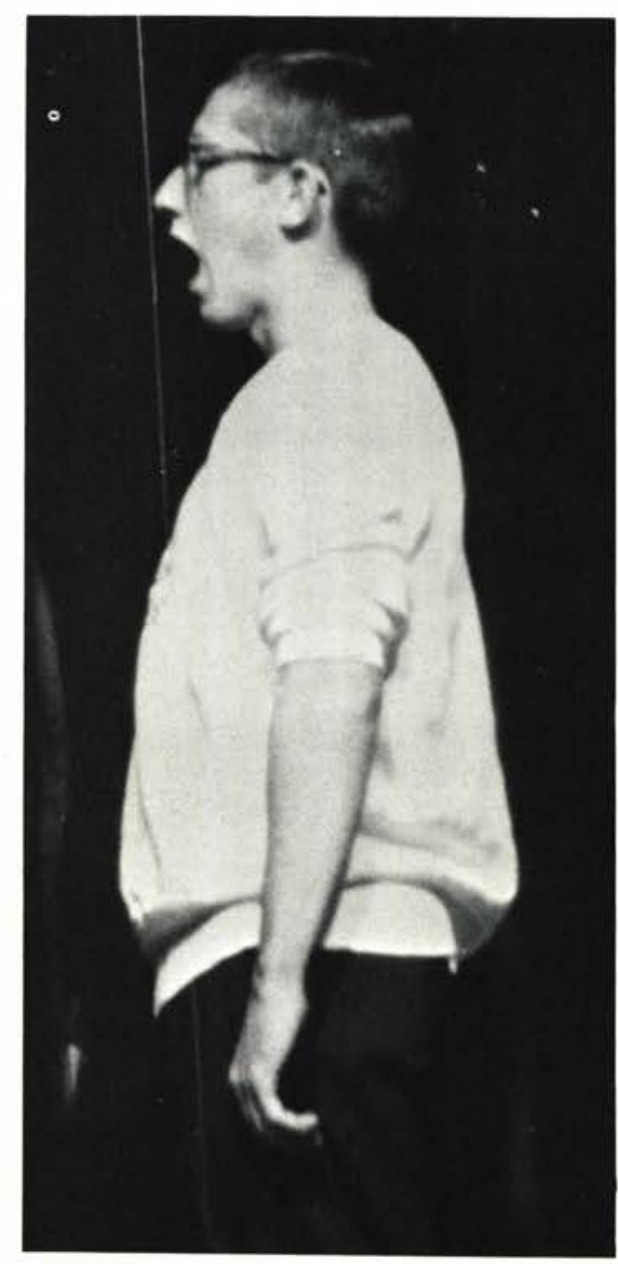

Gordy Griffin 
Abney, Barbara to Abshear, Keith Bryson Abuhl, Barbara Ruth Acker, Donald Lynn : Acker, Elton Dale .

Adams, Richard Robert Akinyemi, Sunday Emmanue

Albright, Charles Edward

Alexander, Pricilla

Allen, Deane Judson Allerton, Ronald Dwight

Amos, Richard M.

Amstutz, Lynn Carroll

Ancel, Lois Marie .

Anderson, Charles Benton

Anderson, David C.

Anderson, Eunice Irene .

Anderson, Margaret Sue

Anderson, Olivia Marie

Arment, Alan K. ...

Asthby, Janie Myrle

Auckland, Gerald Paul ...

Augsburger, Maxine $G$. .

Augspurger, Ruth Ellen

Averitt, Grace Linda

Averitt, Mark Leslie

Babcock, Lynne Charlotte

Baker, Rebecca Lovise.

Baker, Ronald Fred

Baldock, Martha S.

Ballard, Mona Lou

Balogh, Greg Lovise. . .

Bancroft, Phyllis J.

Barcus, Joanna Ruth

Barker, Jon David

Barkley, Linda I.

Barnard, Norman Joseph

Barnhart, Roy Edgar .

Barrett, Donna J. ,

Barto, Constance Lois

Baumann, Carol Elaine .

Beerer, Carol Ann .

Beerer, Mary Ruth

Beikert, Elisabeth Joyce

Beiser, Margaret-ann

Beistline, Harry Edwin III .

Beistline, Sharon Lee.

Beitter, Marilyn Ann ....

Bell, Terry Deaton.

Bellew, Larry Joe .

Bellintani, Loris Jt.

Benedict, Stephen Fred

Bennett, Martha Lucinda.

Bennett, Russell Gordon

Benton, Cheri Lou

Berg, Carolyn Louise

Bergen, Harmon

Bergsma, Marvin John

Berry, James Lee .

Bertram, Nancy Ann

Bertschinger, Betty Jean

Best, Janice Mae.

Biddle, Calvin J.

Bigelow, Judith Anne

Biller, Tom Arthur .

Billman, Donald Robert

Bird, John David . .

Bird, Patricia Marie

Bishop, Joe .

Bixel, Robert Harry

Brake, Carol Ann .

Blaylock, James L

Boblitt, Janice Lee
34509 Jerome St., New Baltimore, Mich.

R. R. 5, Springfield, Ohi

1113 W. 3rd St., Ankeny, low

.75 Jamestown St, Randolph, New York

.75 Jamestown St., Randolph, New York

3 Goldmine Rd., New Milford, Conn.

47 Lagos St., Lagos, Nigeria

R. D. 3, Fenner Rd., Shelby, Ohio

.503 W. Funderburg Rd., Fairborn, Ohio

. Box 342, N. Main St., Hurlock, Mo. Prospect, Pa.

.847 Maple Lane, Waterville, Ohio 321 Sheffield Rd., Cherry Hill, New Jersey .R. R. 1, Taylors Falls, Minn. .. 605 Roberts St., Henry, III.

1108 W. Grand Blanc Rd., Grand Blanc, Mich. 1216 Mackinaw Ave., Cheboygan, Mich. .203 E. Buehl, Forsyth, III. .239 Kimpton Drive, Berea, Ohio 3104 Stanwick Dr., Dayton, Ohio .1107 Park Ave., Chariton, lowa .4 W. Ave., Arkport, New York Baptist Mid-Missions, Cleveland, Ohio 6094 Howe Rd., R. R. 2, Middletown, Ohio

... Trailer Court, Cedarville, Ohio Trailer Court, Cedarville, Ohio R. R. 1, Box 74, Swanton, Ohio 1110 Jerry Ave., Schenectady, N. Y. 1110 Jerry Ave., Schenectady, N. Y. 1319 4th St. S.E., Rochester, Minn. 48 S. Biggs Ave., Belleville, Ohio 1000 Pollock Rd., Delaware, Ohio 403 E. Hunt St., Adrian, Mich.

376 W. Glendale Dr., Valparaiso, Ind. 2438 182nd St, Highland, Ind. 408 W. North St., Arcanum, Ohio Box 237, Kingman, Ind. 8035 Lantis-Geeding Rd., R. 1, Camden, Ohio .. 807 Harding Rd., Elkhart, Ind. 249 Mansion Ave., Audubon, N. J. 201 Palmer Dr., Cedarville, Ohio 1249 Canton St., Elkhart, ind. 1249 Canton St., Elkhart, Ind.

R. D. 4, Saxonburg Rd., Butler, Pa. .. R. D. 3, Jerseyville, III. 62 Xenia Ave., Cedarville, Ohio .62 Xenia Ave., Cedarville, Ohio 317 N. Harmony, Medina, Ohio 366 Farmersville, Pike, Germantown, Ohio 1398 Columbus St., Cincinnati, Ohio Av. 9 de julho 4760 Apt. 91, Sao Paulo, Brazil . R. R. 2. N. Mitchell, Boise, Idaho 5610 Harleston Dr., Lyndhurst, Ohio . Box 253, Homer, Alaska .. R. D. 1, East Springfield, Pa. Sunfield, Mich. 214 Leonard N.W., Grand Rapids, Mich. .439 E. North St, Medina, Otio 2401 N. 26, Boise, Idaho 1203 Indiana St., Martins Ferry, Ohio .6222 W. Spokane St., Milwaukee, Wisc. R. D. 2, Beloit, Ohio Cedarville, Ohio 5810 W. 5 th Ave., Gary, Ind. R. D. 1, Homerville, Ohio .. 834 Iralger $\mathrm{Or}$, Toledo, Ohio 960 Judd Rd., Saline, Mich. 6776 Rosecrest St., Cincinnati, Ohio .516 E. Linn St., Bellefonte, Pa. 275 Melbourne Ave., Akron, Ohio .623 Fairfield Hue, Fairborn, Ohio $6031 \frac{1}{2}$ S. Limestone, Springfield, Ohio
Boblitt, Terry Wayne

Bodden, David Edward .

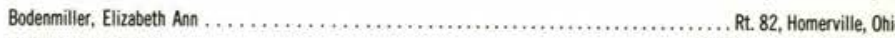

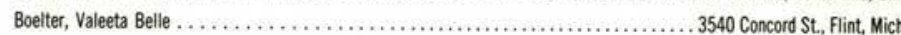

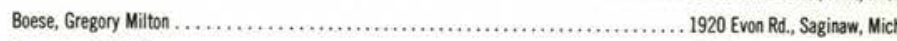

Bonnema, Frieda E. . . . ........................................ 3659 S. Ridge Rd., Perry, Ohio

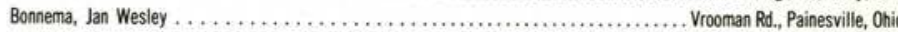

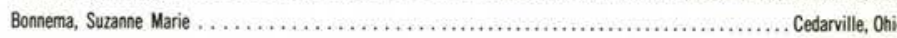

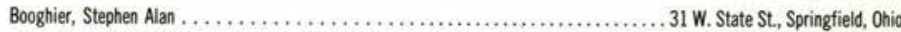

Boonstra, Ruth Ann ............................................. 83 Cedar St, Garfield, N. J.

Bowersox, Bernice W. ........................................... 561 Leslie Dr., Xenia, Ohio

Bowersox, Richard A . . . . . . . . . . . . ................................ 561 Leslie Dr., Xenia, Ohio

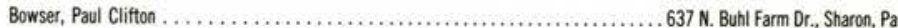

Boyd, Dan Ray. R. R. 1, Argenta, III.

Brake, Donald Lee

Branch, Herbert Gardner

Colchester, Colchester, III.

................................... 14816 Tudor Ave., N.W., Cleveland, Ohio

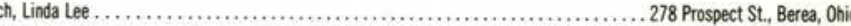

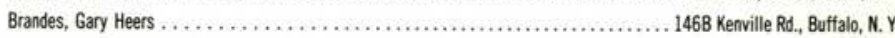

Branon, Jack Kent ..........................................1139 Normandy Dr., Portsmouth, Ohio

Brewer, Margaret Ann

259 Jackson Pike, Gallipolis, Ohio

289 Bennett Rd., Cheektowaga, N. Y.

Brinckerhoff, Carol A

289 Bennett Rd., Cheektowaga, N. Y.

1636 Ongaro Dr. Columbus, Ohio

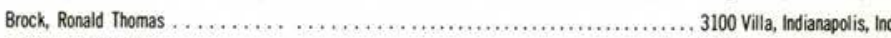

Brookhart, Philip Dean ....................................... 1756 Breese Rd., Lima, Ohio

Brown, Jonathan $E$.

691 Wainut St., Cedarville, Ohio .307 North St, Holly, Mich.

.47 West St., Hornell, N. Y.

Brown, Martha Valorie

Brown, Maynard Ben

Brown, Stephen Paul

Brown, Thomas Wesley

Buckle, Marian Jane.

Burcher, Bryan Jay .

Burcham, Carolyn Fern

Burns, Dennis Lee.

Burns, Warren William

Burroughs, Kristine .

Bush, Deborah Florence.

Bush, Dorinda Rae

4 Lawnview Ave., Springfield, Ohio

208 N. Mantorville Ave., Kasson, Minn.

718 N.E. 4th St., Eagle Grove, lowa

400 Irwin St., Lock Haven, Pa.

.. 3315 Ridgewood Dr., Parma, Ohi R. R. 2, Mattoon, III.

.2131 Main St., Central Lake, Mich.

4527 Hessen Cassel, Fort Wayne, Ind .2615 Wicker St., Highland, Ind.

Cady, Audrey Jean . 2749 Northampton Ro.., Cuyahoga Falls, Ohio 2749 Northampton Rd., Cuyahoga Falls, Ohio

Cagwin, Raymond $\mathrm{H}$.

Campbell, Hugh Wallace Troupsburg, N.Y.

Campbell, Lois Kathleen .

Campbell, Robert DelRoy

Canada, William Richard .

Cannady, Dorothy Margaret.

Caraway, Daniel Guy

Carlberg, Patricia Ann

Carlson, Beverly Jeanne

Carpenter, Timothy James 9961 Jennings Rd., Eden, N.Y.

23 Rio Grande Ave., Irotwood, Ohio

.. 699 Trebisky Rd., Cleveland, Ohio R. D. 3, New Bethlehem, $\mathrm{Pa}$

22701 So. Riverdale Dr., Detroit, Mich.

37 Cadmus Ave., East Patterson, N. J. 3533 Park Dr., Covington, Ky.

145 Hazeltine Ave., Jamestown, N. Y.

2570 Pangborn Circle, Decatur, Georgia

Carroll, William M.

R. D. 1, Gerry, N. Y.

Carter, Dozier...

Carter, Kathy Ann

Cartner, M. Kathleen

Chaffe, Dianne Lovise

Chapman, Judith Ellen . .

Chilton, Betty Jo......

Chumbler, David Willard

ill Ave., Buffalo, N.Y

1126 15th St., Portsmouth, Ohio R. R. 2, Winterset, lowa 16522 Plainview, Markham, III. Cedarview Lane, Cedarville, Ohio 414 Hedgewood Dr., Gallipolis, Ohio

Clark, John Martin

Clark, Steve R.

Clater, Robert Dean

Clater, Theodore $\mathrm{F}$.

Cleckner, Barbara An

Clem, Douglas Rax. ...

Clithero, Donley Wright

Colgan, Esther Lowise

Collins, Cherrill Lynn

Comden, James Anthony

Compton, Gary Lee .

Conant, Joy Katherine

Constant, Harold Eldon . .

Cooper, Delores

Cooper, Donald William
2716 Logan Ave., Des Moines, lowa

5361 Marcella Rd., Gary, Ind.

604 N. Sycamore St., Creston, lowa 110 N. Columbia St., Warsaw, Ind. 110 N. Columbia St., Warsaw, Ind.

.. 1001 E. 10th St., Salem, Ohio

61 Thornton Dr., Fairborn, Ohio 5536 Kentiand Ave., Sciotoville, Ohio 5 Hope St., DuBois, $\mathrm{Pa}$ Caixa Postal 2612, Sao Paulo, Brazi R. R. 1, Lakeview, Mich. 153 10th St., Silvis, III.

425 Terrace Ave., Hasbroock Heights, N. J. 2801 So. Fed., Del Ray Beach, Fla. 626 Lamont Or., Kettering, Ohio R. R. 2, Belle Center, Ohio 
Cooper, Gwytha D. .

1058 Lester St., Ypsilanti, Mich.

Corder, Sandra Gayle .

Coriell, Ronald James

Corn, Larry Allan .

Cornett, James Lowis .

Cosgray, Robert Eugene .

Courtot, Armand Max . .

Cox, Martha Lovise

Crabb, Alvah A.

Crabtree, Janet Lee.

Crampton, Dennis .

Crampton, Michael

Crampton, Roger Gary

Crider, Glenda Lee.

Crow, Judy L

Crown, Patricia Karen

Culberson, Lyle .

Culley, Ronald Eugene.

Culley, Linda R.

Cummings, Marsha Louise

Curtis, David Allyn

Curtis, Mary Jane.

Cushey, William

Cypher, Kay Ellen

Cypher, Richard Earl .

Daab, Donna Sue

Damon, Ann .

Daniel, Kenneth Eric .

Darrow, Gary Robert

Daubert, Russell Eugene

Davis, Andrew Lewis

Davis, Nancy Lynne ...

Davis, Raymond A.

Davis, Richard T. .

Dawes, Dennis F.

Day, Juanita June

DePuy, Dianna Lee

Devaney, Ken Ray .

Dewar, Barbara Jea

Dillon, Berdine

Dillon, Dallas James

Dillon, Leslie S.

Dillon, Robert

Dixon, Larry Lee .........................................., 135 N. Limestone St, Springfield, Ohio

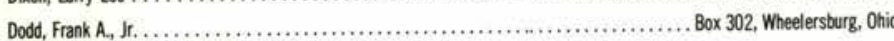

Donaldson, Karen Ann

Donovan, Richard L. .

Draxler, David William

R. R. 2, Continental, Ohio

19 Ohio St., Fairborn, Ohio

263 Oakdale Rd., Berea, Ohio

Drill, Gary Lavern

Du Bois, Janice E.

419 Residence St.. Winchester, Ind.

1215 County Rd, Milan, Mich.

.P. 0. Box 63, Roulette, Pa.

Dudley, Eunice .

Duff, Thomas Michael

719 Stounton Rd., Troy, Ohio

R. R. 1, Shell Rock, lowa

Duftord, Shirley Belle

Dugan, Virginia A.

6965 W. 29th St., Denver, Colo.

Dunn, Ruth Damaris

154 E. 214 St., Euclid, Ohio

Dunstan, Donald Lee

R. D. 2, Randolph, N.Y

Durkee, Dariene Ann

Dutiel, Pamela Lee .

Earl, Pamela V. .

Earles, Nancy Carol

Eaton, Jerry M. .

Edwards, Dorinne

Edwards, George John

Edwards, Suzanne Leslie

Elkin, June Ellen

Elliott, Thomas

Elmore, David Edward .

Emerson, Janice Marie .

Emmons, Carole Sue

Empet, Sara Ellen

Englemann, lames $\mathrm{D}$.

Engle, Sharon Faith ....

Entenman, Emily Jean

Entner, Paul Dwight

Falci, John Joseph

Farrell, Mary Kathleen

S.E., Byron Center, Mich. 628 S. Scott St., Lima, Ohio

10 Garrison Ave., Hasbrouck Hgts., N. J. 3332 Hiwood Ave., Stow, Ohio R. R. 2, Rochester, Ind Sharon Springs., N.Y. .. Cedarville, Ohio .. R. D. 1, Albion, lowa

64 Lyndale Dr. Painesville, Ohio .6541 W. Marlette St., Marlette, Mich.

12130 Dearmyer Rd., Brooklyn, Mich. 14542 Park Ave., Harvey, III. R. D. 1, Kingsley, Pa. .2159 N. Belsay, Flint, Mich. .406 W. George St., Arcanum, Ohio ... Evansville, ind. 304 S. Franklin, Dunkirk, Ind. .49 Hill St., Homell, N. Y 237 W. F. St., Phillipsburg, Kans.
Felton, Mary Louise 1966 Arlene Ave., Dayton, Ohio Fenton, Stephen Orville 1316 Huntington Rd.. Waterloo, lowa

Ferris, Joyce Elaine

Fidler, David

Field, Jennifer Lynn

Fisher, Gerald A. . . . .

Fissel, Nancy Jane

Fitch, Darlene Mae .

Fleming, Barbara Jean

Flury, Gerald George .

Flynn, Elizabeth Jean

Ford, George Lee.

Foster, Dale $\mathrm{H}$.

Foulkrod, L. Beverly

Foulkrod, Thomas F.

Fourman, Nancy Carol

fox, Loris Walter .

Fox, Sheryl .

Francis, William Allan

Fransted, Gary M. .

Freeman, Nancy Faith

French, Gerald Arthur

French, Shirley Joy .

Fries, Stanley Robert.

Frink, Mary Lynn

Frost, Terry Jean

Fruchtenbaum, Arnold Genekovich

Fulop, Patrica June

Gaffner, David Charles

913 N. Elm St., Sandwich, III. . 5956 Minnesota St., Hobart, Ind. 1708 Park Bivd., Cherry Hill, N. J. 324 W. North St., Lebanon, Ind. P. 0. Box 116, West Liberty, Ohio 1733 E. Washington St., New Castle, Pa. .410 N. Maple St, Lancaster, Ohio 3195 W. 112 St. Cleveland, Ohio .. Baumhart R. D. 1, Amherst, Ohio .25 Milburn St. Cedarville, Ohio 25260 akwood Dr., Ft. Wayne, Ind. .83 West Elm, Cedarville, Ohio .83 West Elm, Cedarville, Ohio 3693 Oneida St. Stow, Ohio Cedarville, Ohio Cedarville, Ohio R. R. 1, Koutz, Ind. 420 S. Dettman Rd., Jackson, Mich. Prairie Bible inst. Three Hills, Alberta, Canada .. Box 25, Marathon, N.Y. 905 Craft Ave., Kalamazoo, Mich.

712 E. Woodland Ave., Maple Shade, N. J. .6423 Arizona Ave., Hammond, Ind. .. 93 W. Main St., Ontario, N.Y. 6 Parkside Dr., Levittown, LI., N.Y.

R. D. 1, Huntley, Windsor, Ohio

Gail, Claudia Jean

136 W. Church St., Williamston, Mich.

Galt, Charles Irving .

Gann, W. Joyce

P. 0. Box 116, Fowler, Ohio

4305 English Ave., Erie, $\mathrm{Pa}$.

Gapinski, Donna Grace

Gardiner, Elizabeth Louise .

Garlich, Patricia Lee .

Garlock, Linda Sue

Gatiliff, Gail Sue

Gavitt, E. Leroy.

Geer, Wynona Lee

5 W. High St, Jackson, Mich.

1627 Sixth St., Perv, III.

716 Arcadia Blvd., Englewood, Ohio R. R. 322. Huntsburg, Ohio R. R. 4, Allegan, Mich, . R. R. 2, Rochester, Ind.

1020 Center St. West, Warren, Ohio

Geritz, Elizabeth L.

Gerringer, Charles R.

Gibb, William James, Jr.

Gridley, David

Gierhart, Glenda Sue

Gisel, Donna Marie

Cleveland Heights, Ohio

R. D. 1, Coudersport, Pa.

221 S. 7th St., Upper Sandusky, Ohio

1048 White Ave., Brownsburg, Ind.

Glander, Verna L.

1703 W. 7 th St., Hastings, Nebr, Box 14, R. R. 2. Archbold, Ohio

Goddard, Marilou .

31 Carroll St., Random Lake, Wisc.

Goethals, James Richard

Goins, Diane Marie

Goldboure, Thomas Harold

Good, Patricia C.

Goodenough, Ervin Dale

Goodman, John Walter

Goodwin, George Raymond

Goodwin, James Warren

Gorney, Sonya .

Gordon, E. David .

Gothard, Rebecca

Gower, John Walton

Grable, Marylynne

5537 Wilson Ave., Portsmouth, Ohio 51894 Elm Rd., Granger, Ind. .9815 Spring St, Racine, Wisc. 3130 12th St., N.W., Canton, Ohio 318 Lamont Dr., Eggertsville, N.Y. .6885 Hart Rd., Saginaw, Mich. 2427 Henn-Hyde Rd., Warren, Ohio 5142 W. 151 st St., Oak Forest, ill. 5142 W. 151 st St., Oak Forest, III. .Tuckahoe Rd., Vineland, N. J. Cedarville College, Cedarville, Ohio 2635 Hemlock Dr. Springfield, Ohio 2141 Micklethwaite, Portsmouth, Ohio 2810 Urbana Dr., Wheaton, Md.

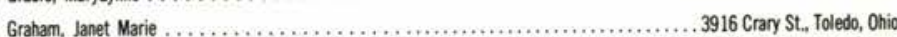

Grant, Ruth Eilene

Gray, Gwendolyn 10.

Greenwood, Thomas Paul 2411 3rd St., East Moline, III. 2408 Blossom St., Joliet, III. 4566 Linda Lane, Lorain, Ohio

Gregg, Julia Ann . R. R. 1, fulton, III.

Gregg, Peggy

Gregory, David E.

.9 Clark St, Honesdale, Pa.

Gregory, Michael Fredric .

9 Clark St, Honesdale, $\mathrm{Pa}$.

Greiner, Miriam Lucille

R. D. 3, Wellinton, Ohio

Griffes, Eunice Mae

4436 River St., Madison, Ohio

Griffin, Gordon D.

Box 504, Carroll, lowa

Griffin, Sylvia Kay

3027 Mounds Rd, Anderson, ind.

Griffiths, Penny Jean

39 Central, Apt. 109, Dayton, Ohio

Grisso, Philip Daniel

1270 Perry St., Springfield, Ohio

Gromacki, Gloria Gay

1823 Kylemore Dr., Xenia, Ohio 
Grosh, Barbara Lee

Grosh, Ronald Milton

Gunlock, James Richard .

Haffey, David Allen

Hall, Floyd Mason

Hall, Terry Lynn

Halvorsen, James

Hamacher, James $G$.

Hamilton, Donna Marie .

Hamilton, Michael Jay

Hamilton, William James

Hammer, Arnold Dayton

Handyside, David Eward

Hanna, Carol Jean

Hannah, Dorthy Elaine ...

Hanson, Carol Jean

Haram, David L.

Hardy, Ruth Ann

Hare, Carol Margaret .

Harper, Nancy Jo

Harris, Gary James

Harris, Jane Ann .

Harrison, Gerald L. .

Hartikainen, Ruth M.

Hartzell, Martin Eugene

Hassennuck, Harold

Hatcher, Franklin R.

Hatton, Alice K.

Hawotte, Paul William . .

Hay, James $R$.

Headlee, Catherine Doris

Healy, Linda Eleanor . .

Heater, William $R$

Helmick, James $R$.

Hendrickson, Rose Emily

Henniger, James Ray ...

Herrin, Gary .

Hes, Nancie 1.

Hess, Jane Harriet .

Hess, John Harvey

Heyer, Raymond Neal

Hiller, Doris Mae

Hillman, Howard Jon

Hockenberry, David Allen

Hoffman, Linda Lou

Hoffman, Robert Francis .

Hogan, Donna Elaine

Holland, Donald Glen ...

Hollaway, Joseph E.

Holloway, Linda $D$.

Holmes, Dale Alton

Holtz, Gary Wayne

Holz, Carl Wayne

Homan, Carolyn $\mathrm{J}$

Hooper, Vernon 1.

Hopkins, Donna Jean

Hopkins, Kenneth Joy

Hooper, Ernest R.

Horwath, Audrey Dianne

Howard, S. Anne

Howard, Charles Kenneth

Howard, Linda Jean .

Hoyte, Arlene Elizabeth

Hubbard, Cathy Elizabeth

Hudson, Nancy Kay .

Huebner, Glen M.

Hull, Charles Olin

Hume, James Douglas

Hunter, Charles Eugene

Hunter, Linda Carroll .

Hunter, Robert Frederick .

Hunter, Sharon Ann .

Husted, Lucinda J.

Hutcherson, Betty Jean

Ice, Lawrence Russell, Jr.

Ingrham, Peggy lane
14921 Bennett Rd., North Royalton, Ohio 14921 Bennett Rd., North Royalton, Ohio 426 Bridle Lane, Wheaton, III.

Gender Rd., Canal Winchester, Ohio 1913 Locust St., Toledo, Ohio .1376 E. Decatur St., Decatur, III. 2014 Nixon Ave., Eau Gallie, Fla. 3123 Brown St., Aiton, III. 1073 Urana Ave., Columbus, Ohio R. R. 3, Jefferson, Ohio 9313 Peterson St., Rockford, Mich. 1271 Turk Hill Rd., Fairport, N. Y. R. R. 1, Cedarville, Ohio 2038 Madison St, Farrel, $\mathrm{Pa}$.

R. R. 1, Box 25, Union Grove, Wisc. 116 Xenia Ave., Cedarville, Ohio 726 S. Jackson St., Mashawaka, Ind. 217 Palmer Dr., Cedarville, Ohio R. R. 1, Lorimor, lowa 247 S. 10th Ave., Beech Grove, Ind. 325 Ivanhoe Rd., Waterloo, lowa 3604 Scribner Dr., End Well, N. Y. 3437 Peach St., Erie, Pa. 646 Pittsburg Ro., Butler, Pa. 4223 Canterbury, N. Olmsted, Ohio 1025 Kumler, Baltimore, Ohio .41 Charles St, Franklin, Ohio 328 40th Ave., East Moline, III. Box 127, Addis Ababa, Ethiopia 700 Sherwood Or., Crawfordsville, Ind. Moscow, lowa 341 Davis St., Findlay, Ohio 4420 Sec. St., Canton, Ohio R. 3, Box 122, Cedar Lake, Ind. 1050 Knolliwood, Canton, Ohio Rt. 1, Kouts, Ind.

6616 Royalton Rd., North Royalton, Ohio 135 Washington, Bunker Hill, III. 19844 Southland, South Bend, Ind. 630 Ohio St., Webster City, lowa 14515 Westropp Ave., Cleveland, Ohio 1045 E. Carleton Rd.. Adrian, Mich. 974 Antioch Dr., Fairfield, Ohio 136 State St., Gallipolis, Ohio 2919 Ensley Ave., Dayton, Ohio 1291 E. 143rd St., E. Cleveland, Ohio 1832 Jefferson Rd., Otsego, Mich. 320 Deerfield Rd., Columbus, Ohio R. R. 2, Mancelona, Mich R. R. 1, Litchfield, Ohio R. R. 2, Muscatine, lowa 4000 E. Cohcotah Rd., Linden Rt. 1, Mich. 1771 Hale Ave., ft. Wayne, Ind R. R. 2, Sabina, Ohio 316 W. Pleasant St., Corry, Pe R. R. 3, Box 159, Parma, Idaho 1805 S. Main St., Corbin, Ky. 3095 Tittabawassee Rd., Saginaw, Mich. .. Traer, lowa 3901 Vannest St, Middletown, Ohio .R. R. 3, Diebler Rd., Bucyrus, Ohio .76 Circut Dr., Binghamton, N. Y 1616 Diamond Lake Rd., Glastonbury, Conn 2310 E. 56th St., Indianapolis, Ind. 3634 N. 24th PI., Milwaukee, Wisc. R. D. 4, Box 88, Cortland, Ohio 3209 Delaney St., Dayton, Ohio R. R. 2, Jesup, lowa 4146 Oxtord Or., Brunswick, Ohio .1293 W. Forest, Decatur, III. 1293 W. Forest, Decatur, III. 219 Scott St., Ankeny, lowa 10717 Oak St., Taylor, Mich. Southland Bible Inst, Pikesville, Ky. Morning Sun, lowa
Jackson, W. Paul

1505 Canadian Ave., Akron, Ohio Jager, Ted, Jr. 92 Nueville S.W., Pine Gate Mobile Vill, Grand Rapids, Mich. Jamison, Sharon Lee . G-3540 Concord St., Flint, Mich. Jefferies, William Garfield 227 Maple Ave., Clarksburg, W. Va. Jenista, Frank L. . Jenista, Ruth Allyn . Jensen, Cynthia Louise . . . Box 119, Iloilo City, Philippines 905 E. Dawes St., Wheaton, III. Jensen, Val Jon . 2016 th St, N.W., Austin, Minn, Johansen, Sharon Alice . 27 North Dr., Buffalo, N.Y.

Johnson, Elaine Marie 1209 Condover Rd., Richmond, Va. Johnson, Gladys I. R. R. 1, Cedarville, Ohio Johnson, Grace Phyllis ........................................ 547 Merrioaks Rd., Barrington, III. Johnson, James Raymond 492 Bridges St., Cedarville, Ohio Johnson, Joyce May

Johnson, Kathleen J. 1772 Fay Ave., East Cleveland, Ohio

Johnson, Philip Roy

718 16th St. East, Seattle, Wash. Jones, David Alan Jones, Mary Elisabeth 3760 E. William, Decatur, III. Jones, Merlyn Earl . 1137 S. Thomas St., Arlington, Va. Jones, Sandra Lee. ... Kaiser, Sheryl M. 729 New St, Stanton, Mich, Kapp, Marion Elizabeth St, Concord, Calif. Kauffold, Ruth Elizabeth . . R. 2, Box 190, Plymouth, Ind. Keating, Janice Ann

Keib, Donna Lee R. 3, Lake Pana, Pana, III.

Keib, Henry Walter.

Keim, Alan L. . . .

Kelley, Samuel Keith

Kensil, Barbara K. .

Kever, Janet Elaine

Kincannon, Donna Rose R. 1, Wausaukee, Wisc. Oak St., Shellby, Ohio Oak St, Shelby, Ohio R. R. 1, 80x 34, Holsobble, Pa. . 70 Champion Rd., Fairborn, Ohio

King, John William 440 S. Westlawn Ave., Decatur, III. King, Marion King, Robert Dennis ville, Ohio

Kingery, Nancy Ann

Kinniburgh, Daniel G.

Kirchner, David .

Kirchner, Dennis E.

Kintz, Michael LeRoy

Kleymeer, Joy Lovise

Knott, Allen Dale

Korlewitz, Elayne

Krueger, Edwin Richard

Kunkle, Kenneth Richard . .

Kunselman, Karen Lee .

Kytle, Laurene Marye .

Lambert, Gerri Anniece

Lambert, Joseph E.

Lane, Lloyd Leon

Larrick, Lynda Carlene

Lautenschlager, Daphne J.

Lavener, Dale

Lawhead, Cheryl Lee

Lawhead, Patti Ann .

Layner, Clifford Franklin

Leach, Donald Paul .

Leach, Roger Dale .

Leasure, Paul Richard

Leiby, Stephen Paul

Lennox, Jessie M.

Lepine, Suzanne Ruth

Lescure, Lynn Lovise

Lewis, Carol Ann .

Lewis, Nancy Lynn .

Lightly, Dougias Arnold

Lloyd, Kathleen May

Lobel, Carol Jane.

Lockhart, Mary Anne

Louwerse, Marsha Marie .

Lowe, Beverly Ann

Lowe, Donald Carl

Lucius, Robert Bruce

Luttrril, Mary Alice.

Maclaren, Wenda June

Macpherson, Bonnie L . .

Maidment, Lee Robert .

Cedarville, Ohio

.926 Worchest, Fenton, Mich.

.7150 Alexander, Hammond, Ind Marion, Mont

610 W. 43 rd St., Chicago, III. 610 W. 43 rd St., Chicago, III. 21 Cherry St., Colfar, lowa

850 Prince St., Grand Rapids, Mich. 158 7th St., Silvis, III.

119 Lindbergh Ave., Johnstown, $\mathrm{Pa}$ 8554 Valley View, R. D. 1, Macedonia, Ohio .22010 Ave., Silvis, III. R. D. 3, Box 225, DuBois, Pa. 22810 Ivan Ave., Euclid, Ohio $761 / 2$ Xenia Ave., Cedarville, Ohio R. R. 1, Box 217, Garland, Tex R. R. 5 , Coldwater, Mich,

515 W. Main St., Blanchester, Ohio .3235 Highway, Highland, ind R. D. 2, Oberlin, Ohio .715 Poplar St., Coshocton, Ohio .715 Poplar St., Coshocton, Ohio Little Hocking, Ohio

126 Ketay Dr. South, East Northport, N. Y. .. R. R. 2. Camp Point, III.

1356 A Coopermill, Zanesville, Ohio 1100 Saratoga St., Fairborn, Ohio 5914 Middlebrook Blvd., Cleveland, Ohic 106 Westbrook Dr., Cheektowaga, N. Y. 1545 Chestnut Ave., Haddon Heights, N. J. 27430 Forestview Ave., Euclio, Ohio 430 South Berkley, Kokomo, Ind. .. R. R. 3, Box 184, Austin, Minn. 3403 13th St., Racine, Wisc. 1955 Short Rd., Saginaw, Mich. .1226 Maro St., Sciotoville, Ohio 
Males, Nancy Jane

524 First St., Du Bois, Pa.

Mansfield, M. Katherine

Marcellus, Charles R.

5191 Broadway, Lorain, Ohio

Marsh, Candace Gail ...

12013 Vaughan, Detroit, Mich.

Marsh, Lynnette Marie

Marshall, Dennis Lyle .

Marshall, Steven Leon

Martens, Ted David .

Marvin, Lois Elaine

Masemore, Jayne Ade

May, John Allen

Mayo, Shelby . ...

McClintock, Judith Anne

McCullough, Mike Thomas .

McDonald, Bruce Wayne.

McDonald, Dave Alan

McGee, Catherine G.

Mcthee, Kenneth Richard

McGillivray, John A. .

McGinnis, Thomas Allen

McGutfey, Manford Earl .

Mclnnes, Roberta Jean

Mcintyre, Ruth Eileen

McKenney, Roger $\mathrm{H}$.

McKnight, Rick James .

Mcleod, Johanna C.

McMahan, Cecil D.

McNeill, Barbara Jeanne

McPheeters, Buster Mayhew

McVey, William Lee

Mehaffey, D. Gregor

Mellish, Donald J.

Mellish, Ronald Norman

Merryman, Valerie Le

Meshew, Helen Viola

Meyers, Betty Mae

Micka, Joyce Elane .

Mikels, Carol Joyce

Miller, Barbara Lou .

Miller, Debra Ellen

Miller, Freddie Lee .

Miller, James $\mathrm{E}$.

Miller, James Richard

Miller, Janice Alene

Miller, Linda Jean

Miller, Priscilla Jean

Miller, Roberta Raye ....

Millikan, Bonnie Louise

Millikan, Irene Alice.

Mitchell, Charles W.

Mitchell, Lola Lovise.

Mitchell, Marilyn Kay

Mogle, Claire Ann .

Montgomery, Lawrence

Moore, Harold James.

Moore, Joyce Wood

Morgan, Carol Kay

Morgan, Donna Rae

Morrell, Thomas Ed . .

Mosley, Beverly Kay

Mossman, Janice Louise

Muck, Kenneth Arlington

Mulford, Arlan Charles

Music, Ernest Wendall

Myers, James Arthur

Myers, Leonard Lee

Myers, Susan Allen

Nance, Karen S.

Nash, Charlotte K.

Navorska, Becky An

Nevitt, Dan P. . . R. D. 4, Corry, Pa.

118 W. Grant St., Bremen, Ind. 118 W. Grant St., Bremen, Ind.

457 Van Dyke Ave., Haledon, N. J. 128th St., Grant, Mich.

R. R. 1, Box 170-A, Scherevville, Ind R. D. 4, Box 158, Stroudsburg, $\mathrm{Pa}$ 1025 Sheffield Dr., Mason, Ohio .3131 Wareham Rd., Columbus, Ohio .501 South Carroll, Michigan City, Ind 3365 Lake George Rd., Lake Orion, Mich. 3365 Lake George Rd., Lake Orion, Mich. .2821 Goolsby Ave., Richmond, Va. 119 15th St., Silvis, III. 134 Carlisle Ave., Springfield, Ohio . 3401 Eichelberger Lane, Springfield, Ohio .5295 Columbia Rd., Mason, Ohio R. R. 3, Columbia City, In 20 South Barrett Ave., Audubon, N. J. 15355 Evergreen, East Detroit, Mich. .638 Virginia Ave., Bucyrus, Ohio .82 North St., Stanford, Conn. 1384 University, Lincoln Park, Mich. 2525 Larwood Dr., Charleston, W. Va. .690 Wainut St., Cedarville, Ohio . 2733 N. 52 nd Ter., Kansas City, Kans. 101 Stuyvesant Rd., W. Englewood, N. J. 1715 Wesley Ave., Evanston, III. 26 Gracey Blvd., Weston, Ontario, Canada 1137 Queen Rd., Teaneck, N. J. 230 Ackerson Lake Dr., Jackson, Mich. 4336 7th St., East Moline, III. 123 Paulson Ave., Ridgefield, N. J. 6102 N. Dearborn, Indianapolis, Ind. 220 Vincent Ave., Troy, Ohio 192 East Second St., Xenia, Ohio 4125 Selma Pike, Springfield, Ohio 2456 Richwood, Pontiac, Mich. R. R. 2, Box 32, Valparaiso, Ind 226 North East St., Medina, Ohis 3282 Revion P1., Kettering, Ohio 3009 E. Ovid, Des Moines, lowa 6570 McGuffey Rd. Ext., Lowellville, Ohi 22605 9th Ave., Bothell, Wash. 226059 th Ave., Bothell, Wash. .40 Highland St., Sharon, Mass. 2113 North Phillippi, Boise, Idaho R. R. 1, Algona, lowa 302 N. Detroit St., Xenia, Ohi 350 Tibet Ro., Columbus, Ohio 506 W. Wisconsin St., Portage, Wisc. 77 Sunset Ave., Mattituck,N.Y. 705 Division St, Albion, Mich.

207 So. Joslin St., Charles City, lowa 201 N. Main St., Arcanum, Ohio .2530 Grace, Melvindale, Mich.

R. R. 1, Box 104, Jamestown, Ohio 126 Harriaon St., Elyria, Ohio Fenner Rd., Troy, Ohio 11428 Beaveriand, Detroit, Mich. .671 High St., Van Wert, Ohio . .6 Warren St., Marathon, N. Y. 3525 Swittwater Lane, Chester, $\mathrm{Pa}$, .13506 Callender, Southgate, Mich. 30 N. Conn. St., Hobart, Ind. R. O. 4, Box 158, Ashland, Ohio .. 612 Henry St., Henry, III.

Newman, Robert Wesley

Newton, David Edwin . .

Nichols, Kenneth Warren ... 21 Packer Ave., Towanda, $\mathrm{Pa}$.

Nicholls, Michael Lee Nicoson, Sydney Kay
237 Euclid Ave., Byesville, Ohio R. R. 2, Carlisle, lowa 1513 W. 12 th St., Loveland, Colo.
Nims, David Arthur

Nelson Star Route, Keene, N. H.

Norris, Maynard Ray

Norton, Nancy Elizabeth

Oakley, Hugh Theodore, II

Ogrodowski, Joseph George

O'keefe, Rebecca Sweet

Okresik, Ronald Wayne

Olsen, Dorothy Anne

Olsen, Fred Paul

Olsen, Philip I.

Osborn, Jerry L.

O'Shell, Constance E.

O'Shell, Suzanne Elizabeth

Oswald, James Oliver

Otto, Shirley Marie

Overholt, Kenneth Gene

Packard, Calvin Porter

Pafford, Alan Ray

Panczenko, Inna

Patterson, Karen Eileen

Patton, Darlene Evelyn ...

Paul, Roland Edward

Payne, Charlotte A.

Pearce, Dennis I.

Pereira, Manny

Pereira, Robert William

Perrin, Bonita Lou

Perry, Keith $D$.

Perryman, Pamela Jean

Pestel, Gary Michael

Pettitt, Richard Norman

Phelps, James R.

Phillips, Judy Evoynne

Phipps, James Ronald . .

Phipps, Ronald D.

Picton, Janet Lee

Pierson, Kenneth Roy

Pinkley, Dale Wayne

Plough, Dianna Kay

Powell, Lorin Alexander

Prather, Eugene Kenneth

Preston, Donna Lee

Price, Daniel Lee.

Price, Nedra Ann

Prisinzano, Dennis Paul

Pritchett, Dale Robert.

Prosser, David H.

Pugh, Catherine Joann

Pugh, Margaret Louise

Pyles, Elizabeth Geraldine

Pysarchyk, Martha Aan

Rader, Rebecca .

Rader, Suranne .

Rakes, David Allen . ...

Ramsey, Joy Ann . .

Rathfon, Yvonne Sue

Rathman, Jill

Rav, Karen Colleen

Redlecki, Jerrold Le . ...

Reed, Carol Sue

Reed, Charles R.

Reed, David Charles

Rejiester, James Leo

Renner, Jill Ann

Ressler, Ruth Ann

Retei, Carolyn

Rexroth, Thomas Allen

Richardson, Gerald $\mathrm{H}$.

Richardson, Lawrence $\mathrm{C}$

Rieken, Marlene Joyce

Riggs, Barbara Gail

Riggs, Linda Sue

Risko, Jeanne Evely

Robinson, Richard Wayn

Rock, Sylvia Surena

Rockwell, David May

R. R. 3, Rochester, Ind.

535 Church Rd., Elkins Park, Pa. 416 Cronkright St., Midland, Mich. Cedarville College, Cedarville, Otio 1203 Brookview Blvd., Parma, Ohio 15554 Ridgeway Ave., Markham, III. Apartado 3550, Lima, Peru, S. Amer. Agartado 5575, Lima, Penu, S. Amer.

12 Chillicothe, Cedarville, Ohio

1136 N. Detroit St., Xenia, Ohio 8970 Lindberg Blvd., OImsted Falls, Ohio .8970 Lindberg Blvd., Olmsted Falls, Ohio

R. R. 5, Millersburg, Ohio Poplar Motel, Carroll, lowa 807 N. Blair, Royal Oak, Mich. 2060 Miami Rd., Euclid, Ohio 1601 "D", Hannibal, Missour 519 Drexel Blvd., So. Milwaukee, Wisc R. R. 3, Coshocton, Ohio 1614 Holyoke Ave., E. Cleveland, Ohio .8131 Chamberhill Rd., Harrisburg, $\mathrm{Pa}$ .4214 S. Logan St., Englewood, Colo. R. R. 2, Box 417, Stanwood, Wash. 50 E. Northport Rd., Kings Park, N. Y. .50 E. Northport Rd., Kings Park, N. Y

4191 W. 23rd St., Cleveland, Ohio 20310 th Ave., Silvis, III. .406 3rd Ave. S.E., Altoona, lowa 516 Sabin Ave. E., Hadysmith, Wisc. 510 W. Front St, Florence, N. J. 30 Jutland Ro., Binghamton, N. Y. 2805 So. 111/2 St., Terre Haute, Ind 3115 lda Dr., Concord, Calif. 1158 Stewart Ave., Xenia, Ohio 3104 W. Glen Ave., Peoria, III. 2649 162nd P.., Hammond, Ind 121 Ridgeway Dr., Decatur, III. R. R. 1. Urbana, ind Washington Court House, Ohi 909 Hortz Dr., Lebanon, Ohio

3705 135th Ave., S.E., Bellevue, Wash. 5335 Lindberg Blvd., Dayton, Ohio 384 Olympic Ave., Buffalo, N. Y. 33 North Dr.. Buffalo, N. Y.

249 W. Summit Ave., Haddonfield, N. J.

1497 North Rd., S. E., Warren, Otio 304 N. East St, Bremen, Ind. 304 N. East St., Bremen, Ind .8330 Lucas Hunt Rd., St. Louis, Mo 3202 Eastlawn St., Lorain, Ohio Yorktown, Ind. 311 Smith St., Yorktown, ind 210 W. Garland Ave., Fairborn, Ohio 1674 Wesleyan Rd., Dayton, Ohio R. R. 2, Rochester, Ind. 707 N. Prospect, Ypsilanti, Mich. ... Palmyra, Mich

R. D. 4, Corry, $\mathrm{Pa}$ 127 Hartzell Ave., Niles, Ohio 322 fitzgerald St. Oglesby, III. 89 Eastwood Dr., Norwalk, Ohio 351 Stanley Ct., Toledo, Ohio 1823 N. Morrison St, Kokomo, Ind. 125 Machen, Toledo, Ohio 
Rockwell, Denis Ross

Roden, Joseph G.

Roderick, Richard A.

Rogers, Geraldine Faye

Rogers, Jean Ann

Rogers, Ruth Ellen

Rohm, Robert K.

Ronk, Joanna

Rooke, John Andrew

Rose, Bonnie Carol

Rose, Mary Lovise

Ross, Diann Odette

Ross, Randall A.

Rothwell, Susan I.

Rotondi, Tony V.

Rouch, Beborah Lee

Rudduck, Joyce Lynn

Rueck, John .

Rulison, Mary Lisa

Rutherford, Ronald D.

Ryan, Colleen Mae

Sadler, Paula Mae

Salisbury, Karen Faye

Sammons, John H., Jr.

Satterblom, Sheryl Rae . .

Schaeffer, James W.

Schafer, John Charles .

Schaffner, David Earl

Schilke, Mary Lowise

Schill, William N., Jr.

Schimkus, Richard C.

Schlechty, Sandy Jane

Schonscheck, Marlene Jea

Schrotberger, Carol Sue .

Schulte, Mary Ellen

Schult, Joan E.

Schult, Robert William

Schut, Mary Ellen

Schwanbeck, Carl Arnold

Schwarm, Sharon Estelle

Scott, Carol Sue .

Scovell, Pat.

Searles, Marshall William

Seigneur, Melvin Dean . .

Seneff, Barbara Darlene

Shamblin, Rebecca I.

Shampoe, Larry Gordon

Sharpless, Larry Dean

Shaw, Lavonne Korine

Shearer, R. Byron

Sheller, Sylvia .

Shepard, Verna Dell

Sherwin, Gail M.

Shriver, Allen Madison

Shubert, Keith Allen

Shue, Donald 0 .

Shufelt, Gary Lee . .

Shugg, Elma Kay

Shultz, Floyd Donald ...

Simonetti, Carol Ann

Simpson, Donna $S$.

Skillman, Jon Charles .

Skinner, Perry Lee

Sleichter, Mary Ann

Smart, Diana Marie

Smart, Monda Lee .

Smelser, Edward Slater

Smith, Beryl Rosamary

Smith, Beverly Ann

Smith, Connie to

Smith, Jerry Lee

Smith, Lois Elaine

Smith, Nancy Lee

Smith, Sharon Kay

Smith, Ted Lyle.

Snider, Joseph Bernard .
2637 Walker, Indianapolis, Ind R. D. 1, Dorset, Astabula, Ohio R. R. 2, Warsaw, Ind

.3380 Orders Rd., Grove City, Ohio 201 Fourth Ave., Haddon Heights, N. J. 201 Fourth Ave., Haddon Heights, N. J.

243 Maple Ave., Audubon, N. J.

2415 Aberdien Rd., Richmond, Va.

546 N. Venice Court, Bay City, Mich.

R. D. 4, Crawfordsville, Ind.

900 E. Main St., Albion, Ind

R. R. 5, Box 397, Chillicothe, Ohio

42 Linwood Ave., Jamestown, N. Y.

17 Winters St., Oakland, N. J.

Spring St, Farmington, N. H.

1171 Sylvania Rd., Cleveland Hgts., Ohio

R. R. 2, Cedar Falls, lowa

670489 Ave., Edmonton, Alberta, Canada

R. D. 2, Amsterdam, N.Y.

1685 Greenwood Rd., Lapeer, Mich.

1715 Bigelow St., Toledo, Ohio

.402 W. Lynn St., Stryker, Ohio

3147 Fisher Rd., Columbus, Ohio

35 E. Xenia St., Jamestown, Ohio 3544 43rd Pl., Highland, Ind. 2840 Lantz Rd., Xenia, Ohio

1691 S. Perry St, Denver, Colo,

R. R 1, Fayette, Ohio

65 W. Possum Rd., Springfield, Ohio

1 Station Ave., Haddon Heights., N. I.

490 Clareridge Lane, Centerville, Ohio

17020 Lakewood Hgts. Blvd., Lakewood, Ohio

. 16188 Thomas, Allen Park, Mich.

3290 Governors Trail, Kettering, Ohio

. R. R. 2, Huntingburg, ind.

18967 Fenemore, Detroit, Mich.

. 4739 N. 51 st St., Milwaukee, Wisc.

.5796 36th Ave., Hudsonville, Mich.

R. R. 3, Norwalk, Ohio

.566 Grandview Ave., Barberton, Ohio

120 N. Haldy Ave., Columbus, Ohio R. R. 3, Brookville, Ohio

R. D. 1, Box 132, Wellington, Ohio

208 W. Curtis, Stryker, Ohio

3872 E. Whittaker Ave., Cudahy, Wisc. Box 352, Clendenin, W. Va. R. D. 2, Clymer, N. Y.

742 Circle Dr., Fostoria, Ohio 1783 Baldwin Rd., Cherry Hill, N. J. .805 Unity Center Rd., Pittsburgh, Pa. . Box 282, Salt Springs Rd., Mineral Ridge, Ohio 211 2nd St., S.W., Waverly, lowa .114 Kraft St., Berea, Ohio . Ursa, III. R. R., Saunemin, III

1385 DeCamp St., Flint, Mich. 5834 Elden Dr. Sylvania, Ohio .8882 Rushside Dr., Pinckney, Mich. 2789 St. Joseph Ave., Columbus, Ohio 3816 English Ave., Erie, Pa

2215 Churchman, Indianapolis, ind.

237 Homer St., Marion, Ohio 500 S. Mulberry St., Creston, lowa Box 142, Scotland, $\mathrm{Pa}$

770 McBee Rd., Bellbrook, Ohio 2031 Hempsted Rd., Kettering, Ohio 125 W. Friendship St., Medina, Ohio 4128 Washington St., Gary, ind . 3845 Bradee Rd., Brookfield, Wisc. .360 Crystal Spring, Adrian, Mich. .512 Dayton St., Xenia, Ohio R. R. 1, Milford, III Cedarville, Ohio

700 Ridge Ave., Bucyrus, Ohio 2111 Hoppes Ave., Springfield, Ohio 2071 McCoy Rd., Barbarton, Ohio

Snook, Barbara Ellen

Snyder, Stephen Russell

R. R. 3, Pataskala, Ohio

Solomon, Sheila Kay.

Southwell, Barbara Elaine

Spear, Judy Ann

Spears, Susan E.

Spencer, Kathryn Lee

Spinks, Linda Lea .

Sprunger, Marcia Ann

Stanbery, Maxine Kay

Staples, David Ward

Stephens, Daniel E.

Stephens, Helen G.

Stephenson, James Lee

Stevens, Duane LeRoy

Stevenson, Connie Jean

Stewart, Gwendolyn Sue ...

Stickle, Fred Emery

Stillwell, Craig Richard

Stockwell, James Earl

Stockwell, John Charles

Stout, Roger

Stowell, Joseph Mishael

Strychalski, JoAnn .

Stuart, Ronald 1.

Stump, Karen Michelle

Stutesman, Lovise E.

Stutzman, Ronald Lee ...

Surber, S. Sharon .

Sweeney, Adrian David . .

Swoverland, Kathleen Faye

Tallman, Sharon Kay

Tanner, Lela Lucy

Tansey, Charles Wesley

Taylor, Delmar Omrie .

Taylor, Gary Willcockson

Taylor, Gordon Allen

Taylor, Karen C.

Taylor, Laura Rae .

Taylor, Linda Kay

Terrill, Douglas Timothy

Terrill, Evelyn Kathleen

Terrill, Linda Irene

Tharp, JoAnn .... Carlisle, Ohio

.. R. R. 3, Argos, Ind.

Box 267, Mancelona, Mich.

7998 Decherville Rd., Decher, Mich. 26207 Harmon, St. Clair Shores, Mich.

393 Wainut St., Cedarville, Ohio R. D. 1. Findley Lake Rd., Sherman, N. Y.

423 High St., Berne, Ind.

4110 Harvard Ave., Peoria, III. Spring Valley, Minn.

Cedarville College, Cedarville, Ohio

. 218 Paimer Dr., Cedarville, Ohio 3268 Withers Ave., Lafayette, Calif. 29011 Cherry Hill Ro., Inkster, Mich. . Baker, Mont.

R. R. 5, B0x 87C, Indianapolis, Ind. Oakland, Minn.

94 Roosevelt Blvd., Oakland, N. J. Box 344, Cedarville, Ohio Box 504, Cedarville, Ohio

60 W. Possum, Springfield, Othio 200 Catalpa Ave., Hackensack, N. J. 521 Ulen Dr., Lebanon, Ind. R. D. 2, Horseheads, N. Y. Box 459, Clendenin, W. Va.

113 Maumie St., Tecumseh, Mich. Box 155, Berlin, Ohio

.833 Mt. Joy, Springfield, Ohio

Folkestone Way, Cherry Hill, N. J.

12956 Hlene, Poway, Calif. 405 S.W. 8th St., Hallandale, Fla. R. R. 1, Sherman, N. Y

1428 W. Jefferson, Kokomo, Ind. .. Cedarville, Ohio

339 Parkside, Bay Village, Ohio Steamboat Rock, lowa 4560 Dayton-Liberty Rd., Dayton, Ohio Steamboat Rock, lowa

Thompson, Brenda Lu . .

Thompson, Connie Lee

Thompson, Patricia Mae

Thomson, Beth Elaine

Thorne, Barbara .

Thorne, Susan J.

Thurlow, William Lee

Tibbett, Jane Ann

Tillotson, Daniel Charles

Timmons, Howard Edwin

Titus, Anna Marthene

Titus, Rick Edward .

Tobias, Judith Elain

Tompkins, Gail Lovise

Towle, Nancy Jean

Townsend, Marcia Jane

Ireadway, Steve Allen

Trimble, Glen Mark

Iripp, Edwin Albert.

Irunnell, David Bruce

Truxton, Charles G. .

Underwood, Norman Lee

Vance, Jeanne Rebecca

Vance, Sharon Elaine .

Vanderground, David John

Vanderground, James Philip

Van Horn, Janice Gale . . .

Vanvoorhis, George Michael

Versluis, Patricia Madge .

Vincent, Beverly R.

Fabius, N.Y

. Fabius, N. Y.

60 Church St., Cedarville, Ohio

202 N. Cherrywood Ave., Dayton, Ohio

830 So. Terrace, Columbus, Ohio

3363 Greensburg Rd., N. Canton, Ohio

... 1101 N. Hazel St., Pontiac, III.

.18610 N.W. 8th Rd., Miami, Fla Stokes Rd., Medford, N. J. Stokes Rd., Medford, N. J. 906 E. Indian, Midland, Mich. . 3127 Elmdale Dr., Fort Wayne, Ind . 1320 Montana, Gary, Ind.

638 W. Main St., Blanchester, Ohio

City Mounted R. 8, Crawfordsville, Ind. .229 3rd St., Clarion, lowa

.1305 Dale St., Muscatine, lowa R. D. 2, Sherman, N. Y. R. R. 2, Pataskala, Ohio

Bermardston Road, Greenfield, Mass. 1919 June Dr., Xenia, Ohio

Caixa 372, Manaos, Amazonas, Brazil, S. Amer. 30240 Powell Rd., Willowick, Ohio R. R. 2, Waterloo, lowa

.511 Graisbury Ave., Haddonfield, N. J. 36 Jones St., Dayton, Ohio

.R. R. 1, Westbrook Rd., Clayton, Ohio 220 Pole Lane Rd., Marion, Ohio 
Votaw, Robert Howard ......................................... R. 1, Box 252, Decatur, II. Wahl, Dona Louise . 143 Main Ave., Warwick, R. I. Waite, Larry Allen

Wakeley, Janice Carol

Walborn, Daniel Lee

Walborn, David .

Walborn, Jon B.

Walker, Kathleen Mary

Walker, Leland Gene

Walker, Lois Adell

Walker, Michael T.

Walker, Roosevelt ....

Wall, David Roy

Wallis, W. Neison .

Ward, Diana Lovise

Warden, Karen Jan

Warren, Timothy Scott

Watson, John H. . .

Weinschenk, Patricia Lynn

32 nd Ave., Hudsonville, Mich.

137 W. Main St, Ripley, N.Y.

239 W. Leggett St., Wauseon, Ohio

239 W. Leggett St., Wauseon, Ohio

13716 Superior Rd., E. Cleveland, Ohio

R. R. 1, Fairbault, Minn

R. R. 2, Carlisle, lowa

7451 Chardon Rd., Kirtiand, Ohio 5605 Farney Ave., Sciotoville, Ohio 5900 Quincy Ave., Cleveland, Ohio

360 N. 34th St., Decatur, III.

Box 78, Thurston, Ohio

15716 0akhill Rd., E. Cleveland, Ohio

159 Victory Highway, Painted Post, N. Y.

303 Pine St., Allegan, Mich.

933 E. Mish Ave., Mish, Ind

Weich, Loren Melvin

Wells, Susan Marie

Wenner, Carol Jean

Werner, Marlene Kay ...

Wessel, Lynda Jayne

Whaley, Teresa Annette

Whattoff, Robert R.

White, Keith Alan .

Whitmire, James William

Whitney, Cheryl Ann

Wilcoxson, Donald Thaine

Wilkins, Charles C.

Williams, David Dale
7 Troon Way Rockledge. F

875 Chopman Ave., E. Cleveland, Ohio

.. 14 Plum St., Shel by, Ohi

713 Leland, Fint, Mich,

.86 Clifton St., New London, Ohio

819 9th St. W., Columbia Falls, Mont.

1301 First St. North, Newton, lowa

16454 McKeever, Granada Hills, Calif. 231 Abraham Dr., Ames, lowa

Trailer Court, Cedarville, Ohio

421 N. Duffy Rd., Butler, $\mathrm{Pa}$

Church St., Bernardston, Mass

R. R. 3, Villisca, lowa

3310 Westline Dr., Columbus, ind

.756 St. Clair, Pontiac, Mich.
Williams, Gay Lynn

Williams, Patricia Joa

3382 Hendon Circle, Cuyahoga Falls, Ohio

Williams, Ruth Marie

Williams, Sarah Esther

Willis, Pamela Jean .

Wilson, Caron Lea

Wilson, Cheryl Lee .

Wilson, Linda Jean

Wing, Twila G. .

Wingate, Jon $B$. .

Wintrow, Linda Carol

Wood, Joyce Arlene.

Woodend, Michael Joseph .

Woodman, Gwendolyn Muriel

Wooster, Michal Ann ....

Work, Dale E.

Worth, Robert C.

Wright, Kenneth Leroy

Wright, Richard James

Wymer, Ralph Ronald . ...

Yeater, Ronald E.

Yoder, Jay Douglas .

Young, Jefferson Theodore

Young, Linda Lou

Young, Elizabeth Maxine

Zanstra, Richard L.

Zazvrskey, Michael E.

Zeigler, James Doran

Zellers, George Russell

Zerbe, Sheryl Ann

Zerbe, Les Paul

Zimmerman, Barry Kent
3015 Orchard Dr. Hammond, Ind.

... 1335 Verbena, Denver, Colo. ... $694 \mathrm{E}$. 124th St., Cleveland, Ohio

1606 Aura Ave., East Wenatchee, Wash. .4917 Wayne Trace, Fort Wayne, ind 12921 Euclid Ave., E. Cleveland, Ohio .30740 Orange Ave., Shaffer, Calif. ...R. D. 1, W. Winfield, N. Y

... 1 S. Elm St, Corning, N.Y. 367 N. Fourth St., Tipp City, Ohio 1110 Seventh St., Clarkston, Wash. 4107 Mesa Way, Alexandria, Va .743 N. Main St.. Cedarville, Ohio 3024 N. College Ave., Indianapolis, Ind R. D. 3, Coudersport, Pa Bella Bridge Rd., Box 178, Medford, N. J. ...66 Pehle Ave., Saddle Brook, N. J. .110 E. Wells Ce., Pennsboro, W. Va. 1503 Julian Lane Dr.. Tampa, Fla 805 Allegheny St., New Brighton, Pa. .. R. R. 1, West Liberty, Ohio .... Lorain, Ohio 1024 So. College Ave., Mt. Pleasant, Mich. 1024 So. College Ave., Mt. Pleasant, Mich. ..2213 Stafford S.W., Grand Rapids, Mich. 2905 Colorado Ave, Lorain, Ohio .708 S. Whitman Dr., Blackwood, N. J. 1619 E. 14th St, Long Beach, Calif. .210 Palmer Or., Cedarville, Ohio 210 Palmer Dr., Cedarville, Ohio Sycamore St., Silver Lake, Ind.

\section{FACULTY}

Jeremiah, James T., D.D.

Ager, Merlin, M.S.

Ballard, Stanley, M.S

Bartholomew, Raymond E., Ph.D.

Baumann, Donald P., Ph.D.

Bergen, Harmon, BA,

Buffham, Charles, MA.

Byrd, Shirley, BA.

Callan, Donald, M.S.

Chaffe, Alberta L., B.A.

Dowell, Max Boyd, M.S.

Elmore, Austin D., M.A.T.

Entner, Esther, M.S.

Fisher, Jean, M.A.
Frye, Burtis L., MA.

Gale, Ralph B., M.A.

Gromacki, Robiert, Ph.D.

Johnson, Clifford, D.Ed.

Johnson, Howard R., B.A.

Kearney, June F., B.S.

Lawior, George L., Th.D.

Mackay, Joy, M.A

Maddox, Clifford R., Ph.D.

Maddox, Miriam B., BA.

Murdoch, J. Murray, M.A.

McDonald, Cleveland, M.Litt.

Mcintosh, Richard T., Th.M.

Olson, Dennis, M.S.
Packard, Carla, M.A.

Reed, John W., M.A.

Riter, William, C.P.A.

Russell, J. Emerson, M.A.

Spencer, Edward E., B.A.

St. Clair, Kenneth, M.S., C.PA.

Thomson, Dale Sterling, Ph.D.

Vanderkoy, Paul, M.M.

Webber, Ardith, BA.

Webber, Warren L., M.M.E.

Wetzel, Daniel, M.S.

White, Deane, M.A.

Williams, Arthur F, D.D.

Wyse, Rodney E., M.B.A. 


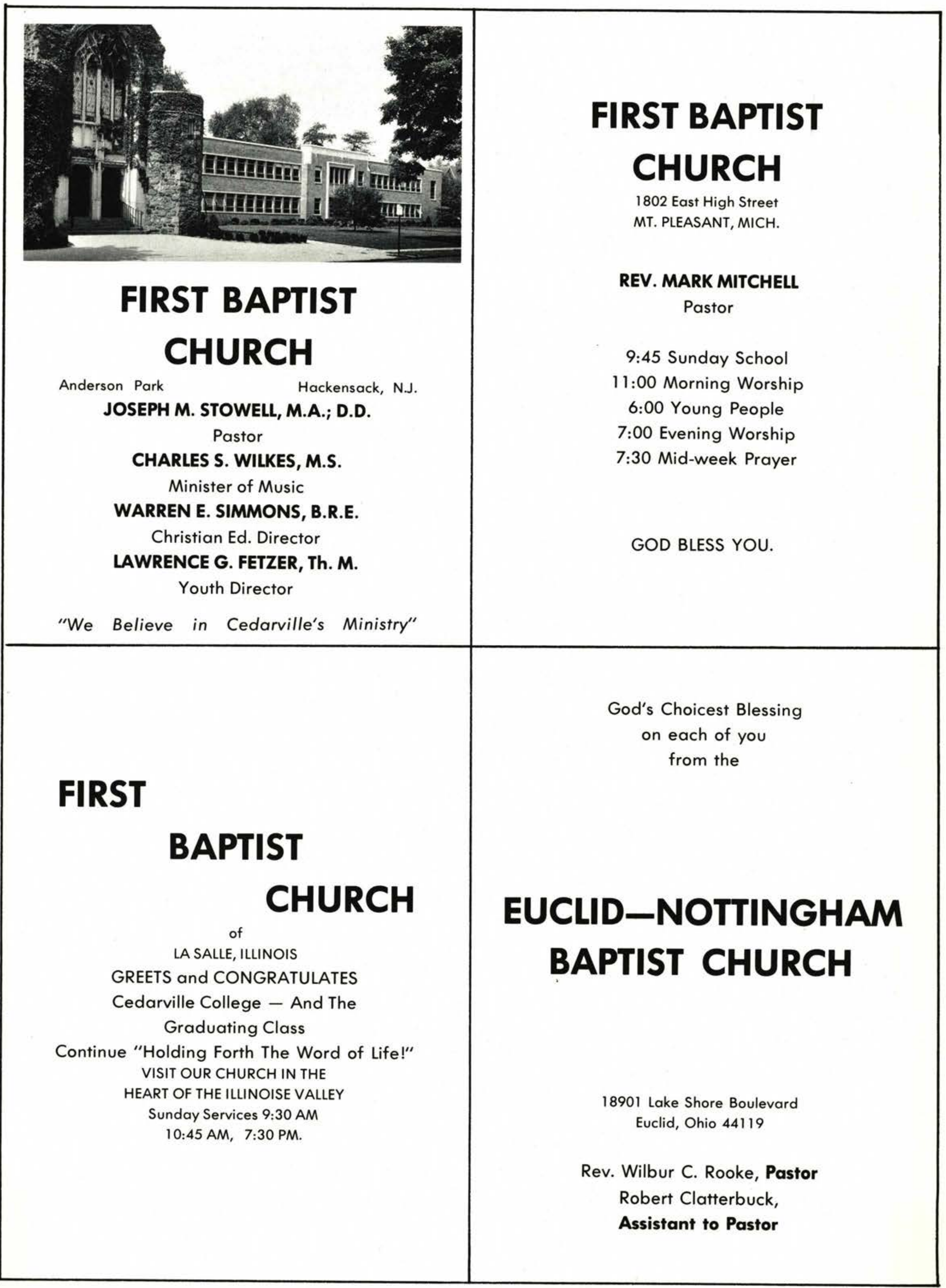




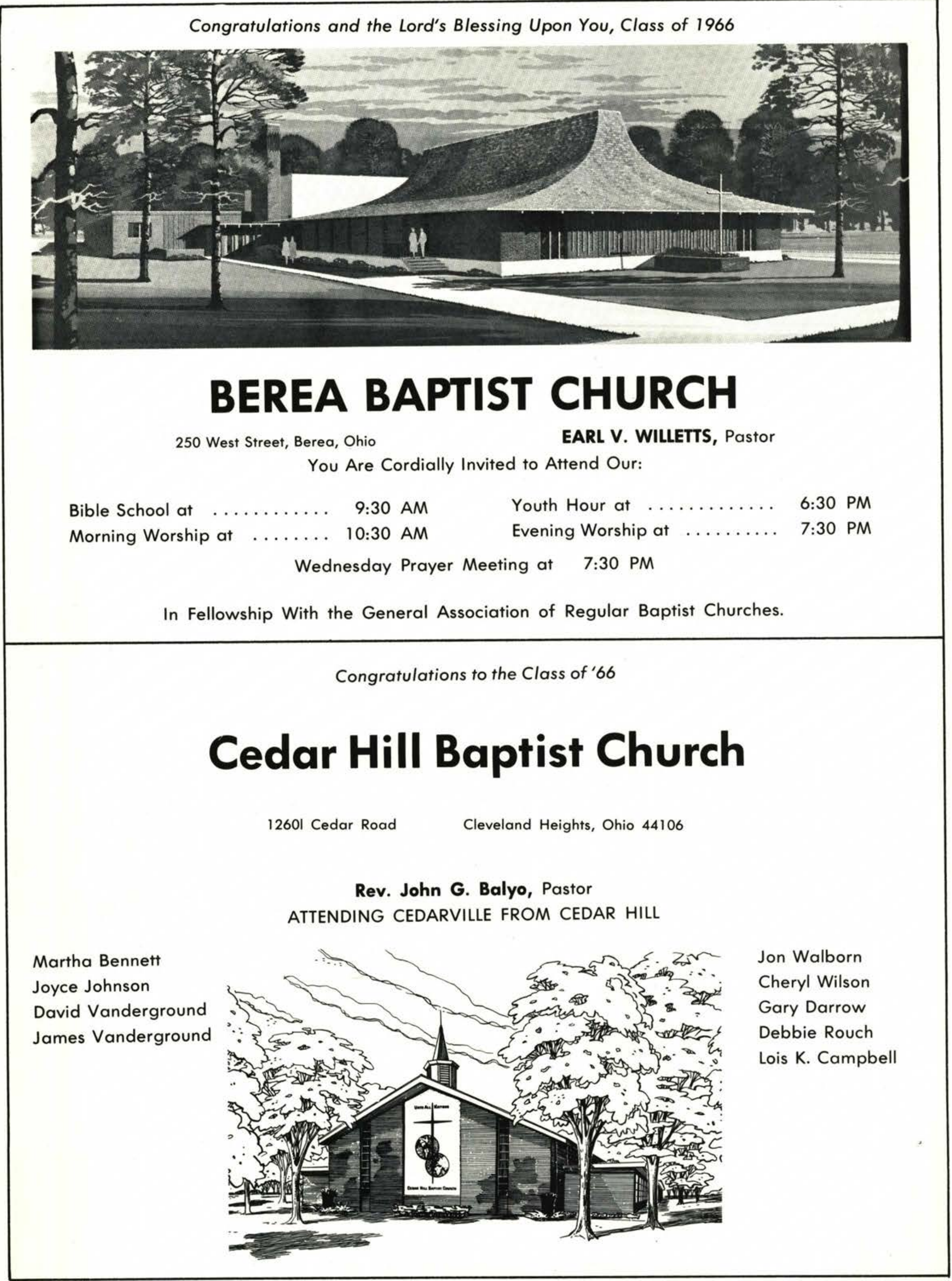




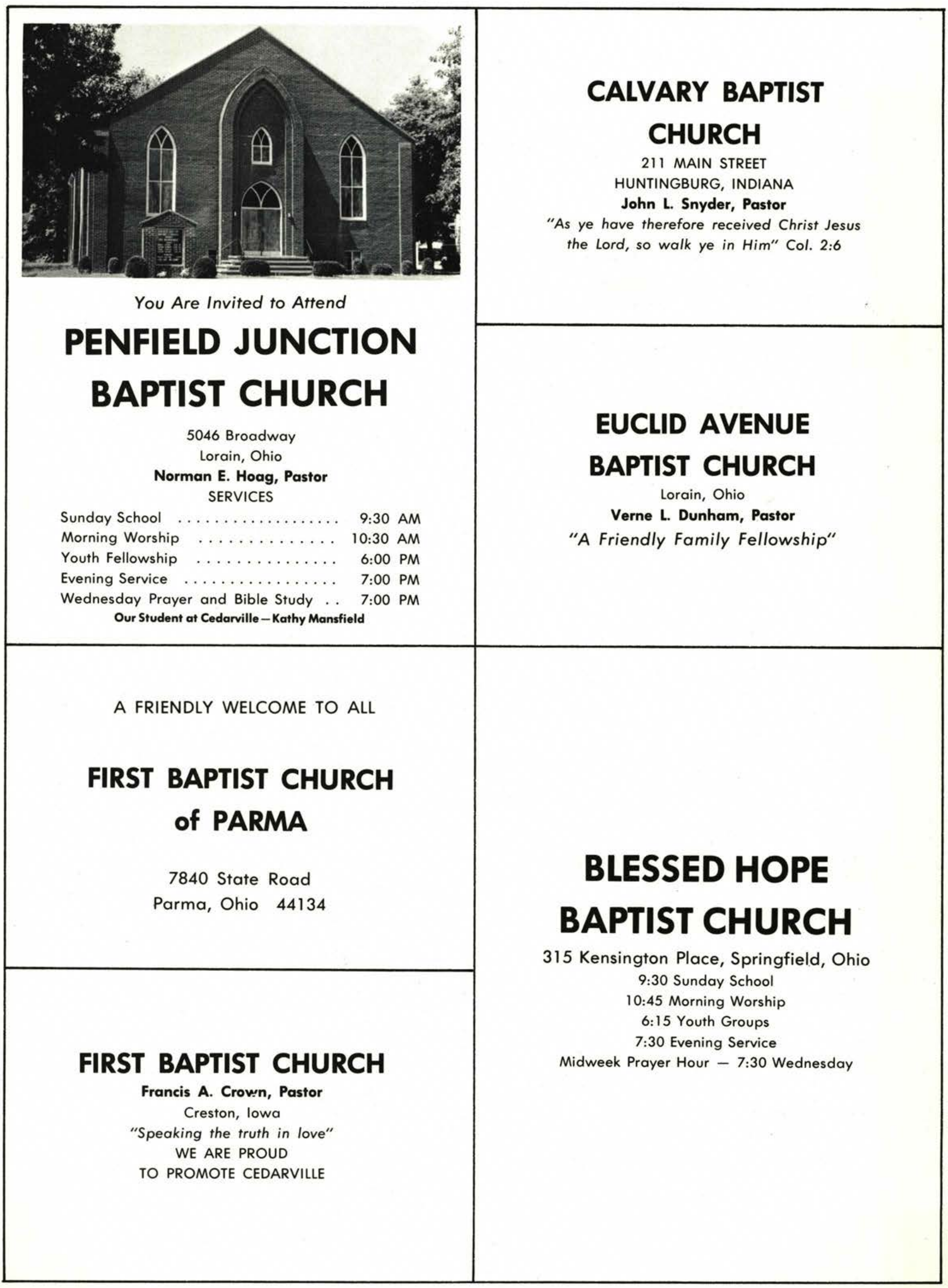


Sincere Christian Wishes

to the

GRADUATING CLASS of 1966

HUNTSBURG

BAPTIST

CHURCH

Huntsburg, Ohio

Ben H. Garlich, Pastor

\section{FIRST BAPTIST}

\section{CHURCH}

221 New Castle Street

Butler, Pennsylvania

RALPH O. BURNS, Pastor

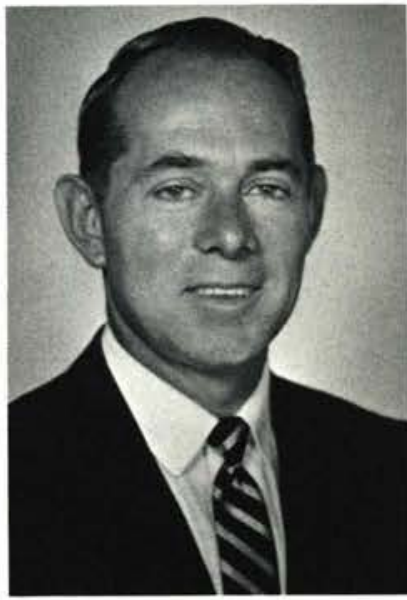

The Home Church

of

Five

Cedarville

Students
BROWN STREET BAPTIST CHURCH

ALTON, ILLINOIS

Gaylord S. Hamilton, Pastor

GRANDVIEW PARK BAPTIST CHURCH

1701 East 33rd Street

Des Moines, lowa
David Nettleton

Steve Boalt

Bernie Payne

Velma George

"Congratulations to the class of 1966"
Pastor

Minister of Music

Minister of Visitation

Church Secretary

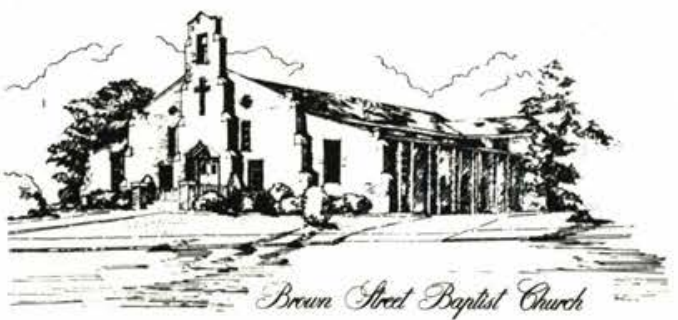

"A friendly church with a warm welcome" Sunday

9:30 AM

Bible School

10:30 AM

6:00 PM

7:00 PM

Wednesday

7:00 PM
Morning Worship

Training Unions

Evening Service

Prayer Meeting 


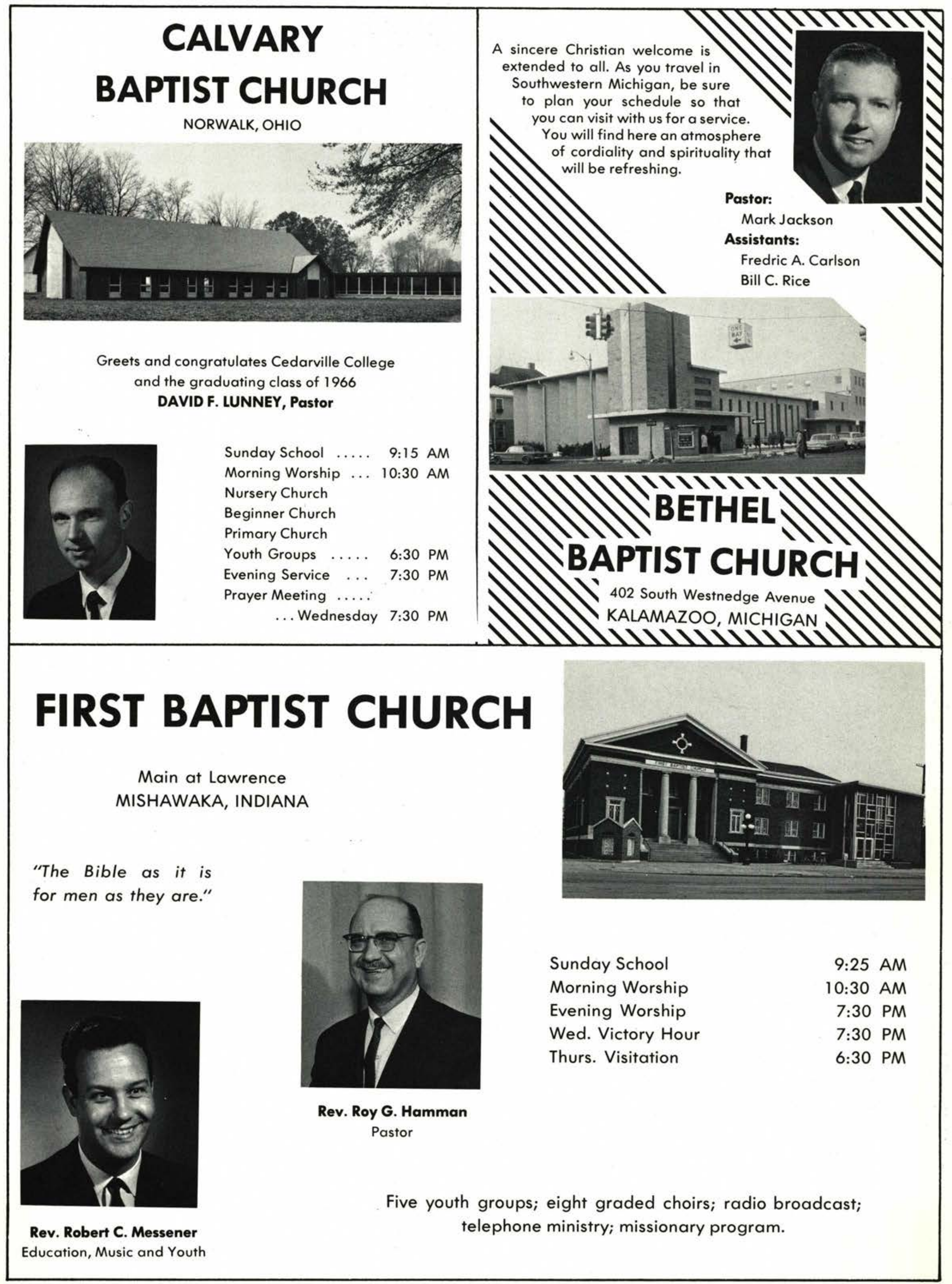




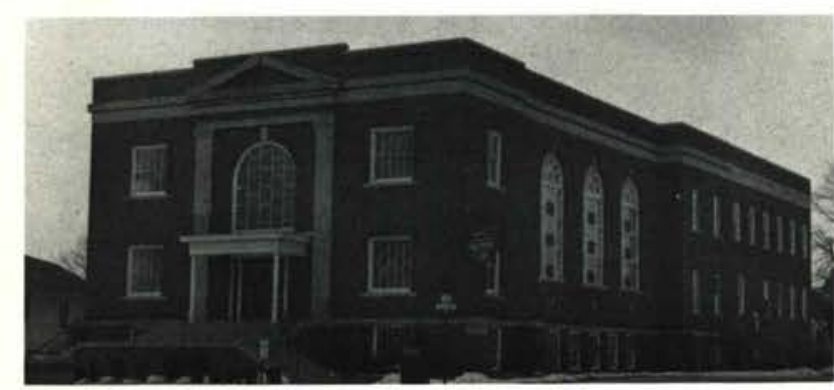

RIVERSIDE BAPTIST CHURCH

S. JASPER \& E. CLEVELAND STS. DECATUR, ILLINOIS 62525

\section{DONALD CURREY PASTOR}

Sunday Services

9:30 AM Sunday School

10:45 AM Morning Worship

6:00 PM Youth Groups

7:00 PM Evening Worship

Wednesday - 7:30 PM Prayer Meeting

Awana Youth Program

In Fellowship with

G.A.R.B.C; A.C.C.C.; I.C.C.C.

\section{FIRST}

\section{BAPTIST CHURCH}

\author{
113 Church Street \\ SHERMAN, NEW YORK
}

Pastor Rev. Fred W. Robb

Sunday

Bible School

10:00 AM

Morning Worship

11:00 AM

Youth Meetings

7:15 PM

Evening Service

8:00 PM

Wednesday

Prayer Meeting 8:00 PM

Choir Practice

9:00 PM

Affiliated with

General Association of Regular Baptist Churches American Council of Christian Churches

International Council of Christian Churches

Psalms 119:89

For ever, O Lord, Thy Word is settled in heaven

\section{CALVARY BAPTIST CHURCH}

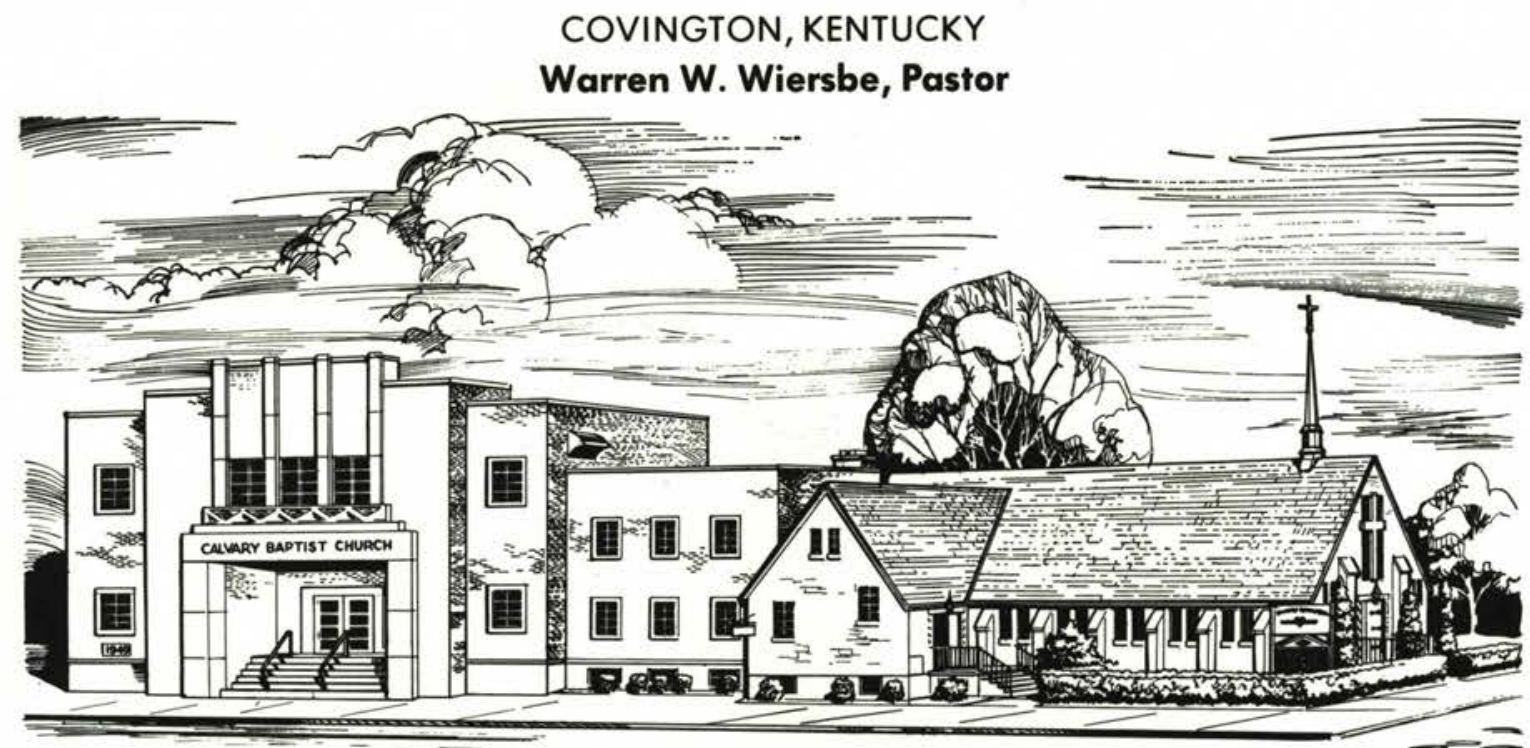

- TEACHING THE WHOLE BIBLE

- MINISTERING TO THE WHOLE FAMILY

Calvary Baptist Church is the home of the "Whole Bible Study Course" developed by Dr. D.B. Eastep, who pastored the church for thirty-five years. The course takes the student through the Bible, book by book and chapter by chapter in seven years.

Calvary Book Room, the literature ministry of the church, publishes and distributes true-to-the-Bible literature across the world.

Write for free information and book list. 


\section{FIRST BAPTIST CHURCH}

Second and Clark Streets

Pasco, Washington

Rev. Elvon Nelson, Pastor

Hugh U. Horner, Pastor

\section{CALVARY BAPTIST}

\section{TABERNACLE}

$$
\text { SANDUSKY, OHIO }
$$

Sunday Services

$$
\begin{aligned}
\text { 9:45 AM } & \text { Bible School } \\
\text { 11:00 AM } & \text { Worship Service } \\
\text { 7:00 PM } & \text { Evening Service }
\end{aligned}
$$

Prayer Meeting, Wed. at 7:30

\section{SILVIS HEIGHTS}

\section{BAPTIST CHURCH}

Middle Road and Tenth Avenue Silvis Heights, Silvis, Illinois

Gordon H. Perry

Pastor

Sunday

9:30

10:30

6:00

7:00

Tuesday

6:00

7:00

Sunday School

Morning Worship

Baptist Youth Fellowships

Evening Gospel Service

Junior Choir Practice

Senior Young People's Bible Study

Wednesday

7:00 Midweek Bible Study and Prayer

8:00 Adult Choir Practice

"Holding forth the word of life; that I may rejoice in the day of Christ"

Phil. 2:16
ONGRATULATIONS

EDARVILLE

ON YOUR ONTINUAL EMPHASIS ON

HRIST

ENTERED

B IBLICALLY

EDUCATION

FROM

\section{WEST CHICAGO BAPTIST CHURCH}

19510 West Chicago Blvd.-Detroit "DETROIT'S OUTSTANDING REGULAR BAPTIST MISSIONARY CHURCH"

Howard K. Miller, Pastor

\section{FIRST BAPTIST CHURCH}

GALION, OHIO

Rev. Wilfred Booth

\section{FIRST BAPTIST CHURCH}

123 W. Liberty Street MEDINA, OHIO

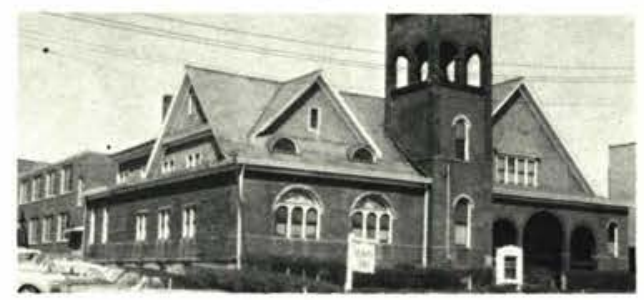

WELCOME

"In the heart of Medina with Medina on its heart" 


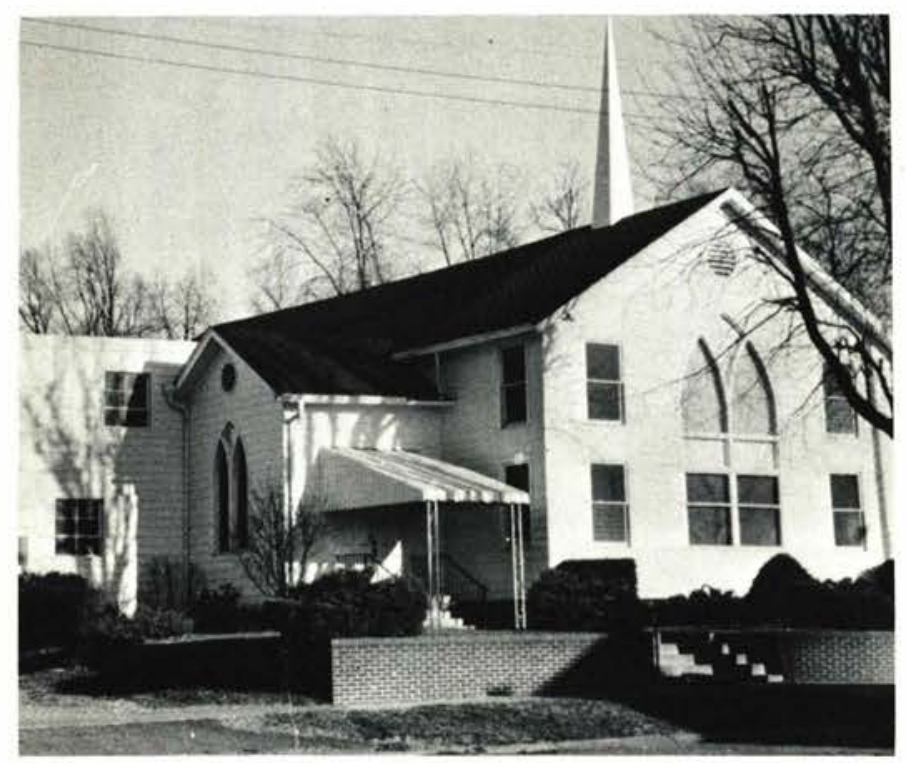

\section{WHEELERSBURG BAPTIST CHURCH}

Gallia at South Street

Wheelersburg, Ohio

THE FRIENDLY CHURCH

with an

ETERNAL MESSAGE

Donald Grollimund

Pastor

Congratulations to the Class of 1966

WELCOME TO

\section{THE ALUMNI ASSOCIATION}

President - William Brown

Vice-President - Tyrone Bryant
Executive Secretary - James Johnson

Treasurer - Stuart Chaffe

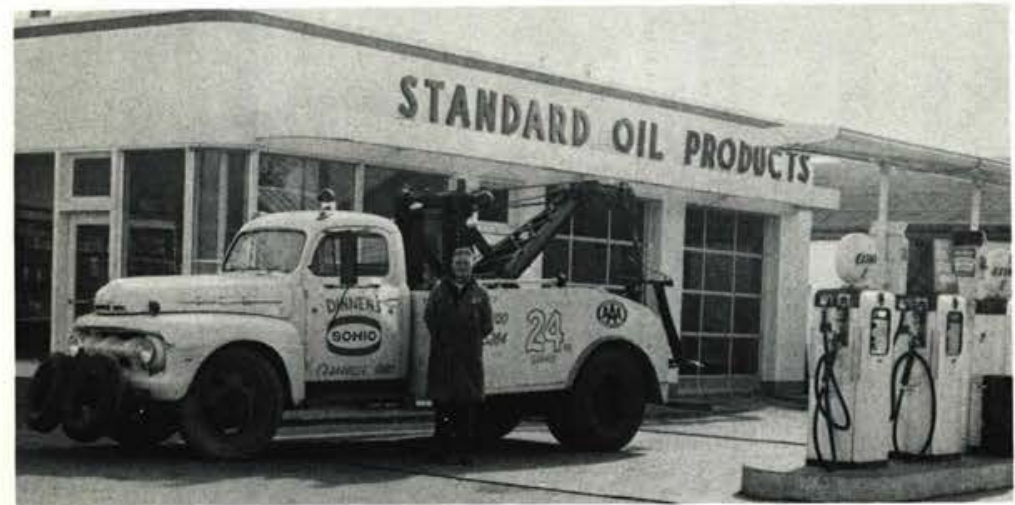

\section{DINNEN'S SOHIO SERVICE \\ BATTERIES \\ LUBRICATION \\ WRECKER SERVICE \\ MYRON DINNEN, Proprietor}




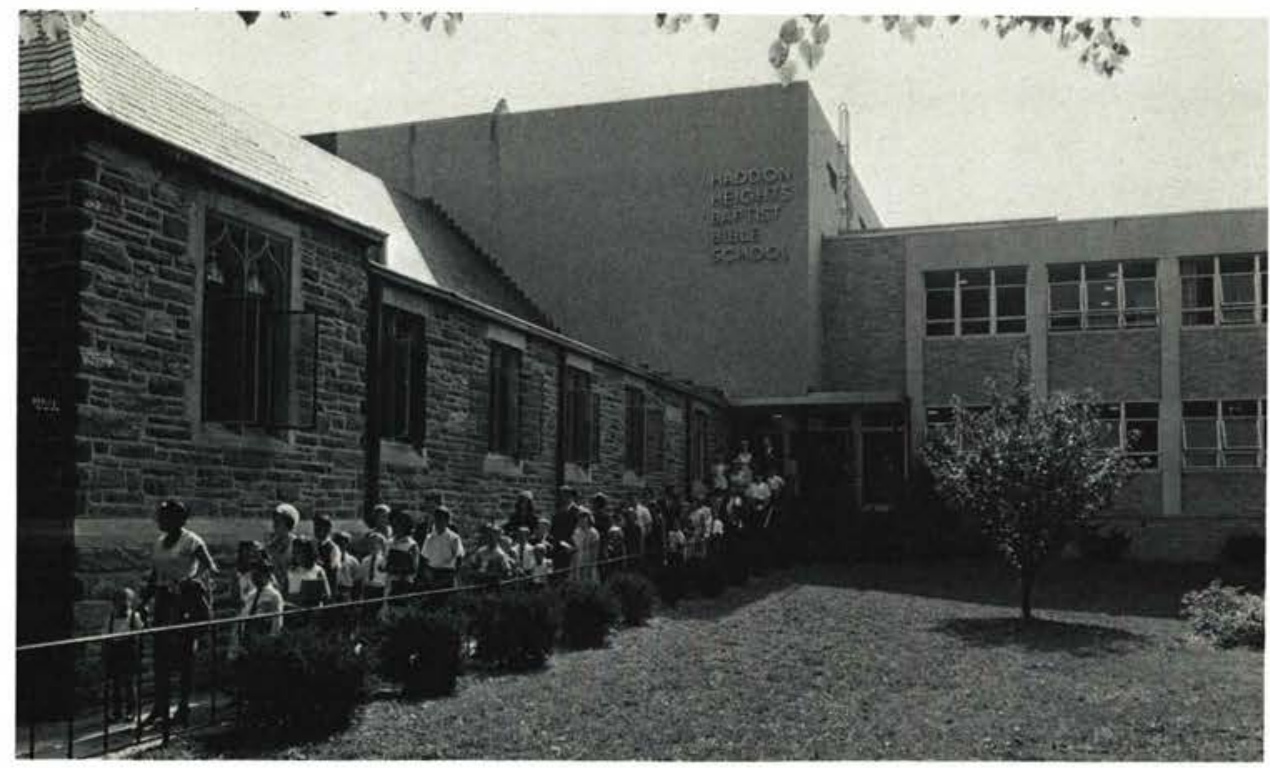

\section{HADDON HEIGHTS BAPTIST CHURCH}

Third and Station Avenues

Haddon Heights, New Jersey

Dr. K. W. Masteller, Pastor

"Holding forth the Word of Life" Rev. F. D. Henzler, Ass't. Pastor

Sincere Best Wishes

to the Class of 1966

\section{TEMPLE BAPTIST CHURCH}

Gallia Street at Waller

Portsmouth, Ohio

Otis R. Holmes, D.D., Pastor

SUNDAY SERVICES

Sunday School

9:15 AM

Trainıng Union

Morning Worship

10:30 AM

Evangelistic Service

6:30 PM

7:30 PM

Radio Broadcasts - WNXT, Portsmouth (1260 KC)

Sunday, 10:30 AM and 9:30 PM

WCDR - FM, Cedarville (88.1)

Sunday, 8:00 to 9:00 PM

\section{WEDNESDAY}

Hour of Power - 7:30 PM

"The Whole Bible for the Whole Family"

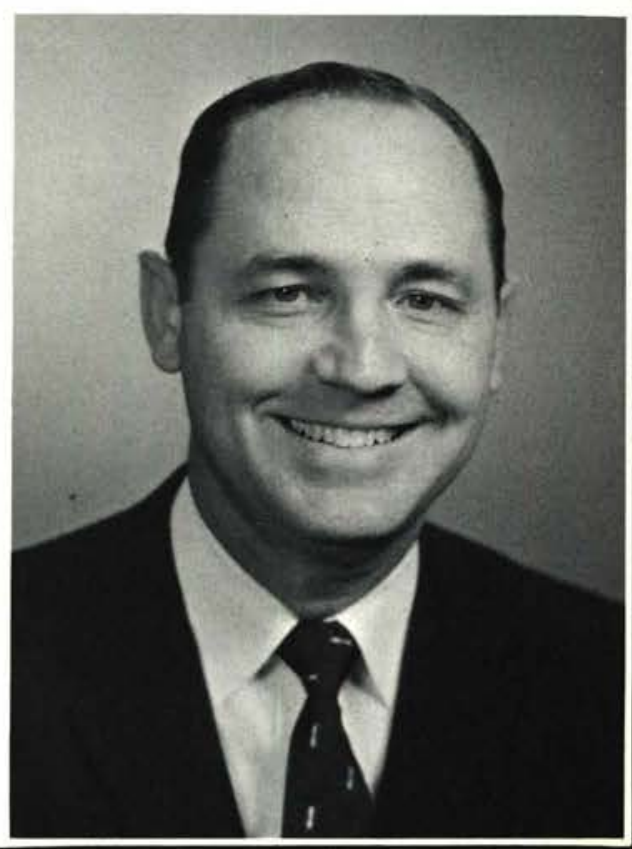




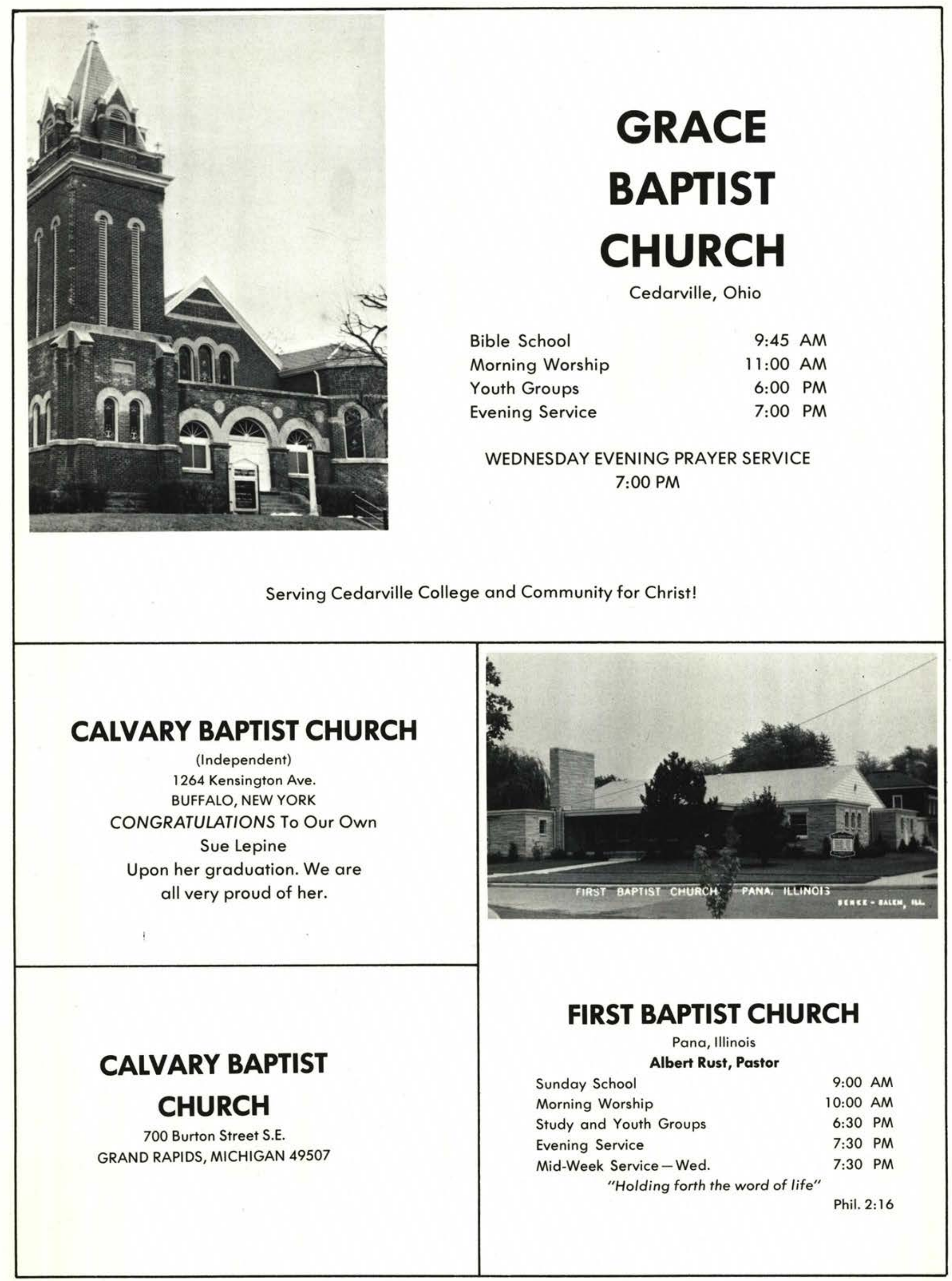




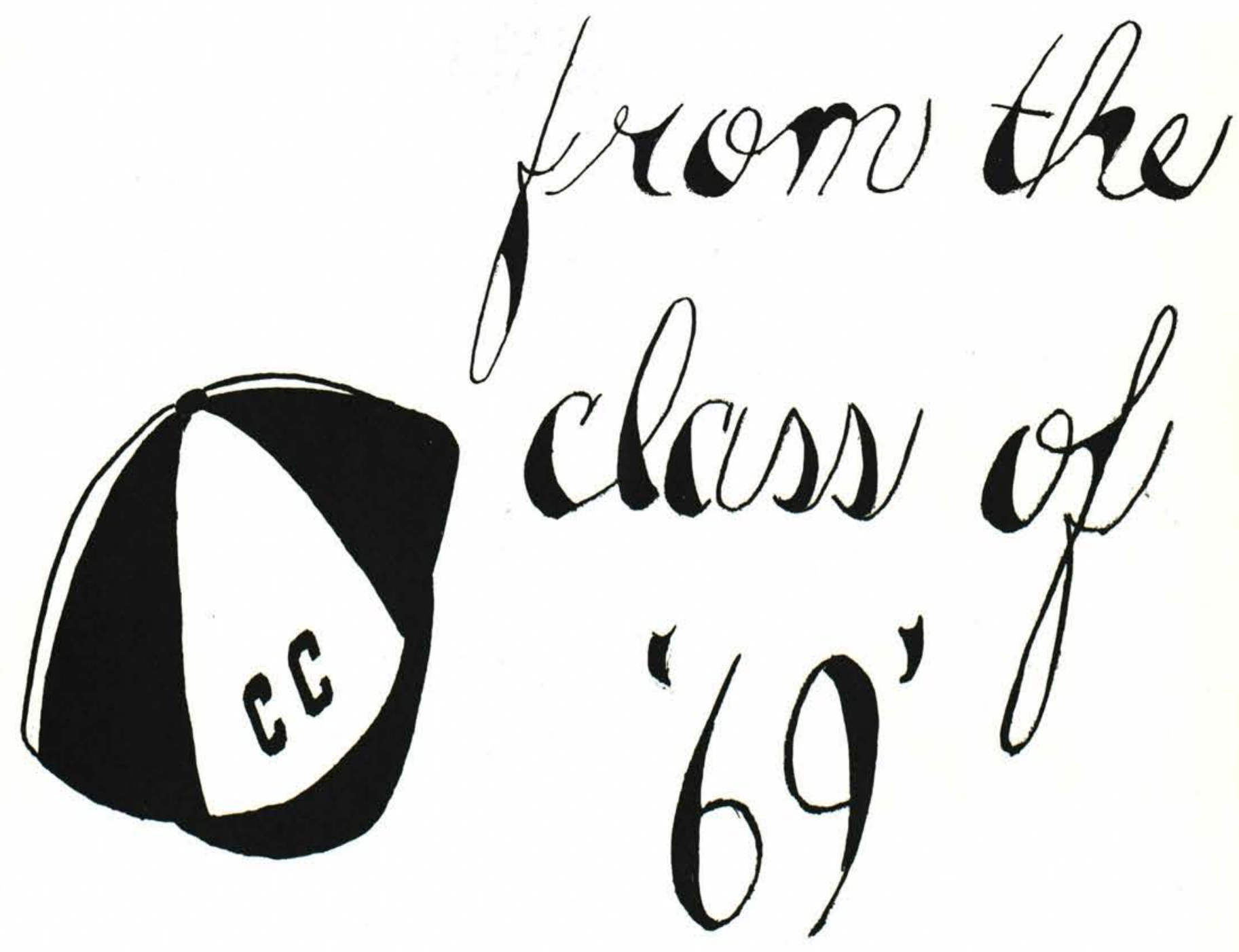

TO THE

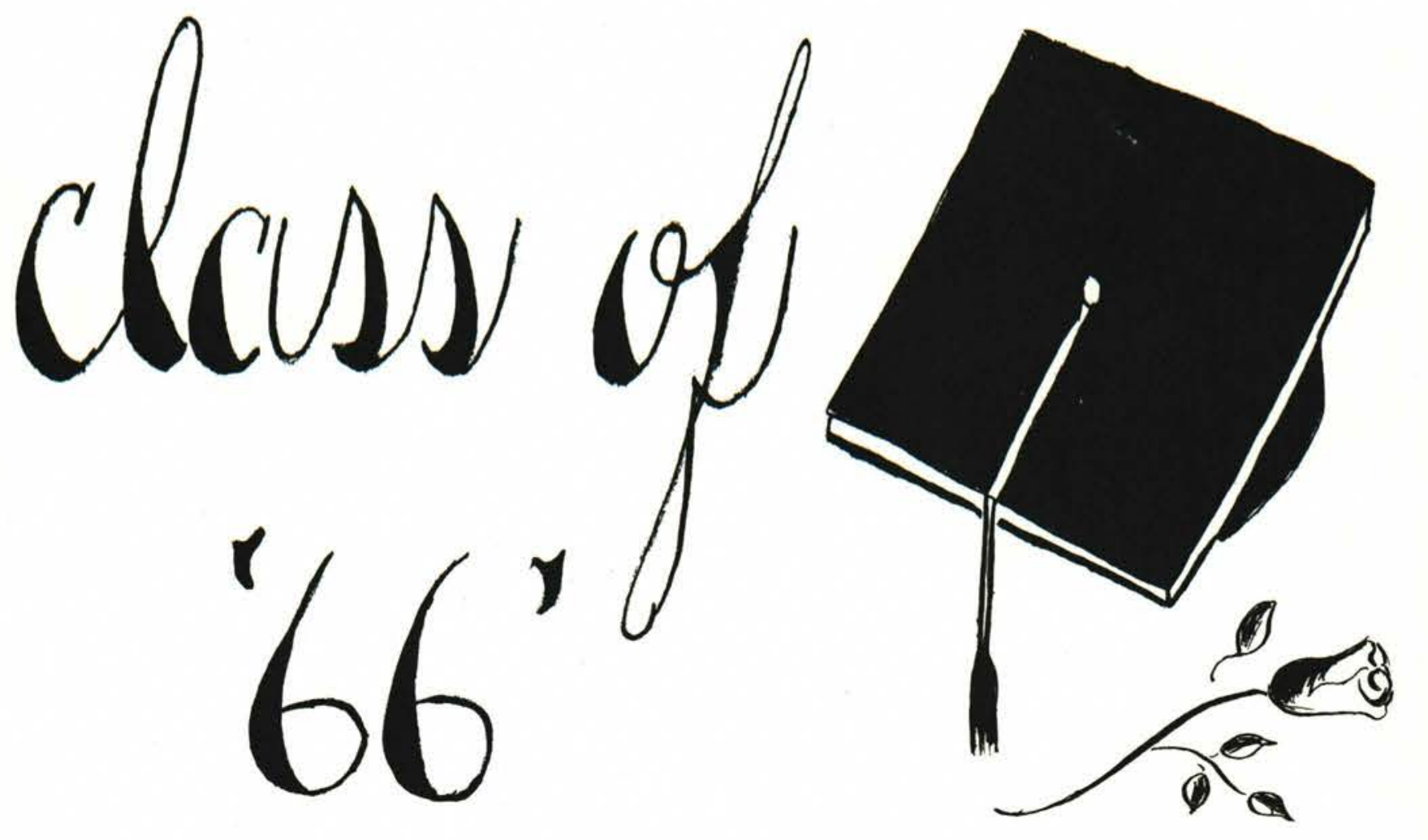

CONGRATULATIOIS 


\title{
CLINTONVILLE BAPTIST CHURCH
}

35 Oakland Park Avenue

Columbus 14, Ohio

\author{
Founded on THE WORD of GOD \\ Grounded on THE WORD of GOD \\ Bounded in THE WORD of GOD
}

"CLINTONVILLE BAPTIST CHURCH CONGRATULATES CEDARVILLE GRADUATES

AND COMMENDS CEDARVILLE COLLEGE TO ONE AND ALL."

Glenn H. Davis, Pastor

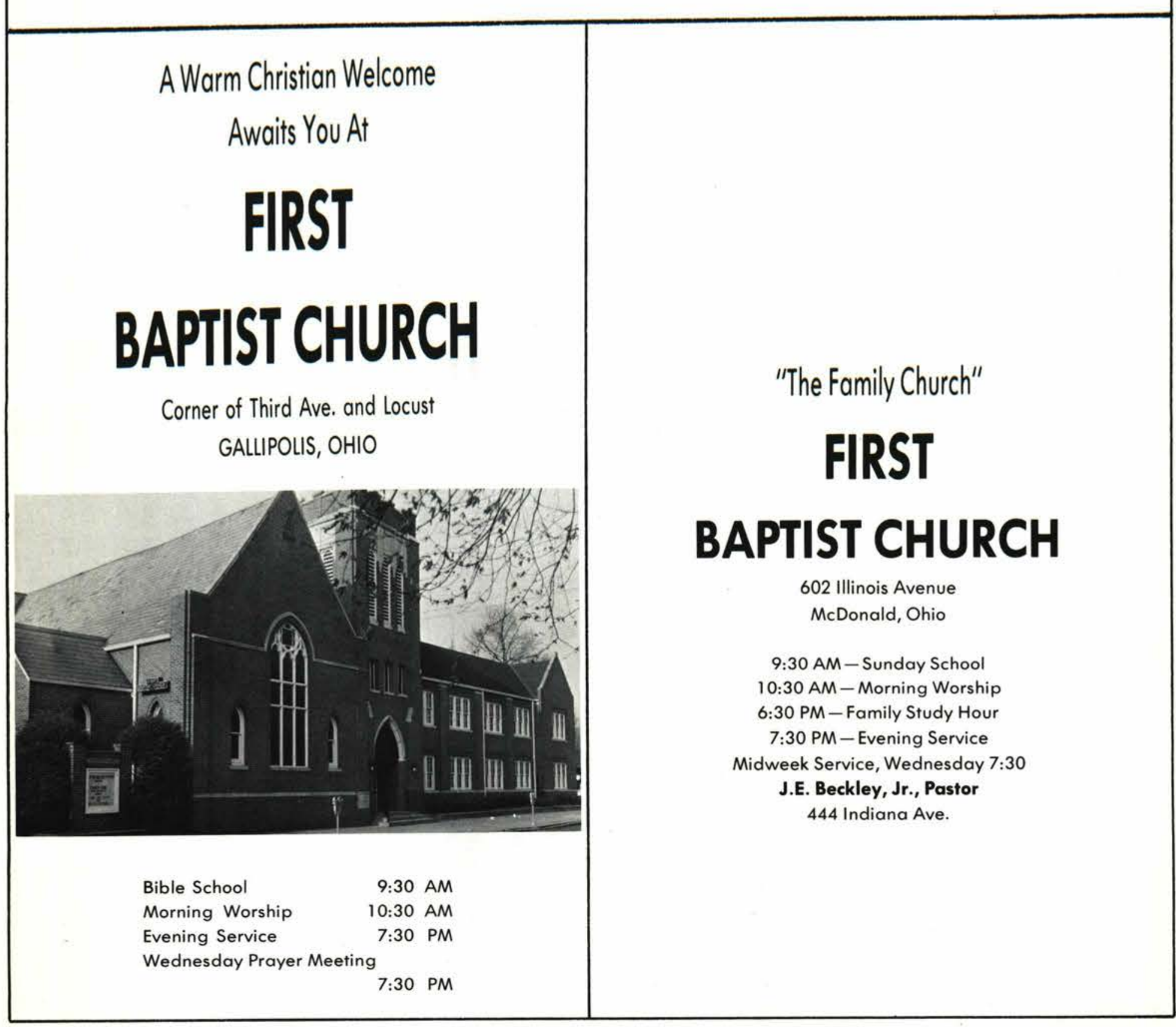




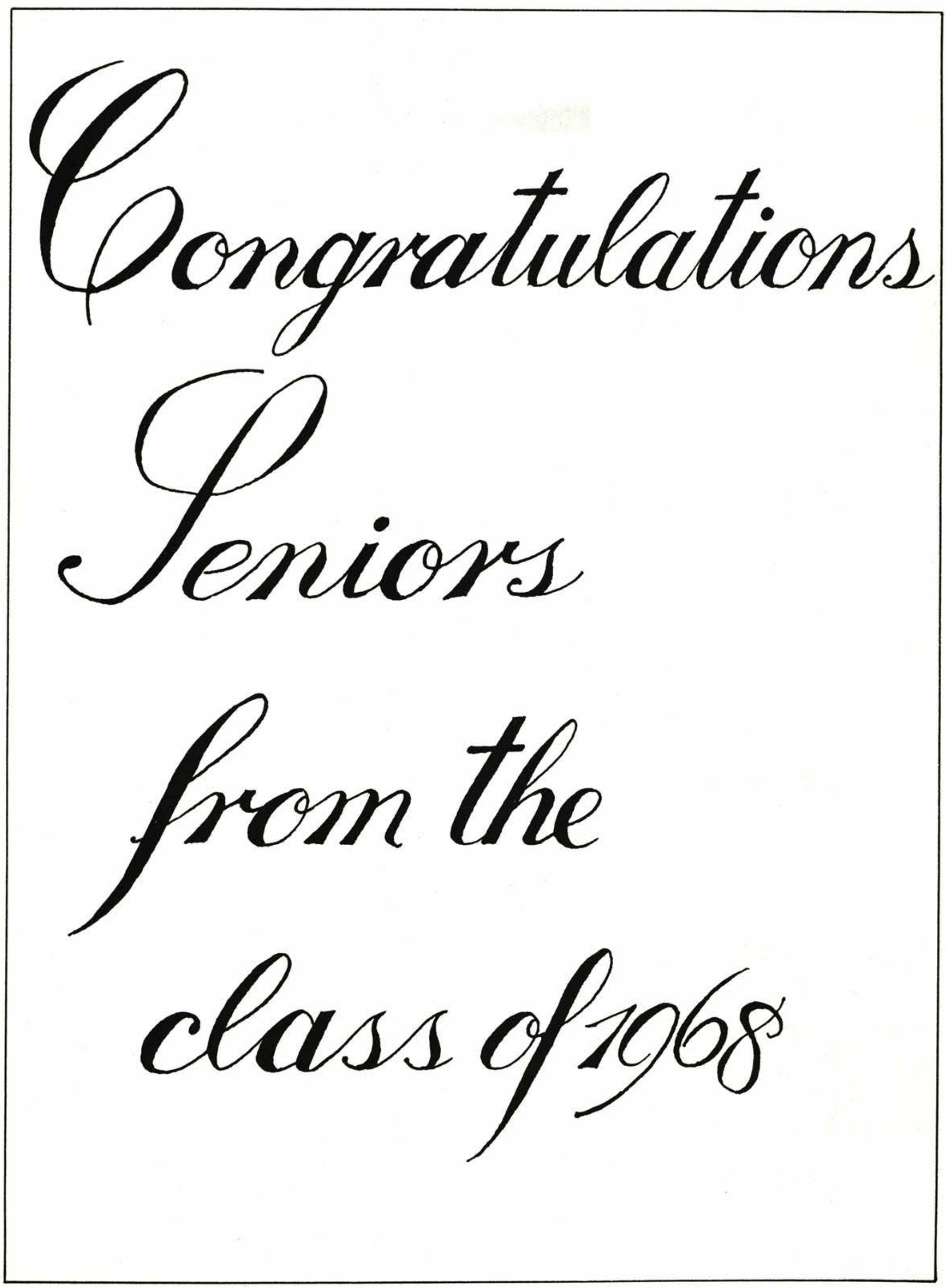




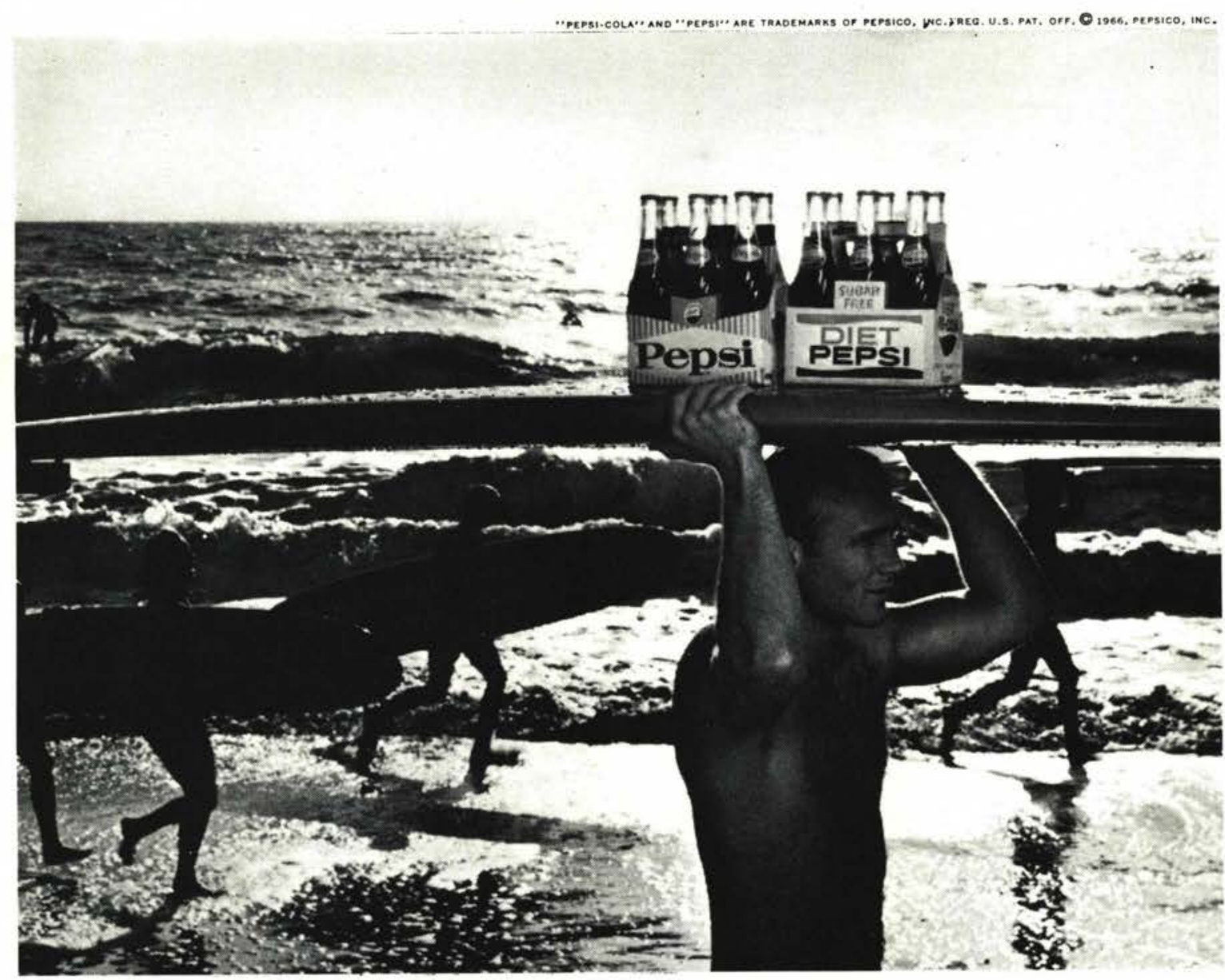

Board members

of the Pepsi generation.

The new wave hitting the beach all over America.

Their drink:

Pepsi-Cola. Regular and Diet.

Faster take-off with one.

Leaner look with the other.

Honest-to-Pepsi taste with both.

Go on in for a sip.

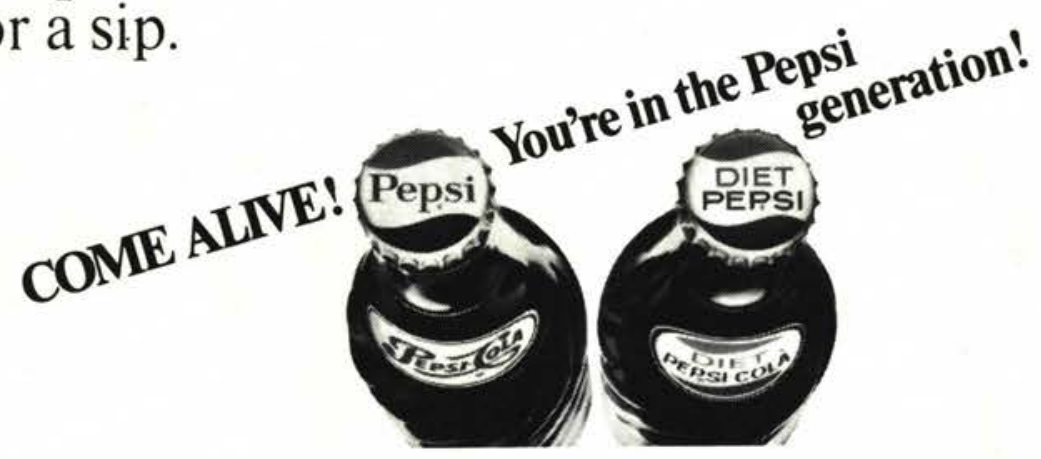

PEPSI-COLA COMPANY OF SPRINGFIELD UNDER AUTHORITY OF PEPSI-CO., NEW YORK, NEW YORK 


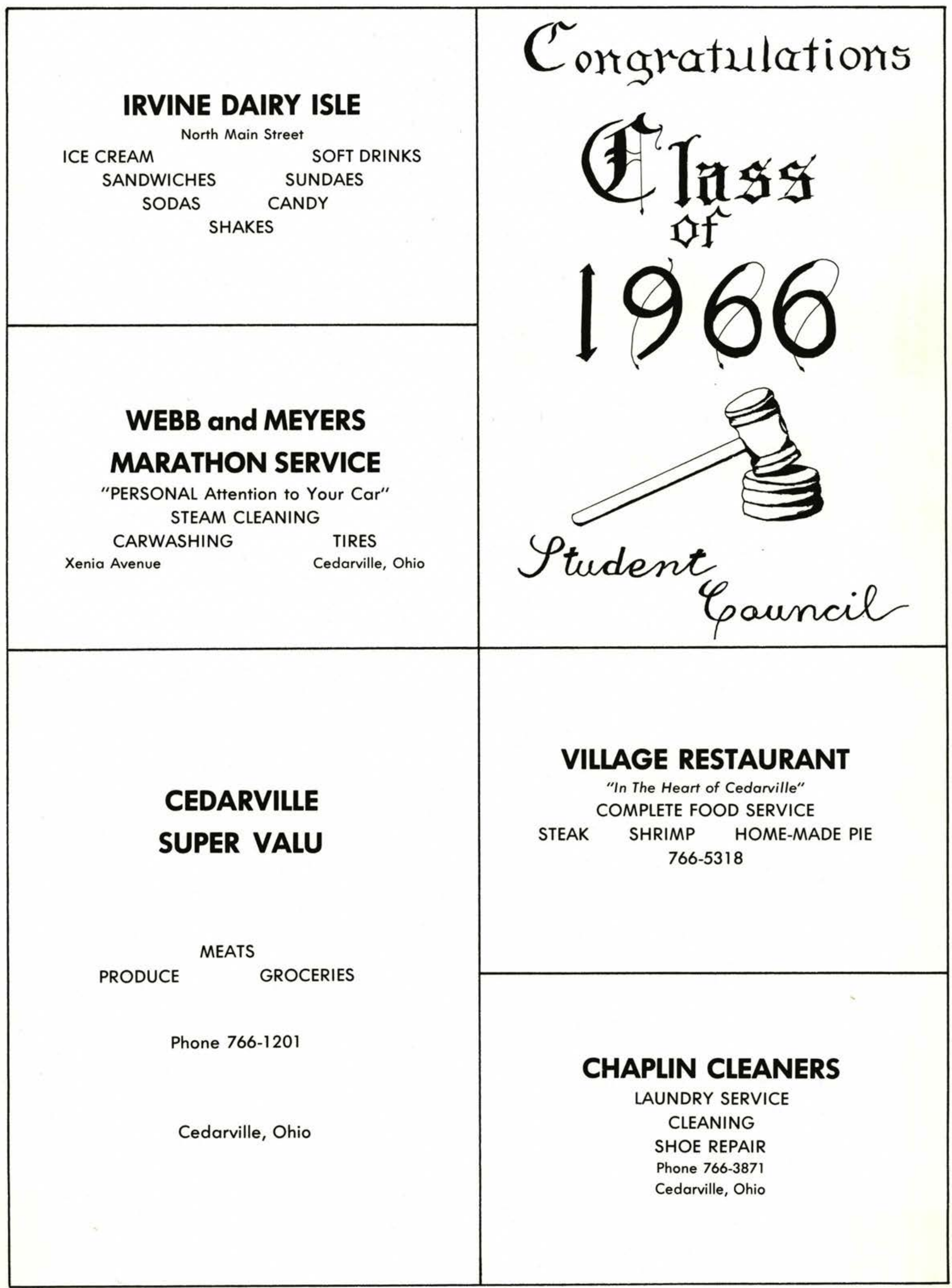




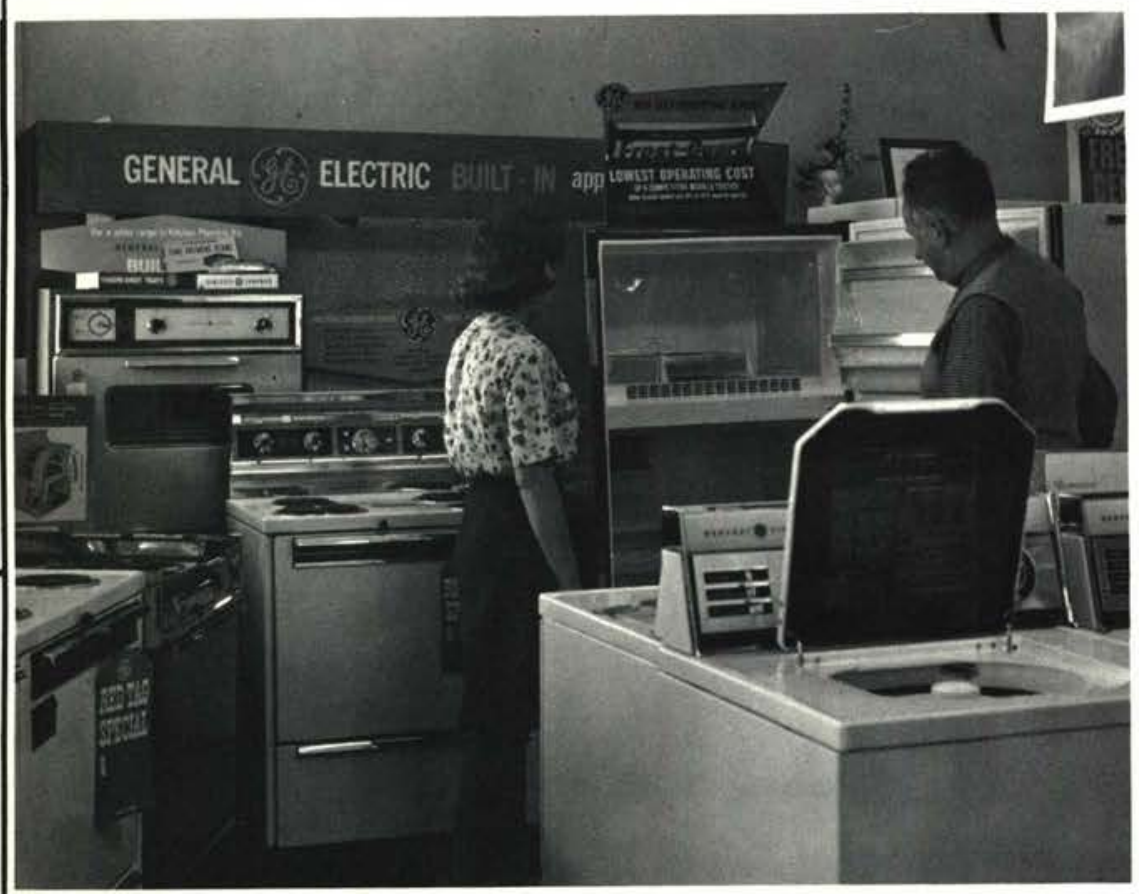

\section{CEDARVILLE HARDWARE}

Progressive Hardware Service Cedarville, Ohio Phone 766-1941

General Electric Appliances

For More Than 20 Years

\section{MIAMI VALLEY \\ Christian Broadcasting, Inc.}

BRAKELL'S LAUNDRAMAT

71 North Main

Cedarville, Ohio

\section{LEACH QUICK WASH}

24-Hour Self-Service Cedarville, Ohio

E. Xenia Street Behind Firehouse Phone 766-5186
7333 Manning Road (Box 222) Miamisburg, Ohio Phone 866-5929

"Witnessing For Christ Jesus" For Millions WFCJ-FM $93.7 \mathrm{mc}$

The First Christian Station in the Ohio Valley Manager 
Morris Bean \& Company producers of high quality Aluminum and Ductile Iron castings Plants in Yellow Springs and Cedarville, Ohio

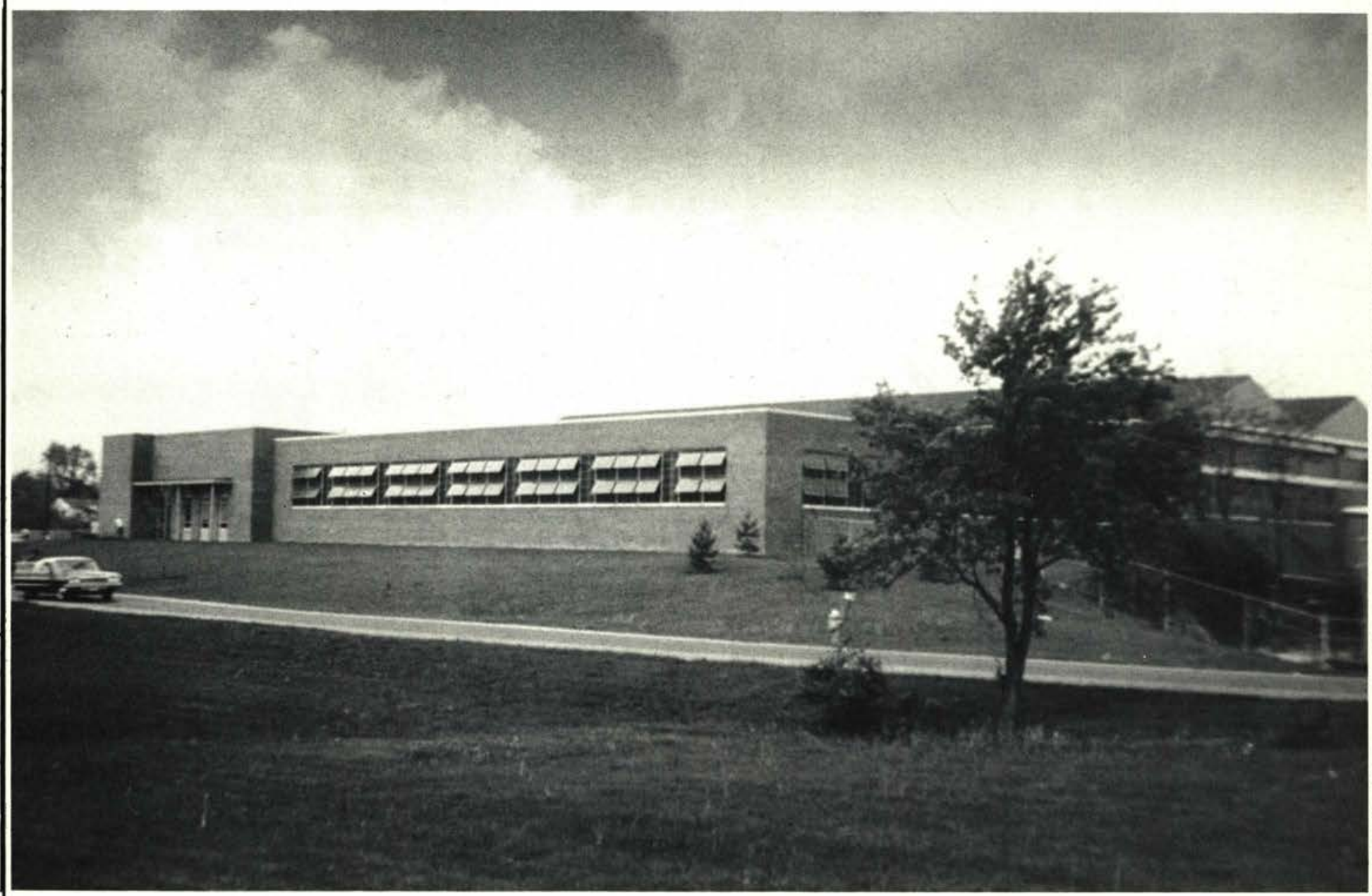

The plant pictured above, located in Cedarville, Ohio, is one of the world's most important sources of tire mold castings used by all the major rubber companies. 


\section{SOUTH \\ BAPTIST CHURCH}

3212 South Saginaw Street FLINT 3, MICHIGAN

Kenneth K. Elgena, Pastor

"An industrious church in an Industrial Center with your Soul as its central business."

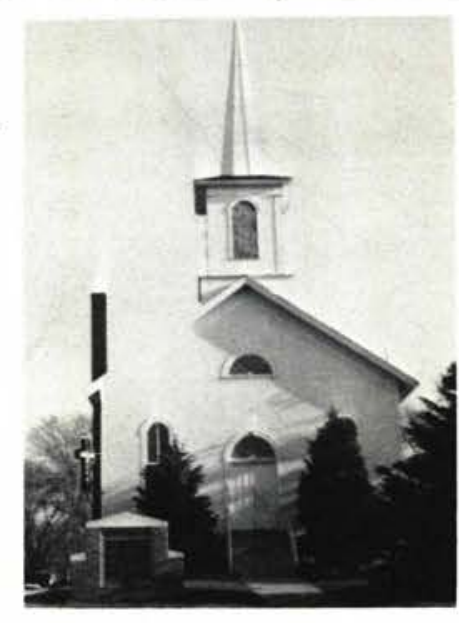

SHELL ROCK BAPTIST CHURCH

Shell Rock, lowa
A COMPLETE COLLEGE LINENS \& TOWEL SERVICE

THE INDEPENDENT TOWEL SUPPLY CO.

212 Willis Avenue

Springfield, Ohio

322-9306

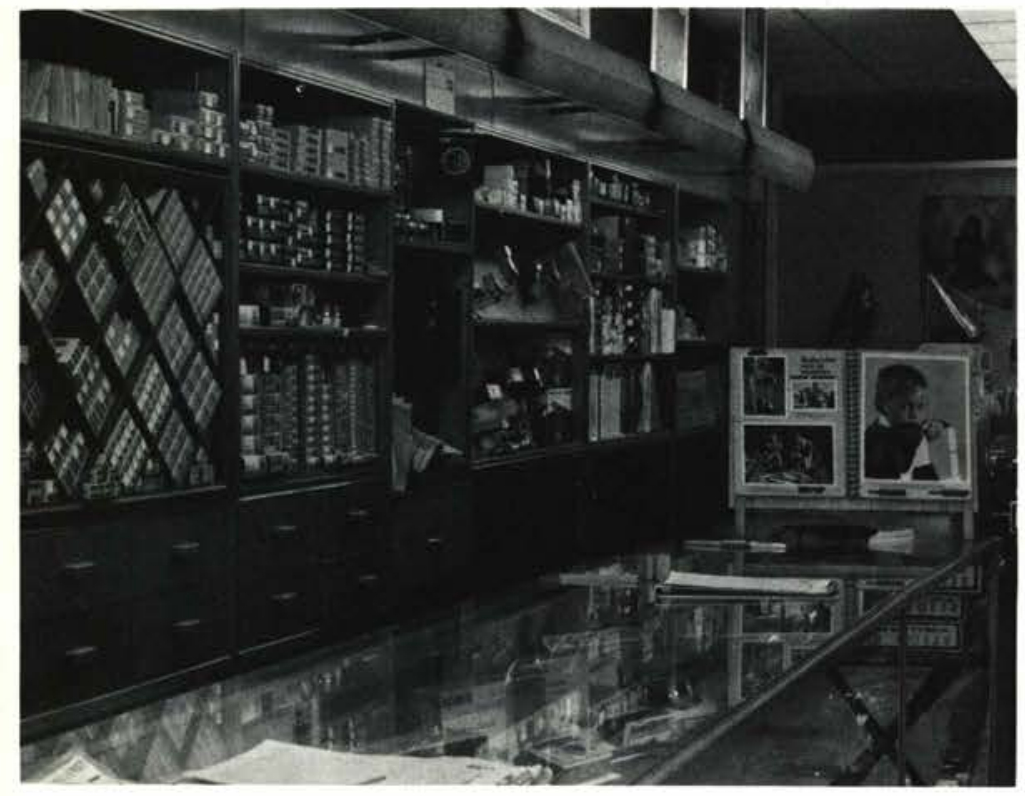

CAMERA SHOP

34 Main Street

Xenia, Ohio

Phone 372-1469

We Desire The Lord's Blessing For The Class of 1966

\section{GRACE BAPTIST CHURCH}

Rocky River, Ohio

Rev. Douglas R. Couch, Pastor
CALVARY BAPTIST CHURCH

Cresaptown, Maryland

Harold Allem, Pastor 


\section{NOW!}

ENJOY THE FINEST

in Dairy Products - Produced

in Springfield's Most Modern Self-Serve

Home-Owned Dairy

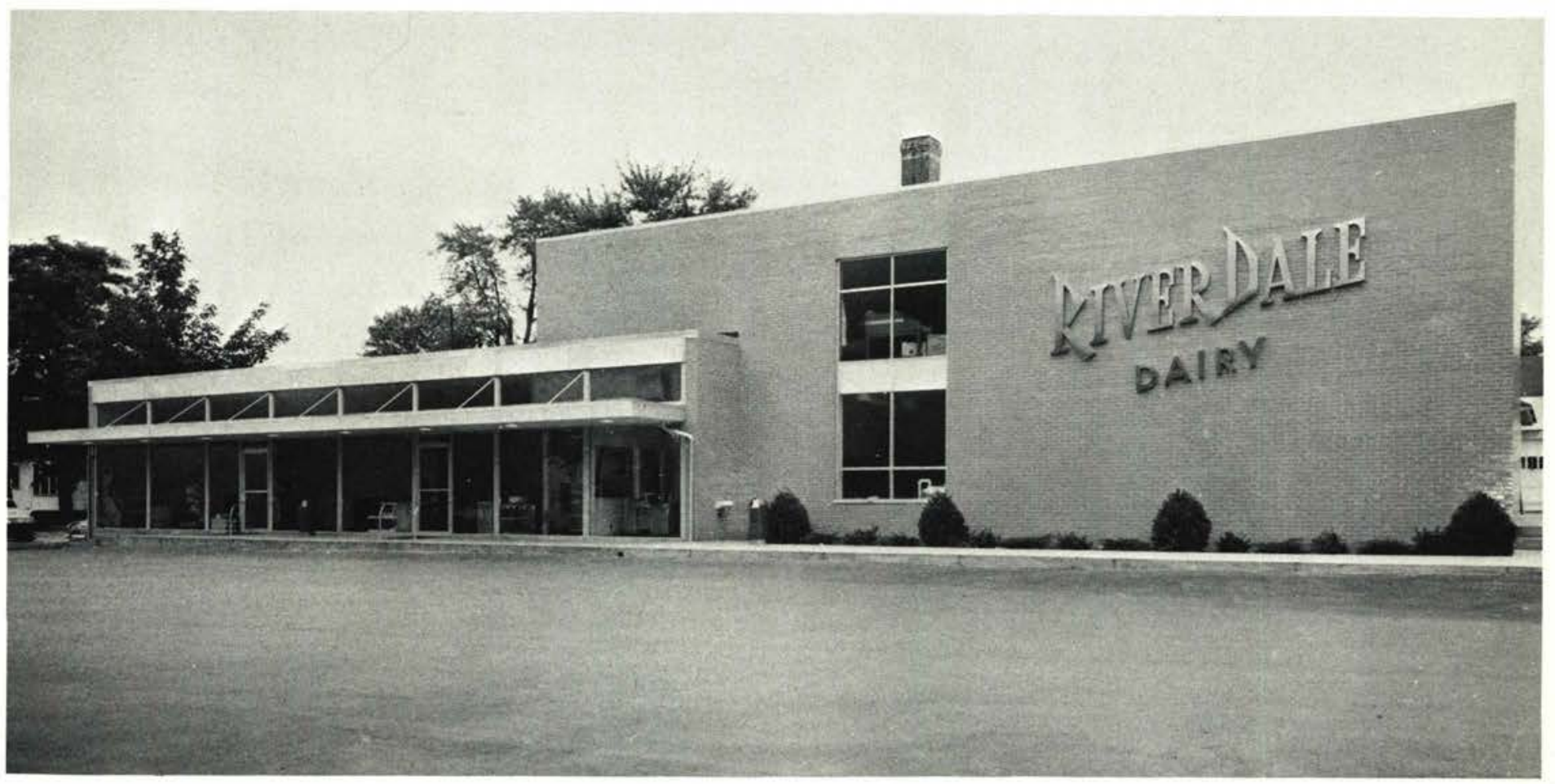

Fruit Punch

Orange Drink Chocolate Milk

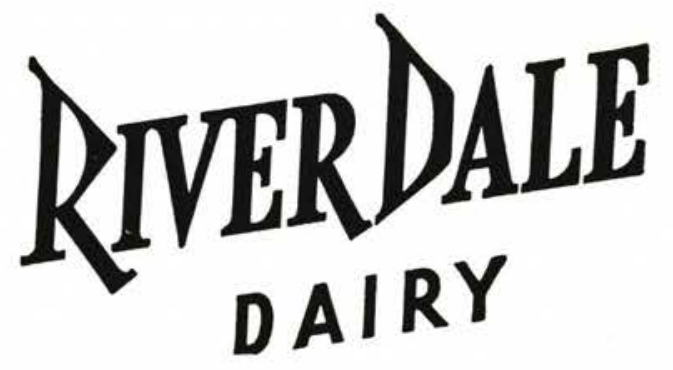

Individual Party Slices Nut Rolls

Anniversary Cakes 


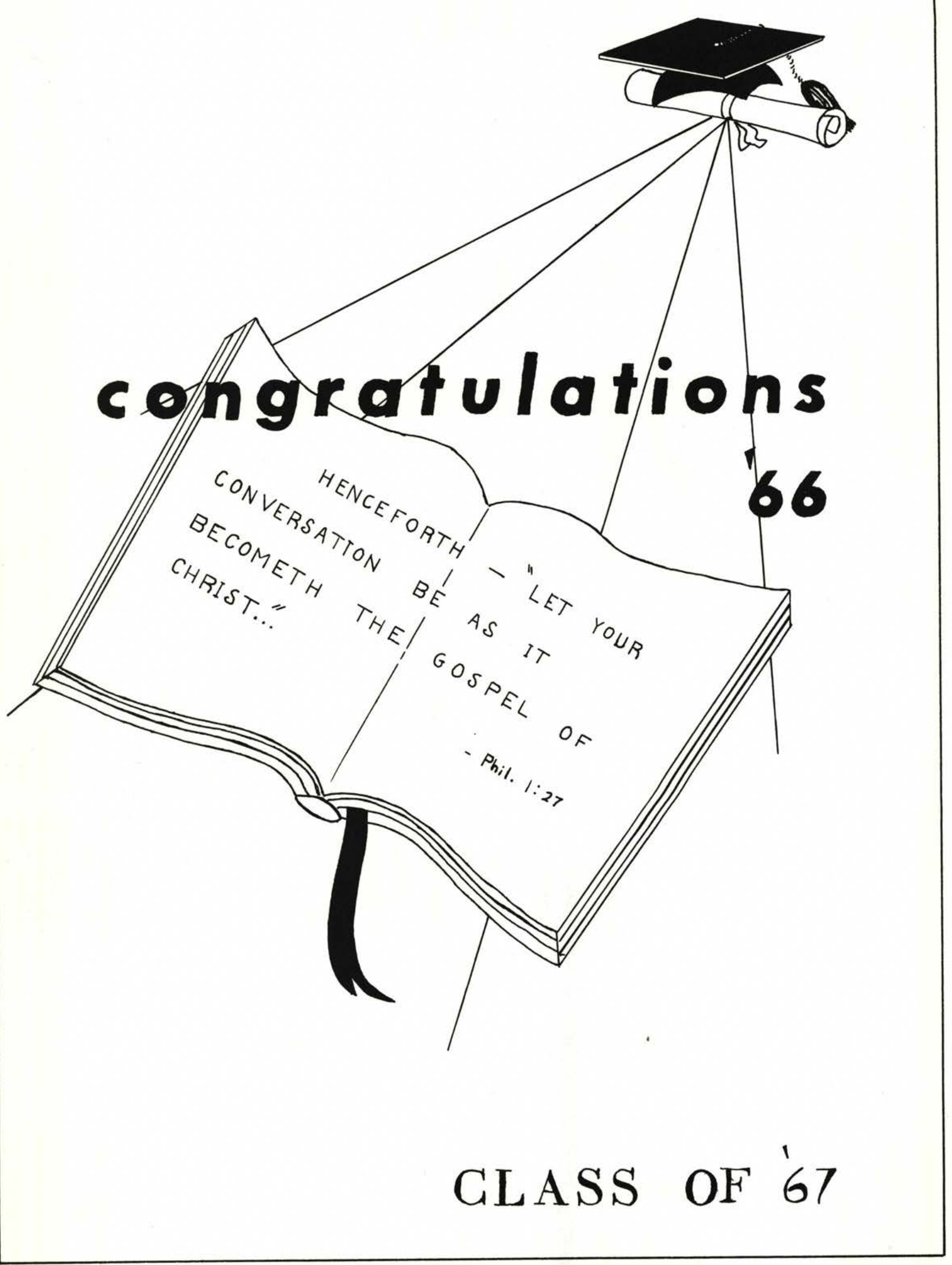




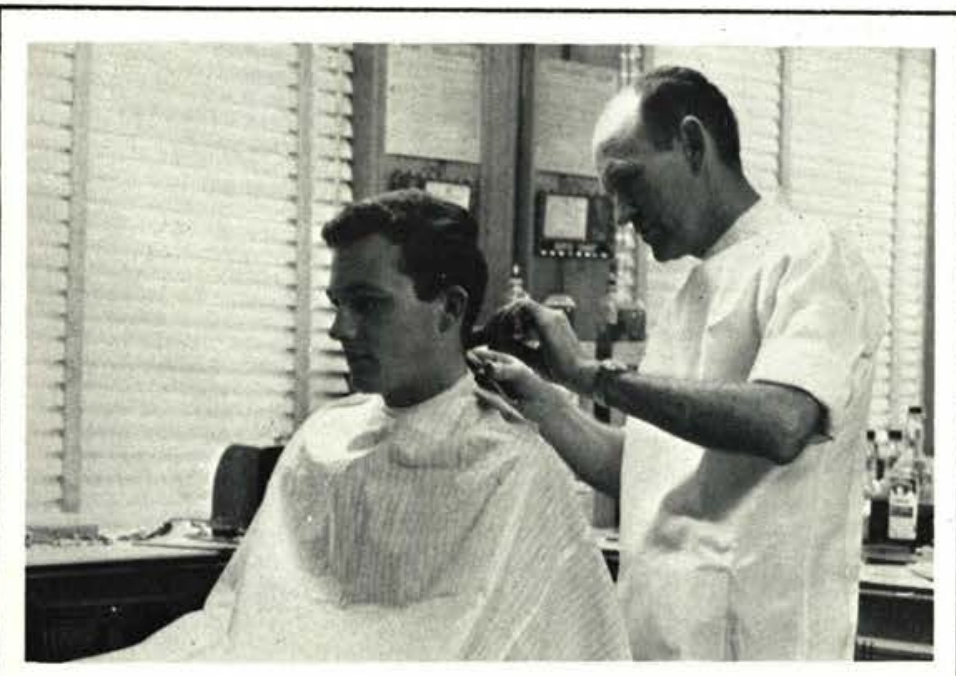

\section{PAUL'S WHOLESALE MDSE., INC.}

\section{Chick's Barbershop}

Cedarville, Ohio

Two Chairs - Air-Conditioned HOURS

Monday, Tuesday-8 A.M.-5 P.M.

Thursday- 8 A.M. -5 P.M.

Friday -8 A.M.-6 P.M.

Saturday-8 A.M.-5 P.M.

\section{"JAMES" REXALL STORE}

Xenia's only Automatic Car Wash Jet Wax Applied-50c

\section{XENIA CAR WASH}

397 West Main Street Xenia, Ohio 372-8611

\section{South Lowry Avenue SPRINGFIELD, OHIO}

Cedarville, Ohio

42 N. Main

Phone 766-1771

397 West Main Street
Xenia, Ohio
$372-8611$

RAY'S RADIO \& TV REPAIR

117 West Main Street

Xenia, Ohio

Ph. $372-7540$

Auto Radio Specialist

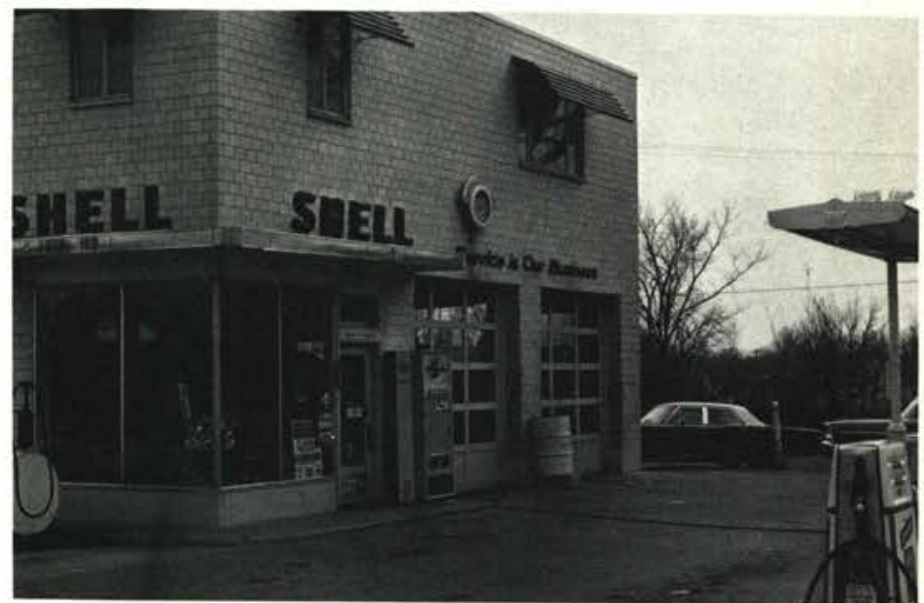

NEIFFER'S SHELL SERVICE

Cedarville, Ohio 766-371

"Pleasing you-Pleases us"

Motor Tune-up Brake Service

Tires \& Batteries Auto Repairing Skilled Mechanic Wheel Balancing Winter Starter Service Mufflers \& Tail Pipes 


\section{CONGRATULATIONS TO THE CLASS OF '66}

BAND BOX MUSIC STORE 57 West Main Street

Xenia - Fairborn

EICHMANS APPLIANCE

82 West Main Street

Xenia, Ohio

JAMES SUPER MARKET

52 East Market Street

Xenia, Ohio

CEDARCLIFF BEAUTY SALON

Cedarville, Ohio

766-4861

\section{SASSEN APPLIANCE}

40 South Main Street

Cedarville, Ohio

\section{CHERRY'S FURNISHINGS}

56 W. Main Street

Xenia-372-3579

\section{CEDARVILLE FEDERAL}

SAVINGS AND LOAN ASSN.

Cedarville, Ohio

\section{CEDARVILLE VARIETY STORE}

Clothes and Gifts for the Family

N. Main Street, Cedarville, Ohio

\section{MCMILLAN FUNERAL HOME}

Congratulations Class of ' 66

Cedarville, Ohio

HILLTOP SERVICE STATION

George Gordon, Mgr.

Cedarville, Ohio

\section{SHERWIN WILLIAMS}

26 N. Detroit

Xenia $-372-6083$

\author{
CORNER PHARMACY \\ 10 S. Detroit \\ Xenia, Ohio \\ STATE FARM INSURANCE \\ Bill Rogers, Agent \\ 338 W. Main-Xenia \\ HOME FURNITURE \\ 84 East Main Street \\ Xenia, Ohio \\ MAIN AUTO SUPPLY \\ 44 West Main Street \\ Xenia, Ohio
}

NILSON-BONE FLORISTS

68 West Main Street

Xenia $-372-4211$

DAUM AND SON

114 S. Detroit St.

372-5231

DEL' 'ABAR HARDWARE

$46 \mathrm{~S}$. Detroit

Xenia-372-0581

FAMOUS AUTO SUPPLY

Sporting Goods

372-4071-64 E. Main

CARRIAGE HOUSE RESTAURANT

37 West Main Street

Xenia, Ohio

TIFFANY JEWELRY STORE

20 South Detroit Street

Xenia, 372-3161

ADAIR'S FINE FURNITURE

Xenia, Ohio

"Worth A Trip to Xenia"
ROGERS JEWELRY

44 S. Detroit Street

372-7681

HITCHCOCK'S JEWELRY STORE

68 S. Detroit Street

Xenia, Ohio

XENIA GAXETTE

"Growing With All Green County"

CENTRAL BARBER SHOP

57 East Main Street

Gary-Phil

DUTCH OVEN BAKERY

$60 \mathrm{~N}$. Detroit Street

Xenia, Ohio

LITT BROTHERS

25 East Main Street

Xenia, Ohio

THE CARD SHOP

Hallmark Cards and Gifts

17 East Main Street

RAY COX INSURANCE

37 S. Detroit-Gazette Bldg.

Xenia, Ohio

\section{SMITH'S BAKERY}

"The Man Who Kneads The Dough"

78 W. Main - Xenia, Ohio

BLACK'S FURNITURE

"For Furniture New"

Northeast on Rt. 42 


\section{BEAL'S}

\section{DEPARTMENT STORE}

$$
\text { GIFT ITEMS }
$$

Clothing and Shoes

S. Main Street

Cedarville, Ohio

Phone 766-5362

\section{CAMPUS \\ BICYCLE RENTAL}

Owned and Operated by

Mr. \& Mrs. Kenneth H. St. Clair

467 Walnut Street

Cedarville, Ohio

While in Cedarville Try a Bike Built for Two

SEE THE BEAUTIFUL OHIO COUNTRYSIDE

ON A BICYCLE

\section{HOAK'S GREENHOUSE}

Greenhouses and Nursery FAIRBORN, OHIO

FLOWERS FOR ALL OCCASIONS

Phone $878-8621$ or $878-8572$

\section{XENIA OFFICE SUPPLIES}

61 Green Street

Xenia, Ohio

372-2381

"For All Your School Needs"

\section{GEORGE MEEK CO., INC.}

36 N. Fountain Ave.

Springfield, Ohio

$323-4918$

Your Complete Sporting Goods Store

We Outfit the Yellow Jackets

GOOD LUCK Cedarville Graduates

\section{RANDALL \& STORMONT,}

\section{INC.}

Purina Chows and Health Aids

Phone 766-1031

Cedarville, Ohio

\section{BIG WHEEL}

\section{RESTAURANT}

N. Main

Cedarville, Ohio

Phone: 766-5220

"Enjoy Your Food in the Rustic

Atmosphere of the BIG WHEEL"
Congratulations Class of 1966

From The

\section{CEDARVILLE COLLEGE FOOD SERVICE}




\title{
MIAMI DEPOSIT BANK
}

\author{
FULL BANKING SERVICES \\ Two Offices Located at \\ Cedarville, Ohio \\ AND \\ Yellow Springs, Ohio \\ Member of \\ Federal Deposit Insurance \\ Corporation
}

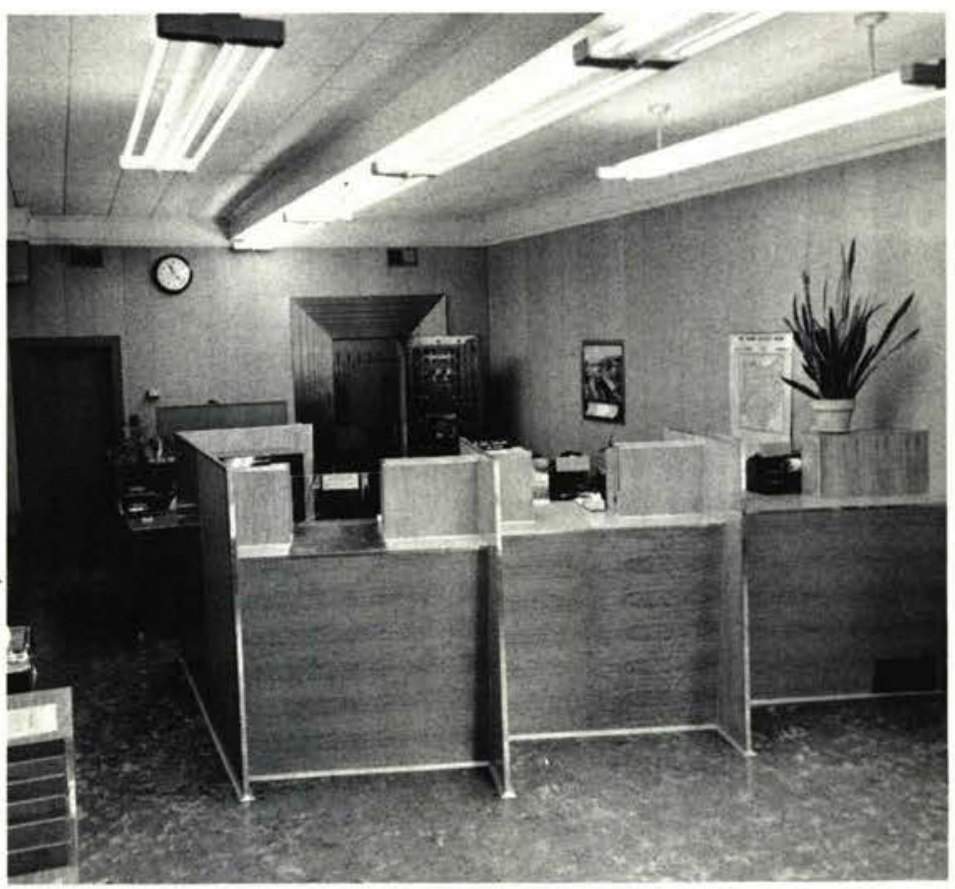

\section{THE CRITERION}

\author{
Xenia, Ohio \\ MEN'S CLOTHING AND \\ FURNISHINGS \\ VARSITY TOWN - ARROW \\ MaCGREGOR - STETSON \\ PALM BEACH
}

Stop in and have lunch with
Pizza Menu
Cheese
Sausage
Mushroom
Pepperoni
Anchovy
Olive
Hamburger
Ham (Diced)
Sausage \& Mushroom
Sausage \& Pepperoni
Sausage \& Pepperoni \& Mushroom
I-T Special

The I-T PIZZA

\section{BRAUN'S JEWELERS}

Registered Jewelers

American Gem Society

22 N. Detroit St.

Xenia, Ohio

"An Education In Diamonds \& Gems

$$
\text { Awaits You" }
$$

Ask To See Our Collection of

Fluorescent Blue Diamonds

Omega-Rolex-Accutron-Bulova Watches

Fine Repair Work

Diamond Setting
"For all your quality meat and grocery needs."

\section{CEDARVILLE LOCKER}

39 Main Street

Phone 766-1141 


\section{RECO \\ SPORTING GOODS \\ and \\ GIFT STORE}

\author{
Uniforms \\ Jackets \\ Sweaters \\ Sports Equipment
}

Come in and Browse Around

TEL. 323-3941

113 E. High St.

Springfield, Ohio

\section{MILNER ELECTRIC COMPANY}

\author{
Sincere Congratulations \\ and \\ Best Wishes to the \\ Class of '66
}

"Electrical Supplies of All Types"

1320 St. Clair Avenue

Cleveland, Ohio 44114
COMPLIMENTS

of

The

\section{CEDARVILLE \\ FEED \& GRAIN}

\author{
Grain, Feed, Seed, Coal, Salt \\ Wool, Fertilizer
}

Builders' Supplies and Fencing

Cedarville, Ohio

Phone 766-2021

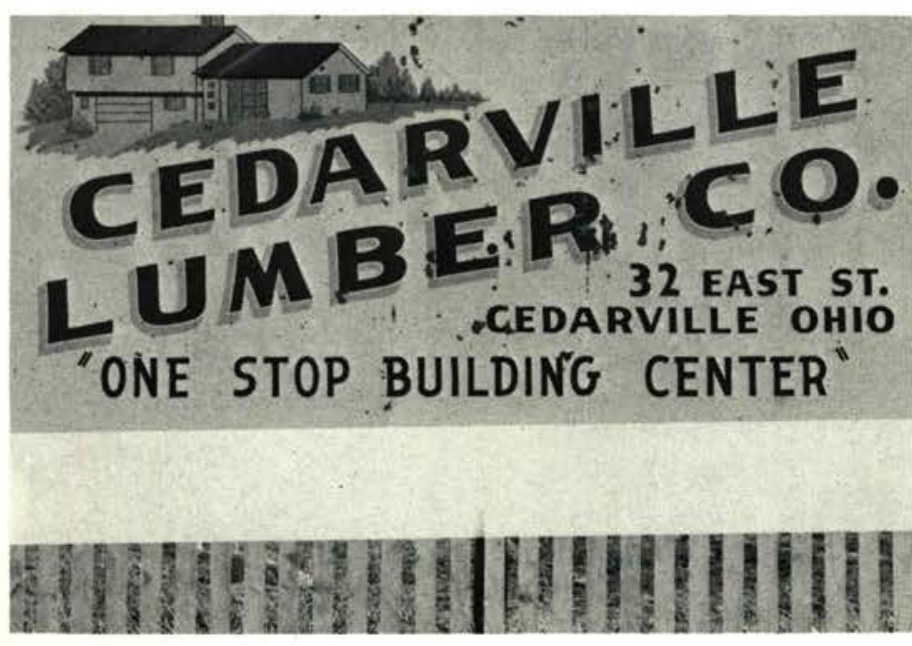




\section{STANDARD LIFE}

of

Lawrence, Kansas

Congratulates the class of ' 66

"providing for his own and especially for

those of his own house .. I Tim. 5:8"

David Gidley

Local Representative

Virgil Sullivan

Cedarville, Ohio

\section{CEDARVILLE COLLEGE BOOKSTORE}

Student Supply Center

Bernice Mick, Manager

\section{Sincere Congratulations Seniors \\ COLLEGE HILL IGA}

"Check-See-Compare-IGA Has Thousands of Lower Prices"

Store Hours:

Monday-Tuesday-Wednesday $8: 30-8: 30$

Thursday-Friday-Saturday $8: 30-9: 00$

Across from College Farm House

Cedarville, Ohio

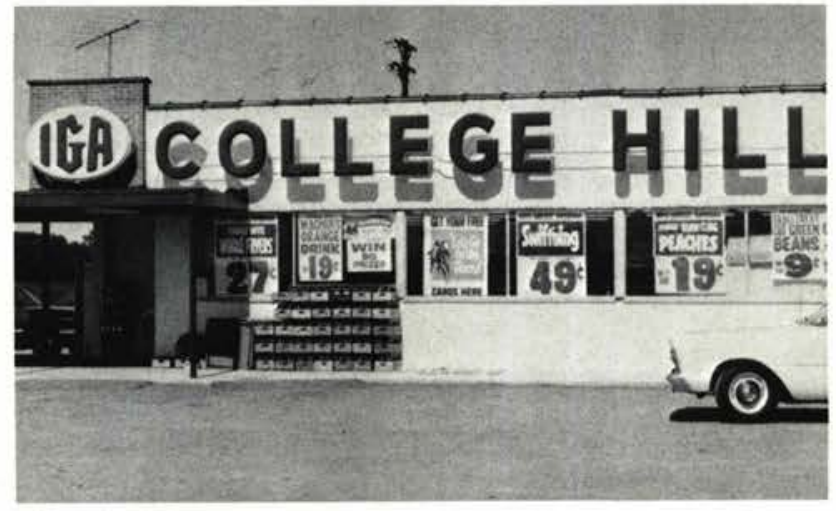

It's The Total That Counts 


\title{
WALNUT STREET BAPTIST CHURCH
}

\author{
415 Walnut Street \\ Waterloo, lowa \\ Rev. David L. Moore, Pastor \\ SUNDAY SERVICES \\ 9:30 A.M. - Sunday School \\ 10:40 A.M. - Morning Worship \\ 6:30 P.M. - Youth Groups \\ 7:30 P.M. - Evening Service \\ WEDNESDAY \\ 7:30 P.M. - Mid-Week Prayer Hour
}

We pray God's blessing and extend congratulations to the Class of 1966.

\section{SIMMS TYPEWRITER}

\section{CO., INC.}

We sell the best-repair the rest.

- Smith-Corona - Underwood-Olivetti

- Royal-Olympia - Standard - Electric

- Portable - SALES \& SERVICE

Adding \& Calculating Machines

- REPAIRS - RENTALS

Office Equipment \& Supplies - Also School Supplies

Phone: 878-5739

401 N. Broad St.

Fairborn, Ohio

\section{JOHN DONLEY GAS \& OIL}

\section{South Main Street}

"First quality products only"

"Lowest gas prices in town"

\section{THE CENTRAL OHIO PAPER COMPANY}

\author{
Dayton \\ Columbus \\ Indianapolis
}

The Finest in Papers for Schools,

Printing, and Industry.

The "Copco" Brand 


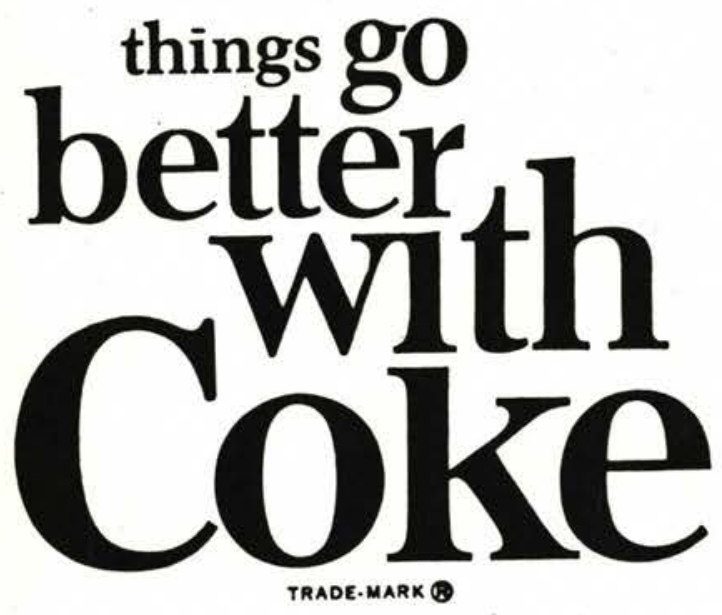

\title{
Congratulations
}

Seniors

\author{
Steve Fenton \\ David Trunnell
}

\section{CLARK'S DISCOUNT STORE}

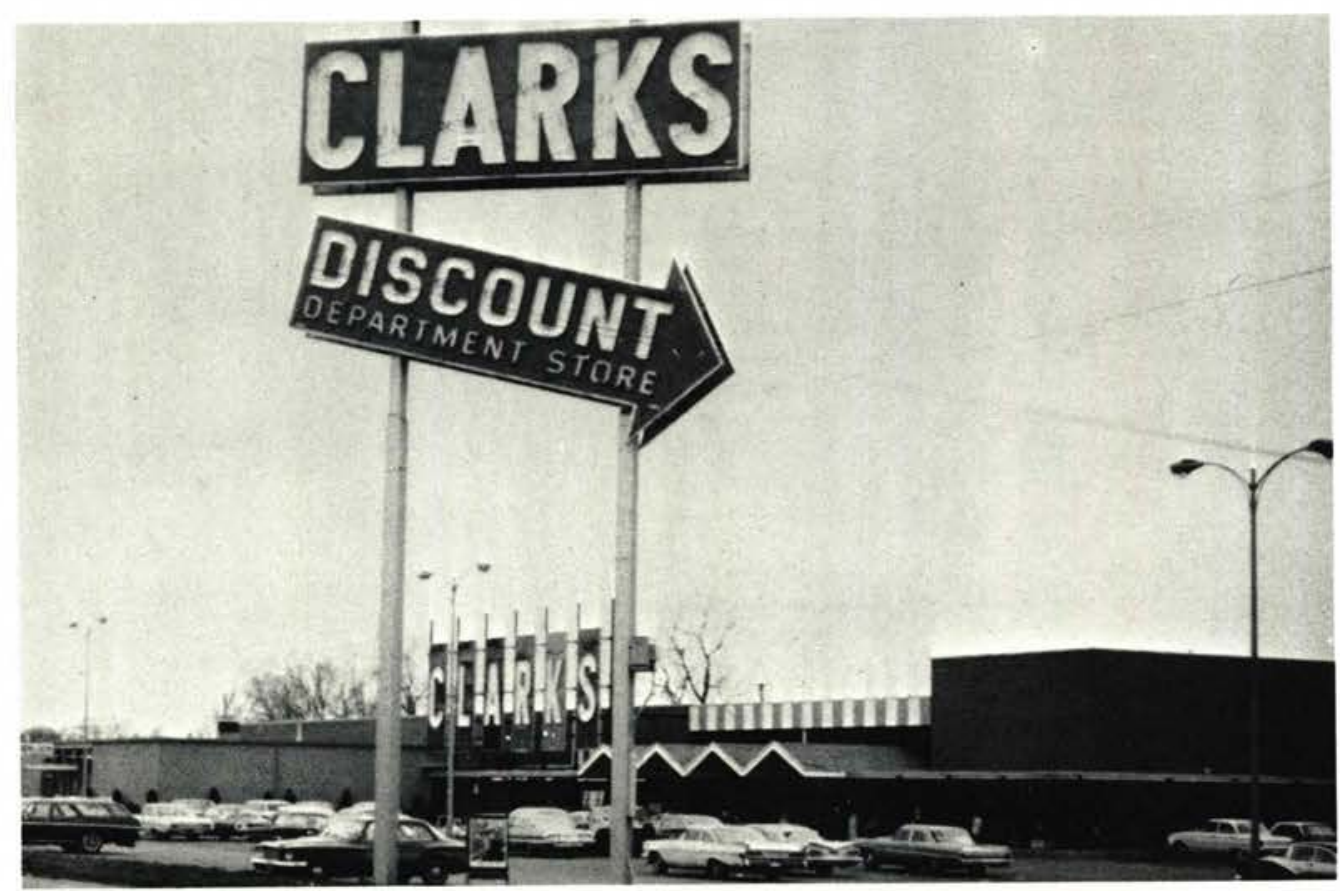

Off Route 72

Springfield, Ohio 




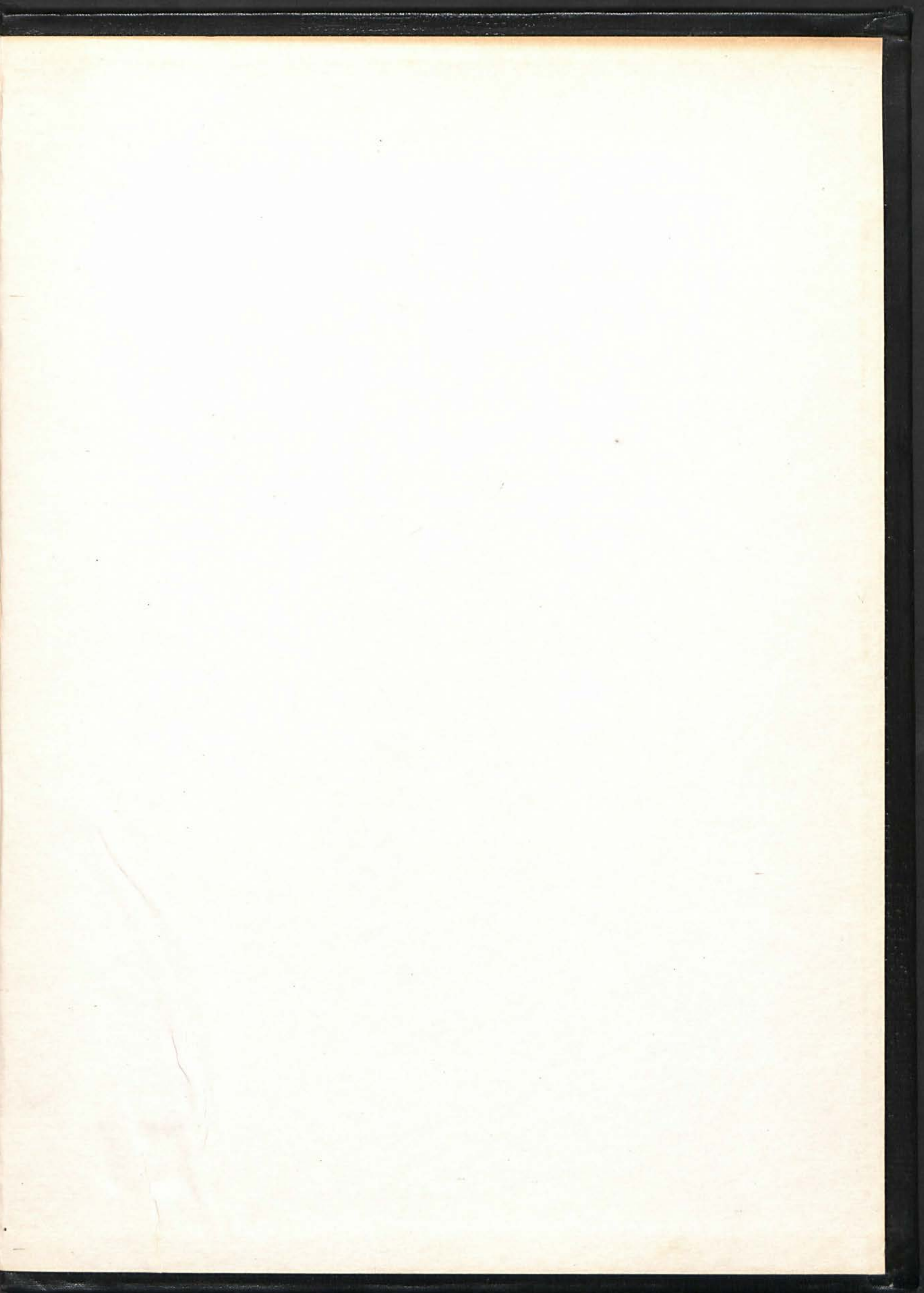




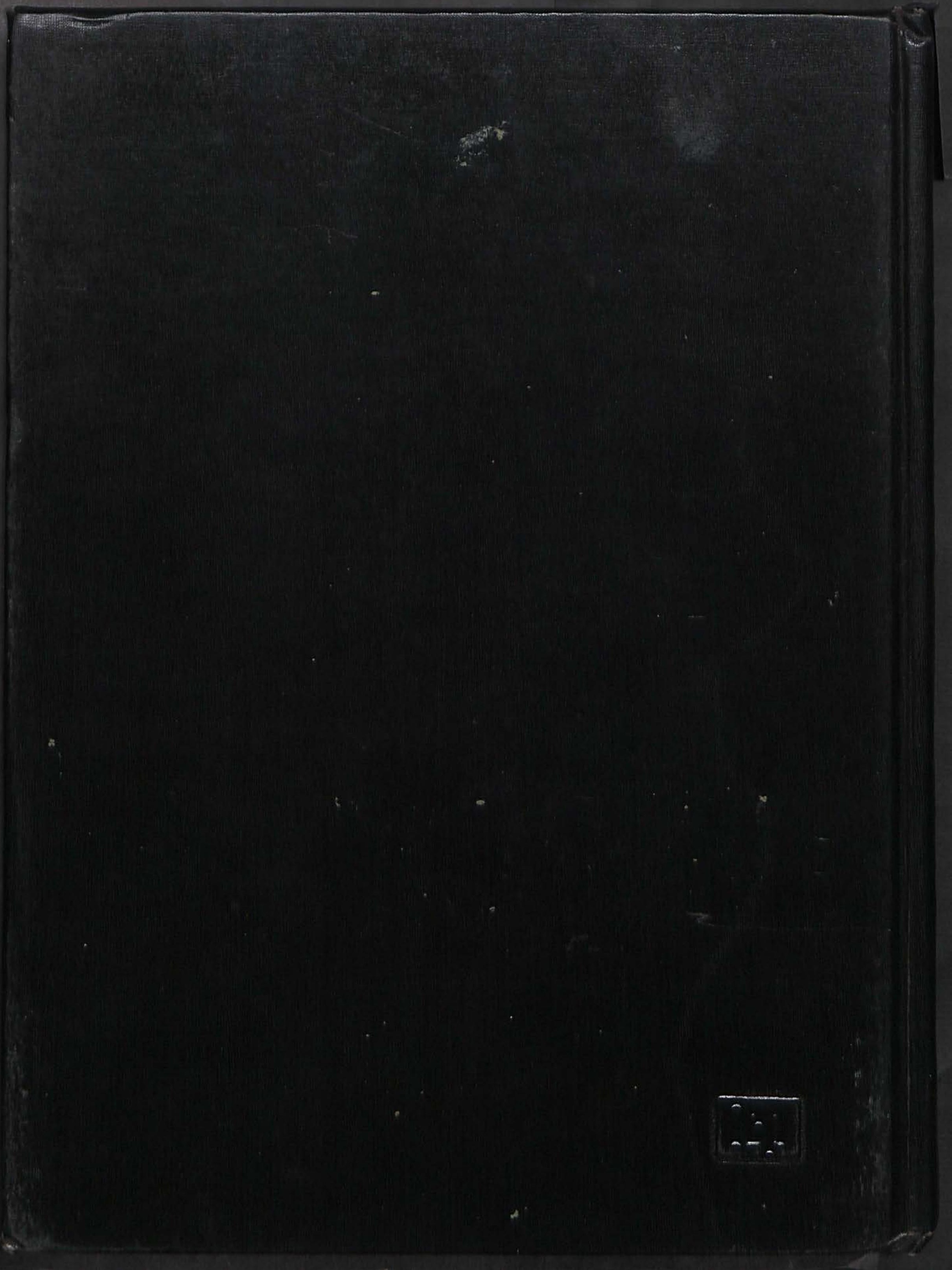

